\title{
SECPOP90: Sector Population, Land Fraction, and Economic Estimation Program
}

Manuscript Completed: July 1997

Date Published: September 1997

Prepared by

S. L. Humphreys

J. A. Rollstin ${ }^{b}$

J. N. Ridgelyc

"Sandia National Laboratories

Albuquerque, NM 87185-0748

Subcontractor:

'GRAM, Inc.

8500 Menaul Blvd., NE

Albuquerque, NM 87112

'J. N. Ridgely, NRC Project Manager

Prepared for

Division of Systems Technology

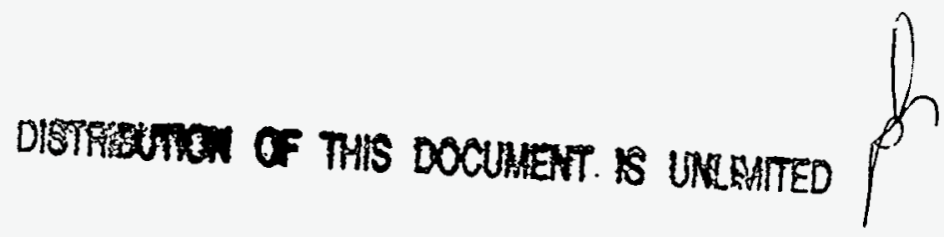

Office of Nuclear Regulatory Research

U.S. Nuclear Regulatory Commission

Washington, DC 20555-0001

NRC Job Code J6024

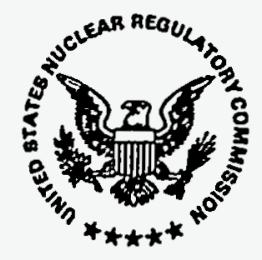


Microsoft and MS-DOS are registered trademarks of the Microsoft Corporation.

Microsoft Windows 3.1, Windows 3.11, Windows NT, Windows 95; Microsoft Visual Basic, Microsoft QuickBasic, Microsoft Excel, and Microsoft Access are trademarks of the Microsoft corporation.

IBM, PS/2, Personal Computer AT, and OS/2 are trademarks of the IBM Corporation.

Intel and 80386 are trademarks of the Intel Corporation.

MapPlan is a trademark of WordTech Systems, Incorporated.

Pizazz Plus is a registered trademark of Application Techniques, Incorporated.

HiJaak PRO is a registered trademark of the Quarterdeck Corporation.

$\mathrm{dBase}$ and $\mathrm{dBase} \mathrm{III}$ is a registered trademark of Borland International, Incorporated. 


\section{DISCLAMIER}

Portions of this document may be illegible in electronic image products. Images are produced from the best available original document. 


\section{CONTENTS}

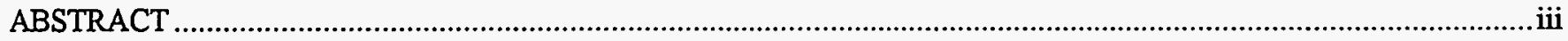

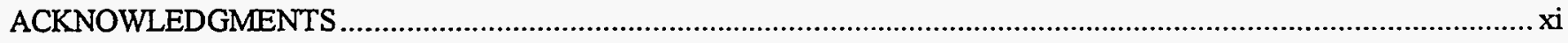

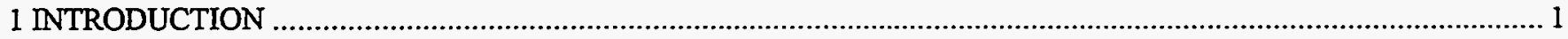

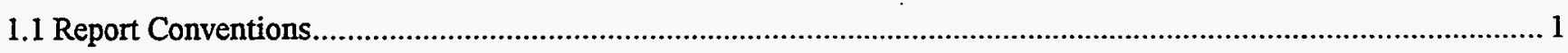

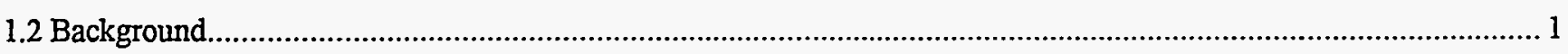

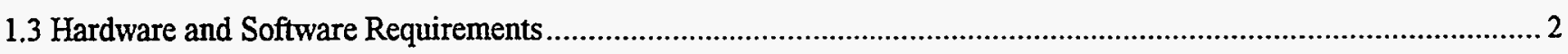

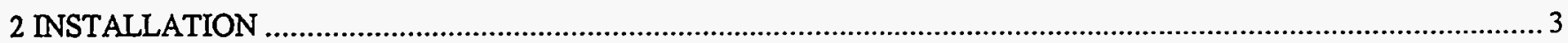

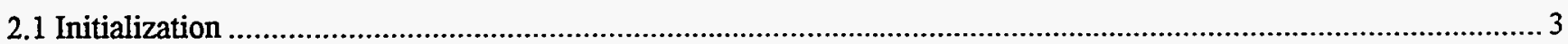

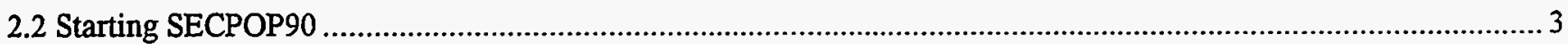

3 USER'S GUIDE

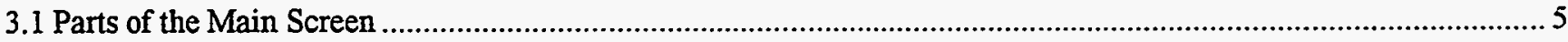

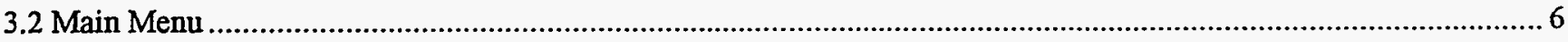

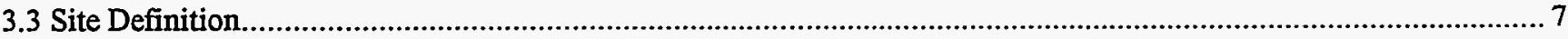

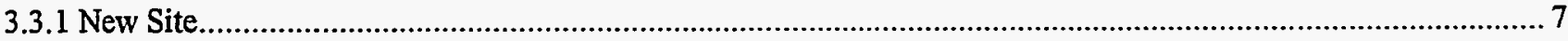

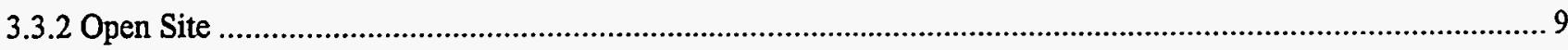

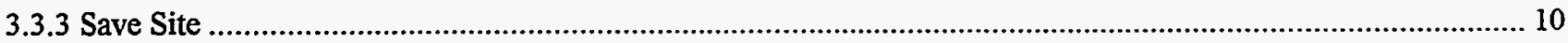

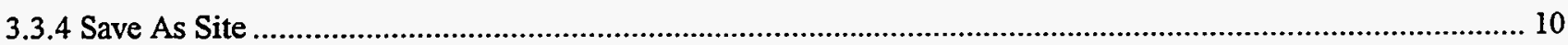

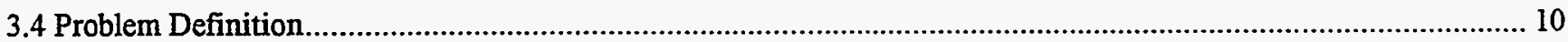

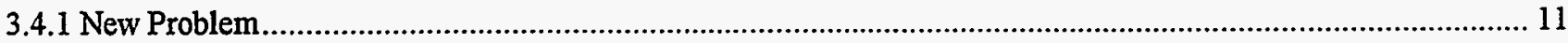

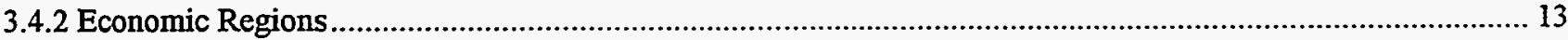

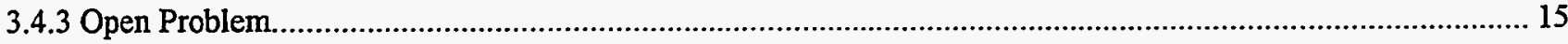

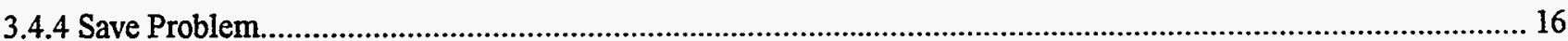

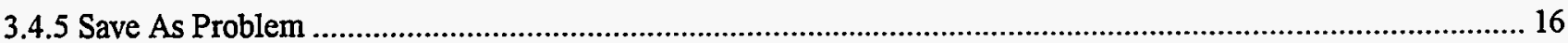

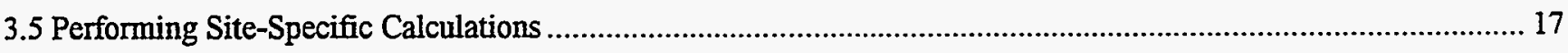

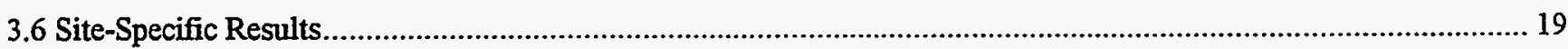

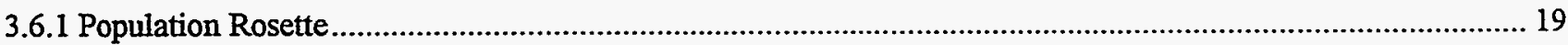

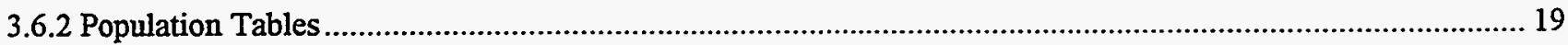

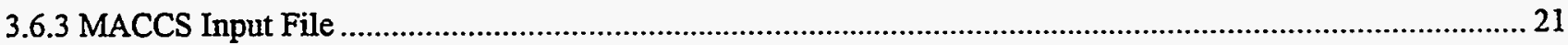

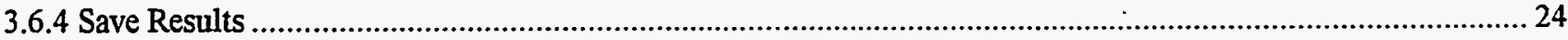

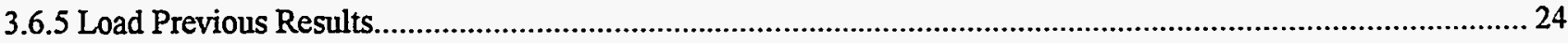

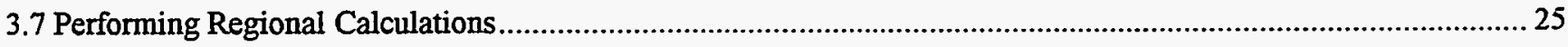

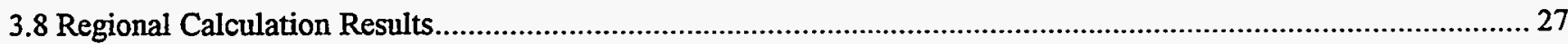

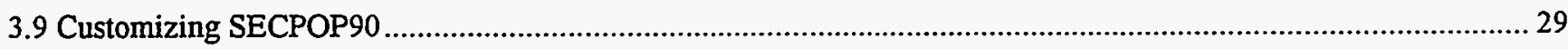

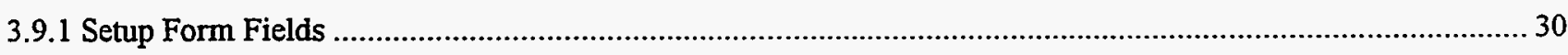

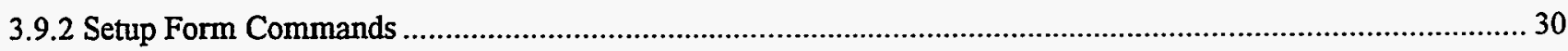

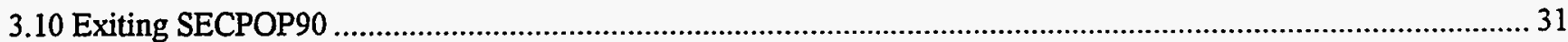




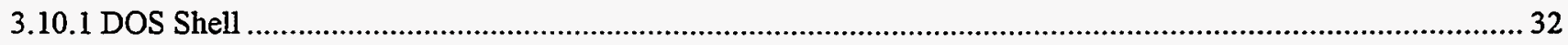

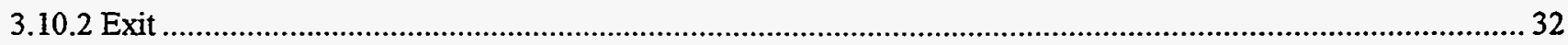

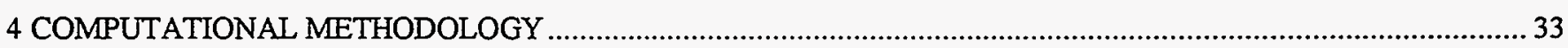

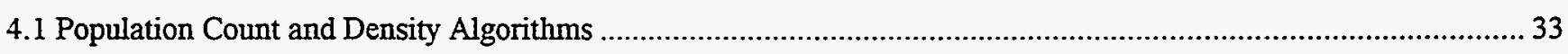

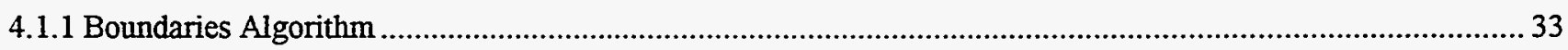

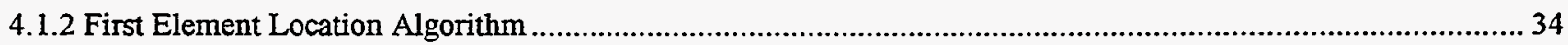

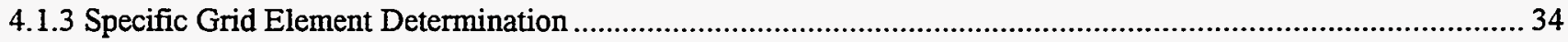

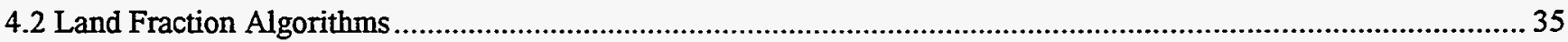

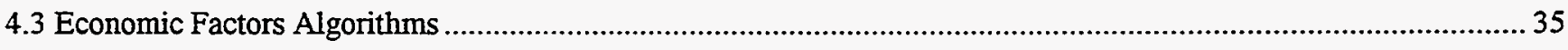

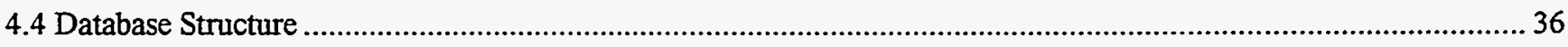

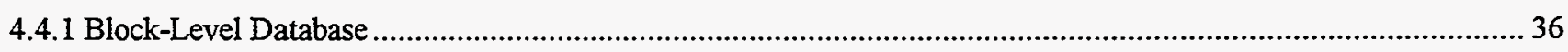

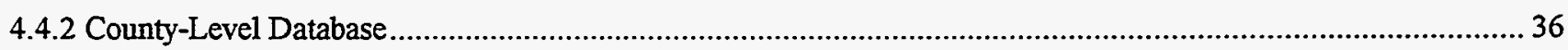

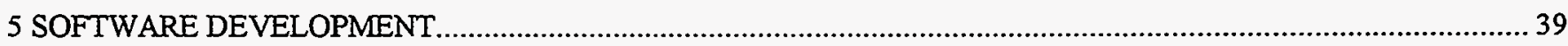

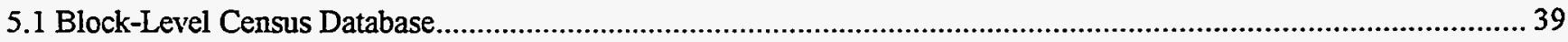

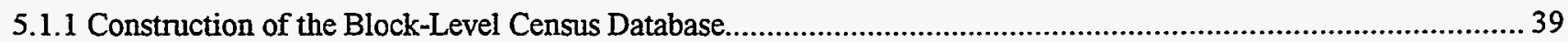

5.1.2 Verification and Validation of the Block-Level Census Database ................................................................. 42

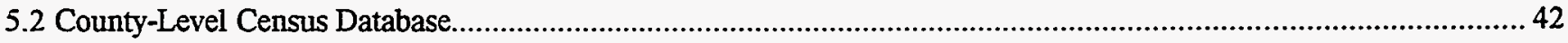

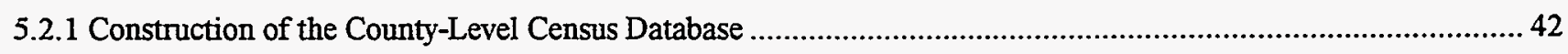

5.2.2 Verification and Validation of the County-Level Census Database................................................................4

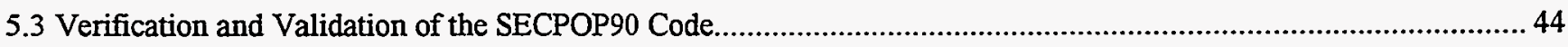

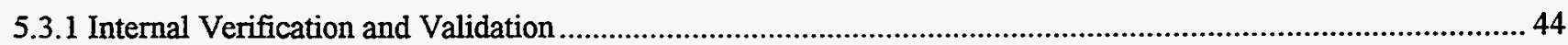

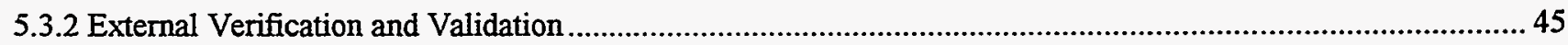

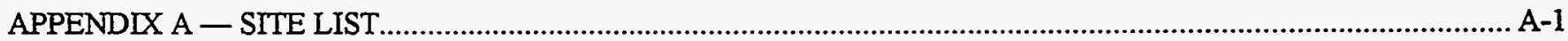

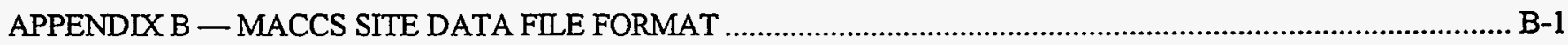

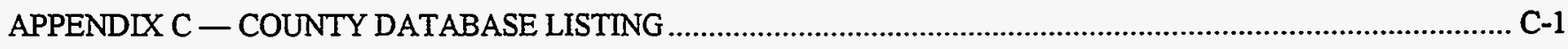

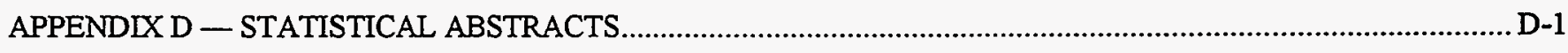

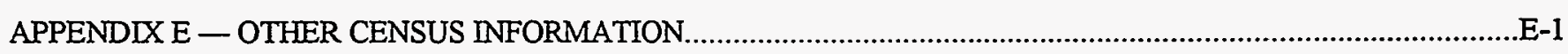

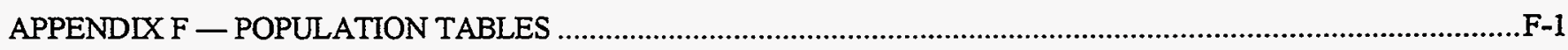

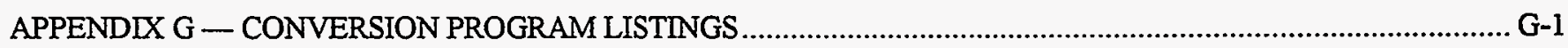

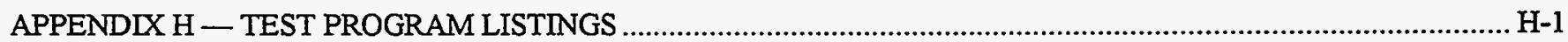

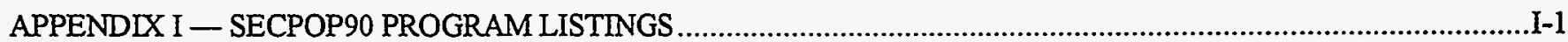




\section{FIGURES}

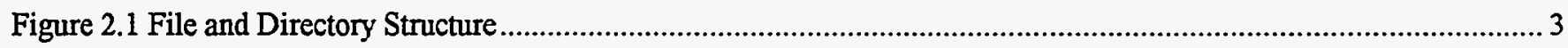

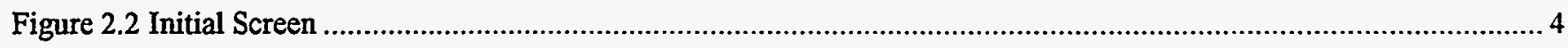

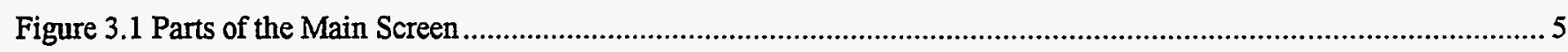

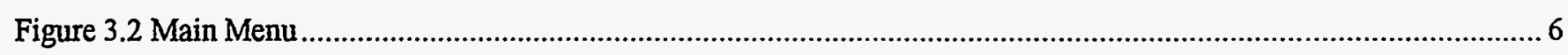

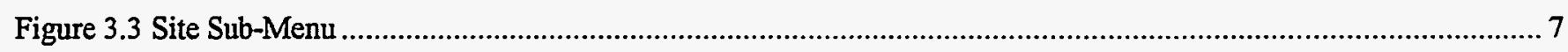

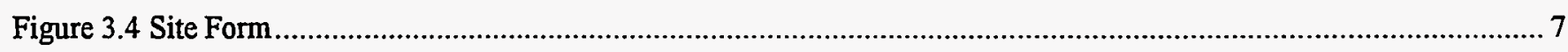

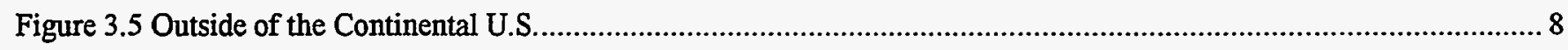

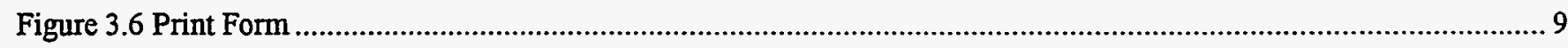

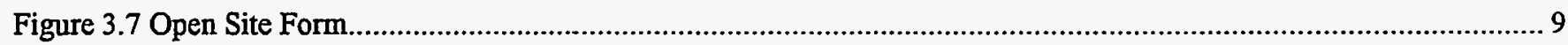

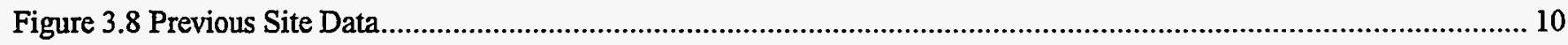

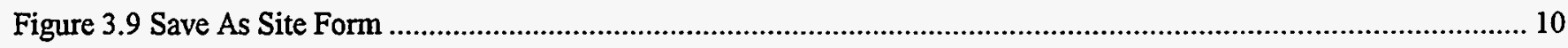

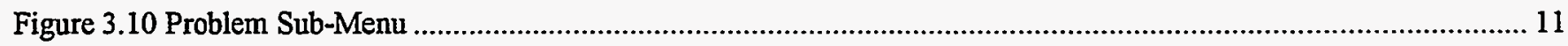

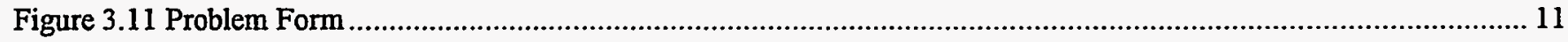

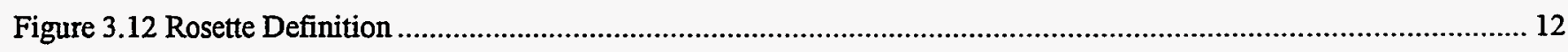

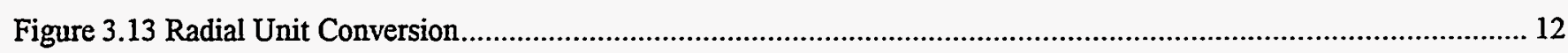

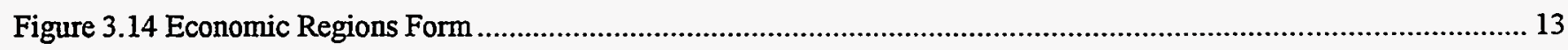

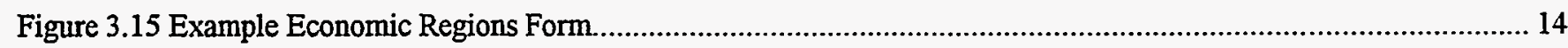

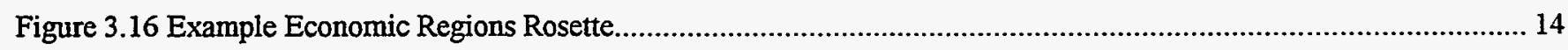

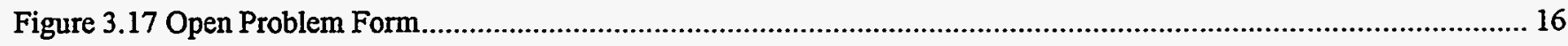

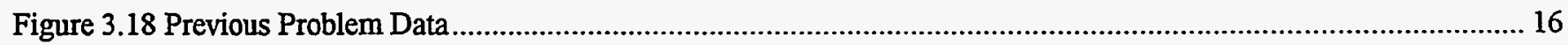

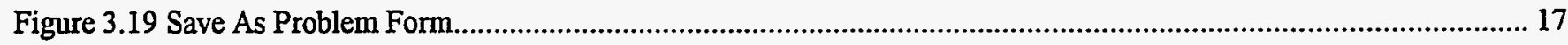

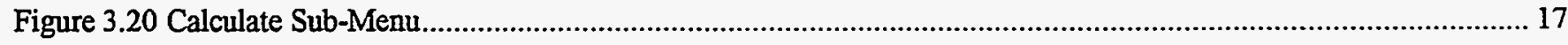

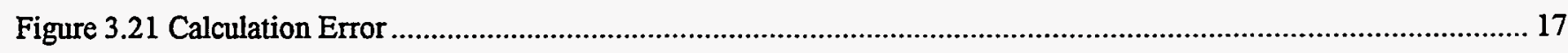

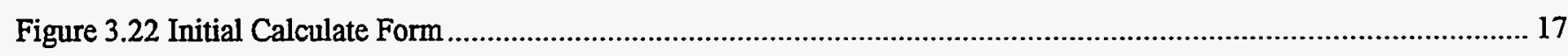

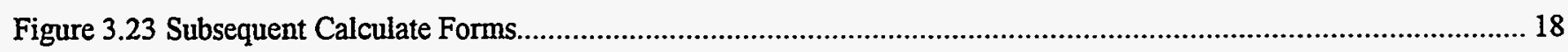

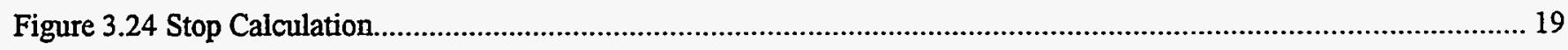

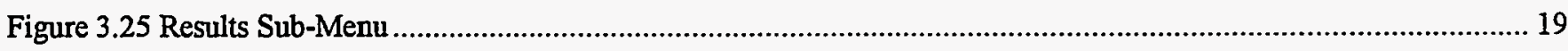

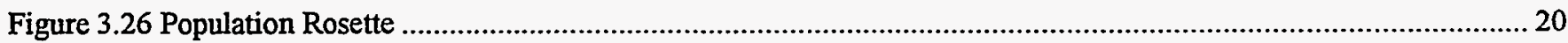

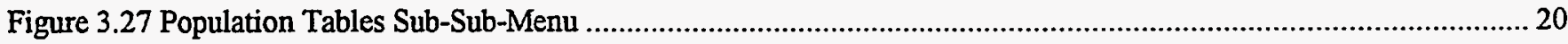

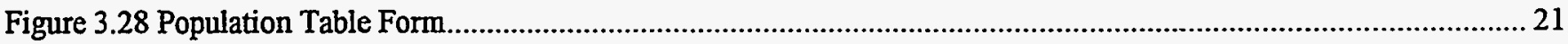

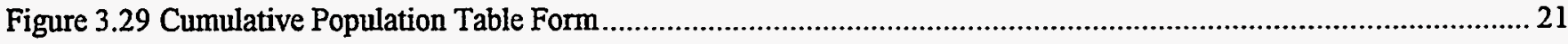

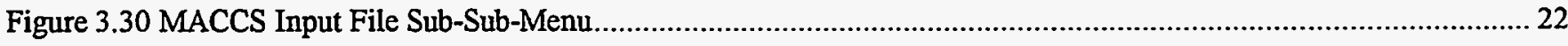

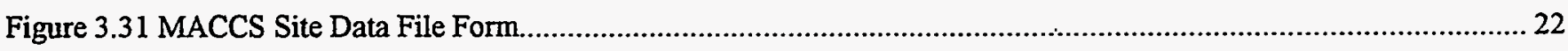

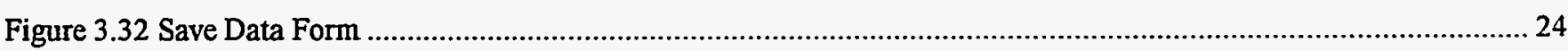

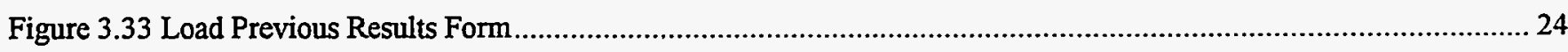

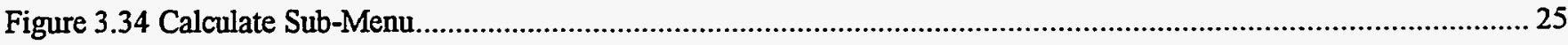




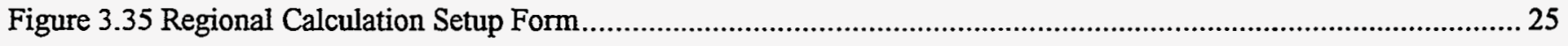

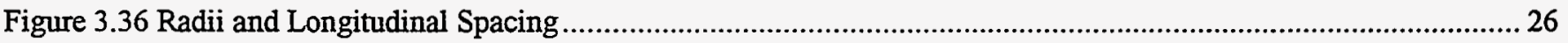

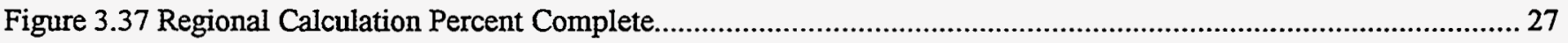

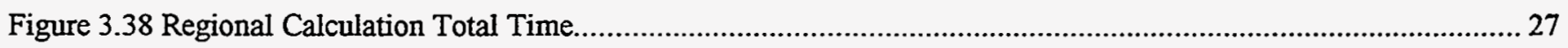

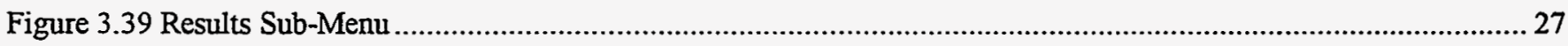

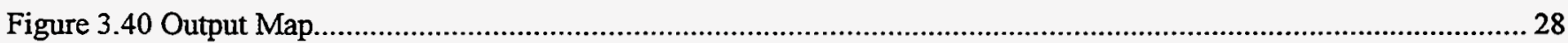

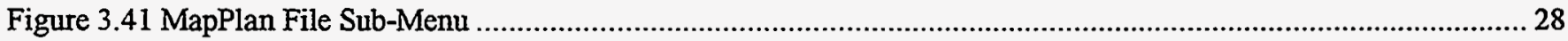

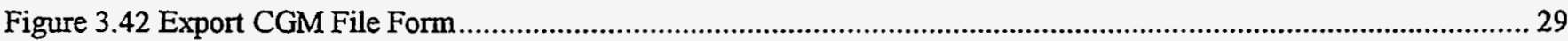

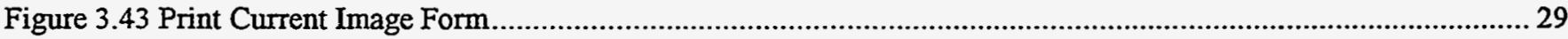

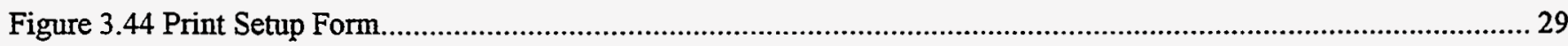

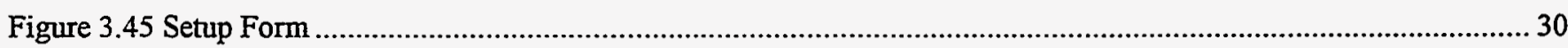

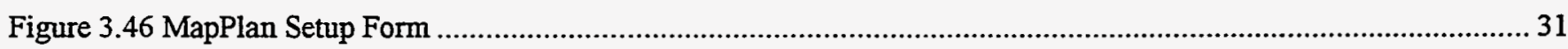

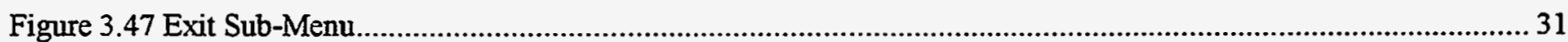

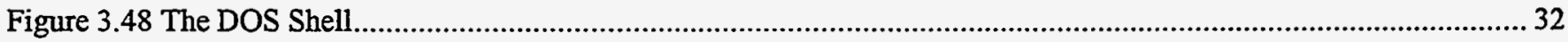

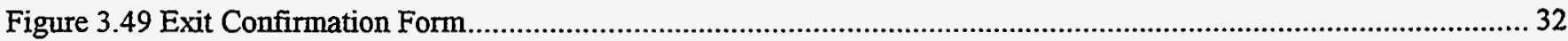

Figure 5.1 PL94-71 README File dBase to ASCII Conversion Program .................................................................. 41

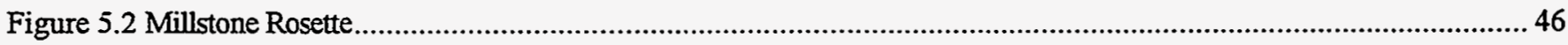

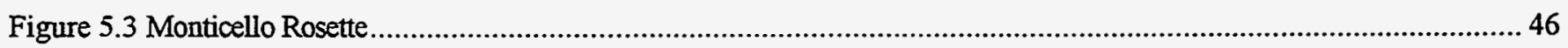

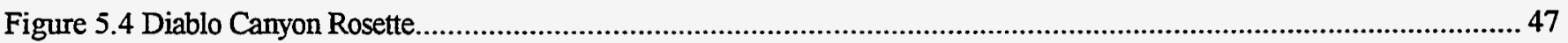




\section{TABLES}

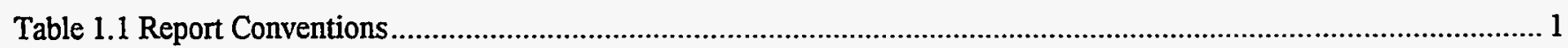

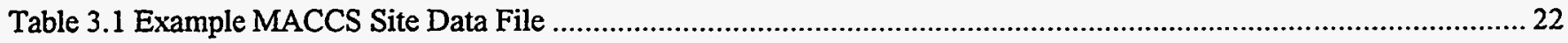

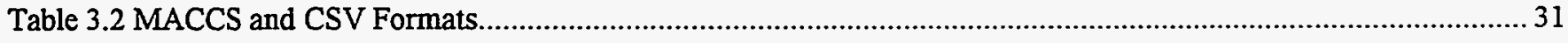

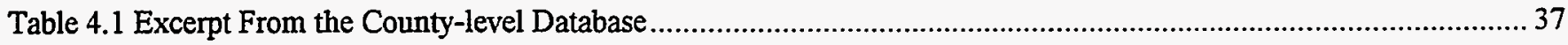

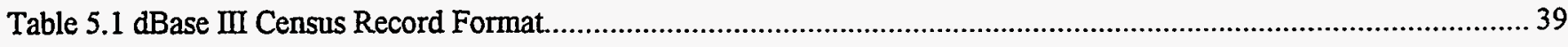

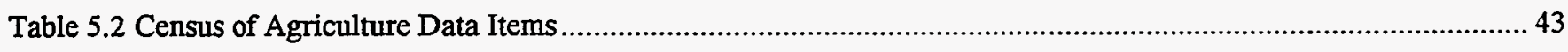

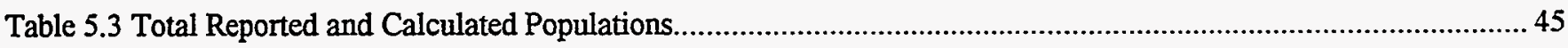


$$
\text { . }
$$ 


\section{ACKNOWLEDGMENTS}

The SECPOP90 team thanks everyone who helped make this report and the SECPOP90 software a reality. In particular we are especially grateful to Brian Lewis, our summer student from Cornell University, who helped make SECPOP90 a more useful program by providing it with many additional features and extensive error checking of all user input; and Donald Mitchell, Sandia National Laboratories, whose assistance with the county-level database effort was invaluable. 


\section{INTRODUCTION}

This report is divided into five sections. The first section is this introduction. The second section describes the installation of the SECPOP90 software. The third section is the user's guide, which explains how to use the software in a step-by-step manner. The fourth section describes the algorithms and methodologies used to perform the population, land fraction, and economic estimates. The fifth section discusses the software design, development, and verification and validation.

Additional appendices describe input and output file formats, the U.S. Bureau of Census CD-ROMs to block census and county census data file conversions, and the complete SECPOP90 Visual Basic code listing.

\subsection{Report Conventions}

The following conventions will be used throughout this report:

\section{Table 1.1 Report Conventions}

\begin{tabular}{|c|c|}
\hline Convention & Explanation \\
\hline SECPOP90 V2.0 MACCS Site Data File & $\begin{array}{l}\text { A monospaced font will be used for example data } \\
\text { files, operating system messages, code fragments, an } \\
\text { the SECPOP } 90 \text { code listings. }\end{array}$ \\
\hline a): install & $\begin{array}{l}\text { A bold monospaced font will be used for input that } \\
\text { the user is expected to type in. }\end{array}$ \\
\hline$\sim$ & $\begin{array}{l}\text { The return or enter key. The user should press this } \\
\text { key where it is indicated in this report. }\end{array}$ \\
\hline Filename & $\begin{array}{l}\text { An italicized monospaced font will be used for input } \\
\text { parameters that the user is to provide and type in. }\end{array}$ \\
\hline HOME, END, PAGE UP & $\begin{array}{l}\text { Text in small caps indicates one of the special keys } \\
\text { on the keyboard. }\end{array}$ \\
\hline$A L T+F l$ & $\begin{array}{l}\text { A plus sign indicates that the first key should be } \\
\text { pressed and held down then the second and } \\
\text { subsequent keys pressed. }\end{array}$ \\
\hline Three-Letter Acronym (TLA) & $\begin{array}{l}\text { Acronyms will be defined where they are first used. } \\
\text { The defining phrase will be capitalized in } \\
\text { correspondence to the letters that make up the } \\
\text { acronym. The definition will be followed by the } \\
\text { acronym in parentheses. }\end{array}$ \\
\hline
\end{tabular}

\subsection{Background}

In $1973 \mathrm{Mr}$. W. Athey of the Environmental Protection Agency wrote a computer program called SECPOP which calculated population estimates. This program was for use on mainframe computers, used the 1970 population census data, and was originally part of a study on air quality. The Nuclear Regulatory Commission used this program as part of the siting review for nuclear power plant construction and operating licensing applications.

Since then, two things have changed which suggested the need for updating the original program - more recent population censuses and the widespread use of personal computers (PCs). The revised computer program uses the 1990 Population Census information and runs on current PCs as "SECPOP90."

SECPOP90 consists of two parts: site and regional. The site analysis provides population and economic data estimates for any location within the continental United States. Calculation results can be displayed, printed, or stored as a file as a rosette, tables, or a MELCOR Accident Consequence Code System (MACCS) site file. Site analysis is relatively fast running. The regional portion assesses site availability for different siting policy decisions; i.e., to assess the impact of available sites given specific population density criteria within the continental United States. Regional analysis is much slower. 
SECPOP90 uses the latest (1990) census data and provides much greater resolution than the 1980 census data. This resolution is achieved by utilizing over six million census data points and performing the sector population estimates using these points directly. SECPOP90 also allows the user to calculate regional population density thresholds and display them via an interface with MapPlan mapping software.

\subsection{Hardware and Software Requirements}

SECPOP90 will run with most personal computers available today. SECPOP90 operates as a stand-alone program in the DOS environment. It also runs in a DOS box in OS/2 Version 2 or Windows Version 3.1 or later. Due to the operational overhead associated with Windows, SECPOP90 will run slower under Windows.

SECPOP90 consists of 422 programs and files requiring approximately 81 megabytes of hard disk storage. This is adequate for running only the "site" portion of SECPOP90. The "regional" calculation can require significantly more hard disk storage space. An additional 35 megabytes may be needed to analyze the entire continental United States in 10 mile rings. One calculation (US NRC Region I with 10 mile increments) took approximately 14 days and left a file of almost 6 megabytes on the hard disk. (More about "site" and "regional" calculations in sections 3.2 and 3.3, respectively.)

An American National Standards Code for Information Interchange (ASCII) compatible printer is required in order for the SECPOP90 print functions to print properly. In addition to printing the standard ASCII characters, it must also be capable of executing a form feed when receiving the ASCII form feed character (CTRL $+L)$.

Minimum System Requirements:

IBM PS/2, IBM Personal Computer AT or compatible;

IBM PC-DOS 5.0, Microsoft MS-DOS 5.0 or compatible;

Intel 80386, or compatible CPU;

620 kilobytes of available conventional RAM, 512 kilobytes expanded RAM;

One $3 \frac{1}{2}$ inch high density (1.44 megabyte) floppy disk drive;

Hard disk with 81 megabytes free (minimum to load programs, 100 megabytes recommend);

VGA monitor, or compatible; and

A Microsoft-compatible mouse (recommended but not required). 


\section{INSTALLATION}

This section describes how to install SECPOP90 under DOS. To install SECPOP90 under Windows 3.1x, Windows 95 , Windows NT, or OS/2, install SECPOP90 in a DOS-compatible window using the instructions provided below. For other operating systems, consult your operating system manual on how to install DOS programs.

To install SECPOP90, first exit or quit all other programs and command shells until you reach the DOS prompt (for example: $\mathrm{C}: \backslash>)$. Then insert disk \# 1 into drive $A$ or $B$ and type:

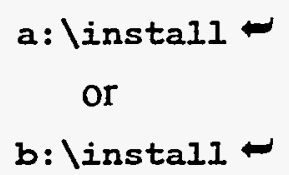

Follow the on-screen prompts to insert the additional disks. When the installation is complete the directory will contain the following files and sub-directories shown in Figure 2.1. (The default directory is ISECPOP90. The root of the directory will be different if you chose another directory during installation.) For more information about these files see sections 3.7,4.4, and Appendix A.

\subsection{Initialization}

All SECPOP90 output files can be printed directly from SECPOP90 or from any word processor or other program capable of printing ASCII text files. The forms themselves and the population rosette (see section 3.6.1) cannot be directly printed. To print or save the forms, the population rosette, or MapPlan output files, thirdparty software capable of performing a graphics screen capture (for example, Pizazz Plus or HiJaak PRO) may need to be loaded before running SECPOP90. Consult the documentation for your screen capture software. If SECPOP90 is being run in the Microsoft Windows environment, then it is possible to print SECPOP90 screen images using ALT+PRINT SCREEN and pasting the image into Microsoft Paintbrush or any Windows program that can print bit mapped (*.BMP) files. Consult your Windows documentation for more information. Other operating systems might have similar features. Consult your operating system documentation to determine if screen capture is supported.

\subsection{Starting SECPOP90}

To start SECPOP90 at the DOS prompt type:

$$
\text { cd Isecpop90 - }
$$

to change to the default SECPOP90 directory. If you installed SECPOP90 on a different hard drive or in a different directory, then type:

$$
\begin{aligned}
& \text { drive letter: } \\
& \text { cd Ipathname }
\end{aligned}
$$

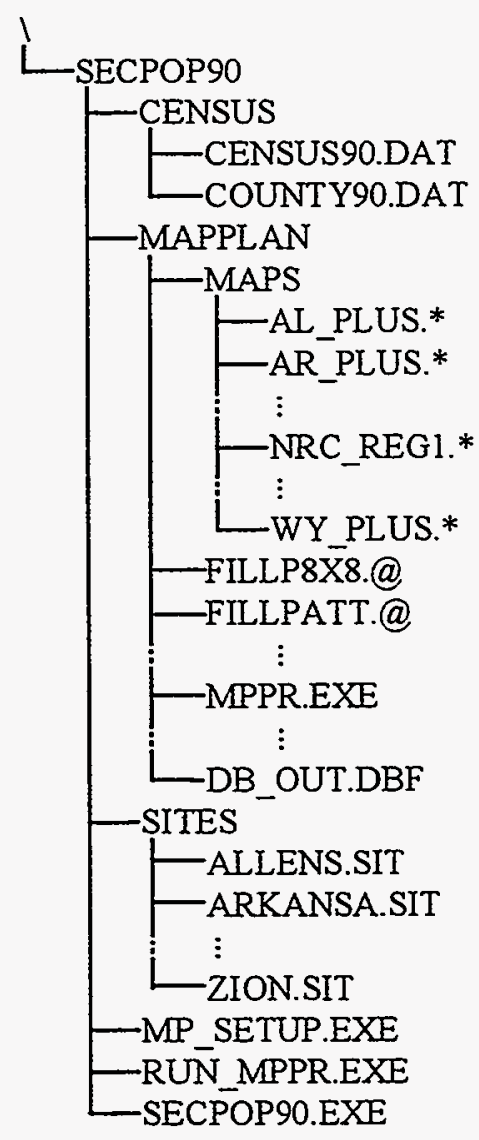

Figure 2.1 File and Directory Structure

where drive letter is the hard drive designator (for example, $\mathrm{C}$ or $\mathrm{D}$ ) of the hard drive on which SECPOP90 was installed and pathname is the complete pathname of the directory in which SECPOP90 was installed. After successfully changing to the appropriate directory, type the following at the DOS prompt:

\section{secpop90}

SECPOP90 will now run and display the first screen as shown in Figure 2.2. To start SECPOP90 from other operating systems, consult your operating system manual on how to run DOS programs. 


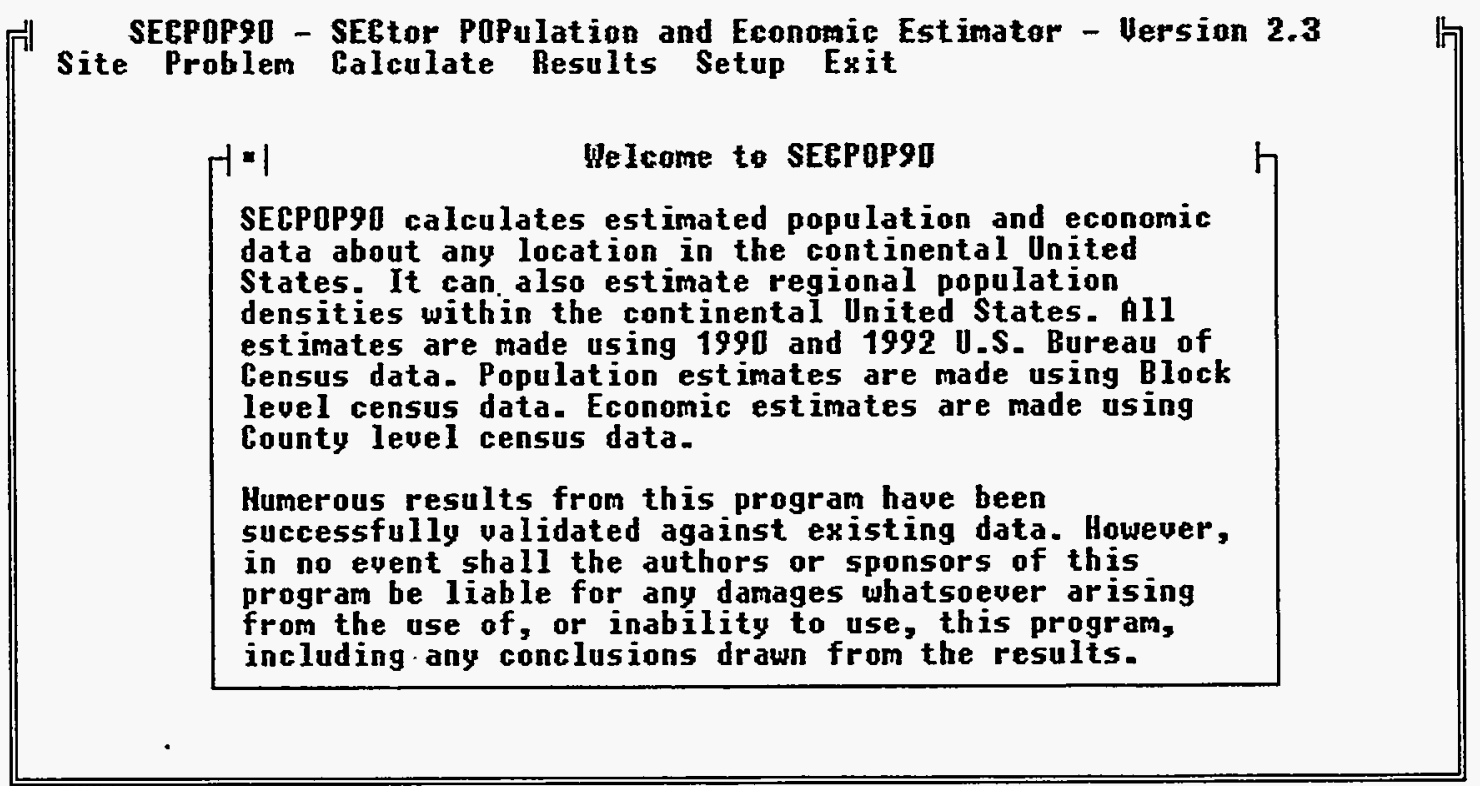

Figure 2.2 Initial Screen 


\section{USER'S GUIDE}

SECPOP90 consists of two main calculation tasks: calculations related to a specific site and calculations related to a region of the continental United States. The site calculation requires, among other things, a specific latitude and longitude from which to work. This is typically the reactor center for a single unit site or the center point between the reactors at a multiunit site. (The variance between these points is not expected to significantly affect the results, especially the results of subsequent MACCS calculations.) The regional calculation requires, among other things, the definition of a region of the continental United States. This region can be the entire continental United States or a subset, such as an NRC region, a state, or the area of Washington, DC.

Calculations for both sections start at the initial screen shown in section 3.1. However, SECPOP90 uses a different approach for each type of calculation and each approach is discussed separately. Site-specific calculations are discussed in sections 3.3 through 3.6 and regional calculations are discussed in section 3.7.

Descriptions on how to navigate through the SECPOP90 menus and forms are given in sections 3.2 and 3.3 respectively. If, while trying to perform one of the operations described later in the User's Guide section, you have trouble with keyboard or mouse usage, refer back to these two sections.

\subsection{Parts of the Main Screen}

The SECPOP90 main screen is divided into three major sections. Section (1), Main Screen Title Bar, displays the name of the SECPOP90 program and the present version number. Section (2), Main Menu, is the SECPOP90 main menu from which all other menu commands, sub-menus and sub-menu commands are selected and executed. It is through this menu that you access all other portions of the SECPOP90 program. Section (3), Area for Additional Forms or Messages, is the area where various other forms and messages will appear. Most of these will require you to provide some information, make a decision, or acknowledge that you have read them. The Welcome to SECPOP90 form below is the only form that will close automatically when you select a command from the main menu. All other forms will require some input before they will close. Note: the Welcome to SECPOP90 form will also close if you click on it with the mouse or select Close from the control box (for more information about the control box, see the Longitude field description in section 3.3.1).

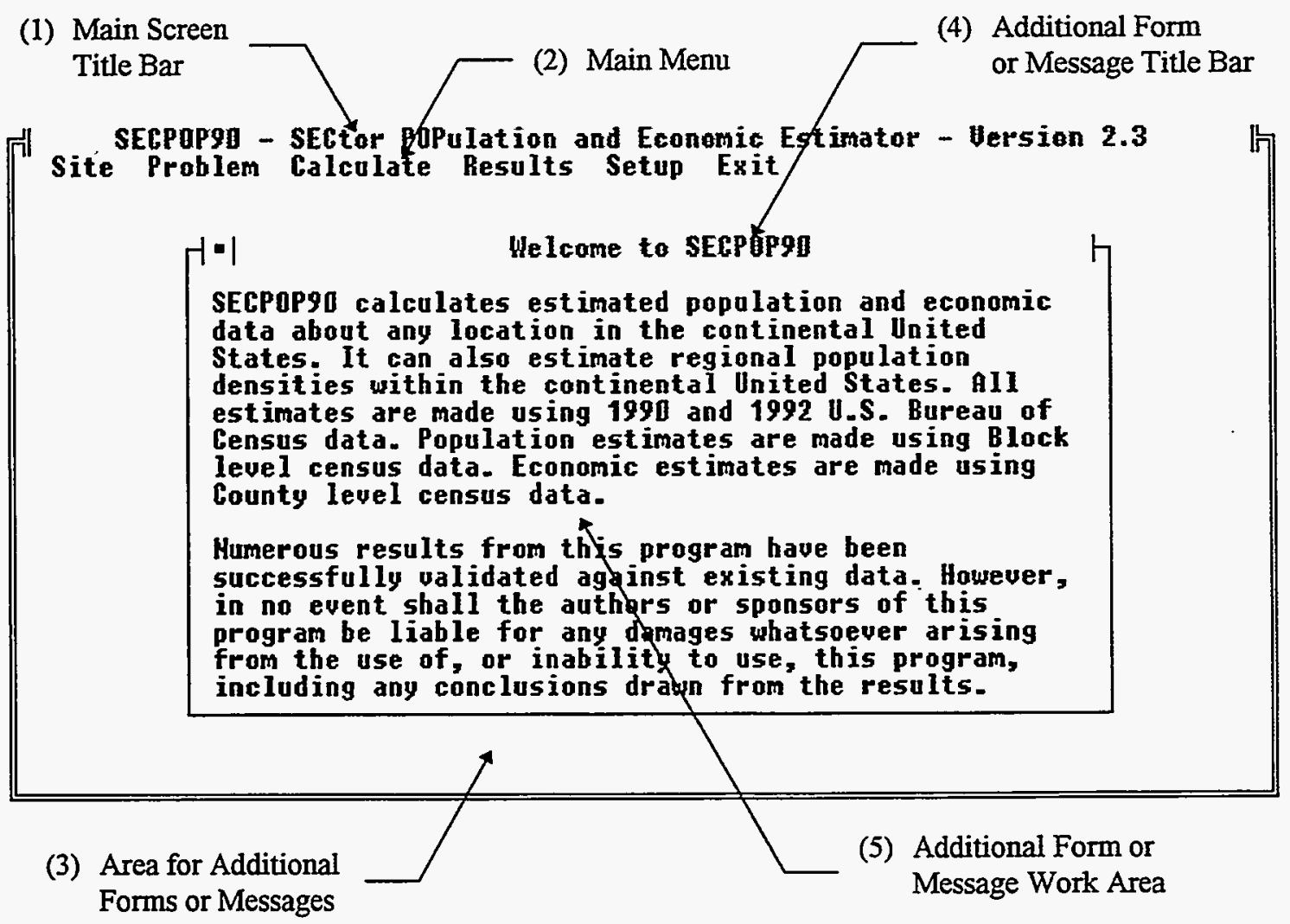

Figure 3.1 Parts of the Main Screen 
In addition to the three major sections of the main screen, each additional form has two sections. Section (4), Additional Form or Message Title Bar, displays the name of the additional form or message. Each form has a unique name to assist the user so that you know exactly where you are in the program. Section (5), Additional Form or Message Work Area, is the area of the additional form or message where fields are filled in, options selected, flags set, and additional commands specific to the form or message may be executed. It is through these additional forms and messages where most of your interaction and work with SECPOP90 will occur.

\subsection{Main Menu}

The main menu consists of six sub-menus or commands:

Site site characteristics for site-specific calculations;

Problem additional non-site-specific parameters for site-specific calculations;

Calculate perform site-specific or regional calculations;

Results results from present or previous calculations;

Setup customize the SECPOP90 environment; and

Exit exit SECPOP90.

The order of the selections above outlines the order of how you might solve a site-specific problem. First, you define the site parameters such as latitude and longitude using the Site sub-menu; next, you define additional problem parameters such as the radius of the exclusion area using the Problem sub-menu; then you perform the calculations using the Calculate submenu; and finally you examine the results using the Results sub-menu.

Regional problems do not require any site or problem specifications and are solved by setting the problem parameters such as population density threshold when the calculation is performed using the Calculate sub-menu. The results are then shown immediately. Previous regional results may be examined using the Results sub-menu.

\section{Site roblem salculate sesults Setsp Exit}

Figure 3.2 Main Menu

The Setup command allows you to modify the SECPOP90 environment. Pathnames for site files and flags to control the output may be set in the Setup form. The Exit sub-menu allows you to execute a DOS shell or exit the SECPOP90 program.

To navigate around the main and sub-menus, there are several methods that can be used. The mouse can be used to reach any command or sub-menu by one of two ways. To use the first method, click (normally with the left button unless you have reversed buttons via your mouse setup software) on the menu item you wish to select. It will then become highlighted (see the selection Site in Figure 3.2) and then either the sub-menu will drop down (see the Site sub-menu in Figure 3.3) or the command selected will execute. To select a command from a sub-menu, click on the command using the mouse and the command will execute. For sub-sub-menus and sub-sub-menu commands, proceed in a similar fashion. Sub-menu commands and sub-sub-menu commands can also be executed using the mouse by moving the mouse to the menu item for the sub-menu you wish to select (for example, the Site menu item if you want the Site sub-menu) then press the left (or right, see comment above) mouse button and keep it held down. With the mouse button held down you can trace through the menus, sub-menus, and sub-sub-menus until you get to the command you wish to execute. Release the mouse button when the appropriate command is highlighted and that command will execute.

The menus can also be accessed using the keyboard. Pressing ALT will highlight the main menu bar Site selection. The left and right arrow keys can then be used to move the highlight from menu item to menu item. Press ENTER (or RETURN) or the down arrow to select a command or sub-menu. Use the up and down arrows to select commands from the sub-menus or subsub-menus. Press ENTER (or RETURN) to execute the desired command. Another way to access the menus is to use the designated "shortcut" keys. Every menu, sub-menu, sub-sub-menu item has a special key associated with it that is highlighted in white (in this report such keys are distinguished in dark gray in the figures and bold at the beginning of each section describing the menu or sub-menu). To use these keys, first press ALT, then press the sequence of shortcut keys that will take you to the command you wish to execute. Typing the last key of the sequence will then execute the command. For example, to begin the site-specifications for a new site type:

ALT $s \mathrm{n}$

and the form to define the site parameters for a new site will be displayed. Compare this sequence of letters to the highlighted letters in Figure 3.3 for a clearer understanding of what sequences to use. 


\subsection{Site Definition}

Site definition consists of specifying the site name, latitude, longitude, and any desired site-specific remarks. The Site submenu has four available commands. Note that some of these commands are not available at all times. For example, Save Site and Save As Site are not enabled if there is no site information to save. The four Site sub-menu commands are:

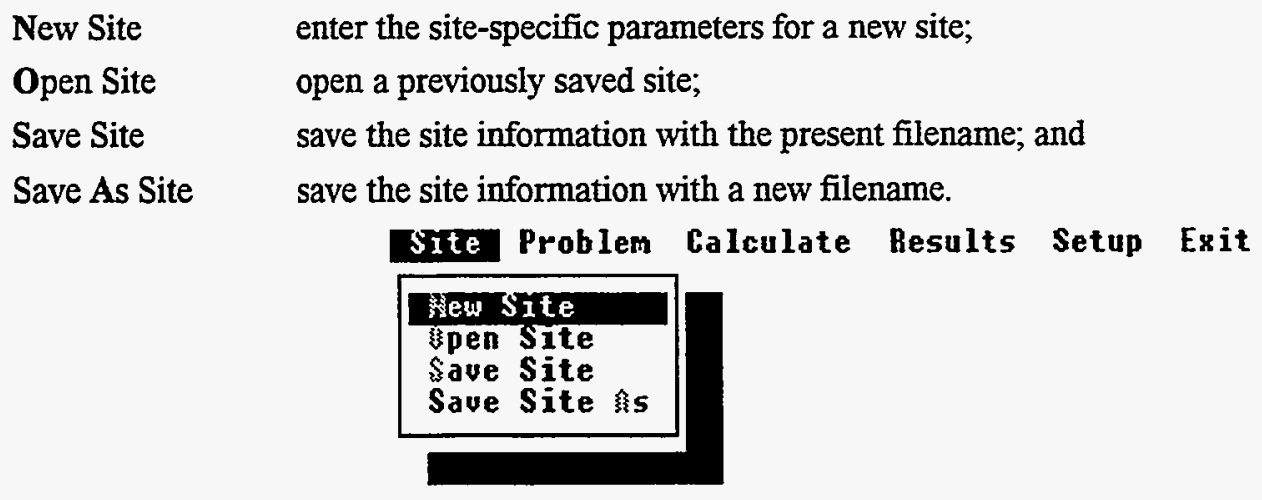

Figure 3.3 Site Sub-Menu

\subsubsection{New Site}

Selecting New Site opens the form shown in Figure 3.4. There are similar methods for moving around the fields (those areas where user input is requested) of the forms as moving through the menus and sub-menus. To move to a field directly with the mouse, move the mouse to the field and click the mouse button. If the field is an option (see the Radial Units field description in section 3.4.1), then the operation is complete. If the field is a text field, you may now enter the information requested. If the field is a command button (for example, the Close button in Figure 3.4), then the command is executed.

You can also move from field to field using the keyboard. Press ALT, then the "shortcut" key of the field you wish to select, move to, or execute (in this report such keys are distinguished in dark gray in the figures and bold at the beginning of each subsequent section describing the form). You can also move from field to field by pressing TAB or SHIFT+TAB. Note that the arrow keys do not work with forms as they do with menus. For example, to move directly to the Site Remarks field of the Site form (see Figure 3.4), type:

ALT+r

while the Site form is showing. Note also that the menus are inactive while forms are showing. To access a menu command all forms must first be closed. Forms and messages can always be closed by pressing ESC.

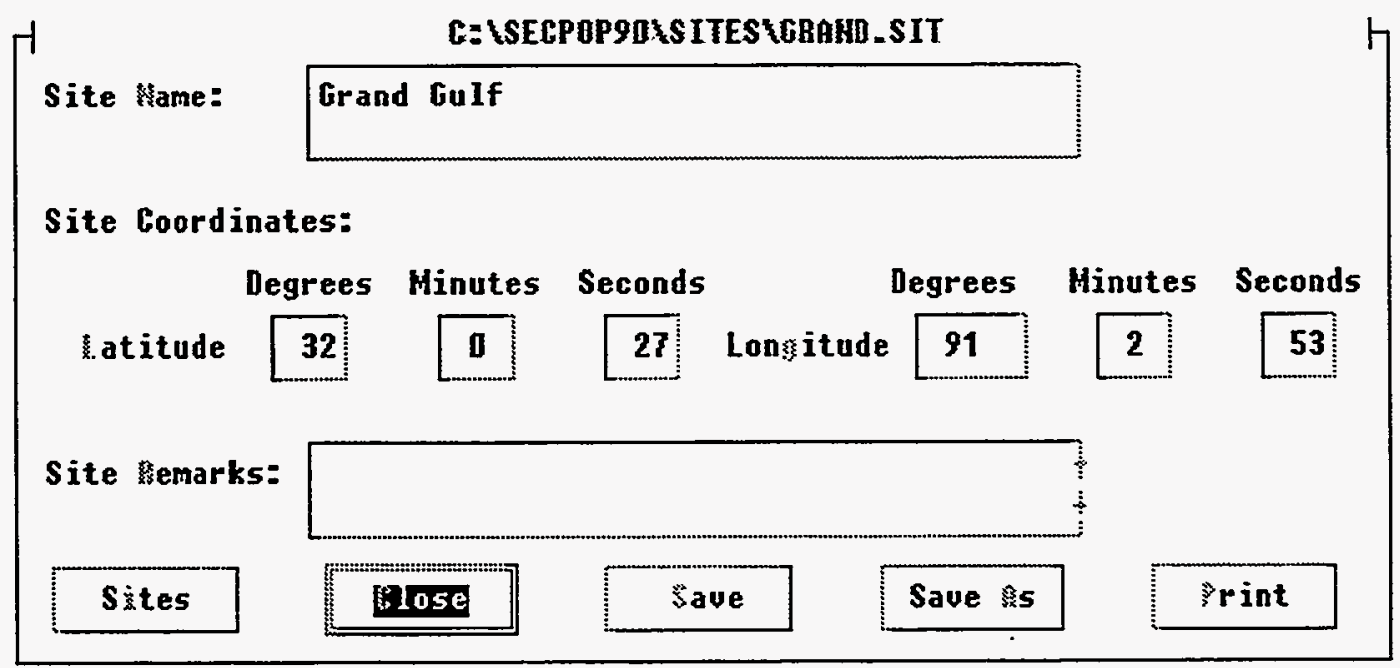

Figure 3.4 Site Form 


\section{Site Form Fields}

The Site form (see Figure 3.4) consists of the following fields which are designed so that the user may easily edit all of the site-specific information (fields are designated required or optional depending on whether they are required to perform sitespecific calculations):

Title Bar

Site Name

Latitude

Longitude

Site Remarks: contains the filename of the site being edited. If this is a new site, then the default filename "NEW_SITE.SIT" will be used. If the Site form is edited, then the phrase "(Modified)" will be appended to the site filename. The filename is automatically updated when the user performs a Save or Save As command (see the next section).

a descriptive name of the site. Up to eighty characters (both rows of the field in the form) may be used to describe the site. This field will be displayed or printed whenever the site-specific information is displayed or printed. Only the first forty of those characters (the first row) will be printed on the first line of the MACCS Site Data File (see Table 3.1). This field is optional.

the latitude of the site in degrees, minutes, and seconds. 0s (zeroes) must be entered if the minutes or seconds are equal to 0 . These fields are required.

the longitude of the site in degrees, minutes, and seconds. 0s (zeroes) must be entered if the minutes or seconds are equal to 0 . These fields are required.

SECPOP 90 checks to see if the coordinates of the site lie outside of the continental U.S. by comparing the coordinates to the minimum and maximum coordinates of the continental U.S. If the coordinates are determined to be outside of the continental U.S., then SECPOP90 displays the following error message:

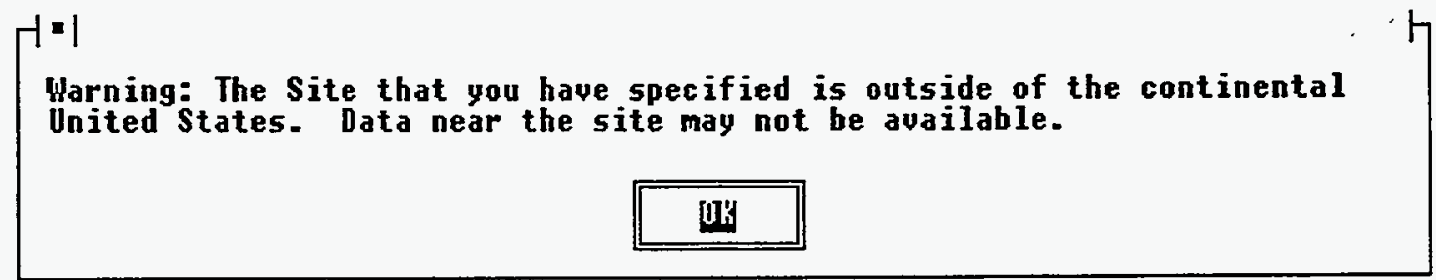

Figure 3.5 Outside of the Continental U.S.

To remove this message select the OK command button using one of the above-mentioned techniques for navigating around forms or click the control box (the small square in the upper lefthand corner of the message) and select Close from the sub-menu that appears. Other messages can be closed in a similar fashion.

Note: this limited checking is meant to alert the user to gross typing errors. The absence of the above message does not guarantee that the site information is correct. The user should always double-check the information in the required fields with the original sources.

descriptive remarks about the site. Up to eighty characters (both rows of the field in the form) may be used to describe the site. This field will be displayed or printed when viewing this form or printing the site-specific information (see the Print command in the next section). One use of this space could be to record plant specific information. For example:

Unit 1 -- GE BWR 6 with Mark III containment, licensed in 1984

This field is optional.

\section{Site Form Commands}

At the bottom of the Site form is a row of five command buttons. The commands are executed when the buttons are selected (see 3.3.1). The commands support the opening, closing, saving, and printing of the Site form. The command buttons are as follows:

Sites opens a previously defined site file. This command is equivalent to the Open Site command of the Site sub-menu (see Figure 3.3). For a more detailed explanation see section 3.3.2.

Close closes the Site form. All information on the form is saved in memory and can be reviewed by opening the Site form again. The information will be lost unless the site information is stored in a file (see the Save and Save As commands below) before SECPOP90 is exited. 
Save

Save As

Print saves the site information using the filename displayed in the Site form Title Bar. This command is equivalent to the Save Site command of the Site sub-menu.

saves the site information using a filename specified by the user. This command is equivalent to the Sove As Site command of the Site sub-menu. For a more detailed explanation see section 3.3.4.

prints the site-specific information to a connected printer or a file. When this button is selected the following form will be displayed:

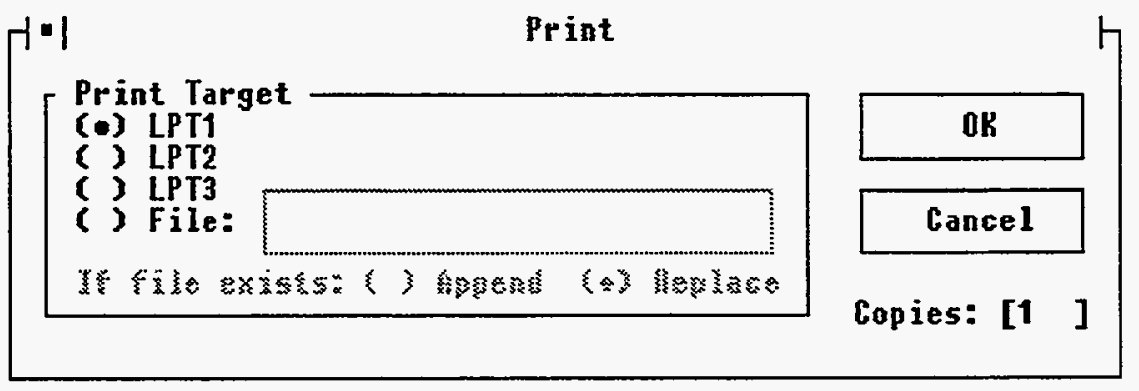

Figure 3.6 Print Form

This form allows the user to select which printer or file the site information is to be printed to. In the case of printing to a file, the user can also select whether to replace an existing file or append this information to the end of it. The user can also specify how many copies of the site-specific information should be printed to either the selected printer or file.

\subsubsection{Open Site}

Selecting Open Site opens the form shown in Figure 3.7. This form allows the user to select a previously defined site from either the list of predefined sites (as shown) or his or her own list of sites. The form has fields for selecting the appropriate disk drive, directory, sub-directories, and filename. Once these are chosen and the $O K$ button is selected, the file is loaded into memory and the Site form will be displayed with the saved information (see Figure 3.4). The user can close the Open Site form by selecting Cancel, pressing ESC, or selecting Close from the control box sub-menu.

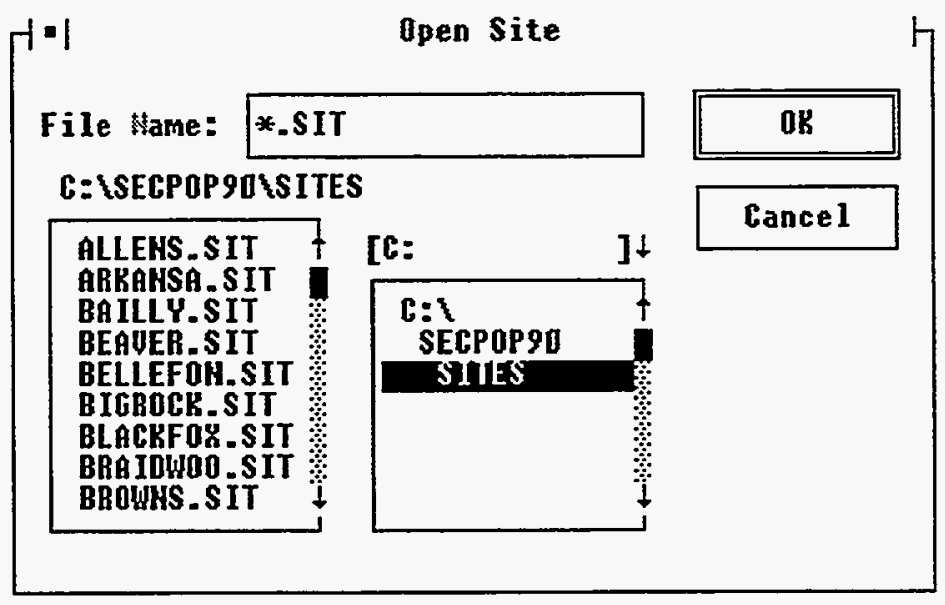

Figure 3.7 Open Site Form

All predefined sites have the default filename extension "SIT." The user is not required to follow this practice. Doing so, however, will make it easier to find previously defined site files. This is especially true when a single directory has a mix of site, problem, and results files.

If either New Site or Open Site is selected from the Site sub-menu and site data have already been entered or loaded from a file, SECPOP90 will first ask the user if the previously entered or loaded data should be used before overwriting it.

SECPOP90 displays the following message: 


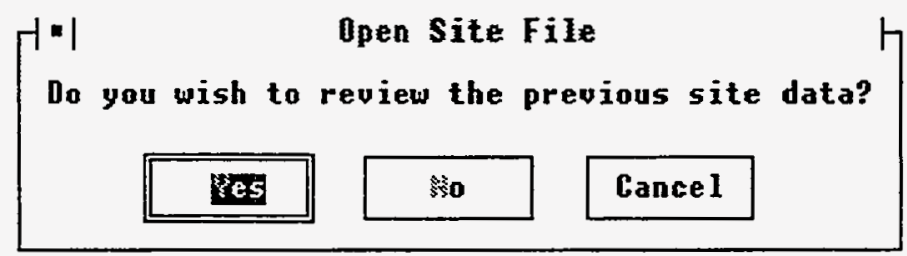

Figure 3.8 Previous Site Data

If the user selects Yes, then the New Site or Open Site operation is canceled and the Site form with the old data is displayed. If the user selects $\mathrm{No}$, then the old data is overwritten with a blank form or the new data. Selecting Cancel cancels the New Site or Open Site operation and returns the user back to the main menu.

\subsubsection{Save Site}

Selecting Save Site saves the site information using the filename displayed in the Site form Title Bar. After the file is saved, the Title Bar of the Site form is updated. This command is equivalent to the Save command found on the Site form (see Figure 3.4).

\subsubsection{Save As Site}

Selecting Save As Site opens the form shown in Figure 3.9 This form allows the user to save the site information using a user-specified filename. The form has fields for selecting the appropriate disk drive, directory, sub-directories, and filename. Once these are chosen and the $O K$ button is selected, the site-specific information is stored in the specified file. The Title Bar of the Site form is then updated with the new filename. Selecting Cancel cancels the Save As Site operation and returns the user back to the main menu. This command is equivalent to the Sove As command found on the Site form (see Figure 3.4).

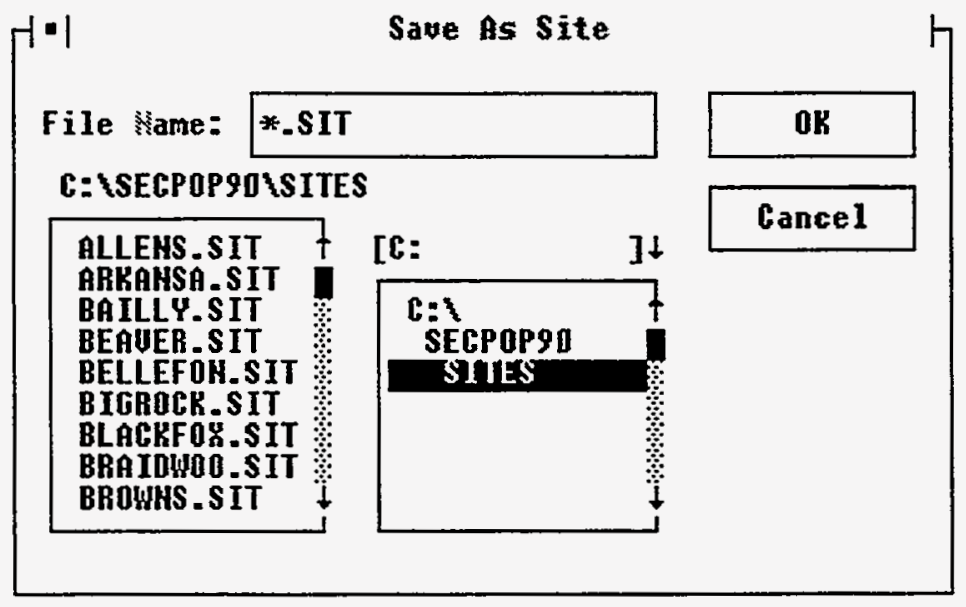

Figure 3.9 Save As Site Form

\subsection{Problem Definition}

Problem definition consists of specifying the radii of interest, the population multiplier, the name of the site file which contains the site-specific information for the problem, mapping the problem sectors to economic regions and any desired problem-specific remarks. The Problem sub-menu has four available commands. Note that some of these commands are not available at all times. For example, Save Problem and Save As Problem are not enabled if there is no problem information to save. The four Problem sub-menu commands are:

New Problem

Open Problem

Save Problem

Save As Problem enter the problem-specific parameters for a new problem; open a previously saved problem; save the problem information with the present filename; and save the problem information with a new filename. 


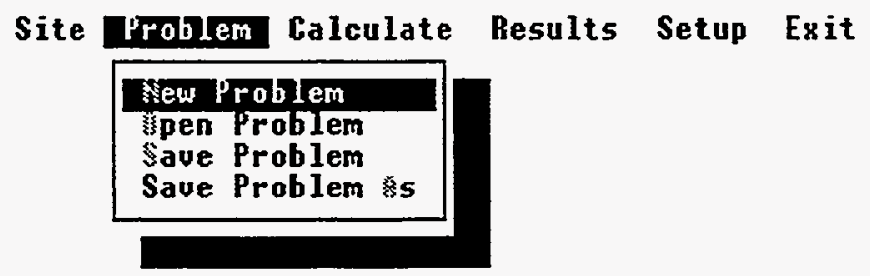

Figure 3.10 Problem Sub-Menu

\subsubsection{New Problem}

Selecting New Problem opens the form shown in Figure 3.11. The Problem form allows the user to enter or edit all problem-specific information.

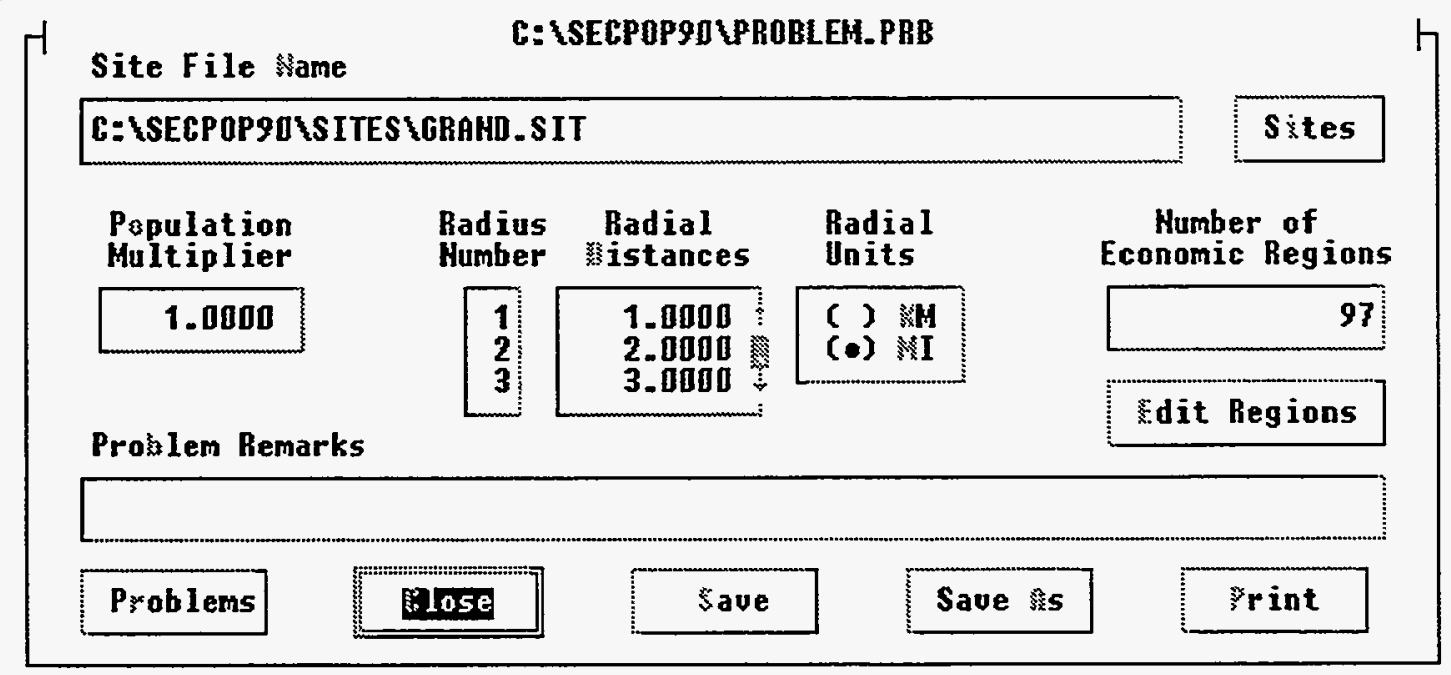

Figure 3.11 Problem Form

\section{Problem Form Fields}

The Problem form (see Figure 3.11) consists of the following fields which are designed so that the user may easily edit all of the problem-specific information (fields are designated required or optional depending on whether they are required to perform site-specific calculations):

Title Bar contains the filename of the problem being edited. If this is a new problem, then the default filename "NEW PROB.SIT" will be used. If the Problem form is edited, then the phrase "(Modified)" will be appended to the problem filename. The filename is automatically updated when the user performs a Save or Save As command (see the next section).

Site File Name the name of the site file associated with this problem. This field is required.

Population

Multiplier a multiplier that is applied to the 1990 census population data. This field allows manipulation of the 1990 census data to estimate changes in the population by a uniform multiplication factor. For example, if it is expected that in the year 2000 the population will increase by $10 \%$ in a given location, a population scale factor of 1.1 could be used to represent this growth. The default value is 1.0 - representing the 1990 census population. A value less than or equal to 0.0 is not allowed. This field is required.

Radial Distances the radii of interest for the problem. The geometry of SECPOP90 problems is based on that of MACCS. The area around a site is divided into sixteen directions that are equivalent to a standard navigational compass rosette. The rosette is then further divided by radial rings specified by the user using the Radial Distances field. Figure 3.12 shows a rosette that has nine user-defined radial rings. As is common in practice, there is a tight collection of "inner" rings surrounded by a looser collections of "outer" rings. The site itself is located at the very center of the rosette.

The collection of directional lines and radii naturally divide the rosette into sections that are defined by two consecutive radii and two neighboring directional lines (except the innermost sections which are defined by the first radius and neighboring directional lines; normally this 
innermost radius is defined to be the exclusion area of the site; the second radius is often defined to be the low population zone). These sections are mapped into economic regions by using the Edit Regions command described below.

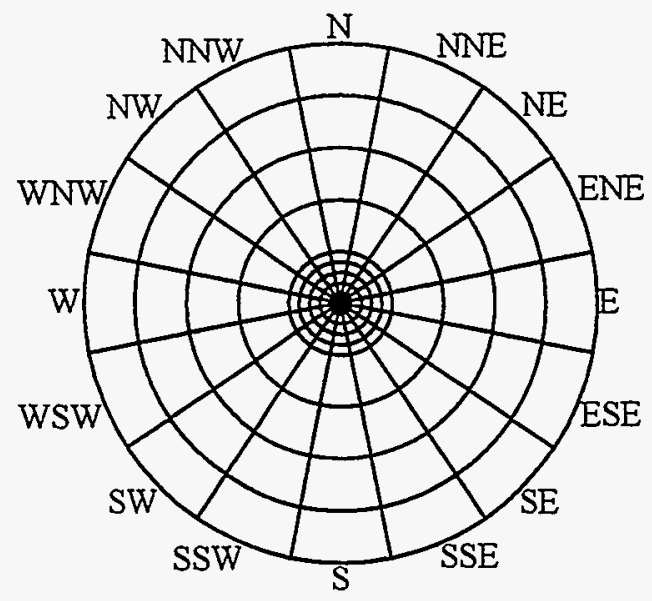

Figure 3.12 Rosette Definition

The radial distances can be expressed in miles or kilometers (see the Radial Units field described below). The user is limited to thirty-five radii to maintain compatibility with the MACCS Site Data File format. This field is required. At least two radii must be defined. All radii must be separated from each other by at least $0.1 \mathrm{~km}(0.06214 \mathrm{mi}$.).

Radial Units, MI indicates that the radial distances are in miles. If the radial distances are in kilometers and this option is selected, then the user is given the option of performing a conversion from miles to kilometers or only changing the unit designation. This is accomplished using the form shown in Figure 3.13. Either miles or kilometers must be selected.

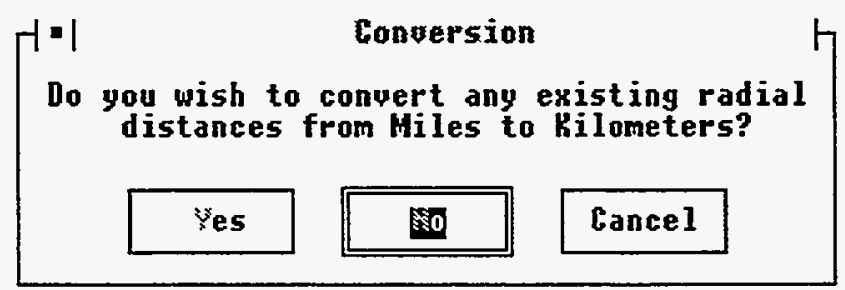

Figure 3.13 Radial Unit Conversion

Radial Units, KM indicates that the radial distances are in kilometers. If the radial distances are in miles and this option is selected, then the user is given the option of performing a conversion from kilometers to miles or only changing the unit designation. This is accomplished using a form similar to that shown in Figure 3.13. Either kilometers or miles must be selected.

Problem Remarks descriptive remarks about the problem. Up to eighty characters (both rows of the field in the form) may be used to describe the problem. This field will be displayed or printed when viewing this form or printing the problem-specific information (see the Print command in the next section). One use of this space could be to record the source of problem-specific information. For example:

Grand Gulf Nuclear Station (GGNS) Unit: 1 Emergency Plan Section 2.2

This field is optional.

\section{Problem Form Commands}

The Problem form has six command buttons: five at the bottom of the Problem form similar to the Site form and an additional command button for editing the economic regions. The commands are executed when the buttons are selected. The commands support editing of the economic regions and the opening, closing, saving, and printing of the Problem form. The command buttons are as follows: 
Edit Regions

Problems

Close

Save

Save As

Print opens the Economic Regions form (see Figure 3.14) and allows editing of the economic regions. This form is described in detail in section 3.4.2.

opens a previously defined problem file. This command is equivalent to the Open Problem command of the Problem sub-menu (see Figure 3.10). For a more detailed explanation, see section 3.4.2.

closes the Problem form. All information on the form is saved in memory and can be reviewed by opening the Problem form again. The information will be lost unless the problem information is stored in a file (see the Save and Save As commands below) before SECPOP90 is exited.

saves the problem information using the filename displayed in the Problem form Title Bar. This command is equivalent to the Save Problem command of the Problem sub-menu.

saves the problem information using a filename specified by the user. This command is equivalent to the Save As Problem command of the Problem sub-menu. For a more detailed explanation, see section 3.4.5.

prints the problem-specific information to a connected printer or a file. When this button is selected, the form in Figure 3.6 will be displayed.

This form allows the user to select which printer or file the problem information is to be printed to. In the case of printing to a file, the user can also select whether to replace an existing file or append this information to the end of it. The user can also specify how many copies of the problem-specific information should be printed to either the selected printer or file.

\subsubsection{Economic Regions}

Selecting Edit Regions from the Problem opens the form shown in Figure 3.14 The Economic Regions form allows the user to assign economic regions to the rosette sections or edit the present assignments.

\begin{tabular}{|c|c|c|c|c|c|c|c|c|}
\hline \multirow[b]{2}{*}{ Sectors } & \multicolumn{7}{|c|}{ Radial Distances in Hiles } & \multirow{2}{*}{$\begin{array}{r}\text { Regions } \\
97\end{array}$} \\
\hline & $\overline{1}$ & $\overline{2}$ & $\overline{3}$ & 4 & $\overline{5}$ & 10 & 20 & \\
\hline H & $\overline{1}$ & $\overline{2} \mid$ & 2 & $\overline{2}$ & 2 & $\overline{3}$ & 4 & \multirow{3}{*}{$\begin{array}{r}\text { Radii } \\
10\end{array}$} \\
\hline HHE & 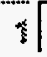 & $\overline{8}$ & $\overline{8}$ & 8 & $\overline{8}$ & 9 & 10 & \\
\hline HE & $\$$ & $\mid 14$ & $\overline{14}$ & $\overline{14}$ & $\overline{14}$ & $\overline{15}$ & 16 & \\
\hline EHE & $\widehat{s}$ & 20 & 20 & 20 & 20 & 21 & 22 & Sort \\
\hline $\mathbf{E}$ & $\hat{\beta}$ & 26 & 26 & 26 & 26 & 27 & 28 & \multirow{2}{*}{ Def̆ault } \\
\hline ESE & $\$$ & $\overline{32}$ & $\overline{32}$ & $\overline{32}$ & 32 & 33 & 34 & \\
\hline SE & $\hat{\xi}$ & 38 & 38 & 38 & 38 & 39 & 40 & Glear \\
\hline \multicolumn{2}{|c|}{ teft } & \multicolumn{2}{|c|}{ fight } & p & \multicolumn{2}{|c|}{ Sown } & & flose \\
\hline
\end{tabular}

Figure 3.14 Economic Regions Form

\section{Economic Regions Form Fields}

The Economic Regions form consists of the following fields which are designed so that the user may assign or edit the economic regions. All of the fields on this form are required. Many of the field are filled automatically by SECPOP90 and cannot be edited by the user. Other fields are editable by the user. These fields will be designated as "User input field," in the descriptions below.

Radial Distances the first row of the economic regions table contains the radial distances in the units specified on the Problem form (see Figure 3.11).

Sectors the first column of the economic regions table contains the rosette direction descriptors. These descriptors correspond to the sixteen standard directions of a navigational compass. 
Regions

Radii

Rosette Sections number of economic regions. The minimum number of allowed economic regions is 1 . The maximum number of allowed regions is 99 .

number of radii. The minimum number of allowed radii is 2 . The maximum number of allowed radii is 35 . The radii are defined on the Problem form.

the remaining values in the economic regions table are the economic region designators for the displayed rosette sections. Economic region \# 1 is automatically assigned to all of the sections that lie within the first innermost radius. This radius is normally thought of as the exclusion area boundary and economic region \# 1 , the exclusion area.

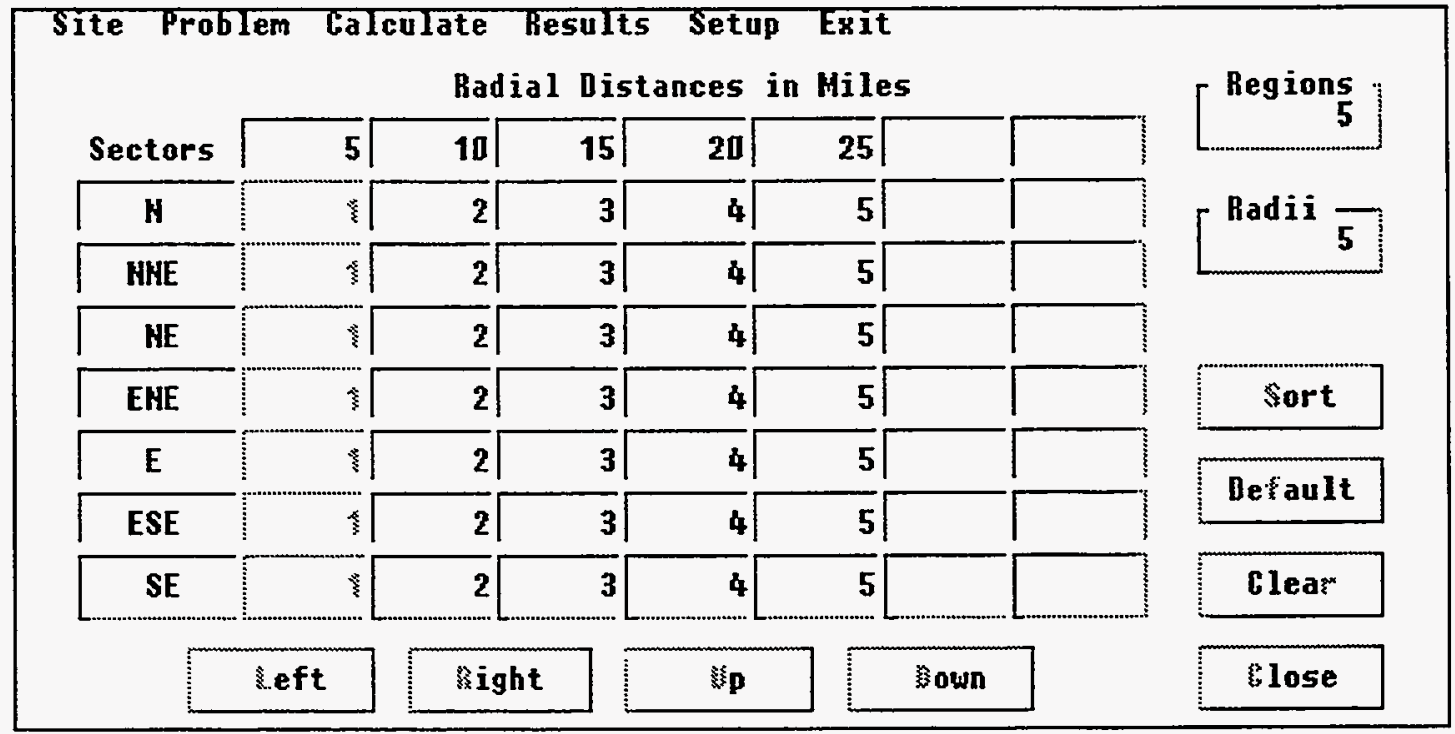

Figure 3.15 Example Economic Regions Form

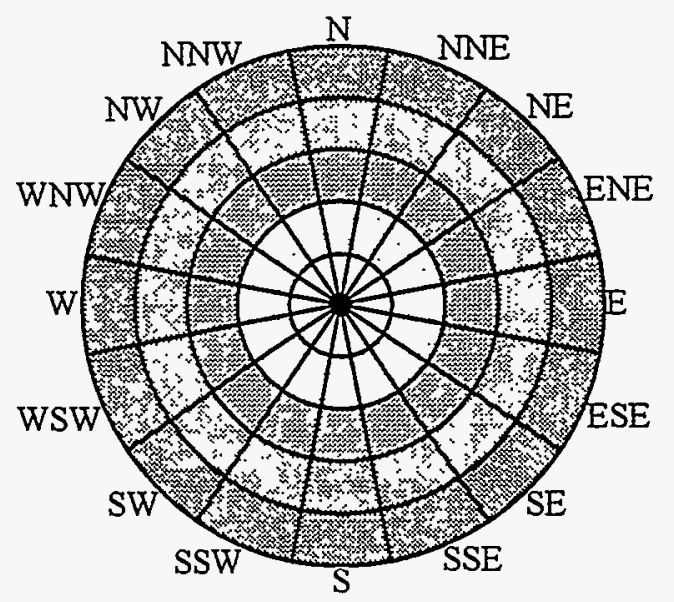

LEGEND - ECONOMIC REGIONS

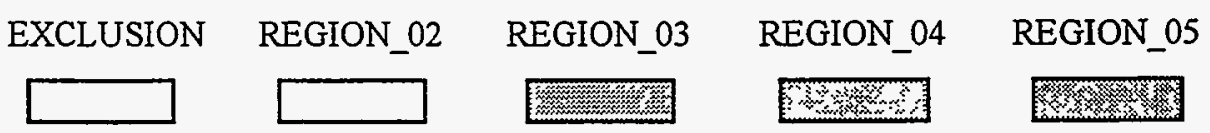

Figure 3.16 Example Economic Regions Rosette

To assign economic regions to the remaining rosette sections, enter a number for that region that is greater than or equal to 2 and less than or equal to 99 . That rosette section then belongs to that economic region. If you enter a 2 in the third column, second row of the economic regions table shown in Figure 3.14, then the rosette section defined by the first and second radii in the northern direction would be part of economic region 2. For example, if you wish to define four economic regions in addition to the exclusion area, and you want them to be simple rings centered around the 
site, then if you have five radii, your Economic Regions should look like Figure 3.15 . This corresponds to the economic regions mapping illustrated in Figure 3.16. (Note: this figure is for illustrative purposes only and does not represent a SECPOP90 screen. Also, actual economic regions are likely to be much more irregular.) The user is not limited to simple geometries such as that shown. Economic regions can be assigned to any rosette section. There is no need for the same economic region to occupy adjacent cells or for all economic regions to occupy the same number of cells. This allows the user to assign a single economic region to features that might occur repeatedly over the rosette. For example, a user could assign the same economic regions to lakes or desert areas.

\section{Economic Regions Form Commands}

The Economic Regions form has eight command buttons: four at the bottom of the Economic Regions form that are used for scrolling through economic region values, three command buttons on the right-hand side of the form for performing various operations on the economic regions, and a button for closing the Economic Regions form. The commands are executed when the buttons are selected. The command buttons are as follows:
Left
scrolls the economic regions table left so that the economic regions of more distant radii can be edited.
Right scrolls the economic regions table right so that the economic regions of less distant radii can be edited.
Up scrolls the economic regions table up so that the economic regions of more northwestern directions can be edited.
Down scrolls the economic regions table down so that the economic regions of more northeastern directions can be edited.
Sort renumbers economic regions so that there are no gaps in the numbering of the regions and the
regions are sorted in row column order. Sorting the economic regions is not required but it
normally makes the economic regions mapping to the rosette sections easier to understand.
Default restores the economic regions to the default values. These values are the same values used if the user does no editing of the economic regions.
Clear clear all values from all of the rosette sections except for economic region 1, which is always the section from the center of the rosette to the first radius - normally considered to be the exclusion area.
Close closes the Economic Regions form. To save the edited economic regions, the user must select Save or Save As from the Problem form (see Figure 3.11) or Save Problem or Save As Problem from the Problem sub-menu (see Figure 3.10).

\subsubsection{Open Problem}

Selecting Open Problem opens the form shown in Figure 3.17. This form allows the user to select a previously defined problem file. The form has fields for selecting the appropriate disk drive, directory, sub-directories, and filename. Once these are chosen and the $O K$ button is selected, the file is loaded into memory and the Problem form will be displayed with the saved information (see Figure 3.11). The user can close the Open Problem form by selecting Cancel, pressing ESC, or selecting Close from the control box sub-menu.

It is recommended that the user use filename extension "PRB" for all problem files. The user is not required to follow this practice. Doing so, however, will make it easier to find previously defined problem files. This is especially true when a single directory has a mix of site, problem, and results files. 


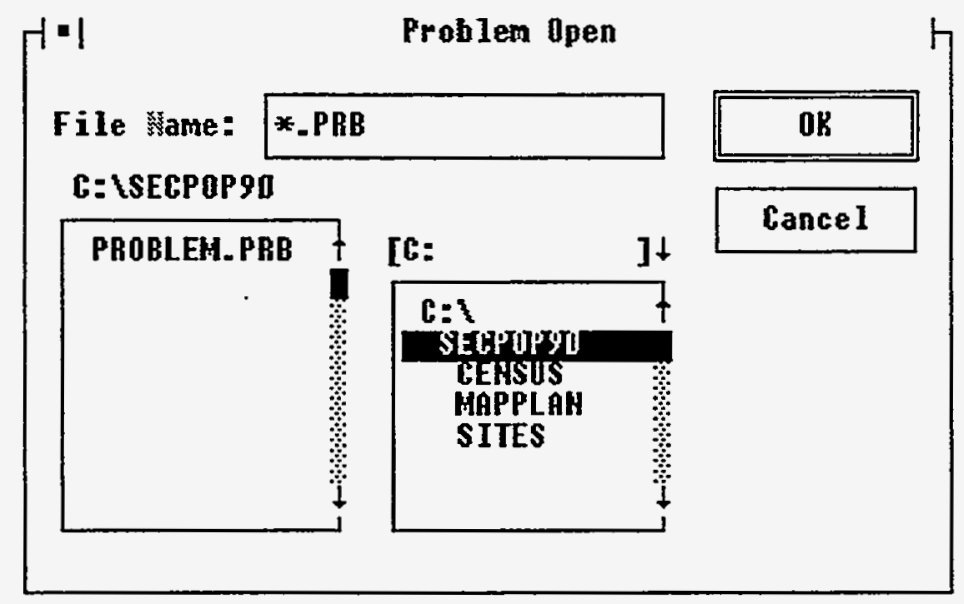

Figure 3.17 Open Problem Form

If either New Problem or Open Problem are selected from the Problem sub-menu and problem data has already been entered or loaded from a file, SECPOP90 will first ask the user if the previously entered or loaded data should be used before overwriting it. SECPOP90 displays the following message:

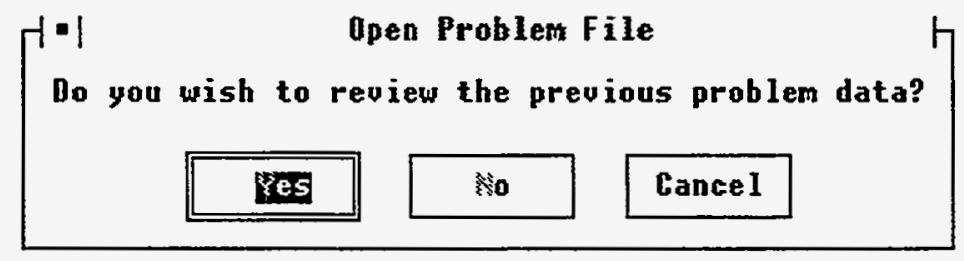

Figure 3.18 Previous Problem Data

If the user selects Yes, then the New Problem or Open Problem operation is canceled and the Problem form with the old data is displayed. If the user selects $N o$, then the old data are overwritten with a blank form or the new data. Selecting Cancel cancels the New Problem or Open Problem operation and returns the user back to the main menu.

\subsubsection{Save Problem}

Selecting Save Problem saves the problem information using the filename displayed in the Problem form Title Bar. After the file is saved, the Title Bar of the Problem form is updated. This command is equivalent to the Save command found on the Problem form (see Figure 3.11).

\subsubsection{Save As Problem}

Selecting Save As Problem opens the form shown in Figure 3.19. This form allows the user to save the problem information using a user-specified filename. The form has fields for selecting the appropriate disk drive, directory, sub-directories, and filename. Once these are chosen and the $O K$ button is selected, the problem-specific information is stored in the specified file. The Title Bar of the Problem form is then updated with the new filename. Selecting Cancel cancels the Save As Problem operation and returns the user back to the main menu. This command is equivalent to the Save As command found on the Problem form (see Figure 3.11). 


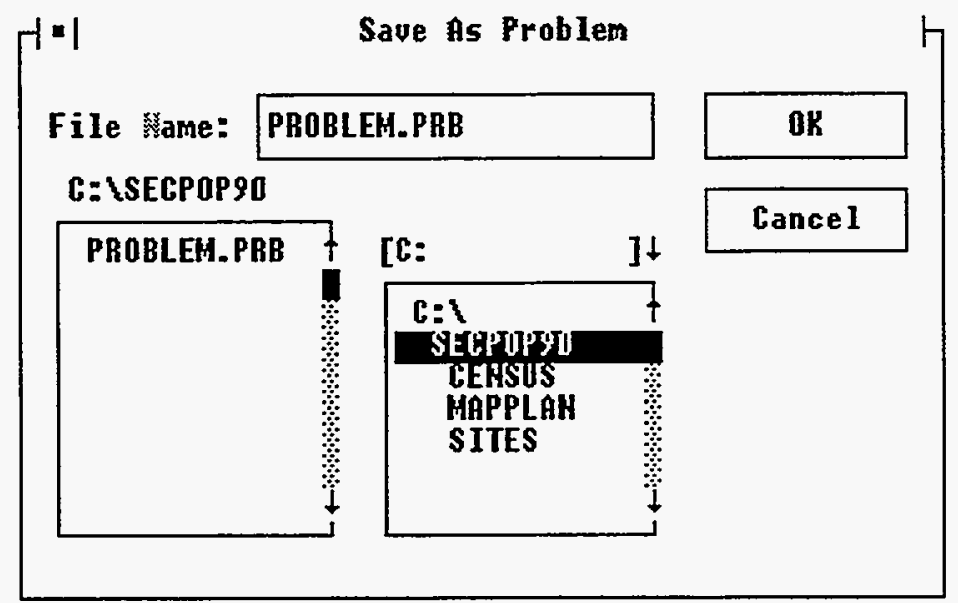

Figure 3.19 Save As Problem Form

\subsection{Performing Site-Specific Calculations}

Once the Site, Problem, and Economic Regions forms are complete, a site-specific calculation may be performed by selecting the Calculate sub-menu (see Figure 3.20) from the main menu and then selecting Site-Specific from the Calculate sub-menu.

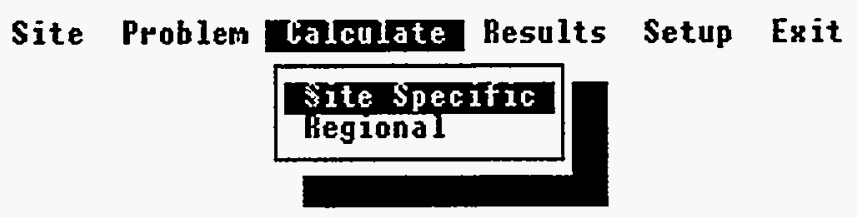

Figure 3.20 Calculate Sub-Menu

If there isn't enough information to perform a site-specific calculation, an error message (see Figure 3.21) is displayed and the user must provide the necessary information before the calculation can proceed.

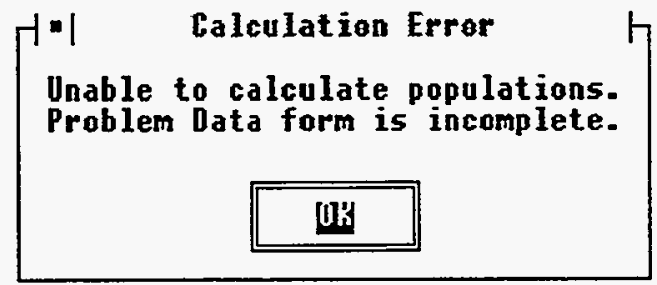

Figure 3.21 Calculation Error

After all of the forms have been sufficiently completed, the Calculate form (see Figure 3.22) will be displayed. To begin the site-specific calculation, select the Calculate button.

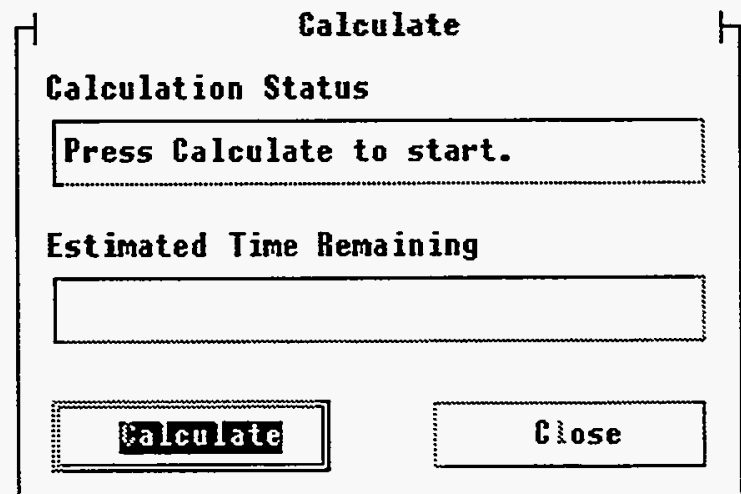

Figure 3.22 Initial Calculate Form 


\section{Calculate Form Fields}

The Calculate form consists of the following fields which are designed so that the user may monitor the progress of the calculation. All of the fields are filled in automatically by SECPOP 90 and cannot be edited by the user.

Calculation the present status of the calculation. Initially this field instructs the user to press the Calculate button.

Status Subsequent phases of the calculation display messages describing the phase of the calculation under way (see Figure 3.23).

Estimated Time an estimate of how much time remains before the calculation is complete.

Remaining

\section{Calculate Form Commands}

The Calculate form has two command buttons that are used to control the calculation and close the Calculate form. The commands are executed when the buttons are selected. The command buttons are as follows:

Calculate begin the site-specific calculation; and

Close stop the calculation if a calculation is under way. Note: the user is asked to confirm that the calculation should be stopped (see Figure 3.24). If a calculation is not under way, then this command closes the Calculate form.

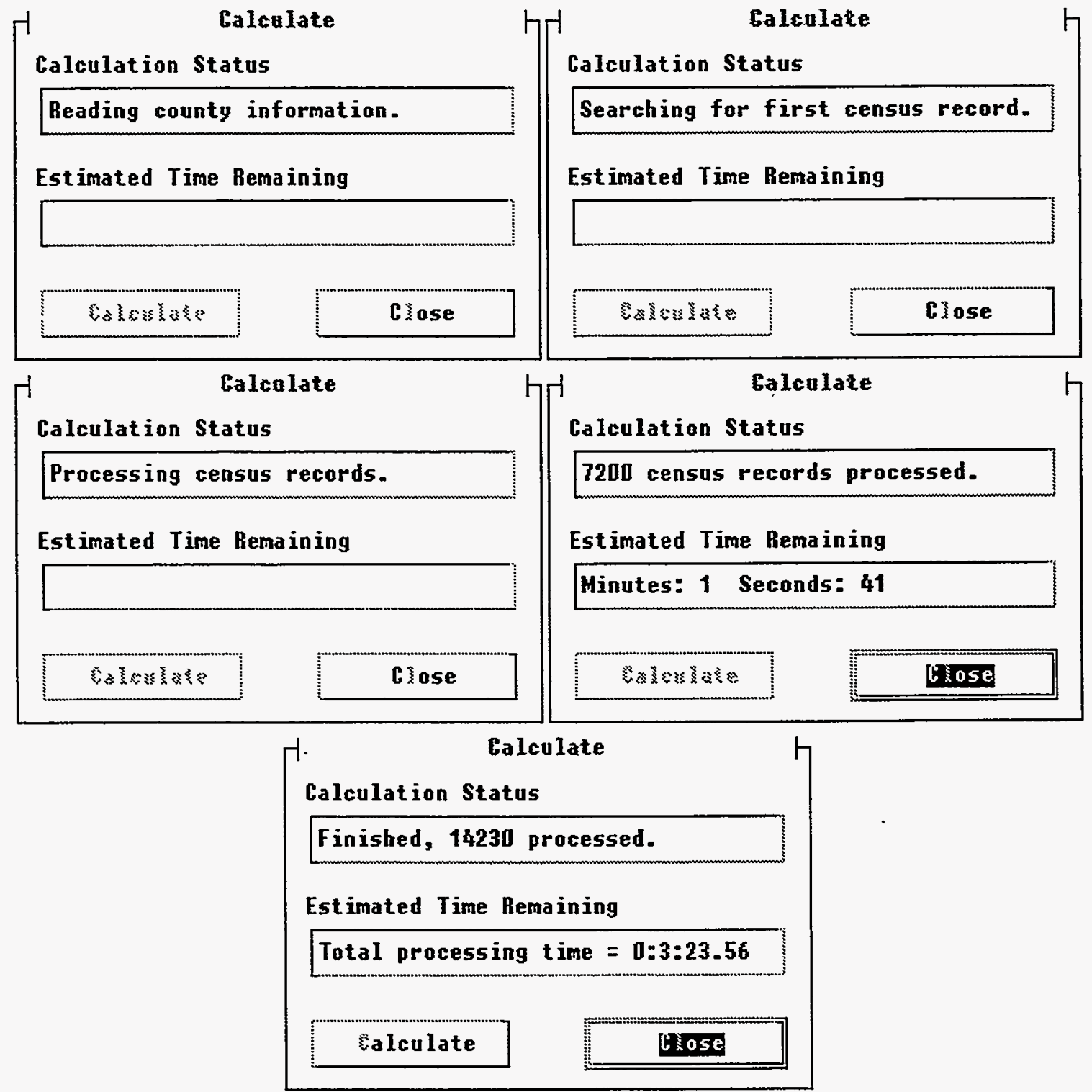

Figure 3.23 Subsequent Calculate Forms 


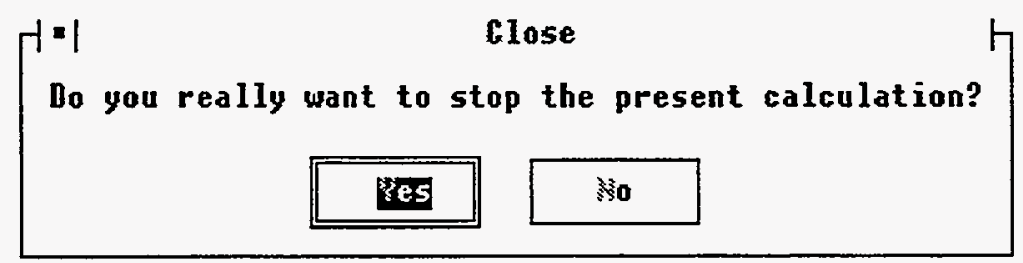

Figure 3.24 Stop Calculation

Note: the population estimates are based on over six million 1990 census block records and are as reasonable estimates as can be made for anywhere in the continental United States. The economic factor and land fraction estimates are aggregated at the county level. This makes these estimates coarser estimates than the population estimates. In addition the various notes and exceptions to the county aggregated data should be reviewed (see section 5.2.1 and Appendix C).

\subsection{Site-Specific Results}

There are several ways to display, print, or save the site-specific calculation results. The results are accessed by selecting the Results sub-menu from the main menu (see Figure 3.25). All of the commands and sub-sub-menus except for Regional apply to site-specific results and will be discussed below. Regional which applies to regional calculation, is discussed in section 3.7.

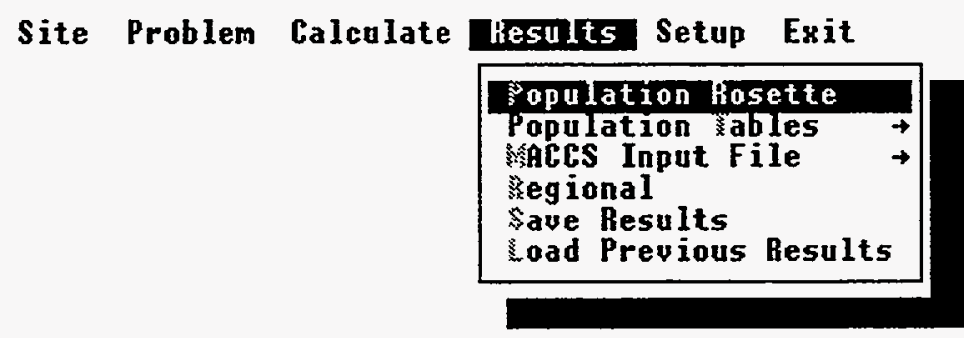

Figure 3.25 Results Sub-Menu

Population displays a rosette with populations per section;

Rosette

Population displays rosette populations in table formats;

Tables

MACCS Input displays or prints the MACCS Site Data File;

File

Save Results saves the calculated results; and

Load Previous loads previously saved calculated results.

Results

\subsubsection{Population Rosette}

Selecting Population Rosette from the Results sub-menu displays a rosette as shown in Figure 3.26 This is a graphics screen. There are no fields or commands since it is not a form. Pressing SPACE returns the user to the main menu. To save or print the rosette, see section 2.1

\subsubsection{Population Tables}

Selecting Population Tables from the Results sub-menu displays the Population Tables sub-sub-menu (see Figure 3.27). This menu provides the user with two methods for displaying the population results, the Population command which displays a table version of the rosette with the population values for each rosette section, and the Cumulative command which displays a similar table except that the population sums along each direction are displayed. 


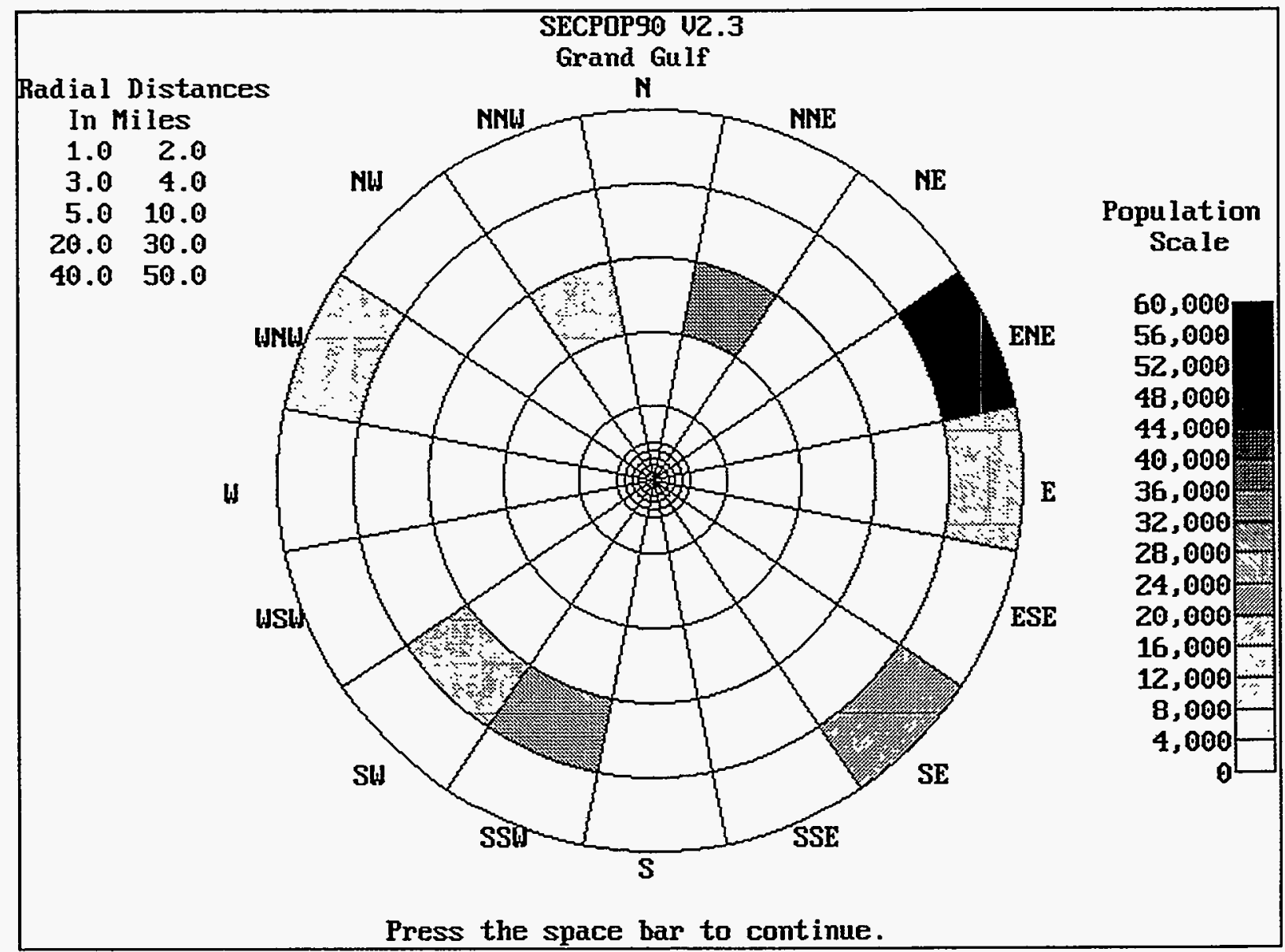

Figure 3.26 Population Rosette

Site Problem Calculate Hesurts Setup Exit

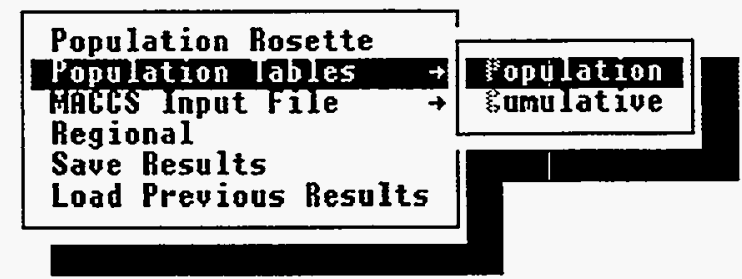

Figure 3.27 Population Tables Sub-Sub-Menu

\section{Population}

Selecting Population from the Population Tables sub-sub-menu displays the table shown in Figure 3.28. The population data are shown in a manner similar to the method used to display the economic regions on the Economic Regions form (see section 3.4.2). The table has no user input fields. There are two command buttons, Close and Print, that perform the following functions:

Close closes the Population Table form; and

Print print the population information to the selected printer or file. Selecting this command displays a Print form similar to Figure 3.6.

\section{Cumulative}

Selecting Cumulative from the Population Tables sub-sub-menu displays the table shown in Figure 3.29. The cumulative population data are shown in a manner similar to the method used to display the economic regions on the Economic 
Regions form (see section 3.4.2). The population is accumulated along each direction. This means that along each direction the radial population includes all of the population up to and including that radius for the given direction.

\begin{tabular}{|c|c|c|c|c|c|c|c|}
\hline$f=1$ & & & Populati & Table & & & \\
\hline & 1.0000 & $\begin{array}{l}\text { Radii } \\
2.0000\end{array}$ & $\begin{array}{l}\text { iles) } \\
\text { 3.0000 }\end{array}$ & 4.0000 & Sum & is & \\
\hline H & D & 21 & D & 0 & 2457 & 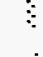 & \\
\hline HME & D & 0 & 0 & 0 & 40758 & o & $\times 1$ \\
\hline NE & 0 & 15 & 0 & 1 & 13649 & $\because$ & \\
\hline EHE & D & 36 & 0 & 74 & 67873 & $\because$ & Frint \\
\hline $\mathbf{E}$ & 2 & 0 & 8 & 80 & 19663 & & \\
\hline ESE & D & 21 & b & 5 & 15621 & & \\
\hline Sum & 2 & 103 & 18 & 228 & 318174 & 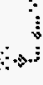 & \\
\hline
\end{tabular}

Figure 3.28 Population Table Form

For example, in Figure 3.29 the cumulative population at the 4.0 -mile radius along the eastern direction is 90 . This value includes the population of 2 found within the 1.0-mile radius, the population of 8 between the 2.0 and 3.0 -mile radii, and the population of 80 found between the 3.0 and 4.0 radii (see also Figure 3.28). The table has no user input fields. There are two command buttons, Close and Print, that perform the following functions:

Close closes the Cumulative Population Table form; and

Print prints the cumulative population information to the selected printer or file. Selecting this command displays a Print form similar to Figure 3.6.

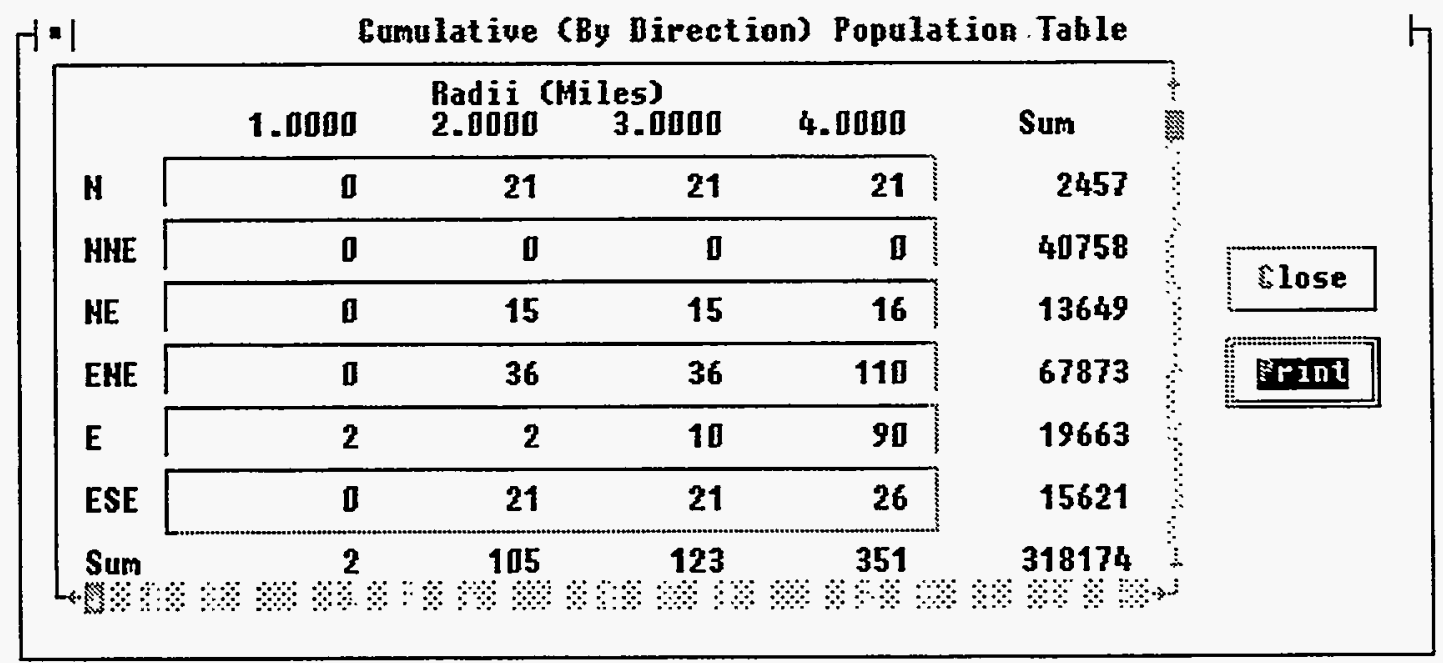

Figure 3.29 Cumulative Population Table Form

\subsubsection{MACCS Input File}

Selecting MACCS Input File from the Result sub-menu displays the MACCS Input File sub-sub-menu (see Figure 3.30). This menu provides the user with two methods for displaying the population results, the Display command which displays the MACCS Site Data File, and the Print command which prints the MACCS Site Data File to the selected printer or file.

\section{Display}

Selecting Display from the MACCS Input File sub-sub-menu displays the MACCS Site File form shown in Figure 3.31. There are no user input fields on this form. The user can scroll through the data by selecting the arrows. The command Close closes the form. The MACCS Input File is normally displayed as ASCII text. If the user has selected the Comma- 
Separated Variables (CSV) option in the Setup form (see section 3.9.1), then the data will be displayed in CSV format. All text strings will be enclosed in double quotes and all variables (including text strings) will be separated by commas. This format is compatible with the import data feature of most spreadsheet programs.

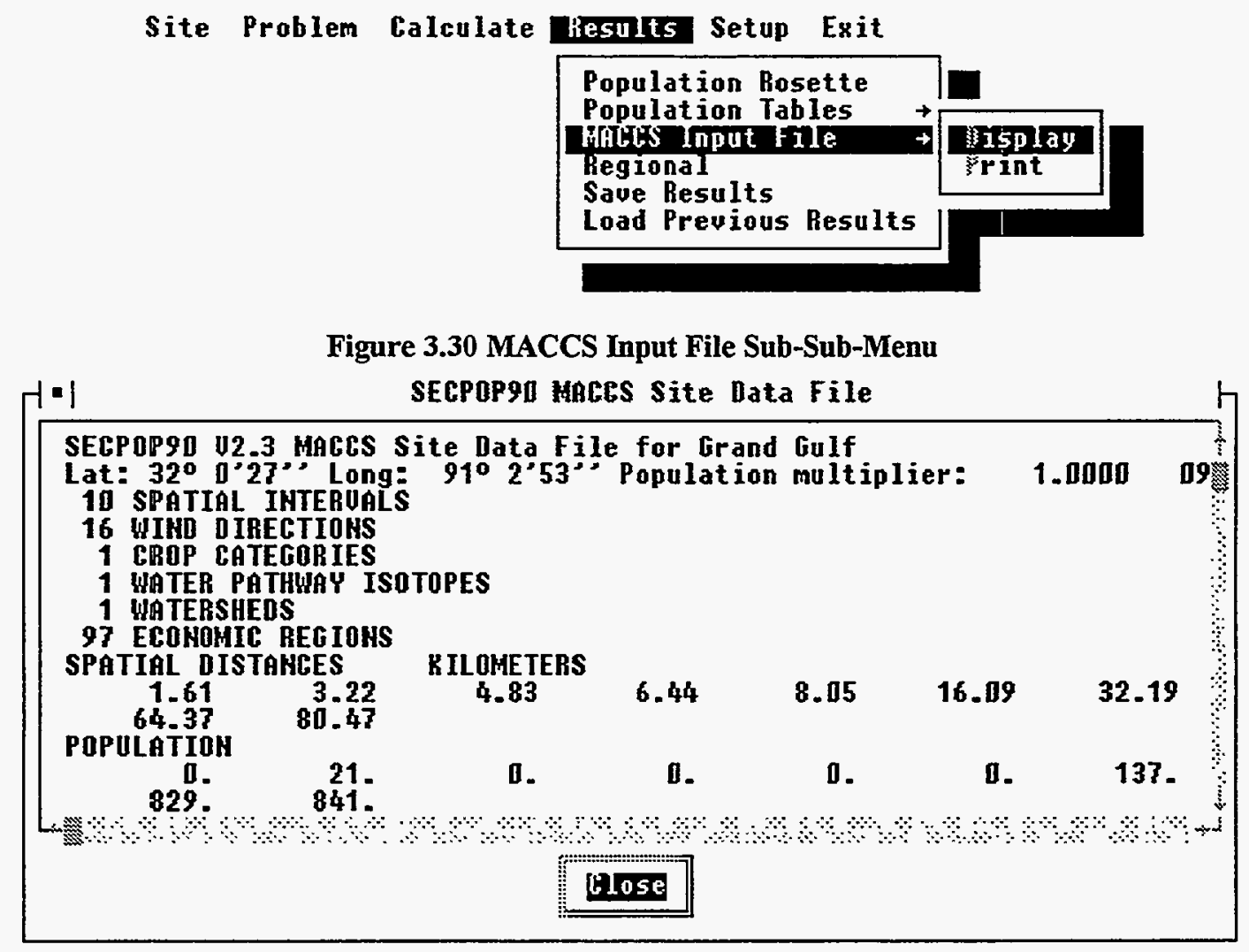

Figure 3.31 MACCS Site Data File Form

Print

Selecting Print from the MACCS Input File sub-sub-menu prints the MACCS Site Data File to the selected printer or file. Selecting this command displays a Print form similar to Figure 3.6. The MACCS Input File is normally printed as ASCII text. If the user has selected the Comma-Separated Variables (CSV) option in the Setup form (see section 3.9.1) then the data will be printed in CSV format. The following is an example MACCS Site Data File. Note: SECPOP90 does not perform any calculations for estimating crop categories, water pathway isotopes, or watersheds. The values below for those categories (watershed index, crop season and share, and watershed definition) are dummy values generated so that the file created will be completely compatible with MACCS. For a full explanation of all of the data fields, see Appendix B.

Table 3.1 Example MACCS Site Data File

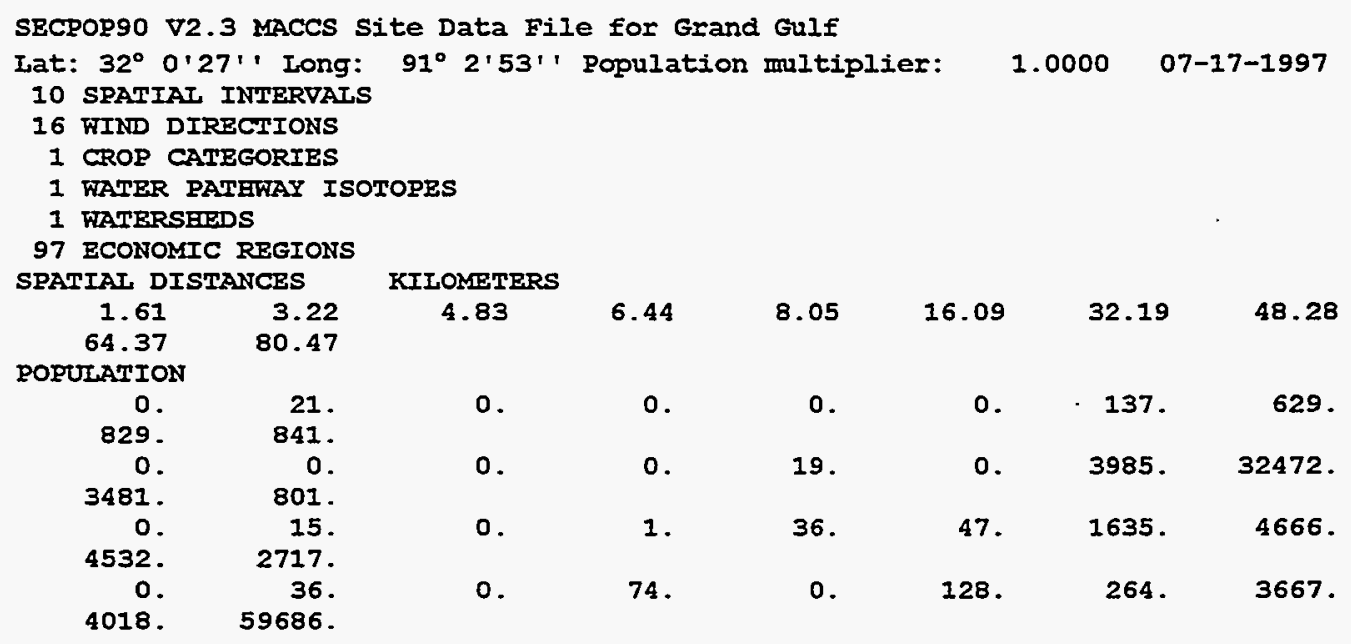




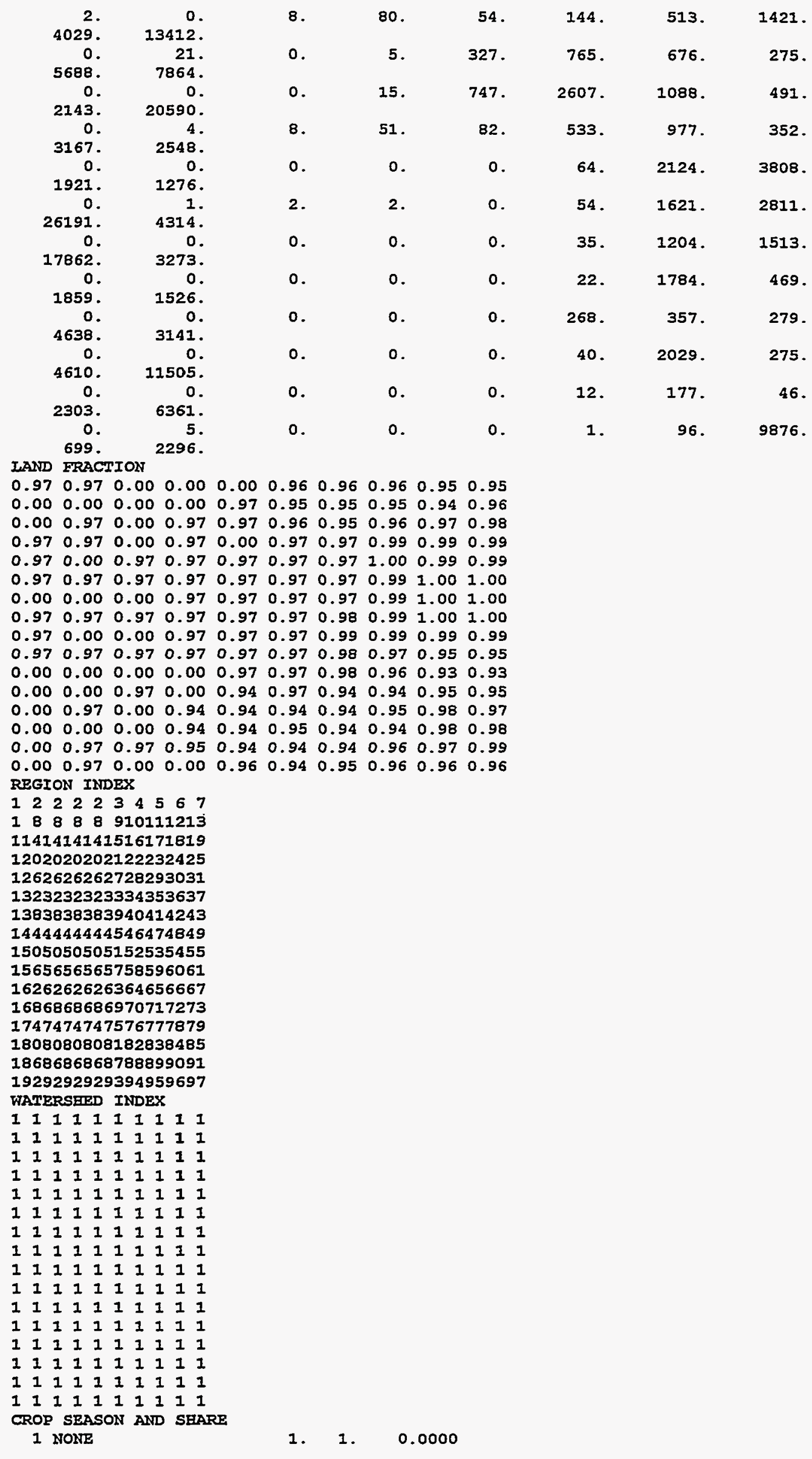




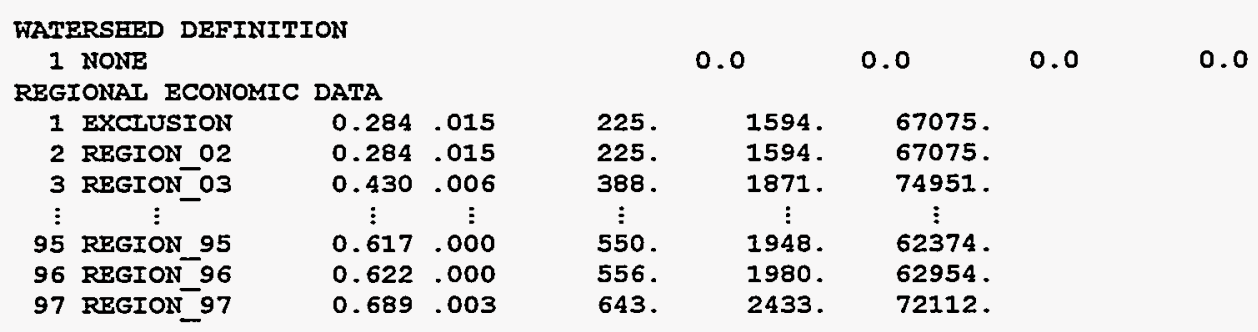

\subsubsection{Save Results}

Selecting Save Results from the Results sub-menu displays the form shown in Figure 3.32. This form allows the user to save the calculation results using a user-specified filename. The form has fields for selecting the appropriate disk drive, directory, sub-directories, and filename. Once these are chosen and the OK button is selected, the calculation results are stored in the specified file. It is recommended that the user save all result files with the filename extension "TXT." The user is not required to follow this practice. Doing so, however, will make it easier to find previously saved calculation results files. This is especially true when a single directory has a mix of site, problem, and results files. It will also allow third-party software to recognize the files as ASCII text files. This also holds true if the files are saved as CSV files (see section 3.6.3).

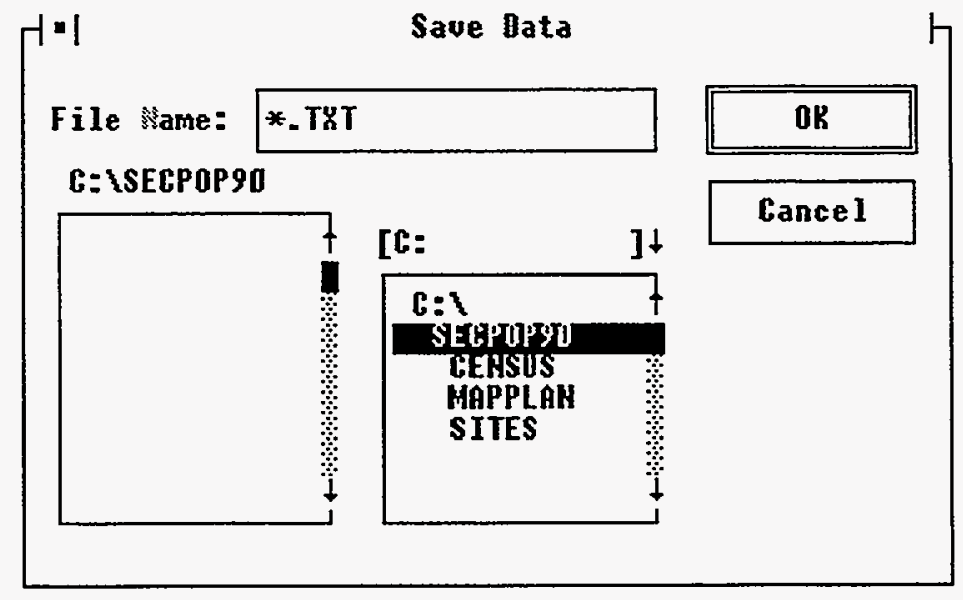

Figure 3.32 Save Data Form

\subsubsection{Load Previous Results}

Selecting Load Previous Results from the Results sub-menu displays the form shown in Figure 3.33. This form allows the user to select previousiy calculated results. The form has fields for selecting the appropriate disk drive, directory, subdirectories, and filename. Once these are chosen and the OK button is selected, the file is loaded into memory and the results can be examined using any of the methods described above.

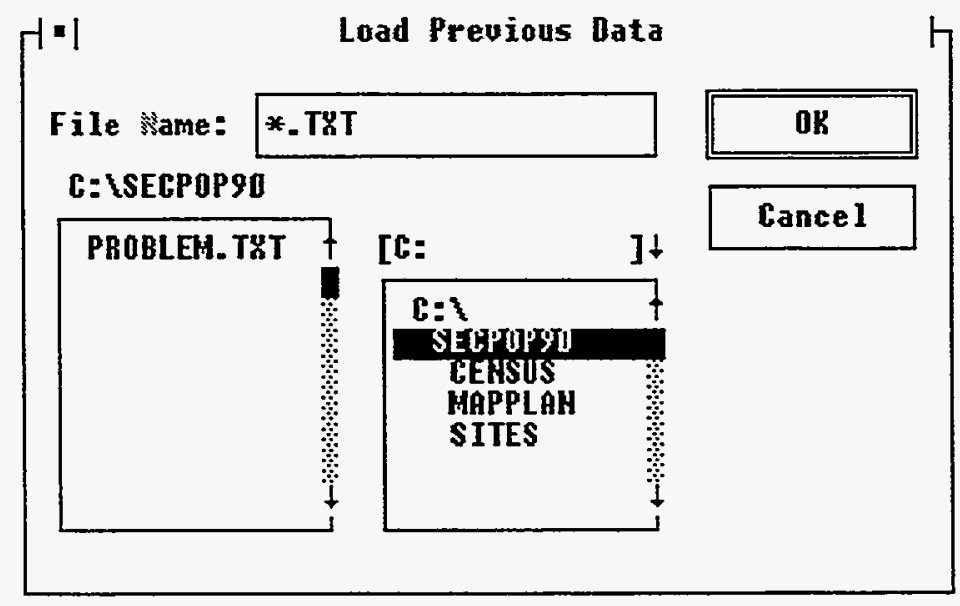

Figure 3.33 Load Previous Results Form 
Note: only results saved as ASCII text can be retrieved. Results saved in CSV format cannot be retrieved by SECPOP90. Also, since the results are saved in a format compatible with MACCS, the radial distances will be in kilometers. The radial distances can be changed back to miles by loading in the problem file for the results file after the results are loaded or opening the problem form after the results are loaded and changing the Radial Units to miles (see section 3.4.1). If the second method is employed, the user should review the radial distances and edit the values, if needed, to compensate for round off error. The user can close the Open Problem form by selecting Cancel, pressing ESC, or selecting Close from the control box sub-menu.

\subsection{Performing Regional Calculations}

A regional calculation may be performed by selecting the Calculate sub-menu (see Figure 3.34) from the main menu then selecting Regional from the Calculate sub-menu. Important note: in order for the regional calculations to be displayed and printed correctly, the MapPlan command from the Setup form (see section 3.9.2) must be run before the first regional calculation. After the MapPlan settings have been saved, it is no longer necessary to run the MapPlan setup command unless the computer's hardware configuration changes.

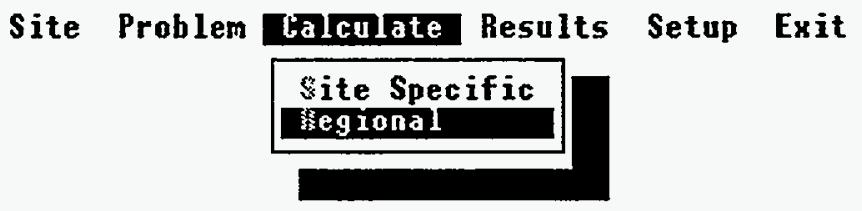

Figure 3.34 Calculate Sub-Menu

Selecting Regional opens the form shown in Figure 3.35. The Regional Calculation Setup form allows the user to enter all of the information necessary to perform regional calculations.

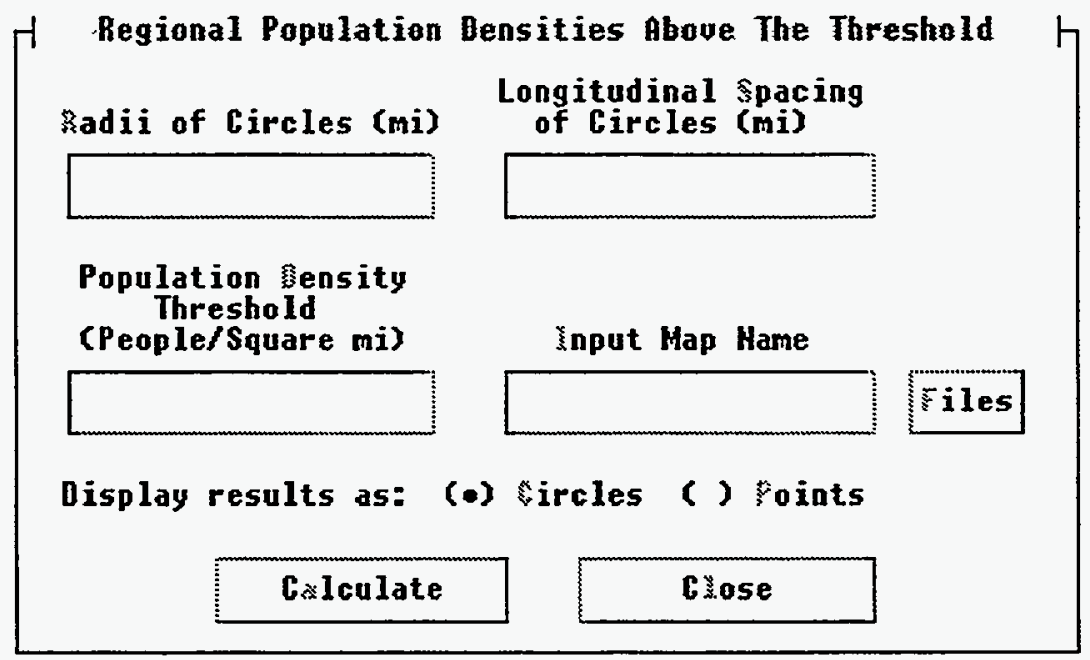

Figure 3.35 Regional Calculation Setup Form

\section{Regional Calculation Setup Form Fields}

The Regional Calculation Setup form (see Figure 3.35) consists of the following fields which are designed so that the user may easily enter all of the regional-calculation specific information (all of the fields are required to perform regional calculations):

Radii of Circles the outermost radii of the circles in miles [see (2) in Figure 3.36] used in the regional population density calculations. Population densities are calculated within each circle in the following manner: population densities are calculated for all persons located within $1,2,3, \ldots 20,30,40, \ldots 170$ miles of the center of each circle. (The last radii will be equal to Radii of Circles which has a minimum value of 1 and a maximum value of 170.) If any one of these population densities equal or exceed the Population Density Threshold, then a circle whose radii is equal to Radii of Circles will be drawn on the output map (see Figure 3.40). 
Longitudinal

Spacing of

Circles

Population

Density

Threshold

Input Map

Name

Display Results the longitudinal spacing of the circles in miles [see (3) in Figure 3.36]. The latitudinal spacing is half the longitudinal spacing and every other row of circles is offset by half of the longitudinal spacing. If the longitudinal spacing is set to twice the radii, then the region will be completely covered.

the population density threshold in people per square mile that when equal or exceeded will cause the circle in which the density was equaled or exceeded to be drawn on the output map [see (1) in Figure 3.36 and Figure 3.40].

the name of the map from the secpop 90 lmapplanlmaps directory (see Figure 2.1). There are maps for each state and its surrounding states (the format of the names are $n n$ _PLUS, where $n n$ is the state's postal abbreviation), the four NRC regions (the format of the names are NRC_REG $n$, where $n$ is from 1 to 4), and a map of the continental United States (the format of the name is CONUS). select Circles to draw circles on the output map (see Figure 3.40) or Points to draw only points at the center of all circles that equal or exceed the population density threshold.

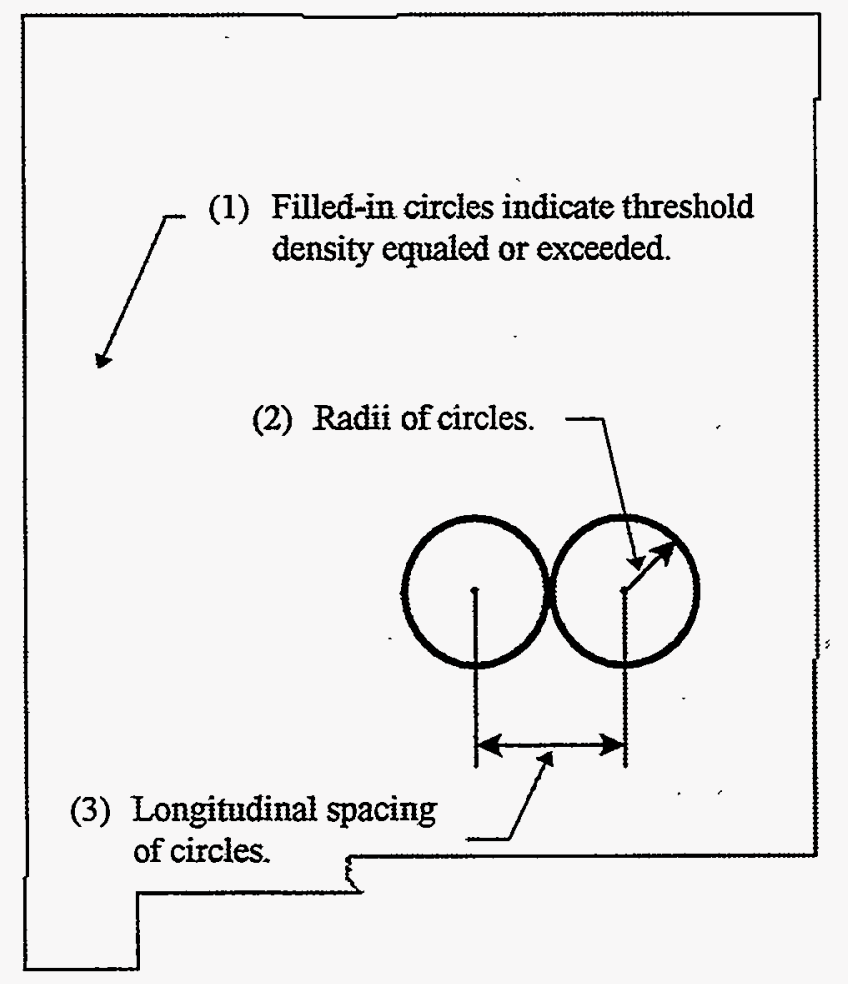

Figure 3.36 Radii and Longitudinal Spacing

\section{Regional Calculation Setup Form Commands}

The Regional Calculation Setup form has two command buttons. Both are located at the bottom of the form and are used to perform the regional calculations or close the Regional Calculation Setup form. The command buttons are as follows:

Calculate begins the regional calculations and displays the Regional Calculation Status form (see Figure 3.37); and

Close closes the Regional Calculation Setup form. 


\section{Regional Calculation Status Form}

There are two Regional Calculation Status forms. The first form displays the percent of the total number of circles calculated (see Figure 3.37). The second form displays the total time (hh:mm:ss.ss) for the calculations to complete (see Figure 3.38). This second form is displayed at the end of the calculations.

Important note: depending on the size of the circles and the size of the input map, calculations can last from minutes to several days! Try not to use very small circles with very large regions.

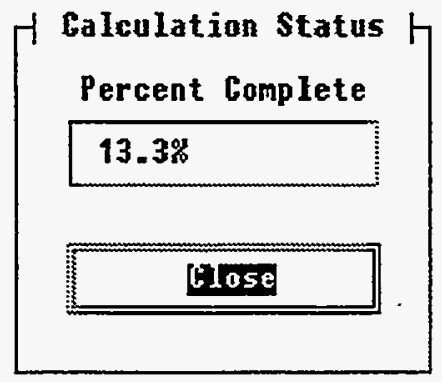

Figure 3.37 Regional Calculation Percent Complete

The command buttons on the Regional Calculation Status form control the calculation and close the Regional Calculation Status form. If the calculation is not complete and the user selects the Close button then the user is asked to confirm that he or she wished to stop the calculation (see Figure 3.24). If confirmation is received, the calculation stops; otherwise, it continues. If the calculation has completed and the user selects the Continue button; then the output map is displayed (see Figure 3.40).

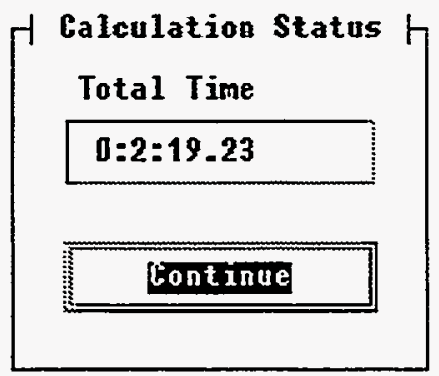

Figure 3.38 Regional Calculation Total Time

\subsection{Regional Calculation Results}

Selecting Continue from the Regional Calculation Status form or Regional from the Results sub-menu (see Figure 3.39) displays the Map Output form (see Figure 3.40). This is a MapPlan form which displays the most recent regional calculation results. The selected input map is displayed overlaid with the circles in which the population density threshold specified in the Regional Calculation Setup form (see Figure 3.35) was equaled or exceeded.

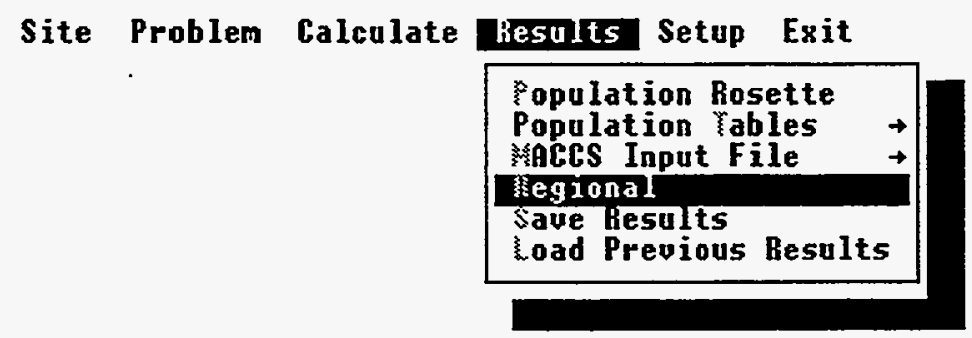

Figure 3.39 Results Sub-Menu

At the top of the output map display is the MapPlan menu bar that operates in a manner similar to the main menu of SECPOP90. For the purposes of SECPOP90, only the File sub-menu of the MapPlan menu bar will be discussed. Other submenus can be accessed and help can be obtained by highlighting the desired selection and pressing the Fl key. The user is warned, however, that the version of MapPlan which is distributed with SECPOP90 lacks the features necessary to save output files that have been changed. 


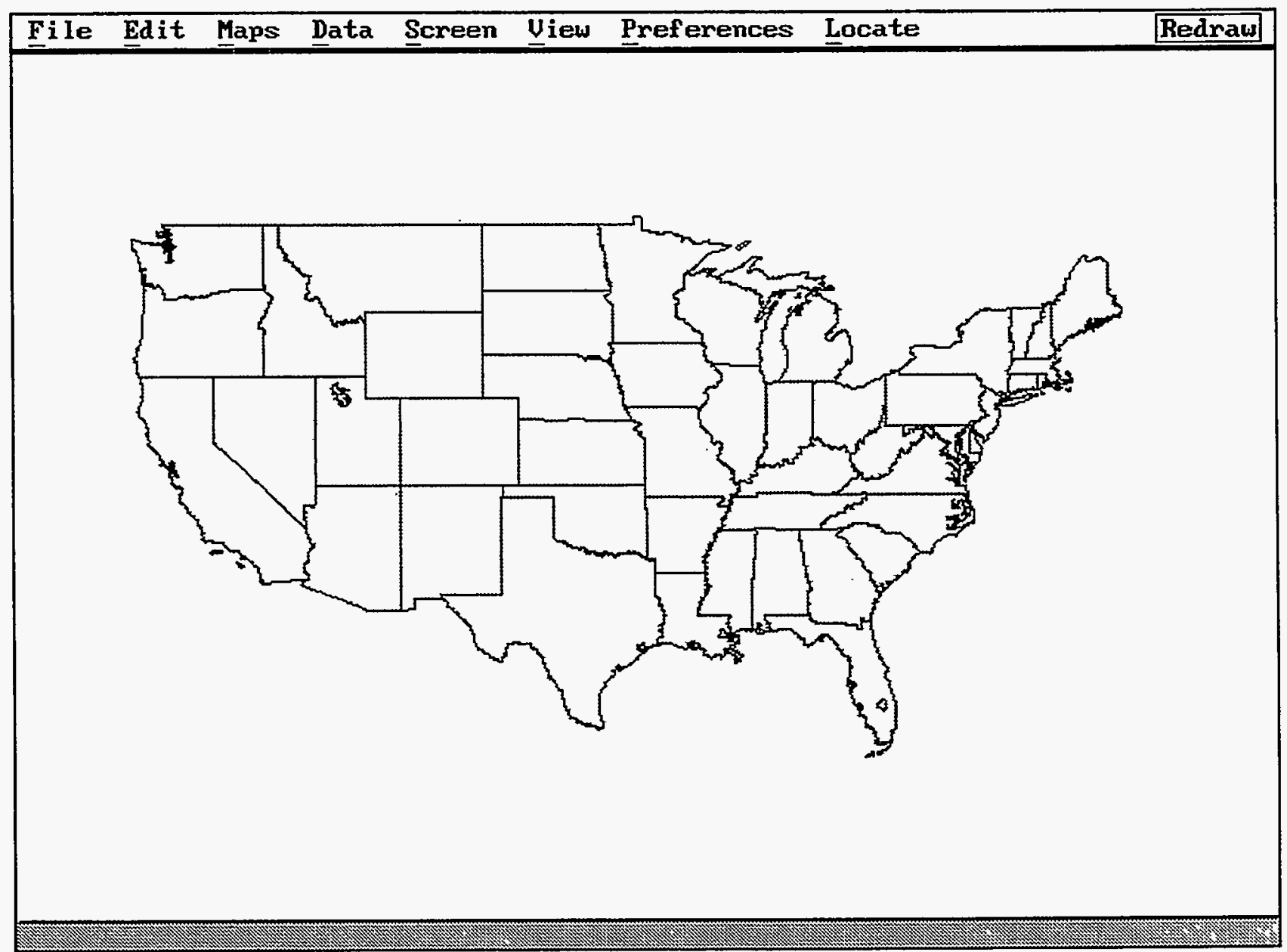

Figure 3.40 Output Map

\section{MapPlan File Sub-Menu}

The MapPlan File sub-menu (see Figure 3.41) has four available commands. The commands are as follows:

\begin{tabular}{l|lll}
\hline File Edit Maps Data Screen Uiew Preferences Locate & Redraw \\
\hline Export CGM & & & \\
\hline Load map & & \\
Print & &
\end{tabular}

Figure 3.41 MapPlan File Sub-Menu

Export CGM exports the output map in Computer Graphics Metafile (CGM) format. The CGM file can then be imported or inserted by other graphics or presentation software. When selected, the Export CGM File form appears (see Figure 3.42) and prompts the user for the filename. It is recommended that the complete pathname be given. If only the filename is given then, the file will be written into the secpop90lmapplan directory. It is important to note that because of the limitations of this version of MapPlan, the map output will be overwritten the next time a regional calculation is executed. The only way to save the output is by exporting it as explained above or saving the screen image as discussed in section 2.1.

Load map loads a previously defined MapPlan map. The maps can be found in the secpop90lmapplanlmaps sub-directory. The maps available are described in section 3.7. The maps are selected by a form similar in function to Figure 3.33. After the map is loaded, it can be exported as a CGM file or printed. 


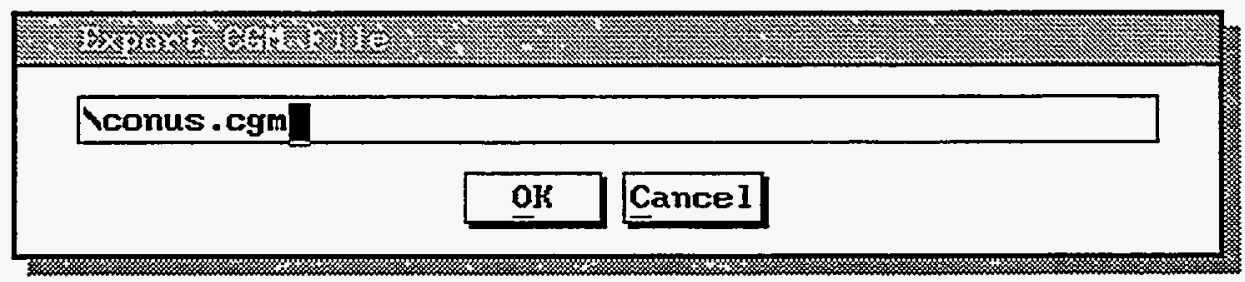

Figure 3.42 Export CGM File Form

Print prints the present output map to the printer. Selecting this command opens the Print Current Image form. The current device is the selected during the MapPlan setup process (see section 3.9.2). The Print Current Image form allows the user to select the paper size - legal or letter; print orientation - portrait or landscape; print resolution - low, medium, or high; the number of copies; and, if the printer supports it, color printing.

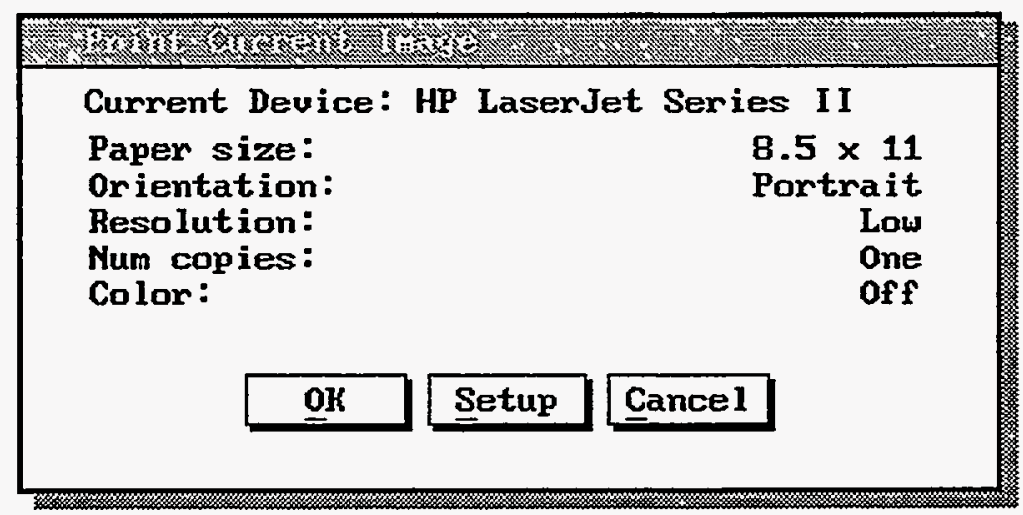

Figure 3.43 Print Current Image Form

Selecting OK starts the print job. Selecting Cancel cancels the print job if it has not started (there is no way to cancel a print job in progress from MapPlan). Selecting Setup displays the Print Setup form (see Figure 3.44). The Print Setup form allows the user to select the printer output port parameters.

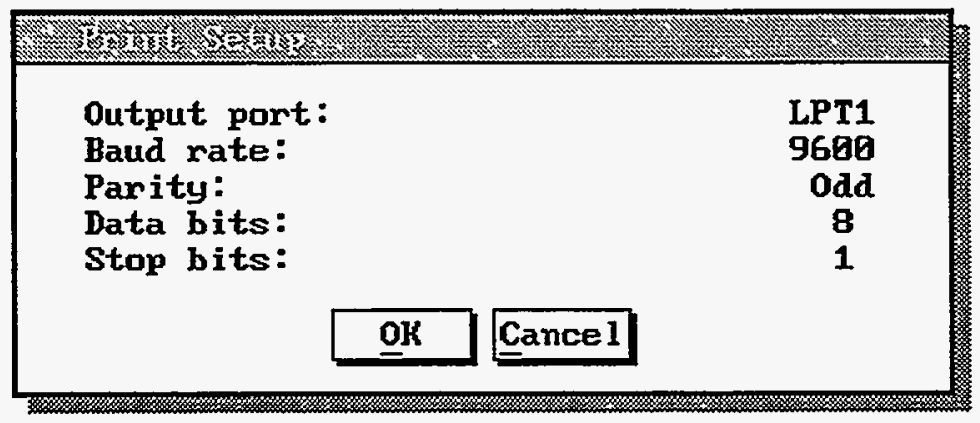

Figure 3.44 Print Setup Form

Quit

exits the regional calculation output map display and returns to the SECPOP90 main screen.

\subsection{Customizing SECPOP90}

Selecting Setup from the main menu displays the Setup form shown in Figure 3.45 This form allows the user to customize various SECPOP90 file paths and the type of site-specific calculation results output that will be generated. 


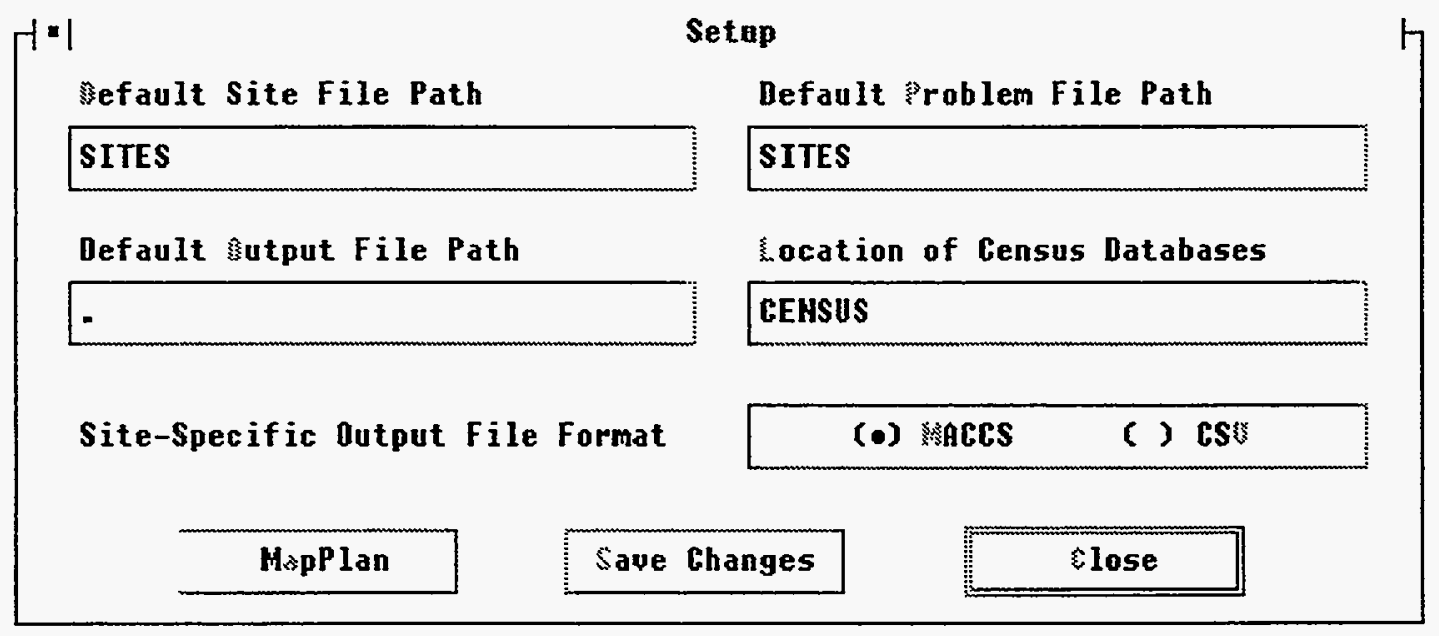

Figure 3.45 Setup Form

\subsubsection{Setup Form Fields}

The Setup form has six fields designed to allow the user to specify where all of the important input and output files are to reside. The user can override many of these values using the File Open and Save As forms discussed earlier but, these pathnames will normally be set to the paths that the user uses most often. For more information about pathnames, see your operating system manual.

Default Site File the default path where site files are located. Relative pathnames start at the directory where Path SECPOP90 is being executed.

Default Problem the default path where problem files are located. Relative pathnames start at the directory where File Path SECPOP90 is being executed.

Default Output the default path where output files will be saved. Relative pathnames start at the directory where File Path SECPOP90 is being executed.

Location of

Census

Databases the default path where the block-level population and county-level land fraction and economic census databases are located. Relative pathnames start at the directory where SECPOP 90 is being executed.

Output File

Format select MACCS to save the site-specific calculation results in MACCS Site Data File compatible format. Select CSV to save the results in comma-separated variable format. This format encloses all text strings within double quotes and separates all variables (including text strings) with commas. Both file formats are ASCII text. The differences between these two formats are illustrated by Table 3.2 .

\subsubsection{Setup Form Commands}

The Setup form has three command buttons: one for executing the MapPlan setup program, one for saving the user-defined defaults, and the other to close the Setup form.

MapPlan

Save Changes executes the MapPlan setup program for specifying the graphics mode, printer type, and printer output options for the MapPlan regional calculation output. This program does not support the mouse. Selections are made using the letters $g, p, 0$, and $e$, along with the arrow keys and the return key. ESC may be pressed at any time to cancel the last operation. Pressing the letter $g$ allows the user to select the graphic display adapter. Pressing the letter $p$ allows the user to select the output printer or plotter. Pressing the letter 0 allows the user to set up the output printer port. Pressing the letter e saves the MapPlan setup information and returns the user back to the SECPOP90 main screen.

immediately changes the default paths, file locations, and file formats. It saves the changes to a special SECPOP90 configuration file. This file will be read the next time SECPOP90 is executed and the default values that have been set by the user will be restored. 
Table 3.2 MACCS and CSV Formats

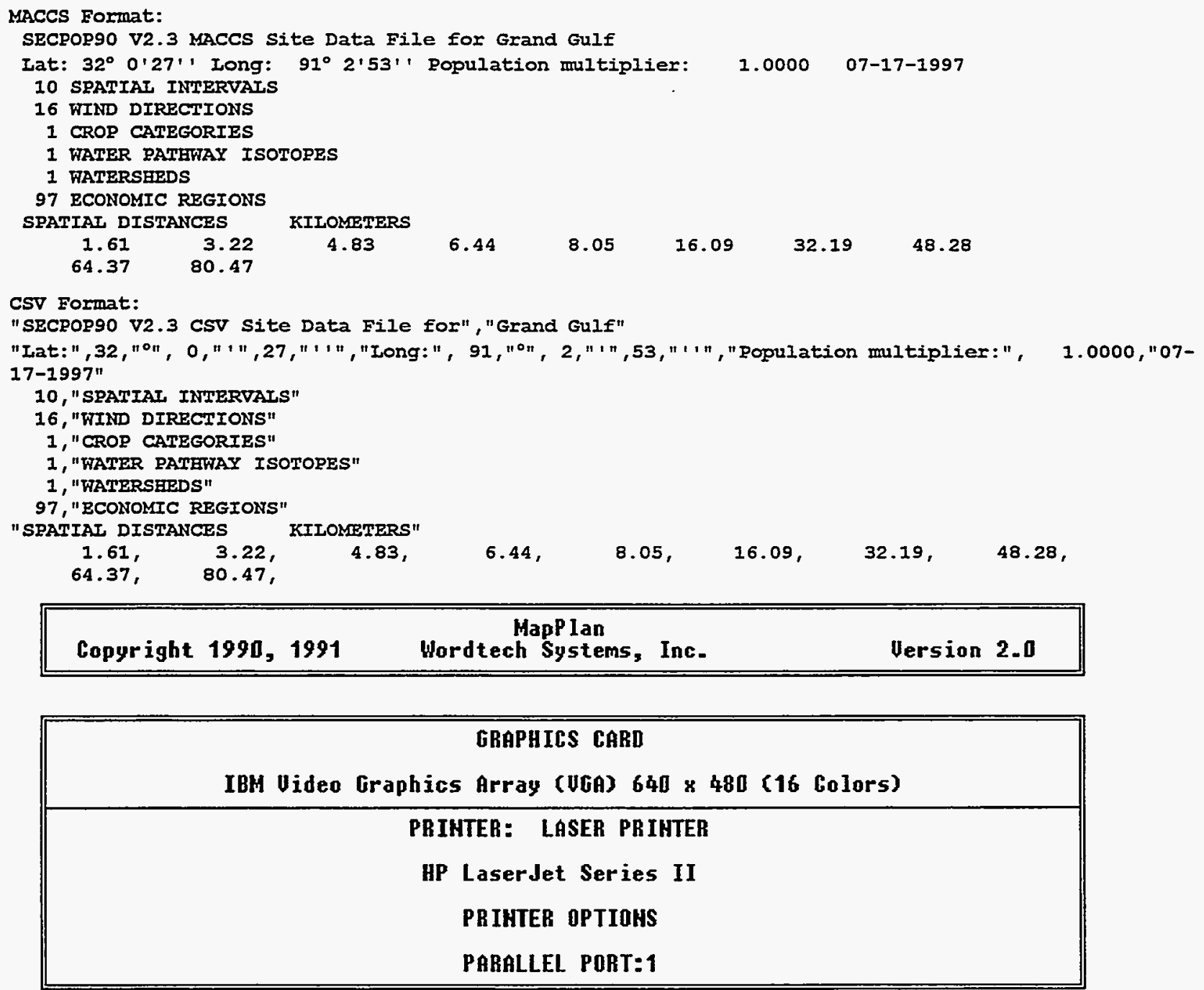

\begin{tabular}{|c|}
\hline GRAPHICS CARD \\
IBH Uideo Graphics Array (UGA) $640 \times 480(16$ Golors) \\
\hline PRINTER: LaSER PRIHTER \\
HP LaserJet Series II \\
PRIHTER OPTIOHS \\
PARALLEL PORT: 1 \\
\hline
\end{tabular}

Change Graphics Card, Printer, Options, or Exit (G/P/O/E)?

Press [Esc] to Quit SETUP without saving changes.

Figure 3.46 MapPlan Setup Form

\subsection{Exiting SECPOP90}

Selecting Exit from the main menu displays the Exit sub-menu (see Figure 3.47). Two commands are available in the Exit sub-menu, DOS Shell and Exit. These commands will take the user back to the DOS shell either temporarily or permanently.

\section{Site Problem Calculate Results Setup Exit}

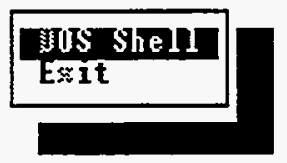

Figure 3.47 Exit Sub-Menu

DOS Shell execute a temporary DOS shell; and

Exit quit the SECPOP90 program: 


\subsubsection{DOS Shell}

Selecting DOS Shell executes a DOS shell, temporarily suspending SECPOP90 and gives the user a DOS prompt (see Figure 3.48). Select this if you wish to do something in DOS but you don't want to stop the program and lose all your information. This is useful for doing such things as printing files, making directories, copying files, etc. Type exit from the DOS prompt to return to SECPOP90. Care should be taken before executing this command if you are not running from DOS. Consult your operating system manual.

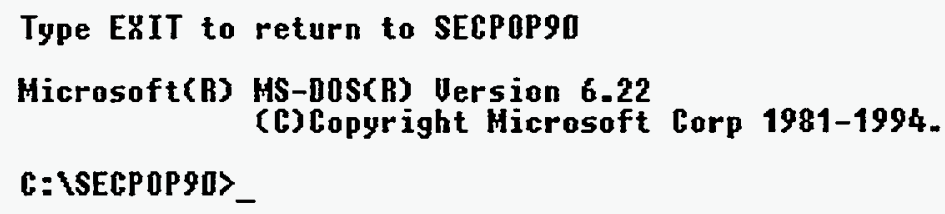

Figure 3.48 The DOS Shell

\subsubsection{Exit}

Selecting Exit will exit the SECPOP90 program. The user will be asked to confirm whether the SECPOP90 program should be exited (see Figure 3.49). Exiting SECPOP90 will cause all unsaved information to be lost.

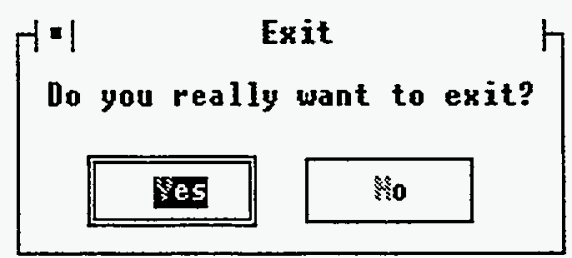

Figure 3.49 Exit Confirmation Form 


\section{COMPUTATIONAL METHODOLOGY}

The SECPOP90 code uses the 1990 block-level census data to calculate the population counts for each of the user-defined MACCS grid (rosette) sections, 1990 county-level census data to calculate land fraction data, and 1990 county-level census data and 1992 county-level agricultural census data to calculate economic data. The methodology involved can be broken down into (1) the algorithms used within the code to accomplish this purpose and (2) the database structures used for the block and county-level databases. Sections 4.1 through 4.3 discuss the algorithms in detail. Section 4.4 discusses the database structures.

\subsection{Population Count and Density Algorithms}

The SECPOP90 code uses the user-defined MACCS grid for a user-defined site to establish a set of longitude-latitude boundaries within which the grid lies. The census data file is a binary file sorted primarily by descending longitude (west to east) and secondarily by descending latitude (north to south). An iterative algorithm is used (within the POINTR subroutine) to find the westernmost point in the census data file which lies on or to the east of the western longitude boundary. For that data point and each subsequent data point read from the census data file, it is determined if the point lies between the north and south latitudinal boundaries. When a point is found to lie between the established boundaries, the distance of that point from the site is calculated using the distance formula to determine if in fact the point lies within the outer limit of the MACCS grid. If the point meets the distance criteria, it is then processed to determine the exact grid element in which it lies based on its radial distance and direction from the site. The population associated with that data point is then added to the population in the appropriate element of the array TOTPOP. The array TOTPOP contains an array element for each grid section.

The algorithm used to determine the longitude-latitude boundaries of the grid will be discussed in section 4.1.1 The iterative algorithm used to determine the westernmost point in the census data file lying on or to the east of the western longitude boundary is discussed in section 4.1.2 Finally, the algorithms used to determine if a data point lies within the MACCS grid and to determine the specific grid element within which any point lies will be discussed in section 4.1.3.

Regional calculations use all of the same algorithms described below except that there is no need to determine where in the circle being calculated the population lies. The population density is calculated directly using the population and area of each census block.

\subsubsection{Boundaries Algorithm}

The heart of the algorithm lies within the subroutine GETDIS which finds the distance $(\mathrm{km})$ per degree latitude and distance per degree longitude for a specific geodetic latitude. Within the GETDIS subroutine, the geodetic latitude is first converted to radians. The corresponding latitude in the master coordinate system, geocentric, is then calculated using the following equation

$$
\theta_{\mathrm{GC}}=\tan ^{-1}\left[\left(\frac{R_{e p}}{R_{e q}}\right)^{2} \tan \theta_{\mathrm{GD}}\right]
$$

where

$\begin{array}{ll}\theta_{G C} & =\text { the geocentric latitude, } \\ \mathrm{R}_{\mathrm{ep}} & =\text { the earth polar radius }(\mathrm{km}), \\ \mathrm{R}_{e q} & =\text { the earth equatorial radius }(\mathrm{km}), \text { and } \\ \theta_{\mathrm{GD}} & =\text { the geodetic latitude. }\end{array}$

The geocentric radius, $\mathrm{R}_{\mathrm{GC}}$, is then calculated using the equation:

$$
R_{G C}=\frac{R_{e q} R_{e p}}{\sqrt{R_{e q}^{2} \sin ^{2} \theta_{G C}+R_{e p}^{2} \cos ^{2} \theta_{G C}}}
$$

The distance $(\mathrm{km})$ per degree latitude, DPDLAT, and the distance $(\mathrm{km})$ per degree longitude, DPDLON are derived using the following equations: 


$$
\begin{aligned}
& \text { DPDLAT }=R_{G C} \frac{\sin \theta_{G C}}{\sin \theta_{G D}} \cdot\left(\frac{\pi}{180}\right) \\
& \text { DPDLON }=R_{G C} \cos \theta_{G C} \cdot\left(\frac{\pi}{180}\right)
\end{aligned}
$$

When finding the west and east longitude boundaries of the grid, the following assumptions are made and can be shown to be valid. If the west-east boundaries are established on a "horizontal" circumference of the earth through the site, the entire circular MACCS grid will lie between those same two longitudinal boundaries.

The distance to those longitude boundaries can be found by dividing the greatest radial distance on the MACCS grid by DPDLON, the distance per degree longitude at the site geodetic latitude.

Finding the north and south latitude boundaries is somewhat more complex. The distance per degree latitude decreases with increasing latitude. To make sure that the boundaries set will encompass the entire grid, distance per degree latitude, DPDLAT, is determined at the Tropic of Cancer for the half of the grid lying below the latitude of the site, and the distance per degree latitude, DPDLAT, determined at the site is used for the half of the grid north of the site latitude. As a result of this conservative approach, the north and south boundaries are well outside the grid, but this is acceptable since the boundaries are used only to determine which of the census data points will undergo further processing to determine if in fact they do lie within the grid.

\subsubsection{First Element Location Algorithm}

An iterative "divide and conquer" algorithm is used in the subroutine POINTR to determine the first element in the census data file which lies on or to the east of the western longitude boundary of the MACCS grid. The set of records in the census data file is divided into two parts, and it is determined in which part the western boundary lies with the procedure then being repeated concentrating on that part of the set. This process is continued until one of two situations occurs. If two adjacent points are found which straddle the boundary, the easternmost record is marked. If a point is found which lies on the western boundary, the records are searched backward sequentially until the first of the data elements is found which lies on that boundary and that point is marked.

\subsubsection{Specific Grid Element Determination}

When a census data element is found to lie within the longitude and latitude boundaries of the grid, both the distance per degree latitude, DPDLAT, and distance per degree longitude, DPDLON, are determined for the geodetic latitude of that data element. An average distance per degree latitude is determined between the census data element and the site. This average is used to calculate an actual distance in kilometers separating the latitudes of the element and the site. In turn, the distance in kilometers between the longitude of the element and the longitude of the site at the latitude of the element is calculated. The distance formula is then applied to determine the straight line distance between the site and the element. If the distance is less than the maximum user-defined radial distance, then each of the radial elements is examined to determine in which radial element the data element lies.

Once it is determined that a data element lies within the MACCS grid, it is then necessary to determine in which of the 16 directional grid elements the data element lies. The angle a line from the site to the data element makes with north is found as follows:

$$
\theta_{\mathrm{de}}=\tan ^{-1}\left(\frac{\mathrm{x}}{\mathrm{y}}\right)
$$

where

$\theta_{\mathrm{de}}$

$\mathbf{x}$

y
$=$ the angle made between a line from the site to the data element and true north,

$=$ the distance from the longitude of the data element to longitude of the site, and

$=$ the distance from the latitude of the data element to the latitude of the site.

This value, $\theta_{\text {de }}$, is then used to determine the specific directional element in which the data element lies. 


\subsection{Land Fraction Algorithms}

In addition to location and population, every record in the block-level database also includes the area of the block and a code to indicate which county in the U.S. the block resides. This additional information is used by both the land fraction algorithm and the economic factors algorithms to estimate land fractions and economic factors respectively.

A county-level database also exists that contains the land fraction data for every county in the continental U.S. These data were obtained from the same 1990 census data files as the block-level data (PL 94-171).

The area of the blocks cannot be used to determine section land fractions directly for two reasons. First, the area given is only the land mass - no area for the water mass is given at the block level. Second, there is no simple way to aggregate the block areas to determine how much of a rosette section they "fill up" since the geometry of the blocks is unknown. Instead the area of the blocks is used to weight the county-level land fraction data.

During a site-specific calculation, a running sum for each rosette section is made of the total area of the blocks that lie within each section and a running sum is made of all of the weighted land fraction data. At the end of the calculation, the sum of the weighted land fractions is divided by the sum of the block areas. This is equivalent to the following formula for the land fraction for rosette section $\mathrm{i}, \mathrm{j}$ :

$$
\operatorname{FRCLND}(i, j)=\frac{\sum_{n} A_{\text {Block }} \times \operatorname{FRCLND}\left(\text { County }_{\text {Block }}\right)}{\sum_{n} A_{\text {Block }}}
$$

where
$\operatorname{FRCLND}(i, j)$ $=$ the estimated land fraction for the rosette section defined by the ith direction and $j$ th and
$j$ th -1 radii (unless $j=1$, then the section is defined only by $i$ and $j-$ i.e., the innermost
sections of the rosette),
FRCLND(County ${ }_{\text {Block}}$ ) = the land fraction of the county that the present census block resides in,
A Block
$=$ the area of the present census block, and
n
$=$ the number of census blocks that reside in section $i, j$ of the rosette.

Note: in areas where census blocks tend to be large - lakes, desert, national and state parks - it is possible that a census block centroid will not lie within a rosette section and the resulting land fraction will be estimated to be 0 . While this is appropriate in a lake, it does not represent desert regions accurately. The user may need to edit the output data manually in such cases to obtain a better estimate.

\subsection{Economic Factors Algorithms}

SECPOP90 estimates the economic factors that are defined in MACCS Site Data File. The economic factors are calculated for the user-defined (or default) economic regions. The region inside the innermost radius is normally considered to be the exclusion region; while SECPOP90 calculates values for this region they are likely to be quite a bit different from the real values for the exclusion area if that area contains most of the nuclear power reactor site.

The algorithm used to calculate the economic factors is very similar to that used in section 4.2. The only difference is that the values are accumulated for each economic region instead of each rosette section. Likewise the county-level database has values for each economic factor for each county. Each Economic Factor (EF) for each economic region i is calculated using the following algorithm:

$$
E F(i)=\frac{\sum_{n} A_{\text {Block }} \times E F\left(\text { County }_{\text {Block }}\right)}{\sum_{n} A_{\text {Block }}}
$$

where
$\mathrm{EF}(\mathrm{i})$
= the estimated economic factor for the ith economic region,
EF(County Block)
$=$ the economic factor of the county that the present census block resides in,
A $_{\text {Block }}$
$=$ the area of the present census block, and
n
$=$ the number of census blocks that reside in economic region $\mathrm{i}$. 
The economic factors estimated and the sources of the data are as follows:

FRMFRC Fraction of land devoted to farming in the region; 1992 Census of Agriculture, Geographic Area Series 1B, County-Level Data, U.S. Department of Commerce, Economics and Statistics Administration, Bureau of the Census.

DPF Fraction of farm sales resulting from dairy production in the region; 1992 Census of Agriculture, Geographic Area Series 1B, County-Level Data, U.S. Department of Commerce, Economics and Statistics Administration, Bureau of the Census.

ASFP Annual average farm sales for the region (\$/hectare); 1992 Census of Agriculture, Geographic Area Series 1B, County-Level Data, U.S. Department of Commerce, Economics and Statistics Administration, Bureau of the Census.

VFRM Average farmland value for the region (\$/hectare); 1992 Census of Agriculture, Geographic Area Series 1B, County-Level Data, U.S. Department of Commerce, Economics and Statistics Administration, Bureau of the Census.

VNFRM Average nonfarm value for the region (\$/person); MACCS methodology using 1990 information from the 1993 and 1994 Statistical Abstract of the United States, U.S. Department of Commerce, Economics and Statistics Administration, Bureau of the Census; and 1990 county per capita income from 1994 County and City Data Book, U.S. Department of Commerce, Bureau of the Census, Data User Services Division.

\subsection{Database Structure}

The two main databases used in SECPOP90 are the block-level census and county-level census databases, CENSUS90.DAT and COUNTY90.DAT The block-level census database contains over six million records - a record for every census block in the continental U.S. The county-level census database contains over three thousand records, one for every county in the continental U.S. The sections below describe the databases in more detail.

\subsubsection{Block-Level Database}

The 1990 block-level census data is stored in a binary file, CENSUS90.DAT, which contains 6,660,337 records. Each record is 12 bytes long and contains the following 5 pieces of information:

(1) a 2-byte integer code for the longitude of the geometric centroid of the census block,

(2) a 2-byte integer code for the latitude of the geometric centroid of the census block,

(3) a 2-byte integer for the residential population that resides within the census block,

(4) a 4-byte integer for the land area $\left(0.001 \mathrm{~km}^{2}\right)$ of the census block, and

(5) a 2-byte integer code for the county that the census block resides in.

The integer codes for the longitude and latitude were derived to reduce the storage required for the block-level database. The longitude can be calculated using the following formula:

$$
\text { longitude }=(\text { integer_code }+91993) / 1000.0
$$

The latitude can be calculated using the following formula:

$$
\text { latitude }=(\text { integer_code }+16610) / 1000.0
$$

The integer code for the county is the index to the county-level data base and is the first field in each record of that database. More information on the county-level database can be found in the next section. Details on how the block-level database was constructed and verified can be found in section 5.1.

\subsubsection{County-Level Database}

The 1990 and 1992 county-level census data is stored in a fixed-width format ASCH text file, COUNTY90.DAT, which contains 3,111 records and one header line. Each record is one line long and contains the following 10 pieces of information:

CountyCode

State the index to the county-level database.

the two-letter abbreviation of the state in which the county lies; 


$\begin{array}{ll}\text { CountyName } & \text { the name of the county; } \\ \text { FRCLND } & \text { the fraction of area that is land in the county; } \\ \text { FRMFRC } & \text { fraction of land devoted to farming in the county; } \\ \text { DPF } & \text { fraction of farm sales resulting from dairy production in the county; } \\ \text { ASFP } & \text { annual average farm sales for the county (\$/hectare) } \\ \text { VFRM } & \text { average farmland value for the county (\$/hectare) } \\ \text { VNFRM } & \text { average nonfarm value for the county (\$/person) } \\ \text { Notes } & \text { various notes concerning exceptions to the data. See Appendix C for a full listing of the } \\ & \text { database and an explanation of all notes. }\end{array}$

Table 4.1 Excerpt From the County-level Database

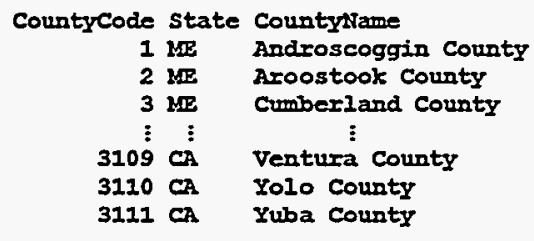

$\begin{array}{lllc}\text { ERCLND } & \text { FRMERC } & \text { DPF } & \text { ASER } \\ 0.945790 & 0.206791 & 0.125332 & 3216 \\ 0.976975 & 0.078229 & 0.027361 & 908 \\ 0.686591 & 0.100777 & 0.233237 & 730 \\ \vdots & \vdots & \vdots & \vdots \\ 0.835891 & 0.271368 & 0.001479 & 5147 \\ 0.989805 & 0.800826 & 0.002811 & 1115 \\ 0.979641 & 0.581876 & 0.029790 & 1142\end{array}$

$\begin{array}{ccr}\text { VERM } & \text { VAFIM } & \text { Notes } \\ 4532 & 109414 & 0 \\ 2062 & 93633 & 0 \\ 6779 & 144689 & 0 \\ \vdots & \vdots & \vdots \\ 17519 & 145082 & 1 \\ 5551 & 133615 & 1 \\ 6226 & 84089 & 0\end{array}$




\section{SOFTWARE DEVELOPMENT}

\subsection{Block-Level Census Database}

The block-level data and some county-level data (area of land and water) were extracted from the CD-ROM set of the Census of Population and Housing, 1990: Public Low (P.L.) 94-171, Data Technical Documentation / prepared by the Bureau of the Census. -Washington: The Bureau, 1991 [sic]. This section describes how the data for the block-level census database were extracted from these CD-ROMs and how after each step of the process the results were verified and validated to ensure the integrity of the intermediate and final data.

\subsubsection{Construction of the Block-Level Census Database}

The PL 94-171 files are stored on ten CD-ROMs in dBase III format (dBase). The first step in the construction of the census-level database (CENSUS90.DAT, located in the secpop90lcensus directory) was to extract the necessary information from the dBase files and store it in an unencoded binary format. There is a dBase file for each state and the District of Columbia. The name of these files is PL9417nn.DBF, where $n n$ is the postal code for the state or district. The format of the dBase census records is specified in another dBase file, PL94STRU.DBF, which is also included on the CD-ROMs. Table 5.1 shows the format of the state files. Note that all fields are of type character string.

Table 5.1 dBase III Census Record Format

\begin{tabular}{|c|c|c|c|c|}
\hline Name & "Type " & Length & $\begin{array}{l}\text { Decimal } \\
\text { Count }\end{array}$ & Doschptan \\
\hline FILEID & $\mathrm{C}$ & 8 & 0 & File ldentification \\
\hline STUSAB* & c & 2 & 0 & State/US Abbreviation \\
\hline SUMLEV* & c & 3 & 0 & Summary Level \\
\hline GEOCOMP & C & 2 & 0 & Geographic Component \\
\hline CHARITER & C & 3 & 0 & Characteristic Iteration \\
\hline LOGRECNU & C & 6 & 0 & Logical Record Number \\
\hline LOGRECPN & C & 4 & 0 & Logical Record Part Number \\
\hline PARTREC & c & 4 & 0 & Total Number of Parts in Record \\
\hline ANRC & $\mathrm{c}$ & 2 & 0 & Alaska Native Regional Corporation \\
\hline AIANACE & C & 4 & 0 & American Indian/Alaska Native Area (Census) \\
\hline AIANAFP & C & 5 & 0 & American Indian/Alaska Native Area (FIPS) \\
\hline AIANACC & c & 2 & 0 & American Indian/Alaska Native Area Class Code \\
\hline ARTLI & C & 1 & 0 & American Indian Reservation Trust Land Indicator Code \\
\hline BLCK & C & 4 & 0 & Block \\
\hline BLCKGR & $\mathrm{C}$ & 1 & 0 & Block Group \\
\hline TRACTBNA & $\mathrm{C}$ & 6 & 0 & Census Tract/Block Numbering Area \\
\hline CONGDIS & $\mathrm{C}$ & 2 & 0 & Congressional District (101st Congress) \\
\hline CONCITCE & $\mathrm{C}$ & 1 & 0 & Consolidated City (Census) \\
\hline CONCITFP & $\mathrm{C}$ & 5 & 0 & Consolidated City (FIPS) \\
\hline CONCITCC & $\mathrm{C}$ & 2 & 0 & Consolidated City Class Code \\
\hline CONCITSC & $\mathrm{C}$ & 2 & 0 & Consolidated City Population Size Code \\
\hline CMSA & $\mathrm{C}$ & 2 & 0 & Consolidated Metropolitan Statistical Area \\
\hline CNTY" & $\mathrm{C}$ & 3 & 0 & County \\
\hline CNTYSC & $\mathrm{C}$ & 2 & 0 & County Population Size Code \\
\hline COUSUBCE & $\mathrm{C}$ & 3 & 0 & County Subdivision (Census) \\
\hline COUSUBFP & C & 5 & 0 & County Subdivision (FIPS) \\
\hline coUSUBCC & $\mathrm{C}$ & 2 & 0 & County Subdivision Class Code \\
\hline COUSUBSC & C & 2 & 0 & County Subdivision Population Size Code \\
\hline DIVIS & $\mathrm{C}$ & 1 & 0 & Division \\
\hline EXTCITIN & $\mathrm{C}$ & 1 & 0 & Extended City Indicator \\
\hline INTUC & C & 15 & 0 & Internal Use Code \\
\hline MSACMSA & $\mathrm{C}$ & 4 & 0 & Metropolitan Statistical Area/Consol. Metro. Statist. Area \\
\hline MSACMSAS & $\mathrm{C}$ & 2 & 0 & MSA CMSA Population Size Code \\
\hline PLACECE & $\mathrm{C}$ & 4 & 0 & Place (Census) \\
\hline PLACEFP & $\mathrm{C}$ & 5 & 0 & Place (FIPS) \\
\hline PLACECC & $C$ & 2 & 0 & Place Class Code \\
\hline
\end{tabular}


Table 5.1 (Continued) dBase III Census Record Format

\begin{tabular}{|c|c|c|c|c|}
\hline Name & Type & Length & Decinal & (1) Description \\
\hline PLACEDC & $\mathrm{C}$ & 1 & 0 & Place Description Code \\
\hline PLACESC & " & 2 & 0 & Place Population Size Code \\
\hline PMSA & C & 4 & 0 & Primary Metropolitan Statistical Area \\
\hline REG & C & 1 & 0 & Region \\
\hline STATECE* & $\mathrm{C}$ & 2 & 0 & State (Census) \\
\hline STATEFP & $\mathrm{C}$ & 2 & 0 & State (FIPS) \\
\hline URBANRUR & $\mathrm{C}$ & 1 & 0 & Urban/Rural \\
\hline URBAREA & $\mathrm{C}$ & 4 & 0 & Urbanized Area \\
\hline UASC & C & 2 & 0 & Urbanized Area Population Size Code \\
\hline $\mathrm{SAC1}$ & C & 5 & 0 & Special Area Code (1) \\
\hline SAC2 & $c$ & 5 & 0 & Special Area Code (2) \\
\hline SAC3 & $\mathrm{C}$ & 4 & 0 & Special Area Code (3)-Voting District Code \\
\hline $\mathrm{SAC}_{4}$ & $\mathrm{C}$ & 4 & 0 & Special Area Code (4) \\
\hline SAC5 & $\mathrm{C}$ & 3 & 0 & Special Area Code (5) \\
\hline SAC6 & ${ }^{3} \mathrm{C}$ & $3:$ & 0 & Special Area Code (6) \\
\hline SAC7 & c & 2 & 0 & Special Area Code $(7)$ \\
\hline SACB & $\mathrm{c}$ & 2 & 0 & Special Area Code (8) \\
\hline SAC9 & $\mathrm{C}$ & 1 & 0 & Special Area Code (9)-Land/Water Area Code \\
\hline SAC10 & $\mathrm{C}$ & 1 & 0 & Special Area Code (10)-Actual/Psuedo Voting District Code \\
\hline AREALAND* & 3 & 10 & 0 & Area (land) \\
\hline AREAWAT & $\cdot \mathbf{N}$ & 10 & 0 & Area (water) \\
\hline ANPSADPI* & $\mathrm{C}$ & 66 & 0 & Area Name/PSAD Term/Part Indicator \\
\hline FUNCSTAT & $\mathrm{C}$ & 1 & 0 & Functional Status Code \\
\hline GCUNI & C & 1 & 0 & Geographic Change User Note Indicator \\
\hline HU100 & in & 9 & 0 & Housing Unit Count (100\%) \\
\hline INTPTLAT & C & 9 & 0 & Internal Point (latitude) \\
\hline INTPTLNG* & IC & 10 & 0 & Internal Point (longitude) \\
\hline PARTFLAG & $\mathrm{C}$ & 1 & 0 & Part Flag \\
\hline PSADC & $\mathrm{C}$ & 2 & 0 & Political/Statistical Area Description Code \\
\hline POP100 & $\mathrm{N}$ & 9 & 0 & Population Count (100\%) \\
\hline SPFLAG & IC & 1 & 0 & Special Flag \\
\hline P001_0001 & $N$ & 9 & 0 & Population Table 1, Item 1 \\
\hline $\mathrm{PO02} 0001$ & $i N$ & 9 & 0 & Population Table 2, Item 1 \\
\hline $\mathrm{POO2} 0002$ & $\mathrm{~N}$ & 9 & 0 & Population Table 2, Item 2 \\
\hline P002 0003 & $N$ & 9 & 0 & Population Table 2, Item 3 \\
\hline$P 002$ 0004 & $\frac{1}{n}$ & 9 & 0 & Population Table 2, Item 4 \\
\hline$P 002 \_0005$ & $N$ & 9 & 0 & Population Table 2, Item 5 \\
\hline P003_0001 & $N$ & 9 & 0 & Population Table 3, Item 1 \\
\hline P003_0002 & $\mathrm{N}$ & 9 & 0 & Population Table 3, Item 2 \\
\hline$P 003 \_0003$ & $N$ & 9 & 0 & Population Table 3 , Item 3 \\
\hline P003_0004 & $\mathrm{N}$ & 9 & 0 & Population Table 3, Item 4 \\
\hline P003_0005 & $\mathrm{N}$ & 9 & 0 & Population Table 3 , Item 5 \\
\hline$P 004 \_0001$ & $N$ & 9 & 0 & Population Table 4, Item 1 \\
\hline P004 0002 & $N$ & 9 & 0 & Population Table 4, Item 2 \\
\hline $\mathrm{POO4} 0003$ & $N$ & 9 & 0 & Population Table 4, Item 3 \\
\hline$P 004 \quad 0004$ & $N$ & 9 & 0 & Population Table 4, Item 4 \\
\hline P004_0005 & $N$ & 9 & 0 & Population Table 4, item 5 \\
\hline$P 004 \quad 0006$ & $\mathrm{~N}$ & 9 & 0 & Population Table 4, Item 6 \\
\hline$P 0050001$ & $N$ & 9 & 0 & Population Table 5, Item 1 \\
\hline$P 0050002$ & $\mathrm{~N}$ & 9 & 0 & Population Table 5, Item 2 \\
\hline P005_0003 & $N$ & 9 & 0 & Population Table 5, ltem 3 \\
\hline P005_0004 & $N$ & 9 & 0 & Population Table 5, Item 4 \\
\hline P005_0005 & $N$ & 9 & 0 & Population Table 5, Item 5 \\
\hline P005_0006 & $\mathrm{N}$ & 9 & 0 & Population Table 5, Item 6 \\
\hline HoO1_0001 & $N$ & 9 & 0 & Housing Table 1, Item 1 \\
\hline
\end{tabular}

* Indicates field used in block or county-level census database. 
To extract the necessary information from the dBase state files, a highly modified version of the Microsoft QuickBasic program included in the CD-ROMs' README file (see Figure 5.1) was written, CONVERT1.BAS (see Appendix G). This Basic program extracted both the block and county-level information and stored it in two separate files, BLOCK_1.BIN and COUNTY_1.TXT respectively. The data stored in BLOCK_1.BIN was converted from the dBase text format to Visual Basic's native binary representation. Each record in BLOCK $1 . B I N$ was 19 bytes long and contained the longitude and latitude of the census block geographical centroid ( 4 bytes each), population ( 4 bytes), area ( 4 bytes), state census number ( 1 byte), and county census number ( 2 bytes).

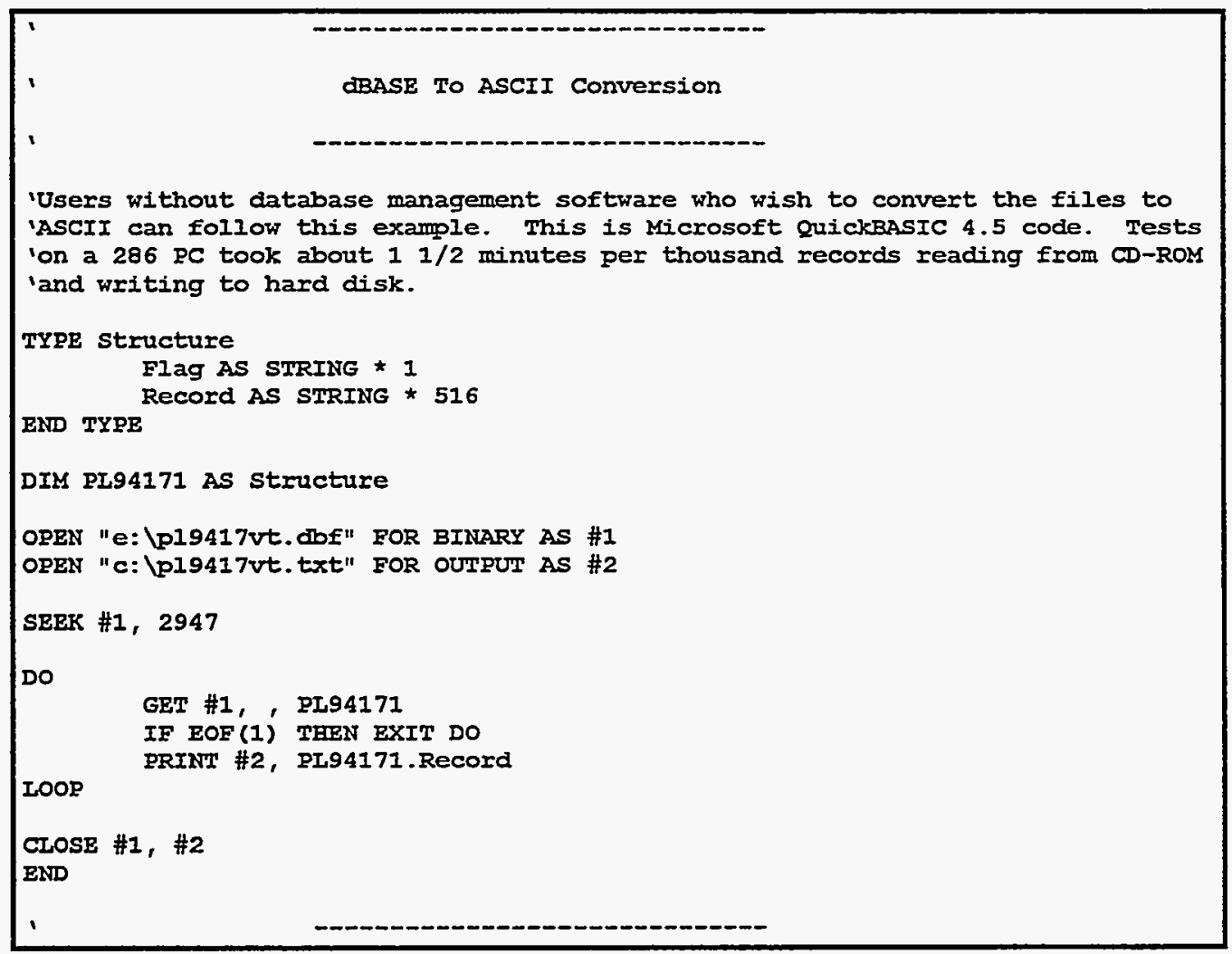

Figure 5.1 PL94-71 README File dBase to ASCII Conversion Program

The second part of the block-level conversion process was to create a second binary file, BLOCK_2.BIN that contained the same information as BLOCK_1.BIN but in an encoded format so that the data records would be smaller (BLOCK_1.BIN was 126 Mbytes, BLOCK_2.BIN was 80 Mbytes) and easier to use (see Appendix G for the CONVERT2.BAS Visual Basic code). The encoding for the latitude and longitude is discussed in section 4.4.1. The encoding of the county code was performed using a lookup table in the CONVERT2.BAS program. This was necessary because the county code used in the census uses designators that are unique within each state but not throughout the country. The code generated for the county designator of each block is also the county index to the county-level database. The codes were ordered by the census statelevel designator as the primary key, and the census county designator as the secondary key. Furthermore, since each county had a unique designator in BLOCK_2.BIN, the state designator was not stored in BLOCK_2.BIN That information can be obtained by performing a table lookup in the county-level database. SECPOP90 has no need for that information at present.

The next part of the creation of the block-level census database was to sort the records by longitude and latitude. This was done in a two-step process using two FORTRAN programs, SORTBIN1.FOR and SORTBIN2.FOR (see Appendix G). FORTRAN was used because it is faster and can support more open files. SORTBIN1.FOR first sorted the BLOCK_2.BIN file by separating it into 30 separate files based on a census block's longitude. SORTBIN2.FOR then individually sorted the 30 files by longitude and latitude, writing the results of each sorted file to BLOCK_3.BIN. The SORTBIN2.FOR program contains an error. It incorrectly sorts files that contain both records with positive and negative longitudes. (Negative and positive longitudes were present in the intermediate BLOCK_n.BIN files because of the encoding of the longitude from 32 to 16 bit integers.) Fortunately, this case only existed in one of the 30, BLOCK_2.BIN files. The database was corrected during the Verification and Validation (V\&V) testing (see section 5.1.2). The SORTBIN2.FOR program was not corrected and still contains the error. 
The final step was the concatenation of duplicate records by the FORTRAN program RMOVEDUP.FOR (see Appendix G). Two records were considered duplicates if they shared the same longitude, latitude, and county code. When such records were found, their areas and population counts were added together and a single record was created to represent the duplicate records. The final resulting database was written to BLOCK_4.BIN, which was then renamed to CENSUS90.DAT.

\subsubsection{Verification and Validation of the Block-Level Census Database}

Upon completion of each of the steps described above, diagnostic output was produced to verify that the conversion programs were operating correctly, and additional testing was performed to validate that the block-level census data integrity was maintained. (No effort was made to determine the integrity of the initial data on the PL 94-171 CD ROMs. For a discussion of the Census Bureau's level of data confidence see Appendix E.)

After the first conversion of the dBase CD-ROM data, the TEST1.BAS Basic program (see Appendix $\mathrm{H}$ ) was executed to read in all of the census block records and print out a summary of all of the county and state total populations and land areas. The summary results agreed with the state and county populations and were always less than or equal to the state and county land areas. (Aggregated block-level census land areas are usually less than county or state land areas because of the differences in tabulating the two area types. See Appendix E.) Comparisons were done using a Microsoft Excel spreadsheet. A sample of Rhode Island census block longitudes and latitudes from the CD-ROM database files was compared with those in BLOCK_1.BIN and were in agreement.

After the second conversion of the BLOCK_1.BIN data and the creation of the BLOCK_2.BIN database, the TEST2.BAS program (see Appendix $\mathrm{H}$ ) was executed to print out a sample of 71 records from both the BLOCK_I.BIN and BLOCK_2.BIN databases. The results were examined by hand and were found to be in agreement. The file lengths of the two databases were also examined to ensure that they contained the same number of records.

After the sorting of the BLOCK_2.BIN database was completed and the BLOCK_3.BIN file was generated, the TEST3.BAS program (see Appendix $\mathrm{H}$ ) was executed to print out the records for Washington County, Maine. These were compared with the same records found in the CD-ROM database files and were in agreement. The file lengths of the two databases, BLOCK_2.BIN and BLOCK_3.BIN, were compared to ensure that they contained the same number of records. The file lengths were equal.

A repeat of testing done after the first conversion was performed on the final BLOCK_4.BIN database using a modified version TEST1.BAS named TEST4.BAS (see Appendix $H$ ). The summary results agreed with the original data files from the PL 94-171 CD-ROMs. The Rhode Island data also agreed and the number of records remaining in BLOCK_4.BIN was equal to the initial number of records minus the duplicate records. BLOCK_4.BIN's name was changed to CENSUS90.DAT, which did not alter the data in any way. A final test to ensure that the database records were sorted correctly, TEST5.BAS (see Appendix $\mathrm{H}$ ), was performed. It was discovered that a section of records were out of order. Two programs, FIX1.BAS and FIX2.BAS (see Appendix H), were run to further diagnose and correct the database. TEST5.BAS was renu. The error was corrected and there were no other records found out of order.

\subsection{County-Level Census Database}

The county-level census database was constructed with data from several sources. The PL 94-171 CD-ROM data files (see 5.1.1); Census of Agriculture, 1992: Final County Files[machine-readable data file] / conducted by the Bureau of the Census. Washington: The Bureau [producer and distributor], 1993 [sic]; County and City Data Book 1994 on CD-ROM [machine-readable data files] / prepared by the Bureau of the Census.-Washington: The Bureau [producer and distributor], 1995 [sic]; and 1993, 1994 Statistical Abstract of the United States, U.S. Department of Commerce, Economics and Statistics Administration, Bureau of the Census.

\subsubsection{Construction of the County-Level Census Database}

Land and water areas were extracted from the PL 94-171 CD-ROM data files (see section 5.1.1 for how this was accomplished). The area data were then imported into a Microsoft Excel spreadsheet and the county land fraction, FRCLND, was then calculated by dividing the land area by the sum of the land and water area. The county population data were also extracted for verification purposes but it was not used in the county-level database. There were no exceptions, omissions, or special treatment for any of the data items extracted from the PL 94-171 CD-ROM data files.

The agricultural data were extracted from the 1992 Census of Agriculture CD-ROM Geographic Area Series 1B using a program that was included on the CD-ROM called EXTRACT. The EXTRACT program allowed the data items described in Table 5.2 to be extracted for each state and saved to a file that was then imported into Microsoft Excel. 
It is important to note that for many of the counties one or more of the above data items was not available due to the confidentiality requirements of the census (see Appendix E). In most cases estimates were made for the undisclosed amount by distributing the excess acreage or dollars evenly among the undisclosed farms. The excess acreage or dollars were determined by subtracting the sum of the disclosed amounts from the state totals provided. This method was chosen because the number of farms and dairy farms in a county was always provided, allowing the estimates to be made in a straightforward manner. Other more complicated methods such as apportioning the excess by acreage or estimating county values by surrounding county values did not provide any more reasonable or trusted estimates. In a small number of cases (particularly when undisclosed values where for small farms in heavily populated areas, e.g.; Queens, New York, or San Francisco, California), it was clear that average dollars per farm or acre were excessive. Those exceptions were handled on a case-by-case basis and are described in the notes at the end of Appendix $C$. The District of Columbia has no agricultural products.

Table 5.2 Census of Agriculture Data Items

\begin{tabular}{l}
\hline fern 1 . Description \\
010001 Farms (number) \\
010002 Land in farms (acres) \\
010004 Est mkt val land \& bldg@1 avg/farm (\$) \\
010006 Est mkt val all mach@1 avg/farm $(\$)$ \\
010019 Mkt val of ag products sold $(\$ 1,000)$ \\
020064 Dairy products, (farms) \\
020065 Dairy products, $(\$ 1,000)$
\end{tabular}

From the Census of Agriculture Data, four agricultural values (these are the agricultural economic values defined for MACCS, see Appendix B) were calculated for each county. They are as follows:

FRMFRC Fraction of land devoted to farming in the county,

DPF Fraction of farm sales resulting from dairy production in the county,

ASFP Annual average farm sales for the county ( $\$ /$ hectare), and

VFRM Average farmland value for the county (\$ / hectare). This includes the average value of farm land, buildings, and machinery.

The fifth economic value calculated for each county is the average of nonfarm value (\$ / person), VNFRM (also a MACCS economic value, see Appendix B). The methodology used to calculate this value for each county is equivalent to that described by Sprung et al. ' First a value of VNFRM is calculated for the entire United States. Then estimates of county values are made using the following equation:

$$
\mathrm{VNFRM}_{\text {County }}=\mathrm{VNFRM}_{\mathrm{US}}\left(\frac{\mathrm{PCI}_{\text {County }}}{\mathrm{PCI}_{\mathrm{US}}}\right)
$$

where

$$
\begin{aligned}
& \mathrm{VNFRM}_{\text {County }}=\text { the average non-farm value for the county (\$/ person), } \\
& \mathrm{VNFRM}_{U S}=\text { the average non-farm value for the United States }(\$ / \text { person), } \\
& \mathrm{PCI}_{\text {County }} \quad \text { = the per capita income for the county ( } \$ \text { / person), and } \\
& \mathrm{PCI}_{U S} \quad=\text { the per capita income for the United States }(\$ / \text { person). }
\end{aligned}
$$

The values for the per capita income were extracted form the 1994 County and City Data Book CD-ROM database files. They were imported directly into Microsoft Access and then modified to contain only the per capita information and the associated footnotes that indicated exceptions and omissions to the data. From Microsoft Access the data were then imported into a Microsoft Excel spreadsheet where they were incorporated into the rest of the county-level database. The exceptions to the data consisted of incidents where two or more counties or independent cities (countylike entities that have no other county affiliation) reported per capita income figures jointly. In all such cases, equal per capita income amounts were applied to all joined counties. For the exact counties affected, see Appendix C.

VNFRM $_{U S}$ is calculated using the equation:

$$
\text { VNFRM }_{U S}=\frac{\text { RTW }_{U S}+V_{S S}-V_{U S}-A_{U S}+V_{F H P}}{P O P_{U S}}
$$


where

RTW $_{\text {US }} \quad=$ the reproducible tangible wealth for the United States (\$),

VSL Us = the value of suburban land in the United States (\$),

$V_{F A} \quad=$ the value of farm assets in the United States $(\$)$,

VFHP US = the value of farm household possessions in the United States (\$), and

$\mathrm{POP}_{U S} \quad=$ the population of the United States (persons).

VSL $_{\text {us }}$ is calculated using the equation:

$$
\mathrm{VSL}_{\mathrm{US}}=\mathrm{UBL}_{\mathrm{US}} \cdot \mathrm{MHV}_{\mathrm{US}} \cdot \mathrm{LPA}_{\mathrm{US}} \cdot \mathrm{FLV}_{\mathrm{US}}
$$

where

UBLus is the amount urban and built-up land in the United States (acres),

$\mathrm{MHV}_{\text {US }}$ is the median housing value for the United States (\$ / house),

LPA $\quad$ is average number of housing lots per acre (houses / acre), and

FLV US is the average fraction of house cost due to land value.

The values and the sources for the variables used in producing VNFRMUs were all obtained from the 1993 and 1994 Statistical Abstract of the United States except for LPA US $_{\text {and }}$ FLV $V_{U S}$ which were obtained from NUREG/CR 4551 (see end note 1) and are equal to 5.0 houses / acre and 0.2 respectively. For details on the remaining values see Appendix D.

\subsubsection{Verification and Validation of the County-Level Census Database}

Unlike the construction of the block-level database, the construction of the county-level database did not require the creation of any additional programs for conversion or sorting. The tools used were Microsoft Excel, Microsoft Access, and the EXTRACT program provided by the Bureau of Census. It is assumed that these programs functioned properly, however, after every operation and / or manipulation of the county-level data, checks were made to ensure that each county had the proper number of records and that all individual county amounts totaled (or other appropriate operation) to the state aggregate amounts.

\subsection{Verification and Validation of the SECPOP90 Code}

The verification and validation of the SECPOP 90 code concentrated on the accuracy of the results of the site-specific population calculations. There are several justifications for this approach. First, the regional calculations use the same algorithms as the site-specific calculations, therefore the regional calculations are indirectly verified and validated by the verification and validation of the site-specific calculations. Second, there is no real way to validate the site-specific economic data that were derived from the county-level database. The economic values are estimates and should only be used for relative evaluations. There are no absolute measures for validating the economic data.

This section pertains to the verification and validation of the SECPOP90 code only. For a discussion of the code's development, see section 4 .

\subsubsection{Internal Verification and Validation}

The first step in the verification of the SECPOP 90 code was to use the first 36 data points on the census data file to enable the code developer to readily verify that the population for each of those data points was correctly placed on a magnified grid close to the "site." The "site" was placed at each of the "corners" of the data as well at the "middle" of the data. SECPOP90 was found to work correctly in each case.

The SECPOP 90 code was then exercised using the 1990 census data and the results contrasted with the results when the SECPOP code was exercised using the 1980 census data for five sites. The sites selected were distributed throughout the continental United States. It was found that the differences were, for the most part, well within differences that would have been predicted simply because of the population growth over the last 10 years.

Final internal verification efforts involved comparing the results obtained from the VAX/VMS system FORTRAN code version with the results obtained using the PC Visual Basic version. The results were in good agreement. 


\subsubsection{External Verification and Validation}

A survey of U.S. commercial nuclear power plants identified those plants that have provided the NRC with updated population information based on the 1990 census data. Licensees for 12 plants have provided this information. Of these 12 plants, four only provided the total population within a specific distance from the plant. The others provided the information in tables, graphs, or both, by rings of 16 segments. The ring distances vary among plants. Licensee submittals may not be accurate; a deviation from the licensee's number(s) is not necessarily an error. In several plants, the licensee's population totals within a ring or sector (or both in one case) have been found to be incorrect. One licensee appears to have averaged the number of people within a ring, which is not accurate.

The following table shows the results for the total populations as reported by the licensees, as calculated by SECPOP 90 , and as percent of reported [((as calculated by SECPOP90) / (as reported by the licensees)) $\times 100]$.

Table 5.3 Total Reported and Calculated Populations

\begin{tabular}{lrrr}
\multicolumn{1}{c}{ Plant Name } & Reported & SECPOP90 & Percent of Reported \\
FitzPatrick $^{2}$ & 44,231 & 39,443 & $89 \%$ \\
Millstone 1 $^{3}$ & $3,363,745$ & $2,780,995$ & $83 \%$ \\
Monticello $^{4}$ & $2,273,213$ & $2,416,872$ & $106 \%$ \\
*Nine Mile Point $^{5}$ & 924,000 & 908,835 & $98 \%$ \\
*Oyster Creek $^{6}$ & 96,718 & 101,433 & $105 \%$ \\
*Peach Bottom APS $^{7}$ & $10,257,315$ & $6,803,644$ & $66 \%$ \\
DC Cook $^{8}$ & 52,953 & 51,070 & $96 \%$ \\
Diablo Canyon $^{9}$ & 374,701 & 376,012 & $100 \%$ \\
Fort Calhoun $^{10}$ & 760,431 & 772,086 & $102 \%$ \\
Prairie Island $^{11}$ & 25,342 & 24,969 & $99 \%$ \\
Turkey Point $^{12}$ & $2,613,535$ & $2,602,834$ & $100 \%$ \\
* These plants only provided total population within a radius. &
\end{tabular}

As shown above, on a total population basis, all of the results are in reasonable agreement. The program also provides the typical rosettes which show the populations in relation to the plant site. Examples of the rosettes for Millstone 1, Monticello, and Diablo Canyon are shown below. The results can also be provided as a MACCS site file for direct use in MACCS calculations. An example of this type of output is shown in section 3.6.3.

Also included are population tables (see Appendix F) generated from licensee information and SECPOP90 output. These tables show the actual population by ring segment and the percent difference from the licensee's reported values. As with all percent results, caution should be used when interpreting the information. For example: at DC Cook, ring 1, south sector, the licensee reported 5 people while SECPOP 90 calculated 20 people, a $400 \%$ difference with no practical effects.

Generally, SECPOP90 calculates the total population in each ring and each sector reasonably well. However, the ringsegment population can have a large deviation from the licensee's values. Assuming that the licensee's values are accurate, this can be caused by the code's identification of the census block centroid. Wherever the census block centroid is located within the rosette, that is where all of the people for that census block will be counted. This can be seen in the Diablo Canyon tables in the NW and NNW segments, rings 10 through 50.

Another factor is the level of detail for the census data that the licensee used. If the level of detail was at the county, city, or block group level, the reported values will be different. As previously mentioned, one licensee (Monticello) used average data for rings 2 through 6 . The reasonableness of the results in both the ring and sector attests to the fact that the ringsegment to ring-segment differences are not significant. The one exception seems to be low population sites with sporadic population, like Turkey Point. In these cases the percent differences become large. If these differences are correct, it is not clear what effect this may have on MACCS calculations. In such cases, engineering judgment is needed to assess the impact of the low population on the health effects of interest, with consideration being given to the meteorology selected (wind rose). 


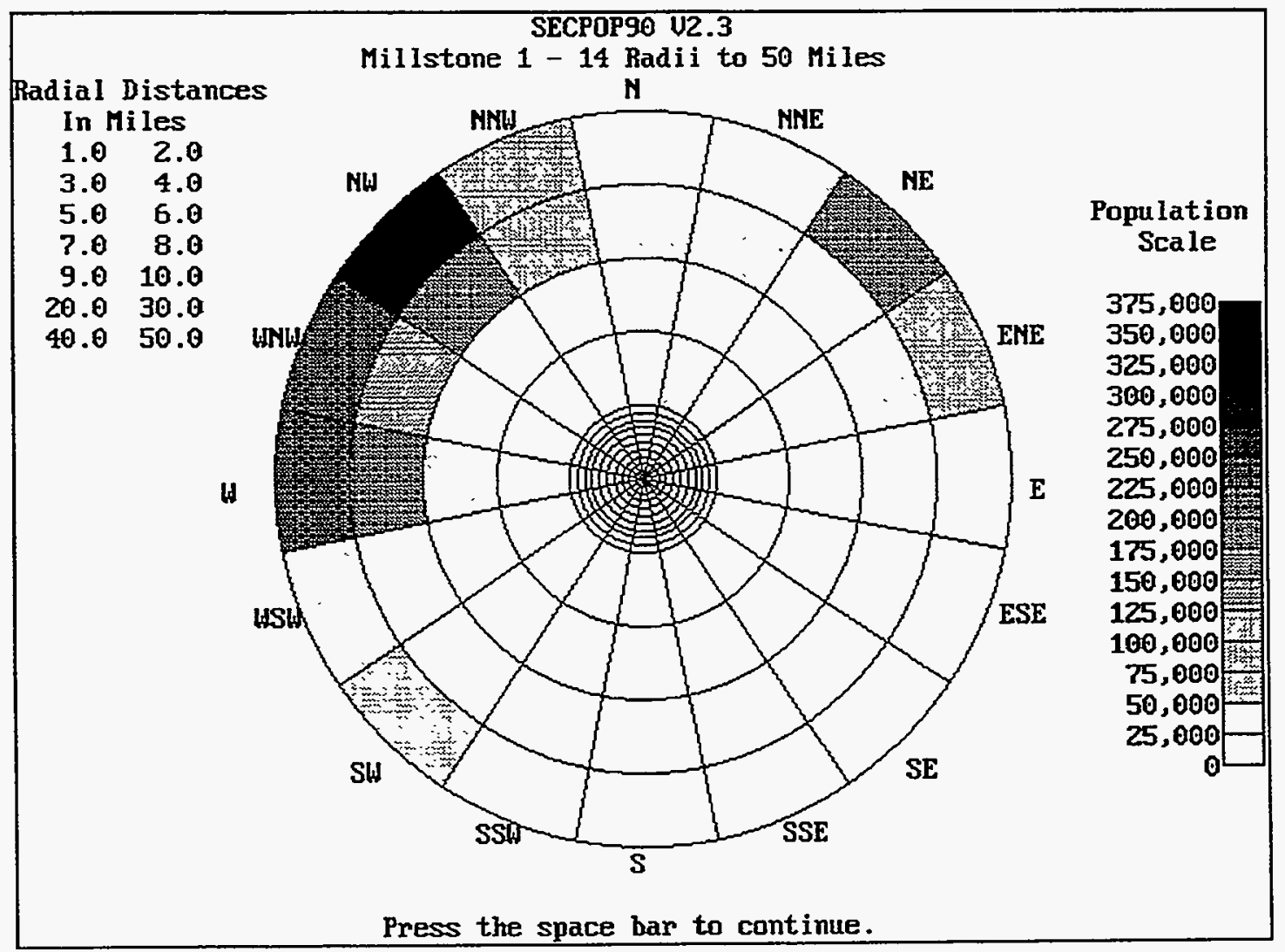

Figure 5.2 Millstone Rosette

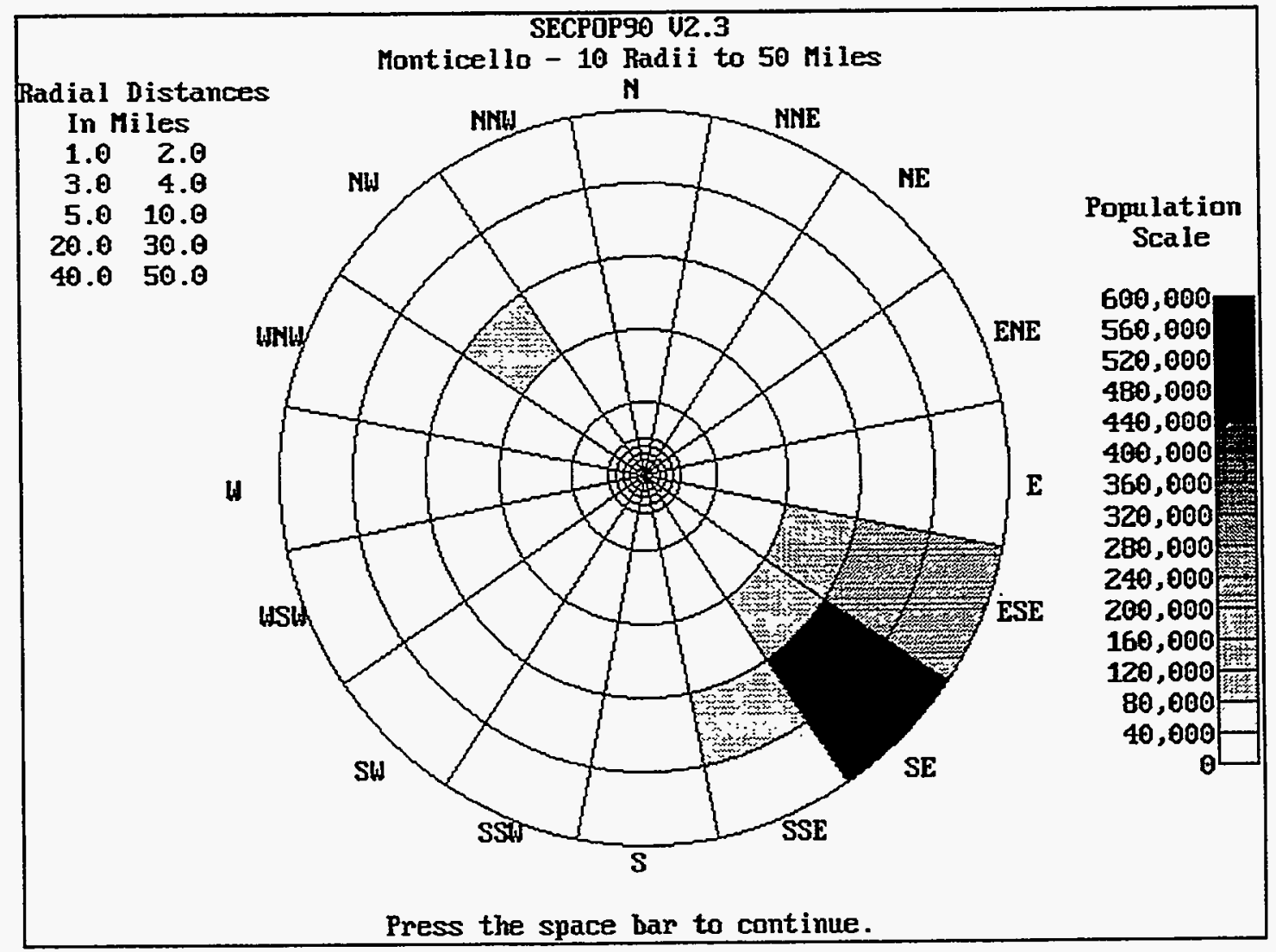

Figure 5.3 Monticello Rosette 


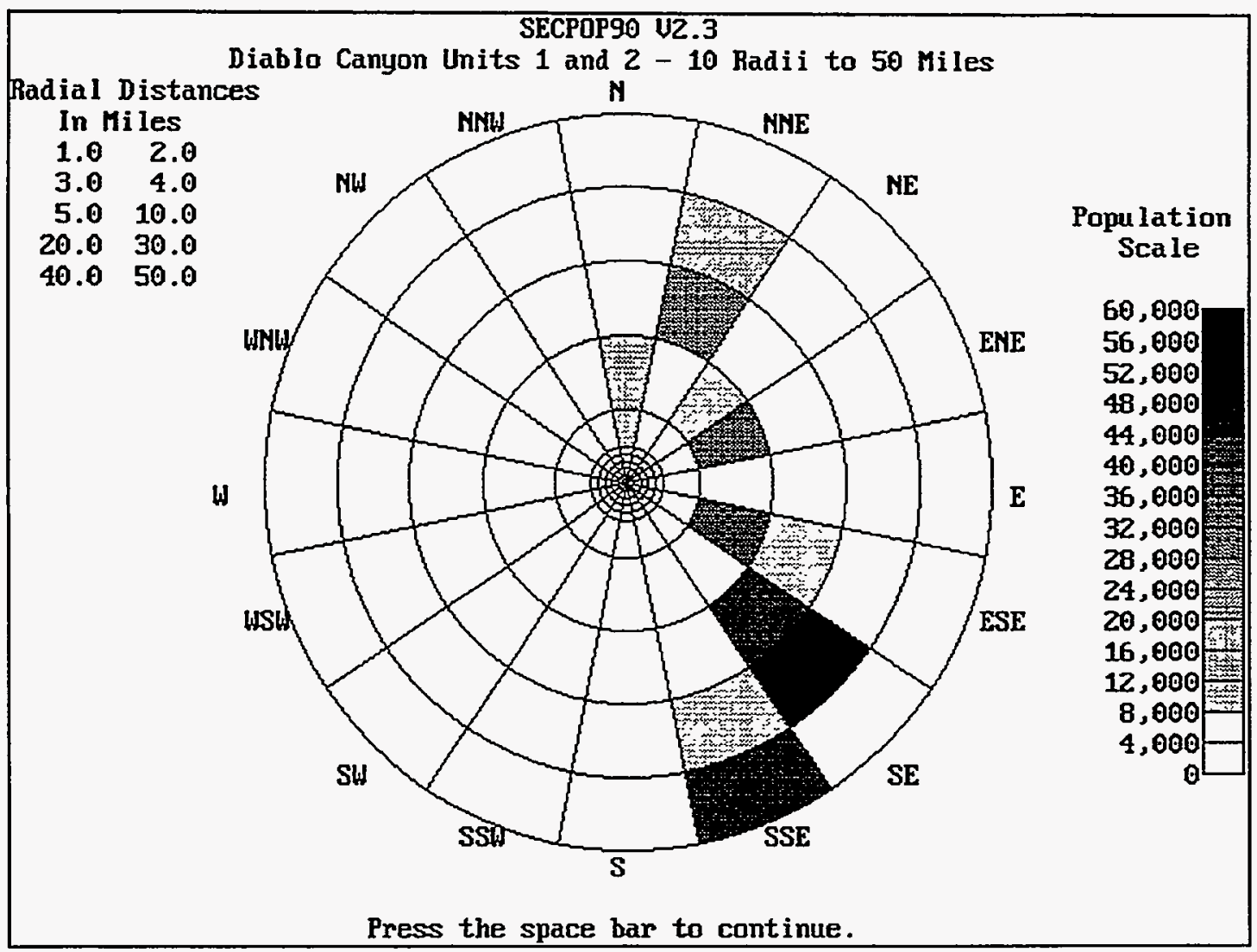

Figure 5.4 Diablo Canyon Rosette

1 Sprung, J. L., Rollstin, J. A, Helton, J. C., Jow, H-N, Evaluation of Severe Accident Risks: Quantification of Major Input Parameters, NUREG/CR - 4551, SAND86 - 1309, Vol2, Rev. 1, Part 7, U.S. Nuclear Regulatory Commission, Washington, DC, December 1990, pg. 5-4.

2 James A. FitzPatrick Emergency Plan, Volume 1, Revision 2, dated September 9, 1992.

3 Millstone 1 Updated Final Safety Analysis Report, Chapter 2, Revision 6, dated May 1993.

${ }^{4}$ Monticello Final Safety Analysis Report, Tables 2.2-1 and 2.2-2, Revision 11, dated December 1991.

5 Nine Mile Point 2 Safety Evaluation Report, dated August 7, 1991, TAC Number 69095 [50 Mile radius].

${ }^{6}$ Oyster Creek, GPU submittal dated May 19, 1992, C321-92-2118 [10 Mile radius].

7 Peach Bottom Atomic Power Station, Philadelphia Electric Company submittal dated May 21, 1992, Accession Number 9206020160 [60 Mile radius].

${ }^{8}$ DC Cook Final Safety Analysis Report, Page 2.1-8 and Figure 2.1-5, updated July 1993.

9 Diablo Canyon Final Safety Analysis Report, Chapter 2, Revision 8, dated September 1992.

${ }^{10}$ Fort Calhoun Station Radiological Emergency Response Plan, Section J, Figures J-6 through J-8, Pages 24 through 26.

${ }^{11}$ Evacuation Time Estimates for the Prairie Island Nuclear Plant Plume Exposure Pathway Emergency Planning Zone, Table 3.1, Page 3-3, dated December 1992.

${ }^{12}$ Turkey Point Final Safety Analysis Report, Tables 2.4-1 and 2.4-3, Revision 10, dated July 1992. 


\section{APPENDIX A - SITE LIST}

This is the list of site files that are available in the secpop90 lsites directory.

$\begin{array}{ll}\text { ALIENS.SIT } & \text { ARKANSA.SIT } \\ \text { BEAVER.SIT } & \text { BELLEFON.SIT } \\ \text { BROWNS.SIT } & \text { BRUNSWI2.SIT } \\ \text { CALIAWAY.SIT } & \text { CALVERT.SIT } \\ \text { COMANCHE.SIT } & \text { COOPER.SIT } \\ \text { DONALD.SIT } & \text { DRESDEN.SIT } \\ \text { EORKED.SIT } & \text { FTCAL.SIT } \\ \text { HADDEM.SIT } & \text { HARTSVIL.SIT } \\ \text { JOSPEH.SIT } & \text { KEWAANEE.SIT } \\ \text { MAINE.SIT } & \text { MARTILE.SIT } \\ \text { MILLSTON.SIT } & \text { MONTICEI.SIT } \\ \text { OYSTER.SIT } & \text { PALISADE.SIT } \\ \text { PERKINS.SIT } & \text { PERRY.SIT } \\ \text { PRAIRIE.SIT } & \text { QUAD.SIT } \\ \text { SALEM.SIT } & \text { SANONO.SIT } \\ \text { SHOREHAM.SIT } & \text { SKAGIT.SIT } \\ \text { SUSQUEHA.SIT } & \text { THREE.SIT } \\ \text { VIRGII.SIT } & \text { VOGTIE.SIT } \\ \text { WPPSS.SIT } & \text { WPPSS35.SIT } \\ \text { ZION.SIT } & \end{array}$

BAILLY.SIT

BIGROCK.SIT BIACKEOX.SIT

BRUNSWIC.SIT

CATAWBA. SIT

CRYSTAL.SIT

DUANE.SIT

FTSTVRA.SIT

HATCH.SIT

LACROSSE. SIT

MCGUIRE.SIT

NINE.SIT

PALO.SIT

PHIPPS.SIT

RANCHO.SIT

SEABROOK. SIT

SOUTHTEX.SIT

TROJAN.SIT

WATERFOR.SIT

YANKEE.SIT

\author{
BYRON STT \\ CHEROKEE.SIT \\ DAVIS.SIT \\ FERMI.SIT \\ GINNA. SIT \\ HOPE. STT \\ IASAILE. SIT \\ MIDLAND.SIT \\ NORTH. SIT \\ PEACH.SIT \\ PIIGRIM. SIT \\ RIVER.SIT \\ SEQUOYA. SIT \\ STLUCIE.SIT \\ TURKEY.SIT \\ WATTS . SIT \\ YELIOW. SIT
}

BRAIDWOO.SIT

CALLAWA2 . SIT

CLINTON.SIT

DIABLO. SIT

FITZPATR.SIT

GRAND. SIT

INDIAN.SIT

LIMERICK. SIT

MILLS.SIT

OCONEE.SIT

PEBBLE.SIT

POINT.SIT

ROBINSON . SIT

SHEARON . SIT

SURRY.SIT

VERMONT. SIT

WOLE.SIT

ZIMMER.SIT 


\section{APPENDIX B - MACCS SITE DATA FILE FORMAT}

The following is taken directly from NUREG/CR 4691, MELCOR Accident Consequence Code System (MACCS), Volume 1, pp. B-6 - B-13, February 1990 for the user's convenience. A listing of a sample Site Data File is supplied in this document as Table 3.1.

\section{B.3 Site Data File Format}

In the Site Data File, the user specifies the population distribution and land use information for the region surrounding the site. Contained in the Site Data File are the geometry data used for the site (spatial intervals), population distribution, fraction of the area that is land, watershed data for the liquid pathways model, information on agricultural land use and growing seasons, and regional economic information. An example of a Site Data File is provided in Appendix D.2.

The decision on whether to use a Site Data File is determined by a flag set in the EARLY User Input File (see Section 2.3). If a Site Data File is not being used, the EARLY User Input File determines the population density to be used by both the FARLY and CHRONC modules. It is not possible for the user to supply differing population data for the two modules.

The Site Data File used in MACCS is a formatted file. The data must appear exactly as described in this Appendix and in exactly the same order. In contrast to the User Input Files for MACCS, which are processed by a free format input processor, the site Data File is processed with fixed format FORTRAN READ statements.

The use of fixed format READ statements requires that the user exercise special attention to line up the data items in their proper fields. Any numeric items specified in exponential format (e.g., l.E-6) must be right-justified in the field.

In contrast to the User Input Files, where every value is validated by the code to ensure that it lies within a range of allowable values, the validation performed on the Site Data is only partially complete. Some of the input parameters on this file are rigorously checked to ensure that they fall within the allowed range, while other values are not checked at all. It is recommended that the user exercise scrupulous care in constructing a site Data File. It is very important that all items appear in their proper fields and that all numeric values lie within the range of acceptable values. Failure to conform to these requirements may lead to the generation of spurious results.

The first two records of the site Data File contain identification information. Up to 80 characters may be used on each line. This header information is printed on the output listing.

Following the descriptive text fields, there are six data cards which specify the amount of data which is being supplied on the file. The values defined on these data cards must be consistent with the MACCs model definition data defined by the ATMOS, EARLY, and CHRONC User Input Files. In other words, the value of the Site Data File input variable end the corresponding comparison variable must be identical. If any inconsistencies are detected, execution of the program will be terminated.

An example of the data cards described up to this point is included for the purpose of illustration. 
MACCS SITE DATA FILE FOR SURRY (JLS, 11/10/88)

SECPOP POP DISTRIBUTION FROM 1980 CENSUS DATA ALTERED USING 010 MI NRC

26 SPATIAL INTERVALS

16 WIND DIRECTIONS

7 CROP CATEGORIES

4 WATER PATHWAY ISOTOPES

2 WATERSHEDS

59 ECONOMIC REGIONS

The format of the data is as follows:

\begin{tabular}{|c|c|c|c|c|c|c|}
\hline Iine & Columns & Format & $\begin{array}{c}\text { Input } \\
\text { Variable }\end{array}$ & Identification & $\begin{array}{l}\text { Comparison } \\
\text { Variable }\end{array}$ & $\begin{array}{c}\text { Allowed } \\
\text { Range }\end{array}$ \\
\hline 3 & $1-4$ & I4 & NSPDTS & $\begin{array}{l}\text { Number of spatial } \\
\text { intervals }\end{array}$ & NUMRAD & $2-35$ \\
\hline 4 & $1-4$ & I4 & NWNDIR & $\begin{array}{l}\text { Number of wind } \\
\text { direction sectors }\end{array}$ & NUMCOR & $16-16$ \\
\hline 5 & $1-4$ & I4 & NCPGZN & $\begin{array}{l}\text { Number of crops } \\
\text { to be considered }\end{array}$ & NFICRP & $1-10$ \\
\hline 6 & $1-4$ & I4 & NWPISO & $\begin{array}{l}\text { Number of water } \\
\text { pathway nuclides }\end{array}$ & NUMWPI & $1-10$ \\
\hline 7 & $1-4$ & I4 & NWTRSH & $\begin{array}{l}\text { Number of watershed } \\
\text { categories }\end{array}$ & NUMWPA & $1-4$ \\
\hline 8 & $1-4$ & I4 & NECRGN & $\begin{array}{l}\text { Number of economic } \\
\text { regions }\end{array}$ & N.A. & $1-99$ \\
\hline
\end{tabular}

Eight blocks of site data follow the introductory block described above. Each of these data blocks is introduced by a separator line that identifies the block of data to follow. The first line of each data block must be the separator for that block. Since each separator is read with a format of $1 \mathrm{x}, \mathrm{A} 22$, the first character of the separator line is ignored and the following 22 characters must match the identification field for that specific data block.

\section{Geometry Data Block}

Geometry information is used to specify the population and land use data. The geometry data consist of the distance in kilometers to the endpoints of the spatial intervals. This grid definition must agree with the grid used in the ATMOS User Input File (see Section 1.3). A relative error of 10 percent in the endpoint distances is allowed. For larger discrepancies in the geometry data, the error flag will be set and execution will terminate upon completion of the Site Data File input processing.

The first line of the geometry information contains the 22 character separator beginning with "SPATIAL DISTANCES" in column 2 . Next, the endpoint distances in kilometers are specified, eight values per line, using the format described below. As many lines as are needed to define the spatial distances are used. The formatting of this information is illustrated below by presentation of the spatial distance data for a single wind direction sector. 
Note: The spacing between adjacent spatial intervals should be at least 0.1 kilometer.

SPATIAL DISTANCES

$\begin{array}{rrrrrrrr}0.16 & 0.52 & 1.21 & 1.61 & 2.13 & 3.22 & 4.02 & 4.83 \\ 5.63 & 8.05 & 11.27 & 16.09 & 20.92 & 25.75 & 32.19 & 40.23 \\ 48.28 & 64.37 & 80.47 & 112.65 & 160.93 & 241.14 & 321.87 & 563.27\end{array}$

$804.67 \quad 1609.34$

The format used to process the data is as follows:

\begin{tabular}{|c|c|c|c|c|c|}
\hline Columns & Format & $\begin{array}{c}\text { Input } \\
\text { Variable }\end{array}$ & Information & $\begin{array}{c}\text { Comparison } \\
\text { Variable }\end{array}$ & $\begin{array}{c}\text { Allowed } \\
\text { Range }\end{array}$ \\
\hline $1-80$ & $8 \mathrm{E} 10.2$ & SPDSTS & $\begin{array}{l}\text { Spatial interval } \\
\text { endpoints }(\mathrm{km})\end{array}$ & SPAEND & $0.001-9999.0$ \\
\hline
\end{tabular}

\section{Population Data Block}

The population data for each element in the spatial grid is defined here. The first line of the data block contains the 22 character separator beginning with "POPULATION" in column 2. Next, the number of people in each element is given for the first sector (The first sector is assumed to be centered on North). The population data are specified, eight values per line, using the format described below. As many lines as needed to cover all the spatial elements in the sector are used. Proceeding in a clockwise rotation, the population data for the second ANNE) and subsequent sectors follow. Data for all 16 wind directions (sectors) must be provided. Data for each sector begins on a new line. The formatting of this information is illustrated below by presentation of the population data for a single wind direction sector for 26 radial distance elements.

POPULATION

0 .

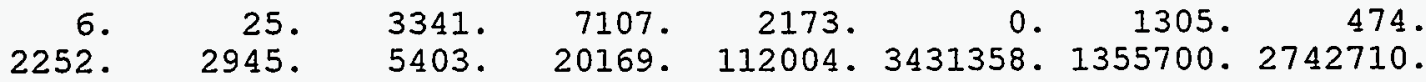

$0 . \quad 0$.

0.

$0 . \quad 0$.

0.

0. $\quad 4 . \quad 5$.

2487346.104331 .

The format used to process the data is as follows:

\begin{tabular}{lllll}
\hline Columns & Format & Variable & Identification & $\frac{\text { Allowed Range }}{1-80}$ \\
\hline $8 E 10.0$ & POPDAT & Population data & $0.0-1 . E 9$ \\
\hline
\end{tabular}

\section{Land Fraction Block}

The fraction of each spatial element that is land (as opposed to lakes, oceans, etc.) must be defined. The first line of the data block contains the 22 character separator beginning with "LAND FRACTION" in column 2. Next, the fraction of area that is land in each radial spatial interval of the first sector is given. All values must be between zero and one. $A$ value of zero means the element has no land, a value of one means the element is all land. The land fraction data are specified, 16 values per line, with the format described below. As many lines as needed to define 
all the spatial intervals in the sector are used. The land fraction data for the second and subsequent sectors follow in a clockwise rotation. Data for all 16 wind direction sectors must be provided. The data for each sector begins on a new line. The formatting of this information is illustrated below by presentation of the land fraction data for a single wind direction sector.

LAND FRACTION

$\begin{array}{lllllllllllllll}1.00 & 1.00 & 0.00 & 0.00 & 0.00 & 0.00 & 0.00 & 0.00 & 0.00 & 0.80 & 1.00 & 1.00 & 0.95\end{array}$

$0.75 \quad 0.70 \quad 0.85$

The format used to process the data is as follows:

\begin{tabular}{llllc}
\hline Columns & Format & Variable & Identification & Allowed Range \\
$1-80$ & $16 \mathrm{~F} 5.2$ & FRCIND & Land fraction & $0.0-1.0$ \\
\hline
\end{tabular}

Region Identification Block

This data block identifies each of the spatial intervals with an economic region. A region can be a state, a county, or any division desired. A description of the economic data used for each region is given later in this Appendix. The first line in the datablock contains the 22 character separator beginning with "REGION INDEX" in column 2. The next line contains two digit integers associating a region index with each of the spatial elements in the first sector. The data are specified with the format defined below. All of the region indices for one sector will fit on one line. The region indices for the second and subsequent sectors are on the following lines, a new line for each sector in a clockwise rotation. A total of 16 lines are required. For example, a region index of 09 means that economic data for region number nine will be used for the spatial element. (See the section of this Appendix on Regional Economic Data.) The formatting of this information is illustrated below by a presentation of the region identification data for a single wind direction sector.

REGION INDEX

$44445050505050505044444444444444444444441818 \quad 7283054$

The format used to process the data is as follows:

\begin{tabular}{lcccc}
\hline Columns & Format & Variable & Identification & Allowed Range \\
\cline { 3 - 4 } & $40 I 2$ & INDREG & Region index & $1-$ NECRGN \\
\hline
\end{tabular}

Watershed Identification BIock

Each of the spatial intervals in the grid must be associated with one of the watershed classes. The definition of the efficiency of these watershed classes is described later in this appendix in the "WATERSHED DEFINITION" block. The watershed identification data block begins with the 22 character separator beginning with "WATERSHED INDEX" in column 2 . The next line contains two digit integers associating a watershed type with each of the spatial elements in the first sector. The data are specified with the format defined below. All of the watershed type 
indices for one sector will fit on a single line. The watershed indices for the second and subsequent sectors are on the following lines, a new line for each sector in a clockwise rotation. A total of 16 lines are required. For example, a watershed index of 1 means that the water ingestion factor for watershed type 1 will be used for all material deposited on that spatial element. A watershed index of 2 means that the water ingestion factor for watershed type 2 will be used for all material deposited on that spatial element. The formatting of this information is illustrated below by the presentation of the watershed data for a single wind direction sector.

WATERSHED INDEX

$\begin{array}{llllllllllllllllllllllllll}1 & 1 & 2 & 2 & 2 & 2 & 2 & 2 & 2 & 1 & 1 & 1 & 1 & 1 & 1 & 1 & 1 & 1 & 1 & 1 & 1 & 1 & 1 & 1 & 3 & 3\end{array}$

The format used to process the data is as follows:

\begin{tabular}{|c|c|c|c|c|}
\hline Columns & Format & Variable & Identification & Allowed Range \\
\hline $1-80$ & $40 I 2$ & INDWTR & Watershed index & 1 - NWTRSH \\
\hline
\end{tabular}

Agricultural Data Block

The length of the growing season and the average fraction of the farmland area at the site devoted to each crop type must be specified. These fractions, FRCLP, need not sum to exactly one, but their sum should not exceed a value of one. If these values sum to a value less than one, that sum indicates the fraction of farmland in production in an average year (some fraction of farmland may be fallow). Data must be given for each of the NCPGZN crop categories. The data block begins with the separator "CROP SEASON AND SHARE" in column 2. The formatting of this information is illustrated below by the presentation of the agricultural data for the seven crop categories used in the example site Data File.

$\begin{array}{lll}\text { CROP SEASON AND SHARE } & & \\ 1 \text { PASTURE } & 90.270 . & 0.41 \\ 2 \text { STORED FORAGE } & 150.240 . & 0.13 \\ 3 \text { GRAINS } & 150.240 . & 0.21 \\ 4 \text { GRN IEAFY VEGETABLES } & 150.240 . & 0.002 \\ 5 \text { OTHER FOOD CROPS } & 150.240 . & 0.004 \\ 6 \text { LEGUMES AND SEEDS } & 150.240 . & 0.15 \\ 7 \text { ROOTS AND TUBERS } & 150.240 . & 0.003\end{array}$


The format used to process the data is as follows:

\begin{tabular}{|c|c|c|c|c|}
\hline Columns & Eormat & Variable & Identification & Allowed Range \\
\hline $1-4$ & I4 & $I$ & Crop index & 1 - NCPGZN \\
\hline $6-25$ & $\mathrm{~A} 20$ & CROP & Crop name & N.A. \\
\hline $26-30$ & E5.0 & GBEG & $\begin{array}{l}\text { Day of the year the growing } \\
\text { season begins }\end{array}$ & 1.0 - GEND \\
\hline $31-35$ & F5.0 & GEND & $\begin{array}{l}\text { Day of the year the growing } \\
\text { season ends }\end{array}$ & GBEG - 365.0 \\
\hline $36-45$ & F10.0 & FRCLCP & $\begin{array}{l}\text { Fraction of the site } \\
\text { averaged farmland devoted } \\
\text { to this crop }\end{array}$ & $0.0-1.0$ \\
\hline
\end{tabular}

Watershed Data Block

The data block begins with the 22 character separator beginning. with "WATERSHED DEFINITION" in column 2. For each of the NUMWPI (NWPISO) nuclides considered in the liquid pathways model, an initial washoff fraction and an annual washoff rate (fraction/year) must be specified together with an ingestion factor (becquerel ingested/becquerel-in-water) for each of the NUMWPA (NWTRSH) watershed classes. The formatting of this information is illustrated below by the presentation of the watershed data for the four water ingestion nuclides used on the example Site Data File.

$\begin{array}{cccc}\text { WATERSHED DEFINITION } & \text { RIVER } & \text { OCEAN } & \text { I.AKE } \\ 1 \text { SR-89 } & 5.0 \mathrm{E} 6 & 0.0 & 2.0 \mathrm{E} 7 \\ 2 \mathrm{SR}-90 & 5.0 \mathrm{E} 6 & 0.0 & 2.0 \mathrm{E} 7 \\ 3 \mathrm{CS}-134 & 5.0 \mathrm{E} 6 & 0.0 & 2.0 \mathrm{E} 6 \\ 4 \mathrm{CS}-137 & 5.0 \mathrm{E} 6 & 0.0 & 4.0 \mathrm{E} 6\end{array}$


The format used to process the data is as follows:

\begin{tabular}{|c|c|c|c|c|}
\hline Columns & Format & Variable & Identification & Allowed Range \\
\hline $1-4$ & I4 & $I$ & Nuclide index & 1 - NWPISO \\
\hline $6-13$ & A8 & NMISO & Nuclide name & N.A. \\
\hline $16-25$ & E10.1 & DUMMYY & No longer used & N.A. \\
\hline $26-35$ & E10.1 & DUMMY & No longer used & N.A. \\
\hline $36-45$ & E10.1 & WTRINE (I) & $\begin{array}{l}\text { Ingestion factor for } \\
\text { watershed class } 1\end{array}$ & $0.0-1.0$ \\
\hline $46-55$ & $\mathrm{E} 10.1$ & WTRINE (2) & $\begin{array}{l}\text { Ingestion factor for } \\
\text { watershed class } 2\end{array}$ & $0.0-1.0$ \\
\hline $56-65$ & E10.1 & WTRINE (3) & $\begin{array}{l}\text { Ingestion factor for } \\
\text { watershed class } 3\end{array}$ & $0.0-1.0$ \\
\hline $66-75$ & E10.1 & WTRINE (4) & $\begin{array}{l}\text { Ingestion factor for } \\
\text { watershed class } 4\end{array}$ & $0.0-1.0$ \\
\hline
\end{tabular}

Regional Economic Data Block

Economic data must be specified for each of the NECRGN economic regions. The data block begins with the separator "REGIONAL ECONOMIC DATA" in column 2. An economic region can be a county, a state or any other userspecified region. The data provided in this block are similar to those contained in the CRAC2 "Economic" subgroup but converted to metric units.

The index of the region is used with the "REGION INDEX" data block (described earlier in this Appendix) to associate each element of the spatial grid with an economic region. The formatting of this information is illustrated below by the presentation of the economic data for the first economic region on the example Site Data File, Alabama.

REGIONAL ECONOMIC DATA
1 AIA
$.354 \quad .040$
459 .
1824 .
62000. 
The format used to process this data is as follows:

\begin{tabular}{|c|c|c|c|c|}
\hline Columns & Format & Variable & Identification & Allowed Range \\
\hline $1-4$ & $I 4$ & I & Region index number & 1 - NECRGN \\
\hline $6-15$ & A10 & NMRGN & Region identification & N.A. \\
\hline $21-25$ & E5.3 & ERMFRC & $\begin{array}{l}\text { Fraction of land devoted } \\
\text { to farming in the region }\end{array}$ & $0.0-1.0$ \\
\hline $26-30$ & F5. 3 & DPE & $\begin{array}{l}\text { Fraction of farm sales } \\
\text { resulting from dairy } \\
\text { production in the region }\end{array}$ & $0.0-1.0$ \\
\hline $31-40$ & F10.1 & ASFP & $\begin{array}{l}\text { Annual average farm sales } \\
\text { for the region ( } \$ / \text { hectare) }\end{array}$ & $0.0-1 . \mathrm{E9}$ \\
\hline $41-50$ & F10.1 & VFRM & $\begin{array}{l}\text { Average farmland value } \\
\text { for the region (\$/hectare) }\end{array}$ & $0.0-1 . E 9$ \\
\hline $51-60$ & F10.1 & VNFRM & $\begin{array}{l}\text { Average non farm value } \\
\text { for the region ( } \$ / \text { person) }\end{array}$ & $0.0-1 . E 9$ \\
\hline
\end{tabular}




\section{APPENDIX C - COUNTY DATABASE LISTING}

This is a complete listing of the county-level census database. An explanation of the notes follows the last record of the database.

\begin{tabular}{|c|c|c|c|c|c|c|c|c|c|}
\hline $\begin{array}{c}\text { County } \\
\text { Code }\end{array}$ & State & $\begin{array}{l}\text { County } \\
\text { Name }\end{array}$ & $\begin{array}{l}\text { FRCLND } \\
\text { Absolute }\end{array}$ & $\begin{array}{l}\text { FRMFRC } \\
\text { Absolute }\end{array}$ & $\begin{array}{c}\text { DPF } \\
\text { Absolute }\end{array}$ & $\begin{array}{l}\text { ASFP } \\
\text { S/ha }\end{array}$ & $\begin{array}{l}\text { VFRM } \\
\text { S/ha }\end{array}$ & $\begin{array}{l}\text { VNFRM } \\
\text { S/person }\end{array}$ & Notes \\
\hline 1 & ME & Androscoggin County & 0.945790 & 0.206791 & 0.125332 & 3216 & 4532 & 109414 & 0 \\
\hline 2 & ME & Aroostook County & 0.976975 & 0.078229 & 0.027361 & 908 & 2062 & 93633 & 0 \\
\hline 3 & ME & Cumberland County & 0.686591 & 0.100777 & 0.233237 & 730 & 6779 & 144689 & 0 \\
\hline 4 & ME & Franklin County & 0.973372 & 0.035752 & 0.595260 & 480 & 2296 & 101618 & 0 \\
\hline 5 & ME & Hancock County & 0.675873 & 0.049238 & 0.013906 & 752 & 3909 & 126083 & 1 \\
\hline 6 & ME & Kennebec County & 0.911939 & 0.171839 & 0.458911 & 947 & 3426 & 116032 & 0 \\
\hline 7 & ME & Knox County & 0.320116 & 0.118053 & 0.223053 & 574 & 5220 & 117346 & 0 \\
\hline 8 & ME & Lincoln County & 0.650885 & 0.083509 & 0.342677 & 482 & 7124 & 131854 & 0 \\
\hline 9 & ME & Oxford County & 0.955295 & 0.047722 & 0.266111 & 565 & 3668 & 100968 & 0 \\
\hline 10 & ME & Penobscot County & 0.954898 & 0.054361 & 0.513882 & 603 & 2904 & 105127 & 0 \\
\hline 11 & ME & Piscataquis County & 0.906075 & 0.014176 & 0.601179 & 384 & 2186 & 93423 & 0 \\
\hline 12 & ME & Sagadahoc County & 0.685980 & 0.115602 & 0.425645 & 372 & 3781 & 127058 & 0 \\
\hline 13 & ME & Somerset County & 0.958764 & 0.042564 & 0.650222 & 452 & 2696 & 92833 & 0 \\
\hline 14 & ME & Waldo County & 0.855772 & 0.153914 & 0.543369 & 589 & 3453 & 88803 & 0 \\
\hline 15 & ME & Washington County & 0.789081 & 0.057641 & 0.003532 & 939 & 2675 & 91770 & 1 \\
\hline 16 & ME & York County & 0.779422 & 0.097436 & 0.179135 & 626 & 5230 & 121294 & 0 \\
\hline 17 & NH & Belknap County & 0.856434 & 0.081411 & 0.354882 & 351 & 6523 & 139609 & 0 \\
\hline 18 & NH & Carroll County & 0.941140 & 0.042561 & 0.242416 & 339 & 4981 & 148692 & 0 \\
\hline 19 & NH & Cheshire County & 0.970187 & 0.074948 & 0.286962 & 1342 & 8003 & 127275 & 0 \\
\hline 20 & $\mathrm{NH}$ & Coos County & 0.983038 & 0.039965 & 0.801689 & 413 & 2442 & 113011 & 0 \\
\hline 21 & $\mathrm{NH}$ & Grafton County & 0.978979 & 0.069059 & 0.713386 & 544 & 3933 & 130641 & 0 \\
\hline 22 & $\mathrm{NH}$ & Hillsborough County & 0.982295 & 0.071030 & 0.292672 & 939 & 10445 & 152946 & 0 \\
\hline 23 & $\mathrm{NH}$ & Merrimack County & 0.976960 & 0.077931 & 0.379773 & 936 & 5685 & 139528 & 0 \\
\hline 24 & $\mathrm{NH}$ & Rockingham County & 0.875568 & 0.077069 & 0.229201 & 834 & 9055 & 147453 & 0 \\
\hline 25 & $\mathrm{NH}$ & Strafford County & 0.960524 & 0.104706 & 0.278765 & 771 & 5980 & 116377 & 0 \\
\hline 26 & NH & Sullivan County & 0.973679 & 0.111339 & 0.445552 & 822 & 5062 & 123049 & 0 \\
\hline 27 & VT & Addison County & 0.952475 & 0.425459 & 0.795092 & 1103 & 3532 & 104605 & 0 \\
\hline 28 & VT & Bennington County & 0.997935 & 0.077816 & 0.649364 & 490 & 4932 & 130391 & 0 \\
\hline 29 & VT & Caledonia County & 0.989694 & 0.232118 & 0.817434 & 628 & 3299 & 96606 & 0 \\
\hline 30 & VT & Chittenden County & 0.869815 & 0.240188 & 0.648925 & 635 & 5739 & 135058 & 0 \\
\hline 31 & VT & Essex County & 0.987327 & 0.041594 & 0.835449 & 824 & 3122 & 80439 & 0 \\
\hline 32 & VT & Franklin County & 0.919758 & 0.499110 & 0.854028 & 1143 & 3211 & 99939 & 0 \\
\hline 33 & $\mathrm{VT}$ & Grand Isle County & 0.424469 & 0.469878 & 0.776269 & 872 & 4961 & 123116 & 0 \\
\hline 34 & VT & Lamoille County & 0.993796 & 0.140267 & 0.759757 & 807 & 3755 & 109882 & 0 \\
\hline 35 & VT & Orange County & 0.995328 & 0.211810 & 0.770961 & 655 & 3982 & 108283 & 0 \\
\hline 36 & VT & Orleans County & 0.967437 & 0.335209 & 0.890911 & 906 & 3104 & 93043 & 0 \\
\hline 37 & VT & Rutland County & 0.987034 & 0.222385 & 0.767982 & 509 & 3563 & 117339 & 0 \\
\hline 38 & VT & Washington County & 0.991004 & 0.133442 & 0.735419 & 535 & 3981 & 120278 & 0 \\
\hline 39 & $\mathrm{vT}$ & Windham County & 0.988228 & 0.087134 & 0.556936 & 791 & 6406 & 122981 & 0 \\
\hline 40 & $V T$ & Windsor County & 0.995116 & 0.144431 & 0.575939 & 358 & 4932 & 124031 & 0 \\
\hline 41 & MA & Barnstable County & 0.303143 & 0.021083 & 0.014410 & 4026 & 27874 & 157592 & 1 \\
\hline 42 & MA & Berkshire County & 0.984207 & 0.102299 & 0.543552 & 728 & 7194 & 135925 & 0 \\
\hline 43 & MA & Bristol County & 0.804381 & 0.096202 & 0.198048 & 2138 & 18080 & 125446 & 0 \\
\hline 44 & MA & Dukes County & 0.211446 & 0.086644 & 0.020024 & 364 & 23899 & 162672 & 0 \\
\hline 45 & MA & Essex County & 0.601028 & 0.079902 & 0.127244 & 1724 & 28381 & 154659 & 0 \\
\hline 46 & MA & Franklin County & 0.968735 & 0.165751 & 0.413335 & 996 & 7244 & 124539 & 1 \\
\hline 47 & MA & Hampden County & 0.975318 & 0.094674 & 0.193698 & 1249 & 8728 & 129185 & 0 \\
\hline 48 & MA & Hampshire County & 0.969861 & 0.157893 & 0.293337 & 1146 & 8717 & 120922 & 0 \\
\hline 49 & MA & Middlesex County & 0.971587 & 0.059922 & 0.055635 & 3512 & 18182 & 171443 & 0 \\
\hline 50 & MA & Nantucket County & 0.157553 & 0.020630 & 0.000000 & 9490 & 35197 & 199017 & 2 \\
\hline 51 & MA & Norfolk County & 0.900073 & 0.038640 & 0.087926 & 2232 & 22306 & 182260 & 0 \\
\hline 52 & MA & Plymouth County & 0.604114 & 0.170887 & 0.015790 & 3275 & 16090 & 145624 & 0 \\
\hline 53 & MA & Suffolk County & 0.487363 & 0.002406 & 0.000000 & 9490 & 35197 & 156800 & 2 \\
\hline 54 & MA & Worcester County & 0.958238 & 0.118548 & 0.287030 & 1068 & 9340 & 134753 & 0 \\
\hline 55 & $\mathbf{R I}$ & Bristol County & 0.552055 & 0.092937 & 0.160382 & 3526 & 31856 & 148773 & 0 \\
\hline 56 & $\mathbf{R I}$ & Kent County & 0.904617 & 0.051779 & 0.121551 & 1097 & 16907 & 136046 & 0 \\
\hline 57 & RI & Newport County & 0.331750 & 0.144624 & 0.148933 & 3004 & 22232 & 137631 & 0 \\
\hline 58 & $\mathbf{R} \mathbf{I}$ & Providence County & 0.948249 & 0.046910 & 0.110992 & 1670 & 15397 & 121010 & 0 \\
\hline 59 & RI & Washington County & 0.591389 & 0.096024 & 0.115392 & 1790 & 13975 & 131732 & 0 \\
\hline 60 & CT & Fairfield County & 0.747724 & 0.024904 & 0.083056 & 2159 & 39050 & 219059 & 0 \\
\hline 61 & CT & Hartford County & 0.979871 & 0.120050 & 0.043448 & 3972 & 20240 & 168483 & 0 \\
\hline 62 & CT & Litchfield County & 0.973931 & 0.147046 & 0.500698 & 859 & 13066 & 170420 & 0 \\
\hline 63 & CT & Middlesex County & 0.841054 & 0.083904 & 0.100521 & 2776 & 15377 & 163451 & 0 \\
\hline
\end{tabular}




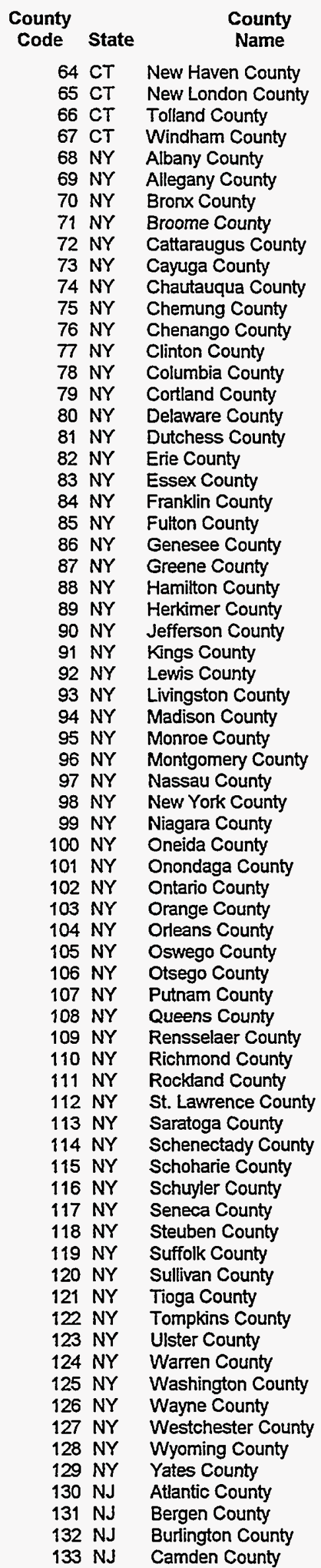

FRCLND FRMFRC DPF ASFP VFRM VNFRM Absolute Absolute Absolute \$/ha \$/ha \$/person Notes

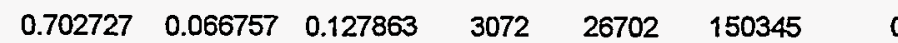

$\begin{array}{lllllll}0.863116 & 0.154793 & 0.140664 & 3658 & 13471 & 137218 & 0\end{array}$

$\begin{array}{lllllll}0.983334 & 0.147512 & 0.638154 & 1368 & 12517 & 148631 & 0\end{array}$

$\begin{array}{rrrrrrr}0.983292 & 0.168386 & 0.520462 & 1510 & 10158 & 122392 & 0 \\ 0.981807 & 0.172694 & 0.002498 & 666 & 6622 & 142257 & 0\end{array}$

$\begin{array}{lllllll}0.995939 & 0.245144 & 0.006992 & 474 & 1711 & 85275 & 0\end{array}$

$\begin{array}{lllllll}0.731929 & 0.000000 & 0.000000 & 0 & 0 & 105520 & 0\end{array}$

$\begin{array}{lllllll}0.987921 & 0.216337 & 0.004848 & 625 & 3004 & 122310 & 0\end{array}$

$\begin{array}{lllllll}0.990631 & 0.242979 & 0.006846 & 680 & 2684 & 92779 & 0\end{array}$

$\begin{array}{lllllll}0.890032 & 0.572469 & 0.002607 & 914 & 3203 & 100914 & 0 \\ 0.708007 & 0.381804 & 0.004889 & 841 & 3210 & 102932 & 0\end{array}$

$\begin{array}{lllllll}0.993630 & 0.225703 & 0.004951 & 499 & 2361 & 107592 & 0\end{array}$

$\begin{array}{lllllll}0.995171 & 0.328439 & 0.006224 & 735 & 2583 & 98916 & 0\end{array}$

$\begin{array}{lllllll}0.929966 & 0.238104 & 0.004210 & 889 & 2748 & 92739 & 0\end{array}$

$\begin{array}{lllllll}0.980669 & 0.275184 & 0.001940 & 1228 & 7247 & 133080 & 0\end{array}$

$\begin{array}{lllllll}0.996270 & 0.433462 & 0.005138 & 770 & 2839 & 95936 & 0\end{array}$

$\begin{array}{rrrrrrr}0.985239 & 0.207531 & 0.006333 & 658 & 3041 & 96396 & 0\end{array}$

$\begin{array}{lllllll}0.971196 & 0.213800 & 0.002025 & 745 & 11073 & 150182 & 0\end{array}$

$\begin{array}{lllllll}0.851419 & 0.217891 & 0.003145 & 1203 & 5177 & 123983 & 0\end{array}$

$\begin{array}{rrrrrrr}0.937565 & 0.047812 & 0.004305 & 376 & 3360 & 102465 & 0 \\ 0.961161 & 0.132441 & 0.006241 & 853 & 2411 & 94147 & 0\end{array}$

$\begin{array}{lllllll}0.931021 & 0.111298 & 0.006473 & 713 & 3369 & 106238 & 0\end{array}$

$\begin{array}{lllllll}0.997511 & 0.543003 & 0.001873 & 1022 & 3145 & 116777 & 0\end{array}$

$\begin{array}{lllllll}0.984297 & 0.110510 & 0.004196 & 437 & 6988 & 111141 & 0\end{array}$

$\begin{array}{lllllll}0.951760 & 0.000015 & 0.000000 & 7562 & 2288 & 112510 & 3\end{array}$

$\begin{array}{lrlrrrr}0.968037 & 0.180473 & 0.006572 & 768 & 2529 & 97256 & 0 \\ 0.685021 & 0.369106 & 0.005745 & 627 & 2027 & 102986 & 0\end{array}$

$\begin{array}{lllllll}0.728098 & 0.000089 & 0.000000 & 85869 & 244261 & 113810 & 4\end{array}$

$\begin{array}{lllllll}0.988826 & 0.207398 & 0.006695 & 911 & 2625 & 84780 & 0\end{array}$

$\begin{array}{lllllll}0.987016 & 0.506944 & 0.001987 & 740 & 3012 & 109577 & 0\end{array}$

$\begin{array}{lllllll}0.991404 & 0.466044 & 0.005285 & 858 & 2878 & 109049 & 0\end{array}$

$\begin{array}{lllllll}0.482662 & 0.261052 & 0.001205 & 931 & 6070 & 146579 & 0\end{array}$

$\begin{array}{lllrrrr}0.986542 & 0.535809 & 0.005736 & 881 & 2993 & 108872 & 0 \\ 0.632598 & 0.010298 & 0.000000 & 2653 & 64475 & 214568 & 0\end{array}$

$\begin{array}{lllllll}0.632598 & 0.010298 & 0.000000 & 2663 & 64475 & 214568 & 0\end{array}$

$\begin{array}{rrrrrrr}0.842529 & 0.000000 & 0.000000 & 0 & 0 & 262759 & 0 \\ 0.458790 & 0.404821 & 0.001499 & 839 & 3451 & 112753 & 0\end{array}$

$\begin{array}{llllll}0.458676 & 0.312610 & 0.006033 & 765 & 2971 & 111602\end{array}$

$\begin{array}{llllll}0.968467 & 0.291002 & 0.002948 & 1055 & 4116 & 131407\end{array}$

$\begin{array}{llllll}0.972754 & 0.440384 & 0.002180 & 767 & 4167 & 124972\end{array}$

$\begin{array}{rrrrrr}0.973522 & 0.196626 & 0.001795 & 1795 & 10604 & 134028\end{array}$

$\begin{array}{llllll}0.478793 & 0.534321 & 0.000989 & 952 & 2927 & 104748\end{array}$

$\begin{array}{llllll}0.726502 & 0.184115 & 0.004570 & 688 & 3246 & 100474\end{array}$

$\begin{array}{llllll}0.987878 & 0.340107 & 0.006748 & 659 & 2972 & 105005\end{array}$

$\begin{array}{llllrr}0.940190 & 0.025664 & 0.000000 & 1251 & 16855 & 182111\end{array}$

$\begin{array}{llllll}0.616847 & 0.000474 & 0.000000 & 7562 & 244261 & 140239\end{array}$

$\begin{array}{llllll}0.982830 & 0.221423 & 0.004105 & 702 & 4071 & 120387\end{array}$

$\begin{array}{llllll}0.571124 & 0.000885 & 0.000000 & 7562 & 244261 & 158811\end{array}$

$\begin{array}{llllll}0.873939 & 0.007453 & 0.000000 & 4333 & 129699 & 181230\end{array}$

$\begin{array}{llllll}0.951839 & 0.230804 & 0.007019 & 587 & 1932 & 86047\end{array}$

$\begin{array}{llllll}0.962231 & 0.135491 & 0.003191 & 836 & 5514 & 127722\end{array}$

$\begin{array}{llllll}0.983231 & 0.145527 & 0.000682 & 1509 & 5469 & 140063\end{array}$

$\begin{array}{llllll}0.993066 & 0.295995 & 0.005793 & 623 & 3564 & 96674\end{array}$

$\begin{array}{llllll}0.949640 & 0.310496 & 0.004412 & 489 & 2421 & 86677\end{array}$

$\begin{array}{llllll}0.832015 & 0.553356 & 0.002491 & 681 & 3309 & 108439\end{array}$

$\begin{array}{llllll}0.991849 & 0.407579 & 0.005079 & 560 & 2276 & 100027\end{array}$

$\begin{array}{llllll}0.383937 & 0.060620 & 0.000000 & 9349 & 26910 & 164331\end{array}$

$\begin{array}{llllll}0.972789 & 0.090228 & 0.003957 & 903 & 6869 & 123922\end{array}$

$\begin{array}{llllll}0.991946 & 0.345978 & 0.005437 & 665 & 2727 & 103440\end{array}$

$\begin{array}{llllll}0.968321 & 0.301362 & 0.002070 & 1365 & 3812 & 103434\end{array}$

$\begin{array}{llllll}0.970504 & 0.096591 & 0.000530 & 1806 & 8135 & 127499\end{array}$

$\begin{array}{llllll}0.933450 & 0.010440 & 0.000656 & 648 & 6953 & 117224\end{array}$

$\begin{array}{llllll}0.987732 & 0.385146 & 0.004086 & 975 & 3519 & 94669\end{array}$

$\begin{array}{llllll}0.436523 & 0.451575 & 0.001288 & 1275 & 3965 & 115998\end{array}$

$\begin{array}{llllll}0.865412 & 0.020607 & 0.000000 & 3083 & 30519 & 225751\end{array}$

$\begin{array}{llllll}0.994126 & 0.553057 & 0.003342 & 1237 & 3207 & 90002\end{array}$

$\begin{array}{llllll}0.909635 & 0.471286 & 0.004848 & 789 & 3228 & 88600\end{array}$

$\begin{array}{llllll}0.835706 & 0.082436 & 0.000000 & 3626 & 12307 & 162794\end{array}$

$\begin{array}{llllll}0.948889 & 0.017586 & 0.000000 & 6211 & 84180 & 218300\end{array}$

$\begin{array}{llllll}0.982049 & 0.188692 & 0.096929 & 1642 & 12922 & 155838\end{array}$

$\begin{array}{lllllll}0.976911 & 0.054810 & 0.000000 & 2592 & 19966 & 134990\end{array}$

0
0
0
0
0
0
0
0
0
0
0
0
0
0
0
0
0
0
0
0
0
0
0
0
0
0
0
0
0
0
0
0
0
0
0
0
0
0
0
0
0
0
0
0
5
0
0
0
0
0
0
0
0
0




\begin{tabular}{|c|c|c|c|c|c|c|c|c|c|}
\hline $\begin{array}{l}\text { County } \\
\text { Code }\end{array}$ & State & $\begin{array}{l}\text { County } \\
\text { Name }\end{array}$ & $\begin{array}{l}\text { FRCLND } \\
\text { Absolute }\end{array}$ & $\begin{array}{l}\text { FRMFRC } \\
\text { Absolute }\end{array}$ & $\begin{array}{c}\text { DPF } \\
\text { Absolute }\end{array}$ & $\begin{array}{l}\text { ASFP } \\
\text { S/ha }\end{array}$ & $\begin{array}{l}\text { VFRM } \\
\text { S/ha }\end{array}$ & $\begin{array}{l}\text { VNFRM } \\
\text { \$/person }\end{array}$ & Notes \\
\hline 134 & NJ & Cape May County & 0.411443 & 0.071288 & 0.000000 & 1191 & 9959 & 152566 & 0 \\
\hline 135 & NJ & Cumberland County & 0.723235 & 0.219141 & 0.024742 & 2628 & 9425 & 113918 & 0 \\
\hline 136 & NJ & Essex County & 0.974518 & 0.007584 & 0.000000 & 3680 & 75792 & 166099 & 0 \\
\hline 137 & NJ & Gloucester County & 0.963994 & 0.296989 & 0.042639 & 2184 & 13895 & 125460 & 0 \\
\hline 138 & NJ & Hudson County & 0.749307 & 0.000000 & 0.000000 & 0 & 0 & 125054 & 0 \\
\hline 139 & NJ & Hunterdon County & 0.982287 & 0.386267 & 0.190300 & 609 & 22180 & 204923 & 0 \\
\hline 140 & NJ & Mercer County & 0.987260 & 0.247461 & 0.053656 & 1096 & 27535 & 176279 & 0 \\
\hline 141 & NJ & Middlesex County & 0.962761 & 0.125808 & 0.011310 & 2324 & 24824 & 168626 & 0 \\
\hline 142 & NJ & Monmouth County & 0.709208 & 0.194567 & 0.013210 & 2142 & 31841 & 185525 & 0 \\
\hline 143 & NJ & Morris County & 0.974473 & 0.079661 & 0.018392 & 1825 & 30889 & 213491 & 1 \\
\hline 144 & NJ & Ocean County & 0.694776 & 0.025450 & 0.069560 & 1203 & 20783 & 142061 & 0 \\
\hline 145 & NJ & Passaic County & 0.939024 & 0.015519 & 0.023743 & 4598 & 31672 & 148827 & 1 \\
\hline 146 & NJ & Salem County & 0.906869 & 0.454421 & 0.160871 & 1369 & 8249 & 120800 & 0 \\
\hline 147 & NJ & Somerset County & 0.998871 & 0.225556 & 0.161104 & 710 & 19066 & 224735 & 0 \\
\hline 148 & NJ & Sussex County & 0.972542 & 0.226416 & 0.440014 & 647 & 13969 & 165300 & 0 \\
\hline 149 & NJ & Union County & 0.979385 & 0.004916 & 0.000000 & 50455 & 148654 & 177783 & 0 \\
\hline 150 & NJ & Warren County & 0.986595 & 0.382590 & 0.264670 & 1126 & 16315 & 146680 & 0 \\
\hline 151 & PA & Adams County & 0.997114 & 0.517874 & 0.143371 & 1773 & 6612 & 113844 & 0 \\
\hline 152 & PA & Aliegheny County & 0.980510 & 0.069599 & 0.053454 & 866 & 7340 & 140077 & 0 \\
\hline 153 & PA & Armstrong County & 0.984185 & 0.285673 & 0.111610 & 1404 & 4641 & 113370 & 0 \\
\hline 154 & PA & Beaver County & 0.980461 & 0.208063 & 0.464782 & 525 & 5487 & 102695 & 0 \\
\hline 155 & PA & Bedford County & 0.997157 & 0.306550 & 0.693447 & 675 & 3727 & 87537 & 0 \\
\hline 156 & PA & Berks County & 0.992537 & 0.403675 & 0.218360 & 2646 & 9077 & 133127 & 0 \\
\hline 157 & PA & Blair County & 0.997511 & 0.227206 & 0.713114 & 1348 & 5322 & 100101 & 0 \\
\hline 158 & PA & Bradford County & 0.991087 & 0.421835 & 0.472403 & 1031 & 3234 & 103264 & 0 \\
\hline 159 & PA & Bucks County & 0.976689 & 0.197458 & 0.142950 & 1989 & 14091 & 152722 & 0 \\
\hline 160 & PA & Butler County & 0.992121 & 0.256239 & 0.359425 & 540 & 5237 & 116844 & 0 \\
\hline 161 & PA & Cambria County & 0.992227 & 0.174844 & 0.271307 & 536 & 3556 & 98029 & 0 \\
\hline 162 & PA & Cameron County & 0.996401 & 0.013036 & 0.861328 & 191 & 4417 & 100921 & 6 \\
\hline 163 & PA & Carbon County & 0.984001 & 0.077707 & 0.151429 & 686 & 7885 & 102797 & 0 \\
\hline 164 & PA & Centre County & 0.996117 & 0.197381 & 0.611454 & 791 & 6274 & 104822 & 0 \\
\hline 165 & PA & Chester County & 0.994941 & 0.365079 & 0.161821 & 3953 & 14121 & 167515 & 0 \\
\hline 166 & PA & Clarion County & 0.989315 & 0.245900 & 0.623767 & 362 & 2750 & 94750 & 0 \\
\hline 167 & PA & Clearfield County & 0.994375 & 0.074930 & 0.518523 & 377 & 3046 & 97636 & 0 \\
\hline 168 & PA & Clinton County & 0.991952 & 0.069123 & 0.516480 & 1071 & 5011 & 88831 & 0 \\
\hline 169 & PA & Columbia County & 0.991350 & 0.327629 & 0.246305 & 691 & 5092 & 97575 & 0 \\
\hline 170 & PA & Crawford County & 0.975989 & 0.325545 & 0.649485 & 619 & 2749 & 97649 & 0 \\
\hline 171 & PA & Cumberland County & 0.998157 & 0.403046 & 0.507543 & 1317 & 7514 & 130411 & 0 \\
\hline 172 & PA & Dauphin County & 0.942166 & 0.268578 & 0.265823 & 1352 & 8348 & 124275 & 0 \\
\hline 173 & PA & Delaware County & 0.965714 & 0.043213 & 0.031759 & 3367 & 36916 & 160240 & 1 \\
\hline 174 & PA & Elk County & 5700 & 0.030868 & 0.353056 & 464 & 3926 & 114277 & 0 \\
\hline 175 & PA & Erie County & 0.514596 & 0.327042 & 0.353668 & 956 & 4835 & 110613 & 0 \\
\hline 176 & PA & Fayette County & 135 & 0.210390 & 0.412694 & 394 & 3718 & 95170 & 0 \\
\hline 177 & PA & Forest County & 0.992398 & 0.017160 & 0.451724 & 305 & 16890 & 88831 & 7 \\
\hline 178 & PA & Franklin County & 0.999071 & 0.474413 & 0.533344 & 1777 & 6498 & 110274 & 0 \\
\hline 179 & PA & Fulton County & 0.998941 & 0.317723 & 0.513678 & 552 & 2906 & 97487 & 0 \\
\hline 180 & PA & Greene County & 0.996402 & 0.341056 & 0.331161 & 133 & 2012 & 85647 & 0 \\
\hline 181 & PA & Huntingdon County & 0.983232 & 0.231158 & 0.661991 & 698 & 3523 & 82349 & 0 \\
\hline 182 & PA & Indiana County & 0.994222 & 0.269423 & 0.331664 & 714 & 3435 & 93748 & 0 \\
\hline 183 & PA & Jefferson County & 0.997864 & 0.189047 & 0.445880 & 463 & 2957 & 100304 & 0 \\
\hline 184 & PA & Juniata County & 0.994972 & 0.339580 & 0.311113 & 1481 & 6054 & 99065 & 0 \\
\hline 185 & PA & Lackawanna County & 0.987555 & 0.125884 & 0.324010 & 765 & 5917 & 114894 & 0 \\
\hline 186 & PA & Lancaster County & 0.964660 & 0.639348 & 0.288362 & 4332 & 13630 & 127864 & 0 \\
\hline 187 & PA & Lawrence County & 0.993697 & 0.374499 & 0.535546 & 686 & 3940 & 100067 & 0 \\
\hline 188 & PA & Lebanon County & 0.997807 & 0.451369 & 0.333374 & 3117 & 9770 & 111758 & 0 \\
\hline 189 & PA & Lehigh County & 0.995235 & 0.374012 & 0.071928 & 1270 & 10369 & 134008 & 0 \\
\hline 190 & PA & Luzerne County & 0.982155 & 0.087424 & 0.256540 & 735 & 6422 & 111114 & 0 \\
\hline 191 & PA & Lycoming County & 0.992834 & 0.168278 & 0.376052 & 722 & 4282 & 106868 & 0 \\
\hline 192 & PA & Mc Kean County & 0.997356 & 0.062972 & 0.581512 & 237 & 2651 & 97588 & 0 \\
\hline 193 & PA & Mercer County & 0.984242 & 0.373964 & 0.528594 & 631 & 3121 & 102797 & 0 \\
\hline 194 & PA & Mifflin County & 0.993358 & 0.309773 & 0.540079 & 1417 & 4737 & 91499 & 0 \\
\hline 195 & PA & Monroe County & 0.985864 & 0.053453 & 0.183507 & 480 & 7358 & 119154 & 0 \\
\hline 196 & PA & Montgomery County & 0.991118 & 0.143672 & 0.202605 & 1542 & 16576 & 192779 & 0 \\
\hline 197 & PA & Montour County & 0.988298 & 0.494087 & 0.366617 & 755 & 5852 & 122703 & 0 \\
\hline 198 & PA & Northampton County & 0.990569 & 0.340538 & 0.310226 & 890 & 8579 & 124193 & 0 \\
\hline 199 & PA & Northumberland County & 0.963407 & 0.371789 & 0.155106 & 1158 & 5087 & 98686 & 0 \\
\hline 200 & PA & Perry County & 0.995957 & 0.294376 & 0.383693 & 960 & 5257 & 101198 & 0 \\
\hline 201 & PA & Philadelphia County & 0.947114 & 0.009196 & 0.000000 & 1588 & 16945 & 113255 & 8 \\
\hline 202 & Pf & Pike County & 0.965437 & 0.017699 & 0.064532 & 834 & 7709 & 129673 & 0 \\
\hline 203 & & Potter County & 0.999779 & 0.130155 & 0.622430 & 519 & 2892 & 94127 & 0 \\
\hline
\end{tabular}




\begin{tabular}{|c|c|c|c|c|c|c|c|c|c|}
\hline $\begin{array}{l}\text { County } \\
\text { Code }\end{array}$ & State & $\begin{array}{l}\text { County } \\
\text { Name }\end{array}$ & $\begin{array}{l}\text { FRCLND } \\
\text { Absolute }\end{array}$ & $\begin{array}{l}\text { FRMFRC } \\
\text { Absolute }\end{array}$ & $\begin{array}{c}\text { DPF } \\
\text { Absolute }\end{array}$ & $\begin{array}{l}\text { ASFP } \\
\text { s/ha }\end{array}$ & $\begin{array}{l}\text { VFRM } \\
\text { S/ha }\end{array}$ & $\begin{array}{l}\text { VNFRM } \\
\text { \$/person }\end{array}$ & Notes \\
\hline 204 & PA & Schuylkill County & 0.994854 & 0.178695 & 0.167082 & & 6041 & 107376 & 0 \\
\hline $\begin{array}{l}205 \\
206\end{array}$ & $\begin{array}{l}\text { PA } \\
\text { PA }\end{array}$ & $\begin{array}{l}\text { Snyder County } \\
\text { Somerset County }\end{array}$ & $\begin{array}{l}0.997137 \\
0.993985\end{array}$ & $\begin{array}{l}0.411600 \\
0.319744\end{array}$ & 0.278921 & $\begin{array}{r}1574 \\
644\end{array}$ & $\begin{array}{l}5597 \\
3184\end{array}$ & $\begin{array}{r}119276 \\
96654\end{array}$ & $\begin{array}{l}0 \\
0\end{array}$ \\
\hline $\begin{array}{l}206 \\
207\end{array}$ & $\begin{array}{l}\text { PA } \\
\text { PA }\end{array}$ & $\begin{array}{l}\text { Somerset County } \\
\text { Sullivan County }\end{array}$ & $\begin{array}{l}0.993985 \\
0.994707\end{array}$ & 0.319744 & 0.673550 & 644 & 3184 & & 0 \\
\hline 207 & & $\begin{array}{l}\text { Sullivan County } \\
\text { Susquehanna County }\end{array}$ & 0.994707 & 0.106300 & 0.569905 & 636 & 3265 & 97094 & 0 \\
\hline 208 & PA & & 0.988667 & 0.336448 & 0.700473 & 587 & 3173 & 94737 & 0 \\
\hline 209 & PA & $\begin{array}{l}\text { Tioga County } \\
\text { Union County }\end{array}$ & 0.996853 & 0.292823 & 0.695515 & 551 & 2658 & 88004 & 0 \\
\hline 210 & PA & Union County & 0.998769 & 0.311553 & 0.375789 & 1463 & 7467 & 103610 & 0 \\
\hline 211 & PA & Venango County & 0.988335 & 0.122110 & 0.505558 & 299 & 2780 & 110180 & 0 \\
\hline 212 & PA & Warren County & 0.984001 & 0.119182 & 0.711556 & 551 & 2384 & 114589 & 0 \\
\hline 213 & PA & Washington County & 0.995521 & 0.370100 & 0.414700 & 328 & 4420 & 112604 & 0 \\
\hline 214 & PA & Wayne County & 0.971720 & 0.261159 & 0.755978 & 530 & 5310 & 110037 & 0 \\
\hline 215 & PA & Westmoreland County & 0.986604 & 0.235156 & 0.361035 & 620 & 5011 & 115612 & 0 \\
\hline 216 & PA & Wyoming County & 0.981238 & 0.246789 & 0.314229 & 1109 & 3721 & 105005 & 0 \\
\hline 217 & PA & York County & 0.993731 & 0.435350 & 0.212071 & 1177 & 7821 & 132091 & 0 \\
\hline 218 & $\mathrm{OH}$ & Adams County & 0.996796 & 0.525421 & 0.170948 & 330 & 2839 & 68159 & 0 \\
\hline 219 & $\mathrm{OH}$ & Allen County & 0.993965 & 0.749540 & 0.043569 & 662 & 4582 & 112042 & 0 \\
\hline 220 & $\mathrm{OH}$ & Ashland County & 0.994207 & 0.630055 & 0.425041 & 673 & 3856 & 101463 & 0 \\
\hline 221 & $\mathrm{OH}$ & Ashtabula County & 0.513388 & 0.346641 & 0.476565 & 577 & 3360 & 90822 & 0 \\
\hline 222 & $\mathrm{OH}$ & Athens County & 0.996496 & 0.248211 & 0.418204 & 178 & 2427 & 78474 & 0 \\
\hline 223 & $\mathrm{OH}$ & Auglaize County & 0.998838 & 0.798652 & 0.202421 & 824 & 4397 & 109523 & 0 \\
\hline 224 & $\mathrm{OH}$ & Belmont County & 0.992421 & 0.366979 & 0.423841 & 216 & 2285 & 95949 & 0 \\
\hline 225 & $\mathrm{OH}$ & Brown County & 0.993020 & 0.642373 & 0.069776 & 425 & 3528 & 88126 & 0 \\
\hline 226 & $\mathrm{OH}$ & Butler County & 0.993768 & 0.462412 & 0.132490 & 616 & 5696 & 113451 & 0 \\
\hline 227 & $\mathrm{OH}$ & Carroll County & 0.989135 & 0.484886 & 0.505121 & 400 & 3027 & 88275 & 0 \\
\hline 228 & $\mathrm{OH}$ & Champaign County & 0.997332 & 0.786713 & 0.133267 & 670 & 3901 & 96938 & 0 \\
\hline 229 & $\mathrm{OH}$ & Clark County & 0.990581 & 0.700269 & 0.024231 & 886 & 4704 & 112950 & 0 \\
\hline 230 & $\mathrm{OH}$ & Clermont County & 0.987582 & 0.342927 & 0.050908 & 457 & 6000 & 109936 & 0 \\
\hline 231 & $\mathrm{OH}$ & Clinton County & 0.996589 & 0.864648 & 0.014912 & 626 & 4346 & 109103 & 0 \\
\hline 232 & $\mathrm{OH}$ & Columbiana County & 0.994972 & 0.421845 & 0.431187 & 759 & 3910 & 85322 & 0 \\
\hline 233 & $\mathrm{OH}$ & Coshocton County & 0.993877 & 0.447932 & 0.283061 & 426 & 3030 & 92969 & 0 \\
\hline 234 & $\mathrm{OH}$ & Crawford County & 0.998711 & 0.866891 & 0.062250 & 616 & 3712 & 94249 & 0 \\
\hline 235 & $\mathrm{OH}$ & Cuyahoga County & 0.367913 & 0.013843 & 0.000000 & 9687 & 26979 & 142820 & 0 \\
\hline 236 & $\mathrm{OH}$ & Darke County & 0.999230 & 0.874012 & 0.080047 & 1467 & 5392 & 109177 & 0 \\
\hline 237 & $\mathrm{OH}$ & Defiance County & 0.992779 & 0.747626 & 0.084824 & 498 & 3340 & 104361 & 0 \\
\hline 238 & $\mathrm{OH}$ & Delaware County & 0.970497 & 0.596758 & 0.060701 & 596 & 6466 & 128549 & 0 \\
\hline 239 & $\mathrm{OH}$ & Erie County & 0.406691 & 0.545797 & 0.103452 & 725 & 5392 & 116093 & 0 \\
\hline 240 & $\mathrm{OH}$ & Fairfield County & 0.994406 & 0.610913 & 0.073027 & 543 & 4517 & 110688 & 0 \\
\hline 241 & $\mathrm{OH}$ & Fayette County & 0.998779 & 0.906776 & 0.008709 & 616 & 3536 & 93192 & 0 \\
\hline 242 & $\mathrm{OH}$ & Franklin County & 0.993779 & 0.280746 & 0.048271 & 1008 & 7469 & 128962 & 0 \\
\hline 243 & $\mathrm{OH}$ & Fulton County & 0.998678 & 0.789785 & 0.038477 & 1003 & 5098 & 113769 & 0 \\
\hline 244 & $\mathrm{OH}$ & Gallia County & 0.994985 & 0.366030 & 0.239265 & 310 & 2583 & 86914 & 0 \\
\hline 245 & $\mathrm{OH}$ & Geauga County & 0.988813 & 0.252333 & 0.474761 & 609 & 7783 & 140320 & 0 \\
\hline 246 & $\mathrm{OH}$ & Greene County & 0.996823 & 0.693966 & 0.031290 & 688 & 5108 & 123008 & 0 \\
\hline 247 & $\mathrm{OH}$ & Guernsey County & 0.988106 & 0.382712 & 0.279282 & 186 & 2381 & 86975 & 0 \\
\hline 248 & $\mathrm{OH}$ & Hamilton County & 0.986874 & 0.111160 & 0.053501 & 1363 & 9286 & 139392 & $\mathbf{0}$ \\
\hline 249 & $\mathrm{OH}$ & Hancock County & 0.995798 & 0.810499 & 0.031032 & 562 & 4857 & 127743 & 0 \\
\hline 250 & $\mathrm{OH}$ & Hardin County & 0.999479 & 0.825261 & 0.041118 & 539 & 3287 & 86751 & 0 \\
\hline 251 & $\mathrm{OH}$ & Harrison County & 0.982362 & 0.438779 & 0.417491 & 183 & 1808 & 73496 & 0 \\
\hline 252 & $\mathrm{OH}$ & Henry County & 0.991736 & 0.919228 & 0.043931 & 646 & 4439 & 109523 & 0 \\
\hline 253 & $\mathrm{OH}$ & Highland County & 0.991826 & 0.652271 & 0.075005 & 473 & 3185 & 89779 & 0 \\
\hline 254 & $\mathrm{OH}$ & Hocking County & 0.998019 & 0.177583 & 0.000000 & 136 & 2729 & 83541 & 0 \\
\hline 255 & $\mathrm{OH}$ & Holmes County & 0.997574 & 0.654511 & 0.372779 & 1101 & 4652 & 74614 & 0 \\
\hline 256 & $\mathrm{OH}$ & Huron County & 0.996292 & 0.694017 & 0.094496 & 717 & 3970 & 111616 & 0 \\
\hline 257 & $\mathrm{OH}$ & Jackson County & 0.997106 & 0.276815 & 0.047491 & 617 & 2379 & 77838 & 0 \\
\hline 258 & $\mathrm{OH}$ & Jefferson County & 0.996941 & 0.260689 & 0.356231 & 232 & 1952 & 98774 & 0 \\
\hline 259 & $\mathrm{OH}$ & Knox County & 0.995449 & 0.624229 & 0.185857 & 680 & 3294 & 101225 & 0 \\
\hline 260 & $\mathrm{OH}$ & Lake County & 0.233123 & 0.117328 & 0.002976 & 7176 & 12755 & 133378 & 1 \\
\hline 261 & $\mathrm{OH}$ & Lawrence County & 0.995947 & 0.212698 & 0.186066 & 191 & 2918 & 80201 & 0 \\
\hline 262 & $\mathrm{OH}$ & Licking County & 0.997275 & 0.517394 & 0.086666 & 1023 & 5129 & 111162 & 0 \\
\hline 263 & $\mathrm{OH}$ & Logan County & 0.982148 & 0.691581 & 0.148281 & 681 & 3445 & 103942 & 0 \\
\hline 264 & $\mathrm{OH}$ & Lorain County & 0.533613 & 0.452421 & 0.131790 & 1223 & 5722 & 108412 & 0 \\
\hline 265 & $\mathrm{OH}$ & Lucas County & 0.607153 & 0.339846 & 0.002428 & 1222 & 6301 & 120665 & 1 \\
\hline 266 & $\mathrm{OH}$ & Madison County & 0.998448 & 0.877624 & 0.045349 & 638 & 4087 & 105716 & 0 \\
\hline 267 & $\mathrm{OH}$ & Mahoning County & 0.980798 & 0.276491 & 0.322282 & 934 & 5444 & 110105 & 0 \\
\hline 268 & $\mathrm{OH}$ & Marion County & 0.999248 & 0.841086 & 0.037738 & 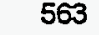 & 3547 & 91777 & 0 \\
\hline 269 & $\mathrm{OH}$ & Medina County & 0.996284 & 0.384945 & 0.350805 & 653 & 6249 & 127959 & 0 \\
\hline 270 & $\mathrm{OH}$ & Meigs County & 0.993165 & 0.316667 & 0.273898 & 293 & 2356 & 78312 & 0 \\
\hline 271 & $\mathrm{OH}$ & Mercer County & 0.978809 & 0.907792 & 0.179281 & 1751 & 5435 & 114636 & 0 \\
\hline 272 & r & Miami County & 0.994721 & 0.769314 & 0.066317 & 767 & 5113 & 114975 & 0 \\
\hline 27 & & Monroe County & 0.995810 & 0.377235 & 0.380866 & 186 & 2123 & 89352 & ? \\
\hline
\end{tabular}




\begin{tabular}{|c|c|c|c|c|c|c|c|c|c|}
\hline $\begin{array}{c}\text { County } \\
\text { Code }\end{array}$ & State & $\begin{array}{c}\text { County } \\
\text { Name }\end{array}$ & $\begin{array}{l}\text { FRCLND } \\
\text { Absolute }\end{array}$ & $\begin{array}{l}\text { FRMFRC } \\
\text { Absolute }\end{array}$ & $\begin{array}{c}\text { DPF } \\
\text { Absolute }\end{array}$ & $\begin{array}{l}\text { ASFP } \\
\text { S/ha }\end{array}$ & $\begin{array}{l}\text { VFRM } \\
\text { \$/ha }\end{array}$ & $\begin{array}{l}\text { VNFRM } \\
\text { \$/person }\end{array}$ & Notes \\
\hline 274 & $\mathrm{OH}$ & Montgomery County & 0.994220 & 0.362640 & 0.053411 & 724 & 6973 & 124695 & 0 \\
\hline 275 & $\mathrm{OH}$ & Morgan County & 0.990082 & 0.426042 & 0.191419 & 231 & 2006 & 87903 & 0 \\
\hline 276 & $\mathrm{OH}$ & Morrow County & 0.995501 & 0.634204 & 0.141746 & 533 & 3390 & 89325 & 0 \\
\hline 277 & $\mathrm{OH}$ & Muskingum County & 0.988158 & 0.441303 & 0.154155 & 334 & 2626 & 101347 & 0 \\
\hline 278 & $\mathrm{OH}$ & Noble County & 0.986197 & 0.408020 & 0.129822 & 114 & 2159 & 84035 & 0 \\
\hline 279 & $\mathrm{OH}$ & Ottawa County & 0.411174 & 0.652775 & 0.045275 & 551 & 4469 & 124810 & 0 \\
\hline 280 & $\mathrm{OH}$ & Paulding County & 0.993703 & 0.822151 & 0.025545 & 620 & 3672 & 100501 & 0 \\
\hline 281 & $\mathrm{OH}$ & Perry County & 0.993746 & 0.366051 & 0.134772 & 364 & 3110 & 79165 & 0 \\
\hline 282 & $\mathrm{OH}$ & Pickaway County & 0.990754 & 0.807111 & 0.036354 & 557 & 3761 & 93186 & 0 \\
\hline 283 & $\mathrm{OH}$ & Pike County & 0.994466 & 0.311262 & 0.149070 & 263 & 2629 & 81366 & 0 \\
\hline 284 & $\mathrm{OH}$ & Portage County & 0.970976 & 0.305549 & 0.290331 & 600 & 5686 & 108581 & 0 \\
\hline 285 & $\mathrm{OH}$ & Preble County & 0.996432 & 0.750590 & 0.073311 & 748 & 4542 & 100900 & 0 \\
\hline 286 & $\mathrm{OH}$ & Putnam County & 0.999248 & 0.925718 & 0.052218 & 753 & 4948 & 110369 & 0 \\
\hline 287 & $\mathrm{OH}$ & Richland County & 0.993358 & 0.505288 & 0.227102 & 680 & 4262 & 109665 & 0 \\
\hline 288 & $\mathrm{OH}$ & Ross County & 0.993439 & 0.575073 & 0.045470 & 428 & 2964 & 91276 & 0 \\
\hline 289 & $\mathrm{OH}$ & Sandusky County & 0.979429 & 0.772552 & 0.039436 & 672 & 4265 & 115138 & 0 \\
\hline 290 & $\mathrm{OH}$ & Scioto County & 0.993839 & 0.244213 & 0.106403 & 420 & 3321 & 85844 & 0 \\
\hline 291 & $\mathrm{OH}$ & Seneca County & 0.996777 & 0.840999 & 0.037909 & 612 & 3605 & 100650 & 0 \\
\hline 292 & $\mathrm{OH}$ & Shelby County & 0.995721 & 0.776844 & 0.235278 & 749 & 4802 & 118917 & 0 \\
\hline 293 & $\mathrm{OH}$ & Stark County & 0.991798 & 0.370471 & 0.327651 & 1021 & 5537 & 114454 & 0 \\
\hline 294 & $\mathrm{OH}$ & Summit County & 0.982640 & 0.072250 & 0.036828 & 1009 & 11165 & 125866 & 0 \\
\hline 295 & $\mathrm{OH}$ & Trumbull County & 0.971235 & 0.305779 & 0.412657 & 515 & 4554 & 109347 & 0 \\
\hline 296 & $\mathrm{OH}$ & Tuscarawas County & 0.993176 & 0.408717 & 0.435107 & 770 & 3813 & 101314 & 0 \\
\hline 297 & $\mathrm{OH}$ & Union County & 0.999255 & 0.797774 & 0.060319 & 862 & 3845 & 130682 & 0 \\
\hline 298 & $\mathrm{OH}$ & Van Wert County & 0.999026 & 0.921196 & 0.027532 & 609 & 4760 & 102682 & 0 \\
\hline 299 & $\mathrm{OH}$ & Vinton County & 0.997880 & 0.157213 & 0.129919 & 125 & 2219 & 74830 & 0 \\
\hline 300 & $\mathrm{OH}$ & Warren County & 0.982155 & 0.505688 & 0.103877 & 610 & 8021 & 116689 & 0 \\
\hline 301 & $\mathrm{OH}$ & Washington County & 0.992281 & 0.343507 & 0.257153 & 339 & 2600 & 99193 & 0 \\
\hline 302 & $\mathrm{OH}$ & Wayne County & 0.998305 & 0.694708 & 0.468025 & 1391 & 5264 & 111514 & 0 \\
\hline 303 & $\mathrm{OH}$ & Williams County & 0.996965 & 0.693372 & 0.051111 & 635 & 3097 & 113295 & 0 \\
\hline 304 & $\mathrm{OH}$ & Wood County & 0.994837 & 0.765472 & 0.012281 & 675 & 4518 & 118680 & 0 \\
\hline 305 & $\mathrm{OH}$ & Wyandot County & 0.995073 & 0.833244 & 0.051345 & 568 & 3463 & 103989 & 0 \\
\hline 306 & IN & Adams County & 0.998308 & 0.910339 & 0.142896 & 1008 & 5134 & 98611 & 0 \\
\hline 307 & IN & Allen County & 0.995679 & 0.679239 & 0.068076 & 690 & 5410 & 129524 & 0 \\
\hline 308 & $\mathbb{I N}$ & Bartholomew County & 0.993843 & 0.634016 & 0.029805 & 669 & 4584 & 116594 & 0 \\
\hline 309 & IN & Benton County & 0.999776 & 1.040629 & 0.003650 & 575 & 4625 & 114535 & 9 \\
\hline 310 & IN & Blackford County & 0.998212 & 0.826373 & 0.010354 & 642 & 2765 & 101754 & 0 \\
\hline 311 & IN & Boone County & 0.998674 & 0.825809 & 0.020779 & 805 & 5067 & 148936 & 0 \\
\hline 312 & IN & Brown County & 0.986237 & 0.112850 & 0.059013 & 260 & 3079 & 88560 & 1 \\
\hline 313 & IN & Carroll County & 0.992541 & 0.923636 & 0.004832 & 1160 & 4978 & 112821 & 0 \\
\hline 314 & IN & Cass County & 0.995046 & 0.861727 & 0.041420 & 780 & 4161 & 110559 & 0 \\
\hline 315 & IN & Clark County & 0.997170 & 0.440046 & 0.095021 & 415 & 3684 & 105709 & 0 \\
\hline 316 & IN & Clay County & 0.992309 & 0.709645 & 0.044909 & 577 & 3289 & 95888 & 0 \\
\hline 317 & IN & Clinton County & 0.999560 & 0.910495 & 0.000673 & 902 & 4455 & 110803 & 0 \\
\hline 318 & IN & Crawford County & 0.989741 & 0.305312 & 0.041180 & 588 & 2543 & 78115 & 0 \\
\hline 319 & IN & Daviess County & 0.985946 & 0.806867 & 0.039309 & 1179 & 4049 & 95841 & 0 \\
\hline 320 & IN & Dearbom County & 0.994014 & 0.441430 & 0.116576 & 264 & 4058 & 103237 & 0 \\
\hline 321 & IN & Decatur County & 0.997822 & 0.848816 & 0.037442 & 905 & 4402 & 107694 & 0 \\
\hline 322 & IN & De Kalb County & 0.997357 & 0.659663 & 0.179433 & 541 & 3315 & 106082 & 0 \\
\hline 323 & IN & Delaware County & 0.993351 & 0.672434 & 0.028253 & 626 & 3788 & 106319 & 0 \\
\hline 324 & IN & Dubois County & 0.988195 & 0.702511 & 0.045328 & 1768 & 3870 & 126876 & 0 \\
\hline 325 & IN & Elkhart County & 0.991389 & 0.647824 & 0.346297 & 1245 & 5854 & 113587 & 0 \\
\hline 326 & IN & Fayette County & 0.999282 & 0.810372 & 0.037704 & 611 & 3457 & 101259 & 0 \\
\hline 327 & IN & Floyd County & 0.997889 & 0.314964 & 0.051388 & 340 & 5200 & 116235 & 0 \\
\hline 328 & IN & Fountain County & 0.994406 & 0.904577 & 0.003971 & 543 & 3680 & 100961 & 0 \\
\hline 329 & IN & Franklin County & 0.986374 & 0.601729 & 0.118187 & 576 & 3489 & 98435 & 0 \\
\hline 330 & IN & Fulton County & 0.992282 & 0.823858 & 0.089932 & 631 & 3224 & 100948 & 0 \\
\hline 331 & IN & Gibson County & 0.979516 & 0.770453 & 0.029507 & 676 & 4303 & 109787 & 0 \\
\hline 332 & IN & Grant County & 0.998054 & 0.741696 & 0.032807 & 716 & 4332 & 107071 & 0 \\
\hline 333 & IN & Greene County & 0.993018 & 0.598805 & 0.055258 & 488 & 3222 & 90686 & 0 \\
\hline 334 & IN & Hamilton County & 0.988256 & 0.638596 & 0.019221 & 763 & 5834 & 173943 & 0 \\
\hline 335 & IN & Hancock County & 0.998133 & 0.833083 & 0.002709 & 698 & 4991 & 128013 & 0 \\
\hline 336 & IN & Harrison County & 0.996589 & 0.520815 & 0.084954 & 695 & 3586 & 96173 & 0 \\
\hline 337 & IN & Hendricks County & 0.998828 & 0.715702 & 0.021472 & 639 & 4929 & 128935 & 0 \\
\hline 338 & IN & Henry County & 0.995009 & 0.758666 & 0.025667 & 690 & 3597 & 105506 & 0 \\
\hline 339 & IN & Howard County & 0.997072 & 0.792270 & 0.025360 & 938 & 5371 & 120163 & 0 \\
\hline 340 & IN & Huntington County & 0.986215 & 0.767575 & 0.042786 & 690 & 4475 & 110566 & 0 \\
\hline 341 & IN & Jackson County & 0.991360 & 0.622413 & 0.056330 & 895 & 3946 & 104659 & 0 \\
\hline 342 & IN & Jasper County & 0.997582 & 0.842677 & 0.004232 & 802 & 3439 & 111358 & 0 \\
\hline 343 & IN & Jay County & 0.999531 & 0.744619 & 0.074106 & 864 & 3509 & 96342 & 0 \\
\hline
\end{tabular}




\begin{tabular}{|c|c|c|c|c|c|c|c|c|c|}
\hline $\begin{array}{c}\text { County } \\
\text { Code }\end{array}$ & State & $\begin{array}{l}\text { County } \\
\text { Name }\end{array}$ & $\begin{array}{l}\text { FRCLND } \\
\text { Absolute }\end{array}$ & $\begin{array}{l}\text { FRMFRC } \\
\text { Absolute }\end{array}$ & $\begin{array}{c}\text { DPF } \\
\text { Absolute }\end{array}$ & $\begin{array}{l}\text { ASFP } \\
\text { \$/ha }\end{array}$ & $\begin{array}{l}\text { VFRM } \\
\text { S/ha }\end{array}$ & $\begin{array}{l}\text { VNFRM } \\
\text { \$/person }\end{array}$ & Notes \\
\hline 344 & $\mathbb{N}$ & Jefferson County & 0.995660 & 0.565635 & 0.078511 & 428 & 3061 & 89007 & 0 \\
\hline 345 & IN & Jennings County & 0.997021 & 0.516458 & 0.022858 & 705 & 3517 & 89596 & 0 \\
\hline 346 & IN & Johnson County & 0.995775 & 0.681354 & 0.051447 & 763 & 4931 & 127025 & 0 \\
\hline 347 & IN & Knox County & 0.984307 & 0.925743 & 0.011510 & 803 & 3430 & 98029 & 0 \\
\hline 348 & IN & Kosciusko County & 0.969621 & 0.731378 & 0.070177 & 1232 & 4058 & 122039 & 0 \\
\hline 349 & $\mathbb{N}$ & Lagrange County & 0.981501 & 0.772030 & 0.167248 & 1295 & 4650 & 90199 & 0 \\
\hline 350 & $\mathbb{N}$ & Lake County & 0.793489 & 0.453653 & 0.062139 & 575 & 4206 & 110586 & 0 \\
\hline 351 & $\mathbb{N}$ & La Porte County & 0.975843 & 0.699130 & 0.131069 & 707 & 3796 & 112808 & 0 \\
\hline 352 & $\mathbb{I N}$ & Lawrence County & 0.992846 & 0.552746 & 0.062329 & 267 & 2451 & 98950 & 0 \\
\hline 353 & IN & Madison County & 0.998277 & 0.771733 & 0.014727 & 727 & 4642 & 108493 & 0 \\
\hline 354 & IN & Marion County & 0.983374 & 0.152868 & 0.013547 & 1319 & 8072 & 129693 & 1 \\
\hline 355 & $\mathbb{N}$ & Marshall County & 0.987444 & 0.771580 & 0.197856 & 655 & 3398 & 104842 & 0 \\
\hline 356 & IN & Martin County & 0.987101 & 0.332786 & 0.008148 & 983 & 3051 & 88634 & 0 \\
\hline 357 & IN & Miami County & 0.995709 & 0.785249 & 0.083955 & 846 & 4043 & 96945 & 0 \\
\hline 358 & $\mathbb{I N}$ & Monroe County & 0.958752 & 0.234873 & 0.059757 & 428 & 4190 & 96626 & 0 \\
\hline 359 & $\mathbb{N}$ & Montgomery County & 0.998402 & 0.875608 & 0.003662 & 799 & 3807 & 121836 & 0 \\
\hline 360 & $\mathbb{I N}$ & Morgan County & 0.992879 & 0.536304 & 0.016435 & 541 & 4070 & 104659 & 0 \\
\hline 361 & IN & Newton County & 0.995826 & 0.804364 & 0.002098 & 799 & 3719 & 102262 & 1 \\
\hline 362 & IN & Noble County & 0.984438 & 0.699736 & 0.161210 & 662 & 3367 & 100277 & 0 \\
\hline 363 & $\mathbb{I N}$ & Ohio County & 0.991464 & 0.582219 & 0.095728 & 336 & 3126 & 91506 & 1 \\
\hline 364 & IN & Orange County & 0.978734 & 0.453900 & 0.061895 & 425 & 2426 & 87408 & 0 \\
\hline 365 & $\mathbb{N}$ & Owen County & 0.993198 & 0.458871 & 0.053295 & 300 & 3016 & 90415 & 0 \\
\hline 366 & IN & Parke County & 0.988232 & 0.638084 & 0.023882 & 489 & 3142 & 100690 & 0 \\
\hline 367 & IN & Perty County & 0.987194 & 0.328048 & 0.073466 & 342 & 3094 & 89061 & 0 \\
\hline 368 & $\mathbb{N}$ & Pike County & 0.985614 & 0.396750 & 0.013925 & 583 & 2601 & 102438 & 1 \\
\hline 369 & IN & Porter County & 0.801732 & 0.532324 & 0.036438 & 602 & 4495 & 118978 & 0 \\
\hline 370 & IN & Posey County & 0.973902 & 0.845117 & 0.036221 & 696 & 3971 & 105574 & 0 \\
\hline 371 & $\mathbb{I N}$ & Pulaski County & 0.997941 & 0.874659 & 0.034842 & 766 & 3579 & 114894 & 0 \\
\hline 372 & IN & Putnam County & 0.995289 & 0.664127 & 0.038882 & 536 & 3374 & 97033 & 0 \\
\hline 373 & $\mathbb{I N}$ & Randolph County & 0.999196 & 0.815700 & 0.033009 & 689 & 3241 & 89704 & 0 \\
\hline 374 & IN & Ripley County & 0.996532 & 0.574124 & 0.078734 & 564 & 3294 & 105987 & 0 \\
\hline 375 & $\mathbb{I N}$ & Rush County & 0.999252 & 0.892253 & 0.046845 & 783 & 4168 & 105330 & 0 \\
\hline 376 & $\mathbb{N}$ & St. Joseph County & 0.992120 & 0.588827 & 0.101528 & 714 & 4737 & 116574 & 0 \\
\hline 377 & IN & Scott County & 0.987837 & 0.519729 & 0.051243 & 388 & 2853 & 82152 & 0 \\
\hline 378 & IN & Shelby County & 0.998858 & 0.822727 & 0.034493 & 720 & 4710 & 113661 & 0 \\
\hline 379 & $\mathbb{N}$ & Spencer County & 0.993670 & 0.686279 & 0.049181 & 644 & 3375 & 98821 & 0 \\
\hline 380 & IN & Starke County & 0.990385 & 0.681732 & 0.011687 & 519 & 2798 & 81698 & 0 \\
\hline 381 & $\mathbb{I N}$ & Steuben County & 0.957332 & 0.615970 & 0.245508 & 508 & 3120 & 120963 & 0 \\
\hline 382 & IN & Sullivan County & 0.984913 & 0.632442 & 0.006032 & 588 & 3440 & 101869 & 0 \\
\hline 383 & IN & Switzeriand County & 0.989531 & 0.559692 & 0.162546 & 411 & 3821 & 72419 & 0 \\
\hline 384 & $\mathbb{N}$ & Tippecanoe County & 0.993483 & 0.804483 & 0.010542 & 696 & 4684 & 107335 & 0 \\
\hline 385 & IN & Tipton County & 0.999913 & 0.965618 & 0.004813 & 895 & 5588 & 122676 & 1 \\
\hline 386 & IN & Union County & 0.977609 & 0.774380 & 0.043556 & 726 & 4552 & 94974 & 0 \\
\hline 387 & IN & Vanderburgh County & 0.995039 & 0.539235 & 0.042686 & 680 & 4905 & 122547 & 0 \\
\hline 388 & IN & Vermillion County & 0.988277 & 0.725698 & 0.002290 & 561 & 3378 & 97988 & 0 \\
\hline 389 & IN & Vigo County & 0.982524 & 0.560661 & 0.020682 & 518 & 3368 & 101842 & 0 \\
\hline 390 & IN & Wabash County & 0.981233 & 0.748539 & 0.038606 & 1213 & 4210 & 104449 & 0 \\
\hline 391 & IN & Warren County & 0.995315 & 0.863827 & 0.002235 & 532 & 3691 & 107057 & 0 \\
\hline 392 & IN & Warrick County & 0.982641 & 0.391426 & 0.037880 & 508 & 4081 & 123130 & 0 \\
\hline 393 & $\mathbb{I N}$ & Washington County & 0.995904 & 0.574442 & 0.139293 & 569 & 2821 & 84821 & 0 \\
\hline 394 & IN & Wayne County & 0.998019 & 0.733548 & 0.067080 & 763 & $3 \uparrow 42$ & 103772 & 0 \\
\hline 395 & IN & Wells County & 0.998795 & 0.839050 & 0.058166 & 688 & 4348 & 109570 & 0 \\
\hline 396 & IN & White County & 0.992993 & 0.881878 & 0.009089 & 907 & 4336 & 109421 & 0 \\
\hline 397 & IN & Whitley County & 0.992956 & 0.755536 & 0.098252 & 683 & 3623 & 110200 & 0 \\
\hline 398 & IL & Adams County & 0.983221 & 0.847790 & 0.051900 & 585 & 2975 & 113214 & 0 \\
\hline 399 & IL & Alexander County & 0.936044 & 0.458399 & 0.009069 & 487 & 2512 & 79646 & 0 \\
\hline 400 & IL & Bond County & 0.993565 & 0.750294 & 0.127589 & 591 & 3239 & 107660 & 0 \\
\hline 401 & IL & Boone County & 0.997976 & 0.750532 & 0.159228 & 738 & 5511 & 117380 & 0 \\
\hline 402 & !L & Brown County & 0.994823 & 0.738308 & 0.003311 & 457 & 2641 & 93524 & 1 \\
\hline 403 & IL & Bureau County & 0.994577 & 0.867337 & 0.005796 & 867 & 4555 & 113234 & 0 \\
\hline 404 & IL & Calhoun County & 0.894759 & 0.613524 & 0.006035 & 435 & 2825 & 104111 & 0 \\
\hline 405 & IL & Carroll County & 0.953720 & 0.840274 & 0.061224 & 1026 & 4158 & 101422 & 0 \\
\hline 406 & IL & Cass County & 0.979680 & 0.870424 & 0.000000 & 730 & 3960 & 102661 & 0 \\
\hline 407 & IL & Champaign County & 0.999630 & 0.895961 & 0.004417 & 692 & 5530 & 114853 & 0 \\
\hline 408 & IL & Christian County & 0.990704 & 0.859743 & 0.000000 & 749 & 5315 & 116194 & 0 \\
\hline 409 & $1 \mathrm{lL}$ & Clark County & 0.993311 & 0.809771 & 0.004596 & 548 & 3582 & 98848 & 1 \\
\hline 410 & IL & Clay County & 0.998743 & 0.745040 & 0.004762 & 410 & 2682 & 102831 & 1 \\
\hline 411 & IL & Clinton County & 0.941918 & 0.754860 & 0.275238 & 1025 & 3951 & 115016 & 0 \\
\hline 412 & IL & Coles County & 0.996511 & 0.809732 & 0.007795 & 610 & 4860 & 103014 & 0 \\
\hline 413 & IL & Cook County & 0.578432 & 0.067606 & 0.007683 & 1234 & 14850 & 147175 & 0 \\
\hline
\end{tabular}




\begin{tabular}{|c|c|c|c|c|c|c|c|c|c|}
\hline $\begin{array}{l}\text { County } \\
\text { Code }\end{array}$ & State & $\begin{array}{l}\text { County } \\
\text { Name }\end{array}$ & $\begin{array}{l}\text { FRCLND } \\
\text { Absolute }\end{array}$ & $\begin{array}{l}\text { FRMFRC } \\
\text { Absolute }\end{array}$ & $\begin{array}{c}\text { DPF } \\
\text { Absolute }\end{array}$ & $\begin{array}{l}\text { ASFP } \\
\text { S/ha }\end{array}$ & $\begin{array}{l}\text { VFRM } \\
\text { \$/ha }\end{array}$ & $\begin{array}{l}\text { VNFRM } \\
\text { \$/person }\end{array}$ & Notes \\
\hline 414 & IL & Crawford County & 0.995054 & 0.787517 & 0.008232 & 585 & 3401 & 108954 & 0 \\
\hline 415 & IL & Cumberland County & 0.997175 & 0.794764 & 0.098696 & 714 & 3826 & 91770 & 0 \\
\hline 416 & IL & DeKalb County & 0.998682 & 0.930116 & 0.015321 & 1033 & 6199 & 111460 & 0 \\
\hline 417 & IL & De Witt County & 0.981295 & 0.810615 & 0.001607 & 659 & 5734 & 122818 & 1 \\
\hline 418 & IL & Douglas County & 0.998662 & 0.972610 & 0.030653 & 755 & 5215 & 98801 & 0 \\
\hline 419 & IL & DuPage County & 0.992783 & 0.085058 & 0.004343 & 2762 & 8382 & 181332 & 1 \\
\hline 420 & IL & Edgar County & 0.998991 & 0.888209 & 0.002877 & 642 & 4551 & 105330 & 1 \\
\hline 421 & IL & Edwards County & 0.998642 & 0.817289 & 0.004202 & 551 & 2437 & 104578 & 0 \\
\hline 422 & IL & Effingham County & 0.997494 & 0.841293 & 0.147956 & 684 & 4177 & 118599 & 0 \\
\hline 423 & IL & Fayette County & 0.987772 & 0.744204 & 0.053737 & 512 & 2777 & 87584 & 0 \\
\hline 424 & IL & Ford County & 0.998951 & 0.965063 & 0.003394 & 643 & 4457 & 116533 & 1 \\
\hline 425 & IL & Franklin County & 0.955203 & 0.608672 & 0.014432 & 396 & 2818 & 94127 & 0 \\
\hline 426 & IL & Fulton County & 0.980773 & 0.778667 & 0.003726 & 490 & 2992 & 96362 & 0 \\
\hline 427 & IL & Gallatin County & 0.985754 & 0.829839 & 0.004690 & 542 & 3021 & 105215 & 1 \\
\hline 428 & IL & Greene County & 0.994056 & 0.873747 & 0.007300 & 557 & 2946 & 90652 & 0 \\
\hline 429 & IL & Grundy County & 0.976063 & 0.838660 & 0.003339 & 580 & 6156 & 132714 & 1 \\
\hline 430 & IL & Hamilton County & 0.998453 & 0.723702 & 0.002718 & 399 & 2189 & 91079 & 1 \\
\hline 431 & IL & Hancock County & 0.975551 & 0.851864 & 0.004089 & 598 & 3228 & 99898 & 0 \\
\hline 432 & IL & Hardin County & 0.982375 & 0.332715 & 0.000000 & 133 & 1684 & 77350 & 0 \\
\hline 433 & IL & Henderson County & 0.958716 & 0.841283 & 0.005091 & 631 & 3515 & 100934 & 1 \\
\hline 434 & IL & Henry County & 0.997112 & 0.861562 & 0.003127 & 930 & 4358 & 108039 & 0 \\
\hline 435 & IL & Iroquois County & 0.998566 & 0.927318 & 0.018908 & 701 & 4227 & 104571 & 0 \\
\hline 436 & IL & Jackson County & 0.976101 & 0.495270 & 0.025206 & 422 & 3056 & 87584 & 0 \\
\hline 437 & IL & Jasper County & 0.992739 & 0.815360 & 0.052370 & 668 & 3674 & 106908 & 0 \\
\hline 438 & IL & Jefferson County & 0.978300 & 0.594253 & 0.025796 & 341 & 2522 & 110749 & 0 \\
\hline 439 & IL & Jersey County & 0.979265 & 0.764573 & 0.035073 & 632 & 3451 & 102634 & 0 \\
\hline 440 & IL & Jo Daviess County & 0.971528 & 0.754916 & 0.321967 & 649 & 3146 & 112801 & 0 \\
\hline 441 & IL & Johnson County & 0.991838 & 0.427523 & 0.027205 & 278 & 1985 & 68247 & 0 \\
\hline 442 & IL & Kane County & 0.993480 & 0.610926 & 0.055233 & 1038 & 10008 & 146870 & 0 \\
\hline 443 & IL & Kankakee County & 0.994158 & 0.827759 & 0.014771 & 724 & 5224 & 110288 & 0 \\
\hline 444 & IL & Kendall County & 0.993692 & 0.868446 & 0.009765 & 679 & 7163 & 136561 & 0 \\
\hline 445 & IL & Knox County & 0.995251 & 0.841019 & 0.008128 & 698 & 3925 & 105716 & 0 \\
\hline 446 & IL & Lake County & 0.327316 & 0.255222 & 0.035874 & 1073 & 8794 & 185437 & 0 \\
\hline 447 & IL & La Salle County & 0.988613 & 0.842633 & 0.006246 & 638 & 5688 & 109177 & 0 \\
\hline 448 & IL & Lawrence County & 0.994757 & 0.711063 & 0.019004 & 643 & 3237 & 107707 & 0 \\
\hline 449 & IL & Lee County & 0.994617 & 0.892688 & 0.012731 & 750 & 4918 & 113153 & 0 \\
\hline 450 & IL & Livingston County & 0.998400 & 0.954352 & 0.022391 & 679 & 4524 & 113187 & 0 \\
\hline 451 & IL & Logan County & 0.998602 & 0.935095 & 0.000865 & 733 & 5525 & 110044 & 0 \\
\hline 452 & IL & McDonough County & 0.998727 & 0.913798 & 0.001342 & 620 & 3863 & 88614 & 0 \\
\hline 453 & IL & McHenry County & 0.988494 & 0.644607 & 0.124103 & 945 & 7112 & 148028 & 0 \\
\hline 454 & IL & McLean County & 0.997733 & 0.936076 & 0.008072 & 691 & 5707 & 128427 & 0 \\
\hline 455 & IL & Macon County & 0.991719 & 0.835724 & 0.000958 & 734 & 5620 & 122060 & 1 \\
\hline 456 & IL & Macoupin County & 0.995420 & 0.727830 & 0.025287 & 712 & 3731 & 108242 & 0 \\
\hline 457 & IL & Madison County & 0.979306 & 0.645867 & 0.092233 & 689 & 4859 & 121782 & 0 \\
\hline 458 & IL & Marion County & 0.994021 & 0.693261 & 0.028101 & 415 & 2394 & 101239 & 0 \\
\hline 459 & IL & Marshall County & 0.968828 & 0.824523 & 0.009394 & 608 & 5155 & 114806 & 0 \\
\hline 460 & IL & Mason County & 0.956644 & 0.818156 & 0.002425 & 638 & 3745 & 103908 & 1 \\
\hline 461 & IL & Massac County & 0.987297 & 0.646025 & 0.016466 & 403 & 2184 & 89596 & 1 \\
\hline 462 & IL & Menard County & 0.996429 & 0.816163 & 0.001860 & 715 & 4510 & 118849 & 1 \\
\hline 463 & IL & Mercer County & 0.986186 & 0.869263 & 0.007130 & 650 & 3074 & 105940 & 0 \\
\hline 464 & IL & Monroe County & 0.976389 & 0.752568 & 0.052782 & 545 & 4051 & 140053 & 0 \\
\hline 465 & IL & Montgomery County & 0.991632 & 0.825675 & 0.022856 & 651 & 3598 & 102810 & 0 \\
\hline 466 & IL & Morgan County & 0.993919 & 0.855033 & 0.004116 & 675 & 4592 & 116797 & 0 \\
\hline 467 & IL & Moultrie County & 0.974245 & 0.859442 & 0.029346 & 746 & 5894 & 104057 & 0 \\
\hline 468 & IL & Ogle County & 0.994190 & 0.808406 & 0.035609 & 832 & 4529 & 108439 & 0 \\
\hline 469 & $\mathbb{I L}$ & Peoria County & 0.981991 & 0.659454 & 0.017239 & 604 & 4784 & 124234 & 0 \\
\hline 470 & IL & Perry County & 0.986949 & 0.593818 & 0.043696 & 342 & 2215 & 100577 & 0 \\
\hline 471 & IL & Piatt County & 0.999289 & 0.892253 & 0.004939 & 691 & 5386 & 123572 & 0 \\
\hline 472 & IL & Pike County & 0.978117 & 0.834513 & 0.004873 & 542 & 2654 & 96897 & 0 \\
\hline 473 & IL & Pope County & 0.989925 & 0.286474 & 0.000000 & 180 & 2218 & 70536 & 0 \\
\hline 474 & IL & Pulaski County & 0.987561 & 0.641343 & 0.024739 & 468 & 2209 & 91181 & 0 \\
\hline 475 & IL & Putnam County & 0.927744 & 0.763449 & 0.007457 & 1035 & 4600 & 124071 & 0 \\
\hline 476 & IL & Randolph County & 0.968450 & 0.730935 & 0.100583 & 411 & 2807 & 101307 & 0 \\
\hline 477 & IL & Richland County & 0.994841 & 0.819930 & 0.021377 & 596 & 3298 & 106854 & 0 \\
\hline 478 & IL & Rock Island County & 0.945900 & 0.643800 & 0.008049 & 735 & 4118 & 120685 & 0 \\
\hline 479 & IL & St. Clair County & 0.985032 & 0.621659 & 0.032770 & 614 & 4805 & 109658 & 0 \\
\hline 480 & IL & Saline County & 0.990532 & 0.577603 & 0.003111 & 471 & 2322 & 111778 & D \\
\hline 481 & IL & Sangamon County & 0.989926 & 0.803946 & 0.001074 & 762 & 5532 & 132761 & 0 \\
\hline 482 & IL & Schuyier County & 0.990776 & 0.740916 & 0.005134 & 399 & 2115 & 96979 & 0 \\
\hline 483 & IL & Scott County & 0.992765 & 0.802338 & 0.000000 & 592 & 3408 & 112537 & D \\
\hline
\end{tabular}




\begin{tabular}{|c|c|c|c|c|c|c|c|c|c|}
\hline $\begin{array}{l}\text { County } \\
\text { Code }\end{array}$ & State & $\begin{array}{l}\text { County } \\
\text { Name }\end{array}$ & $\begin{array}{l}\text { FRCLND } \\
\text { Absolute }\end{array}$ & $\begin{array}{l}\text { FRMFRC } \\
\text { Absolute }\end{array}$ & $\begin{array}{c}\text { DPF } \\
\text { Absolute }\end{array}$ & $\begin{array}{l}\text { ASFP } \\
\text { \$/ha }\end{array}$ & $\begin{array}{l}\text { VFRM } \\
\text { \$/ha }\end{array}$ & $\begin{array}{l}\text { VNFRM } \\
\text { S/person }\end{array}$ & Notes \\
\hline 484 & IL & Shelby County & 0.987584 & 0.828495 & 0.042253 & 630 & 4067 & 106570 & $v$ \\
\hline 485 & IL & Stark County & 0.998985 & 0.920471 & 0.004353 & 679 & 5085 & 115551 & 0 \\
\hline 486 & $\mathrm{IL}$ & Stephenson County & 0.999107 & 0.871927 & 0.357337 & 1019 & 4012 & 122744 & 0 \\
\hline 487 & IL & Tazewell County & 0.986282 & 0.810131 & 0.016924 & 759 & 5724 & 121972 & 0 \\
\hline 488 & IL & Union County & 0.985875 & 0.448158 & 0.042443 & 408 & 2281 & 96809 & 0 \\
\hline 489 & IL & Vermilion County & 0.996614 & 0.848423 & 0.003622 & 632 & 4431 & 100379 & 0 \\
\hline 490 & IL & Wabash County & 0.981156 & 0.807639 & 0.028910 & 565 & 3361 & 116499 & 0 \\
\hline 491 & IL & Warren County & 0.998824 & 0.914245 & 0.006181 & 724 & 4454 & 102478 & 0 \\
\hline 492 & IL & Washington County & 0.997340 & 0.824784 & 0.200873 & 608 & 3820 & 128379 & 0 \\
\hline 493 & IL & Wayne County & 0.997678 & 0.729311 & 0.020455 & 421 & 2206 & 102912 & 0 \\
\hline 494 & IL & White County & 0.986484 & 0.741802 & 0.000000 & 493 & 2639 & 113729 & 0 \\
\hline 495 & IL & Whiteside County & 0.982438 & 0.911078 & 0.027928 & 890 & 4580 & 110633 & 0 \\
\hline 496 & IL & Will County & 0.985654 & 0.606940 & 0.026391 & 695 & 7144 & 127248 & 0 \\
\hline 497 & IL & Williamson County & 0.954473 & 0.330028 & 0.004331 & 293 & 3234 & 100325 & 0 \\
\hline 498 & IL & Winnebago County & 0.989427 & 0.618589 & 0.126839 & 728 & 4283 & 124464 & 0 \\
\hline 499 & IL. & Woodford County & 0.972844 & 0.875410 & 0.020122 & 819 & 5503 & 116018 & 0 \\
\hline 500 & $\mathrm{Ml}$ & Alcona County & 0.376681 & 0.098624 & 0.282110 & 253 & 2122 & 88614 & 0 \\
\hline 501 & Ml & Alger County & 0.181975 & 0.027403 & 0.472829 & 246 & 1749 & 75846 & 0 \\
\hline 502 & $\mathrm{Ml}$ & Allegan County & 0.451341 & 0.465240 & 0.204416 & 1545 & 4342 & 109096 & 0 \\
\hline 503 & MI & Alpena County & 0.338732 & 0.210883 & 0.440294 & 330 & 1939 & 99505 & 0 \\
\hline 504 & MI & Antrim County & 0.792348 & 0.168771 & 0.144520 & 626 & 2916 & 97724 & 0 \\
\hline 505 & MI & Arenac County & 0.538920 & 0.351017 & 0.240191 & 589 & 2809 & 92664 & 0 \\
\hline 506 & MI & Baraga County & 0.845707 & 0.024373 & 0.630952 & 177 & 1535 & 86460 & 0 \\
\hline 507 & MI & Barry County & 0.963997 & 0.464600 & 0.372634 & 573 & 3014 & 111602 & 0 \\
\hline 508 & MI & Bay County & 0.704087 & 0.636770 & 0.054178 & 832 & 4103 & 111751 & 0 \\
\hline 509 & MI & Benzie County & 0.373772 & 0.096491 & 0.022548 & 569 & 3259 & 90774 & 1 \\
\hline 510 & MI & Berrien County & 0.361078 & 0.456643 & 0.040881 & 1010 & 4519 & 109943 & 0 \\
\hline 511 & MI & Branch County & 0.976707 & 0.701051 & 0.114179 & 719 & 3176 & 90781 & 0 \\
\hline 512 & MI & Calhoun County & 0.986627 & 0.539862 & 0.180081 & 533 & 2798 & 107992 & 0 \\
\hline 513 & MI & Cass County & 0.967997 & 0.591782 & 0.038115 & 966 & 3088 & 105791 & 0 \\
\hline 514 & MI & Charlevoix County & 0.299705 & 0.153821 & 0.385775 & 295 & 2296 & 107457 & 0 \\
\hline 515 & MI & Cheboygan County & 0.808348 & 0.089241 & 0.578444 & 278 & 1883 & 89115 & 0 \\
\hline 516 & MI & Chippewa County & 0.578605 & 0.092893 & 0.413945 & 163 & 1636 & 73584 & 0 \\
\hline 517 & $\mathrm{Ml}$ & Clare County & 0.985414 & 0.176642 & 0.495634 & 411 & 1661 & 83100 & 0 \\
\hline 518 & MI & Clinton County & 0.994577 & 0.700576 & 0.405672 & 698 & 3073 & 108053 & 0 \\
\hline 519 & MI & Crawford County & 0.990699 & 0.003925 & 0.000000 & 148 & 3685 & 69791 & 10 \\
\hline 520 & MI & Delta County & 0.587519 & 0.097176 & 0.450054 & 284 & 1527 & 97879 & 0 \\
\hline 521 & Ml & Dickinson County & 0.986129 & 0.057929 & 0.246551 & 347 & 1993 & 126015 & 0 \\
\hline 522 & MI & Eaton County & 0.995649 & 0.633947 & 0.149117 & 499 & 3232 & 120319 & 0 \\
\hline 523 & $\mathrm{Ml}$ & Emmet County & 0.530362 & 0.134779 & 0.403053 & 297 & 3103 & 126354 & 0 \\
\hline 524 & $\mathrm{Ml}$ & Genesee County & 0.985056 & 0.334848 & 0.147134 & 557 & 4073 & 113986 & 0 \\
\hline 525 & MI & Gladwin County & 0.981375 & 0.189700 & 0.343258 & 286 & 2325 & 86006 & 0 \\
\hline 526 & MI & Gogebic County & 0.746296 & 0.008458 & 0.248792 & 172 & 2219 & 87957 & 1 \\
\hline 527 & $\mathrm{Ml}$ & Grand Traverse County & 0.773649 & 0.224380 & 0.091599 & 551 & 4606 & 113844 & 0 \\
\hline 528 & M! & Gratiot County & 0.997408 & 0.760200 & 0.201292 & 700 & 3059 & 98882 & 0 \\
\hline 529 & MI & Hillsdale County & 0.986293 & 0.604162 & 0.271225 & 598 & 2864 & 100311 & 0 \\
\hline 530 & MI & Houghton County & 0.673765 & 0.045035 & 0.499209 & 214 & 1679 & 88194 & 0 \\
\hline 531 & MI & Huron County & 0.391430 & 0.819765 & 0.172370 & 1015 & 3571 & 120597 & 0 \\
\hline 532 & MI & Ingham County & 0.996879 & 0.541165 & 0.301432 & 583 & 3559 & 118247 & 0 \\
\hline 533 & MI & Ionia County & 0.987905 & 0.694498 & 0.314953 & 735 & 2890 & 86541 & 0 \\
\hline 534 & MI & losco County & 0.290445 & 0.134606 & 0.292981 & 500 & 2063 & 89372 & 0 \\
\hline 535 & MI & Iron County & 0.963117 & 0.039867 & 0.196507 & 152 & 1468 & 98990 & 0 \\
\hline 536 & MI & Isabella County & 0.993978 & 0.543409 & 0.422175 & 608 & 2595 & 97263 & 0 \\
\hline 537 & MI & Jackson County & 0.976279 & 0.465751 & 0.224213 & 524 & 2871 & 106678 & 0 \\
\hline 538 & MI & Kalamazoo County & 0.968411 & 0.429577 & 0.053540 & 1402 & 4862 & 123868 & 0 \\
\hline 539 & MI & Kalkaska County & 0.982924 & 0.044772 & 0.149548 & 476 & 2197 & 73570 & 0 \\
\hline 540 & MI & Kent County & 0.981682 & 0.348009 & 0.200170 & 1373 & 5566 & 126483 & 0 \\
\hline 541 & MI & Keweenaw County & 0.089300 & 0.000762 & 0.000000 & 139 & 2269 & 92698 & 10 \\
\hline 542 & MI & Lake County & 0.987717 & 0.049582 & 0.443380 & 157 & 2082 & 73631 & 0 \\
\hline 543 & MI & Lapeer County & 0.986741 & 0.463156 & 0.290668 & 613 & 4266 & 116458 & 0 \\
\hline 544 & MI & Leelanau County & 0.137562 & 0.291312 & 0.051639 & 952 & 4731 & 119581 & 0 \\
\hline 545 & MI & Lenawee County & 0.985809 & 0.700033 & 0.129673 & 654 & 3582 & 110898 & 0 \\
\hline 546 & MI & Livingston County & 0.970861 & 0.326474 & 0.291171 & 607 & 4395 & 143273 & 0 \\
\hline 547 & MI & Luce County & 0.472341 & 0.016248 & 0.000000 & 263 & 1638 & 124586 & 0 \\
\hline 548 & $\mathrm{Ml}$ & Mackinac County & 0.486340 & 0.033920 & 0.692455 & 349 & 1789 & 93064 & 0 \\
\hline 549 & $\mathrm{MI}$ & Macomb County & 0.843107 & 0.228664 & 0.069076 & 1203 & 8068 & 141722 & 0 \\
\hline 550 & MI & Manistee County & 0.424597 & 0.137985 & 0.038590 & 400 & 2831 & 96234 & 0 \\
\hline 551 & M! & Marquette County & 0.531475 & 0.019980 & 0.494550 & 185 & 1496 & 92414 & 0 \\
\hline 552 & MI & Mason County & 0.398710 & 0.231719 & 0.255191 & 686 & 3058 & 97859 & 0 \\
\hline 553 & MI & Mecosta County & 0.973123 & 0.340600 & 0.464418 & 450 & 2304 & 82111 & 0 \\
\hline
\end{tabular}




\begin{tabular}{|c|c|c|c|c|c|c|c|c|c|}
\hline $\begin{array}{l}\text { County } \\
\text { Code }\end{array}$ & State & $\begin{array}{l}\text { County } \\
\text { Name }\end{array}$ & $\begin{array}{l}\text { FRCLND } \\
\text { Absolute }\end{array}$ & $\begin{array}{l}\text { FRMFRC } \\
\text { Absolute }\end{array}$ & $\begin{array}{c}\text { DPF } \\
\text { Absolute }\end{array}$ & $\begin{array}{l}\text { ASFP } \\
\text { \$/ha }\end{array}$ & $\begin{array}{c}\text { VFRM } \\
\text { \$/ha }\end{array}$ & $\begin{array}{l}\text { VNFRM } \\
\text { S/person }\end{array}$ & Notes \\
\hline 554 & MI & Menominee County & 0.779935 & 0.164706 & 0.773368 & 376 & 1915 & 95272 & 0 \\
\hline 555 & MI & Midland County & 0.987314 & 0.267322 & 0.050966 & 564 & 3480 & 133649 & 0 \\
\hline 556 & MI & Missaukee County & 0.987668 & 0.243487 & 0.741791 & 814 & 2312 & 78285 & 0 \\
\hline 557 & MI & Monroe County & 0.810374 & 0.615478 & 0.026987 & 755 & 4977 & 117027 & 0 \\
\hline 558 & MI & Montcalm County & 0.982062 & 0.494356 & 0.239375 & 716 & 2803 & 92359 & 0 \\
\hline 559 & MI & Montmorency County & 0.973640 & 0.062930 & 0.614665 & 287 & 2293 & 79991 & 0 \\
\hline 560 & MI & Muskegon County & 0.348936 & 0.226032 & 0.209421 & 995 & 3768 & 100020 & 0 \\
\hline 561 & M! & Newaygo County & 0.977924 & 0.213924 & 0.384147 & 845 & 3114 & 93301 & 0 \\
\hline 562 & $\mathrm{Mi}$ & Oakland County & 0.961058 & 0.086362 & 0.031629 & 1281 & 7446 & 182091 & 0 \\
\hline 563 & MI & Oceana County & 0.413586 & 0.373166 & 0.135447 & 843 & 2951 & 98591 & 0 \\
\hline 564 & MI & Ogemaw County & 0.982057 & 0.208601 & 0.674765 & 516 & 2415 & 74194 & 0 \\
\hline 565 & MI & Ontonagon County & 0.350574 & 0.039288 & 0.479556 & 148 & 1148 & 96518 & 0 \\
\hline 566 & MI & Osceola County & 0.987729 & 0.300087 & 0.704769 & 389 & 2206 & 80547 & 0 \\
\hline 567 & MI & Oscoda County & 0.988516 & 0.038939 & 0.667914 & 328 & 2057 & 78217 & 0 \\
\hline 568 & MI & Otsego County & 0.978318 & 0.110134 & 0.256572 & 194 & 2280 & 101171 & 0 \\
\hline 569 & MI & Ottawa County & 0.346614 & 0.486952 & 0.121231 & 3264 & 6321 & 124356 & 0 \\
\hline 570 & MI & Presque Isle County & 0.256509 & 0.189191 & 0.350748 & 337 & 2387 & 91594 & 0 \\
\hline 571 & MI & Roscommon County & 0.899204 & 0.011345 & 0.000000 & 155 & 2717 & 90131 & 0 \\
\hline 572 & MI & Saginaw County & 0.991606 & 0.614449 & 0.099446 & 579 & 3527 & 114494 & 0 \\
\hline 573 & MI & St. Clair County & 0.870166 & 0.391607 & 0.156607 & 496 & 3884 & 118396 & 0 \\
\hline 574 & MI & St. Joseph County & 0.966550 & 0.728369 & 0.084068 & 701 & 3543 & 100806 & 0 \\
\hline 575 & $\mathrm{MI}$ & Sanilac County & 0.605954 & 0.720423 & 0.415141 & 650 & 2783 & 104538 & 0 \\
\hline 576 & MI & Schoolcraft County & 0.625429 & 0.018445 & 0.067674 & 270 & 1451 & 84157 & 1 \\
\hline 577 & MI & Shiawassee County & 0.996419 & 0.686657 & 0.264353 & 440 & 3245 & 106766 & 0 \\
\hline 578 & MI & Tuscola County & 0.889218 & 0.623217 & 0.124181 & 742 & 3930 & 98787 & 0 \\
\hline 579 & MI & Van Buren County & 0.560412 & 0.528792 & 0.032626 & 1015 & 3798 & 92773 & 0 \\
\hline 580 & MI & Washtenaw County & 0.982676 & 0.415800 & 0.249292 & 688 & 5664 & 154307 & 0 \\
\hline 581 & MI & Wayne County & 0.913554 & 0.057214 & 0.000000 & 2359 & 9530 & 118267 & 0 \\
\hline 582 & MI & Wexford County & 0.982047 & 0.086827 & 0.501599 & 320 & 2398 & 90165 & 0 \\
\hline 583 & WI & Adams County & 0.940777 & 0.287885 & 0.118357 & 824 & 3233 & 74715 & 0 \\
\hline 584 & WI & Ashland County & 0.455041 & 0.076647 & 0.751264 & 239 & 1308 & 92278 & 0 \\
\hline 585 & WI & Barron County & 0.969448 & 0.635341 & 0.538489 & 970 & 2278 & 102038 & 0 \\
\hline 586 & WI & Bayfield County & 0.723109 & 0.103207 & 0.705791 & 295 & 1474 & 87591 & 0 \\
\hline 587 & WI & Brown County & 0.859040 & 0.606910 & 0.647611 & 1311 & 3904 & 125236 & 0 \\
\hline 588 & WI & Buffalo County & 0.964613 & 0.738388 & 0.634108 & 622 & 1795 & 111385 & 0 \\
\hline 589 & WI & Burnett County & 0.933112 & 0.159933 & 0.656820 & 424 & 1603 & 84455 & $\mathbf{0}$ \\
\hline 590 & WI & Calumet County & 0.805594 & 0.792349 & 0.697330 & 1176 & 3731 & 112191 & 0 \\
\hline 591 & WI & Chippewa County & 0.970337 & 0.598199 & 0.733361 & 736 & 2283 & 104578 & 0 \\
\hline 592 & WI & Clark County & 0.997174 & 0.548660 & 0.784958 & 813 & 2418 & 90573 & 0 \\
\hline 593 & WI & Columbia County & 0.972461 & 0.660613 & 0.358539 & 790 & 3396 & 115862 & 0 \\
\hline 594 & WI & Crawford County & 0.955724 & 0.679166 & 0.560449 & 482 & 1892 & 89196 & 0 \\
\hline 595 & WI & Dane County & 0.970727 & 0.700009 & 0.410577 & 1241 & 4276 & 136053 & 0 \\
\hline 596 & WI & Dodge County & 0.972794 & 0.733513 & 0.543775 & 1129 & 4001 & 104612 & 0 \\
\hline 597 & WI & Door County & 0.203696 & 0.420933 & 0.634213 & 737 & 3374 & 116899 & 0 \\
\hline 598 & WI & Douglas County & 0.884614 & 0.084193 & 0.468282 & 189 & 1313 & 100582 & 0 \\
\hline 599 & WI & Dunn County & 0.986241 & 0.672241 & 0.570996 & 719 & 2363 & 90544 & 0 \\
\hline 600 & WI & Eau Claire County & 0.988153 & 0.465335 & 0.593274 & 689 & 2533 & 105459 & 0 \\
\hline 601 & WI & Florence County & 0.981041 & 0.067253 & 0.591308 & 281 & 1729 & 83486 & 0 \\
\hline 602 & WI & Fond du Lac County & 0.943989 & 0.759952 & 0.615353 & 1052 & 3529 & 117732 & 0 \\
\hline 603 & WI & Forest County & 0.969092 & 0.040761 & 0.275388 & 409 & 1621 & 71362 & 1 \\
\hline 604 & WI & Grant County & 0.969971 & 0.845227 & 0.509891 & 814 & 2713 & 104524 & 0 \\
\hline 605 & WI & Green County & 0.999015 & 0.784221 & 0.626323 & 996 & 3189 & 122893 & 0 \\
\hline 606 & WI & Green Lake County & 0.931253 & 0.719476 & 0.476705 & 848 & 3380 & 104863 & 0 \\
\hline 607 & WI & lowa County & 0.992955 & 0.741396 & 0.530793 & 763 & 2847 & 101354 & 0 \\
\hline 608 & WI & Iron County & 0.823767 & 0.021176 & 0.255343 & 679 & 1594 & 82335 & 11 \\
\hline 609 & WI & Jackson County & 0.987214 & 0.345239 & 0.449766 & 717 & 2648 & 92251 & 0 \\
\hline 610 & WI & Jefferson County & 0.955842 & 0.652391 & 0.347568 & 1129 & 3714 & 114291 & 0 \\
\hline 611 & WI & Juneau County & 0.954580 & 0.397470 & 0.406154 & 705 & 2622 & 94493 & 0 \\
\hline 612 & WI & Kenosha County & 0.361659 & 0.531261 & 0.303922 & 816 & 5022 & 117434 & 0 \\
\hline 613 & WI & Kewaunee County & 0.315925 & 0.776237 & 0.706847 & 1157 & 3853 & 106874 & 0 \\
\hline 614 & WI & La Crosse County & 0.943372 & 0.629238 & 0.585397 & 651 & 3109 & 113864 & 0 \\
\hline 615 & WI & Lafayette County & 0.998402 & 0.879511 & 0.502392 & 940 & 3126 & 102885 & 0 \\
\hline 616 & WI & Langlade County & 0.982881 & 0.215524 & 0.373029 & 879 & 2691 & 91560 & 0 \\
\hline 617 & WI & Lincoln County & 0.973617 & 0.151288 & 0.665757 & 487 & 2110 & 97886 & 0 \\
\hline 618 & WI & Manitowoc County & 0.395999 & 0.657321 & 0.698904 & 1228 & 3702 & 108818 & 0 \\
\hline 619 & WI & Marathon County & 0.980226 & 0.535942 & 0.576136 & 1030 & 2757 & 110437 & 0 \\
\hline 620 & WI & Marinette County & 0.904344 & 0.162695 & 0.617953 & 683 & 2623 & 97311 & 0 \\
\hline 621 & WI & Marquette County & 0.980788 & 0.464902 & 0.430073 & 512 & 2078 & 99146 & 0 \\
\hline 622 & WI & Menominee County & 0.980747 & 0.000956 & 0.000000 & 578 & 1358 & 84245 & 25 \\
\hline 623 & WI & Milwaukee County & 0.203028 & 0.056682 & 0.008057 & 1918 & 7521 & 124837 & 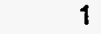 \\
\hline
\end{tabular}




\begin{tabular}{|c|c|c|c|c|c|c|c|c|c|}
\hline $\begin{array}{c}\text { County } \\
\text { Code }\end{array}$ & State & $\begin{array}{l}\text { County } \\
\text { Name }\end{array}$ & $\begin{array}{l}\text { FRCLND } \\
\text { Absolute }\end{array}$ & $\begin{array}{l}\text { FRMFRC } \\
\text { Absolute }\end{array}$ & $\begin{array}{c}\text { DPF } \\
\text { Absolute }\end{array}$ & $\begin{array}{l}\text { ASFP } \\
\text { \$/ha }\end{array}$ & $\begin{array}{l}\text { VFRM } \\
\text { \$/ha }\end{array}$ & $\begin{array}{l}\text { VNFRM } \\
\text { \$/person }\end{array}$ & Notes \\
\hline 624 & WI & Monroe County & 0.991697 & 0.600763 & 0.608095 & 688 & 2870 & 87659 & 0 \\
\hline 625 & WI & Oconto County & 0.868526 & 0.327012 & 0.713432 & 738 & 2520 & 92556 & 0 \\
\hline 626 & $W !$ & Oneida County & 0.909893 & 0.044145 & 0.028190 & 605 & 2231 & 105913 & 1 \\
\hline 627 & WI & Outagamie County & 0.993691 & 0.642949 & 0.629770 & 1221 & 3625 & 116377 & 0 \\
\hline 628 & WI & Ozaukee County & 0.207796 & 0.530607 & 0.558680 & 975 & 4458 & 169716 & 0 \\
\hline 629 & WI & Pepin County & 0.934136 & 0.763691 & 0.658518 & 610 & 2196 & 99566 & 0 \\
\hline 630 & WI & Pierce County & 0.974479 & 0.739559 & 0.566909 & 659 & 2796 & 115293 & 0 \\
\hline 631 & WI & Polk County & 0.959210 & 0.481026 & 0.665064 & 571 & 2440 & 96349 & 0 \\
\hline 632 & WI & Portage County & 0.980004 & 0.514907 & 0.306200 & 847 & 3338 & 104294 & 0 \\
\hline 633 & WI & Price County & 0.979762 & 0.117991 & 0.666608 & 299 & 1684 & 100230 & 0 \\
\hline 634 & WI & Racine County & 0.420631 & 0.624739 & 0.176466 & 1059 & 4382 & 124803 & 0 \\
\hline 635 & WI & Richland County & 0.994597 & 0.722077 & 0.577614 & 578 & 2228 & 86961 & 0 \\
\hline 636 & WI & Rock County & 0.992128 & 0.744044 & 0.298580 & 832 & 3803 & 113397 & 0 \\
\hline 637 & WI & Rusk County & 0.980909 & 0.287975 & 0.734812 & 494 & 1581 & 83331 & 0 \\
\hline 638 & WI & St. Croix County & 0.981121 & 0.667584 & 0.592979 & 750 & 2840 & 127594 & 0 \\
\hline 639 & WI & Sauk County & 0.987274 & 0.625803 & 0.516358 & 837 & 2947 & 111690 & 0 \\
\hline 640 & WI & Sawyer County & 0.930458 & 0.058912 & 0.588121 & 508 & 1963 & 82565 & 0 \\
\hline 641 & WI & Shawano County & 0.981501 & 0.521640 & 0.651669 & 1033 & 3199 & 84245 & 23 \\
\hline 642 & WI & Sheboygan County & 0.404120 & 0.630056 & 0.630239 & 1071 & 3691 & 122331 & 0 \\
\hline 643 & WI & Taylor County & 0.990228 & 0.370892 & 0.709633 & 627 & 1825 & 96173 & 0 \\
\hline 644 & WI & Trempealeau County & 0.989350 & 0.741935 & 0.501632 & 751 & 2282 & 104626 & 0 \\
\hline 645 & WI & Vernon County & 0.973609 & 0.718421 & 0.646133 & 654 & 2499 & 89643 & 0 \\
\hline 646 & $W 1$ & Vilas County & 0.857436 & 0.014508 & 0.000000 & 1096 & 5486 & 94764 & 12 \\
\hline 647 & WI & Walworth County & 0.963316 & 0.636070 & 0.291054 & 1007 & 4537 & 115930 & 0 \\
\hline 648 & WI & Washburn County & 0.949150 & 0.166123 & 0.538450 & 376 & 1532 & 87557 & 0 \\
\hline 649 & WI & Washington County & 0.988369 & 0.533855 & 0.568445 & 1074 & 5237 & 132260 & 0 \\
\hline 650 & WI & Waukesha County & 0.957081 & 0.321114 & 0.323690 & 952 & 6534 & 155811 & 0 \\
\hline 651 & WI & Waupaca County & 0.981398 & 0.502940 & 0.683982 & 819 & 3048 & 105567 & 0 \\
\hline 652 & WI & Waushara County & 0.982180 & 0.417253 & 0.233678 & 948 & 3536 & 104829 & 0 \\
\hline 653 & WI & Winnebago County & 0.757888 & 0.605137 & 0.597764 & 851 & 3511 & 119513 & 0 \\
\hline 654 & $W !$ & Wood County & 0.979427 & 0.436233 & 0.528971 & 921 & 3421 & 122683 & 0 \\
\hline 655 & MN & Aitkin County & 0.911790 & 0.144343 & 0.279633 & 232 & 1206 & 86507 & 0 \\
\hline 656 & MN & Anoka County & 0.950055 & 0.227864 & 0.061307 & 827 & 6451 & 120421 & 0 \\
\hline 657 & MN & Becker County & 0.906811 & 0.450322 & 0.236521 & 494 & 1596 & 95617 & 0 \\
\hline 658 & MN & Beltrami County & 0.819905 & 0.140275 & 0.268278 & 187 & 1111 & 86365 & 0 \\
\hline 659 & MN & Benton County & 0.988562 & 0.703229 & 0.307408 & 1048 & 2542 & 95461 & 0 \\
\hline 660 & $M N$ & Big Stone County & 0.941416 & 0.824348 & 0.076928 & 421 & 2034 & 99464 & 0 \\
\hline 661 & MN & Blue Earth County & 0.982321 & 0.796158 & 0.028631 & 813 & 4101 & 101307 & 0 \\
\hline 662 & MN & Brown County & 0.987486 & 0.888620 & 0.129195 & 864 & 3746 & 112049 & 0 \\
\hline 663 & $M N$ & Cartton County & 0.982969 & 0.205981 & 0.540515 & 206 & 1722 & 95963 & 0 \\
\hline 664 & MN & Carver County & 0.949172 & 0.726154 & 0.473180 & 958 & 5190 & 138763 & 0 \\
\hline 665 & MN & Cass County & 0.835723 & 0.155033 & 0.322334 & 206 & 1055 & 81461 & 0 \\
\hline 666 & MN & Chippewa County & 0.991451 & 0.876138 & 0.025146 & 623 & 3116 & 109204 & 0 \\
\hline 667 & MN & Chisago County & 0.943838 & 0.518473 & 0.324198 & 423 & 3198 & 108649 & 0 \\
\hline 668 & MN & Clay County & 0.992882 & 0.847526 & 0.062607 & 525 & 2495 & 100955 & 0 \\
\hline 669 & MN & Clearwater County & 0.965937 & 0.331260 & 0.134254 & 296 & 1094 & 78298 & 0 \\
\hline 670 & MN & Cook County & 0.434446 & 0.001345 & 0.000000 & 87 & 2267 & 113817 & 0 \\
\hline 671 & MN & Cottonwood County & 0.986240 & 0.915311 & 0.032518 & 838 & 3464 & 109482 & 0 \\
\hline 672 & MN & Crow Wing County & 0.861728 & 0.204869 & 0.358056 & 265 & 1462 & 101408 & 0 \\
\hline 673 & MN & Dakota County & 0.971613 & 0.606642 & 0.127995 & 897 & 4500 & 143070 & 0 \\
\hline 674 & MN & Dodge County & 0.999722 & 0.857286 & 0.204112 & 759 & 3517 & 106881 & 0 \\
\hline 675 & MN & Douglas County & 0.881040 & 0.640757 & 0.439861 & 475 & 2080 & 96356 & 0 \\
\hline 676 & $\mathrm{MN}$ & Faribault County & 0.988936 & 0.907978 & 0.034202 & 715 & 3749 & 109326 & 0 \\
\hline 677 & MN & Fillmore County & 0.998957 & 0.804562 & 0.260043 & 650 & 2668 & 103014 & 0 \\
\hline 678 & $M N$ & Freeborn County & 0.979257 & 0.809293 & 0.060712 & 763 & 3537 & 103982 & 0 \\
\hline 679 & MN & Goodhue County & 0.971963 & 0.781886 & 0.393166 & 801 & 357.0 & 113512 & 0 \\
\hline 680 & MN & Grant County & 0.950010 & 0.769583 & 0.061248 & 478 & 2272 & 112598 & 0 \\
\hline 681 & MN & Hennepin County & 0.917888 & 0.222269 & 0.214677 & 1184 & 8076 & 160559 & 0 \\
\hline 682 & MN & Houston County & 0.981543 & 0.761178 & 0.289458 & 730 & 2531 & 102573 & 0 \\
\hline 683 & MN & Hubbard County & 0.923077 & 0.190387 & 0.090525 & 338 & 1584 & 84292 & 0 \\
\hline 684 & $M N$ & Isanti County & 0.971654 & 0.468169 & 0.199593 & 369 & 3375 & 101429 & 0 \\
\hline 685 & $M N$ & Itasca County & 0.910304 & 0.063202 & 0.162397 & 145 & 1484 & 90754 & 0 \\
\hline 686 & $\mathrm{MN}$ & Jackson County & 0.975596 & 0.892694 & 0.018139 & 782 & 4002 & 113431 & 0 \\
\hline 687 & MN & Kanabec County & 0.984149 & 0.433208 & 0.404207 & 320 & 1893 & 87720 & 0 \\
\hline 688 & MN & Kandiyohi County & 0.923669 & 0.707455 & 0.092776 & 1276 & 2955 & 111108 & 0 \\
\hline 689 & MN & Kittson County & 0.994169 & 0.687878 & 0.008604 & 315 & 1735 & 128549 & 0 \\
\hline 690 & MN & Koochiching County & 0.983526 & 0.034640 & 0.167034 & 126 & 950 & 95942 & 1 \\
\hline 691 & MN & Lac qui Parle County & 0.983050 & 0.827370 & 0.034986 & 452 & 2403 & 106150 & 0 \\
\hline 692 & MN & Lake County & 0.701872 & 0.003916 & 0.000000 & 54 & 2262 & 86494 & 0 \\
\hline 693 & MN & Lake of the Woods County & 0.730503 & 0.124914 & 0.081647 & 133 & 950 & 90287 & 0 \\
\hline
\end{tabular}




\begin{tabular}{|c|c|c|c|c|c|c|c|c|c|}
\hline $\begin{array}{l}\text { County } \\
\text { Code }\end{array}$ & State & $\begin{array}{l}\text { County } \\
\text { Name }\end{array}$ & $\begin{array}{l}\text { FRCLND } \\
\text { Absolute }\end{array}$ & $\begin{array}{l}\text { FRMFRC } \\
\text { Absolute }\end{array}$ & $\begin{array}{c}\text { DPF } \\
\text { Absolute }\end{array}$ & $\begin{array}{l}\text { ASFP } \\
\text { \$/ha }\end{array}$ & $\begin{array}{l}\text { VFRM } \\
\text { \$/ha }\end{array}$ & $\begin{array}{l}\text { VNFRM } \\
\text { \$/person }\end{array}$ & Notes \\
\hline 694 & MN & Le Sueur County & 0.946487 & 0.714235 & 0.141268 & 788 & 3632 & 109983 & 0 \\
\hline 695 & MN & Lincoln County & 0.979197 & 0.743207 & 0.109379 & 491 & 1698 & 108656 & 0 \\
\hline 696 & MN & Lyon County & 0.989960 & 0.864158 & 0.041098 & 690 & 2870 & 112245 & 0 \\
\hline 697 & MN & McLeod County & 0.972791 & 0.795665 & 0.309049 & 732 & 3529 & 114305 & 0 \\
\hline 698 & MN & Mahnomen County & 0.953949 & 0.524154 & 0.192941 & 285 & 1592 & 88695 & 0 \\
\hline 699 & MN & Marshall County & 0.977632 & 0.656562 & 0.029137 & 333 & 1666 & 110816 & 0 \\
\hline 700 & MN & Martin County & 0.972297 & 0.908935 & 0.021702 & 845 & 4360 & 119127 & 0 \\
\hline 701 & MN & Meeker County & 0.943353 & 0.773103 & 0.159996 & 819 & 2857 & 107613 & 0 \\
\hline 702 & MN & Mille Lacs County & 0.842618 & 0.387383 & 0.513037 & 486 & 2232 & 102052 & 0 \\
\hline 703 & MN & Morrison County & 0.975012 & 0.587619 & 0.406929 & 714 & 2090 & 87144 & 0 \\
\hline 704 & MN & Mower County & 0.999705 & 0.862167 & 0.098472 & 718 & 3353 & 114345 & 0 \\
\hline 705 & MN & Murray County & 0.979031 & 0.833130 & 0.059715 & 647 & 2786 & 106854 & 0 \\
\hline 706 & MN & Nicollet County & 0.968621 & 0.835722 & 0.110103 & 1116 & 3847 & 98570 & 0 \\
\hline 707 & MN & Nobles County & 0.990438 & 0.909759 & 0.054392 & 741 & 3257 & 115849 & 0 \\
\hline 708 & MN & Norman County & 0.999373 & 0.816056 & 0.044596 & 421 & 2093 & 121112 & 0 \\
\hline 709 & MN & Olmsted County & 0.997719 & 0.731749 & 0.285655 & 763 & 3468 & 138952 & 0 \\
\hline 710 & MN & Otter Tail County & 0.889803 & 0.647994 & 0.364077 & 514 & 1763 & 98672 & 0 \\
\hline 711 & MN & Pennington County & 0.997084 & 0.709813 & 0.083563 & 259 & 1225 & 106698 & 0 \\
\hline 712 & MN & Pine County & 0.983605 & 0.291508 & 0.458014 & 384 & 1608 & 78379 & 0 \\
\hline 713 & MN & Pipestone County & 0.999444 & 0.847331 & 0.137716 & 574 & 2415 & $107 t 66$ & 0 \\
\hline 714 & MN & Polk County & 0.986288 & 0.826926 & 0.039682 & 466 & 2359 & 111013 & 0 \\
\hline 715 & MN & Pope County & 0.934285 & 0.723061 & 0.273997 & 464 & 1975 & 93490 & 0 \\
\hline 716 & MN & Ramsey County & 0.915724 & 0.021479 & 0.010931 & 6179 & 15256 & 137516 & 1 \\
\hline 717 & MN & Red Lake County & 0.999812 & 0.661966 & 0.166458 & 324 & 1280 & 101876 & 0 \\
\hline 718 & MN & Redwood County & 0.998402 & 0.873219 & 0.038427 & 773 & 3597 & 104260 & 0 \\
\hline 719 & MN & Renville County & 0.995644 & 0.953920 & 0.036486 & 777 & 3841 & 117224 & 0 \\
\hline 720 & MN & Rice County & 0.964050 & 0.714450 & 0.228944 & 960 & 3910 & 106793 & 0 \\
\hline 721 & MN & Rock County & 0.999524 & 0.875190 & 0.058657 & 827 & 3380 & 108188 & 0 \\
\hline 722 & MN & Roseau County & 0.990579 & 0.503990 & 0.128189 & 249 & 1330 & 111649 & 0 \\
\hline 723 & MN & St. Louis County & 0.907461 & 0.038447 & 0.269956 & 215 & 1542 & 104436 & 0 \\
\hline 724 & MN & Scott County & 0.968091 & 0.576959 & 0.363559 & 835 & 6070 & $\$ 29097$ & 0 \\
\hline 725 & MN & Sherburne County & 0.967987 & 0.421259 & 0.094866 & 840 & 4015 & 92088 & 0 \\
\hline 726 & $\mathrm{MN}$ & Sibley County & 0.980439 & 0.827765 & 0.138753 & 1047 & 3774 & 106461 & 0 \\
\hline 727 & MN & Stearns County & 0.967336 & 0.748096 & 0.439537 & 1032 & 2724 & 99952 & 0 \\
\hline 728 & MN & Steele County & 0.993947 & 0.842459 & 0.162386 & 790 & 3729 & 119154 & 0 \\
\hline 729 & MN & Stevens County & 0.977103 & 0.795943 & 0.021373 & 681 & 2425 & 103447 & 0 \\
\hline 730 & MN & Swift County & 0.988275 & 0.819310 & 0.057858 & 533 & 2433 & 106258 & 0 \\
\hline 731 & MN & Todd County & 0.961884 & 0.655263 & 0.507057 & 598 & 1821 & 85749 & 0 \\
\hline 732 & MN & Traverse County & 0.979742 & 0.844173 & 0.009468 & 480 & 2421 & 138661 & 0 \\
\hline 733 & MN & Wabasha County & 0.954886 & 0.731145 & 0.473087 & 712 & 2899 & 100182 & 0 \\
\hline 734 & MN & Wadena County & 0.986070 & 0.500194 & 0.303896 & 548 & 1505 & 83764 & 0 \\
\hline 735 & MN & Waseca County & 0.977916 & 0.875776 & 0.104726 & 708 & 3803 & 112266 & 0 \\
\hline 736 & MN & Washington County & 0.925667 & 0.401951 & 0.094731 & 1181 & 6860 & 140083 & 0 \\
\hline 737 & $M N$ & Watonwan County & 0.987716 & 0.898001 & 0.021110 & 746 & 3409 & 106915 & 0 \\
\hline 738 & MN & Wilkin County & 0.999740 & 0.874905 & 0.018943 & 451 & 2348 & 114603 & 0 \\
\hline 739 & MN & Winona County & 0.976173 & 0.725008 & 0.540247 & 787 & 3057 & 104456 & 0 \\
\hline 740 & MN & Wright County & 0.924987 & 0.644404 & 0.348824 & 735 & 4035 & 114054 & 0 \\
\hline 741 & MN & Yellow Medicine County & 0.992885 & 0.840947 & 0.042125 & 568 & 2790 & 108168 & 0 \\
\hline 742 & IA & Adair County & 0.998243 & 0.902845 & 0.016359 & 565 & 2321 & 107897 & 0 \\
\hline 743 & IA & Adams County & 0.995613 & 0.884621 & 0.005438 & 476 & 1892 & 112814 & 0 \\
\hline 744 & IA & Allamakee County & 0.970936 & 0.785949 & 0.328593 & 687 & 2495 & 103515 & 0 \\
\hline 745 & IA & Appanoose County & 0.961066 & 0.751233 & 0.017301 & 284 & 1795 & 100697 & 0 \\
\hline 746 & IA & Audubon County & 0.998999 & 0.946715 & 0.002453 & 863 & 3471 & 105926 & 0 \\
\hline 747 & IA & Benton County & 0.997132 & 0.931703 & 0.024856 & 878 & 3959 & 115896 & 0 \\
\hline 748 & IA & Black Hawk County & 0.991983 & 0.824804 & 0.030499 & 929 & 4466 & 112652 & 0 \\
\hline 749 & IA & Boone County & 0.996419 & 0.902469 & 0.004196 & 771 & 4435 & 108046 & 0 \\
\hline 750 & IA & Bremer County & 0.996128 & 0.844404 & 0.164681 & 931 & 4476 & 112889 & 0 \\
\hline 751 & IA & Buchanan County & 0.996348 & 0.911084 & 0.058752 & 865 & 3963 & 105181 & 0 \\
\hline 752 & IA & Buena Vista County & 0.990896 & 0.929428 & 0.004171 & 1137 & 4480 & 109015 & 0 \\
\hline 753 & IA & Butler County & 0.997970 & 0.849191 & 0.044717 & 895 & 3720 & 105777 & 0 \\
\hline 754 & IA & Calhoun County & 0.996287 & 0.946944 & 0.000882 & 803 & 4714 & 107294 & 0 \\
\hline 755 & IA & Carroll County & 0.998390 & 0.987299 & 0.002872 & 1346 & 4153 & 114223 & 0 \\
\hline 756 & IA & Cass County & 0.998797 & 0.961707 & 0.005032 & 686 & 2891 & 118158 & 0 \\
\hline 757 & IA & Cedar County & 0.995815 & 0.913391 & 0.012896 & 859 & 3953 & 120068 & 0 \\
\hline 758 & IA & Cerro Gordo County & 0.988259 & 0.848001 & 0.007197 & 731 & 4108 & 117501 & 0 \\
\hline 759 & IA & Cherokee County & 0.999685 & 0.910249 & 0.016606 & 940 & 3980 & 106143 & 0 \\
\hline 760 & IA & Chickasaw County & 0.998363 & 0.851072 & 0.081408 & 900 & 3547 & 111934 & 0 \\
\hline 761 & IA & Clarke County & 0.998609 & 0.856746 & 0.016323 & 291 & 1678 & 106143 & 0 \\
\hline 762 & IA & Clay County & 0.993667 & 0.864587 & 0.001256 & 825 & 4125 & 114643 & 0 \\
\hline 763 & IA & Clayton County & 0.982337 & 0.916724 & 0.283145 & 842 & 3283 & 102729 & 0 \\
\hline
\end{tabular}




\begin{tabular}{|c|c|c|c|c|c|c|c|c|c|}
\hline $\begin{array}{l}\text { County } \\
\text { Code }\end{array}$ & State & $\begin{array}{l}\text { County } \\
\text { Name }\end{array}$ & $\begin{array}{l}\text { FRCLND } \\
\text { Absolute }\end{array}$ & $\begin{array}{l}\text { FRMFRC } \\
\text { Absolute }\end{array}$ & $\begin{array}{c}\text { DPF } \\
\text { Absolute }\end{array}$ & $\begin{array}{l}\text { ASFP } \\
\$ / h a\end{array}$ & $\begin{array}{l}\text { VFRM } \\
\text { \$/ha }\end{array}$ & $\begin{array}{l}\text { VNFRM } \\
\text { \$/person }\end{array}$ & Notes \\
\hline 764 & IA & Clinton County & 0.978581 & 0.827543 & 0.034845 & 909 & 3692 & 110708 & \\
\hline 765 & IA & Crawford County & 0.999119 & 0.907880 & 0.011585 & 740 & 3101 & 98645 & \\
\hline 766 & IA & Dallas County & 0.991104 & 0.831655 & 0.003446 & 691 & 4398 & 120008 & \\
\hline 767 & IA & Davis County & 0.996735 & 0.854756 & 0.062016 & 359 & 1677 & 94385 & \\
\hline 768 & IA & Decatur County & 0.997920 & 0.767653 & 0.012533 & 388 & 1402 & 83520 & \\
\hline 769 & IA & Delaware County & 0.997936 & 0.908840 & 0.199925 & 1315 & 4248 & 106292 & \\
\hline 770 & IA & Des Moines County & 0.968244 & 0.722602 & 0.026864 & 661 & 4120 & 115822 & \\
\hline 771 & IA & Dickinson County & 0.943924 & 0.829282 & 0.010817 & 875 & 3833 & 127438 & \\
\hline 772 & IA & Dubuque County & 0.986365 & 0.883394 & 0.311392 & 1329 & 4061 & 110559 & \\
\hline 773 & IA & Emmet County & 0.983513 & 0.887554 & 0.001697 & 713 & 4115 & 98821 & \\
\hline 774 & IA & Fayette County & 0.999326 & 0.858489 & 0.176250 & 933 & 3362 & 101043 & \\
\hline 775 & IA & Floyd County & 0.998530 & 0.897635 & 0.007781 & 793 & 3482 & 111243 & \\
\hline 776 & IA & Franklin County & 0.999017 & 0.921066 & 0.010472 & 850 & 3876 & 109746 & \\
\hline 777 & IA & Fremont County & 0.989420 & 0.923935 & 0.003414 & 675 & 2673 & 118192 & \\
\hline 778 & IA & Greene County & 0.995236 & 1.008589 & 0.000350 & 705 & 3847 & 115720 & 13 \\
\hline 779 & IA & Grundy County & 0.999936 & 0.986230 & 0.009951 & 999 & 4707 & 121816 & \\
\hline 780 & IA & Guthrie County & 0.995857 & 0.870056 & 0.007203 & 633 & 3089 & 106603 & \\
\hline 781 & IA & Hamilton County & 0.998677 & 0.900520 & 0.004620 & 1059 & 4927 & 132592 & \\
\hline 782 & IA & Hancock County & 0.996451 & 0.900468 & 0.007410 & 816 & 4279 & 109089 & \\
\hline 783 & IA & Hardin County & 0.998813 & 0.912171 & 0.001753 & 1069 & 4060 & 118599 & \\
\hline 784 & IA & Harrison County & 0.994104 & 0.894992 & 0.001232 & 623 & 2726 & 103176 & \\
\hline 785 & IA & Henry County & 0.994864 & 0.812175 & 0.009874 & 711 & 3801 & 119811 & \\
\hline 786 & IA & Howard County & 0.999244 & 0.860664 & 0.109331 & 700 & 3136 & 114061 & \\
\hline 787 & IA & Humboldt County & 0.996979 & 1.009926 & 0.006695 & 803 & 4435 & 118382 & \\
\hline 788 & IA & Ida County & 0.998801 & 0.987401 & 0.007997 & 801 & 3146 & 105594 & \\
\hline 789 & IA & lowa County & 0.998486 & 0.855933 & 0.017511 & 735 & 2884 & 117915 & \\
\hline 790 & IA & Jackson County & 0.978975 & 0.851236 & 0.112330 & 739 & 2772 & 107308 & \\
\hline 791 & IA & Jasper County & 0.995991 & 0.922868 & 0.020033 & 776 & 3364 & 123211 & \\
\hline 792 & IA & Jefferson County & 0.996850 & 0.814915 & 0.016176 & 576 & 2730 & 108276 & \\
\hline 793 & IA & Johnson County & 0.985773 & 0.723453 & 0.049442 & 864 & 4451 & 122304 & \\
\hline 794 & IA & Jones County & 0.997600 & 0.874325 & 0.052290 & 985 & 3569 & 99918 & \\
\hline 795 & IA & Keokuk County & 0.998707 & 0.869681 & 0.004075 & 638 & 2864 & 110383 & \\
\hline 796 & IA & Kossuth County & 0.998579 & 0.987584 & 0.015028 & 764 & 4547 & 109604 & \\
\hline 797 & IA & Lee County & 0.960314 & 0.803517 & 0.045955 & 683 & 2999 & 107186 & \\
\hline 798 & IA & Linn County & 0.990195 & 0.760552 & 0.037957 & 724 & 4444 & 129693 & \\
\hline 799 & IA & Louisa County & 0.962330 & 0.743622 & 0.001590 & 596 & 3394 & 112706 & \\
\hline 800 & IA & Lucas County & 0.991666 & 0.796030 & 0.024712 & 286 & 1653 & 108358 & \\
\hline 801 & IA & Lyon County & 0.999772 & 0.924385 & 0.035682 & 1233 & 3802 & 106170 & \\
\hline 802 & IA & Madison County & 0.997937 & 0.851139 & 0.002642 & 561 & 2580 & 113729 & \\
\hline 803 & IA & Mahaska County & 0.995638 & 0.861745 & 0.023850 & 910 & 3907 & 105520 & \\
\hline 804 & $\mathbb{A}$ & Marion County & 0.971396 & 0.756979 & 0.033093 & 600 & 2992 & 118511 & \\
\hline 805 & IA & Marshall County & 0.998780 & 0.854067 & 0.014080 & 802 & 3788 & 122642 & \\
\hline 806 & IA & Mills County & 0.992873 & 0.851325 & 0.002191 & 673 & 3314 & 109949 & \\
\hline 807 & IA & Mitchell County & 0.998992 & 0.876446 & 0.049672 & 1002 & 3717 & 124898 & \\
\hline 808 & IA & Monona County & 0.991798 & 0.885518 & 0.002008 & 626 & 2634 & 103914 & \\
\hline 809 & IA & Monroe County & 0.998389 & 0.806187 & 0.044018 & 328 & 1630 & 107294 & \\
\hline 810 & IA & Montgomery County & 0.997809 & 0.885000 & 0.006614 & 652 & 2561 & 113905 & \\
\hline 811 & IA & Muscatine County & 0.976840 & 0.782982 & 0.039235 & 735 & 3929 & 126997 & \\
\hline 812 & IA & O'Brien County & 0.999701 & 0.987197 & 0.009215 & 998 & 4496 & 106942 & \\
\hline 813 & IA & Osceola County & 0.998193 & 1.021741 & 0.032591 & 897 & 4451 & 110613 & \\
\hline 814 & IA & Page County & 0.999021 & 0.931255 & 0.005491 & 634 & 2435 & 110735 & \\
\hline 815 & IA & Palo Alto County & 0.990217 & 0.938619 & 0.004677 & 786 & 3840 & 112571 & \\
\hline 816 & IA & Plymouth County & 0.999554 & 0.937623 & 0.014576 & 1055 & 3681 & 113038 & \\
\hline 817 & IA & Pocahontas County & 0.997539 & 0.972106 & 0.003862 & 757 & 4359 & 107911 & \\
\hline 818 & IA & Polk County & 0.962062 & 0.630556 & 0.009397 & 664 & 4096 & 136405 & \\
\hline 819 & IA & Pottawattamie County & 0.994086 & 0.888804 & 0.003748 & 820 & 3385 & 112740 & \\
\hline 820 & IA & Poweshiek County & 0.998046 & 0.910621 & 0.014505 & 626 & 2995 & 119730 & \\
\hline 821 & IA & Ringgold County & 0.997683 & 0.852185 & 0.001917 & 323 & 1458 & 96295 & \\
\hline 822 & IA & Sac County & 0.995530 & 0.988159 & 0.007685 & 993 & 4165 & 107897 & \\
\hline 823 & IA & Scott County & 0.978023 & 0.795786 & 0.047835 & 965 & 5650 & 130025 & \\
\hline 824 & IA & Shelby County & 0.999179 & 0.934909 & 0.004707 & 867 & 3473 & 110004 & \\
\hline 825 & IA & Sioux County & 0.999090 & 1.008712 & 0.041501 & 2104 & 4825 & 107166 & \\
\hline 826 & IA & Story County & 0.998540 & 0.903348 & 0.019025 & 792 & 4348 & 107436 & \\
\hline 827 & IA & Tama County & 0.998519 & 0.870450 & 0.017513 & 674 & 3894 & 108703 & \\
\hline 828 & IA & Taylor County & 0.998369 & 0.816166 & 0.014307 & 414 & 1910 & 98333 & \\
\hline 829 & IA & Union County & 0.996322 & 0.869825 & 0.005260 & 507 & 2153 & 105560 & \\
\hline 830 & IA & Van Buren County & 0.989125 & 0.777372 & 0.025127 & 375 & 1951 & 93707 & \\
\hline 831 & IA & Wapello County & 0.990431 & 0.705623 & 0.017685 & 484 & 2765 & 102567 & \\
\hline 832 & IA & Warren County & 0.997274 & 0.826748 & 0.039354 & 466 & 3059 & 114548 & \\
\hline 833 & IA & Washington County & 0.996525 & 0.850183 & 0.015409 & 1029 & 3755 & 113309 & \\
\hline
\end{tabular}




\begin{tabular}{|c|c|c|c|c|c|c|c|c|c|}
\hline $\begin{array}{l}\text { County } \\
\text { Code }\end{array}$ & State & $\begin{array}{l}\text { County } \\
\text { Name }\end{array}$ & $\begin{array}{l}\text { FRCLND } \\
\text { Absolute }\end{array}$ & $\begin{array}{l}\text { FRMFRC } \\
\text { Absolute }\end{array}$ & $\begin{array}{c}\text { DPF } \\
\text { Absolute }\end{array}$ & $\begin{array}{l}\text { ASFP } \\
\text { S/ha }\end{array}$ & $\begin{array}{l}\text { VFRM } \\
\text { S/ha }\end{array}$ & $\begin{array}{l}\text { VNFRM } \\
\text { S/person }\end{array}$ & Notes \\
\hline 834 & IA & Wayne County & 0.997199 & 0.840410 & 0.012621 & 296 & 1574 & 97907 & 0 \\
\hline 835 & IA & Webster County & 0.996070 & 0.892276 & 0.004648 & 768 & 4401 & 111378 & 0 \\
\hline 836 & IA & Winnebago County & 0.997248 & 0.905067 & 0.019499 & 645 & 3761 & 122283 & 0 \\
\hline 837 & IA & Winneshiek County & 0.999609 & 0.810372 & 0.299449 & 864 & 2821 & 107755 & 0 \\
\hline 838 & IA & Woodbury County & 0.994554 & 0.791848 & 0.002080 & 843 & 3039 & 113817 & 0 \\
\hline 839 & IA & Worth County & 0.995729 & 0.877434 & 0.008399 & 723 & 3514 & 125175 & 0 \\
\hline 840 & IA & Wright County & 0.996882 & 0.951560 & 0.002304 & 694 & 4566 & 125914 & 0 \\
\hline 841 & MO & Adair County & 0.997134 & 0.735025 & 0.030400 & 196 & 1339 & 91242 & 0 \\
\hline 842 & MO & Andrew County & 0.996980 & 0.815542 & 0.087225 & 418 & 2488 & 100507 & 0 \\
\hline 843 & MO & Atchison County & 0.995172 & 0.872062 & 0.002415 & 512 & 2410 & 112584 & 0 \\
\hline 844 & MO & Audrain County & 0.995015 & 0.849715 & 0.019900 & 498 & 2472 & 102201 & 0 \\
\hline 845 & MO & Barry County & 0.985041 & 0.586441 & 0.105550 & 829 & 2311 & 91208 & 0 \\
\hline 846 & MO & Barton County & 0.995934 & 0.818018 & 0.060261 & 307 & 1965 & 99512 & 0 \\
\hline 847 & MO & Bates County & 0.996592 & 0.792625 & 0.056804 & 388 & 1710 & 101754 & 0 \\
\hline 848 & MO & Benton County & 0.937578 & 0.529930 & 0.106382 & 289 & 1612 & 76327 & 0 \\
\hline 849 & MO & Bollinger County & 0.999270 & 0.497197 & 0.028189 & 215 & 1780 & 75562 & 0 \\
\hline 850 & MO & Boone County & 0.991599 & 0.619897 & 0.008427 & 327 & 2883 & 116330 & 1 \\
\hline 851 & MO & Buchanan County & 0.988329 & 0.691301 & 0.063070 & 390 & 2656 & 108317 & 0 \\
\hline 852 & MO & Butler County & 0.997966 & 0.572283 & 0.000638 & 455 & 2814 & 86663 & 0 \\
\hline 853 & MO & Caldwell County & 0.999123 & 0.844933 & 0.021723 & 280 & 1606 & 92488 & 0 \\
\hline 854 & MO & Callaway County & 0.990358 & 0.631954 & 0.025124 & 281 & 2438 & 100507 & 0 \\
\hline 855 & MO & Camden County & 0.924234 & 0.388903 & 0.185498 & 188 & 1475 & 97426 & 0 \\
\hline 856 & MO & Cape Girardeau County & 0.986917 & 0.682856 & 0.143157 & 424 & 3101 & 111460 & 0 \\
\hline 857 & MO & Carroll County & 0.988952 & 0.848076 & 0.010751 & 407 & 2280 & 93660 & 0 \\
\hline 858 & MO & Carter County & 0.997171 & 0.168578 & 0.044845 & 122 & 1475 & 63993 & 1 \\
\hline 859 & MO & Cass County & 0.994860 & 0.728141 & 0.055725 & 383 & 3338 & 115124 & 0 \\
\hline 860 & MO & Cedar County & 0.954723 & 0.616689 & 0.067176 & 215 & 1805 & 82599 & 0 \\
\hline 861 & MO & Chariton County & 0.983881 & 0.834250 & 0.009443 & 464 & 2268 & 98374 & 0 \\
\hline 862 & MO & Christian County & 0.998423 & 0.585790 & 0.322899 & 331 & 3520 & 97609 & 0 \\
\hline 863 & MO & Clark County & 0.991043 & 0.753960 & 0.009234 & 321 & 1917 & 82288 & 0 \\
\hline 864 & MO & Clay County & 0.968957 & 0.513761 & 0.005428 & 423 & 3767 & 124593 & 1 \\
\hline 865 & MO & Clinton County & 0.988879 & 0.774594 & 0.041408 & 400 & 2269 & 106705 & 0 \\
\hline 866 & MO & Cole County & 0.981083 & 0.747164 & 0.113317 & 245 & 2448 & 110505 & 0 \\
\hline 867 & MO & Cooper County & 0.990888 & 0.825996 & 0.022350 & 451 & 2077 & 92563 & 0 \\
\hline 868 & MO & Crawford County & 0.998317 & 0.424349 & 0.033875 & 118 & 1675 & 94974 & 0 \\
\hline 869 & MO & Dade County & 0.968478 & 0.805443 & 0.073819 & 272 & 1522 & 87801 & 0 \\
\hline 870 & MO & Dallas County & 0.997636 & 0.657160 & 0.411141 & 340 & 1951 & 80472 & 0 \\
\hline 871 & MO & Daviess County & 0.996284 & 0.768407 & 0.028121 & 287 & 1634 & 85180 & 0 \\
\hline 872 & MO & Dekalb County & 0.996270 & 0.776552 & 0.067878 & 310 & 1837 & 75487 & 0 \\
\hline 873 & MO & Dent County & 0.998710 & 0.454993 & 0.053991 & 126 & 1557 & 90659 & 0 \\
\hline 874 & MO & Douglas County & 0.999900 & 0.577303 & 0.445827 & 245 & 1721 & 63404 & 0 \\
\hline 875 & MO & Dunklin County & 0.997272 & 0.827024 & 0.000000 & 706 & 3044 & 77878 & 0 \\
\hline 876 & MO & Franklin County & 0.991858 & 0.502064 & 0.127990 & 313 & 3221 & 110884 & 0 \\
\hline 877 & MO & Gasconade County & 0.990374 & 0.592416 & 0.069316 & 184 & 1906 & 106603 & 0 \\
\hline 878 & MO & Gentry County & 0.999418 & 0.781410 & 0.014098 & 312 & 1562 & 94324 & 0 \\
\hline 879 & MO & Greene County & 0.995829 & 0.660855 & 0.308635 & 361 & 3887 & 112638 & 0 \\
\hline 880 & MO & Grundy County & 0.995065 & 0.811399 & 0.028646 & 290 & 1759 & 96687 & 0 \\
\hline 881 & MO & Harrison County & 0.998197 & 0.860100 & 0.011792 & 233 & 1473 & 95915 & 0 \\
\hline 882 & MO & Henry County & 0.958890 & 0.714374 & 0.050032 & 284 & 1963 & 93341 & 0 \\
\hline 883 & MO & Hickory County & 0.968159 & 0.683201 & 0.203416 & 224 & 1541 & 70651 & 0 \\
\hline 884 & MO & Holt County & 0.984683 & 0.786801 & 0.002607 & 494 & 2684 & 96742 & 1 \\
\hline 885 & MO & Howard County & 0.989747 & 0.801478 & 0.011960 & 289 & 2108 & 91465 & 0 \\
\hline 886 & MO & Howell County & 0.999392 & 0.626955 & 0.258800 & 263 & 1796 & 83249 & 0 \\
\hline 887 & MO & Iron County & 0.998791 & 0.194371 & 0.000000 & 188 & 1733 & 76544 & 0 \\
\hline 888 & MO & Jackson County & 0.981595 & 0.346685 & 0.024381 & 479 & 4970 & 126056 & 0 \\
\hline 889 & MO & Jasper County & 0.997578 & 0.687031 & 0.101724 & 529 & 2439 & 99823 & 0 \\
\hline 890 & MO & Jefferson County & 0.989017 & 0.284499 & 0.298079 & 222 & 3797 & 107667 & 0 \\
\hline 891 & MO & Johnson County & 0.997045 & 0.697930 & 0.033823 & 383 & 2364 & 82708 & 0 \\
\hline 892 & MO & Krox County & 0.997894 & 0.829389 & 0.038162 & 276 & 1656 & 91249 & 0 \\
\hline 893 & MO & Laclede County & 0.997211 & 0.621338 & 0.477052 & 282 & 1975 & 90774 & 0 \\
\hline 894 & MO & Lafayette County & 0.985080 & 0.884235 & 0.038966 & 579 & 2771 & 109645 & 0 \\
\hline 895 & MO & Lawrence County & 0.999506 & 0.848400 & 0.212837 & 571 & 2796 & 87787 & 0 \\
\hline 896 & MO & Lewis County & 0.988597 & 0.770485 & 0.016198 & 350 & 1845 & 87144 & 0 \\
\hline 897 & MO & Lincoln County & 0.984497 & 0.627667 & 0.049467 & 509 & 3249 & 102722 & 0 \\
\hline 898 & MO & Linn County & 0.998211 & 0.851010 & 0.054104 & 300 & 1512 & 95712 & 0 \\
\hline 899 & MO & Livingston County & 0.992805 & 0.790767 & 0.021177 & 297 & 2097 & 103190 & 0 \\
\hline 900 & MO & McDonald County & 0.999644 & 0.577141 & 0.033075 & 1013 & 2476 & 77404 & 0 \\
\hline 901 & MO & Macon County & 0.989214 & 0.742426 & 0.017445 & 211 & 1456 & 100704 & 0 \\
\hline 902 & MO & Madison County & 0.998251 & 0.350861 & 0.008255 & 163 & 1693 & 86907 & 1 \\
\hline 903 & MO & Maries County & 0.995822 & 0.690684 & 0.113155 & 181 & 1311 & 85573 & 0 \\
\hline
\end{tabular}




\begin{tabular}{|c|c|c|c|c|c|c|c|c|c|}
\hline $\begin{array}{l}\text { County } \\
\text { Code }\end{array}$ & State & $\begin{array}{l}\text { County } \\
\text { Name }\end{array}$ & $\begin{array}{l}\text { FRCLND } \\
\text { Absolute }\end{array}$ & $\begin{array}{l}\text { FRMFRC } \\
\text { Absolute }\end{array}$ & $\begin{array}{c}\text { DPF } \\
\text { Absolute }\end{array}$ & $\begin{array}{l}\text { ASFP } \\
\$ / h a\end{array}$ & $\begin{array}{l}\text { VFRM } \\
\text { \$/ha }\end{array}$ & $\begin{array}{l}\text { VNFRM } \\
\text { \$/person }\end{array}$ & Notes \\
\hline 904 & MO & Marion County & 0.986553 & 0.784267 & 0.059833 & 375 & 2259 & 101225 & 0 \\
\hline 905 & MO & Mercer County & 0.998347 & 0.722882 & 0.007618 & 296 & 1576 & 84855 & 0 \\
\hline & & Miller County & 0.987151 & 0.638474 & 0.012265 & 541 & 1855 & 89867 & 0 \\
\hline 907 & MO & Mississippi County & 0.963279 & 1.003056 & 0.000801 & 705 & 3592 & 81949 & 13 \\
\hline 908 & MO & Moniteau County & 0.994438 & 0.814424 & 0.047309 & 428 & 2008 & 105262 & 0 \\
\hline 909 & MO & Monroe County & 0.963838 & 0.740529 & 0.027497 & 362 & 2112 & 107294 & 0 \\
\hline 910 & MO & Montgomery County & 0.993994 & 0.651774 & 0.003409 & 391 & 2701 & 100826 & 1 \\
\hline 911 & MO & Morgan County & 0.973191 & 0.527510 & 0.072972 & 566 & 1816 & 86379 & 0 \\
\hline 912 & MO & New Madrid County & 0.971339 & 0.849960 & 0.000000 & 641 & 3265 & 80242 & 0 \\
\hline 913 & MO & Newton County & 0.999611 & 0.638553 & 0.086552 & 849 & 2798 & 87787 & 0 \\
\hline 914 & MO & Nodaway County & 0.998714 & 0.905181 & 0.014332 & 399 & 1972 & 91878 & 0 \\
\hline 915 & MO & Oregon County & 0.999768 & 0.497640 & 0.118160 & 256 & 1514 & 68098 & 0 \\
\hline 916 & MO & Osage County & 0.988104 & 0.816658 & 0.044117 & 417 & 1534 & 107680 & 0 \\
\hline 917 & MO & Ozark County & 0.989024 & 0.524030 & 0.338215 & 257 & 1555 & 81129 & 0 \\
\hline 918 & MO & Pemiscot County & 0.962278 & 0.924759 & 0.000000 & 637 & 3012 & 81861 & 0 \\
\hline 919 & MO & Perry County & 0.980166 & 0.689426 & 0.110738 & 394 & 2542 & 103264 & 0 \\
\hline 920 & MO & Pettis County & 0.998026 & 0.819841 & 0.028568 & 385 & 2124 & 107396 & 0 \\
\hline 921 & MO & Phelps County & 0.997883 & 0.466191 & 0.023793 & 123 & 1840 & 94161 & 0 \\
\hline 922 & MO & Pike County & 0.982608 & 0.751068 & 0.006241 & 432 & 2371 & 94784 & 0 \\
\hline 923 & MO & Platte County & 0.983873 & 0.700999 & 0.004576 & 347 & 2970 & 129856 & 1 \\
\hline 924 & MO & Polk County & 0.991811 & 0.847575 & 0.324205 & 376 & 2116 & 89372 & 0 \\
\hline 925 & MO & Pulaski County & 0.992042 & 0.396975 & 0.119023 & 152 & 1560 & 74146 & 0 \\
\hline 926 & MO & Putnam County & 0.996646 & 0.767722 & 0.011206 & 267 & 1158 & 86541 & 0 \\
\hline 927 & MO & Ralls County & 0.973515 & 0.759435 & 0.011220 & 350 & 2289 & 100968 & 0 \\
\hline 928 & MO & Randolph County & 0.989204 & 0.716186 & 0.013737 & 254 & 1702 & 91973 & 0 \\
\hline 929 & MO & Ray County & 0.992837 & 0.760858 & 0.007199 & 377 & 2544 & 103312 & 0 \\
\hline 930 & MO & Reynolds County & 0.996055 & 0.172732 & 0.021025 & 96 & 1823 & 74790 & 0 \\
\hline 931 & MO & Ripley County & 0.996521 & 0.378589 & 0.013322 & 147 & 1551 & 64792 & 1 \\
\hline 932 & MO & St. Charles County & 0.945923 & 0.568210 & 0.057459 & 531 & 5125 & 127018 & 0 \\
\hline 933 & MO & St. Clair County & 0.964061 & 0.593892 & 0.048662 & 201 & 1412 & 80093 & 0 \\
\hline 934 & MO & Ste. Genevieve County & 0.987142 & 0.524294 & 0.022366 & 268 & 2113 & 97426 & 0 \\
\hline 935 & MO & St. Francois County & 0.993515 & 0.406391 & 0.042593 & 358 & 2844 & 89169 & 0 \\
\hline 936 & MO & St. Louis County & 0.969727 & 0.166425 & 0.000000 & 752 & 5869 & 164040 & 0 \\
\hline 937 & MO & Saline County & 0.988179 & 0.856944 & 0.001390 & 520 & 2505 & 103400 & 1 \\
\hline 938 & MO & Schuyler County & 0.999065 & 0.838477 & 0.058161 & 211 & 1172 & 89576 & 0 \\
\hline 939 & MO & Scotland County & 0.998210 & 0.772143 & 0.096079 & 334 & 1823 & 90415 & 0 \\
\hline 940 & MO & Scott County & 0.988218 & 0.812867 & 0.012812 & 584 & 3055 & 93836 & 0 \\
\hline 941 & MO & Shannon County & 0.999847 & 0.186828 & 0.071799 & 111 & 1191 & 59015 & 0 \\
\hline 942 & MO & Shelby County & 0.996932 & 0.852800 & 0.001302 & 420 & 1914 & 102275 & 1 \\
\hline 943 & MO & Stoddard County & 0.997832 & 0.827612 & 0.001356 & 661 & 3464 & 88648 & 0 \\
\hline 944 & MO & Stone County & 0.906691 & 0.464587 & 0.446066 & 317 & 2355 & 89982 & 0 \\
\hline 945 & MO & Sullivan County & 0.999265 & 0.792044 & 0.013984 & 193 & 1160 & 89156 & 0 \\
\hline 946 & MO & Taney County & 0.970657 & 0.396734 & 0.117372 & 115 & 1536 & 101253 & 0 \\
\hline 947 & MO & Texas County & 0.999412 & 0.609378 & 0.445284 & 224 & 1652 & 75867 & 0 \\
\hline 948 & MO & Vernon County & 0.996311 & 0.753505 & 0.069337 & 254 & 1872 & 98902 & 0 \\
\hline 949 & MO & Warren County & 0.986153 & 0.457762 & 0.005391 & 439 & 4550 & 109658 & 1 \\
\hline 950 & MO & Washington County & 0.996519 & 0.230001 & 0.017565 & 305 & 1718 & 73320 & 1 \\
\hline 951 & MO & Wayne County & 139 & 0.191038 & 536 & 119 & 1300 & 67102 & 1 \\
\hline 952 & MO & er County & 0.999441 & 0.762889 & 0.423597 & 445 & 2259 & 80269 & 0 \\
\hline 953 & MO & Worth County & 0.999126 & 0.785702 & 0.012621 & 226 & 1566 & 91309 & 0 \\
\hline 954 & MO & Wright County & 0.998695 & 0.725019 & 0.619215 & 376 & 1819 & 71769 & 0 \\
\hline 955 & MO & St. Louis city & 0.936142 & 0.000000 & 0.000000 & 0 & 0 & 122683 & 0 \\
\hline 956 & ND & Adams County & 0.999067 & 0.940357 & 0.012619 & 97 & 549 & 112469 & 0 \\
\hline 957 & ND & Bames County & 0.985740 & 0.898963 & 0.025023 & 263 & 1259 & 102858 & 0 \\
\hline 958 & ND & Benson County & 0.964695 & 0.875082 & 0.021521 & 151 & 1024 & 80418 & 0 \\
\hline 959 & ND & Billings County & 0.998287 & 1.111182 & 0.048065 & 31 & 427 & 116770 & 9 \\
\hline 960 & ND & Bottineau County & 0.982829 & 0.889582 & 0.009700 & 147 & 1013 & 113810 & 0 \\
\hline 961 & ND & Bowman County & 0.995761 & 0.911546 & 0.020248 & 71 & 504 & 113160 & 1 \\
\hline 962 & ND & Burke County & 0.977216 & 0.791986 & 0.009021 & 105 & 765 & 110125 & 0 \\
\hline 963 & ND & Burleigh County & 0.979051 & 0.839411 & 0.046116 & 106 & 741 & 113715 & 0 \\
\hline 964 & ND & Cass County & 0.998728 & 0.947303 & 0.008512 & 371 & 2063 & 110234 & 0 \\
\hline 965 & ND & Cavalier County & 0.986002 & 0.897636 & 0.002487 & 250 & 1347 & 131231 & 0 \\
\hline 966 & ND & Dickey County & 0.990664 & 0.867206 & 0.036509 & .240 & 893 & 106217 & 0 \\
\hline 967 & ND & Divide County & 0.973101 & 0.900678 & 0.009728 & 92 & 858 & 113851 & 1 \\
\hline 968 & ND & Dunn County & 0.965244 & 1.051566 & 0.052667 & 68 & 568 & 87679 & 9 \\
\hline 969 & ND & Eddy County & 0.981110 & 0.912498 & 0.037130 & 147 & 858 & 108595 & 0 \\
\hline 970 & ND & Emmons County & 0.971163 & 0.863307 & 0.219787 & 145 & 724 & 91370 & 0 \\
\hline 971 & ND & Foster County & 0.982239 & 0.900932 & 0.010715 & 212 & 1124 & 105330 & 0 \\
\hline 972 & ND & Golden Valley County & 0.999626 & 0.788185 & 0.056375 & 80 & 525 & 130100 & 0 \\
\hline 973 & ND & Grand Forks Cour & 0.998595 & 0.835889 & 0.005533 & 429 & 1991 & 93010 & 0 \\
\hline
\end{tabular}




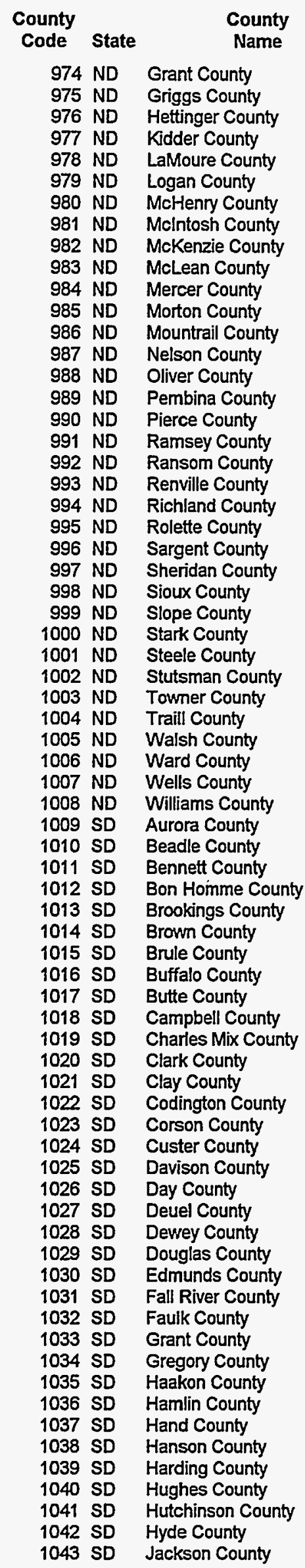

FRCLND FRMFRC DPF ASFP VFRM VNFRM Absolute Absolute Absolute \$/ha \$/ha \$/person Notes

\begin{tabular}{|c|c|c|c|c|c|}
\hline 0.996125 & 0.959682 & 0.116877 & 94 & 586 & 83994 \\
\hline 0.989296 & 0.873609 & 0.024441 & 211 & 1053 & 100982 \\
\hline 0.998720 & 0.950015 & 0.059190 & 138 & 769 & 109170 \\
\hline 0.943029 & 0.836728 & 0.086128 & 102 & 669 & 81400 \\
\hline 0.996846 & 0.911225 & 0.081321 & 256 & 1165 & 101882 \\
\hline 0.981773 & 0.942542 & 0.120436 & 156 & 774 & 90463 \\
\hline 0.980324 & 0.874286 & 0.040379 & 111 & 675 & 87903 \\
\hline 0.980060 & 0.872771 & 0.092147 & 158 & 766 & 104118 \\
\hline 0.958449 & 0.664218 & 0.006237 & 104 & 853 & 102025 \\
\hline 0.906438 & 0.835393 & 0.039696 & 132 & 947 & 98184 \\
\hline 0.939720 & 0.794591 & 0.055201 & 103 & 787 & 112388 \\
\hline 0.990250 & 1.000607 & 0.187885 & 124 & 644 & 92989 \\
\hline 0.939649 & 0.857211 & 0.020923 & 101 & 908 & 93253 \\
\hline 0.973118 & 0.879717 & 0.028526 & 216 & 1127 & 109184 \\
\hline 0.989508 & 0.829692 & 0.139547 & 116 & 725 & 90754 \\
\hline 0.997338 & 0.839132 & 0.000578 & 470 & 2378 & 129334 \\
\hline 0.940544 & 0.899888 & 0.052579 & 131 & 880 & 98042 \\
\hline 0.911833 & 0.842623 & 0.010051 & 196 & 1141 & 112625 \\
\hline 0.998398 & 0.878347 & 0.037508 & 261 & 1177 & 98299 \\
\hline 0.980576 & 0.899464 & 0.015624 & 159 & 1017 & 98611 \\
\hline 0.993848 & 0.869495 & 0.019029 & 404 & 2185 & 95434 \\
\hline 0.960762 & 0.904624 & 0.051934 & 137 & 897 & 74112 \\
\hline 0.990386 & 0.901516 & 0.036878 & 272 & 1188 & 128698 \\
\hline 0.966173 & 0.838234 & 0.079475 & 102 & 808 & 113295 \\
\hline 0.969708 & 1.065017 & 0.073349 & 46 & 564 & 63736 \\
\hline 0.998931 & 1.007974 & 0.008615 & 59 & 478 & 101049 \\
\hline 0.998284 & 0.982783 & 0.152617 & 124 & 840 & 96085 \\
\hline 0.995626 & 0.964695 & 0.009749 & 279 & 1396 & 111263 \\
\hline 0.966558 & 0.892960 & 0.061470 & 193 & 956 & 107172 \\
\hline 0.984399 & 0.900876 & 0.002927 & 188 & 1026 & 124850 \\
\hline 0.999250 & 0.908298 & 0.000772 & 421 & 2381 & 105174 \\
\hline 0.990598 & 0.898583 & 0.001429 & 462 & 1945 & 121653 \\
\hline 0.978911 & 0.901112 & 0.035167 & 154 & 1024 & 101171 \\
\hline 0.985013 & 0.922874 & 0.045496 & 180 & 979 & 118423 \\
\hline 0.963909 & 0.892468 & 0.004306 & 96 & 765 & 112476 \\
\hline 0.993895 & 0.838296 & 0.028897 & 285 & 763 & 96538 \\
\hline 0.995645 & 0.899194 & 0.055926 & 281 & 1084 & 109760 \\
\hline 0.995549 & 1.038492 & 0.004195 & 68 & 476 & 110749 \\
\hline 0.969176 & 0.894140 & 0.045044 & 558 & 1770 & 103156 \\
\hline 0.987202 & 0.874038 & 0.074316 & 438 & 1748 & 92292 \\
\hline 0.989294 & 0.936276 & 0.027643 & 305 & 1220 & 115131 \\
\hline 0.967501 & 0.947745 & 0.031406 & 207 & 840 & 102059 \\
\hline 0.965478 & 0.926944 & 0.009761 & 145 & 644 & 58317 \\
\hline 0.992123 & 0.863856 & 0.146387 & 78 & 432 & 91282 \\
\hline 0.953924 & 0.886940 & 0.086895 & 159 & 1017 & 116269 \\
\hline 0.954800 & 0.978917 & 0.042257 & 298 & 1113 & 94642 \\
\hline 0.989805 & 0.872297 & 0.022527 & 333 & 968 & 121247 \\
\hline 0.987780 & 0.898113 & 0.005923 & 460 & 2344 & 88973 \\
\hline 0.959048 & 0.892699 & 0.205704 & 368 & 1454 & 103339 \\
\hline 0.977680 & 1.075332 & 0.029745 & 54 & 373 & 78799 \\
\hline 0.999070 & 0.463643 & 0.101622 & 54 & 501 & 108392 \\
\hline 0.996898 & 0.971205 & 0.080118 & 308 & 1418 & 111053 \\
\hline 0.942589 & 0.852631 & 0.163696 & 211 & 992 & 119303 \\
\hline 0.979316 & 0.854764 & 0.242241 & 314 & 1249 & 95299 \\
\hline 0.941528 & 1.261474 & 0.063883 & 34 & 435 & 67861 \\
\hline 0.998745 & 0.909568 & 0.111281 & 397 & 1297 & 78610 \\
\hline 0.995154 & 0.875472 & 0.062248 & 208 & 805 & 109177 \\
\hline 0.994676 & 0.875407 & 0.002402 & 169 & 324 & 102296 \\
\hline 0.994497 & 0.874887 & 0.008521 & 188 & 791 & 113885 \\
\hline 0.992113 & 0.855703 & 0.166253 & 384 & 1442 & 104199 \\
\hline 0.964391 & 0.924334 & 0.114608 & 155 & 797 & 101015 \\
\hline 0.992198 & 1.037982 & 0.001465 & 66 & 545 & 124512 \\
\hline 0.950197 & 0.845879 & 0.155041 & 384 & 1556 & 90490 \\
\hline 0.997478 & 0.936527 & 0.049472 & 179 & 700 & 133209 \\
\hline 0.997761 & 0.881271 & 0.069604 & 356 & 1626 & 84062 \\
\hline 0.997356 & 0.969659 & 0.003189 & 37 & 293 & 108480 \\
\hline 0.925810 & 0.823919 & 0.002373 & 176 & 834 & 113485 \\
\hline 0.998257 & 0.965653 & 0.093764 & 423 & 1784 & 110891 \\
\hline 0.993553 & 0.989074 & 0.009850 & 111 & 558 & 127119 \\
\hline 0.998868 & 1.137718 & 0.011487 & 51 & 371 & 70313 \\
\hline
\end{tabular}




\begin{tabular}{|c|c|c|}
\hline $\begin{array}{l}\text { County } \\
\text { Code }\end{array}$ & State & $\begin{array}{l}\text { County } \\
\text { Name }\end{array}$ \\
\hline 1044 & SD & Jerauld County \\
\hline 1045 & SD & Jones County \\
\hline 1046 & SD & Kingsbury County \\
\hline 1047 & SD & Lake County \\
\hline 1048 & SD & Lawrence County \\
\hline 1049 & SD & Lincoln County \\
\hline 1050 & SD & Lyman County \\
\hline 1051 & SD & McCook County \\
\hline 1052 & SD & McPherson County \\
\hline 1053 & SD & Marshall County \\
\hline 1054 & SD & Meade County \\
\hline 1055 & $S D$ & Mellette County \\
\hline 1056 & SD & Miner County \\
\hline 1057 & SD & Minnehaha County \\
\hline 1058 & SD & Moody County \\
\hline 1059 & SD & Pennington County \\
\hline 1060 & SD & Perkins County \\
\hline 1061 & SD & Potter County \\
\hline 1062 & SD & Roberts County \\
\hline 1063 & SD & Sanborn County \\
\hline 1064 & SD & Shannon County \\
\hline 1065 & SD & Spink County \\
\hline 1066 & SD & Stanley County \\
\hline 1067 & SD & Sully County \\
\hline 1068 & SD & Todd County \\
\hline 1069 & SD & Tripp County \\
\hline 1070 & SD & Turner County \\
\hline 1071 & SD & Union County \\
\hline 1072 & SD & Walworth County \\
\hline 1073 & SD & Yankton County \\
\hline 1074 & SD & Ziebach County \\
\hline 1075 & NE & Adams County \\
\hline 1076 & NE & Antelope County \\
\hline 1077 & NE & Arthur County \\
\hline 1078 & NE & Banner County \\
\hline 1079 & NE & Blaine County \\
\hline 1080 & NE & Boone County \\
\hline 1081 & NE & Box Butte County \\
\hline 1082 & NE & Boyd County \\
\hline 1083 & NE & Brown County \\
\hline 1084 & NE & Buffalo County \\
\hline 1085 & NE & Burt County \\
\hline 1086 & NE & Butler County \\
\hline 1087 & NE & Cass County \\
\hline 1088 & NE & Cedar County \\
\hline 1089 & NE & Chase County \\
\hline 1090 & NE & Cherry County \\
\hline 1091 & NE & Cheyenne County \\
\hline 1092 & NE & Clay County \\
\hline 1093 & NE & Colfax County \\
\hline 1094 & NE & Cuming County \\
\hline 1095 & NE & Custer County \\
\hline 1096 & NE & Dakota County \\
\hline 1097 & NE & Dawes County \\
\hline 1098 & NE & Dawson County \\
\hline 1099 & $N E$ & Deuel County \\
\hline 1100 & NE & Dixon County \\
\hline 1101 & NE & Dodge County \\
\hline 1102 & NE & Douglas County \\
\hline 1103 & NE & Dundy County \\
\hline 1104 & NE & Fillmore County \\
\hline 1105 & NE & Franklin County \\
\hline 1106 & NE & Frontier County \\
\hline 1107 & NE & Furnas County \\
\hline 1108 & NE & Gage County \\
\hline 1109 & $N E$ & Garden County \\
\hline 1110 & $\mathrm{NE}$ & Garfield County \\
\hline 1111 & NE & Gosper County \\
\hline 1112 & NE & Grant County \\
\hline 1113 & $\mathrm{NE}$ & Greeley County \\
\hline
\end{tabular}

FRCLND FRMFRC DPF

$\begin{array}{lll}0.995517 & 0.984313 & 0.012952\end{array}$

$\begin{array}{llll}0.998856 & 0.940474 & 0.017368\end{array}$

$\begin{array}{llll}0.970701 & 0.857380 & 0.029078\end{array}$

$\begin{array}{llll}0.979432 & 0.826147 & 0.023902\end{array}$

$\begin{array}{llll}0.999651 & 0.380981 & 0.193912\end{array}$

$\begin{array}{llll}0.999131 & 0.872414 & 0.036406\end{array}$

$\begin{array}{lll}0.960696 & 0.806395 & 0.024755\end{array}$

$\begin{array}{llll}0.995424 & 0.886536 & 0.104639\end{array}$

$\begin{array}{llll}0.987057 & 0.909006 & 0.074362\end{array}$

$\begin{array}{llll}0.947181 & 0.904747 & 0.022088\end{array}$

$\begin{array}{llll}0.996605 & 0.934660 & 0.036744\end{array}$

$\begin{array}{llll}0.997509 & 0.838705 & 0.022576\end{array}$

$\begin{array}{llll}0.997115 & 0.858626 & 0.054567\end{array}$

$\begin{array}{llll}0.994386 & 0.821222 & 0.085211\end{array}$

$\begin{array}{llll}0.997265 & 0.856520 & 0.041193\end{array}$

$\begin{array}{llll}0.997094 & 0.599962 & 0.034328\end{array}$

$\begin{array}{llll}0.994001 & 0.938692 & 0.014908\end{array}$

$\begin{array}{llll}0.964449 & 0.914389 & 0.019439\end{array}$

$\begin{array}{llll}0.969986 & 0.857223 & 0.074028\end{array}$

$\begin{array}{llll}0.997897 & 0.886302 & 0.023217\end{array}$

$\begin{array}{llll}0.998681 & 1.057744 & 0.000000\end{array}$

$\begin{array}{llll}0.995895 & 0.925365 & 0.010944\end{array}$

$\begin{array}{lll}0.951427 & 0.978587 & 0.008976\end{array}$

$\begin{array}{llll}0.940712 & 0.955040 & 0.001503\end{array}$

$\begin{array}{llll}0.998001 & 1.214754 & 0.018073\end{array}$

$\begin{array}{llll}0.997603 & 0.974924 & 0.062380\end{array}$

$\begin{array}{lll}0.998984 & 0.930188 & 0.089203\end{array}$

$\begin{array}{llll}0.985654 & 0.880737 & 0.017009\end{array}$

$\begin{array}{llll}0.951082 & 0.990758 & 0.043669\end{array}$

$\begin{array}{llll}0.979270 & 0.812375 & 0.029034\end{array}$

$\begin{array}{llll}0.995577 & 1.119733 & 0.012310\end{array}$

$\begin{array}{llll}0.998661 & 0.930299 & 0.000703\end{array}$

$\begin{array}{llll}0.998411 & 0.892122 & 0.062925\end{array}$

$\begin{array}{llll}0.995897 & 1.005003 & 0.000000\end{array}$

$\begin{array}{lll}0.999794 & 0.853567 & 0.000000\end{array}$

$\begin{array}{llll}0.994969 & 1.012324 & 0.008315\end{array}$

$\begin{array}{lll}0.999190 & 0.996158 & 0.015171\end{array}$

$\begin{array}{llll}0.997641 & 0.943876 & 0.000793\end{array}$

$\begin{array}{llll}0.991656 & 0.856805 & 0.071082\end{array}$

$\begin{array}{llll}0.996901 & 0.831087 & 0.009834\end{array}$

$\begin{array}{llll}0.992575 & 0.948396 & 0.009289\end{array}$

$\begin{array}{llll}0.991288 & 0.856110 & 0.017300\end{array}$

$\begin{array}{llll}0.998596 & 0.899167 & 0.005994\end{array}$

$\begin{array}{llll}0.987701 & 0.826969 & 0.026867\end{array}$

$\begin{array}{lll}0.992511 & 0.905003 & 0.065669\end{array}$

$\begin{array}{lll}0.996503 & 0.910722 & 0.000668\end{array}$

$\begin{array}{lll}0.991827 & 1.019071 & 0.004248\end{array}$

$\begin{array}{llll}0.999905 & 1.008794 & 0.001570\end{array}$

$\begin{array}{llll}0.999141 & 0.973566 & 0.001629\end{array}$

$\begin{array}{llll}0.987000 & 0.865977 & 0.005876\end{array}$

$\begin{array}{lll}0.995580 & 0.944371 & 0.008475\end{array}$

$\begin{array}{llll}0.999854 & 0.864609 & 0.009370\end{array}$

$\begin{array}{lll}0.987124 & 0.817002 & 0.013076\end{array}$

$\begin{array}{lll}0.996704 & 0.942357 & 0.005049\end{array}$

$\begin{array}{lll}0.993722 & 1.015873 & 0.000595\end{array}$

$\begin{array}{llll}0.997968 & 0.941353 & 0.000000\end{array}$

$\begin{array}{lll}0.986879 & 0.796633 & 0.005835\end{array}$

$\begin{array}{llll}0.982540 & 0.873671 & 0.002261\end{array}$

$\begin{array}{llll}0.974648 & 0.453560 & 0.019253\end{array}$

$\begin{array}{llll}0.998985 & 0.898074 & 0.000635\end{array}$

$\begin{array}{llll}0.999725 & 0.922788 & 0.003201\end{array}$

$\begin{array}{llll}0.999673 & 0.877135 & 0.021290\end{array}$

$\begin{array}{llll}0.994377 & 0.844061 & 0.005827\end{array}$

$\begin{array}{lll}0.996636 & 0.937729 & 0.004505\end{array}$

$\begin{array}{llll}0.994587 & 0.929372 & 0.100988\end{array}$

$\begin{array}{lll}0.984635 & 0.980527 & 0.000000\end{array}$

$\begin{array}{lll}0.997709 & 0.926819 & 0.001551\end{array}$

$\begin{array}{llll}0.990125 & 0.783306 & 0.002752\end{array}$

$\begin{array}{lll}0.991061 & 1.098600 & 0.000000\end{array}$

$\begin{array}{llll}0.998479 & 0.834010 & 0.024168\end{array}$
ASFP VFRM VNFRM

S/ha S/ha \$/person Notes

$\begin{array}{rrr}258 & 712 & 109577 \\ 78 & 439 & 134942\end{array}$

339

543

114

593

93
415

415
179

442

54

65

592

592

93

58

216

309

382

20

333

49
214

53
141

141
690

741

157

560
34

1131

1131
614

63

219

97
677

677

495
215

306

\section{6}

869

641

535
725

725
366

366
64

316

877

1915

3107

380

492

70
1211

118

1243

962

954
380

800

355

200

453

468

108

240

404

50

322
1230

115300

$1691 \quad 108195$

1093100690

$699 \quad 106942$

$1460 \quad 108039$

$\begin{array}{ll}769 & 110004\end{array}$

$1069 \quad 107646$

$476 \quad 102634$

$417 \quad 89928$

$1133 \quad 108852$

$2498 \quad 125480$

$2250 \quad 104111$

$548 \quad 107152$

$357 \quad 138580$

$966 \quad 121071$

$1426 \quad 89264$

$983 \quad 100968$

$371 \quad 49194$

$1197 \quad 143321$

$459 \quad 114142$

$951 \quad 204862$

$460 \quad 51517$

$754 \quad 106068$

$2319 \quad 106732$

$2599 \quad 119249$

$828 \quad 107220$

$2052 \quad 101374$

$419 \quad 89982$

$2946 \quad 121897$

$2060 \quad 101490$

$457 \quad 148949$

$837 \quad 134936$

$418 \quad 121708$

$2033 \quad 110078$

$1316 \quad 127858$

$847 \quad 101408$

$804 \quad 124031$

$2168 \quad 99905$

$2925 \quad 115205$

$2780 \quad 110369$

$3467 \quad 106719$

$2294 \quad 96796$

$1495 \quad 131664$

$481 \quad 113553$

$889 \quad 132789$

$2916 \quad 126747$

$2959 \quad 103650$

$3195 \quad 116858$

$1083 \quad 118206$

$2514 \quad 102831$

100101

$2346 \quad 113207$

$1066 \quad 143145$

$2254 \quad 105472$

$3941 \quad 107308$

$5023 \quad 132951$

$1065 \quad 159834$

$3004 \quad 142528$

$2231 \quad 109739$

$1034 \quad 108933$

$1285 \quad 123367$

$2073 \quad 106481$

$503 \quad 160295$

$679 \quad 97019$

$1907 \quad 136792$

$524 \quad 114054$

$1232 \quad 112239$
$3043 \quad 100467$

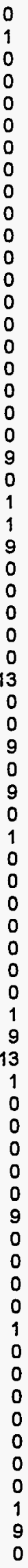




\begin{tabular}{|c|c|c|c|c|c|c|c|c|c|}
\hline $\begin{array}{l}\text { County } \\
\text { Code }\end{array}$ & State & $\begin{array}{l}\text { County } \\
\text { Name }\end{array}$ & $\begin{array}{l}\text { FRCLND } \\
\text { Absolute }\end{array}$ & $\begin{array}{l}\text { FRMFRC } \\
\text { Absolute }\end{array}$ & $\begin{array}{c}\text { DPF } \\
\text { Absolute }\end{array}$ & $\begin{array}{c}\text { ASFP } \\
\text { \$/ha }\end{array}$ & $\begin{array}{l}\text { VFRM } \\
\$ / h a\end{array}$ & $\begin{array}{l}\text { VNFRM } \\
\text { \$/person }\end{array}$ & Notes \\
\hline 1114 & NE & Hall County & 0.989468 & 0.905156 & 0.013092 & 1186 & 2942 & 109496 & 0 \\
\hline 1115 & NE & Hamilton County & 0.994363 & 0.922759 & 0.004764 & 989 & 4081 & 112008 & 0 \\
\hline 1116 & NE & Harlan County & 0.962672 & 0.864189 & 0.009679 & 601 & 1602 & 106177 & 0 \\
\hline 1117 & NE & Hayes County & 0.999691 & 0.880729 & 0.002957 & 482 & 818 & 153887 & 0 \\
\hline 1118 & NE & Hitchcock County & 0.988111 & 0.888069 & 0.003171 & 187 & 1083 & 110396 & 0 \\
\hline 1119 & NE & Holt County & 0.997938 & 0.898678 & 0.037844 & 304 & 1086 & 111263 & 0 \\
\hline 1120 & NE & Hooker County & 0.999527 & 0.812859 & 0.006343 & 54 & 314 & 116316 & 1 \\
\hline 1121 & NE & Howard County & 0.989102 & 0.892590 & 0.022716 & 550 & 1763 & 98272 & 0 \\
\hline 1122 & NE & Jefferson County & 0.995690 & 0.891148 & 0.078698 & 466 & 2031 & 118429 & 0 \\
\hline 1123 & NE & Johnson County & 0.998237 & 0.775926 & 0.042200 & 325 & 1613 & 103542 & 0 \\
\hline 1124 & NE & Kearney County & 0.999806 & 0.938739 & 0.000331 & 1241 & 3307 & 118964 & 1 \\
\hline 1125 & NE & Keith County & 0.956288 & 0.984838 & 0.003000 & 305 & 864 & 113790 & 0 \\
\hline 1126 & NE & Keya Paha County & 0.998857 & 0.901155 & 0.108061 & 114 & 662 & 142447 & 0 \\
\hline 1127 & NE & Kimball County & 0.999388 & 0.829272 & 0.007762 & 100 & 665 & 135457 & 0 \\
\hline 1128 & NE & Knox County & 0.972327 & 0.863857 & 0.059579 & 480 & 1408 & 94486 & 0 \\
\hline 1129 & NE & Lancaster County & 0.990742 & 0.772532 & 0.036463 & 417 & 2837 & 120671 & 0 \\
\hline 1130 & NE & Lincoln County & 0.995689 & 0.883864 & 0.010154 & 257 & 950 & 114548 & 0 \\
\hline 1131 & NE & Logan County & 0.999185 & 0.919408 & 0.000000 & 121 & 590 & 138912 & 0 \\
\hline 1132 & NE & Loup County & 0.997710 & 0.906016 & 0.000000 & 112 & 505 & 116790 & 0 \\
\hline 1133 & NE & McPherson County & 0.998796 & 0.844956 & 0.004460 & 62 & 391 & 130608 & 1 \\
\hline 1134 & NE & Madison County & 0.995708 & 0.878962 & 0.028954 & 784 & 2596 & 105750 & 0 \\
\hline 1135 & NE & Merrick County & 0.980304 & 0.936916 & 0.006283 & 1131 & 2640 & 102628 & 0 \\
\hline 1136 & NE & Morrill County & 0.995760 & 0.794996 & 0.000978 & 360 & 767 & 121301 & 1 \\
\hline 1137 & NE & Nance County & 0.984983 & 0.838869 & 0.021511 & 570 & 1735 & 104016 & 0 \\
\hline 1138 & NE & Nemaha County & 0.993718 & 0.862872 & 0.010937 & 488 & 2133 & 112388 & 0 \\
\hline 1139 & NE & Nuckolls County & 0.998855 & 0.905674 & 0.035034 & 351 & 1534 & 106928 & 0 \\
\hline 1140 & NE & Otoe County & 0.994731 & 0.826596 & 0.029777 & 444 & 2672 & 105547 & 0 \\
\hline 1141 & NE & Pawnee County & 0.997108 & 0.810535 & 0.058544 & 327 & 1792 & 108073 & 0 \\
\hline 1142 & NE & Perkins County & 0.998685 & 0.942805 & 0.008305 & 232 & 1402 & 175643 & 0 \\
\hline 1143 & NE & Phelps County & 0.998836 & 1.087316 & 0.001411 & 1510 & 3428 & 140212 & 9 \\
\hline 1144 & NE & Pierce County & 0.997660 & 0.809406 & 0.032340 & 880 & 2350 & 111446 & 0 \\
\hline 1145 & NE & Platte County & 0.983952 & 0.944118 & 0.026502 & 1008 & 3202 & 110261 & 0 \\
\hline 1146 & NE & Polk County & 0.995432 & 0.889869 & 0.004084 & 1171 & 3265 & 119947 & 0 \\
\hline 1147 & NE & Red Willow County & 0.998011 & 0.958154 & 0.003638 & 456 & 1307 & 112252 & 0 \\
\hline 1148 & NE & Richardson County & 0.995494 & 0.851191 & 0.064252 & 427 & 1935 & 100223 & 0 \\
\hline 1149 & NE & Rock County & 0.996644 & 1.019301 & 0.011687 & 196 & 579 & 119256 & 9 \\
\hline 1150 & NE & Saline County & 0.998747 & 0.847511 & 0.016969 & 456 & 2151 & 108649 & 0 \\
\hline 1151 & NE & Sarpy County & 0.973225 & 0.682238 & 0.008545 & 1420 & 4441 & 109299 & 0 \\
\hline 1152 & NE & Saunders County & 0.993612 & 0.906079 & 0.015564 & 792 & 3394 & 102180 & 0 \\
\hline 153 & NE & Scotts Bluff County & 0.991656 & 0.882740 & 0.002912 & 1132 & 1873 & 113539 & 0 \\
\hline 1154 & NE & Seward County & 0.998331 & 0.856166 & 0.026778 & 784 & 2758 & 104781 & 0 \\
\hline 1155 & NE & Sheridan County & 0.988284 & 0.948250 & 0.010570 & 101 & 544 & 120536 & 0 \\
\hline 1156 & NE & Sherman County & 0.989884 & 0.823158 & 0.039266 & 289 & 1387 & 101632 & 0 \\
\hline 1157 & NE & Sioux County & 0.999648 & 0.760480 & 0.000000 & 161 & 584 & 126164 & 0 \\
\hline 1158 & NE & Stanton County & 0.997144 & 0.789617 & 0.021076 & 1105 & 2215 & 104260 & 0 \\
\hline 1159 & NE & Thayer County & 0.998647 & 0.945250 & 0.006654 & 572 & 2215 & 116668 & 0 \\
\hline 1160 & NE & Thomas County & 0.998894 & 0.789476 & 0.005735 & 62 & 429 & 116689 & 1 \\
\hline 1161 & NE & Thurston County & 0.993845 & 0.767911 & 0.025391 & 695 & 2129 & 72270 & 0 \\
\hline 1162 & NE & Valley County & 0.995783 & 0.933311 & 0.022706 & 525 & 1461 & 120292 & 0 \\
\hline 1163 & NE & Washington County & 0.991590 & 0.913000 & 0.060558 & 843. & 3697 & 116276 & 0 \\
\hline 1164 & NE & Wayne County & 0.999895 & 0.875562 & 0.034401 & 1062 & 2369 & 97534 & 0 \\
\hline 165 & NE & Webster County & 0.999776 & 0.835805 & 0.007210 & 744 & 1481 & 121599 & 0 \\
\hline 1166 & NE & Wheeler County & 0.999289 & 0.717078 & 0.018053 & 1150 & 811 & 243408 & 0 \\
\hline 1167 & NE & York County & 0.999377 & 0.937765 & 0.000682 & 1083 & 4231 & 118958 & 1 \\
\hline 1168 & KS & Allen County & 0.995695 & 0.878562 & 0.147362 & 254 & 1227 & 92366 & 0 \\
\hline 1169 & KS & Anderson County & 0.997647 & 1.014506 & 0.042863 & 299 & 1419 & 97554 & 9 \\
\hline 1170 & KS & Atchison County & 0.993784 & 0.885764 & 0.054754 & 360 & 1854 & 101070 & 0 \\
\hline 1171 & KS & Barber County & 0.998153 & 0.880774 & 0.022753 & 193 & 929 & 125331 & 0 \\
\hline 1172 & KS & Barton County & 0.992829 & 1.014000 & 0.008687 & 588 & 1450 & 118131 & 9 \\
\hline 1173 & KS & Bourbon County & 0.997283 & 0.827182 & 0.053333 & 198 & 1115 & 100033 & 0 \\
\hline 1174 & KS & Brown County & 0.997342 & 0.928506 & 0.039091 & 558 & 2151 & 107660 & 0 \\
\hline 1175 & KS & Butler County & 0.987325 & 0.837716 & 0.008335 & 403 & 1413 & 117522 & 0 \\
\hline 1176 & KS & Chase County & 0.997272 & 0.708699 & 0.009891 & 324 & 1128 & 120346 & 0 \\
\hline 1177 & KS & Chautauqua County & 0.995128 & 0.941986 & 0.014408 & 190 & 815 & 92298 & 0 \\
\hline 1178 & KS & Cherokee County & 0.993487 & 0.721189 & 0.013596 & 327 & 1605 & 86372 & 0 \\
\hline 1179 & KS & Cheyenne County & 0.999069 & 0.907232 & 0.000000 & 215 & 1044 & 129246 & 0 \\
\hline 1180 & KS & Clark County & 0.997421 & 0.906120 & 0.000812 & 362 & 714 & 164155 & 1 \\
\hline 1181 & KS & Clay County & 0.982323 & 0.924499 & 0.031218 & 308 & 1414 & 105635 & 0 \\
\hline 1182 & KS & Cloud County & 0.996050 & 0.889604 & 0.015657 & 204 & 1432 & 108168 & 0 \\
\hline 1183 & KS & Coffey County & 0.962781 & 0.876050 & 0.008080 & 292 & 1420 & 108378 & 0 \\
\hline
\end{tabular}




\begin{tabular}{|c|c|c|c|c|c|c|c|c|c|}
\hline $\begin{array}{l}\text { County } \\
\text { Code }\end{array}$ & State & $\begin{array}{l}\text { County } \\
\text { Name }\end{array}$ & $\begin{array}{l}\text { FRCLND } \\
\text { Absolute }\end{array}$ & $\begin{array}{l}\text { FRMFRC } \\
\text { Absolute }\end{array}$ & $\begin{array}{c}\text { DPF } \\
\text { Absolute }\end{array}$ & $\begin{array}{l}\text { ASFP } \\
\text { \$/ha }\end{array}$ & $\begin{array}{l}\text { VFRM } \\
\text { S/ha }\end{array}$ & $\begin{array}{l}\text { UNFRM } \\
\text { \$/person }\end{array}$ & Notes \\
\hline 1184 & KS & Comanche County & 0.998242 & 0.965208 & 0.023633 & 157 & 724 & 121586 & 0 \\
\hline 1185 & KS & Cowley County & 0.994411 & 0.870642 & 0.018980 & 263 & 1332 & 104402 & 0 \\
\hline 1186 & KS & Crawford County & 0.996423 & 0.798007 & 0.044861 & 274 & 1332 & 105946 & 0 \\
\hline 1187 & KS & Decatur County & 0.999291 & 0.919852 & 0.003829 & 391 & 1056 & 174857 & 0 \\
\hline 1188 & KS & Dickinson County & 0.995054 & 0.947391 & 0.040485 & 381 & 1474 & 110633 & 0 \\
\hline 1189 & KS & Doniphan County & 0.987687 & 0.803938 & 0.022556 & 455 & 2069 & 95570 & 0 \\
\hline 1190 & KS & Douglas County & 0.963040 & 0.759179 & 0.086169 & 386 & 2628 & 95746 & 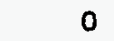 \\
\hline 1191 & KS & Edwards County & 0.999870 & 1.013217 & 0.002058 & 479 & 1382 & 137767 & ( \\
\hline 1192 & KS & Elk County & 0.996078 & 0.781575 & 0.007335 & 153 & 848 & 99390 & 0 \\
\hline 1193 & KS & Ellis County & 0.999443 & 0.950472 & 0.048434 & 235 & 1092 & 113708 & 0 \\
\hline 1194 & KS & Ellsworth County & 0.989582 & 0.965438 & 0.003359 & 112 & 1101 & 103027 & 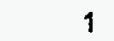 \\
\hline 1195 & KS & Finney County & 0.998058 & 0.895771 & 0.000200 & 1111 & 1489 & 103901 & 4 \\
\hline 1196 & KS & Ford County & 0.999283 & 0.954688 & 0.001321 & 1195 & 1302 & 117380 & 0 \\
\hline 1197 & KS & Franklin County & 0.995153 & 0.861201 & 0.105419 & 316 & 2025 & 97785 & 0 \\
\hline 1198 & KS & Geary County & 0.951286 & 0.667171 & 0.065328 & 278 & 1322 & 82369 & 0 \\
\hline 1199 & KS & Gove County & 0.999916 & 0.979246 & 0.006238 & 514 & 1066 & 143362 & 0 \\
\hline 1200 & KS & Graham County & 0.999573 & 0.891805 & 0.020769 & 187 & 878 & 110999 & 0 \\
\hline 1201 & KS & Grant County & 0.999682 & 0.928421 & 0.000465 & 2089 & 1479 & 144459 & 1 \\
\hline 1202 & $\mathrm{KS}$ & Gray County & 0.999494 & 0.930763 & 0.000509 & 1260 & 1500 & 123462 & 1 \\
\hline 1203 & KS & Greeley County & 1.000000 & 0.851692 & 0.000708 & 552 & 1254 & 185518 & 1 \\
\hline 1204 & KS & Greenwood County & 0.988858 & 0.827646 & 0.026787 & 205 & 950 & 105770 & 0 \\
\hline 1205 & KS & Hamilton County & 0.998868 & 0.835535 & 0.001202 & 518 & 853 & 158669 & 1 \\
\hline 1206 & KS & Harper County & 0.998116 & 0.973021 & 0.005646 & 264 & 1376 & 127573 & 0 \\
\hline 1207 & $\mathrm{KS}$ & Harvey County & 0.997944 & 0.925997 & 0.049903 & 446 & 2140 & 103996 & 0 \\
\hline 1208 & KS & Haskell County & 0.999376 & 0.992513 & 0.000371 & 2440 & 1891 & 129869 & 4 \\
\hline 1209 & ks & Hodgeman County & 0.999631 & 0.871939 & 0.004273 & 558 & 887 & 120759 & 0 \\
\hline 1210 & KS & Jackson County & 0.998413 & 0.808863 & 0.069866 & 235 & 1494 & 101408 & 0 \\
\hline 1211 & KS & Jefferson County & 0.962603 & 0.791844 & 0.081989 & 326 & 1945 & 105242 & 0 \\
\hline 1212 & KS & Jewell County & 0.994210 & 0.833176 & 0.015720 & 256 & 1335 & 112198 & 0 \\
\hline 1213 & KS & Johnson County & 0.992924 & 0.463350 & 0.044899 & 494 & 4621 & 177160 & 0 \\
\hline 1214 & KS & Kearny County & 0.998251 & 0.929145 & 0.000331 & 968 & 1183 & 151462 & 1 \\
\hline 1215 & KS & Kingman County & 0.996505 & 0.984242 & 0.037592 & 220 & 1422 & 108432 & \\
\hline 1216 & KS & Kowa County & 0.999682 & 0.864768 & 0.001930 & 215 & 1120 & 132457 & \\
\hline 1217 & KS & Labette County & 0.993187 & 0.834381 & 0.030158 & 461 & 1428 & 103942 & D \\
\hline 1218 & KS & Lane County & 0.999703 & 0.913679 & 0.000000 & 738 & 1034 & 125182 & 0 \\
\hline 1219 & KS & Leavenworth County & 0.989203 & 0.696520 & 0.192995 & 382 & 2777 & 97304 & 0 \\
\hline 1220 & $\mathrm{KS}$ & Lincoln County & 0.998513 & 1.048550 & 0.023314 & 165 & 1132 & 111047 & 9 \\
\hline 1221 & KS & Linn County & 0.987493 & 0.714596 & 0.074521 & 224 & 1433 & 93877 & 0 \\
\hline 1222 & KS & Logan County & 0.999901 & 0.878290 & 0.007355 & 112 & 799 & 110112 & 1 \\
\hline 1223 & $\mathrm{KS}$ & Lyon County & 0.995070 & 0.891709 & 0.019775 & 320 & 1284 & 101598 & 0 \\
\hline 1224 & KS & McPherson County & 0.998363 & 0.934068 & 0.047768 & 481 & 2012 & 118145 & 0 \\
\hline 1225 & $\mathrm{KS}$ & Marion County & 0.989061 & 0.974219 & 0.104808 & 320 & 1506 & 100467 & 0 \\
\hline 1226 & KS & Marshall County & 0.998027 & 0.991878 & 0.043473 & 307 & 1635 & 112489 & 0 \\
\hline 1227 & KS & Meade County & 0.998733 & 0.951897 & 0.002091 & 266 & 1092 & 121782 & 1 \\
\hline 1228 & KS & Miami County & 0.977228 & 0.777485 & 0.071888 & 280 & 2839 & 103779 & 0 \\
\hline 1229 & $\mathrm{KS}$ & Mitchell County & 0.973931 & 1.070019 & 0.009850 & 362 & 1450 & 118795 & 9 \\
\hline 1230 & KS & Montgomery County & 0.990495 & 0.784007 & 0.045824 & 234 & 1480 & 101774 & 0 \\
\hline 1231 & KS & Morris County & 0.992233 & 0.918181 & 0.041320 & 293 & 1285 & 98604 & 0 \\
\hline 1232 & KS & Morton County & 0.999971 & 0.914855 & 0.000486 & 143 & 1003 & 118592 & 0 \\
\hline 1233 & KS & Nemaha County & 0.999504 & 0.959170 & 0.106661 & 437 & 1852 & 113471 & 0 \\
\hline 1234 & KS & Neosho County & 0.989319 & 0.892608 & 0.074773 & 259 & 1409 & 106001 & 0 \\
\hline 1235 & KS & Ness County & 0.999760 & 0.971711 & 0.008523 & 124 & 847 & 148645 & 0 \\
\hline 1236 & KS & Norton County & 0.995977 & 0.828576 & 0.004305 & 203 & 1117 & 113952 & 0 \\
\hline 1237 & KS & Osage County & 0.978181 & 0.775686 & 0.015555 & 247 & 1480 & 99078 & 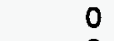 \\
\hline 1238 & KS & Osborne County & 0.998101 & 0.958160 & 0.018102 & 175 & 941 & 119608 & \\
\hline 1239 & KS & Ottawa County & 0.998945 & 0.824182 & 0.012216 & 281 & 1410 & 99248 & 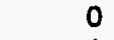 \\
\hline 1240 & KS & Pawnee County & 0.999491 & 0.930505 & 0.001390 & 797 & 1264 & 124071 & \\
\hline 1241 & KS & Phillips County & 0.990408 & 1.026177 & 0.013607 & 195 & 985 & 116303 & \\
\hline 1242 & KS & Pottawatomie County & 0.979319 & 0.835320 & 0.025230 & 281 & 1428 & 98049 & 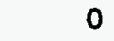 \\
\hline 1243 & $\mathrm{KS}$ & Pratt County & 0.998964 & 0.919029 & 0.000968 & 821 & 1548 & 125243 & \\
\hline 1244 & KS & Rawlins County & 0.999899 & 0.936474 & 0.020164 & 129 & 903 & 132789 & 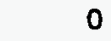 \\
\hline 1245 & KS & Reno County & 0.986766 & 0.872940 & 0.058641 & 388 & 1875 & 116479 & \\
\hline 1246 & KS & Republic County & 0.994711 & 0.966638 & 0.007129 & 610 & 1669 & 109611 & \\
\hline 1247 & KS & Rice County & 0.997642 & 0.930475 & 0.005436 & 470 & 1494 & 111907 & \\
\hline 1248 & KS & Riley County & 0.979890 & 0.584820 & 0.045757 & 291 & 1503 & 88783 & \\
\hline 1249 & KS & Rooks County & 0.992164 & 1.017086 & 0.035100 & 152 & 929 & 103549 & \\
\hline 1250 & KS & Rush County & 0.999708 & 0.929913 & 0.015828 & 135 & 1024 & 125866 & \\
\hline 1251 & KS & Russell County & 0.984101 & 0.818906 & 0.035997 & 115 & 950 & 121240 & \\
\hline 1252 & KS & Saline County & 0.997690 & 0.875591 & 0.056146 & 213 & 1617 & 128704 & \\
\hline 1253 & KS & Scott County & 0.999836 & 1.054787 & 0.000000 & 2067 & 1252 & 156231 & \\
\hline
\end{tabular}




\begin{tabular}{|c|c|c|c|c|c|c|c|c|c|}
\hline $\begin{array}{l}\text { County } \\
\text { Code }\end{array}$ & State & $\begin{array}{l}\text { County } \\
\text { Name }\end{array}$ & $\begin{array}{l}\text { FRCLND } \\
\text { Absolute }\end{array}$ & $\begin{array}{l}\text { FRMFRC } \\
\text { Absolute }\end{array}$ & $\begin{array}{c}\text { DPF } \\
\text { Absolute }\end{array}$ & $\begin{array}{c}\text { ASFP } \\
\text { \$/ha }\end{array}$ & $\begin{array}{c}\text { VFRM } \\
\text { \$/ha }\end{array}$ & $\begin{array}{l}\text { VNFRM } \\
\text { \$/person }\end{array}$ & Notes \\
\hline 1254 & KS & Sedgwick County & 0.990858 & 0.797176 & 0.179726 & 337 & 2557 & 130560 & 0 \\
\hline 1255 & KS & Seward County & 0.998457 & 0.801553 & 0.000000 & 1641 & 1430 & 116228 & 0 \\
\hline 1256 & KS & Shawnee County & 0.988315 & 0.646052 & 0.036272 & 322 & 2485 & 132470 & 0 \\
\hline 1257 & KS & Sheridan County & 0.999724 & 0.933159 & 0.015997 & 330 & 1282 & 116391 & 0 \\
\hline 1258 & KS & Sherman County & 0.999799 & 0.917700 & 0.001883 & 284 & 1352 & 126571 & 1 \\
\hline 1259 & KS & Smith County & 0.998769 & 0.937817 & 0.012438 & 248 & 1130 & 109800 & 0 \\
\hline 1260 & KS & Stafford County & 0.996639 & 0.860536 & 0.000000 & 523 & 1417 & 134495 & 0 \\
\hline 1261 & KS & Stanton County & 0.999917 & 0.946110 & 0.000503 & 801 & 1391 & 176076 & 1 \\
\hline 1262 & KS & Stevens County & 0.999754 & 0.968145 & 0.000670 & 1098 & 1317 & 167420 & 1 \\
\hline 1263 & KS & Sumner County & 0.997492 & 0.909020 & 0.037173 & 241 & 1559 & 112171 & 0 \\
\hline 1264 & KS & Thomas County & 0.999897 & 1.021278 & 0.000000 & 351 & 1461 & 123787 & 9 \\
\hline 1265 & KS & Trego County & 0.988066 & 0.851451 & 0.016401 & 316 & 1083 & 112354 & 0 \\
\hline 1266 & KS & Wabaunsee County & 0.997000 & 0.828930 & 0.035033 & 232 & 989 & 103677 & 0 \\
\hline 1267 & KS & Wallace County & 0.999942 & 0.806252 & 0.002205 & 160 & 1080 & 115104 & 1 \\
\hline 1268 & KS & Washington County & 0.999578 & 0.906211 & 0.045168 & 329 & 1620 & 99519 & 0 \\
\hline 1269 & KS & Wichita County & 0.999967 & 0.965030 & 0.000000 & 1849 & 1381 & 159678 & 0 \\
\hline 1270 & KS & Wilson County & 0.998031 & 0.851395 & 0.007721 & 247 & 1307 & 97040 & 0 \\
\hline 1271 & KS & Woodson County & 0.990414 & 0.830090 & 0.000729 & 229 & 1009 & 33538 & 0 \\
\hline 1272 & KS & Wyandotte County & 0.972370 & 0.232758 & 0.013749 & 535 & 5243 & 95732 & 1 \\
\hline 1273 & $D E$ & Kent County & 0.738196 & 0.522125 & 0.083485 & 1399 & 5687 & 101232 & 0 \\
\hline 1274 & $\overline{D E}$ & New Castle County & 0.863773 & 0.319369 & 0.073593 & 1143 & 8849 & 934 & 0 \\
\hline 1275 & $\mathrm{DE}$ & Sussex County & 0.784189 & 0.507715 & 0.013127 & 3307 & 6214 & 112428 & 0 \\
\hline 1276 & MD & Allegany County & 0.989772 & 0.138865 & 0.353833 & 224 & 3026 & 100149 & 0 \\
\hline 1277 & MD & Anne Arundel County & 0.707515 & 0.162729 & 0.015845 & 641 & 9964 & 154409 & 1 \\
\hline 1278 & MD & Baltimore County & 0.877594 & 0.217262 & 0.152840 & 1206 & 13905 & 158967 & 0 \\
\hline 1279 & MD & Calvert County & 0.623547 & 0.270976 & 0.000000 & 450 & 10646 & 148286 & 0 \\
\hline 1280 & MD & Caroline County & 0.981852 & 0.619727 & 0.045242 & 1655 & 5490 & 95644 & 0 \\
\hline 1281 & MD & Carroll County & 0.992770 & 0.547917 & 0.317609 & 1051 & 9686 & 149024 & 0 \\
\hline 1282 & MD & Cecil County & 0.833137 & 0.360109 & 0.223721 & 1093 & 8412 & 126957 & 0 \\
\hline 1283 & MD & Charles County & 0.716787 & 0.201254 & 0.010464 & 414 & 8410 & 130838 & 0 \\
\hline 1284 & MD & Dorchester County & 0.567244 & 0.346813 & 0.002777 & 1280 & 5373 & 115361 & 1 \\
\hline 1285 & MD & Frederick County & 0.993326 & 0.525042 & 0.620383 & 1211 & 9403 & 135152 & 0 \\
\hline 1286 & MD & Garrett County & 0.987908 & 0.266865 & 0.547585 & 456 & 3239 & 89217 & 0 \\
\hline 1287 & MD & Harford County & 0.836028 & 0.345275 & 0.364608 & 730 & 9986 & 141838 & 0 \\
\hline 1288 & MD & Howard County & 0.994540 & 0.276485 & 0.156948 & 1049 & 11199 & 186575 & 0 \\
\hline 1289 & MD & Kent County & 0.674459 & 0.734067 & 0.174232 & 1025 & 7058 & 130459 & 0 \\
\hline 1290 & MD & Montgomery County & 0.976988 & 0.260546 & 0.163402 & 830 & 14078 & 203745 & 0 \\
\hline 1291 & MD & Prince George's County & 0.973938 & 0.174940 & 0.024308 & 997 & 10383 & 132538 & 1 \\
\hline 1292 & MD & Queen Anne's County & 0.730119 & 0.694076 & 0.092130 & 825 & 6861 & 144567 & 0 \\
\hline 1293 & MD & St. Mary's County & 0.472558 & 0.335132 & 0.033152 & 521 & 6819 & 113126 & 0 \\
\hline 1294 & MD & Somerset County & 0.535720 & 0.265762 & 0.006921 & 4568 & 6099 & 80527 & 1 \\
\hline 1295 & MD & Talbot County & 0.564547 & 0.633328 & 0.051407 & 804 & 8968 & 170800 & 0 \\
\hline 1296 & MD & Washington County & 0.979875 & 0.422654 & 0.590580 & 1163 & 7277 & 109983 & 0 \\
\hline 1297 & MD & Wicomico County & 0.943472 & 0.377996 & 0.001081 & 4459 & 7287 & 112306 & 1 \\
\hline 1298 & MD & Worcester County & 0.681152 & 0.354990 & 0.002711 & 3018 & 5266 & 128386 & 1 \\
\hline 1299 & MD & Baltimore city & 0.877610 & 0.000000 & 0.000000 & 0 & 0 & 116926 & 0 \\
\hline 1300 & DC & District of Columbia & 0.898382 & 0.000000 & 0.000000 & 0 & 0 & 159868 & 0 \\
\hline 1301 & VA & Accomack County & 0.346984 & 0.314718 & 0.000000 & 1938 & 4386 & 100386 & 0 \\
\hline 1302 & VA & Albemarle County & 0.995200 & 0.407658 & 0.123163 & 286 & 7559 & 136798 & 23 \\
\hline 1303 & VA & Alleghany County & 0.997671 & 0.090445 & 0.070519 & 196 & 3420 & 98354 & 24 \\
\hline 1304 & VA & Amelia County & 0.995144 & 0.308317 & 0.132636 & 1362 & 3533 & 103149 & 0 \\
\hline 1305 & VA & County & 0.992576 & 0.296866 & 0.161980 & 146 & 2652 & 85024 & 1 \\
\hline 1306 & VA & Appomattox Caunty & 0.996869 & 0.368453 & 0.365510 & 216 & 4061 & 98990 & 0 \\
\hline 1307 & VA & Arlington County & 0.996373 & 0.010060 & 0.000000 & 155 & 3077 & 204835 & 2 \\
\hline 1308 & VA & Augusta County & 0.999413 & 0.462204 & 0.227345 & 955 & 5152 & 108480 & 23 \\
\hline 1309 & VA & Bath County & 0.994921 & 0.138101 & 0.081127 & 93 & 2522 & 124681 & 1 \\
\hline 1310 & VA & Bedford County & 0.981134 & 0.415042 & 0.406847 & 256 & 3355 & 111169 & 23 \\
\hline 1311 & VA & Bland County & 0.999823 & 0.356209 & 0.306144 & 200 & 2054 & 85844 & 0 \\
\hline 1312 & VA & Botetourt County & 0.993995 & 0.278795 & 0.349988 & 320 & 3746 & 118274 & 0 \\
\hline 1313 & VA & Brunswick County & 0.994324 & 0.233691 & 0.120647 & 462 & 2490 & 86887 & 0 \\
\hline 1314 & - VA & Buchanan County & 1.000000 & 0.026751 & 0.460800 & 179 & 3031 & 94445 & 14 \\
\hline 1315 & VA & Buckingham County & 0.995368 & 0.177620 & 0.050029 & 581 & 2566 & 88431 & 0 \\
\hline 1316 & VA & Campbell County & 0.994521 & 0.416482 & 0.233164 & 274 & 2592 & 113526 & 23 \\
\hline 1317 & VA & Caroline County & 0.988283 & 0.151386 & 0.034749 & 397 & 3868 & 108459 & 1 \\
\hline 1318 & VA & Carroll County & 0.997271 & 0.371055 & 0.184521 & 431 & 3149 & 85776 & 23 \\
\hline 1319 & VA & Charles City County & 0.894919 & 0.246843 & 0.000000 & 462 & 4148 & 123448 & 0 \\
\hline 1320 & VA & Charlotte County & 0.994915 & 0.371503 & 0.196346 & 319 & 2479 & 89582 & 0 \\
\hline 1321 & VA & Chesterfield County & 0.970979 & 0.063835 & 0.000000 & 997 & 7316 & 134922 & 0 \\
\hline 1322 & VA & Clarke County & 0.991197 & 0.604415 & 0.238838 & 642 & 8896 & 153460 & 0 \\
\hline 1323 & VA & Craig County & 0.999986 & 0.215158 & 0.123596 & 140 & 2403 & 90063 & 0 \\
\hline
\end{tabular}




\begin{tabular}{|c|c|c|c|c|c|c|c|c|c|}
\hline $\begin{array}{l}\text { County } \\
\text { Code }\end{array}$ & State & $\begin{array}{l}\text { County } \\
\text { Name }\end{array}$ & $\begin{array}{l}\text { FRCLND } \\
\text { Absolute }\end{array}$ & $\begin{array}{l}\text { FRMFRC } \\
\text { Absolute }\end{array}$ & $\begin{array}{c}\text { DPF } \\
\text { Absolute }\end{array}$ & $\begin{array}{l}\text { ASFP } \\
\$ / h a\end{array}$ & $\begin{array}{l}\text { VFRM } \\
\text { \$/ha }\end{array}$ & $\begin{array}{l}\text { VNFRM } \\
\text { \$/person }\end{array}$ & Notes \\
\hline 1324 & VA & Culpeper County & 0.997001 & 0.472621 & 0.296438 & 516 & 5896 & 117847 & 0 \\
\hline 1325 & VA & Cumberland County & 0.995871 & 0.322784 & 0.067594 & 1091 & 3078 & 90178 & 0 \\
\hline 1326 & VA & Dickenson County & 0.994288 & 0.040697 & 0.022077 & 155 & 3773 & 91641 & 15 \\
\hline 1327 & VA & Dinwiddie County & 0.993528 & 0.266602 & 0.110966 & 484 & 3012 & 109211 & 23 \\
\hline 1328 & VA & Essex County & 0.901571 & 0.341132 & 0.000000 & 424 & 3321 & 113803 & 0 \\
\hline 1329 & VA & Fairfax County & 0.972373 & 0.062069 & 0.043204 & 524 & 13797 & 196416 & 24 \\
\hline 1330 & VA & Fauquier County & 0.997666 & 0.565916 & 0.298232 & 396 & 8374 & 165076 & 0 \\
\hline 1331 & VA & Floyd County & 0.999965 & 0.477212 & 0.228122 & 453 & 3135 & 99011 & 0 \\
\hline 1332 & VA & Fluvanna County & 0.990349 & 0.317066 & 0.031648 & 193 & 3147 & 100826 & 1 \\
\hline 1333 & VA & Franklin County & 0.972627 & 0.375832 & 0.658034 & 596 & 3899 & 98157 & 0 \\
\hline 1334 & VA & Frederick County & 0.997403 & 0.369883 & 0.059776 & 596 & 7722 & 110999 & 23 \\
\hline 1335 & VA & Giles County & 0.991492 & 0.319123 & 0.071058 & 137 & 2022 & 98232 & 1 \\
\hline 1336 & VA & Gloucester County & 0.751995 & 0.176588 & 0.030625 & 475 & 7322 & 107084 & 1 \\
\hline 1337 & VA & Goochland County & 0.980843 & 0.282569 & 0.185014 & 228 & 5299 & 160532 & 0 \\
\hline 1338 & VA & Grayson County & 0.992679 & 0.481178 & 0.265199 & 306 & 3257 & 84889 & 0 \\
\hline 1339 & VA & Greene County & 0.997654 & 0.369579 & 0.310774 & 295 & 5155 & 94520 & 0 \\
\hline 1340 & VA & Greensville County & 0.995378 & 0.271684 & 0.018378 & 754 & 2194 & 105526 & 24 \\
\hline 1341 & VA & Halifax County & 0.987557 & 0.447078 & 0.027101 & 354 & 2307 & 97527 & 23 \\
\hline 1342 & VA & Hanover County & 0.997155 & 0.318215 & 0.152681 & 537 & 6632 & 144913 & 0 \\
\hline 1343 & VA & Henrico County & 0.979079 & 0.158796 & 0.000000 & 700 & 7328 & 161771 & 0 \\
\hline 1344 & VA & Henry County & 0.994701 & 0.200097 & 0.072162 & 201 & 2418 & 112157 & 24 \\
\hline 1345 & VA & Highland County & 000 & 0.364097 & 0.029361 & 250 & 2353 & 87259 & 1 \\
\hline 1346 & VA & Isle of Wight County & 727 & 0.426609 & 0.000000 & 1102 & 4725 & 121450 & 0 \\
\hline 1347 & VA & James City County & 0.795163 & 0.104511 & 0.118616 & 628 & 8074 & 116546 & 24 \\
\hline 1348 & VA & King and Queen County & 0.969157 & 0.259396 & 0.047793 & 425 & 2955 & 98882 & 1 \\
\hline 1349 & VA & King George County & 0.958495 & 0.327898 & 0.000000 & 343 & 4653 & 118707 & 0 \\
\hline 1350 & VA & King William County & 0.964223 & 0.336529 & 0.167369 & 494 & 3808 & 119046 & 0 \\
\hline 1351 & VA & Lancaster County & 0.575503 & 0.230672 & 0.005376 & 327 & 3357 & 153440 & 0 \\
\hline 1352 & VA & Lee County & 9573 & 0.462141 & 0.037032 & 294 & 2772 & 77601 & 0 \\
\hline 1353 & VA & Loudoun County & 0.997651 & 0.587461 & 0.122411 & 338 & 10973 & 180722 & 0 \\
\hline 1354 & VA & Louisa County & 0.973883 & 0.255742 & 0.126833 & 300 & 4073 & 101523 & 0 \\
\hline 1355 & VA & Lunenburg County & 0.998564 & 0.309762 & 0.002253 & 384 & 2559 & 84834 & 0 \\
\hline 1356 & VA & Madison County & 0.998884 & 0.488999 & 0.307339 & 460 & 4964 & 97900 & 0 \\
\hline 1357 & VA & Mathews County & 0.340081 & 0.112172 & 0.000000 & 971 & 7242 & 126950 & 0 \\
\hline 1358 & VA & Mecklenburg County & 0.918510 & 0.420329 & 0.115123 & 469 & 2490 & 96078 & 0 \\
\hline 1359 & VA & Middlesex County & 0.618245 & 0.257898 & 0.075969 & 436 & 5320 & 122737 & 1 \\
\hline 1360 & VA & Montgomery County & 0.996912 & 0.398088 & 0.412118 & 379 & 4260 & 89697 & 23 \\
\hline 1361 & VA & Nelson County & 0.995881 & 0.240407 & 0.024832 & 197 & 3915 & 102512 & 1 \\
\hline 1362 & VA & New Kent County & 0.937724 & 0.136767 & 0.000000 & 458 & 5039 & 141750 & 0 \\
\hline $13 \widetilde{3}$ & VA & Northampton County & 726 & 324 & 0.000000 & 1260 & 5093 & 96430 & 0 \\
\hline 1364 & VA & Northumberland County & 182 & 0.335474 & 0.000000 & 476 & 4085 & 120048 & 0 \\
\hline 1365 & VA & ay County & 571 & 687 & 639 & 952 & 3433 & 102133 & 0 \\
\hline 1366 & VA & Orange County & 040 & 0.492434 & 157 & 465 & 5023 & 118605 & 0 \\
\hline 1367 & VA & Page County & 54 & 992 & 3466 & 2682 & 6161 & 99857 & 0 \\
\hline 1368 & VA & Patrick County & 397 & 0.256930 & 3414 & 348 & 3129 & 102892 & 0 \\
\hline 1369 & VA & Pittsylvani & 0.992475 & 0.478098 & 0.067601 & 466 & 2942 & 100894 & 23 \\
\hline 1370 & VA & Powhatan County & 0.995721 & 0.258207 & 0.451047 & 454 & 5764 & 120956 & 0 \\
\hline 1371 & VA & Prince Edward County & 0.996994 & 0.303741 & 0.240860 & 426 & 3437 & 82274 & 0 \\
\hline 1372 & VA & Prince George County & 0.943389 & 0.287599 & 0.000000 & 388 & 3780 & 90822 & 23 \\
\hline 1373 & VA & Prince William County & 0.969556 & 0.152234 & 0.444188 & 422 . & 11973 & 139142 & 23 \\
\hline 1374 & VA & Pulaski County & 0.972611 & 0.349973 & 0.282857 & 401 & 3837 & 90666 & 0 \\
\hline 1375 & VA & Rappahannock County & 026 & 0.462873 & 1582 & 193 & 7694 & 131563 & 0 \\
\hline 1376 & VA & Richmond County & 0.884835 & 0.317763 & 0.017161 & 532 & 3541 & 103779 & 1 \\
\hline 1377 & VA & Roanoke County & 0.999290 & 0.155361 & 0.027111 & 1276 & 6968 & 132971 & 23 \\
\hline 1378 & VA & Rockbridge County & 0.997752 & 385 & 0.246921 & 251 & 3979 & 99464 & 23 \\
\hline 1379 & VA & Rockingham County & 486 & 0.433344 & 0.143494 & 3883 & 7827 & 106847 & 23 \\
\hline 30 & VA & Russell County & 565 & 0.529888 & 0.075828 & 300 & 2405 & 87882 & 0 \\
\hline 1381 & VA & Scott County & 0.996205 & 0.390735 & 0.076005 & 299 & 3373 & 81441 & 0 \\
\hline 1382 & VA & Shenandoah County & 0.999370 & 0.382494 & 0.093539 & 1103 & 5928 & 115822 & 0 \\
\hline 1383 & VA & Smyth County & 0.999517 & 0.414631 & 0.278144 & 406 & 2938 & 96965 & 0 \\
\hline 1384 & VA & Southampton County & 0.995360 & 0.464556 & 0.000000 & 777 & 3297 & 90984 & 23 \\
\hline 1385 & VA & Spotsylvania County & 0.972344 & 0.206192 & 0.389865 & 277 & 8258 & 112706 & 23 \\
\hline 1386 & VA & Stafford County & 0.965849 & 0.116357 & 0.077088 & 230 & 10475 & 147060 & 1 \\
\hline 1387 & VA & Surry County & 0.899402 & 0.295418 & 0.015266 & 883 & 4339 & 121606 & 1 \\
\hline 1388 & VA & Sussex County & 0.995734 & 0.263421 & 0.000000 & 807 & 2762 & 97297 & 0 \\
\hline 1389 & VA & Tazewell County & 0.999716 & 0.416446 & 0.158660 & 242 & 2631 & 98929 & 0 \\
\hline 1390 & VA & n County & 0.987877 & 0.284883 & 0.039066 & 258 & 5942 & 103596 & 0 \\
\hline 1391 & VA & Washington County & 0.994041 & 0.526354 & 0.245090 & 618 & 4280 & 103393 & 23 \\
\hline 1392 & VA & Westmoreland County & 0.907156 & 0.383738 & 0.028475 & 666 & 4440 & 114914 & 1 \\
\hline 1393 & $V$ & ounty & 9 & 0.05 & 3 & 1 & 4028 & 8 & 23 \\
\hline
\end{tabular}




\begin{tabular}{|c|c|c|c|c|c|c|c|c|c|}
\hline $\begin{array}{l}\text { County } \\
\text { Code }\end{array}$ & State & $\begin{array}{l}\text { County } \\
\text { Name }\end{array}$ & $\begin{array}{l}\text { FRCLND } \\
\text { Absolute }\end{array}$ & $\begin{array}{l}\text { FRMFRC } \\
\text { Absolute }\end{array}$ & $\begin{array}{c}\text { DPF } \\
\text { Absolute }\end{array}$ & $\begin{array}{c}\text { ASFP } \\
\text { \$/ha }\end{array}$ & $\begin{array}{l}\text { VFRM } \\
\text { \$/ha }\end{array}$ & $\begin{array}{l}\text { VNFRM } \\
\text { \$/person }\end{array}$ & Notes \\
\hline 1394 & VA & Wythe County & 0.997045 & 0.443075 & 0.400944 & 482 & 3172 & 93267 & 0 \\
\hline 1395 & VA & York County & 0.490095 & 0.034885 & 0.050157 & 3009 & 19563 & 140117 & 24 \\
\hline 1396 & VA & Alexandria city & 0.993195 & 0.000000 & 0.000000 & 0 & 0 & 214704 & 0 \\
\hline 1397 & VA & Bedford city & 0.997917 & 0.000000 & 0.000000 & 0 & 0 & 111169 & 23 \\
\hline 1398 & VA & Bristol city & 1.000000 & 0.000000 & 0.000000 & 0 & 0 & 103393 & 23 \\
\hline 1399 & VA & Buena Vista city & 1.000000 & 0.000000 & 0.000000 & 0 & 0 & 99464 & 23 \\
\hline 1400 & VA & Charlottesville city & 1.000000 & 0.000000 & 0.000000 & 0 & 0 & 136798 & 23 \\
\hline 1401 & VA & Chesapeake city & 0.970831 & 0.248996 & 0.058490 & 1075 & 6459 & 114630 & 0 \\
\hline 1402 & VA & Clifton Forge city & 0.998628 & 0.000000 & 0.000000 & 0 & 0 & 98354 & 23 \\
\hline 1403 & VA & Colonial Heights city & 0.958395 & 0.000000 & 0.000000 & 0 & 0 & 109211 & 23 \\
\hline 1404 & VA & Covington city & 1.000000 & 0.000000 & 0.000000 & 0 & 0 & 98354 & 23 \\
\hline 1405 & VA & Danville city & 0.980046 & 0.000000 & 0.000000 & 0 & 0 & 100894 & 23 \\
\hline 1406 & VA & Emporia city & 0.990230 & 0.000000 & 0.000000 & 0 & 0 & 105526 & 23 \\
\hline 1407 & VA & Faiffax city & 1.000000 & 0.000000 & 0.000000 & 0 & 0 & 196416 & 23 \\
\hline 1408 & VA & Falls Church city & 1.000000 & 0.000000 & 0.000000 & 0 & 0 & 196416 & 23 \\
\hline 1409 & VA & Franklin city & 0.993951 & 0.000000 & 0.000000 & 0 & 0 & 90984 & 23 \\
\hline 1410 & VA & Fredericksburg city & 0.999120 & 0.000000 & 0.000000 & 0 & 0 & 112706 & 23 \\
\hline 1411 & VA & Galax city & 1.000000 & 0.000000 & 0.000000 & 0 & 0 & 85776 & 23 \\
\hline 1412 & VA & Hampton city & 0.403078 & 0.000000 & 0.000000 & 0 & 0 & 99857 & 0 \\
\hline 1413 & VA & Harrisonburg city & 0.998332 & 0.000000 & 0.000000 & 0 & 0 & 106847 & 23 \\
\hline 1414 & VA & Hopewell city & 0.955812 & 0.000000 & 0.000000 & 0 & 0 & 90822 & 23 \\
\hline 1415 & VA & Lexington city & 1.000000 & 0.000000 & 0.000000 & 0 & 0 & 99464 & 23 \\
\hline 1416 & VA & Lynchburg city & 0.992653 & 0.000000 & 0.000000 & 0 & 0 & 113526 & 23 \\
\hline 1417 & VA & Manassas city & 0.997768 & 0.000000 & 0.000000 & 0 & 0 & 139142 & 23 \\
\hline 1418 & VA & Manassas Park city & 1.000000 & 0.000000 & 0.000000 & 0 & 0 & 139142 & 23 \\
\hline 1419 & VA & Martinsville city & 0.995265 & 0.000000 & 0.000000 & 0 & 0 & 112157 & 23 \\
\hline 1420 & VA & Newport News city & 0.574113 & 0.000000 & 0.000000 & 0 & 0 & 103813 & 0 \\
\hline 1421 & VA & Norfolk city & 0.558239 & 0.000000 & 0.000000 & 0 & 0 & 111162 & 0 \\
\hline 1422 & VA & Norton city & 1.000000 & 0.000000 & 0.000000 & 0 & 0 & 105018 & 23 \\
\hline 1423 & VA & Petersburg city & 0.985284 & 0.000000 & 0.000000 & 0 & 0 & 109211 & 23 \\
\hline 1424 & VA & Poquoson city & 0.197859 & 0.000000 & 0.000000 & 0 & 0 & 140117 & 23 \\
\hline 1425 & VA & Portsmouth city & 0.710980 & 0.000000 & 0.000000 & 0 & 0 & 100264 & 0 \\
\hline 1426 & VA & Radford city & 0.963547 & 0.000000 & 0.000000 & 0 & 0 & 89697 & 23 \\
\hline 1427 & VA & Richmond city & 0.960401 & 0.000000 & 0.000000 & 0 & 0 & 157253 & 0 \\
\hline 1428 & VA & Roanoke city & 0.999290 & 0.000000 & 0.000000 & 0 & 0 & 128474 & 0 \\
\hline 1429 & VA & Salem city & 1.000000 & 0.000000 & 0.000000 & 0 & 0 & 132971 & 23 \\
\hline 1430 & VA & South Boston city & 0.997566 & 0.000000 & 0.000000 & 0 & 0 & 97527 & 23 \\
\hline 1431 & VA & Staunton city & 1.000000 & 0.000000 & 0.000000 & 0 & 0 & 108480 & 23 \\
\hline 1432 & VA & Suffolk city & 0.932382 & 0.324338 & 0.003886 & 1103 & 4833 & 113837 & 1 \\
\hline 1433 & VA & Virginia Beach city & 0.499294 & 0.272653 & 0.008470 & 970 & 6611 & 119865 & 1 \\
\hline 1434 & VA & Waynesboro city & 0.998682 & 0.000000 & 0.000000 & 0 & 0 & 108480 & 23 \\
\hline 1435 & VA & Williamsburg city & 0.985241 & 0.000000 & 0.000000 & 0 & 0 & 116546 & 23 \\
\hline 1436 & VA & Winchester city & 1.000000 & 0.000000 & 0.000000 & 0 & 0 & 110999 & 23 \\
\hline 1437 & WV & Barbour County & 0.994269 & 0.348802 & 0.133532 & 120 & 1723 & 65937 & 0 \\
\hline 1438 & WV & Berkeley County & 0.998659 & 0.357233 & 0.176248 & 722 & 5063 & 90388 & 0 \\
\hline 1439 & WV & Boone County & 0.999609 & 0.007862 & 0.000000 & 77 & 3589 & 97670 & 0 \\
\hline 1440 & WV & Braxton County & 0.994794 & 0.223360 & 0.026685 & 65 & 1057 & 84340 & 1 \\
\hline 1441 & WV & Brooke County & 0.963024 & 0.214101 & 0.621622 & 165 & 1713 & 90923 & 0 \\
\hline 1442 & WV & Cabell County & 0.977686 & 0.197451 & 0.069942 & 157 & 2202 & 105888 & 0 \\
\hline 1443 & WV & Calhoun County & 0.999944 & 0.194419 & 0.088148 & 42 & 1197 & 60634 & 1 \\
\hline 1444 & WV & Clay County & 0.995867 & 0.067843 & 0.093496 & 82 & 2429 & 59293 & 0 \\
\hline 1445 & WV & Doddridge County & 0.999848 & 0.288572 & 0.000000 & 69 & 1513 & 67380 & 0 \\
\hline 1446 & WV & Fayette County & 0.993365 & 0.048136 & 0.041361 & 151 & 2339 & 76767 & 1 \\
\hline 1447 & WV & Gilmer County & 0.999932 & 0.242357 & 0.000000 & 87 & 1330 & 68687 & 0 \\
\hline 1448 & WV & Grant County & 0.993783 & 0.348112 & 0.011592 & 529 & 2218 & 90768 & 0 \\
\hline 1449 & WV & Greenbrier County & 0.996903 & 0.274975 & 0.078917 & 468 & 2246 & 88648 & 0 \\
\hline 1450 & WV & Hampshire County & 0.995508 & 0.330081 & 0.010084 & 187 & 2534 & 83615 & 1 \\
\hline 1451 & WV & Hancock County & 0.939819 & 0.145065 & 0.000000 & 282 & 3820 & 113234 & 0 \\
\hline 1452 & WV & Hardy County & 0.998144 & 0.379599 & 0.015646 & 1178 & 3428 & 91323 & 0 \\
\hline 1453 & WV & Harrison County & 0.998704 & 0.332605 & 0.184268 & 116 & 2333 & 97961 & 0 \\
\hline 1454 & WV & Jackson County & 0.987671 & 0.339513 & 0.076737 & 101 & 2134 & 78583 & 0 \\
\hline 1455 & WV & Jefferson County & 0.990416 & 0.553742 & 0.415489 & 690 & 7269 & 96809 & 0 \\
\hline 1456 & WV & Kanawha County & 0.991333 & 0.034524 & 0.055734 & 231 & 2700 & 116445 & 0 \\
\hline 1457 & WV & Lewis County & 0.997566 & 0.325875 & 0.055301 & 113 & 1598 & 78867 & 0 \\
\hline 1458 & WV & Lincoln County & 0.997366 & 0.107207 & 0.031980 & 133 & 2624 & 64406 & 1 \\
\hline 1459 & WV & Logan County & 0.996936 & 0.010708 & 0.631010 & 130 & 2035 & 89711 & 1 \\
\hline 1460 & WV & McDowell County & 0.999661 & 0.003179 & 0.000000 & 343 & 2777 & 68152 & 0 \\
\hline 1461 & WV & Marion County & 0.994130 & 0.206040 & 0.070017 & 89 & 2572 & 96464 & 1 \\
\hline 1462 & WV & Marshall County & 0.983429 & 0.327394 & 0.393981 & 139 & 1844 & 89298 & 0 \\
\hline 1463 & WV & Mason County & 0.970889 & 0.423895 & 0.363624 & 301 & 2210 & 82403 & \\
\hline
\end{tabular}




\begin{tabular}{|c|c|c|c|c|c|c|c|c|c|}
\hline $\begin{array}{l}\text { County } \\
\text { Code }\end{array}$ & State & $\begin{array}{l}\text { County } \\
\text { Name }\end{array}$ & $\begin{array}{l}\text { FRCLND } \\
\text { Absolute }\end{array}$ & $\begin{array}{l}\text { FRMFRC } \\
\text { Absolute }\end{array}$ & $\begin{array}{c}\text { DPF } \\
\text { Absolute }\end{array}$ & $\begin{array}{c}\text { ASFP } \\
\$ / h a\end{array}$ & $\begin{array}{l}\text { VFRM } \\
\text { \$/ha }\end{array}$ & $\begin{array}{l}\text { VNFRM } \\
\text { \$/person }\end{array}$ & Notes \\
\hline 1464 & WV & Mercer County & 0.999510 & 0.210148 & 0.018434 & 123 & 2605 & 102052 & 1 \\
\hline 1465 & WV & Mineral County & 0.995757 & 0.356391 & 0.209848 & 131 & 2110 & 86961 & 0 \\
\hline 1466 & $w v$ & Mingo County & 0.997562 & 0.000954 & 0.000000 & 699 & 4784 & 87198 & 0 \\
\hline 1467 & wV & Monongalia County & 0.987102 & 0.236290 & 0.143192 & 116 & 2939 & 102804 & 0 \\
\hline 1468 & WV & Monroe County & 0.999376 & 0.491281 & 0.218020 & 267 & 2190 & 80344 & 0 \\
\hline 1469 & WV & Morgan County & 0.997073 & 0.149217 & 0.097079 & 181 & 3644 & 89271 & 1 \\
\hline 1470 & WV & Nicholas County & 0.991181 & 0.079694 & 0.022468 & 172 & 3095 & 80303 & 1 \\
\hline 1471 & WV & Ohio County & 0.975393 & 0.311426 & 0.569444 & 235 & 2230 & 123144 & 0 \\
\hline 1472 & WV & Pendleton County & 0.999702 & 0.398818 & 0.004616 & 703 & 1980 & 72893 & 0 \\
\hline 1473 & WV & Pleasants County & 0.971309 & 0.187043 & 0.061379 & 133 & 2244 & 88600 & 1 \\
\hline 1474 & WV & Pocahontas County & 0.998347 & 0.191895 & 0.054925 & 117 & 2066 & 87029 & 0 \\
\hline 1475 & WV & Preston County & 0.995295 & 0.334215 & 0.326210 & 179 & 1955 & 69317 & 0 \\
\hline 1476 & WV & Putnam County & 0.988102 & 0.251924 & 0.000000 & 137 & 2477 & 93680 & 0 \\
\hline 1477 & WV & Raleigh County & 0.996049 & 0.084005 & 0.060731 & 129 & 2896 & 89860 & 1 \\
\hline 1478 & WN & Randolph County & 0.999808 & 0.156572 & 0.164878 & 133 & 2183 & 83324 & 0 \\
\hline 1479 & WV & Ritchie County & 0.999853 & 0.244464 & 0.013667 & 132 & 1433 & 75982 & 1 \\
\hline 1480 & wV & Roane County & 0.999634 & 0.265423 & 0.000000 & 73 & 1551 & 67353 & 0 \\
\hline 1481 & WV & Summers County & 0.982202 & 0.249687 & 0.095761 & 139 & 2420 & 61575 & 1 \\
\hline 1482 & Wv & Taylor County & 0.983455 & 0.378258 & 0.059971 & 255 & 2114 & 68965 & 1 \\
\hline 1483 & WV & Tucker County & 0.994688 & 0.119709 & 0.080408 & 99 & 2627 & 81522 & 1 \\
\hline 1484 & wN & Tyler County & 0.987964 & 0.287333 & 0.292419 & 87 & 1359 & 86352 & 0 \\
\hline 1485 & wN & Upshur County & 0.999714 & 0.258423 & 0.049030 & 119 & 3213 & 72764 & 0 \\
\hline 1486 & wV & Wayne County & 0.987455 & 0.088418 & 0.202966 & 93 & 1852 & 80195 & 0 \\
\hline 1487 & WV & Webster County & 0.999919 & 0.026227 & 0.212061 & 65 & 2474 & 65077 & 1 \\
\hline 1488 & WV & Wetzel County & 0.994065 & 0.161494 & 0.048251 & 55 & 1435 & 91398 & 0 \\
\hline 1489 & wV & Wirt County & 0.992168 & 0.240304 & 0.155836 & 229 & 1815 & 57037 & 0 \\
\hline 1490 & WV & Wood County & 0.974479 & 0.254548 & 0.128701 & 105 & 2975 & 105784 & 0 \\
\hline 149$\}$ & wV & Wyoming County & 0.998120 & 0.017759 & 0.389044 & 115 & 2919 & 68809 & 1 \\
\hline 1492 & NC & Alamance County & 0.990543 & 0.366683 & 0.267643 & 794 & 5175 & 117217 & 0 \\
\hline 1493 & NC & Alexander County & 0.988735 & 0.317955 & 0.193832 & 1643 & 4522 & 101564 & 0 \\
\hline 1494 & NC & Alleghany County & 0.996420 & 0.483510 & 0.594266 & 566 & 4160 & 87713 & 0 \\
\hline 1495 & NC & Anson County & 0.989653 & 0.207790 & 0.012200 & 2172 & 3981 & 93152 & 0 \\
\hline 1496 & NC & Ashe County & 0.998482 & 0.382872 & 0.086198 & 264 & 3822 & 84448 & 0 \\
\hline 1497 & NC & Avery County & 0.999177 & 0.124686 & 0.059798 & 782 & 6938 & 84780 & 1 \\
\hline$\uparrow 498$ & NC & Beaufort County & 0.863896 & 0.272865 & 0.003465 & 921 & 3293 & 93646 & 1 \\
\hline 1499 & NC & Bertie County & 0.943365 & 0.379892 & 0.000000 & 1102 & 3382 & 91255 & 0 \\
\hline 1500 & NC & Bladen County & 0.986319 & 0.228139 & 0.006521 & 1107 & 2948 & 82220 & 1 \\
\hline 1501 & NC & Brunswick County & 0.814141 & 0.072500 & 0.000000 & 724 & 4085 & 84848 & 0 \\
\hline 1502 & NC & Buncombe County & 0.994291 & 0.222808 & 0.366309 & 587 & 9317 & 114697 & 0 \\
\hline 1503 & NC & Burke County & 0.984139 & 0.097654 & 0.033928 & 1566 & 6168 & 102248 & 0 \\
\hline 1504 & NC & Cabarrus County & 0.998376 & 0.270402 & 0.097152 & 488 & 6107 & 112902 & 0 \\
\hline 1505 & NC & Caldwell County & 0.994418 & 0.103302 & 0.157119 & 1000 & 5576 & 101225 & 0 \\
\hline 1506 & NC & Camden County & 0.787308 & 0.279503 & 0.000000 & 954 & 3190 & 100399 & 0 \\
\hline 1507 & NC & Carteret County & 0.393150 & 0.188283 & 0.000000 & 489 & 5057 & 97653 & 0 \\
\hline 1508 & NC & Caswell County & 0.993608 & 0.460340 & 0.066234 & 413 & 2481 & 77133 & 0 \\
\hline 1509 & NC & Catawba County & 0.967257 & 0.245533 & 0.261952 & 573 & 6842 & 117827 & 0 \\
\hline 1510 & NC & Chatham County & 0.963198 & 0.247873 & 0.055529 & 2112 & 4772 & 117468 & 0 \\
\hline 1511 & NC & Cherokee County & 0.975385 & 0.082137 & 0.063161 & 1133 & 5548 & 83114 & 0 \\
\hline 1512 & NC & Chowan County & 0.739922 & 0.487847 & 0.000000 & 1115 & 3567 & 95881 & 0 \\
\hline 1513 & NC & Clay County & 0.973276 & 0.119386 & 0.197198 & 699 & 5895 & 81224 & 0 \\
\hline 1514 & NC & Cleveland County & 0.991527 & 0.316206 & 0.084838 & 650 & 4162 & 106712 & 0 \\
\hline 1515 & NC & Columbus County & 0.982388 & 0.271247 & 0.013655 & 831 & 3537 & 83818 & 1 \\
\hline 1516 & NC & Craven County & 0.913020 & 0.198552 & 0.004113 & 1268 & 3594 & 100196 & 1 \\
\hline 1517 & NC & Cumberland County & 0.991842 & 0.235719 & 0.000000 & 869 & 4219 & 87530 & 0 \\
\hline 1518 & NC & Currituck County & 0.497844 & 0.249266 & 0.000000 & 855 & 4289 & 95793 & 0 \\
\hline 1519 & NC & Dare County & 0.244412 & 0.028845 & 0.000000 & 194 & 1734 & 103264 & 0 \\
\hline 1520 & NC & Davidson County & 0.974279 & 0.260851 & 0.242615 & 548 & 5867 & 102133 & 0 \\
\hline 1521 & NC & Davie County & 0.993697 & 0.404857 & 0.347081 & 495 & 4057 & 124214 & 0 \\
\hline 1522 & NC & Duplin County & 0.998394 & 0.474803 & 0.000522 & 4307 & 3916 & 82247 & 0 \\
\hline 1523 & NC & Durham County & 0.974891 & 0.105783 & 0.056230 & 834 & 11251 & 130303 & 1 \\
\hline 1524 & NC & Edgecombe County & 0.996952 & 0.558097 & 0.002551 & 1002 & 2910 & 91039 & 1 \\
\hline 1525 & NC & Forsyth County & 0.992138 & 0.182454 & 0.050672 & 692 & 10621 & 139833 & 0 \\
\hline 1526 & NC & Franklin County & 0.994835 & 0.375982 & 0.007971 & 978 & 3962 & 86223 & 1 \\
\hline 1527 & NC & Gaston County & 0.979889 & 0.152147 & 0.595739 & 535 & 4321 & 110532 & 0 \\
\hline 1528 & NC & Gates County & 0.985546 & 0.296013 & 0.000000 & 1132 & 3471 & 99072 & 0 \\
\hline 1529 & NC & Graham County & 0.968397 & 0.047515 & 0.301211 & 345 & 5806 & 62469 & 1 \\
\hline 1530 & NC & Granville County & 0.989880 & 0.458973 & 0.076712 & 490 & 3334 & 91621 & 0 \\
\hline 1531 & NC & Greene County & 0.998269 & 0.660976 & 0.000000 & 2599 & 4980 & 86297 & 0 \\
\hline 1532 & NC & Guilford County & 0.988449 & 0.273152 & 0.129361 & 850 & 8300 & 137828 & 0 \\
\hline 1533 & NC & Halifax County & 0.992440 & 0.440340 & 0.009988 & 1038 & 2587 & 86941 & 0 \\
\hline
\end{tabular}




\begin{tabular}{|c|c|c|c|c|c|c|c|c|c|}
\hline $\begin{array}{l}\text { County } \\
\text { Code }\end{array}$ & State & $\begin{array}{l}\text { County } \\
\text { Name }\end{array}$ & $\begin{array}{l}\text { FRCLND } \\
\text { Absolute }\end{array}$ & $\begin{array}{l}\text { FRMFRC } \\
\text { Absolute }\end{array}$ & $\begin{array}{c}\text { DPF } \\
\text { Absolute }\end{array}$ & $\begin{array}{c}\text { ASFP } \\
\text { S/ha }\end{array}$ & $\begin{array}{c}\text { VFRM } \\
\text { \$/ha }\end{array}$ & $\begin{array}{l}\text { VNFRM } \\
\text { S/person }\end{array}$ & Notes \\
\hline 534 & NC & Harnett County & 0.989586 & 0.335226 & 0.027950 & 1198 & 4927 & 81326 & 0 \\
\hline 1535 & NC & Haywood County & 0.998269 & 0.197357 & 0.275243 & 508 & 6402 & 96416 & 0 \\
\hline 1536 & NC & Henderson County & 0.997317 & 0.218512 & 0.197487 & 2005 & 8485 & 123103 & 0 \\
\hline 1537 & NC & Hertford County & 0.980146 & 0.333538 & 0.000000 & 1264 & 3142 & 86528 & 0 \\
\hline 1538 & NC & Hoke County & 0.997155 & 0.226410 & 0.000000 & 1392 & 2709 & 68795 & 0 \\
\hline 1539 & NC & Hyde County & 0.430354 & 0.238975 & 0.000000 & 657 & 2618 & 84536 & 0 \\
\hline 1540 & NC & Iredell County & 0.967951 & 0.402959 & 0.362296 & 1061 & 5954 & 115910 & 0 \\
\hline 1541 & $\mathrm{NC}$ & Jackson County & 0.992346 & 0.042392 & 0.063093 & 665 & 6417 & 87171 & 0 \\
\hline 1542 & NC & Johnston County & 0.995073 & 0.454560 & 0.006567 & 1219 & 4817 & 100609 & 1 \\
\hline 1543 & NC & Jones County & 0.996962 & 0.226391 & 0.000000 & 1045 & 3505 & 81333 & 0 \\
\hline 1544 & NC & Lee County & 0.992004 & 0.227338 & 0.011945 & 1031 & 4318 & 112930 & 1 \\
\hline 1545 & NC & Lenoir County & 0.994439 & 0.553931 & 0.003768 & 1727 & 4519 & 101131 & 1 \\
\hline 1546 & NC & Lincoln County & 0.973171 & 0.305287 & 0.349539 & 597 & 4138 & 103610 & 0 \\
\hline 1547 & NC & McDowell County & 0.989397 & 0.075058 & 0.127522 & 1010 & 5549 & 84435 & 0 \\
\hline 1548 & $\mathrm{NC}$ & Macon County & 0.994248 & 0.066829 & 0.346570 & 434 & 6527 & 95455 & 0 \\
\hline 1549 & NC & Madison County & 0.995199 & 0.324427 & 0.054390 & 273 & 5144 & 82565 & 0 \\
\hline 1550 & NC & Martin County & 0.999331 & 0.445076 & 0.000000 & 1071 & 3473 & 96633 & 0 \\
\hline 1551 & NC & Mecklenburg County & 0.959416 & 0.082657 & 0.069948 & 3110 & 15308 & 141844 & 0 \\
\hline 1552 & NC & Mitchell County & 0.996948 & 0.162314 & 0.118213 & 339 & 4583 & 82552 & 1 \\
\hline 1553 & NC & Montgomery County & 0.979971 & 0.117650 & 0.029543 & 2527 & 3642 & 87984 & 0 \\
\hline 1554 & NC & Moore County & 0.989330 & 0.194503 & 0.000468 & 2248 & 4463 & 129050 & 0 \\
\hline 1555 & NC & Nash County & 0.995580 & 0.517777 & 0.001499 & 1718 & 4162 & 111805 & 1 \\
\hline 1556 & NC & New Hanover County & 0.606659 & 0.027379 & 0.000000 & 1797 & 16076 & 115950 & 0 \\
\hline 1557 & NC & Northampton County & 0.974341 & 0.452343 & 0.005220 & 1138 & 2452 & 93612 & 1 \\
\hline 1558 & NC & Onslow County & 0.844141 & 0.130384 & 0.015897 & 2266 & 4214 & 69019 & 1 \\
\hline 1559 & NC & Orange County & 0.996610 & 0.263776 & 0.220202 & 1234 & 7760 & 134495 & 0 \\
\hline 1560 & NC & Pamlico County & 0.595059 & 0.204037 & 0.000000 & 943 & 3142 & 91878 & 0 \\
\hline 1561 & NC & Pasquotank County & 0.783872 & 0.573079 & 0.000000 & 837 & 3706 & 89711 & 0 \\
\hline 1562 & NC & Pender County & 0.933557 & 0.116886 & 0.005017 & 1411 & 3729 & 90544 & 1 \\
\hline 1563 & NC & Perquimans County & 0.751440 & 0.434491 & 0.000000 & 1063 & 4265 & 85410 & 0 \\
\hline 1564 & NC & Person County & 0.970901 & 0.461385 & 0.035384 & 549 & 3110 & 94994 & 0 \\
\hline 1565 & NC & Pitt County & 0.995125 & 0.465221 & 0.000000 & 1708 & 4653 & 107369 & 0 \\
\hline 1566 & NC & Polk County & 0.996872 & 0.152021 & 0.310368 & 316 & 4695 & 136182 & 0 \\
\hline 1567 & NC & Randolph County & 0.996829 & 0.287428 & 0.148553 & 1756 & 5162 & 99708 & 0 \\
\hline 1568 & NC & Richmond County & 0.988325 & 0.171122 & 0.000000 & 1825 & 2791 & 87875 & 0 \\
\hline 1569 & NC & Robeson County & 0.997714 & 0.481069 & 0.001728 & 913 & 2859 & 73482 & 1 \\
\hline 1570 & NC & Rockingham County & 0.989727 & 0.361003 & 0.055793 & 588 & 3957 & 101855 & 0 \\
\hline 1571 & NC & Rowan County & 0.976157 & 0.320424 & 0.339602 & 616 & 4251 & 106278 & 0 \\
\hline 1572 & NC & Rutherford County & 0.996889 & 0.153168 & 0.126165 & 216 & 3423 & 96166 & 0 \\
\hline 1573 & NC & Sampson County & 0.997894 & 0.439685 & 0.007039 & 3298 & 3891 & 94852 & 0 \\
\hline 1574 & NC & Scotland County & 0.995347 & 0.262846 & 0.000000 & 1182 & 2532 & 89264 & 0 \\
\hline 1575 & NC & Stanly County & 0.977249 & 0.352218 & 0.073925 & 1245 & 3892 & 99762 & 0 \\
\hline 1576 & NC & Stokes County & 0.991119 & 0.362166 & 0.031211 & 651 & 4245 & 96565 & 0 \\
\hline 1577 & NC & Surry County & 0.997670 & 0.353537 & 0.098157 & 1196 & 4885 & 104714 & 0 \\
\hline 1578 & NC & Swain County & 0.976839 & 0.017447 & 0.092836 & 842 & 6647 & 67258 & 1 \\
\hline 1579 & NC & Transylvania County & 0.994193 & 0.051159 & 0.000000 & 1243 & 8714 & 104544 & 0 \\
\hline 1580 & NC & Tyrrell County & 0.649549 & 0.271271 & 0.000000 & 828 & 2626 & 76185 & 0 \\
\hline 1581 & NC & Union County & 0.996527 & 0.410294 & 0.005721 & 3556 & 5969 & 122520 & 0 \\
\hline 1582 & NC & Vance County & 0.939586 & 0.417306 & 0.030721 & 665 & 3565 & 88634 & 1 \\
\hline 1583 & NC & Wake County & 0.972494 & 0.224571 & 0.033989 & 1362 & 8276 & 139921 & 0 \\
\hline 1584 & NC & Warren County & 0.966043 & 0.318794 & 0.037489 & 843 & 2632 & 80181 & 0 \\
\hline 1585 & NC & Washington County & 0.819891 & 0.462421 & 0.000000 & 1285 & 3185 & 85336 & 0 \\
\hline 1586 & NC & Watauga County & 0.999346 & 0.233586 & 0.039698 & 400 & 5849 & 86250 & 0 \\
\hline 1587 & NC & Wayne County & 0.992597 & 0.507695 & 0.004486 & 2669 & 4564 & 90422 & 0 \\
\hline 1588 & NC & Wilkes County & 0.996399 & 0.237517 & 0.020867 & 3367 & 5486 & 99559 & 0 \\
\hline 1589 & NC & Wilson County & 0.991467 & 0.599208 & 0.000000 & 1557 & 4565 & 106062 & 0 \\
\hline 1590 & NC & Yadkin County & 0.994338 & 0.483130 & 0.186029 & 1027 & 5208 & 103366 & 0 \\
\hline 1591 & NC & Yancey County & 0.997840 & 0.192001 & 0.109561 & 351 & 5305 & 81739 & 0 \\
\hline 1592 & sc & Abbeville County & 0.994028 & 0.276594 & 0.218125 & 184 & 2752 & 79064 & 0 \\
\hline 1593 & sc & Aiken County & 0.993100 & 0.198851 & 0.023476 & 590 & 3194 & 121227 & 1 \\
\hline 1594 & sc & Allendale County & 0.989440 & 0.311009 & 0.000000 & 397 & 1799 & 73442 & 0 \\
\hline 1595 & Sc & Anderson County & 0.947944 & 0.349602 & 0.159713 & 550 & 4794 & 96213 & 0 \\
\hline 1596 & sc & Bamberg County & 0.994391 & 0.347065 & 0.281193 & 372 & 2079 & 71213 & 0 \\
\hline 1597 & Sc & Barnwell County & 0.984227 & 0.212891 & 0.021953 & 329 & 2671 & 95319 & 1 \\
\hline 1598 & Sc & Beaufort County & 0.635997 & 0.119245 & 0.000000 & 346 & 2474 & 123320 & 0 \\
\hline 1599 & Sc & Berkeley County & 0.894502 & 0.072501 & 0.008678 & 610 & 4217 & 87489 & 1 \\
\hline 1600 & Sc & Calhoun County & 0.969306 & 0.373841 & 0.013023 & 456 & 2233 & 83182 & 1 \\
\hline 1601 & Sc & Charleston County & 0.675997 & 0.055168 & 0.000000 & 1274 & 8681 & 108473 & 0 \\
\hline 1602 & SC & Cherokee County & 0.988441 & 0.263257 & 0.022415 & 364 & 2961 & 99451 & 1 \\
\hline 1603 & sc & Chester County & 0.990397 & 0.253506 & 0.205646 & 196 & 2347 & 80208 & 0 \\
\hline
\end{tabular}




\begin{tabular}{|c|c|c|c|c|c|c|c|c|c|}
\hline $\begin{array}{l}\text { County } \\
\text { Code }\end{array}$ & State & $\begin{array}{l}\text { County } \\
\text { Name }\end{array}$ & $\begin{array}{l}\text { FRCLND } \\
\text { Absolute }\end{array}$ & $\begin{array}{l}\text { FRMFRC } \\
\text { Absolute }\end{array}$ & $\begin{array}{c}\text { DPF } \\
\text { Absolute }\end{array}$ & $\begin{array}{l}\text { ASFP } \\
\text { \$/ha }\end{array}$ & $\begin{array}{l}\text { VFRM } \\
\$ / h a\end{array}$ & $\begin{array}{l}\text { VNFRM } \\
\text { \$/person }\end{array}$ & Notes \\
\hline 1604 & SC & Chesterfield County & 0.991237 & 0.214492 & 0.003803 & 1363 & 2653 & 88309 & 0 \\
\hline 1605 & SC & Clarendon County & 0.872851 & 0.349336 & 0.000000 & 670 & 2301 & 70543 & 0 \\
\hline 1606 & SC & Colleton County & 0.933243 & 0.186898 & 0.000000 & 241 & 2805 & 80215 & 0 \\
\hline 1607 & SC & Darlington County & 0.990326 & 0.436041 & 0.025012 & 600 & 2556 & 90984 & 0 \\
\hline 1608 & SC & Dillon County & 0.995892 & 0.420057 & 0.000000 & 790 & 2858 & 78258 & \\
\hline 1609 & SC & Dorchester County & 0.996607 & 0.168834 & 0.051003 & 541 & 3580 & 95096 & 0 \\
\hline 1610 & SC & Edgefield County & 0.990836 & 0.214361 & 0.091487 & 610 & 3227 & 80628 & 0 \\
\hline 1611 & SC & Fairfield County & 0.967011 & 0.126792 & 0.107373 & 90 & 2233 & 89406 & \\
\hline 1612 & SC & Florence County & 0.995219 & 0.380887 & 0.004282 & 648 & 2683 & 101334 & \\
\hline 1613 & SC & Georgetown County & 0.787232 & 0.071498 & 0.000000 & 631 & 3021 & 99288 & 0 \\
\hline 1614 & SC & Greenville County & 833 & 0.131789 & 0.219012 & 489 & 7555 & 121064 & 0 \\
\hline 1615 & SC & Greenwood County & 0.983976 & 0.241054 & 0.011585 & 332 & 2771 & 102641 & \\
\hline 1616 & SC & Hampton County & 0.995052 & 0.271352 & 0.000000 & 317 & 2726 & 86345 & \\
\hline 1617 & SC & Horry County & 0.903357 & 0.269712 & 0.001853 & 804 & 3853 & 99519 & \\
\hline 1618 & SC & Jasper County & 0.954051 & 0.173126 & 0.000000 & 131 & 2310 & 77634 & \\
\hline 1619 & SC & Kershaw County & 0.981081 & 0.113972 & 0.000000 & 928 & 2687 & 101368 & \\
\hline 1620 & SC & Lancaster County & 0.988652 & 0.165986 & 0.020663 & 448 & 3652 & 92644 & 1 \\
\hline 1621 & SC & Laurens County & 0.987723 & 0.280715 & 0.255296 & 317 & 3612 & 94039 & 0 \\
\hline 1622 & SC & Lee County & 565 & 450 & 093 & 490 & 2011 & 67454 & 1 \\
\hline 1623 & SC & Lexington County & 0.922749 & 0.184236 & 0.008119 & 1937 & 5363 & 122771 & 0 \\
\hline 1624 & SC & McCormick County & 0.912939 & 0.084670 & 0.000000 & 356 & 2698 & 74349 & \\
\hline 1625 & SC & Marion County & 0.989873 & 0.250401 & 0.004473 & 770 & 3675 & 79687 & \\
\hline 1626 & SC & Marlboro County & 0.988510 & 0.341546 & 0.000000 & 493 & 1968 & 76090 & 0 \\
\hline 1627 & SC & Newberry County & 0.974489 & 0.232762 & 0.291120 & 926 & 3227 & 93247 & \\
\hline 1628 & SC & Oconee County & 0.927959 & 0.174713 & 0.037480 & 1057 & 4603 & 106217 & 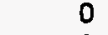 \\
\hline 1629 & SC & Orangeburg County & 0639 & 0274 & 0212 & 598 & 2380 & 88241 & 0 \\
\hline 1630 & SC & Pickens County & 0.970788 & 0.138982 & 0.048397 & 253 & 5425 & 100941 & 1 \\
\hline 1631 & SC & Richland County & 0.980298 & 0.137045 & 0.000000 & 298 & 4312 & 116072 & 0 \\
\hline 1632 & SC & Saluda County & 0.979884 & 0.383131 & 0.109200 & 880 & 3005 & 86785 & 0 \\
\hline 1633 & SC & Spartanburg County & 0.989965 & 0.206263 & 0.154725 & 538 & 5427 & 106854 & 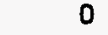 \\
\hline 1634 & SC & Sumter County & 0.975633 & 0.325368 & 0.010574 & 922 & 2457 & 79944 & \\
\hline 1635 & SC & Union County & 0.996616 & 0.170138 & 0.164369 & 88 & 3206 & 80608 & \\
\hline 1636 & SC & Williamsburg County & 0.996832 & 9728 & 5379 & 580 & 2725 & 69459 & 1 \\
\hline 1637 & SC & York County & 0.980999 & 0.276433 & 0317 & 449 & 4200 & 113200 & 0 \\
\hline 1638 & GA & Appling County & 0.993412 & 0.324118 & 0.059700 & 728 & 2138 & 87828 & \\
\hline 1639 & GA & Atkinson County & 0.982508 & 0.358891 & 0.000000 & 1217 & 2378 & 91486 & 0 \\
\hline 1640 & GA & Bacon County & 0.996611 & 0.431721 & 0.019993 & 583 & 2076 & 85525 & \\
\hline 1641 & GA & Baker County & 0.982964 & 0.495454 & 0.007779 & 542 & 2264 & 87313 & \\
\hline 1642 & GA & Baldwin County & 0.966265 & 0.199329 & 0.147374 & 189 & 2319 & 99112 & \\
\hline 1643 & GA & Banks County & 0.999145 & 0.330287 & 0.003171 & 3045 & 7477 & 94188 & \\
\hline 1644 & GA & Barrow County & 0.996135 & 0.345316 & 0.001540 & 2775 & 9277 & 97006 & \\
\hline 1645 & GA & Bartow County & 0.977287 & 0.289057 & 0.045767 & 769 & 4623 & 94872 & 0 \\
\hline 1646 & GA & Ben Hill County & 0.991317 & 0.292049 & 0.013037 & 748 & 3173 & 91282 & 1 \\
\hline 1647 & GA & Berrien County & 0.988373 & 0.446204 & 0.010791 & 658 & 2378 & 86670 & 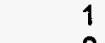 \\
\hline 1648 & GA & Bibb County & 0.979738 & 0.106906 & 0.180791 & 511 & 4183 & 118856 & \\
\hline 1649 & GA & Bleckley County & 0.992105 & 0.452643 & 0.028522 & 511 & 2144 & 96735 & \\
\hline 1650 & GA & Brantley County & 0.993262 & 0.096898 & 0.014354 & 1160 & 3517 & 71688 & \\
\hline 1651 & GA & Brooks County & 798 & 0.53 & 0.068744 & 637 & 2582 & 71545 & \\
\hline 1652 & GA & Bryan County & 373 & 409 & 000 & 303 & 2509 & 87740 & \\
\hline 1653 & GA & Bulloch County & 0.990825 & 0.489728 & 0.000187 & 618 & 2652 & 82538 & \\
\hline 254 & GA & Burke County & 0.994619 & 249 & 70 & 438 & 1717 & 76049 & \\
\hline 1655 & GA & Butts County & 0.982093 & 0.244594 & 0.049743 & 316 & 3902 & 97473 & \\
\hline 1656 & GA & Calhoun County & 0.987993 & 0.634955 & 0.000000 & 686 & 2307 & 97595 & \\
\hline 1657 & GA & Camden County & 0.805027 & 0.044508 & 0.520145 & 49 & 1723 & 75765 & \\
\hline 1658 & GA & Candler County & 0.992546 & 0.361049 & 0.011795 & 682 & 2952 & 86670 & \\
\hline 1659 & GA & Carroll County & 0.990887 & 0.258342 & 0.020731 & 1377 & 4554 & 97961 & \\
\hline 1660 & GA & Catoosa County & 0.997297 & 0.283648 & 0.149633 & 1497 & 5081 & 87103 & \\
\hline 1661 & GA & Chartton County & 0.997192 & 0.043418 & 0.000000 & 427 & 2838 & 80831 & \\
\hline 1662 & GA & Chatham County & 0.681377 & 0.030222 & 0.000000 & 619 & 3913 & 120400 & \\
\hline 1663 & GA & ochee County & 0.990467 & 0.037060 & 0.000000 & 139 & 1423 & 78061 & \\
\hline 1664 & GA & Chattooga County & 0.999303 & 0.262204 & 0.152036 & 207 & 2097 & 80378 & \\
\hline 1665 & GA & Cherokee County & 0.976318 & 0.124045 & 0.012188 & 3453 & 11850 & 122155 & \\
\hline 1666 & GA & Clarke County & 0.996202 & 0.149473 & 0.010438 & 3803 & 6212 & 102506 & \\
\hline 1667 & GA & Clay County & 0.899600 & 0.341583 & 0.034419 & 625 & 2144 & 68125 & \\
\hline 1668 & GA & Clayton County & 0.988609 & 0.049499 & 0.000000 & 344 & 8454 & 109529 & \\
\hline 1669 & GA & Clinch County & 0.981941 & 0.026184 & 0.000000 & 555 & 3050 & 73855 & \\
\hline 1670 & GA & Cobb County & 0.987472 & 0.046808 & 0.000000 & 3818 & 13542 & 148557 & \\
\hline 1671 & GA & Coffee County & 0.994116 & 0.466464 & 0.013657 & 1291 & 2775 & 90571 & \\
\hline 1672 & GA & Colquitt County & 0.992312 & 0.560665 & 0.030325 & 1031 & 2812 & 94547 & \\
\hline 1673 & GA & Columbia County & 0.942287 & 0.145370 & 0.085849 & 198 & 6052 & 113756 & \\
\hline
\end{tabular}




\begin{tabular}{|c|c|c|c|c|c|c|c|c|c|}
\hline $\begin{array}{l}\text { County } \\
\text { Code }\end{array}$ & State & $\begin{array}{l}\text { County } \\
\text { Name }\end{array}$ & $\begin{array}{l}\text { FRCLND } \\
\text { Absolute }\end{array}$ & $\begin{array}{l}\text { FRMFRC } \\
\text { Absolute }\end{array}$ & $\begin{array}{c}\text { DPF } \\
\text { Absolute }\end{array}$ & $\begin{array}{l}\text { ASFP } \\
\text { \$/ha }\end{array}$ & $\begin{array}{l}\text { VFRM } \\
\text { \$/ha }\end{array}$ & $\begin{array}{l}\text { VNFRM } \\
\text { S/person }\end{array}$ & Notes \\
\hline 1674 & GA & Cook County & 0.982302 & 0.495370 & 0.000000 & 735 & 2596 & 84455 & 0 \\
\hline 1675 & GA & Coweta County & 0.993384 & 0.148019 & 0.183670 & 309 & 5186 & 108473 & 0 \\
\hline 1676 & GA & Crawford County & 0.995886 & 0.182485 & 0.032166 & 751 & 2042 & 84543 & 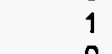 \\
\hline 1677 & GA & Crisp County & 0.973761 & 0.627218 & 0.000000 & 677 & 2405 & 84787 & 0 \\
\hline 1678 & GA & Dade County & 0.998958 & 0.231802 & 0.032193 & 552 & 4465 & 72663 & \\
\hline 1679 & GA & Dawson County & 0.986444 & 0.141111 & 0.006690 & 3598 & 8654 & 90172 & \\
\hline 1680 & $G A$ & Decatur County & 0.957694 & 0.441372 & 0.016125 & 675 & 3119 & 91743 & 1 \\
\hline 1681 & GA & DeKalb County & 0.990213 & 0.017740 & 0.000000 & 522 & 11524 & 142271 & 0 \\
\hline 1682 & GA & Dodge County & 0.994930 & 0.303404 & 0.019448 & 485 & 1789 & 85098 & 1 \\
\hline 1683 & $G A$ & Dooly County & 0.989461 & 0.623482 & 0.007813 & 749 & 2223 & 90192 & 1 \\
\hline 1684 & $G A$ & Dougherty County & 0.985130 & 0.337126 & 0.000000 & 454 & 3351 & 101774 & 0 \\
\hline 1685 & GA & Douglas County & 0.995219 & 0.063899 & 0.167290 & 337 & 6598 & 116614 & 1 \\
\hline 1686 & GA & Early County & 0.990249 & 0.562742 & 0.000000 & 542 & 2399 & 93200 & 0 \\
\hline 1687 & GA & Echols County & 0.960382 & 0.063257 & 0.000000 & 845 & 2673 & 75508 & 0 \\
\hline 1688 & GA & Effingham County & 0.992960 & 0.142649 & 0.071201 & 294 & 2946 & 84584 & 1 \\
\hline 1689 & GA & Elbert County & 0.984563 & 0.229775 & 0.327900 & 475 & 2804 & 96992 & 0 \\
\hline 1690 & $G A$ & Emanuel County & 0.993577 & 0.281771 & 0.062421 & 357 & 1623 & 82653 & 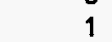 \\
\hline 1691 & GA & Evans County & 0.989966 & 0.342930 & 0.029294 & 771 & 2538 & 101422 & 1 \\
\hline 1692 & GA & Fannin County & 0.985466 & 0.063092 & 0.042462 & 1181 & 5866 & 83398 & 0 \\
\hline 1693 & GA & Fayette County & 0.990603 & 0.175822 & 0.194137 & 213 & 7790 & 157890 & 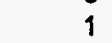 \\
\hline 1694 & GA & Floyd County & 0.989943 & 0.224228 & 0.042031 & 593 & 3436 & 108791 & \\
\hline 1695 & GA & Forsyth County & 0.912786 & 0.250904 & 0.025004 & 3543 & 12573 & 138546 & 1 \\
\hline 1696 & GA & Franklin County & 0.988510 & 0.442912 & 0.007463 & 2719 & 5408 & 99972 & 0 \\
\hline 1697 & GA & Fulton County & 0.988966 & 0.064945 & 0.183309 & 456 & 9559 & 154558 & 1 \\
\hline 1698 & GA & Gilmer County & 0.988109 & 0.092919 & 0.026245 & 4211 & 6714 & 103196 & 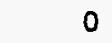 \\
\hline 1699 & $\mathrm{GA}$ & Glascock County & 0.998212 & 0.309188 & 0.000000 & 137 & 2011 & 98726 & 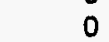 \\
\hline 1700 & GA & Glynn County & 0.724453 & 0.035811 & 0.000000 & 66 & 3542 & 118572 & 0 \\
\hline 1701 & GA & Gordon County & 0.993071 & 0.324989 & 0.017226 & 2208 & 4580 & 100819 & 0 \\
\hline 1702 & GA & Grady County & 0.995189 & 0.469399 & 0.043241 & 989 & 3779 & 79849 & 0 \\
\hline 1703 & GA & Greene County & 0.955919 & 0.188079 & 0.351799 & 855 & 3000 & 92190 & 0 \\
\hline 1704 & GA & Gwinnett County & 0.991121 & 0.087495 & 0.014730 & 1285 & 12853 & 134523 & \\
\hline 1705 & GA & Habersham County & 0.996395 & 0.202605 & 0.006068 & 6289 & 11250 & 102445 & \\
\hline 1706 & GA & Hall County & 0.917191 & 0.214110 & 0.031729 & 5684 & 8886 & 114758 & \\
\hline 1707 & GA & Hancock County & 0.988569 & 0.116816 & 0.281564 & 92 & 1534 & 79362 & \\
\hline 1708 & GA & Haralson County & 0.996383 & 0.174592 & 0.052402 & 1080 & 3938 & 90327 & 0 \\
\hline 1709 & $G A$ & Harris County & 0.980508 & 0.104572 & 0.000000 & 166 & 3507 & 104416 & 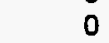 \\
\hline 1710 & GA & Hart County & 0.905601 & 0.393803 & 0.128360 & 1192 & 3902 & 103718 & 0 \\
\hline 1711 & GA & Heard County & 0.983219 & 0.127942 & 0.000000 & 958 & 3742 & 75399 & 0 \\
\hline 1712 & $\mathrm{GA}$ & Henry County & 0.994437 & 0.220916 & 0.134494 & 411 & 7380 & 121965 & 0 \\
\hline 1713 & GA & Houston County & 0.991903 & 0.304450 & 0.108419 & 629 & 3926 & 106454 & \\
\hline 1714 & GA & Irwin County & 0.983684 & 0.592216 & 0.005230 & 649 & 2653 & 79660 & 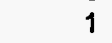 \\
\hline 1715 & GA & Jackson County & 0.998175 & 0.379106 & 0.008359 & 2630 & 6424 & 103820 & 0 \\
\hline 1716 & $G A$ & Jasper County & 0.991602 & 0.256480 & 0.103772 & 1104 & 2421 & 98645 & 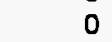 \\
\hline 1717 & $G A$ & Jeff Davis County & 0.993921 & 0.340350 & 0.013883 & 455 & 1830 & 95617 & \\
\hline 1718 & $G A$ & Jefferson County & 0.996388 & 0.402941 & 0.165544 & 388 & 1903 & 86297 & 0 \\
\hline 1719 & $G A$ & Jenkins County & 0.992517 & 0.346279 & 0.2 & 573 & 1851 & 78610 & 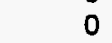 \\
\hline 1720 & GA & Johnson County & 0.993094 & 0.366359 & 0.029201 & 220 & 1332 & 80533 & \\
\hline 1721 & GA & Jones County & & 0.12 & 0.512936 & 681 & 3162 & 91513 & 0 \\
\hline 1722 & $G A$ & Lamar County & 0.994786 & 0.335697 & 0.224577 & 669 & 3821 & 95624 & 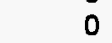 \\
\hline 1723 & GA & Lanier County & 0.934847 & 0.342535 & 0.000000 & 981 & 2366 & 87909 & 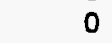 \\
\hline 1724 & GA & Laurens County & 0.992756 & 0.323123 & 0.066943 & 359 & 2006 & 95638 & 0 \\
\hline 1725 & $G A$ & Lee County & 781 & 89 & & 581 & 2360 & 86392 & \\
\hline 1726 & GA & Liberty County & 0.861492 & 0.046904 & 0.000000 & 45 & 1486 & 69737 & 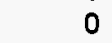 \\
\hline 1727 & GA & Linco & & 0.26 & & 281 & 2478 & 84441 & \\
\hline 1728 & GA & Long County & 0.993826 & 0.046633 & 0.000000 & 745 & 2662 & 54727 & 0 \\
\hline 1729 & GA & Lowndes County & 0.987470 & 0.226267 & 0.037969 & 512 & 3456 & 95976 & 0 \\
\hline 1730 & GA & Lumpkin County & 0.99 & 0.127883 & 0.020684 & 3811 & 9016 & 97202 & \\
\hline 1731 & GA & McDuffie County & & 0.203194 & & 854 & 3792 & 92407 & 0 \\
\hline 1732 & GA & Molntosh County & 0.754165 & 0.028848 & 0.000000 & 40 & 1515 & 84441 & 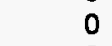 \\
\hline 1733 & $G A$ & Macon Count & 0.993433 & 0.468138 & 0.388938 & 1124 & 2287 & 84787 & 0 \\
\hline 1734 & GA & Madison County & 0.99 & 0.339240 & 0.010538 & 2286 & 4885 & 88438 & 0 \\
\hline 1735 & GA & County & 709 & 0.193 & 0.047435 & 639 & 1872 & 76937 & \\
\hline 1736 & GA & Meriwether County & 0.995974 & 0.213347 & 0.237847 & 166 & 3206 & 79334 & 0 \\
\hline 1737 & GA & Miller County & & 0.671164 & 0.0 & 671 & 2429 & 87591 & \\
\hline 1738 & $G A$ & Mitchell Cou & & 0.627325 & & 1010 & 2708 & 78962 & 0 \\
\hline 1739 & GA & Monroe County & 0.994553 & 0.176124 & 0.225886 & 790 & 3311 & 97629 & 0 \\
\hline 1740 & GA & ery County & 0.99 & 0.413 & 0.003597 & 339 & 1643 & 86805 & \\
\hline 1741 & GA & Morgan County & 0.986017 & 0.415854 & 0.313216 & 1296 & 2979 & 109997 & \\
\hline 1742 & GA & Murray County & 0.992909 & 0.149471 & 0.091927 & 757 & 3957 & 83981 & \\
\hline 174 & $A$ & Muscogee County & 0.978690 & 0.035178 & 0.000000 & 263 & 7323 & 105018 & \\
\hline
\end{tabular}




\begin{tabular}{|c|c|c|c|c|c|c|c|c|c|}
\hline $\begin{array}{l}\text { County } \\
\text { Code }\end{array}$ & State & $\begin{array}{l}\text { County } \\
\text { Name }\end{array}$ & $\begin{array}{l}\text { FRCLND } \\
\text { Absolute }\end{array}$ & $\begin{array}{l}\text { FRMFRC } \\
\text { Absolute }\end{array}$ & $\begin{array}{c}\text { DPF } \\
\text { Absolute }\end{array}$ & $\begin{array}{l}\text { ASFP } \\
\text { S/ha }\end{array}$ & $\begin{array}{l}\text { VFRM } \\
\text { \$/ha }\end{array}$ & $\begin{array}{l}\text { VNFRM } \\
\text { \$/person }\end{array}$ & Notes \\
\hline 1744 & GA & Newton County & 0.990124 & 0.259119 & 0.196769 & 577 & 4944 & 107050 & 0 \\
\hline 1745 & GA & Oconee County & 0.997932 & 0.435993 & 0.020467 & 2373 & 5498 & 110322 & 0 \\
\hline 1746 & GA & Oglethorpe County & 0.997605 & 0.195903 & 0.087739 & 1669 & 3407 & 93159 & 0 \\
\hline 1747 & GA & Paulding County & 0.995155 & 0.092905 & 0.060237 & 1226 & 7438 & 92204 & 1 \\
\hline 1748 & GA & Peach County & 0.997448 & 0.459895 & 0.146019 & 1160 & 3771 & 112435 & 0 \\
\hline 1749 & GA & Pickens County & 0.997210 & 0.122861 & 0.005909 & 4254 & 7963 & 106813 & 1 \\
\hline 1750 & $\mathrm{GA}$ & Pierce County & 0.998126 & 0.368530 & 0.169642 & 611 & 2925 & 77059 & 0 \\
\hline 1751 & GA & Pike County & 0.995366 & 0.325109 & 0.151025 & 289 & 3574 & 88079 & 0 \\
\hline 1752 & GA & Polk County & 0.996815 & 0.231055 & 0.065730 & 699 & 4550 & 86473 & 0 \\
\hline 1753 & GA & Pulaski County & 0.990131 & 0.507756 & 0.008606 & 663 & 2433 & 99979 & 1 \\
\hline 1754 & GA & Putnam County & 0.955200 & 0.157592 & 0.852644 & 1373 & 2521 & 81475 & 0 \\
\hline 1755 & GA & Quitman County & 0.941725 & 0.119171 & 0.000000 & 370 & 1771 & 86697 & 0 \\
\hline 1756 & GA & Rabun County & 0.984244 & 0.053616 & 0.006267 & 1889 & 7276 & 85728 & 0 \\
\hline 1757 & GA & Randolph County & 0.996187 & 0.348970 & 0.025312 & 567 & 2284 & 80391 & 1 \\
\hline 1758 & GA & Richmond County & 0.986508 & 0.077019 & 0.120267 & 478 & 3775 & 107972 & 1 \\
\hline 1759 & GA & Rockdale County & 0.989147 & 0.153458 & 0.144395 & 248 & 6231 & 130682 & 1 \\
\hline 1760 & GA & Schley County & 0.998739 & 0.353508 & 0.070833 & 414 & 1928 & 82470 & 0 \\
\hline 1761 & GA & Screven County & 0.989149 & 0.334410 & 0.034270 & 386 & 2247 & 81278 & 1 \\
\hline 1762 & GA & Seminole County & 0.927800 & 0.715203 & 0.012272 & 686 & 2683 & 87124 & 1 \\
\hline $17 \approx 3$ & GA & Spalding County & 0.991780 & 0.190098 & 0.200261 & 381 & 8770 & 99790 & 1 \\
\hline 1764 & $\mathrm{GA}$ & Stephens County & 0.973029 & 0.135282 & 0.027250 & 2170 & 4252 & 91350 & 1 \\
\hline 1765 & GA & Stewart County & 0.990240 & 0.167043 & 0.019540 & 479 & 1952 & 73029 & 1 \\
\hline 1766 & GA & Sumter County & 0.985254 & 0.547287 & 0.044997 & 652 & 2298 & 90991 & 0 \\
\hline 1767 & GA & Talbot County & 0.996134 & 0.152231 & 0.101360 & 118 & 1872 & 82098 & 1 \\
\hline 1768 & GA & Taliaferro County & 0.999615 & 0.154440 & 0.787627 & 416 & 2111 & 107213 & 0 \\
\hline 1769 & GA & Tattnall County & 0.990736 & 0.387188 & 0.005077 & 1508 & 2951 & 91973 & 1 \\
\hline 1770 & GA & Tayior County & 0.994291 & 0.224997 & 0.000000 & 616 & 2025 & 88356 & 0 \\
\hline 1771 & GA & Telfair County & 0.993355 & 0.251805 & 0.000000 & 431 & 1948 & 81014 & 0 \\
\hline 1772 & GA & Terrell County & 0.993563 & 0.665213 & 0.012717 & 505 & 2002 & 73902 & 1 \\
\hline 1773 & GA & Thomas County & 0.993320 & 0.495783 & 0.046404 & 416 & 2872 & 100149 & 0 \\
\hline 1774 & GA & Tift County & 0.986074 & 0.674691 & 0.007116 & 1126 & 3673 & 102919 & 1 \\
\hline 1775 & GA & Toombs County & 0.994733 & 0.378388 & 0.005789 & 485 & 2512 & 99627 & 0 \\
\hline 1776 & GA & Towns County & 0.968888 & 0.092988 & 0.003373 & 444 & 7844 & 83771 & 0 \\
\hline 1777 & GA & Treutlen County & 0.992626 & 0.255323 & 0.000000 & 338 & 2333 & 71498 & 0 \\
\hline 1778 & GA & Troup County & 0.928154 & 0.153940 & 0.326729 & 180 & 2336 & 102025 & 0 \\
\hline 1779 & GA & Turner County & 0.986932 & 0.539747 & 0.009727 & 859 & 3082 & 78894 & 0 \\
\hline 1780 & GA & Twiggs County & 0.992974 & 0.135108 & 0.058726 & 251 & 1776 & 76727 & 1 \\
\hline 1781 & GA & Union County & 0.980330 & 0.106386 & 0.016411 & 3399 & 8380 & 78535 & 10 \\
\hline 1782 & GA & Upson County & 0.993464 & 0.157748 & 0.000000 & 626 & 3449 & 89501 & 0 \\
\hline 1783 & GA & Walker County & 0.998992 & 0.310999 & 0.118569 & 444 & 3908 & 89061 & 0 \\
\hline 1784 & GA & Walton County & 0.997534 & 0.264704 & 0.017179 & 771 & 5943 & 92515 & 0 \\
\hline 1785 & GA & Ware County & 0.995568 & 0.093298 & 0.039019 & 655 & 2447 & 93362 & 1 \\
\hline 1786 & GA & Warren County & 0.995756 & 0.257186 & 0.510281 & 266 & 1863 & 76334 & 0 \\
\hline 1787 & GA & Washington County & 0.994218 & 0.256719 & 0.142085 & 282 & 1709 & 99607 & 0 \\
\hline 1788 & GA & Wayne County & 0.993666 & 0.131944 & 0.076115 & 441 & 2473 & 92535 & 0 \\
\hline 1789 & GA & Webster County & 0.996570 & 0.397361 & 0.000000 & 441 & 1856 & 94391 & 0 \\
\hline 1790 & GA & Wheeler County & 0.991926 & 0.255867 & 0.026313 & 358 & 1679 & 74431 & 1 \\
\hline 1791 & GA & White County & 0.997699 & 0.156025 & 0.018265 & 4682 & 9490 & 102126 & 0 \\
\hline 1792 & GA & Whitfield County & 0.997719 & 0.208453 & 0.024143 & 2243 & 5549 & 120969 & 0 \\
\hline 1793 & GA & Wilcox County & 0.992332 & 0.474505 & 0.022775 & 548 & 2030 & 90056 & 0 \\
\hline 1794 & GA & Wilkes County & 0.994470 & 0.308511 & 0.219326 & 406 & 2526 & 101544 & 0 \\
\hline 1795 & GA & Wilkinson County & 0.987968 & 0.111392 & 0.000000 & 95 & 1235 & 97399 & 0 \\
\hline 1796 & GA & Worth County & 0.991663 & 0.548586 & 0.029790 & 769 & 2468 & 76883 & 0 \\
\hline 1797 & FL & Alachua County & 0.902099 & 0.341595 & 0.155847 & 513 & 4182 & 108764 & 0 \\
\hline 1798 & $\mathrm{FL}$ & Baker County & 0.993762 & 0.065381 & 0.261540 & 2807 & 4877 & 88431 & 0 \\
\hline 1799 & FL & Bay County & 0.739094 & 0.018689 & 0.865022 & 225 & 4042 & 100338 & 1 \\
\hline 1800 & FL & Bradford County & 0.976974 & - 0.193106 & 0.127604 & 1153 & 4809 & 80168 & 1 \\
\hline 1801 & FL & Brevard County & 0.654033 & 0.306402 & 0.040917 & 435 & 3669 & 119669 & 1 \\
\hline 1802 & FL & Broward County & 0.916005 & 0.030678 & 0.157936 & 3617 & 14064 & 151415 & 0 \\
\hline 1803 & $\mathrm{FL}$ & Calhoun County & 0.987824 & 0.119280 & 0.049212 & 865 & 3077 & 64176 & 0 \\
\hline 1804 & FL & Charlotte County & 0.807267 & 0.511774 & 0.018965 & 412 & 3146 & 110823 & 1 \\
\hline 1805 & $\mathrm{FL}$ & Citrus County & 0.754754 & 0.189213 & 0.000000 & 194 & 4688 & 93064 & 0 \\
\hline 1806 & FL & Clay County & 0.933845 & 0.223600 & 0.479524 & 976 & 5547 & 108568 & 0 \\
\hline 1807 & FL & Collier County & 0.878688 & 0.232954 & 0.000000 & 2134 & 5128 & 173319 & 0 \\
\hline 1808 & FL & Columbia County & 0.995060 & 0.190065 & 0.174170 & 526 & 3537 & 84922 & 1 \\
\hline 1809 & FL & Dade County & 0.800324 & 0.067243 & 0.002014 & 10541 & 23985 & 120719 & 1 \\
\hline 1810 & L & Desoto County & 0.996524 & 0.820376 & 0.005587 & 950 & 5289 & 82531 & 1 \\
\hline 181 & $L$ & Dixie County & 0.815152 & 0.070335 & 0.536843 & 209 & 2007 & 69622 & 1 \\
\hline 1812 & 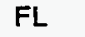 & Duval County & 0.842713 & 0.080844 & 0.374772 & 1385 & 7611 & 119628 & 0 \\
\hline 1813 & $F L$ & Escambia County & 0.742382 & 0.134624 & 0.250303 & 676 & 3807 & 104253 & 0 \\
\hline
\end{tabular}




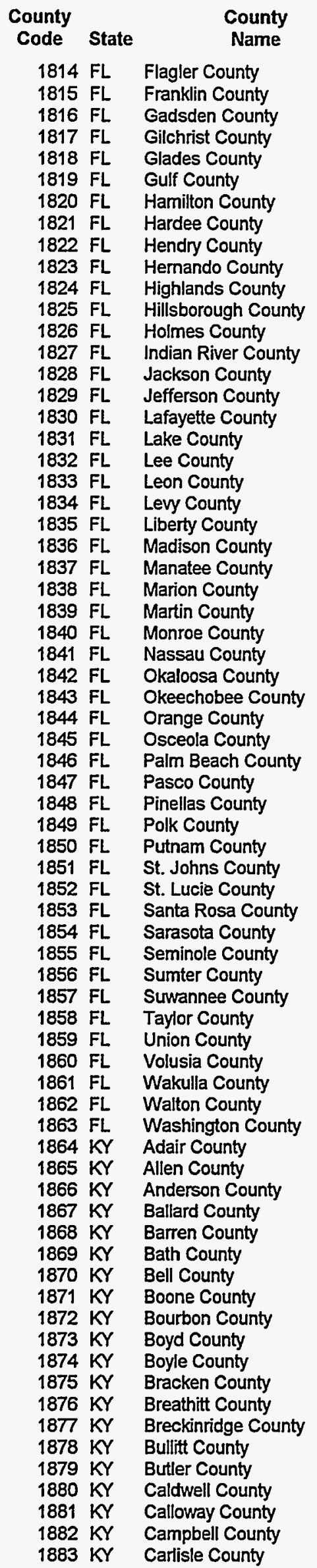

FRCLND FRMFRC DPF ASFP VFRM VNFRM

Absolute Absolute Absolute S/ha \$/ha \$/person Notes

$0.849741 \quad 0.168353 \quad 0.000000$

$\begin{array}{lll}0.520224 & 0.010386 & 0.000000\end{array}$

$\begin{array}{lll}0.976623 & 0.175131 & 0.000000\end{array}$

$\begin{array}{lll}0.981353 & 0.317940 & 0.623361 \\ 0.784338 & 0.747340 & 0.025353\end{array}$

$\begin{array}{llll}0.747652 & 0.039274 & 0.000000\end{array}$

$\begin{array}{lll}0.991390 & 0.210622 & 0.099527\end{array}$

$\begin{array}{lll}0.998395 & 0.803148 & 0.141004\end{array}$

$\begin{array}{llll}0.968744 & 0.718198 & 0.002986\end{array}$

$\begin{array}{lll}0.811877 & 0.199337 & 0.319143\end{array}$

$\begin{array}{lll}0.929609 & 0.735057 & 0.085350\end{array}$

$\begin{array}{lll}0.829956 & 0.394620 & 0.067556\end{array}$

$\begin{array}{lll}0.987300 & 0.280757 & 0.106968\end{array}$

$\begin{array}{lll}0.815675 & 0.542329 & 0.014866\end{array}$

$\begin{array}{lll}0.959259 & 0.416629 & 0.131038\end{array}$

$\begin{array}{llll}0.938917 & 0.309349 & 0.136621\end{array}$

$\begin{array}{lll}0.990638 & 0.275849 & 0.506761\end{array}$

$\begin{array}{lll}0.824122 & 0.326397 & 0.068936\end{array}$

$\begin{array}{lll}0.663037 & 0.207505 & 0.008814\end{array}$

$\begin{array}{lll}0.950081 & 0.236120 & 0.374100\end{array}$

$\begin{array}{lll}0.791870 & 0.266209 & 0.489003\end{array}$

$\begin{array}{llll}0.991350 & 0.021940 & 0.000000\end{array}$

$\begin{array}{lll}0.966565 & 0.298537 & 0.072288\end{array}$

$\begin{array}{llll}0.830162 & 0.631784 & 0.053492\end{array}$

$\begin{array}{llll}0.949425 & 0.293148 & 0.128227\end{array}$

$\begin{array}{llll}0.738088 & 0.536477 & 0.026961\end{array}$

$\begin{array}{llll}0.266832 & 0.000050 & 0.000000 & 1586\end{array}$

$\begin{array}{llll}0.897667 & 0.107814 & 0.343399\end{array}$

$\begin{array}{lll}0.864795 & 0.094680 & 0.115698\end{array}$

$\begin{array}{llll}0.868102 & 0.710056 & 0.606845\end{array}$

$\begin{array}{llll}0.903732 & 0.238290 & 0.013838\end{array}$

$\begin{array}{llll}0.877552 & 0.846882 & 0.004042\end{array}$

$\begin{array}{lll}0.852424 & 0.489974 & 0.003226\end{array}$

$\begin{array}{llll}0.858267 & 0.463997 & 0.182480\end{array}$

$\begin{array}{lll}0.460971 & 0.022995 & 0.000000\end{array}$

$\begin{array}{llll}0.932712 & 0.509466 & 0.043005\end{array}$

$\begin{array}{llll}0.873010 & 0.228522 & 0.086300\end{array}$

$\begin{array}{llll}0.741407 & 0.125296 & 0.000000\end{array}$

$\begin{array}{llll}0.831950 & 0.820487 & 0.003471\end{array}$

$\begin{array}{llll}0.879261 & 0.121930 & 0.033496\end{array}$

$\begin{array}{llll}0.788331 & 0.413317 & 0.076055\end{array}$

$\begin{array}{llll}0.893667 & 0.302349 & 0.105716\end{array}$

$\begin{array}{lll}0.940249 & 0.725389 & 0.072292\end{array}$

$\begin{array}{llll}0.993846 & 0.367939 & 0.133985\end{array}$

$\begin{array}{llll}0.845704 & 0.110887 & 0.546641\end{array}$

$\begin{array}{llll}0.962262 & 0.313932 & 0.088265\end{array}$

$\begin{array}{lll}0.771986 & 0.195275 & 0.009113\end{array}$

$\begin{array}{llll}0.824557 & 0.022352 & 0.474164\end{array}$

$\begin{array}{lll}0.854250 & 0.142899 & 0.096895\end{array}$

$\begin{array}{llll}0.941615 & 0.121830 & 0.278970\end{array}$

$\begin{array}{llll}0.986916 & 0.683048 & 0.349087\end{array}$

$\begin{array}{llll}0.983186 & 0.706858 & 0.066713\end{array}$

$\begin{array}{lll}0.992106 & 0.694062 & 0.153306\end{array}$

$\begin{array}{llll}0.917981 & 0.696188 & 0.053777\end{array}$

$\begin{array}{lll}0.982012 & 0.791284 & 0.260856\end{array}$

$\begin{array}{llll}0.984100 & 0.743533 & 0.096800\end{array}$

$\begin{array}{llll}0.998387 & 0.023469 & 0.000000\end{array}$

$\begin{array}{llll}0.958328 & 0.513058 & 0.065937\end{array}$

$\begin{array}{lll}0.999210 & 1.109124 & 0.011170\end{array}$

$\begin{array}{llll}0.989844 & 0.271518 & 0.000000\end{array}$

$\begin{array}{lll}0.994028 & 0.931622 & 0.085739\end{array}$

$\begin{array}{llll}0.972839 & 0.761193 & 0.162328\end{array}$

$\begin{array}{lll}0.999809 & 0.134414 & 0.016608\end{array}$

$\begin{array}{llll}0.977595 & 0.728069 & 0.012070\end{array}$

$\begin{array}{llll}0.996399 & 0.318168 & 0.146152\end{array}$

$\begin{array}{llll}0.992021 & 0.513942 & 0.038657\end{array}$

$\begin{array}{lll}0.996563 & 0.579995 & 0.071410\end{array}$

$\begin{array}{llll}0.940284 & 0.555530 & 0.072431\end{array}$

$\begin{array}{lll}0.950607 & 0.447899 & 0.053167\end{array}$

$\begin{array}{lll}0.967169 & 0.640965 & 0.090423\end{array}$

\begin{tabular}{|c|c|c|}
\hline $\begin{array}{r}479 \\
55\end{array}$ & $\begin{array}{r}3759 \\
355\end{array}$ & $\begin{array}{l}90097 \\
79097\end{array}$ \\
\hline 3035 & 5363 & 87347 \\
\hline 982 & 3982 & 78217 \\
\hline 379 & 1787 & 62110 \\
\hline 643 & 3591 & 84184 \\
\hline 514 & 2244 & 70861 \\
\hline 963 & 5335 & 96593 \\
\hline 1275 & 4848 & 97900 \\
\hline 756 & 5974 & 94276 \\
\hline 950 & 5355 & 100609 \\
\hline 2413 & 10108 & 114305 \\
\hline 893 & 2407 & 76700 \\
\hline 2052 & 9349 & 164535 \\
\hline 533 & 2307 & 85451 \\
\hline 439 & 3736 & 90835 \\
\hline 1127 & 2678 & 83331 \\
\hline 1120 & 8285 & 118504 \\
\hline
\end{tabular}

7616

$4463 \quad 111229$

$552 \quad 2477 \quad 78874$

$\begin{array}{lll}136 & 3049 & 77120\end{array}$

$372 \quad 2082 \quad 74200$

1730

534

$5217 \quad 121897$

$6459 \quad 93484$

$7869 \quad 183723$

58632

1529

256628

133784

$4152 \quad 122527$

$\begin{array}{lll}271 & 3445 & 107037\end{array}$

$936 \quad 3926 \quad 86731$

3709

183

3452

6868

822

779

2319

1703

669

309

845

360

1420

88

417
1410

1410

432

569

601
397

397
387

387
375

650

570

456

88

427

948

400

598

438

118

319

339

246

369

601

269

10615

121098

$2635 \quad 111426$

$9200 \quad 181508$

$5521 \quad 94445$

$18616 \quad 146369$

$6591 \quad 100480$

$3602 \quad 77587$

$5885 \quad 133053$

$7798 \quad 95753$

$3208 \quad 91878$

$4858 \quad 168978$

$7223 \quad 121139$

$3640 \quad 82823$

$3666 \quad 82057$

$1555 \quad 95062$

3396

7221

4052

2459

2462

2459

2127

3682

2709

3113

2482

3006

7038

4961

3026

4186

2448

1516

2194

4396

1958

2010

2856

7875

$562 \quad 2636$

66581

105987

88661

78488

76713

79944

78793

98211

105425

88614

76016

72663

123780

100223

113952

98394

76036

71329

83879

95028

77011

82823

95136

108486

85498 


\begin{tabular}{|c|c|c|c|c|c|c|c|c|c|}
\hline $\begin{array}{l}\text { County } \\
\text { Code }\end{array}$ & State & $\begin{array}{l}\text { County } \\
\text { Name }\end{array}$ & $\begin{array}{l}\text { FRCLND } \\
\text { Absolute }\end{array}$ & $\begin{array}{l}\text { FRMFRC } \\
\text { Absolute }\end{array}$ & $\begin{array}{c}\text { DPF } \\
\text { Absolute }\end{array}$ & $\begin{array}{l}\text { ASFP } \\
\text { \$/ha }\end{array}$ & $\begin{array}{l}\text { VFRM } \\
\text { S/ha }\end{array}$ & $\begin{array}{l}\text { VNFRM } \\
\text { \$/person }\end{array}$ & Notes \\
\hline $\begin{array}{l}1884 \\
1885\end{array}$ & $\begin{array}{l}K Y \\
K Y\end{array}$ & $\begin{array}{l}\text { Carroll County } \\
\text { Carter County }\end{array}$ & $\begin{array}{l}0.947697 \\
0.996247\end{array}$ & $\begin{array}{l}0.730338 \\
0.429341\end{array}$ & $\begin{array}{l}0.028705 \\
0.047581\end{array}$ & $\begin{array}{l}355 \\
220\end{array}$ & $\begin{array}{l}2613 \\
2204\end{array}$ & $\begin{array}{l}97073 \\
70238\end{array}$ & $\begin{array}{l}0 \\
0\end{array}$ \\
\hline $\begin{array}{l}1885 \\
1886\end{array}$ & KY & Casey County & 0.999739 & 0.673861 & 0.219884 & 327 & $\begin{array}{l}2204 \\
2390\end{array}$ & $\begin{array}{l}70238 \\
69364\end{array}$ & $\begin{array}{l}0 \\
0\end{array}$ \\
\hline 1887 & KY & Christian County & 0.996282 & 0.648342 & 0.010129 & 491 & 2584 & 77208 & 0 \\
\hline 1888 & KY & Clark County & 0.996685 & 0.890228 & 0.009462 & 553 & 4255 & 99830 & 0 \\
\hline 1889 & $\mathrm{KY}$ & Clay County & 0.999852 & 0.226811 & 0.025617 & 196 & 1943 & 58879 & 0 \\
\hline 1890 & KY & Clinton County & 0.960655 & 0.596710 & 0.155835 & 318 & 2641 & 60112 & 0 \\
\hline 1891 & KY & Crittenden County & 0.976252 & 0.539867 & 0.052575 & 183 & 1562 & 75569 & 0 \\
\hline 1892 & KY & Cumberland County & 0.983834 & 0.555725 & 0.097621 & 177 & 1393 & 67055 & 0 \\
\hline 1893 & $\mathrm{Kr}$ & Daviess County & 0.970883 & 0.845154 & 0.028735 & 661 & 4279 & 107166 & 0 \\
\hline 1894 & $K Y$ & Edmonson County & 0.982495 & 0.477509 & 0.185515 & 345 & 2185 & 53664 & 0 \\
\hline 1895 & $K Y$ & Elliott County & 0.994775 & 0.402623 & 0.000000 & 180 & 2022 & 53373 & 0 \\
\hline 1896 & $\mathrm{KY}$ & Estill County & 0.993353 & 0.426339 & 0.019494 & 185 & 2300 & 68091 & 0 \\
\hline 1897 & $K \gamma$ & Fayette County & 0.996455 & 0.808124 & 0.000537 & 2117 & 8495 & 130858 & 1 \\
\hline 1898 & KY & Fleming County & 0.998800 & 0.862797 & 0.283024 & 525 & 2458 & 77966 & 0 \\
\hline 1899 & $\mathrm{KY}$ & Floyd County & 0.997056 & 0.043266 & 0.000000 & 138 & 3968 & 85146 & 0 \\
\hline 1900 & KY & Franklin County & 0.992232 & 0.638957 & 0.012317 & 475 & 3947 & 114989 & 0 \\
\hline 1901 & KY & Fulton County & 0.906313 & 0.724030 & 0.000000 & 526 & 2882 & 95502 & 0 \\
\hline 1902 & $\mathrm{KY}$ & Gallatin County & 0.944217 & 0.653701 & 0.073508 & 367 & 3577 & 94026 & 0 \\
\hline 1903 & $K Y$ & Garrard County & 0.988593 & 0.932963 & 0.065211 & 550 & 2853 & 90273 & 0 \\
\hline 1904 & $\mathrm{KY}$ & Grant County & 0.996698 & 0.764523 & 0.037247 & 312 & 3052 & 87476 & 0 \\
\hline 1905 & $\mathrm{KY}$ & Graves County & 0.998482 & 0.591297 & 0.036247 & 801 & 2843 & 97094 & 0 \\
\hline 1906 & KY & Grayson County & 0.985967 & 0.639310 & 0.201512 & 362 & 2348 & 73313 & 0 \\
\hline 1907 & KY & Green County & 0.999829 & 0.729505 & 0.238861 & 454 & 2309 & 82206 & 0 \\
\hline 1908 & $\mathrm{KY}$ & Greenup County & 0.976568 & 0.453414 & 0.051009 & 215 & 2339 & 94838 & 0 \\
\hline 1909 & $\mathrm{Kr}$ & Hancock County & 0.949113 & 0.576895 & 0.001904 & 391 & 2430 & 98679 & 0 \\
\hline 1910 & Kr & Hardin County & 0.997033 & 0.562797 & 0.165118 & 430 & 3512 & 91160 & 0 \\
\hline 1911 & KY & Harlan County & 0.998334 & 0.016234 & 0.162806 & 106 & 2125 & 78637 & 1 \\
\hline 1912 & $\mathrm{KY}$ & Harrison County & 0.999433 & 0.899085 & 0.027510 & 461 & 2938 & 89101 & 0 \\
\hline 1913 & $\mathrm{KY}$ & Hart County & 0.995257 & 0.752997 & 0.234189 & 440 & 2445 & 74404 & 0 \\
\hline 1914 & $\mathrm{KY}$ & Henderson County & 0.941974 & 0.702267 & 0.000845 & 501 & 3144 & 108879 & 1 \\
\hline 1915 & $\mathrm{KY}$ & Henry County & 0.993846 & 0.863843 & 0.162599 & 537 & 3024 & 93091 & 0 \\
\hline 1916 & $\mathrm{KY}$ & Hickman County & 0.966458 & 0.633173 & 0.027640 & 541 & 2398 & 88539 & 0 \\
\hline 1917 & KY & Hopkins County & 0.993366 & 0.411012 & 0.001681 & 344 & 2667 & 114697 & 1 \\
\hline 1918 & $\mathrm{KY}$ & Jackson County & 0.999304 & 0.364030 & 0.155884 & 321 & 2407 & 56834 & 0 \\
\hline 1919 & KY & Jefferson County & 0.966149 & 0.181396 & 0.052751 & 731 & 9258 & 129639 & 0 \\
\hline 1920 & KY & Jessamine County & 0.992549 & 0.889188 & 0.011364 & 647 & 5663 & 104179 & 0 \\
\hline 1921 & KY & Johnson County & 0.990911 & 0.137766 & 0.018930 & 164 & 3200 & 83127 & 0 \\
\hline 1922 & KY & Kenton County & 0.985457 & 0.424694 & 0.109589 & 310 & 5107 & 114704 & 0 \\
\hline 1923 & $\mathrm{KY}$ & Knott County & 0.997678 & 0.014302 & 0.236809 & 110 & 1978 & 62212 & 1 \\
\hline 1924 & $\mathrm{KY}$ & Knox County & 0.999898 & 0.186693 & 0.069869 & 159 & 2933 & 67332 & 0 \\
\hline 1925 & KY & Larue County & 0.998883 & 0.717412 & 0.276716 & 487 & 3046 & 92976 & 0 \\
\hline 1926 & KY & Laurel County & 0.981808 & 0.356927 & 0.089133 & 375 & 3880 & 81746 & 0 \\
\hline 1927 & KY & Lawrence County & 0.996903 & 0.180958 & 0.031760 & 168 & 1763 & 66073 & 0 \\
\hline 1928 & Kr & Lee County & 0.993575 & 0.154881 & 0.048830 & 165 & 1603 & 59523 & 1 \\
\hline 1929 & KY & Leslie County & 0.999182 & 0.005805 & 0.000000 & 110 & 2042 & 67251 & D \\
\hline 1930 & KY & Letcher County & 0.999783 & 0.015590 & 0.000000 & 45 & 2471 & 73774 & D \\
\hline 1931 & $\mathrm{KY}$ & Lewis County & 0.977374 & 0.515047 & 0.211947 & 234 & 1839 & 71207 & 0 \\
\hline 1932 & $K Y$ & Lincoln County & 0.999369 & 0.807312 & 0.193152 & 553 & 3344 & 69872 & 0 \\
\hline 1933 & KY & Livingston County & 0.923707 & 0.589286 & 0.064868 & 222 & 1557 & 87354 & 0 \\
\hline 1934 & $K Y$ & Logan County & 0.997464 & 0.783540 & 0.104401 & 543 & 2780 & 88959 & 0 \\
\hline 1935 & $K Y$ & Lyon County & 0.841240 & 0.358550 & 0.022985 & 304 & 2258 & 79389 & 0 \\
\hline 1936 & $\mathrm{KY}$ & McCracken County & 0.936758 & 0.390502 & 0.060829 & 508 & 3120 & 118192 & 0 \\
\hline 1937 & $K Y$ & McCreary County & 0.993179 & 0.050729 & 0.068829 & 88 & 2223 & 51903 & 1 \\
\hline 1938 & KY & McLean County & 0.992667 & 0.830544 & 0.004917 & 587 & 3177 & 90090 & 0 \\
\hline 1939 & $\mathrm{K \gamma}$ & Madison County & 0.994529 & 0.876651 & 0.021881 & 461 & 3395 & 85830 & 0 \\
\hline 1940 & KY & Magoffin County & 0.999980 & 0.227886 & 0.012798 & 145 & 1873 & 59895 & 1 \\
\hline 1941 & KY & Marion County & 0.999393 & 0.791173 & 0.259091 & 504 & 2970 & 84035 & 0 \\
\hline 1942 & KY & Marshall County & 0.895939 & 0.390179 & 0.009399 & 468 & 2925 & 98868 & 1 \\
\hline 1943 & $K Y$ & Martin County & 0.999523 & 0.035596 & 0.000000 & 287 & 3239 & 81698 & 0 \\
\hline 1944 & $\mathrm{KY}$ & Mason County & 0.977728 & 0.934760 & 0.232200 & 543 & 3217 & 95658 & 0 \\
\hline 1945 & $\mathrm{KY}$ & Meade County & 0.951562 & 0.605355 & 0.041207 & 369 & 2936 & 77397 & 0 \\
\hline 1946 & $K Y$ & Menifee County & 0.989658 & 0.326749 & 0.099379 & 271 & 2846 & 55581 & 0 \\
\hline 1947 & $K Y$ & Mercer County & 0.991321 & 0.829228 & 0.161601 & 662 & 3704 & 107640 & 0 \\
\hline 1948 & $\mathrm{KY}$ & Metcalfe County & 0.999762 & 0.735128 & 0.282599 & 436 & 2161 & 71478 & 0 \\
\hline 1949 & $\mathrm{~K} \gamma$ & Monroe County & 0.996095 & 0.783625 & 0.341494 & 369 & 2244 & 82687 & 0 \\
\hline 1950 & $K Y$ & Montgomery County & 0.998936 & 0.892031 & 0.065209 & 476 & 2850 & 89508 & 0 \\
\hline 1951 & $K Y$ & Morgan County & 0.993633 & 0.430533 & 0.020673 & 220 & 1880 & 64325 & 0 \\
\hline 1952 & $K Y$ & Muhlenberg County & 0.990131 & 0.387938 & 0.012408 & 520 & 2154 & 82443 & 0 \\
\hline 1953 & $K Y$ & Nelson County & 0.996652 & 0.706059 & 0.284644 & 527 & 3258 & 98753 & 0 \\
\hline
\end{tabular}




\begin{tabular}{|c|c|c|c|c|c|c|c|c|c|}
\hline $\begin{array}{l}\text { County } \\
\text { Code }\end{array}$ & State & $\begin{array}{l}\text { County } \\
\text { Name }\end{array}$ & $\begin{array}{l}\text { FRCLND } \\
\text { Absolute }\end{array}$ & $\begin{array}{l}\text { FRMFRC } \\
\text { Absolute }\end{array}$ & $\begin{array}{c}\text { DPF } \\
\text { Absolute }\end{array}$ & $\begin{array}{l}\text { ASFP } \\
\text { S/ha }\end{array}$ & $\begin{array}{l}\text { VFRM } \\
\text { \$/ha }\end{array}$ & $\begin{array}{l}\text { VNFRM } \\
\text { \$/person }\end{array}$ & Notes \\
\hline 1954 & $\mathrm{KY}$ & Nicholas County & 0.998786 & 0.893288 & 0.020060 & 398 & 1934 & 90510 & 0 \\
\hline 1955 & $K \gamma$ & Ohio County & 0.995069 & 0.420466 & 0.005997 & 325 & 2227 & 77214 & 0 \\
\hline 1956 & $\mathrm{KY}$ & Oldham County & 0.962576 & 0.697280 & 0.151166 & 501 & 6771 & 138620 & 0 \\
\hline 1957 & $\mathrm{KY}$ & Owen County & 0.994326 & 0.784540 & 0.103900 & 302 & 2371 & 78901 & 0 \\
\hline 1958 & $\mathrm{KY}$ & Owsley County & 1.000000 & 0.281672 & 0.018204 & 228 & 2234 & 57918 & 0 \\
\hline 1959 & $\mathrm{KY}$ & Pendleton County & 0.995286 & 0.710842 & 0.072023 & 296 & 3312 & 81881 & 0 \\
\hline 1960 & $\mathrm{KY}$ & Perry County & 0.998578 & 0.020408 & 0.000000 & 187 & 2531 & 91865 & 0 \\
\hline 1961 & $\mathrm{KY}$ & Pike County & 0.998529 & 0.012215 & 0.000000 & 102 & 2569 & 88790 & 0 \\
\hline 1962 & $\mathrm{KY}$ & Powell County & 0.999617 & 0.287571 & 0.000000 & 210 & 2831 & 66940 & 0 \\
\hline 1963 & $\mathrm{KY}$ & Pulaski County & 0.977179 & 0.515165 & 0.197751 & 421 & 3602 & 87659 & 0 \\
\hline 1964 & $\mathrm{KY}$ & Robertson County & 0.999630 & 0.827861 & 0.071722 & 318 & 2257 & 67468 & 0 \\
\hline 1965 & $\mathrm{KY}$ & Rockcastle County & 0.998310 & 0.456536 & 0.141908 & 289 & 2767 & 71796 & 0 \\
\hline 1966 & $\mathrm{KY}$ & Rowan County & 0.981034 & 0.276581 & 0.036883 & 239 & 3302 & 70421 & 0 \\
\hline 1967 & $K Y$ & Russell County & 0.896430 & 0.563051 & 0.220093 & 601 & 3487 & 86196 & 0 \\
\hline 1968 & $K Y$ & Scott County & 0.999568 & 0.844191 & 0.006318 & 739 & 5000 & 121897 & 0 \\
\hline 1969 & KY & Shelby County & 0.996182 & 0.934732 & 0.222315 & 643 & 4624 & 115849 & 0 \\
\hline 1970 & KY & Simpson County & 0.999982 & 0.779039 & 0.059353 & 597 & 2871 & 89677 & 0 \\
\hline 1971 & KY & Spencer County & 0.969272 & 0.789071 & 0.279336 & 579 & 3476 & 86995 & 0 \\
\hline 1972 & KY & Taylor County & 0.973947 & 0.745341 & 0.181535 & 524 & 2915 & 89650 & 0 \\
\hline 1973 & KY & Todd County & 0.998356 & 0.685037 & 0.103411 & 712 & 2622 & 78136 & 0 \\
\hline 1974 & $\mathrm{KY}$ & Trigg County & 0.921522 & 0.394241 & 0.005451 & 412 & 1973 & 89332 & 1 \\
\hline 1975 & $\mathrm{KY}$ & Trimble County & 0.952737 & 0.748646 & 0.071915 & 387 & 3131 & 95414 & 0 \\
\hline 1976 & $\mathrm{KY}$ & Union County & 0.949562 & 0.890564 & 0.000677 & 628 & 3501 & 100406 & 1 \\
\hline 1977 & KY & Warren County & 0.995473 & 0.724505 & 0.131149 & 533 & 3739 & 99884 & 0 \\
\hline 1978 & $\mathrm{KY}$ & Washington County & 0.996926 & 0.859642 & 0.198754 & 482 & 2606 & 88411 & 0 \\
\hline 1979 & $\mathrm{KY}$ & Wayne County & 0.948778 & 0.462030 & 0.076723 & 419 & 2019 & 64528 & 0 \\
\hline 1980 & $\mathrm{KY}$ & Webster County & 0.997084 & 0.655553 & 0.001385 & 430 & 2452 & 114352 & 1 \\
\hline 1981 & KY & Whitley County & 0.988786 & 0.158134 & 0.033808 & 156 & 2633 & 83920 & 0 \\
\hline 1982 & $\mathrm{KY}$ & Wolfe County & 0.999652 & 0.428805 & 0.004400 & 175 & 1969 & 60898 & 0 \\
\hline 1983 & $K Y$ & Woodford County & 0.993209 & 1.013209 & 0.000895 & 1512 & 6792 & 159123 & 13 \\
\hline 1984 & TN & Anderson County & 0.978792 & 0.193965 & 0.089798 & 333 & 5603 & 111094 & 0 \\
\hline 1985 & $\mathrm{TN}$ & Bedford County & 0.997541 & 0.704543 & 0.158948 & 684 & 2957 & 97615 & 0 \\
\hline 1986 & TN & Benton County & 0.905054 & 0.249284 & 0.045419 & 170 & 2357 & 89982 & 1 \\
\hline 1987 & TN & Bledsoe County & 0.999026 & 0.357995 & 0.203942 & 307 & 2570 & 70218 & 0 \\
\hline 1988 & TN & Blount County & 0.985732 & 0.269048 & 0.238596 & 447 & 6500 & 103657 & 0 \\
\hline 1989 & $T N$ & Bradley County & 0.991657 & 0.436580 & 0.199393 & 1144 & 5004 & 99329 & 0 \\
\hline 1990 & $T N$ & Campbell County & 0.963500 & 0.098613 & 0.203886 & 315 & 4555 & 73909 & 0 \\
\hline 1991 & TN & Cannon County & 0.999760 & 0.567851 & 0.222589 & 349 & 2489 & 92027 & 0 \\
\hline 1992 & TN & Carroll County & 0.998608 & 0.431792 & 0.049544 & 323 & 2085 & 86880 & 0 \\
\hline 1993 & TN & Carter County & 0.981098 & 0.167825 & 0.191027 & 472 & 5555 & 77147 & 0 \\
\hline 1994 & TN & Cheatham County & 0.985433 & 0.300892 & 0.015075 & 287 & 3786 & 94405 & 0 \\
\hline 1995 & TN & Chester County & 0.999233 & 0.387259 & 0.068754 & 243 & 1889 & 78095 & 0 \\
\hline 1996 & TN & Claiborne County & 0.983537 & 0.513508 & 0.083469 & 414 & 4047 & 86467 & 0 \\
\hline 1997 & TN & Clay County & 0.910727 & 0.466420 & 0.115330 & 222 & 2218 & 81488 & 0 \\
\hline 1998 & TN & Cocke County & 0.980261 & 0.302230 & 0.178788 & 436 & 3934 & 73110 & 0 \\
\hline 1999 & TN & Coffee County & 0.987146 & 0.482285 & 0.229275 & 547 & 3355 & 105107 & 0 \\
\hline 2000 & TN & Crockett County & 0.999221 & 0.853541 & 0.005331 & 629 & 3103 & 89948 & 1 \\
\hline 2001 & TN & Cumberland County & 0.995125 & 0.222064 & 0.202056 & 412 & 3653 & 84123 & 0 \\
\hline 2002 & $T N$ & Davidison County & 0.954574 & 0.147207 & 0.049406 & 312 & 8357 & 132111 & 1 \\
\hline 2003 & TN & Decatur County & 0.968063 & 0.406435 & 0.000000 & 164 & 1725 & 77492 & 0 \\
\hline 2004 & TN & DeKalb County & 0.925796 & 0.491535 & 0.079836 & 477 & 2967 & 88174 & 0 \\
\hline 2005 & TN & Dickson County & 0.997113 & 0.460112 & 0.089401 & 196 & 3118 & 101334 & 0 \\
\hline 2006 & TN & Dyer County & 0.969737 & 0.706621 & 0.017758 & 523 & 2871 & 100609 & 0 \\
\hline 2007 & TN & Fayette County & 0.997553 & 0.572765 & 0.045014 & 479 & 2619 & 90382 & 0 \\
\hline 2008 & $T N$ & Fentress County & 0.999327 & 0.220770 & 0.058702 & 660 & 2669 & 65138 & 0 \\
\hline 2009 & TN & Franklin County & 0.963024 & 0.382675 & 0.180160 & 759 & 4123 & 79903 & 0 \\
\hline 2010 & TN & Gibson County & 0.998483 & 0.705448 & 0.017215 & 567 & 2661 & 95333 & 0 \\
\hline 2011 & $T N$ & Giles County & 0.999593 & 0.655393 & 0.195019 & 290 & 2622 & 91994 & 0 \\
\hline 2012 & $T N$ & Grainger County & 0.926918 & 0.582168 & 0.110868 & 368 & 3435 & 71146 & 0 \\
\hline 2013 & TN & Greene County & 0.996118 & 0.595286 & 0.312200 & 549 & 4633 & 88167 & 0 \\
\hline 2014 & $T N$ & Grundy County & 0.998398 & 0.185180 & 0.042587 & 1346 & 3625 & 74641 & 0 \\
\hline 2015 & TN & Hamblen County & 0.916137 & 0.555135 & 0.146390 & 604 & 6010 & 93328 & 0 \\
\hline 2016 & $T N$ & Hamilton County & 0.942223 & 0.180134 & 0.212552 & 448 & 5010 & 123557 & 0 \\
\hline 2017 & $T N$ & Hancock County & 0.994593 & 0.564740 & 0.022803 & 258 & 2163 & 60932 & 0 \\
\hline 2018 & TN & Hardeman County & 0.995736 & 0.374313 & 0.006328 & 240 & 2149 & 79314 & 1 \\
\hline 2019 & TN & Hardin County & 0.969059 & 0.297991 & 0.023401 & 188 & 2067 & 78589 & 1 \\
\hline 2020 & $T N$ & Hawkins County & 0.974032 & 0.499552 & 0.086829 & 302 & 3920 & 81692 & 0 \\
\hline 2021 & TN & Haywood County & 0.998225 & 0.657081 & 0.000000 & 554 & 2656 & 87408 & 0 \\
\hline 2022 & TN & Henderson County & 0.988799 & 0.441258 & 0.007743 & 343 & 2376 & 83425 & 0 \\
\hline 2023 & 11 & Henry County & 0.946519 & 0.532612 & 0.148927 & 403 & 2389 & 90307 & 0 \\
\hline
\end{tabular}




\begin{tabular}{|c|c|c|c|c|c|c|c|c|c|}
\hline $\begin{array}{l}\text { County } \\
\text { Code }\end{array}$ & State & $\begin{array}{l}\text { County } \\
\text { Name }\end{array}$ & $\begin{array}{l}\text { FRCLND } \\
\text { Absolute }\end{array}$ & $\begin{array}{l}\text { FRMFRC } \\
\text { Absolute }\end{array}$ & $\begin{array}{c}\text { DPF } \\
\text { Absolute }\end{array}$ & $\begin{array}{l}\text { ASFP } \\
\$ / h a\end{array}$ & $\begin{array}{l}\text { VFRM } \\
\text { S/ha }\end{array}$ & $\begin{array}{l}\text { VNFRM } \\
\text { \$/person }\end{array}$ & Notes \\
\hline 2024 & TN & Hickman County & 0.999916 & 0.331951 & 0.009174 & 166 & 2632 & 82945 & 0 \\
\hline 2025 & TN & Houston County & 0.967569 & 0.346603 & 0.057883 & 189 & 2217 & 80222 & 1 \\
\hline 2026 & TN & Humphreys County & 0.955988 & 0.350576 & 0.092523 & 187 & 3406 & 87374 & 0 \\
\hline 2027 & TN & Jackson County & 0.966601 & 0.441578 & 0.068864 & 162 & 1952 & 66689 & 1 \\
\hline 2028 & TN & Jefferson County & 0.871122 & 0.563020 & 0.224568 & 465 & 5949 & 90145 & 0 \\
\hline 2029 & TN & Johnson County & 0.985923 & 0.285380 & 0.123340 & 365 & 4327 & 67061 & 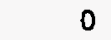 \\
\hline 2030 & TN & Knox County & 0.967057 & 0.289625 & 0.131858 & 364 & 8389 & 117732 & 0 \\
\hline 2031 & TN & Lake County & 0.843245 & 0.873303 & 0.000000 & 647 & 3137 & 74668 & 0 \\
\hline 2032 & TN & Lauderdale County & 0.927655 & 0.606938 & 0.013172 & 502 & 3131 & 83256 & 0 \\
\hline 2033 & TN & Lawrence County & 0.998801 & 0.498035 & 0.255995 & 323 & 3439 & 89359 & 0 \\
\hline 2034 & TN & Lewis County & 0.998646 & 0.204802 & 0.040149 & 164 & 2393 & 70888 & 1 \\
\hline 2035 & TN & Lincoln County & 0.999315 & 0.754052 & 0.239699 & 347 & 2698 & 93504 & 0 \\
\hline 2036 & TN & Loudon County & 0.925414 & 0.503395 & 0.201992 & 1293 & 6750 & 93687 & 0 \\
\hline 2037 & TN & McMinn County & 0.995508 & 0.448661 & 0.361566 & 659 & 3886 & 91133 & n \\
\hline 2038 & TN & McNairy County & 0.998589 & 0.339928 & 0.002056 & 266 & 2329 & 82850 & 0 \\
\hline 2039 & TN & Macon County & 0.999813 & 0.705170 & 0.035224 & 308 & 2665 & 78576 & 0 \\
\hline 2040 & TN & Madison County & 0.997209 & 0.396452 & 0.013694 & 475 & 3179 & 103373 & 0 \\
\hline 2041 & TN & Marion County & 0.972832 & 0.158698 & 0.033287 & 431 & 3375 & 81441 & 1 \\
\hline 2042 & TN & Marshall County & 0.998018 & 0.673892 & 0.507454 & 352 & 3346 & 97947 & 0 \\
\hline 2043 & TN & Maury County & 0.995694 & 0.626307 & 0.216450 & 260 & 3593 & 113830 & 0 \\
\hline 2044 & $T N$ & Meigs County & 0.898876 & 0.451042 & 0.384402 & 221 & 2653 & 79016 & 0 \\
\hline 2045 & $\mathrm{TN}$ & Monroe County & 0.972988 & 0.246400 & 0.483438 & 580 & 4501 & 79734 & 0 \\
\hline 2046 & TN & Montgomery County & 0.991336 & 0.506593 & 0.026096 & 431 & 3356 & 89196 & 0 \\
\hline 2047 & $T N$ & Moore County & 0.990754 & 0.581812 & 0.335016 & 397 & 2701 & 80289 & 0 \\
\hline 2048 & TN & Morgan County & 0.999344 & 0.129299 & 0.117974 & 298 & 2455 & 72060 & 0 \\
\hline 2049 & TN & Obion County & 0.981218 & 0.736880 & 0.012498 & 499 & 2719 & 97649 & 0 \\
\hline 2050 & TN & Overton County & 0.996628 & 0.380451 & 0.232745 & 248 & 2442 & 65307 & - \\
\hline 2051 & TN & Perry County & 0.981079 & 0.199682 & 0.000280 & 167 & 1731 & 81190 & 0 \\
\hline 2052 & TN & Pickett County & 0.933205 & 0.360121 & 0.020383 & 326 & 2928 & 58554 & 0 \\
\hline 2053 & TN & Polk County & 0.983544 & 0.112645 & 0.182014 & 1500 & 4842 & 74363 & 0 \\
\hline 2054 & TN & Putnam County & 0.996337 & 0.454745 & 0.206088 & 282 & 3896 & 98550 & 0 \\
\hline 2055 & $T N$ & Rhea County & 0.939202 & 0.259446 & 0.104831 & 372 & 2917 & 93748 & 0 \\
\hline 2056 & TN & Roane County & 0.913927 & 0.226947 & 0.136580 & 227 & 3807 & 104382 & 0 \\
\hline 2057 & $T N$ & Robertson County & 0.999573 & 0.765036 & 0.112109 & 604 & 3716 & 93308 & 0 \\
\hline 2058 & $T N$ & Rutherford County & 0.991946 & 0.505113 & 0.306519 & 261 & 4344 & 104991 & 0 \\
\hline 2059 & TN & Scott County & 0.997889 & 0.096060 & 0.076786 & 387 & 3458 & 75311 & \\
\hline 2060 & TN & Sequatchie County & 0.999332 & 0.145939 & 0.118617 & 308 & 3063 & 75840 & 0 \\
\hline 2061 & $T N$ & Sevier County & 0.990902 & 0.195486 & 0.027423 & 319 & 8237 & 91960 & 0 \\
\hline 2052 & $T N$ & Shelby County & 0.963229 & 0.300036 & 0.010417 & 483 & 5499 & 124830 & 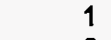 \\
\hline 2063 & $T N$ & Smith County & 0.966385 & 0.746912 & 0.093587 & 243 & 2558 & 99742 & 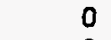 \\
\hline 2064 & $\mathrm{TN}$ & Stewart County & 0.927984 & 0.183646 & 0.000000 & 240 & 3217 & 78576 & 0 \\
\hline 2065 & TN & Sullivan County & 0.961219 & 0.350947 & 0.171700 & 594 & 7815 & 106658 & 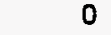 \\
\hline 2066 & TN & Sumner County & 0.974580 & 0.523996 & 0.065670 & 425 & 5153 & 111853 & 0 \\
\hline 2067 & TN & Tipton County & 0.967639 & 0.623031 & 0.006365 & 538 & 3154 & 94676 & ? \\
\hline 2068 & TN & Trousdale County & 0.979390 & 0.753516 & 0.034067 & 342 & 3357 & 87936 & 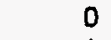 \\
\hline 2069 & $T N$ & Unicoi County & 0.998120 & 0.094786 & 0.063206 & 340 & 7361 & 85904 & \\
\hline 2070 & TN & Union County & 0.904625 & 0.345608 & 0.072845 & 270 & 4726 & 66669 & ( \\
\hline 2071 & TN & Van Buren County & 0.995862 & 0.187929 & 0.386755 & 305 & 2308 & 66059 & 0 \\
\hline 2072 & TN & Warren County & 0.996743 & 0.596937 & 0.105539 & 844 & 3447 & 89555 & \\
\hline 2073 & TN & Washington County & 0.989424 & 0.563303 & 0.275470 & 941 & 7703 & 108249 & \\
\hline 2074 & TN & Wayne County & 0.997779 & 0.266281 & 0.006539 & 127 & 1783 & 72006 & \\
\hline 2075 & $T N$ & Weakley County & 0.997295 & 0.549698 & 0.104463 & 560 & 2232 & 94635 & \\
\hline 2076 & TN & White County & 0.992614 & 0.513415 & 0.370269 & 356 & 3090 & 86541 & \\
\hline 2077 & TN & Williamson County & 0.998391 & 0.548044 & 0.143323 & 308 & 5994 & 163126 & \\
\hline 2078 & $\mathrm{TN}$ & Wilson County & 0.978346 & 0.587330 & 0.135767 & 220 & 4143 & 113512 & \\
\hline 2079 & $\mathrm{AL}$ & Autauga County & 0.985972 & 0.281190 & 0.024913 & 326 & 1931 & 102113 & \\
\hline 2080 & $A L$ & Baldwin County & 0.787602 & 0.164254 & 0.027608 & 704 & 3825 & 98909 & \\
\hline 2081 & $A L$ & Barbour County & 0.978340 & 0.312836 & 0.011133 & 442 & 2199 & 88505 & \\
\hline 2082 & $A L$ & Bibb County & 0.994982 & 0.120564 & 0.089310 & 136 & 2516 & 82051 & \\
\hline 2083 & $A L$ & Blount County & 0.992354 & 0.332566 & 0.023420 & 1743 & 3983 & 88966 & \\
\hline 2084 & $A L$ & Bullock County & 0.998333 & 0.361960 & 0.022319 & 326 & 1940 & 66113 & \\
\hline 2085 & $A L$ & Butler County & 0.998648 & 0.193925 & 0.017695 & 578 & 2032 & 73421 & \\
\hline 2086 & $A L$ & Calhoun County & 0.993721 & 0.189605 & 0.026250 & 1265 & 3888 & 93308 & \\
\hline 2087 & $A L$ & Chambers County & 0.990472 & 0.286538 & 0.099783 & 94 & 1648 & 87835 & \\
\hline 2088 & $A L$ & Cherokee County & 0.921953 & 0.343203 & 0.012866 & 808 & 2604 & 85627 & \\
\hline 2089 & $A L$ & Chitton County & 0.990384 & 0.223921 & 0.017596 & 299 & 3018 & 81570 & \\
\hline 2090 & $A L$ & Choctaw County & 0.992036 & 0.116216 & 0.043897 & 195 & 2316 & 77289 & \\
\hline 2091 & $A L$ & Clarke County & 0.988723 & 0.077497 & 0.013906 & 67 & 2137 & 86006 & \\
\hline 2092 & $A L$ & Clay County & 0.998469 & 0.176818 & 0.028893 & 794 & 2283 & 87699 & \\
\hline 2093 & $A L$ & Cleburne County & 0.998468 & 0.131648 & 0.004511 & 1799 & 3771 & 83222 & \\
\hline
\end{tabular}




\begin{tabular}{|c|c|c|c|c|c|c|c|c|c|}
\hline $\begin{array}{c}\text { County } \\
\text { Code }\end{array}$ & State & $\begin{array}{l}\text { County } \\
\text { Name }\end{array}$ & $\begin{array}{l}\text { FRCLND } \\
\text { Absolute }\end{array}$ & $\begin{array}{l}\text { FRMFRC } \\
\text { Absolute }\end{array}$ & $\begin{array}{c}\text { DPF } \\
\text { Absolute }\end{array}$ & $\begin{array}{l}\text { ASFP } \\
\text { \$/ha }\end{array}$ & $\begin{array}{l}\text { VFRM } \\
\text { \$/ha }\end{array}$ & $\begin{array}{l}\text { VNFRM } \\
\text { \$/person }\end{array}$ & Notes \\
\hline 2094 & $A L$ & Coffee County & 0.998043 & 0.403076 & 0.015210 & 1334 & 2513 & 95211 & 0 \\
\hline 2095 & $\mathrm{AL}$ & Colbert County & 0.953369 & 0.363009 & 0.000000 & 492 & 2936 & 90625 & 0 \\
\hline 2096 & $A L$ & Conecuh County & 0.997990 & 0.151437 & 0.084815 & 199 & 2820 & 83534 & 0 \\
\hline 2097 & $\mathrm{AL}$ & Coosa County & 0.979119 & 0.097779 & 0.168632 & 84 & 2170 & 72229 & 1 \\
\hline 2098 & $A L$ & Covington County & 0.991169 & 0.251410 & 0.033281 & 666 & 2257 & 86663 & 0 \\
\hline 2099 & $A L$ & Crenshaw County & 0.997886 & 0.285308 & 0.027963 & 1027 & 2041 & 80723 & 1 \\
\hline 2100 & $\mathrm{AL}$ & Cullman County & 0.978299 & 0.416520 & 0.012245 & 2981 & 4947 & 93430 & 0 \\
\hline 2101 & $A L$ & Dale County & 0.997164 & 0.374683 & 0.002642 & 639 & 2564 & 92603 & 0 \\
\hline 2102 & AL & Dallas County & 0.987253 & 0.371864 & 0.049779 & 264 & 1678 & 80913 & 0 \\
\hline 2103 & $\mathrm{AL}$ & DeKalb County & 0.999059 & 0.423236 & 0.018832 & 1902 & 4169 & 97358 & 0 \\
\hline 2104 & $A L$ & Elmore County & 0.945710 & 0.262346 & 0.152503 & 423 & 2681 & 100717 & 0 \\
\hline 2105 & $A L$ & Escambia County & 0.994158 & 0.141613 & 0.038201 & 433 & 2604 & 82633 & 0 \\
\hline 2106 & $A L$ & Etowah County & 0.974548 & 0.250721 & 0.044011 & 939 & 3446 & 90720 & 0 \\
\hline 2107 & AL & Fayette County & 0.997505 & 0.161162 & 0.204614 & 251 & 2279 & 83663 & 0 \\
\hline 2108 & $\mathrm{AL}$ & Franklin County & 0.983194 & 0.319693 & 0.030426 & 942 & 2812 & 93037 & 0 \\
\hline 2109 & AL & Geneva County & 0.995549 & 0.530073 & 0.020900 & 1015 & 2501 & 94296 & 0 \\
\hline 2110 & $A L$ & Greene County & 0.978746 & 0.310481 & 0.056789 & 173 & 1801 & 66086 & 0 \\
\hline 2111 & $A L$ & Hale County & 0.980595 & 0.406734 & 0.096129 & 379 & 1794 & 61264 & 1 \\
\hline 2112 & $\mathrm{AL}$ & Henry County & 0.988700 & 0.464198 & 0.011427 & 609 & 2176 & 86961 & 1 \\
\hline 2113 & $A L$ & Houston County & 0.997769 & 0.516365 & 0.042571 & 729 & 3104 & 106651 & 0 \\
\hline 2114 & $A L$ & Jackson County & 0.957388 & 0.296171 & 0.031606 & 574 & 2489 & 86473 & 0 \\
\hline 2115 & $\mathrm{AL}$ & Jefferson County & 0.990038 & 0.050200 & 0.073071 & 334 & 6159 & 126144 & 1 \\
\hline 2116 & $A L$ & Lamar County & 0.998980 & 0.144918 & 0.178982 & 203 & 2413 & 85586 & 0 \\
\hline 2117 & $A L$ & Lauderdale County & 0.931390 & 0.471166 & 0.040781 & 329 & 2963 & 97554 & 0 \\
\hline 2118 & $A L$ & Lawrence County & 0.965629 & 0.390865 & 0.023707 & 803 & 3009 & 79673 & 0 \\
\hline 2119 & $A L$ & Lee County & 0.988916 & 0.174429 & 0.010935 & 391 & 3324 & 91235 & 1 \\
\hline 2120 & AL & Limestone County & 0.935707 & 0.569964 & 0.017695 & 642 & 4204 & 99004 & 0 \\
\hline 2121 & $A L$ & Lowndes County & 0.990224 & 0.434613 & 0.020799 & 284 & 1636 & 75413 & 0 \\
\hline 2122 & $A L$ & Macon County & 0.995568 & 0.354269 & 0.162408 & 163 & 1966 & 66553 & 0 \\
\hline 2123 & $A L$ & Madison County & 0.990248 & 0.435523 & 0.034444 & 533 & 4420 & 128623 & 0 \\
\hline 2124 & $A L$ & Marengo County & 0.994095 & 0.318405 & 0.245353 & 173 & 1516 & 83527 & 0 \\
\hline 2125 & AL & Marion County & 0.997101 & 0.188031 & 0.034068 & 435 & 2061 & 74512 & 0 \\
\hline 2126 & $A L$ & Marshall County & 0.909973 & 0.393646 & 0.014054 & 2346 & 5343 & 102980 & 0 \\
\hline 2127 & $A L$ & Mobile County & 0.750157 & 0.132187 & 0.038497 & 1296 & 5319 & 97460 & 0 \\
\hline 2128 & AL & Monroe County & 0.991639 & 0.167625 & 0.030646 & 322 & 2241 & 84191 & 0 \\
\hline 2129 & $A L$ & Montgomery County & 0.987577 & 0.457438 & 0.042283 & 325 & 2875 & 118321 & 0 \\
\hline 2130 & $A L$ & Morgan County & 0.971885 & 0.418406 & 0.096047 & 913 & 3925 & 111473 & 0 \\
\hline 2131 & $A L$ & Perry County & 0.993654 & 0.313120 & 0.167354 & 229 & 1480 & 62632 & 0 \\
\hline 2132 & $A L$ & Pickens County & 0.990297 & 0.188264 & 0.027404 & 1142 & 2133 & 77336 & 0 \\
\hline 2133 & $A L$ & Pike County & 0.998420 & 0.417511 & 0.014453 & 563 & 1999 & 87632 & 0 \\
\hline 2134 & AL & Randolph County & 0.994757 & 0.259304 & 0.020153 & 802 & 2339 & 81746 & 0 \\
\hline 2135 & $A L$ & Russell County & 0.990268 & 0.274464 & 0.065527 & 158 & 1736 & 87191 & 1 \\
\hline 2136 & $A L$ & St. Clair County & 0.969909 & 0.192669 & 0.023563 & 1261 & 3952 & 88431 & 0 \\
\hline 2137 & $A L$ & Shelby County & 0.981868 & 0.140928 & 0.225212 & 432 & 5877 & 107931 & 0 \\
\hline 2138 & $A L$ & Sumter County & 0.990840 & 0.289926 & 0.009984 & 159 & 1379 & 70888 & 0 \\
\hline 2139 & $A L$ & Talladega County & 0.972742 & 0.220134 & 0.083389 & 352 & 2922 & 82714 & 0 \\
\hline 2140 & $A L$ & Tallapoosa County & 0.937022 & 0.171667 & 0.005960 & 184 & 1969 & 96416 & 0 \\
\hline 2141 & $A L$ & oosa County & 205 & 0.113414 & 399 & 528 & 3129 & 102363 & 0 \\
\hline 2142 & $A L$ & Walker County & 0.986458 & 0.098844 & 0.003367 & 1460 & 3311 & 98591 & 0 \\
\hline 2143 & AL & Washington County & 0.992756 & 0.123015 & 0.001842 & 473 & 2424 & 84712 & 0 \\
\hline 2144 & AL & Wilcox County & 0.979303 & 0.248346 & 0.032550 & 126 & 1539 & 70922 & 1 \\
\hline 2145 & $A L$ & Winston County & 0.972433 & 0.144125 & 0.048529 & 2398 & 3547 & 81434 & 0 \\
\hline 2146 & MS & Adams County. & 0.946561 & 0.271503 & 0.000000 & 230 & 1860 & 91865 & 0 \\
\hline 2147 & MS & Alcorn County & 0.996466 & 0.307267 & 0.051791 & 203 & 2135 & 85959 & 0 \\
\hline 2148 & MS & Amite County & 0.997208 & 0.241757 & 0.603258 & 238 & 2230 & 63885 & 0 \\
\hline 2149 & MS & Attala County & 0.997331 & 0.228527 & 0.026289 & 206 & 1404 & 71796 & 0 \\
\hline 2150 & MS & Benton County & 0.995451 & 0.350923 & 0.004739 & 360 & 1523 & 68971 & 0 \\
\hline 2151 & MS & Bolivar County & 0.967460 & 0.760591 & 0.002123 & 673 & 2149 & 76300 & 1 \\
\hline 2152 & MS & Calhoun County & 0.997578 & 0.339231 & 0.014143 & 358 & 1782 & 74444 & 0 \\
\hline 2153 & MS & Carroll County & 0.989240 & 0.377688 & 0.027829 & 433 & 1740 & 69324 & 1 \\
\hline 2154 & MS & Chickasaw County & 0.994631 & 0.464231 & 0.051434 & 401 & 1565 & 79057 & 0 \\
\hline 2155 & MS & Choctaw County & 0.998305 & 0.159229 & 0.115676 & 308 & 1464 & 65937 & 1 \\
\hline 2156 & MS & Claiborne County & 0.970839 & 0.284119 & 0.015256 & 225 & 1594 & 67075 & 1 \\
\hline 2157 & MS & Clarke County & 0.996909 & 0.155190 & 0.057107 & 116 & 1880 & 71870 & 0 \\
\hline 2158 & MS & Clay County & 0.981986 & 0.483231 & 0.081026 & 300 & 1675 & 83249 & 0 \\
\hline 2159 & MS & Coahoma County & 0.950288 & 0.830451 & 0.000000 & 746 & 2712 & 85640 & 0 \\
\hline 2160 & MS & Copiah County & 0.996429 & 0.254721 & 0.084644 & 539 & 1869 & 70631 & 0 \\
\hline 2161 & MS & Covington County & 0.997238 & 0.301920 & 0.008892 & 857 & 3313 & 67725 & 1 \\
\hline 2162 & MS & DeSoto County & 0.962677 & 0.456035 & 0.049638 & 428 & 3014 & 109523 & 0 \\
\hline 2163 & & Forrest County & 0.992619 & 0.124358 & 0.057630 & 569 & 3036 & 89589 & 1 \\
\hline
\end{tabular}




\begin{tabular}{|c|c|c|c|c|c|c|c|c|c|}
\hline $\begin{array}{l}\text { County } \\
\text { Code }\end{array}$ & State & $\begin{array}{l}\text { County } \\
\text { Name }\end{array}$ & $\begin{array}{l}\text { FRCLND } \\
\text { Absolute }\end{array}$ & $\begin{array}{l}\text { FRMFRC } \\
\text { Absolute }\end{array}$ & $\begin{array}{c}\text { DPF } \\
\text { Absolute }\end{array}$ & $\begin{array}{l}\text { ASFP } \\
\text { \$/ha }\end{array}$ & $\begin{array}{l}\text { VFRM } \\
\text { \$/ha }\end{array}$ & $\begin{array}{l}\text { VNFRM } \\
\text { \$/person }\end{array}$ & Notes \\
\hline $\begin{array}{l}2164 \\
2165\end{array}$ & $\begin{array}{l}\text { MS } \\
\text { MS }\end{array}$ & $\begin{array}{l}\text { Franklin County } \\
\text { George County }\end{array}$ & $\begin{array}{l}0.996219 \\
0.988961\end{array}$ & $\begin{array}{l}0.128766 \\
0.142096\end{array}$ & $\begin{array}{l}0.239676 \\
0.101348\end{array}$ & $\begin{array}{l}137 \\
392\end{array}$ & $\begin{array}{l}1813 \\
2971\end{array}$ & $\begin{array}{l}76686 \\
71024\end{array}$ & 0 \\
\hline 2166 & MS & Greene County & 0.991940 & 0.108008 & 0.024346 & 508 & 2556 & 53732 & 1 \\
\hline 2167 & MS & Grenada County & 0.938820 & 0.369322 & 0.000000 & 363 & 1446 & 85132 & 0 \\
\hline 2168 & MS & Hancock County & 0.863096 & 0.098453 & 0.481890 & 186 & 2926 & 84143 & 0 \\
\hline 2169 & MS & Harrison County & 0.595202 & 0.044816 & 0.143004 & 289 & 6872 & 92732 & 0 \\
\hline 2170 & MS & Hinds County & 0.990713 & 0.414932 & 0.010469 & 378 & 2632 & 109827 & 1 \\
\hline 2171 & MS & Holmes County & 0.989295 & 0.461697 & 0.000770 & 431 & 2226 & 63953 & 0 \\
\hline 2172 & MS & Humphreys County & 0.969680 & 0.673028 & 0.000000 & 1251 & 2552 & 96477 & 0 \\
\hline 2173 & MS & Issaquena County & 0.935662 & 0.430283 & 0.000000 & 607 & 1987 & 76706 & 0 \\
\hline 2174 & MS & Itawamba County & 0.985043 & 0.225030 & 0.006918 & 657 & 1906 & 77871 & 0 \\
\hline 2175 & MS & Jackson County & 0.696441 & 0.053424 & 0.050282 & 488 & 4830 & 91269 & 1 \\
\hline 2176 & MS & Jasper County & 0.997870 & 0.206088 & 0.067846 & 429 & 1617 & 68592 & 0 \\
\hline 2177 & MS & Jefferson County & 0.985194 & 0.199308 & 0.000000 & 182 & 1377 & 55120 & 0 \\
\hline 2178 & MS & Jefferson Davis County & 0.998299 & 0.309493 & 0.021873 & 278 & 2400 & 59997 & 0 \\
\hline 2179 & MS & Jones County & 0.991556 & 0.218244 & 0.024431 & 1706 & 3526 & 83933 & 0 \\
\hline 2180 & MS & Kemper County & 0.998851 & 0.190380 & 0.055656 & 117 & 1481 & 60850 & 0 \\
\hline 2181 & MS & Lafayette County & 0.929081 & 0.244632 & 0.027515 & 112 & 1575 & 78705 & 1 \\
\hline 2182 & MS & Lamar County & 0.993784 & 0.167747 & 0.038987 & 620 & 3578 & 69940 & 0 \\
\hline 2183 & MS & Lauderdale County & 0.983545 & 0.179184 & 0.062088 & 158 & 2130 & 103142 & 0 \\
\hline 2184 & MS & Lawrence County & 0.988253 & 0.227976 & 0.093809 & 272 & 1803 & 71227 & 0 \\
\hline 2185 & MS & Leake County & 5422 & 0.256692 & 591 & 1348 & 2921 & 74614 & 0 \\
\hline 2186 & MS & Lee County & 0.992259 & 0.487200 & 0.139573 & 353 & 2136 & 99072 & 0 \\
\hline 2187 & MS & Leflore County & 0.976215 & 0.692517 & 0.000000 & 856 & 2593 & 90158 & 0 \\
\hline 2188 & MS & Lincoln County & 0.995806 & 0.263854 & 0.467175 & 380 & 2198 & 79626 & 0 \\
\hline 2189 & MS & Lowndes County & 0.972557 & 0.391049 & 0.022634 & 321 & 1848 & 94825 & 1 \\
\hline 2190 & MS & Madison County & 0.969148 & 0.432278 & 0.003039 & 323 & 3003 & 100067 & 0 \\
\hline 2191 & MS & Marion County & 0.988649 & 0.258743 & 0.430401 & 264 & 2116 & 71322 & 0 \\
\hline 2192 & MS & Marshall County & 0.995119 & 0.402593 & 0.125461 & 214 & 2474 & 77445 & 0 \\
\hline 2193 & MS & Monroe County & 0.989808 & 0.358267 & 0.054740 & 288 & 1858 & 83392 & 0 \\
\hline 2194 & MS & Montgomery County & 0.997511 & 0.308257 & 0.008701 & 248 & 1446 & 73245 & 0 \\
\hline 2195 & MS & Neshoba County & 0.997126 & 0.376251 & 0.053337 & 744 & 2277 & 72602 & 0 \\
\hline 2196 & MS & Newton County & 0.997331 & 0.260764 & 0.118482 & 899 & 1909 & 85153 & 0 \\
\hline 2197 & MS & Noxubee County & 0.992553 & 0.453675 & 0.138186 & 331 & 1616 & 60566 & 0 \\
\hline 2198 & MS & Oktibbeha County & 0.990950 & 0.275669 & 0.494844 & 291 & 2175 & 76090 & 0 \\
\hline 2199 & MS & Panola County & 0.970306 & 0.498158 & 0.005509 & 358 & 1940 & 76300 & 0 \\
\hline 2200 & MS & Pearl River County & 0.990920 & 0.179404 & 0.096476 & 264 & 2994 & 76361 & 0 \\
\hline 2201 & MS & Perry County & 0.995352 & 0.076257 & 0.015434 & 625 & 2665 & 69635 & 1 \\
\hline 2202 & MS & Pike County & 0.995402 & 0.306988 & 0.662467 & 530 & 2858 & 77133 & 0 \\
\hline 2203 & MS & Pontotoc County & 0.992831 & 0.390139 & 0.094509 & 247 & 1986 & 86446 & 0 \\
\hline 2204 & MS & Prentiss County & 0.992111 & 0.324175 & 0.119868 & 236 & 1868 & 77546 & 0 \\
\hline 2205 & MS & Quitman County & 0.995928 & 0.718975 & 0.000000 & 547 & 2353 & 71356 & 0 \\
\hline 2206 & MS & Rankin County & 0.960857 & 0.239339 & 0.023550 & 833 & 2461 & 98381 & 0 \\
\hline 2207 & MS & Scott County & 0.997867 & 0.282482 & 0.010949 & 3031 & 3320 & 87991 & 1 \\
\hline 2208 & MS & Sharkey County & 0.983505 & 0.664631 & 0.000000 & 810 & 2376 & 81292 & 0 \\
\hline 2209 & MS & Simpson County & 0.996957 & 0.256199 & 0.005892 & 2155 & 3296 & 74072 & 0 \\
\hline 2210 & MS & Smith County & 0.997816 & 0.233717 & 0.009225 & 2326 & 3533 & 76408 & 0 \\
\hline 2211 & MS & Stone County & 0.993966 & 0.114598 & 0.011479 & 343 & 3342 & 77397 & 0 \\
\hline 2212 & MS & Sunflower County & 0.981016 & 0.812975 & 0.000000 & 885 & 2262 & 83541 & 0 \\
\hline 2213 & MS & Tallahatchie County & 0.987487 & 0.652678 & 0.000420 & 560 & 2113 & 71735 & 0 \\
\hline 2214 & MS & Tate County & 0.984258 & 0.545583 & 0.276967 & 362 & 2149 & 91445 & 0 \\
\hline 2215 & MS & Tippah County & 0.995510 & 0.369573 & 0.165110 & 227 & 1862 & 80046 & 0 \\
\hline 2216 & MS & Tishomingo County & 0.954084 & 0.14 & 0.127977 & 117 & 1965 & 76070 & 1 \\
\hline 2217 & MS & Tunica County & 0.945979 & 0.791906 & 0.000000 & 674 & 2379 & 77275 & 0 \\
\hline 2218 & MS & Union County & 0.996673 & 0.377662 & 0.062399 & 275 & 2003 & 80987 & 0 \\
\hline 2219 & MS & Walthall County & 0.998469 & 0.418767 & 0.688482 & 482 & 2637 & 58636 & 0 \\
\hline 2220 & MS & Warren County & 0.948038 & 0.303843 & 0.000000 & 343 & 2144 & 101869 & 0 \\
\hline 2221 & MS & Washington County & 0.951043 & 0.738563 & 0.000000 & 821 & 2632 & 83554 & 0 \\
\hline 2222 & MS & Wayne County & 0.996115 & 0.139816 & 0.008731 & 962 & 2582 & 69852 & 1 \\
\hline 2223 & MS & Webster County & 0.998759 & 0.279173 & 0.032834 & 282 & 1508 & 77025 & 0 \\
\hline 2224 & MS & Wilkinson County & 0.984074 & 0.207348 & 0.213455 & 144 & 1591 & 65829 & 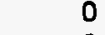 \\
\hline 2225 & MS & Winston County & 0.994914 & 0.214783 & 0.157141 & 186 & 1894 & 77817 & 0 \\
\hline 2226 & MS & Yalobusha County & 0.943688 & 0.261657 & 0.047340 & 216 & 1524 & 80181 & 0 \\
\hline 2227 & MS & Yazoo County & 0.984327 & 0.614484 & 0.008246 & 511 & 1969 & 83121 & \\
\hline 2228 & AR & Arkansas County & 0.956166 & 0.650385 & 0.000000 & 646 & 2759 & 99810 & $c$ \\
\hline 2229 & AR & Ashley County & 0.981044 & 0.256627 & 0.001398 & 738 & 2360 & 97168 & \\
\hline 2230 & AR & Baxter County & 0.944842 & 0.261276 & 0.013111 & 604 & 2256 & 99769 & \\
\hline 2231 & AR & Benton County & 0.961788 & 0.544255 & 0.033786 & 2287 & 4880 & 107944 & \\
\hline 2232 & AR & Boone County & 0.982316 & 0.662872 & 0.047089 & 402 & 2410 & 95699 & 0 \\
\hline 2233 & AR & Bradley County & 0.994267 & 0.072511 & 0.000000 & 978 & 3262 & 94655 & \\
\hline
\end{tabular}




\begin{tabular}{|c|c|c|c|c|c|c|c|c|c|}
\hline $\begin{array}{l}\text { County } \\
\text { Code }\end{array}$ & State & $\begin{array}{l}\text { County } \\
\text { Name }\end{array}$ & $\begin{array}{l}\text { FRCLND } \\
\text { Absolute }\end{array}$ & $\begin{array}{l}\text { FRMFRC } \\
\text { Absolute }\end{array}$ & $\begin{array}{c}\text { DPF } \\
\text { Absolute }\end{array}$ & $\begin{array}{l}\text { ASFP } \\
\text { S/ha }\end{array}$ & $\begin{array}{c}\text { VFRM } \\
\text { \$/ha }\end{array}$ & $\begin{array}{l}\text { VNFRM } \\
\text { \$/person }\end{array}$ & Notes \\
\hline 2234 & AR & Calhoun County & 0.993261 & 0.046796 & 0.000000 & 118 & 2559 & 65822 & 0 \\
\hline 2235 & AR & Carroll County & 0.986709 & 0.606924 & 0.041181 & 1102 & 2448 & 93179 & 0 \\
\hline 2236 & AR & Chicot County & 0.932176 & 0.652885 & 0.003598 & 644 & 2352 & 69879 & 1 \\
\hline 2237 & AR & Clark County & 0.980547 & 0.178579 & 0.094957 & 279 & 1653 & 87720 & 0 \\
\hline 2238 & AR & Clay County & 0.996696 & 0.766339 & 0.000960 & 518 & 2633 & 86961 & 1 \\
\hline 2239 & AR & Cleburne County & 0.934350 & 0.305232 & 0.068637 & 820 & 2569 & 83906 & 0 \\
\hline 2240 & AR & Cleveland County & 0.998184 & 0.089175 & 0.009390 & 1948 & 2549 & 90659 & 1 \\
\hline 2241 & AR & Columbia County & 0.999025 & 0.116757 & 0.002209 & 1234 & 3038 & 99044 & 1 \\
\hline 2242 & AR & Conway County & 0.981490 & 0.470732 & 0.096750 & 932 & 2482 & 88621 & 0 \\
\hline 2243 & AR & Craighead County & 0.996869 & 0.770261 & 0.001363 & 653 & 2973 & 92298 & 1 \\
\hline 2244 & AR & Crawford County & 0.985532 & 0.382411 & 0.028224 & 806 & 3555 & 83961 & 0 \\
\hline 2245 & AR & Crittenden County & 0.958795 & 0.836427 & 0.000000 & 596 & 2322 & 84164 & 0 \\
\hline 2246 & AR & Cross County & 0.989583 & 0.823342 & 0.000000 & 548 & 2612 & 87666 & 0 \\
\hline 2247 & $A R$ & Dallas County & 0.998893 & 0.048197 & 0.000000 & 143 & 2368 & 88018 & 0 \\
\hline 2248 & AR & Desha County & 0.933461 & 0.535147 & 0.000000 & 679 & 2295 & 83337 & 0 \\
\hline 2249 & AR & Drew County & 0.991059 & 0.208008 & 0.009104 & 466 & 2297 & 82267 & 1 \\
\hline 2250 & AR & Faulkner County & 0.974872 & 0.508530 & 0.304465 & 223 & 2359 & 96897 & 0 \\
\hline 2251 & AR & Franklin County & 0.983657 & 0.432548 & 0.050222 & 1197 & 2460 & 87042 & 0 \\
\hline 2252 & AR & Fulton County & 0.996571 & 0.565846 & 0.300029 & 155 & 1292 & 66946 & 0 \\
\hline 2253 & AR & Garland County & 0.923013 & 0.098614 & 0.037006 & 477 & 3521 & 107924 & 0 \\
\hline 2254 & AR & Grant County & 0.998053 & 0.093000 & 0.033782 & 368 & 3068 & 96227 & 1 \\
\hline 2255 & AR & Greene County & 0.996313 & 0.680968 & 0.012192 & 498 & 2667 & 81990 & 0 \\
\hline 2256 & AR & Hempstead County & 0.983044 & 0.361974 & 0.003924 & 1712 & 2615 & 84557 & 0 \\
\hline 2257 & AR & Hot Spring County & 0.988387 & 0.199445 & 0.086898 & 302 & 2454 & 79727 & 0 \\
\hline 2258 & AR & Howard County & 0.986943 & 0.281190 & 0.004332 & 2093 & 3083 & 106583 & 0 \\
\hline 2259 & AR & Independence County & 0.989909 & 0.538354 & 0.011587 & 512 & 1999 & 89948 & 0 \\
\hline 2260 & AR & Izard County & 0.994308 & 0.494775 & 0.059619 & 302 & 1339 & 89738 & 0 \\
\hline 2261 & $A R$ & Jackson County & 0.987648 & 0.907475 & 0.001933 & 439 & 2033 & 85803 & 1 \\
\hline 2262 & AR & Jefferson County & 0.968303 & 0.497758 & 0.000000 & 656 & 2281 & 93531 & 0 \\
\hline 2263 & AR & Johnson County & 0.969868 & 0.256982 & 0.001277 & 1404 & 2714 & 80337 & 0 \\
\hline 2264 & AR & Lafayette County & 0.965932 & 0.320015 & 0.002729 & 1060 & 1634 & 96234 & 1 \\
\hline 2265 & AR & Lawrence County & 0.990250 & 0.750854 & 0.006091 & 452 & 2320 & 82951 & 0 \\
\hline 2266 & AR & Lee County & 0.971261 & 0.775272 & 0.000000 & 541 & 1931 & 69642 & 0 \\
\hline 2267 & $A R$ & Lincoln County & 0.980832 & 0.519735 & 0.003178 & 789 & 2166 & 63187 & 1 \\
\hline 2268 & AR & Little River County & 0.941338 & 0.420486 & 0.010712 & 407 & 1794 & 95218 & 1 \\
\hline 2269 & AR & Logan County & 0.970430 & 0.411202 & 0.095737 & 757 & 2339 & 86934 & 0 \\
\hline 2270 & AR & Lonoke County & 0.953916 & 0.781174 & 0.035916 & 678 & 2664 & 98503 & 0 \\
\hline 2271 & AR & Madison County & 0.999798 & 0.500465 & 0.039429 & 769 & 2566 & 88749 & 0 \\
\hline 2272 & AR & Marion County & 0.933402 & 0.373430 & 0.057600 & 284 & 2057 & 85417 & 0 \\
\hline 2273 & AR & Miller County & 0.978896 & 0.435298 & 0.005294 & 502 & 2264 & 92522 & 0 \\
\hline 2274 & AR & Mississippi County & 0.976644 & 0.843160 & 0.000000 & 731 & 2914 & 81339 & 0 \\
\hline 2275 & AR & Monroe County & 0.976289 & 0.565138 & 0.000000 & 523 & 2365 & 84082 & 0 \\
\hline 2276 & AR & Montgomery County & 0.975801 & 0.159662 & 0.013793 & 1001 & 3026 & 78874 & 0 \\
\hline 2277 & AR & Nevada County & 0.998672 & 0.174952 & 0.001982 & 772 & 1931 & 82945 & 0 \\
\hline 2278 & AR & Newton County & 0.999746 & 0.194708 & 0.112489 & 138 & 1892 & 66242 & 0 \\
\hline 2279 & AR & Ouachita County & 0.990290 & 0.068264 & 0.000000 & 400 & 2518 & 85207 & 0 \\
\hline 2280 & AR & Perry County & 0.982966 & 0.190134 & 0.006476 & 956 & 2430 & 77912 & 0 \\
\hline 2281 & AR & Phillips County & 0.952404 & 0.806185 & 0.000000 & 645 & 1996 & 77059 & 0 \\
\hline 2282 & AR & Pike County & 0.982290 & 0.183627 & 0.005035 & 1312 & 2989 & 89169 & 1 \\
\hline 2283 & $A R$ & Poinsett County & 0.992604 & 0.834217 & 0.000000 & 670 & 2770 & 83676 & 0 \\
\hline 2284 & AR & Polk County & 0.996501 & 0.223381 & 0.004433 & 1511 & 3417 & 79931 & 0 \\
\hline 2285 & AR & Pope County & 0.977266 & 0.300900 & 0.015099 & 1474 & 3797 & 92271 & 0 \\
\hline 2286 & AF & Prairie County & 0.955891 & 0.757621 & 0.019610 & 550 & 2264 & 80730 & 0 \\
\hline 2287 & AF & Pulaski County & 0.954257 & 0.226778 & 0.005608 & 370 & 2794 & 123442 & 0 \\
\hline 2288 & AR & Randolph County & 0.993584 & 0.608699 & 0.007742 & 283 & 2326 & 73110 & 0 \\
\hline 2289 & AR & St. Francis County & 0.986677 & 0.752803 & 0.000585 & 484 & 1908 & 70272 & 0 \\
\hline 2290 & AR & Saline County & 0.992140 & 0.098328 & 0.034268 & 200 & 3861 & 94676 & 1 \\
\hline 2291 & A & Scott County & 0.995310 & 0.200591 & 0.008750 & 1181 & 2553 & 81102 & 0 \\
\hline 2292 & AF & Searcy County & 0.997967 & 0.457860 & 0.313708 & 152 & 1937 & 73557 & 0 \\
\hline 2293 & $\mathrm{AF}$ & Sebastian County & 0.982231 & 0.335065 & 0.070430 & 588 & 3503 & 106143 & 0 \\
\hline 2294 & $A F$ & Sevier County & 0.970059 & 0.363915 & 0.003442 & 1678 & 2837 & 95468 & 0 \\
\hline 2295 & AR & Sharp County & 0.996715 & 0.411081 & 0.023517 & 374 & 1721 & 78190 & 0 \\
\hline 2296 & Af & Stone County & 0.995346 & 0.351087 & 0.006418 & 565 & 1957 & 70895 & 0 \\
\hline 2297 & Al & Union County & 0.984489 & 0.046905 & 0.001604 & 3118 & 4866 & 110579 & 1 \\
\hline 2298 & A & Van Buren County & 0.982325 & 0.263354 & 0.349105 & 350 & 2114 & 84238 & 0 \\
\hline 2299 & Af & Washington County & 0.993906 & 0.579328 & 0.026474 & 1995 & 4468 & 99810 & 0 \\
\hline 2300 & A & White County & 0.992056 & 0.542269 & 0.100121 & 359 & 2037 & 80527 & 0 \\
\hline 2301 & $A F$ & Woodruff County & 0.987392 & 0.732086 & 0.000000 & 472 & 2017 & 81339 & 0 \\
\hline 2302 & A & Yell County & 0.977871 & 0.320556 & 0.010463 & 1177 & 2538 & 85593 & 0 \\
\hline 2303 & $\mathrm{~L}$ & Acadia Parish & 0.996452 & 0.637841 & 0.004581 & 417 & 2671 & 74397 & 1 \\
\hline
\end{tabular}




\begin{tabular}{|c|c|c|c|c|c|c|c|c|c|}
\hline $\begin{array}{l}\text { County } \\
\text { Code }\end{array}$ & State & $\begin{array}{l}\text { County } \\
\text { Name }\end{array}$ & $\begin{array}{l}\text { FRCLND } \\
\text { Absolute }\end{array}$ & $\begin{array}{l}\text { FRMFRC } \\
\text { Absolute }\end{array}$ & $\begin{array}{c}\text { DPF } \\
\text { Absolute }\end{array}$ & $\begin{array}{l}\text { ASFP } \\
\text { \$/ha }\end{array}$ & $\begin{array}{l}\text { VFRM } \\
\text { \$/ha }\end{array}$ & $\begin{array}{l}\text { VNFRM } \\
\text { \$/person }\end{array}$ & Notes \\
\hline 2304 & LA & Allen Parish & 0.998502 & 0.240327 & 0.025641 & 219 & 2372 & 66262 & 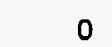 \\
\hline 2305 & LA & Ascension Parish & 0.962522 & 0.340017 & 0.013729 & 586 & 6147 & 90930 & 1 \\
\hline 2306 & LA & Assumption Parish & 0.929047 & 0.313350 & 0.000000 & 742 & 2989 & 77391 & 0 \\
\hline 2307 & LA & Avoyelles Parish & 0.961605 & 0.480703 & 0.011808 & 481 & 2679 & 68267 & 0 \\
\hline 2308 & LA & Beauregard Parish & 0.994948 & 0.183883 & 0.242999 & 188 & 2477 & 83974 & 0 \\
\hline 2309 & LA & Bienville Parish & 0.986471 & 0.099066 & 0.185084 & 265 & 2764 & 81827 & 0 \\
\hline 2310 & LA & Bossier Parish & 0.967072 & 0.206176 & 0.046507 & 298 & 2489 & 94981 & 1 \\
\hline 2311 & LA & Caddo Parish & 0.941418 & 0.301748 & 0.047170 & 318 & 2245 & 110654 & 1 \\
\hline 2312 & LA & Calcasieu Parish & 0.978786 & 0.477720 & 0.000000 & 117 & 2318 & 101381 & 0 \\
\hline 2313 & LA & Caldwell Parish & 0.979094 & 0.195895 & 0.000000 & 387 & 2495 & 79456 & 0 \\
\hline 2314 & LA & Cameron Parish & 0.679709 & 0.307059 & 0.028065 & 71 & 2013 & 85566 & 1 \\
\hline 2315 & LA & Catahoula Parish & 0.951606 & 0.556441 & 0.000000 & 468 & 1980 & 68497 & 0 \\
\hline 2316 & LA & Claiborne Parish & 0.983185 & 0.131843 & 0.113607 & 720 & 2219 & 75792 & 0 \\
\hline 2317 & LA & Concordia Parish & 0.930075 & 0.510896 & 0.000000 & 489 & 2011 & 84773 & 0 \\
\hline 2318 & LA & De Soto Parish & 0.980673 & 0.263294 & 0.694077 & 312 & 2284 & 79931 & 0 \\
\hline 2319 & LA & East Baton Rouge Parish & 0.968300 & 0.271407 & 0.266113 & 278 & 6333 & 121112 & 0 \\
\hline 2320 & LA & East Camoll Parish & 0.952510 & 0.719364 & 0.000000 & 666 & 2562 & 73665 & 0 \\
\hline 2321 & LA & East Feliciana Parish & 0.994985 & 0.471440 & 0.191288 & 153 & 2726 & 82335 & 0 \\
\hline 2322 & LA & Evangeline Parish & 0.977443 & 0.416200 & 0.014152 & 418 & 2576 & 75840 & 0 \\
\hline 2323 & LA & Franklin Parish & 0.981022 & 0.662476 & 0.002750 & 612 & 2057 & 65937 & 0 \\
\hline 2324 & LA & Grant Parish & 0.970685 & 0.107756 & 0.000000 & 252 & 2271 & 68992 & 0 \\
\hline 2325 & LA & Iberia Parish & 0.557918 & 0.299291 & 0.009711 & 954 & 5169 & 83317 & \\
\hline 2326 & LA & Iberville Parish & 0.947609 & 0.205581 & 0.000000 & 824 & 3820 & 87110 & 0 \\
\hline 2327 & LA & Jackson Parish & 0.982050 & 0.046839 & 0.013545 & 2206 & 3452 & 81996 & 1 \\
\hline 2328 & LA & Jefferson Parish & 0.476207 & 0.021078 & 0.000000 & 850 & 4932 & 115828 & 0 \\
\hline 2329 & LA & Jefferson Davis Parish & 0.990488 & 0.698250 & 0.000000 & 322 & 2208 & 75609 & 0 \\
\hline 2330 & LA & Lafayette Parish & 0.998313 & 044 & 0.022436 & 517 & 4777 & 114250 & 0 \\
\hline 2331 & LA & Lafourche Parish & 0.736817 & 0.191101 & 0.007630 & 504 & 3328 & 87300 & 1 \\
\hline 2332 & LA & La Salle Parish & 0.941830 & 0.068795 & 0.000000 & 123 & 2569 & 78528 & 0 \\
\hline 2333 & LA & Lincoin Parish & 0.998111 & 0.194664 & 0.044521 & 1053 & 3074 & 86108 & 0 \\
\hline 2334 & LA & Livingston Parish & 0.922007 & 0.086939 & 0.059805 & 674 & 3563 & 75955 & 0 \\
\hline 2335 & LA & Madison Parish & 0.959344 & 0.617192 & 0.000000 & 550 & 1948 & 62374 & 0 \\
\hline 2336 & LA & Morehouse Parish & 0.986401 & 0.475510 & 0.005692 & 742 & 2411 & 83669 & 1 \\
\hline 2337 & $\overrightarrow{L A}$ & Natchitoches Parish & 0.966939 & 0.227089 & 0.024781 & 564 & 2038 & 72575 & 1 \\
\hline 2338 & LA & Orleans Parish & 5796 & 0.000865 & 0.000000 & 10798 & 81900 & 112286 & 0 \\
\hline 2339 & LA & Ouachita Parish & 0.965600 & 0.190975 & 0.011272 & 607 & 3055 & 93937 & \\
\hline 2340 & LA & Plaquemines Parish & 0.347772 & 0.085298 & 0.000000 & 134 & 3617 & 100149 & 0 \\
\hline 2341 & $\overrightarrow{L A}$ & Pointe Coupee Parish & 0.943554 & 0.541403 & 0.000000 & 558 & 2887 & 82728 & 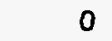 \\
\hline 2342 & LA & Rapides Parish & 0.971090 & 0.248752 & 0.019445 & 531 & 3017 & 98990 & 0 \\
\hline 2343 & $\overrightarrow{L A}$ & Red River Parish & 0.966421 & 0.392611 & 0.000000 & 214 & 1498 & 75460 & 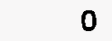 \\
\hline 2344 & LA & Richland Parish & 9281 & 318 & 7084 & 583 & 2418 & 82647 & 1 \\
\hline 2345 & LA & Sabine Parish & 426 & 46 & 1732 & 1603 & 3218 & 78989 & 0 \\
\hline 2346 & LA & St. Bernard Parish & 0.259312 & 0.020712 & 1000 & 180 & 5209 & 93023 & , \\
\hline 2347 & LA & St. Charles Parish & 0.691539 & 0.127712 & 0.000000 & 432 & 3090 & 109502 & 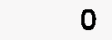 \\
\hline 2348 & LA & St. Helena Parish & 0.997326 & 0.192519 & 0.460822 & 1113 & 3146 & 59584 & 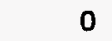 \\
\hline 2349 & LA & St. James Parish & 0.954767 & 0.272460 & 0.000000 & 1124 & 4874 & 100088 & 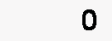 \\
\hline 2350 & LA & St. John the Baptist Parish & 0.629306 & 0.123815 & 0.000000 & 635 & 3635 & 90402 & 0 \\
\hline 2351 & LA & St. Landry Parish & 0.989158 & 0.476363 & 262 & 416 & 2593 & 76273 & 0 \\
\hline 2352 & LA & St. Martin Parish & 179 & 799 & 336 & 689 & 3595 & 73184 & 0 \\
\hline 2353 & LA & St. Mary Parish & 0.547788 & 0.208390 & 0.000000 & 803 & 4057 & 84177 & 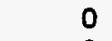 \\
\hline 2354 & LA & St. Tamm & 0.760092 & 0.073478 & 0.124129 & 679 & 6640 & 116018 & \\
\hline 2355 & LA & Tangipahoa Parish & 0.960062 & 0.250863 & 0.715459 & 1200 & 4382 & 79274 & 0 \\
\hline 2356 & LA & Tensas Parish & 0.939619 & 0.637895 & 0.003573 & 581 & 2085 & 81610 & \\
\hline 2357 & LA & Terrebonne Parish & 0.603373 & 0.054960 & 0.000000 & 464 & 3418 & 85566 & 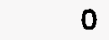 \\
\hline 2358 & LA & Union Parish & 0.969428 & 0.110168 & 0.026775 & 2383 & 3302 & 83974 & 0 \\
\hline 2359 & LA & Vermilion Parish & 3034 & 0.421539 & 0.005909 & 357 & $289+2$ & 79870 & $u$ \\
\hline 2360 & LA & Ver & 236 & 922 & 255 & 498 & 3099 & 65043 & 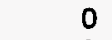 \\
\hline 2361 & LA & Washington Parish & 0.990532 & 0.271196 & 0.736909 & 823 & 3197 & 77099 & 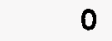 \\
\hline 2362 & LA & Webster Parish & 0.968788 & 0.154145 & 0.229665 & 149 & 2389 & 88959 & \\
\hline 2363 & LA & West Baton Rouge Parish & 0.938998 & 0.315128 & 0.000000 & 759 & 4117 & 94567 & 0 \\
\hline 2364 & LA & West Carroll Parish & 0.997442 & 0.551394 & 0.020784 & 581 & 1952 & 64921 & \\
\hline 2365 & LA & West Feliciana Parish & 0.953037 & 0.334237 & 0.060502 & 194 & 2719 & 66330 & \\
\hline 2366 & LA & Winn Parish & 0.993281 & 0.037953 & 0.000000 & 315 & 2338 & 68897 & \\
\hline 2367 & OK & Adair County & 0.997643 & 0.562717 & 0.094086 & 1053 & 2073 & 74309 & 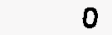 \\
\hline 2368 & OK & Alfalfa County & 0.983216 & 0.879113 & 0.006104 & 467 & 1860 & 129388 & 0 \\
\hline 2369 & OK & Atoka County & 0.988194 & 0.599986 & 0.039436 & 117 & 1002 & 60200 & \\
\hline 2370 & OK & Beaver County & 0.998227 & 0.849616 & 0.016307 & 199 & 785 & 131773 & \\
\hline 2371 & OK & Beckham County & 0.997447 & 0.855212 & 0.063305 & 129 & 1137 & 88593 & \\
\hline 2372 & OK & Blaine County & 0.988963 & 0.864545 & 0.008407 & 342 & 1693 & 103847 & 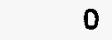 \\
\hline 2373 & OK & Bryan County & 0.963290 & 0.709469 & 0.060914 & 204 & 1440 & 77750 & \\
\hline
\end{tabular}




\begin{tabular}{|c|c|c|c|c|c|c|c|c|c|}
\hline $\begin{array}{l}\text { County } \\
\text { Code }\end{array}$ & State & $\begin{array}{l}\text { County } \\
\text { Name }\end{array}$ & $\begin{array}{l}\text { FRCLND } \\
\text { Absolute }\end{array}$ & $\begin{array}{l}\text { FRMFRC } \\
\text { Absolute }\end{array}$ & $\begin{array}{c}\text { DPF } \\
\text { Absolute }\end{array}$ & $\begin{array}{l}\text { ASFP } \\
\text { \$/ha }\end{array}$ & $\begin{array}{l}\text { VFRM } \\
\text { S/ha }\end{array}$ & $\begin{array}{l}\text { VNFRM } \\
\text { \$/person }\end{array}$ & Notes \\
\hline $\begin{array}{l}2374 \\
2375\end{array}$ & $\begin{array}{l}\text { OK } \\
\text { OK }\end{array}$ & Caddo County & 0.990717 & 0.887908 & 0.007909 & $\begin{array}{l}302 \\
356\end{array}$ & 1689 & $\begin{array}{r}89474 \\
105662\end{array}$ & 0 \\
\hline $\begin{array}{l}2375 \\
2376\end{array}$ & $\begin{array}{l}\text { OK } \\
\text { OK }\end{array}$ & $\begin{array}{l}\text { Canadian County } \\
\text { Carter County }\end{array}$ & $\begin{array}{l}0.994089 \\
0.988094\end{array}$ & $\begin{array}{l}0.867940 \\
0.707235\end{array}$ & 0.053348 & $\begin{array}{l}356 \\
132\end{array}$ & $\begin{array}{l}2209 \\
1317\end{array}$ & $\begin{array}{l}105662 \\
105039\end{array}$ & 0 \\
\hline $\begin{array}{l}2376 \\
2377\end{array}$ & $\begin{array}{l}\text { OK } \\
\text { OK }\end{array}$ & $\begin{array}{l}\text { Carter County } \\
\text { Cherokee County }\end{array}$ & & 0.707235 & 0.004543 & 132 & 1317 & 105039 & 1 \\
\hline 2377 & OK & & 0.967331 & 0.455179 & 0.049212 & 878 & 1901 & 77323 & $\mathbf{0}$ \\
\hline 2378 & OK & Choctaw County & 0.966590 & 0.607317 & 0.030152 & 185 & 1155 & 73408 & $\mathbf{0}$ \\
\hline 2379 & OK & Cimarron County & 0.996671 & 0.881214 & 0.000512 & 424 & 568 & 166661 & 1 \\
\hline 2380 & OK & Cleveland County & 0.960199 & 0.457833 & 0.137787 & 199 & 2732 & 97825 & 0 \\
\hline 2381 & OK & Coal County & 0.994102 & 0.798632 & 0.105639 & 124 & 1034 & 69588 & 0 \\
\hline 2382 & OK & Comanche County & 0.986641 & 0.592942 & 0.162405 & 187 & 1480 & 89596 & 0 \\
\hline 2383 & OK & Cotton County & 0.991738 & 0.879662 & 0.003009 & 208 & 1271 & 99431 & 1 \\
\hline 2384 & OK & Craig County & 0.997792 & 0.918111 & 0.031580 & 298 & 1197 & 97128 & 0 \\
\hline 2385 & OK & Creek County & 0.985332 & 0.549862 & 0.147087 & 91 & 1382 & 98733 & 0 \\
\hline 2386 & OK & Custer County & 0.984541 & 1.003900 & 0.016665 & 254 & 1534 & 98076 & 9 \\
\hline 2387 & OK & Delaware County & 0.934848 & 0.510667 & 0.072922 & 752 & 2055 & 72995 & 0 \\
\hline 2388 & OK & Dewey County & 0.991936 & 0.910895 & 0.016520 & 149 & 1056 & 111534 & 0 \\
\hline 2389 & OK & Ellis County & 0.997808 & 0.889163 & 0.057426 & 119 & 762 & 115050 & 0 \\
\hline 2390 & OK & Garfield County & 0.998584 & 0.977384 & 0.013820 & 288 & 1813 & 112889 & 0 \\
\hline 2391 & OK & Garvin County & 0.994391 & 0.810561 & 0.050400 & 190 & 1570 & 97791 & 0 \\
\hline 2392 & OK & Grady County & 0.996081 & 0.803425 & 0.342118 & 345 & 1642 & 87469 & 0 \\
\hline 2393 & OK & Grant County & 0.996964 & 0.936189 & 0.012319 & 224 & 1507 & 146816 & 0 \\
\hline 2394 & OK & Greer County & 0.993312 & 0.827251 & 0.002514 & 132 & 1000 & 88438 & 1 \\
\hline 2395 & OK & Harmon County & 0.998632 & 0.831934 & 0.004550 & 172 & 931 & 103704 & 1 \\
\hline 2396 & OK & Harper County & 0.998117 & 0.913051 & 0.009757 & 359 & 811 & 132612 & 0 \\
\hline 2397 & OK & Haskell County & 0.922863 & 0.725762 & 0.007878 & 165 & 1330 & 80940 & 0 \\
\hline 2398 & OK & Hughes County & 0.990298 & 0.671202 & 0.017688 & 112 & 1055 & 77011 & 0 \\
\hline 2399 & OK & Jackson County & 0.998169 & 0.914596 & 0.001996 & 263 & 1405 & 90734 & 0 \\
\hline 2400 & OK & Jefferson County & 0.980592 & 0.834453 & 0.001366 & 183 & 1082 & 88891 & 0 \\
\hline 2401 & OK & Johnston County & 0.979072 & 0.784301 & 0.056588 & 207 & 1169 & 70211 & 0 \\
\hline 2402 & OK & Kay County & 0.972061 & 0.812298 & 0.018111 & 243 & 1777 & 118172 & 0 \\
\hline 2403 & OK & Kingfisher County & 0.996733 & 0.902861 & 0.044774 & 361 & 1813 & 109252 & 0 \\
\hline 2404 & OK & Kjowa County & 0.984420 & 0.859741 & 0.004949 & 203 & 1307 & 105174 & 1 \\
\hline 2405 & OK & Latimer County & 0.990471 & 0.420264 & 0.000000 & 112 & 1272 & 73584 & 0 \\
\hline 2406 & OK & Le Flore County & 0.986251 & 0.374883 & 0.008665 & 431 & 1916 & 82233 & 0 \\
\hline 2407 & OK & Lincoln County & 0.992713 & 0.648553 & 0.224954 & 129 & 1527 & 81983 & 0 \\
\hline 2408 & OK & Logan County & 0.994202 & 0.722420 & 0.035062 & 260 & 1799 & 90761 & 0 \\
\hline 2409 & OK & Love County & 0.968865 & 0.774649 & 0.004715 & 186 & 1472 & 88986 & 1 \\
\hline 2410 & OK & McClain County & 0.981920 & 0.695700 & 0.149984 & 309 & 2072 & 95387 & 0 \\
\hline 2411 & OK & McCurtain County & 0.974195 & 0.265694 & 0.001257 & 718 & 1936 & 75724 & 0 \\
\hline 2412 & OK & Melntosh County & 0.870212 & 0.596668 & 0.105869 & 132 & 1418 & 74248 & 0 \\
\hline 2413 & OK & Major County & 0.998846 & 0.807165 & 0.038070 & 233 & 1453 & 105770 & 0 \\
\hline 2414 & OK & Marshall County & 0.869207 & 0.684929 & 0.000000 & 145 & 1766 & 87869 & 0 \\
\hline 2415 & OK & Mayes County & 0.959959 & 0.661927 & 0.310805 & 245 & 2039 & 86020 & 0 \\
\hline 2416 & OK & Murray County & 0.984407 & 0.862194 & 0.156216 & 205 & 1231 & 77878 & 0 \\
\hline 2417 & OK & Muskogee County & 0.970035 & 0.667106 & 0.085371 & 264 & 1788 & 91784 & 0 \\
\hline 2418 & OK & Noble County & 0.985811 & 0.834565 & 0.015426 & 209 & 1354 & 99654 & 0 \\
\hline 2419 & OK & Nowata County & 0.972563 & 0.781702 & 0.053489 & 231 & 1152 & 86026 & 0 \\
\hline 2420 & OK & Okfuskee County & 0.993393 & 0.644162 & 0.009567 & 118 & 1054 & 71843 & 0 \\
\hline 2421 & OK & Oklahoma County & 0.987153 & 0.345367 & 0.054477 & 171 & 2986 & 120163 & 0 \\
\hline 2422 & OK & Okmulgee County & 0.992394 & 0.628869 & 0.019806 & 144 & 1503 & 81150 & 0 \\
\hline 2423 & OK & Osage County & 0.976999 & 0.774735 & 0.012996 & 183 & 854 & 78820 & 0 \\
\hline 2424 & OK & Ottawa County & 0.972324 & 0.686583 & 0.072513 & 505 & 2088 & 82877 & 0 \\
\hline 2425 & OK & Pawnee County & 0.957254 & 0.774298 & 0.002374 & 306 & 1213 & 94710 & 0 \\
\hline 2426 & OK & Payne County & 0.984522 & 0.748881 & 0.220820 & 201 & 1748 & 94344 & 0 \\
\hline 2427 & OK & Pittsburg County & 0.947816 & 0.575752 & 0.038476 & 127 & 1091 & 83541 & 0 \\
\hline 2428 & OK & Pontotoc County & 0.992015 & 0.766467 & 0.080141 & 207 & 1368 & 90104 & 0 \\
\hline 2429 & OK & Pottawatomie County & 0.993061 & 0.593484 & 0.124968 & 130 & 1695 & 92095 & 0 \\
\hline 2430 & OK & Pushmataha County & 0.982100 & 0.268324 & 0.018209 & 105 & 1099 & 66201 & 0 \\
\hline 2431 & OK & Roger Mills County & 0.996002 & 0.903357 & 0.106380 & 107 & 975 & 102174 & 0 \\
\hline 2432 & OK & Rogers County & 0.948712 & 0.716701 & 0.132203 & 184 & 2460 & 100555 & 0 \\
\hline 2433 & OK & Seminole County & 0.987467 & 0.619932 & 0.065244 & 95 & 1240 & 82261 & 0 \\
\hline 2434 & OK & Sequoyah County & 0.942581 & 0.499030 & 0.026002 & 131 & 1642 & 72676 & 0 \\
\hline 2435 & OK & Stephens County & 0.984244 & 0.750357 & 0.055140 & 132 & 1169 & 94154 & 0 \\
\hline 2436 & OK & Texas County & 0.994312 & 0.806349 & 0.000393 & 1185 & 977 & 130431 & 0 \\
\hline 2437 & OK & Tillman County & 0.992151 & 0.861410 & 0.003368 & 208 & 1233 & 97006 & \\
\hline 2438 & OK & Tulsa County & 0.971523 & 0.367375 & 0.013408 & 252 & 3899 & 130689 & 0 \\
\hline 2439 & OK & Wagoner County & 0.952708 & 0.601172 & 0.091730 & 227 & 2347 & 99329 & te \\
\hline 2440 & OK & Washington County & 0.982717 & 0.810624 & 0.004049 & 206 & 1795 & 135213 & 0 \\
\hline 2441 & Or & Washita County & 0.994402 & 0.899509 & 0.014415 & 298 & 1601 & 99796 & 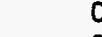 \\
\hline 2442 & 0 & Woods County & 0.997294 & 0.902979 & 0.001409 & 226 & 1166 & 126591 & 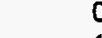 \\
\hline 2443 & & Woodward County & 0.997021 & 0.864391 & 0.001357 & 120 & 890 & 101205 & \\
\hline
\end{tabular}




\begin{tabular}{|c|c|c|c|c|c|c|c|c|c|}
\hline $\begin{array}{l}\text { County } \\
\text { Code }\end{array}$ & State & $\begin{array}{l}\text { County } \\
\text { Name }\end{array}$ & $\begin{array}{l}\text { FRCLND } \\
\text { Absolute }\end{array}$ & $\begin{array}{l}\text { FRMFRC } \\
\text { Absolute }\end{array}$ & $\begin{array}{c}\text { DPF } \\
\text { Absolute }\end{array}$ & $\begin{array}{l}\text { ASFP } \\
\text { \$/ha }\end{array}$ & $\begin{array}{l}\text { VFRM } \\
\text { \$/ha }\end{array}$ & $\begin{array}{l}\text { VNFRM } \\
\text { \$/person }\end{array}$ & Notes \\
\hline 2444 & $\mathrm{TX}$ & Anderson County & 0.993400 & 0.514284 & 0.086027 & 182 & 2363 & 84800 & 0 \\
\hline 2445 & $T X$ & Andrews County & 0.999760 & 1.002194 & 0.015915 & 26 & 317 & 103738 & 13 \\
\hline 2446 & $T X$ & Angelina County & 0.927255 & 0.200887 & 0.010473 & 424 & 3756 & 102174 & 0 \\
\hline 2447 & $T X$ & Aransas County & 0.477181 & 0.118641 & 0.000000 & 46 & 2960 & 95956 & 0 \\
\hline 2448 & TX & Archer County & 0.982624 & 1.052326 & 0.349618 & 212 & 874 & 110559 & 9 \\
\hline 2449 & $\mathrm{TX}$ & Armstrong County & 0.999806 & 0.856201 & 0.000000 & 153 & 548 & 147249 & 0 \\
\hline 2450 & $T X$ & Atascosa County & 0.997156 & 0.970231 & 0.065235 & 176 & 1528 & 78948 & 0 \\
\hline 2451 & $T X$ & Austin County & 0.994240 & 0.807641 & 0.002805 & 256 & 4261 & 122730 & 0 \\
\hline 2452 & $T X$ & Bailey County & 0.999172 & 0.818225 & 0.013679 & 399 & 984 & 133148 & 1 \\
\hline 2453 & $T X$ & Bandera County & 0.992689 & 0.782479 & 0.067219 & 30 & 1821 & 105824 & 1 \\
\hline 2454 & TX & Bastrop County & 0.991588 & 0.694542 & 0.012385 & 143 & 3036 & 89677 & 0 \\
\hline 2455 & $T X$ & Baylor County & 0.966437 & 0.642223 & 0.000000 & 204 & 815 & 116526 & 0 \\
\hline 2456 & $\mathrm{TX}$ & Bee County & 0.999807 & 0.784908 & 0.041026 & 109 & 1558 & 85038 & 1 \\
\hline 2457 & $T X$ & Bell County & 0.974060 & 0.614724 & 0.011326 & 202 & 2849 & 91682 & 0 \\
\hline 2458 & $T X$ & Bexar County & 0.992176 & 0.512147 & 0.038881 & 274 & 3903 & 105039 & 0 \\
\hline 2459 & $T X$ & Blanco County & 0.996966 & 0.815537 & 0.032561 & 98 & 2127 & 120902 & 1 \\
\hline 2460 & $T X$ & Borden County & 0.992004 & 1.094580 & 0.006847 & 91 & 430 & 168632 & 13 \\
\hline 2461 & $T X$ & Bosque County & 0.986596 & 0.865261 & 0.039399 & 174 & 1920 & 99051 & 0 \\
\hline 2462 & $\mathrm{TX}$ & Bowie County & 0.962193 & 0.462934 & 0.121977 & 340 & 2082 & 102621 & 0 \\
\hline 2463 & $\mathrm{TX}$ & Brazoria County & 0.868137 & 0.635408 & 0.007360 & 190 & 2713 & 121586 & 1 \\
\hline 2464 & $T X$ & Brazos County & 0.992345 & 0.788418 & 0.023845 & 278 & 2723 & 85620 & 0 \\
\hline 2465 & $\mathrm{TX}$ & Brewster County & 0.999971 & 0.606784 & 0.026220 & 12 & 277 & 82105 & 1 \\
\hline 2466 & $T X$ & Briscoe County & 0.998521 & 0.709525 & 0.021363 & 90 & 527 & 135030 & 1 \\
\hline 2467 & $\mathrm{TX}$ & Brooks County & 0.999653 & 0.938151 & 0.052148 & 83 & 1172 & 67651 & 0 \\
\hline 2468 & $\mathrm{TX}$ & Brown County & 0.986374 & 0.850005 & 0.195161 & 153 & 1323 & 93998 & 0 \\
\hline 2469 & $T X$ & Burleson County & 0.981974 & 0.744580 & 0.009105 & 241 & 2633 & 89115 & 0 \\
\hline 2470 & $T X$ & Burnet County & 0.975609 & 0.860850 & 0.008010 & 59 & 2455 & 109137 & 0 \\
\hline 2471 & $T X$ & Caldwell County & 0.996946 & 0.755574 & 0.004643 & 288 & 2380 & 86643 & 0 \\
\hline 2472 & $T X$ & Calhoun County & 0.496356 & 0.634541 & 0.022508 & 168 & 1670 & 104422 & 1 \\
\hline 2473 & $T X$ & Callahan County & 0.997072 & 0.853213 & 0.026161 & 102 & 1353 & 87991 & 0 \\
\hline 2474 & $T X$ & Cameron County & 0.709458 & 0.568165 & 0.000170 & 617 & 3281 & 65890 & 0 \\
\hline 2475 & TX & Camp County & 0.972015 & 0.541445 & 0.032682 & 4813 & 2908 & 122277 & 0 \\
\hline 2476 & TX & Carson County & 0.999018 & 1.052898 & 0.006915 & 275 & 1042 & 121159 & 13 \\
\hline 2477 & $\mathrm{TX}$ & Cass County & 0.976113 & 0.278242 & 0.006701 & 276 & 2428 & 92637 & 0 \\
\hline 2478 & TX & Castro County & 0.998929 & 0.901434 & 0.002417 & 2343 & 1445 & 117698 & 0 \\
\hline 2479 & $T X$ & Chambers County & 0.690041 & 0.654989 & 0.000000 & 147 & 1425 & 89975 & 0 \\
\hline 2480 & $T X$ & Cherokee County & 0.990863 & 0.399632 & 0.296883 & 769 & 3187 & 89664 & 0 \\
\hline 2481 & $\mathrm{TX}$ & Childress County & 0.995414 & 0.989719 & 0.000000 & 108 & 676 & 97615 & 0 \\
\hline 2482 & $T X$ & Clay County & 0.983553 & 0.954200 & 0.221002 & 169 & 1081 & 101327 & 0 \\
\hline 2483 & TX & Cochran County & 0.999887 & 0.746880 & 0.000000 & 300 & 858 & 116729 & 0 \\
\hline 2484 & $T X$ & Coke County & 0.968570 & 0.909183 & 0.000000 & 51 & 820 & 101618 & 0 \\
\hline 2485 & $\mathrm{TX}$ & Coleman County & 0.993283 & 0.835375 & 0.014696 & 70 & 931 & 99248 & 0 \\
\hline 2486 & TX & Collin County & 0.956883 & 0.508054 & 0.084832 & 250 & 4610 & 151063 & 0 \\
\hline 2487 & $T X$ & Collingsworth County & 0.999301 & 0.786543 & 0.000888 & 144 & 891 & 93985 & 0 \\
\hline 2488 & $T X$ & Colorado County & 0.989068 & 0.891011 & 0.012404 & 202 & 2258 & 109435 & 0 \\
\hline 2489 & $\mathrm{TX}$ & Comal County & 0.977119 & 0.577001 & 0.002626 & 59 & 3408 & 120854 & 0 \\
\hline 2490 & $\mathrm{TX}$ & Comanche County & 0.989470 & 0.905995 & 0.352257 & 463 & 1699 & 98591 & 0 \\
\hline 2491 & $\mathrm{TX}$ & Concho County & 0.997755 & 0.900892 & 0.002962 & 74 & 1070 & 105079 & 0 \\
\hline 2492 & $T X$ & Cooke County & 0.972099 & 0.769593 & 0.249629 & 224 & 2459 & 93795 & 0 \\
\hline 2493 & $T X$ & Coryell County & 0.995307 & 0.899085 & 0.018095 & 108 & 1612 & 82091 & 1 \\
\hline 2494 & $T X$ & Cottle County & 0.999538 & 0.817441 & 0.013934 & 60 & 679 & 125128 & 1 \\
\hline 2495 & $\mathrm{TX}$ & Crane County & 0.999963 & 0.785212 & 0.000000 & 18 & 290 & 92603 & 0 \\
\hline 2496 & $T X$ & Crockett County & 0.999996 & 1.113672 & 0.000000 & 19 & 323 & 106170 & 9 \\
\hline 2497 & $T X$ & Crosby County & 0.997588 & 0.784378 & 0.004496 & 194 & 1599 & 108114 & 1 \\
\hline 2498 & $\mathrm{TX}$ & Culberson County & 0.999936 & 0.649297 & 0.011585 & 8 & 267 & 75609 & 0 \\
\hline 2499 & $\mathrm{TX}$ & Dallam County & 0.999625 & 0.810867 & 0.003191 & 895 & 991 & 133534 & 0 \\
\hline 2500 & $\mathrm{TX}$ & Dallas County & 0.968107 & 0.219766 & 0.059472 & 312 & 4336 & 142244 & 0 \\
\hline 2501 & $\mathrm{TX}$ & Dawson County & 0.999934 & 0.957911 & 0.000000 & 219 & 1182 & 98767 & 0 \\
\hline 2502 & $T X$ & Deaf Smith County & 0.999383 & 0.893929 & 0.000494 & 1860 & 990 & 117556 & 1 \\
\hline 2503 & $T X$ & Delta County & 0.997243 & 0.606375 & 0.191185 & 196 & 2029 & 93165 & 0 \\
\hline 2504 & $T X$ & Denton County & 0.927716 & 0.642976 & 0.019462 & 296 & 4583 & 120570 & 0 \\
\hline 2505 & $T X$ & DeWitt County & 0.998602 & 0.978130 & 0.115277 & 135 & 1701 & 93646 & 0 \\
\hline 2508 & $T X$ & Dickens County & 0.998891 & 0.970269 & 0.026477 & 53 & 395 & 110552 & 1 \\
\hline 2507 & TX & Dimmit County & 0.997323 & 0.795108 & 0.011389 & 51 & 1082 & 55628 & 1 \\
\hline 2508 & $T X$ & Donley County & 0.996490 & 1.007659 & 0.002929 & 224 & 742 & 135782 & 13 \\
\hline 2509 & $T X$ & Duval County & 0.998347 & 0.698222 & 0.120636 & 55 & 1077 & 80818 & 0 \\
\hline 2510 & TX & Eastland County & 0.993681 & 0.832174 & 0.015052 & 148 & 1383 & 87042 & 0 \\
\hline 2511 & TX & Ector County & 0.999306 & 0.899543 & 0.000000 & 23 & 307 & 100013 & 0 \\
\hline 2512 & TX & Edwards County & 0.999909 & 0.823381 & 0.000000 & 21 & 566 & 86751 & 0 \\
\hline 2513 & $T X$ & Ellis County & 0.987658 & 0.708437 & 0.098998 & 181 & 3454 & 107951 & 0 \\
\hline
\end{tabular}




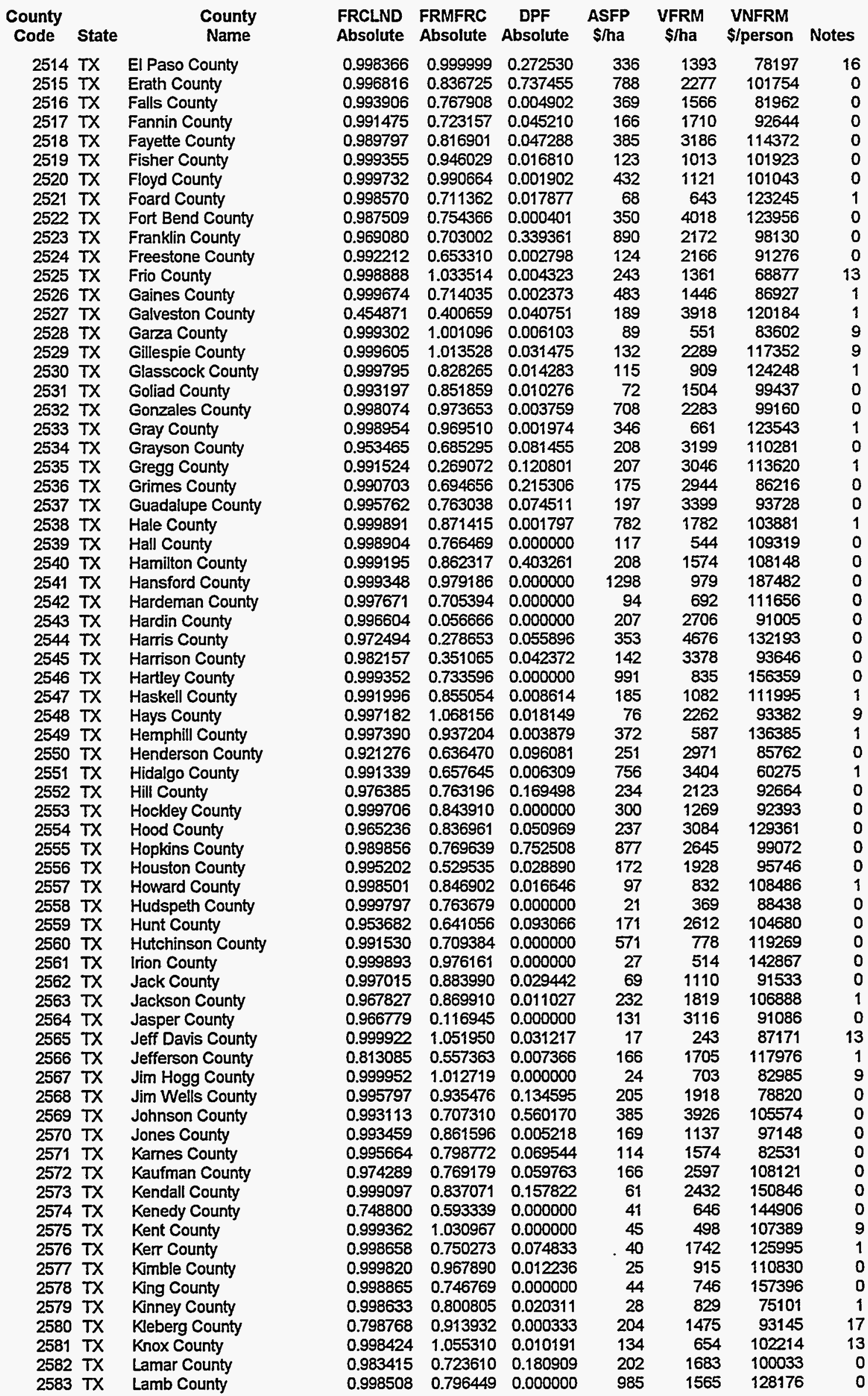




\begin{tabular}{|c|c|c|c|c|c|c|c|c|c|}
\hline $\begin{array}{l}\text { County } \\
\text { Code }\end{array}$ & State & $\begin{array}{l}\text { County } \\
\text { Name }\end{array}$ & $\begin{array}{l}\text { FRCLND } \\
\text { Absolute }\end{array}$ & $\begin{array}{l}\text { FRMFRC } \\
\text { Absolute }\end{array}$ & $\begin{array}{c}\text { DPF } \\
\text { Absolute }\end{array}$ & $\begin{array}{l}\text { ASFP } \\
\text { S/ha }\end{array}$ & $\begin{array}{l}\text { VFRM } \\
\text { \$/ha }\end{array}$ & $\begin{array}{l}\text { UNFRM } \\
\text { \$/person }\end{array}$ & Notes \\
\hline 2584 & TX & Lampasas County & 0.997308 & 0.948736 & 0.056658 & 70 & 1675 & 94175 & 0 \\
\hline 2585 & TX & La Salle County & 0.996401 & 0.803857 & 0.013414 & 38 & 877 & 62354 & 1 \\
\hline 2586 & TX & Lavaca County & 0.999539 & 0.850262 & 0.064430 & 178 & 2322 & 108581 & 0 \\
\hline 2587 & $\mathrm{TX}$ & Lee County & 0.991311 & 0.792112 & 0.015681 & 193 & 2788 & 95841 & 0 \\
\hline 2588 & $T X$ & Leon County & 0.992283 & 0.702698 & 0.022342 & 122 & 2348 & 99776 & 0 \\
\hline 2589 & TX & Liberty County & 0.985971 & 0.461024 & 0.004077 & 156 & 2027 & 95597 & 0 \\
\hline 2590 & $T X$ & Limestone County & 0.973919 & 0.735909 & 0.024874 & 171 & 1670 & 88648 & 0 \\
\hline 2591 & $T X$ & Lipscomb County & 0.999880 & 0.986444 & 0.004003 & 167 & 519 & 124214 & 1 \\
\hline 2592 & $T X$ & Live Oak County & 0.960576 & 0.842087 & 0.013352 & 72 & 1261 & 93355 & 0 \\
\hline 2593 & $\mathrm{TX}$ & Llano County & 0.967511 & 0.847610 & 0.027747 & 56 & 1708 & 124539 & 1 \\
\hline 2594 & TX & Loving County & 0.994433 & 0.804683 & 0.000000 & 7 & 466 & 170658 & 0 \\
\hline 2595 & $T X$ & Lubbock County & 0.998736 & 0.836378 & 0.000278 & 684 & 2063 & 104599 & 0 \\
\hline 2596 & TX & Lynn County & 0.998237 & 0.860171 & 0.006435 & 249 & 1178 & 114806 & 1 \\
\hline 2597 & TX & McCulloch County & 0.996230 & 0.987615 & 0.007466 & 78 & 1156 & 90829 & 1 \\
\hline 2598 & $\mathrm{TX}$ & McLennan County & 0.982698 & 0.708322 & 0.124392 & 358 & 2301 & 101090 & 0 \\
\hline 2599 & $T X$ & McMullen County & 0.974090 & 0.724273 & 0.000000 & 48 & 967 & 188864 & 0 \\
\hline 2600 & $\mathrm{TX}$ & Madison County & 0.994082 & 0.811678 & 0.015059 & 432 & 2685 & 95509 & 0 \\
\hline 2601 & TX & Marion County & 0.906870 & 0.203199 & 0.000000 & 79 & 2521 & 77790 & 0 \\
\hline 2602 & $\mathrm{TX}$ & Martin County & 0.999085 & 0.875261 & 0.000000 & 146 & 1134 & 122405 & 0 \\
\hline 2603 & $T X$ & Mason County & 0.999879 & 0.917618 & 0.000000 & 101 & 1390 & 112239 & 0 \\
\hline 2604 & $T X$ & Matagorda County & 0.691267 & 0.788732 & 0.000000 & 196 & 1647 & 108066 & 0 \\
\hline 2605 & $T X$ & Maverick County & 0.990970 & 0.828245 & 0.000000 & 135 & 998 & 51686 & 0 \\
\hline 2606 & TX & Medina County & 0.994932 & 0.774507 & 0.024221 & 197 & 2155 & 88749 & 0 \\
\hline 2607 & TX & Menard County & 0.999625 & 0.844618 & 0.010829 & 75 & 859 & 113377 & 1 \\
\hline 2608 & $T X$ & Midland County & 0.998099 & 1.257725 & 0.027580 & 59 & 548 & 131028 & 13 \\
\hline 2609 & $\mathrm{TX}$ & Milam County & 0.995153 & 0.846949 & 0.034308 & 208 & 1990 & 96078 & 0 \\
\hline 2610 & TX & Mills County & 0.997631 & 0.894349 & 0.046172 & 106 & 1323 & 109062 & 0 \\
\hline 2611 & TX & Mitchell County & 0.993605 & 1.008301 & 0.000980 & 90 & 522 & 92474 & 9 \\
\hline 2612 & $T X$ & Montague County & 0.991714 & 0.831612 & 0.086989 & 134 & 1566 & 91100 & 0 \\
\hline 2613 & $T X$ & Montgomery County & 0.969745 & 0.290087 & 0.035976 & 113 & 3546 & 115713 & 0 \\
\hline 2614 & $T X$ & Moore County & 0.989060 & 1.019864 & 0.001202 & 1116 & 1148 & 116519 & 13 \\
\hline 2615 & $T X$ & Morris County & 0.984037 & 0.453957 & 0.019670 & 542 & 1847 & 91845 & 1 \\
\hline 2616 & TX & Motley County & 0.999565 & 0.757829 & 0.000000 & 69 & 556 & 121545 & 0 \\
\hline 2617 & $T X$ & Nacogdoches County & 0.964782 & 0.363633 & 0.059415 & 1414 & 3310 & 91303 & 0 \\
\hline 2618 & TX & Navarro County & 0.986131 & 0.767079 & 0.023739 & 155 & 1807 & 95319 & 0 \\
\hline 2619 & $T X$ & Newton County & 0.992745 & 0.050703 & 0.000000 & 99 & 3428 & 67746 & 0 \\
\hline 2620 & TX & Nolan County & 0.997872 & 0.918760 & 0.010815 & 204 & 1021 & 96660 & 1 \\
\hline 2621 & TX & Nueces County & 0.716533 & 0.828502 & 0.003470 & 256 & 2395 & 102438 & 1 \\
\hline 2622 & TX & Ochiltree County & 0.999440 & 1.011131 & 0.000000 & 515 & 1000 & 120814 & 9 \\
\hline 2623 & $T X$ & Oldham County & 0.999476 & 0.882488 & 0.001954 & 238 & 425 & 188512 & 1 \\
\hline 2624 & $T X$ & Orange County & 0.939035 & 0.249771 & 0.054656 & 126 & 2382 & 101388 & 1 \\
\hline 2625 & $\mathrm{TX}$ & Palo Pinto County & 0.966952 & 0.848109 & 0.048183 & 63 & 1425 & 94019 & 0 \\
\hline 2626 & TX & Panola County & 0.975141 & 0.380682 & 0.102168 & 415 & 2036 & 105770 & 0 \\
\hline 2627 & $T X$ & Parker County & 0.992777 & 0.718836 & 0.238636 & 248 & 3527 & 112198 & 0 \\
\hline 2628 & $T X$ & Parmer County & 0.996040 & 0.932612 & 0.005841 & 2059 & 1690 & 175426 & 0 \\
\hline 2629 & TX & Pecos County & 0.999775 & 0.948395 & 0.011267 & 24 & 250 & 73855 & 1 \\
\hline 2630 & TX & Polk County & 0.952658 & 0.208678 & 0.037099 & 105 & 2300 & 85738 & 0 \\
\hline 2631 & $T X$ & Potter County & 0.986324 & 0.690687 & 0.000000 & 135 & 562 & 110119 & 0 \\
\hline 2632 & $T X$ & Presidio County & 0.999806 & 0.687068 & 0.011662 & 20 & 415 & 62036 & 1 \\
\hline 2633 & $\mathrm{TX}$ & Rains County & 0.896394 & 0.652850 & 0.486137 & 375 & 2498 & 80906 & 0 \\
\hline 2634 & TX & Randall County & 0.991302 & 0.849382 & 0.008443 & 888 & 967 & 120035 & 0 \\
\hline 2635 & $T X$ & Reagan County & 0.999420 & 0.821327 & 0.000000 & 49 & 551 & 88302 & 0 \\
\hline 2636 & $T X$ & Real County & 0.999814 & 0.809517 & 0.000000 & 29 & 1347 & 94452 & 0 \\
\hline 2637 & $T X$ & Red River County & 0.992976 & 0.632711 & 0.047457 & 217 & 1480 & 86622 & 0 \\
\hline 2638 & TX & Reeves County & 0.997701 & 0.922251 & 0.005853 & 130 & 319 & 72487 & 1 \\
\hline 2639 & $T X$ & Refugio County & 0.940899 & 1.353270 & 0.015115 & 78 & 1209 & 118050 & 13 \\
\hline 2640 & $T X$ & Roberts County & 0.999894 & 0.862412 & 0.000000 & 97 & 485 & 135660 & 0 \\
\hline 2641 & $\mathrm{TX}$ & Robertson County & 0.987174 & 0.716395 & 0.021062 & 197 & 2258 & 95123 & 0 \\
\hline 2642 & $T X$ & Rockwall County & 0.866084 & 0.575598 & 0.114003 & 146 & 4315 & 144865 & 1 \\
\hline 2643 & $\mathrm{TX}$ & Runnels County & 0.997432 & 0.848453 & 0.102509 & 153 & 1311 & 112665 & 0 \\
\hline 2644 & TX & Rusk County & 0.983960 & 0.453465 & 0.101678 & 199 & 2250 & 97168 & 0 \\
\hline 2645 & $T X$ & Sabine County & 0.850257 & 0.106899 & 0.025835 & 454 & 2772 & 84313 & 1 \\
\hline 2646 & $\mathrm{TX}$ & San Augustine County & 0.891366 & 0.161544 & 0.002120 & 683 & 2428 & 85586 & 0 \\
\hline 2647 & $T X$ & San Jacinto County & 0.908830 & 0.226477 & 0.114604 & 125 & 2026 & 65876 & 1 \\
\hline 2648 & $T X$ & San Patricio County & 0.978299 & 0.809093 & 0.005652 & 389 & 2289 & 89880 & 1 \\
\hline 2649 & TX & San Saba County & 0.996678 & 1.024138 & 0.006033 & 116 & 1178 & 105967 & 9 \\
\hline 2650 & TX & Schleicher County & 0.999967 & 0.911636 & 0.011810 & 44 & 551 & 92163 & 1 \\
\hline 2651 & TX & Scurry County & 0.994452 & 0.897383 & 0.030957 & 98 & 959 & 98171 & 1 \\
\hline 2652 & $T X$ & Shackelford County & 0.998268 & 0.964792 & 0.059984 & 58 & 691 & 130858 & 1 \\
\hline 2653 & $T X$ & Shelby County & 0.951590 & 0.369334 & 0.005998 & 1505 & 3302 & 92244 & 0 \\
\hline
\end{tabular}




\begin{tabular}{|c|c|c|c|c|c|c|c|c|c|}
\hline $\begin{array}{c}\text { County } \\
\text { Code }\end{array}$ & State & $\begin{array}{l}\text { County } \\
\text { Name }\end{array}$ & $\begin{array}{l}\text { FRCLND } \\
\text { Absolute }\end{array}$ & $\begin{array}{l}\text { FRMFRC } \\
\text { Absolute }\end{array}$ & $\begin{array}{c}\text { DPF } \\
\text { Absolute }\end{array}$ & $\begin{array}{l}\text { ASFP } \\
\text { S/ha }\end{array}$ & $\begin{array}{l}\text { VFRM } \\
\text { \$/ha }\end{array}$ & $\begin{array}{l}\text { VNFRM } \\
\text { \$iperson }\end{array}$ & Notes \\
\hline 2654 & TX & Sherman County & 0.999823 & 0.858939 & 0.000735 & 1056 & 936 & 211100 & 1 \\
\hline 2655 & TX & Smith County & 0.977828 & 0.416713 & 0.061123 & 336 & 3984 & 118605 & 0 \\
\hline 2656 & TX & Somervell County & 0.975349 & 0.524639 & 0.067791 & 92 & 2758 & 97094 & 1 \\
\hline 2657 & $T X$ & Starr County & 0.994912 & 0.808151 & 0.003384 & 275 & 1183 & 37652 & 0 \\
\hline 2658 & $\mathrm{TX}$ & Stephens County & 0.970881 & 0.936941 & 0.028784 & 51 & 1118 & 92393 & 1 \\
\hline 2659 & $T X$ & Sterling County & 0.999857 & 1.413412 & 0.000000 & 25 & 521 & 102106 & 9 \\
\hline 2660 & TX & Stonewall County & 0.998302 & 0.871172 & 0.024848 & 62 & 614 & 132443 & 1 \\
\hline 2661 & TX & Sutton County & 0.999565 & 0.995278 & 0.000000 & 30 & 525 & 106116 & 0 \\
\hline 2662 & TX & Swisher County & 0.999724 & 0.876497 & 0.000759 & 1185 & 1196 & 143626 & 0 \\
\hline 2663 & $\mathrm{TX}$ & Tarrant County & 0.962070 & 0.303211 & 0.114371 & 320 & 6002 & 127505 & 0 \\
\hline 2664 & $\mathrm{TX}$ & Taylor County & 0.996044 & 0.868554 & 0.006244 & 314 & 1469 & 108513 & 0 \\
\hline 2665 & $T X$ & Terrell County & 0.999986 & 0.925261 & 0.000000 & 10 & 270 & 133114 & 0 \\
\hline 2666 & $T X$ & Terry County & 0.998812 & 0.806133 & 0.005998 & 286 & 1266 & 98536 & 1 \\
\hline 2667 & $\mathrm{TX}$ & Throckmorton County & 0.996579 & 0.995835 & 0.023773 & 85 & 660 & 139731 & 1 \\
\hline 2668 & TX & Titus County & 0.964431 & 0.686272 & 0.070508 & 421 & 2735 & 104524 & 0 \\
\hline 2669 & TX & Tom Green County & 0.988033 & 1.047782 & 0.037251 & 190 & 1251 & 107220 & 9 \\
\hline 2670 & $\mathrm{TX}$ & Travis County & 0.967930 & 0.525607 & 0.036092 & 142 & 3701 & 124220 & 0 \\
\hline 2671 & TX & Trinity County & 0.970367 & 0.247233 & 0.062484 & 172 & 2580 & 82565 & 1 \\
\hline 2672 & $T X$ & Tyler County & 0.986321 & 0.099356 & 0.003805 & 133 & 4090 & 97521 & 0 \\
\hline 2673 & TX & Upshur County & 0.991567 & 0.515780 & 0.385342 & 530 & 2531 & 87848 & 0 \\
\hline 2674 & $\mathrm{TX}$ & Upton County & 0.999873 & 0.874393 & 0.000000 & 30 & 320 & 99742 & 0 \\
\hline 2675 & $\mathrm{TX}$ & Uvalde County & 0.998680 & 0.920633 & 0.010327 & 166 & 1139 & 87144 & 1 \\
\hline 2676 & $T X$ & Val Verde County & 0.980814 & 0.890307 & 0.000000 & 19 & 419 & 69940 & 0 \\
\hline 2677 & TX & Van Zandt County & 0.987497 & 0.696225 & 0.194882 & 340 & 3619 & 97846 & 0 \\
\hline 2678 & $\mathrm{TX}$ & Victoria County & 0.992994 & 0.762560 & 0.037966 & 144 & 1873 & 112930 & 1 \\
\hline 2679 & $\mathrm{TX}$ & Walker County & 0.982548 & 0.424441 & 0.018984 & 164 & 2914 & 87273 & 0 \\
\hline 2680 & TX & Waller County & 0.990608 & 0.738930 & 0.005416 & 280 & 5140 & 95929 & 0 \\
\hline 2681 & $\mathrm{TX}$ & Ward County & 0.999699 & 0.852490 & 0.000000 & 11 & 313 & 98171 & 0 \\
\hline 2682 & $T X$ & Washington County & 0.980490 & 0.842101 & 0.108535 & 219 & 4330 & 128278 & 0 \\
\hline 2683 & TX & Webb County & 0.994460 & 0.796869 & 0.002604 & 33 & 1050 & 60776 & 0 \\
\hline 2684 & $\mathrm{TX}$ & Wharton County & 0.996070 & 0.924033 & 0.002810 & 435 & 2229 & 103183 & 1 \\
\hline 2685 & $\mathrm{TX}$ & Wheeler County & 0.998779 & 0.857397 & 0.002378 & 330 & 582 & 126584 & 1 \\
\hline 2686 & TX & Wichita County & 0.991555 & 0.766152 & 0.020960 & 162 & 1501 & 115950 & 0 \\
\hline 2687 & $\mathrm{TX}$ & Wilbarger County & 0.992809 & 1.389143 & 0.009793 & 93 & 792 & 102912 & 13 \\
\hline 2688 & $\mathrm{TX}$ & Willacy County & 0.760854 & 0.683145 & 0.000000 & 414 & 2468 & 59895 & 0 \\
\hline 2689 & TX & Williamson County & 0.989331 & 0.758296 & 0.041806 & 203 & 2822 & 101151 & 0 \\
\hline 2690 & $T X$ & Wilson County & 0.998196 & 0.922369 & 0.147676 & 352 & 2365 & 86460 & 0 \\
\hline 2691 & $\mathrm{TX}$ & Winkler County & 0.999770 & 0.804167 & 0.000000 & 19 & 241 & 86467 & 0 \\
\hline 2692 & $\mathrm{TX}$ & Wise County & 0.980311 & 0.796438 & 0.363915 & 211 & 2441 & 91431 & 0 \\
\hline 2693 & TX & Wood County & 0.934505 & $0.4893 \widetilde{3}$ & 0.428419 & 636 & 3170 & 94994 & 0 \\
\hline 2694 & $\mathrm{TX}$ & Yoakum County & 0.999984 & 0.673362 & 0.000000 & 261 & 930 & 117752 & 0 \\
\hline 2695 & $T X$ & Young County & 0.990855 & 0.954015 & 0.007073 & 99 & 890 & 120658 & 1 \\
\hline 2696 & TX & Zapata County & 0.942036 & 0.760067 & 0.001639 & 59 & 1213 & 51334 & 0 \\
\hline 2697 & $\mathrm{TX}$ & Zavala County & 0.997513 & 0.869963 & 0.012813 & 128 & 874 & 51490 & 1 \\
\hline 2698 & MT & Beaverhead County & 0.994664 & 0.378455 & 0.001241 & 120 & 799 & 101374 & 0 \\
\hline 2699 & MT & Big Hom County & 0.996043 & 0.939204 & 0.000682 & 76 & 617 & 82267 & 0 \\
\hline 2700 & MT & Blaine County & 0.996995 & 0.864710 & 0.003145 & 45 & 456 & 86074 & 1 \\
\hline 2701 & MT & Broadwater County & 0.961698 & 0.590085 & 0.012241 & 90 & 835 & 86521 & 1 \\
\hline 2702 & MT & Carbon County & 0.993117 & 0.456752 & 0.008990 & 180 & 1098 & 97094 & 0 \\
\hline 2703 & MT & Carter County & 0.997393 & 0.757689 & 0.000000 & 35 & 243 & 100623 & 0 \\
\hline 2704 & MT & Cascade County & 0.994942 & 0.824811 & 0.040188 & 92 & 742 & 114907 & 0 \\
\hline 2705 & MT & Chouteau County & 0.994072 & 0.895767 & 0.000682 & 62 & 809 & 115693 & 0 \\
\hline 2706 & MT & Custer County & 0.997341 & 0.861181 & 0.000502 & 52 & 372 & 109577 & 0 \\
\hline 2707 & MT & Daniels County & 0.999698 & 0.834267 & 0.000000 & 75 & 534 & 121362 & 0 \\
\hline 2708 & MT & Dawson County & 0.995820 & 0.878300 & 0.018009 & 63 & 409 & 99248 & 0 \\
\hline 2709 & MT & Deer Lodge County & 0.994150 & 0.286502 & 0.014289 & 86 & 926 & 82626 & 1 \\
\hline 2710 & MT & Fallon County & 0.998343 & 0.910765 & 0.010680 & 49 & 354 & 114372 & 1 \\
\hline 2711 & MT & Fergus County & 0.997426 & 0.803904 & 0.008546 & 66 & 528 & 99173 & 0 \\
\hline 2712 & MT & Flathead County & 0.969942 & 0.084904 & 0.124783 & 236 & 3583 & 104571 & 0 \\
\hline 2713 & MT & Gallatin County & 0.989782 & 0.435935 & 0.178665 & 206 & 1861 & 99234 & 0 \\
\hline 2714 & MT & Garfield County & 0.962975 & 0.669515 & 0.005355 & 31 & 287 & 120157 & 1 \\
\hline 2715 & MT & Glacier County & 0.986048 & 0.902901 & 0.019266 & 51 & 549 & 79490 & 0 \\
\hline 2716 & MT & Golden Valley County & 0.999068 & 0.846192 & 0.011981 & 43 & 475 & 114203 & 1 \\
\hline 2717 & MT & Granite County & 0.996741 & 0.316510 & 0.006534 & 71 & 799 & 106312 & 1 \\
\hline 2718 & MT & Hill County & 0.993249 & 0.886882 & 0.008451 & 62 & 681 & 93687 & 0 \\
\hline 2719 & MT & Jefferson County & 0.998678 & 0.346587 & 0.036954 & 69 & 809 & 110898 & 0 \\
\hline 2720 & MT & Judith Basin County & 0.999539 & 0.725364 & 0.002107 & 90 & 623 & 100529 & 1 \\
\hline 2721 & MT & Lake County & 0.903289 & 0.660406 & 0.107597 & 144 & 1632 & 86101 & 0 \\
\hline 2722 & MT & Lewis and Clark County & 0.989537 & 0.398851 & 0.021675 & 53 & 693 & 111487 & 0 \\
\hline 2723 & MT & Liberty County & 0.987994 & 1.040113 & 0.022465 & 80 & 653 & 132728 & 9 \\
\hline
\end{tabular}




\begin{tabular}{|c|c|c|c|c|c|c|c|c|c|}
\hline $\begin{array}{l}\text { County } \\
\text { Code }\end{array}$ & State & $\begin{array}{l}\text { County } \\
\text { Name }\end{array}$ & $\begin{array}{l}\text { FRCLND } \\
\text { Absolute }\end{array}$ & $\begin{array}{l}\text { FRMFRC } \\
\text { Absolute }\end{array}$ & $\begin{array}{c}\text { DPF } \\
\text { Absolute }\end{array}$ & $\begin{array}{c}\text { ASFP } \\
\text { S/ha }\end{array}$ & $\begin{array}{c}\text { VFRM } \\
\text { S/ha }\end{array}$ & $\begin{array}{l}\text { VNFRM } \\
\text { \$/person }\end{array}$ & Notes \\
\hline 2724 & MT & Lincoln County & 0.983014 & 0.021720 & 0.005326 & 111 & 3951 & 85566 & 0 \\
\hline 2725 & MT & McCone County & 0.984999 & 0.762822 & 0.004363 & 59 & 472 & 100006 & 1 \\
\hline 2726 & MT & Madison County & 0.995476 & 0.553775 & 0.000000 & 71 & 821 & 89332 & 0 \\
\hline 2727 & MT & Meagher County & 0.998739 & 0.595873 & 0.003639 & 50 & 481 & 96674 & 1 \\
\hline 2728 & MT & Mineral County & 0.997093 & 0.024539 & 0.000000 & 125 & 2698 & 80452 & 0 \\
\hline 2729 & MT & Missoula County & 0.992294 & 0.149270 & 0.017280 & 77 & 1953 & 105357 & 1 \\
\hline 2730 & MT & Musselshell County & 0.997988 & 0.863495 & 0.014223 & 34 & 411 & 100345 & 1 \\
\hline 2731 & MT & Park County & 0.996037 & 0.457546 & 0.019650 & 63 & 933 & 89447 & 23 \\
\hline 2732 & MT & Petroleum County & 0.987986 & 0.632503 & 0.000000 & 43 & 367 & 95522 & 0 \\
\hline 2733 & MT & Phillips County & 0.986121 & 0.598523 & 0.002982 & 56 & 407 & 104314 & 1 \\
\hline 2734 & MT & Pondera County & 0.990755 & 0.859667 & 0.021468 & 92 & 1007 & 95773 & 0 \\
\hline 2735 & MT & Powder River County & 0.999770 & 0.772118 & 0.005222 & 39 & 272 & 103142 & 1 \\
\hline 2736 & MT & Powell County & 0.997108 & 0.453822 & 0.003685 & 66 & 783 & 89752 & 1 \\
\hline 2737 & MT & Prairie County & 0.996554 & 0.614604 & 0.003530 & 69 & 374 & 113173 & 1 \\
\hline 2738 & MT & Ravalii County & 0.997462 & 0.157702 & 0.204360 & 234 & 3542 & 88898 & 0 \\
\hline 2739 & MT & Richland County & 0.990999 & 0.897418 & 0.007293 & 114 & 560 & 101293 & 1 \\
\hline 2740 & MT & Roosevelt County & 0.994073 & 0.938178 & 0.000000 & 65 & 507 & 82159 & 0 \\
\hline 2741 & MT & Rosebud County & 0.997102 & 0.806075 & 0.003105 & 41 & 287 & 94642 & 1 \\
\hline 2742 & MT & Sanders County & 0.990003 & 0.215573 & 0.044641 & 78 & 1004 & 75426 & 0 \\
\hline 2743 & MT & Sheridan County & 0.982578 & 0.896923 & 0.004314 & 80 & 713 & 112279 & 1 \\
\hline 2744 & MT & Silver Bow County & 0.999075 & 0.216968 & 0.000000 & 61 & 920 & 107003 & 0 \\
\hline 2745 & MT & Stillwater County & 0.994473 & 0.774223 & 0.006449 & 86 & 624 & 112638 & 1 \\
\hline 2746 & MT & Sweet Grass County & 0.996284 & 0.705712 & 0.003711 & 53 & 755 & 114765 & 1 \\
\hline 2747 & MT & Teton County & 0.991291 & 0.810528 & 0.013902 & 122 & 786 & 106949 & 0 \\
\hline 2748 & MT & Toole County & 0.982058 & 0.869257 & 0.008674 & 72 & 687 & 118545 & 1 \\
\hline 2749 & MT & Treasure County & 0.994673 & 0.956133 & 0.000000 & $7 \overline{8}$ & 358 & 128786 & 0 \\
\hline 2750 & MT & Valley County & 0.972118 & 0.535997 & 0.004940 & 59 & 550 & 105885 & 1 \\
\hline 2751 & MT & Wheatland County & 0.996363 & 0.933867 & 0.032714 & 62 & 505 & 105312 & 0 \\
\hline 2752 & MT & Wibaux County & 0.999124 & 0.862623 & 0.024030 & 51 & 370 & 97148 & 0 \\
\hline 2753 & MT & Yellowstone County & 0.994754 & 0.862518 & 0.038621 & 170 & 751 & 116987 & 0 \\
\hline 2754 & MT & Yellowstone National Park & 0.997044 & 0.000000 & 0.000000 & 0 & 0 & 89447 & 23 \\
\hline 2755 & ID & Ada County & 0.994966 & 0.344889 & 0.185576 & 1031 & 3831 & 127241 & 0 \\
\hline 2756 & ID & Adams County & 0.996044 & 0.253274 & 0.025961 & 120 & 1157 & 99431 & 0 \\
\hline 2757 & ID & Bannock County & 0.970130 & 0.456640 & 0.106703 & 197 & 1256 & 90443 & 0 \\
\hline 2758 & ID & Bear Lake County & 0.925622 & 0.433373 & 0.227533 & 131 & 1034 & 81224 & 0 \\
\hline 2759 & ID & Benewah County & 0.989825 & 0.224519 & 0.011373 & 279 & 2266 & 94330 & 1 \\
\hline 2760 & ID & Bingham County & 0.987974 & 1.023054 & 0.073475 & 388 & 1490 & 92007 & 9 \\
\hline 2761 & ID & Blaine County & 0.993898 & 0.157315 & 0.045739 & 247 & 1906 & 157023 & 1 \\
\hline 2762 & ID & Boise County & 0.997743 & 0.065976 & 0.000000 & 109 & 1710 & 77661 & 0 \\
\hline 2763 & ID & Bonner County & 0.905189 & 0.134902 & 0.162822 & 99 & 2807 & 86981 & 0 \\
\hline 2764 & ID & Bonneville County & 0.983084 & 0.379337 & 0.034346 & 554 & 2294 & 112184 & 0 \\
\hline 2765 & ID & Boundary County & 0.992645 & 0.089484 & 0.055882 & 405 & 3305 & 77675 & 0 \\
\hline 2766 & ID & Butte County & 0.999669 & 0.111512 & 0.046801 & 301 & 1388 & 106522 & 0 \\
\hline 2767 & ID & Camas County & 0.996234 & 0.188212 & 0.000000 & 82 & 1040 & 130933 & 0 \\
\hline 2768 & ID & Canyon County & 0.977201 & 1.036039 & 0.107633 & 1657 & 4316 & 89068 & 9 \\
\hline 2769 & ID & Caribou County & 0.981868 & 0.519938 & 0.091678 & 155 & 870 & 116912 & 0 \\
\hline 2770 & ID & Cassia County & 0.994628 & 0.405658 & 0.054728 & 1054 & 2049 & 106840 & 0 \\
\hline 2771 & ID & Clark County & 0.999671 & 0.253859 & 0.000000 & 316 & 878 & 178447 & 0 \\
\hline 2772 & ID & Cleanwater County & 0.989269 & 0.065536 & 0.077686 & 110 & 1427 & 97554 & 1 \\
\hline 2773 & ID & Custer County & 0.997707 & 0.044633 & 0.005079 & 247 & 2268 & 115050 & 1 \\
\hline 2774 & ID & Elmore County & 0.992609 & 0.179477 & 0.001090 & 1853 & 1321 & 91127 & 0 \\
\hline 2775 & 10 & Franklin County & 0.995627 & 0.540230 & 0.465390 & 483 & 1905 & 70550 & 0 \\
\hline 2776 & ID & Fremont County & 0.984788 & 0.318829 & 0.027239 & 559 & 1878 & 95563 & 0 \\
\hline 2777 & ID & Gem County & 0.994397 & 0.547613 & 0.161742 & 370 & 2415 & 86121 & 0 \\
\hline 2778 & ID & Gooding County & 0.995852 & 0.485557 & 0.289474 & 2197 & 3178 & 96511 & 0 \\
\hline 2779 & ID & Idaho County & 0.997931 & 0.137058 & 0.023206 & 99 & 1277 & 91506 & 0 \\
\hline 2780 & ID & Jefferson County & 0.990523 & 0.444142 & 0.073231 & 724 & 2529 & 86426 & 0 \\
\hline 2781 & ID & Jerome County & 0.996625 & 0.540606 & 0.367528 & 2075 & 4084 & 85356 & 0 \\
\hline 2782 & ID & Kootenai County & 0.946365 & 0.164733 & 0.004637 & 321 & 4097 & 101083 & 0 \\
\hline 2783 & ID & Latah County & 0.999779 & 0.503988 & 0.003607 & 282 & 2008 & 94276 & 1 \\
\hline 2784 & ID & Lemhi County & 0.998830 & 0.066380 & 0.047223 & 238 & 1718 & 92833 & 0 \\
\hline 2785 & ID & Lewis County & 0.998404 & 0.688319 & 0.003664 & 229 & 1775 & 134800 & 1 \\
\hline 2786 & ID & Lincoln County & 0.999728 & 0.171634 & 0.246973 & .712 & 1955 & 117095 & 0 \\
\hline 2787 & ID & Madison County & 0.996113 & 0.743454 & 0.040302 & 806 & 3654 & 74688 & 0 \\
\hline 2788 & ID & Minidoka County & 0.995599 & 0.428145 & 0.075650 & 1534 & 4002 & 89393 & 0 \\
\hline 2789 & ID & Nez Perce County & 0.991499 & 0.879294 & 0.002109 & 175 & 1456 & 110186 & 1 \\
\hline 2790 & ID & Oneida County & 0.998938 & 0.352930 & 0.104641 & 120 & 990 & 87781 & 0 \\
\hline 2791 & ID & Owyhee County & 0.997569 & 0.153033 & 0.090195 & 317 & 1129 & 84177 & 0 \\
\hline 2792 & ID & Payette County & 0.993490 & 0.570426 & 0.214099 & 718 & 2533 & 83710 & 0 \\
\hline 2793 & ID & Power County & 0.974331 & 0.483613 & 0.011509 & 559 & 1783 & 134231 & 0 \\
\hline
\end{tabular}




\begin{tabular}{|c|c|c|c|c|c|c|c|c|c|}
\hline $\begin{array}{l}\text { County } \\
\text { Code }\end{array}$ & State & $\begin{array}{l}\text { County } \\
\text { Name }\end{array}$ & $\begin{array}{l}\text { FRCLND } \\
\text { Absolute }\end{array}$ & $\begin{array}{l}\text { FRMFRC } \\
\text { Absolute }\end{array}$ & $\begin{array}{c}\text { DPF } \\
\text { Absolute }\end{array}$ & $\begin{array}{l}\text { ASFP } \\
\text { \$/ha }\end{array}$ & $\begin{array}{l}\text { VFRM } \\
\text { \$/ha }\end{array}$ & $\begin{array}{l}\text { VNFRM } \\
\text { \$/person }\end{array}$ & Notes \\
\hline 2794 & ID & Shoshone County & 0.999397 & 0.002627 & 0.199257 & 200 & 5121 & 93978 & 1 \\
\hline 2795 & ID & Teton County & 0.999538 & 0.467606 & 0.101768 & 370 & 2823 & 91174 & 0 \\
\hline 2796 & ID & Twin Falls County & 0.998218 & 0.397694 & 0.191315 & 860 & 2940 & 106204 & 0 \\
\hline 2797 & $\overline{I D}$ & Valley County & 0.985095 & 0.033480 & 0.000000 & 204 & 2249 & 118145 & 0 \\
\hline 2798 & ID & Washington County & 0.988310 & 0.596657 & 0.082910 & 153 & 1382 & 88560 & 0 \\
\hline 2799 & WY & Albany County & 0.991834 & 0.683054 & 0.000000 & 44 & 387 & 92610 & 0 \\
\hline 2800 & WY & Big Horn County & 0.993045 & 0.219808 & 0.007661 & 224 & 950 & 90009 & 0 \\
\hline 2801 & WY & Campbell County & 0.999000 & 0.880832 & 0.000957 & 29 & 215 & 123293 & 1 \\
\hline 2802 & $W Y$ & Carbon County & 0.991479 & 0.538386 & 0.000563 & 42 & 351 & 114393 & 0 \\
\hline 2803 & WY & Converse County & 0.997560 & 0.867844 & 0.000765 & 26 & 275 & 93978 & 0 \\
\hline 2804 & WY & Crook County & 0.995844 & 0.842970 & 0.002151 & 46 & 402 & 106312 & 1 \\
\hline 2805 & WY & Fremont County & 0.990985 & 0.411078 & 0.016847 & 57 & 461 & 86223 & 0 \\
\hline 2806 & WY & Goshen County & 0.996936 & 0.866769 & 0.001392 & 255 & 619 & 89176 & 0 \\
\hline 2807 & WY & Hot Springs County & 0.998842 & 0.708210 & 0.000000 & 28 & 378 & 112930 & 0 \\
\hline 2808 & wr & Johnson County & 0.997982 & 0.770863 & 0.000000 & 28 & 272 & 112997 & 0 \\
\hline 2809 & WY & Laramie County & 0.999414 & 0.989040 & 0.007673 & 89 & 494 & 112130 & 0 \\
\hline 2810 & Wr & Lincoln County & 0.995130 & 0.214627 & 0.210819 & 111 & 1254 & 91309 & 0 \\
\hline 2811 & WY & Natrona County & 0.993330 & 0.734060 & 0.004519 & 24 & 285 & 121626 & 0 \\
\hline 2812 & Wr & Niobrara County & 0.999206 & 0.800057 & 0.002808 & 40 & 271 & 110329 & 1 \\
\hline 2813 & Wr & Park County & 0.996253 & 0.179430 & 0.023543 & 177 & 912 & 110417 & 0 \\
\hline 2814 & WY & Platte County & 0.987699 & 1.022868 & 0.017312 & 114 & 503 & 100792 & 9 \\
\hline 2815 & Wr & Sheridan County & 0.998526 & 0.748473 & 0.022268 & 66 & 662 & 122913 & 0 \\
\hline 2816 & WY & Sublette County & 0.988988 & 0.189728 & 0.000000 & 108 & 996 & 114040 & 0 \\
\hline 2817 & Wr & Sweetwater County & 0.993722 & 0.257883 & 0.016092 & 11 & 328 & 118314 & 1 \\
\hline 2818 & wr & Teton County & 0.949308 & 0.024290 & 0.013750 & 353 & 4358 & 198014 & 1 \\
\hline 2819 & Wr & Uinta County & 0.997169 & 0.660271 & 0.015324 & 47 & 473 & 82857 & 0 \\
\hline 2820 & WY & Washakie County & 0.998801 & 0.277521 & 0.000000 & 205 & 726 & 104761 & 0 \\
\hline 2821 & Wr & Weston County & 0.999078 & 0.967365 & 0.000000 & 43 & 280 & 107944 & 0 \\
\hline 2822 & $\mathrm{co}$ & Adams County & 0.995151 & 0.898964 & 0.068682 & 308 & 1387 & 109157 & 0 \\
\hline 2823 & co & Alamosa County & 0.998916 & 0.448444 & 0.010039 & 540 & 2234 & 86311 & 0 \\
\hline 2824 & $\mathrm{CO}$ & Arapahoe County & 0.997143 & 0.628029 & 0.004812 & 114 & 1273 & 152444 & 1 \\
\hline 2825 & $\mathrm{CO}$ & Archuleta County & 0.996119 & 0.180017 & 0.000000 & 108 & 2238 & 80019 & 0 \\
\hline 2826 & co & Baca County & 0.999465 & 0.768591 & 0.001401 & 101 & 563 & 141479 & 1 \\
\hline 2827 & $\mathrm{co}$ & Bent County & 0.982338 & 0.822407 & 0.005530 & 161 & 381 & 104097 & 1 \\
\hline 2828 & $\mathrm{co}$ & Boulder County & 0.988100 & 0.331444 & 0.186684 & 1058 & 7993 & 145089 & 0 \\
\hline 2829 & $\mathrm{CO}$ & Chaffee County & 0.998469 & 0.129764 & 0.018692 & 113 & 2627 & 97283 & 1 \\
\hline 2830 & $\mathrm{co}$ & Cheyenne County & 0.999987 & 0.801732 & 0.005716 & 102 & 638 & 201482 & 1 \\
\hline 2831 & co & Clear Creek County & 0.997442 & 0.028167 & 0.000000 & 9 & 3982 & 108798 & 18 \\
\hline 2832 & co & Conejos County & 0.997170 & 0.369696 & 0.030535 & 185 & 1368 & 66743 & 0 \\
\hline 2833 & $\mathrm{CO}$ & Costilla County & 0.997286 & 0.421210 & 0.000000 & 102 & 965 & 86067 & 0 \\
\hline 2834 & co & Crowley County & 0.985836 & 0.839195 & 0.012072 & 552 & 475 & 82288 & 0 \\
\hline 2835 & co & Custer County & 0.998627 & 0.331559 & 0.016366 & 69 & 1480 & 102709 & 1 \\
\hline 2836 & $\mathrm{co}$ & Delta County & 0.994428 & 0.356667 & 0.089498 & 423 & 2543 & 90307 & 0 \\
\hline 2837 & $\mathrm{co}$ & Denver County & 0.989672 & 0.046589 & 0.000000 & 1023 & 5471 & 150067 & 12 \\
\hline 2838 & co & Dolores County & 0.998944 & 0.244704 & 0.000000 & 103 & 1078 & 107491 & 0 \\
\hline 2839 & Co & Douglas County & 0.996869 & 0.430278 & 0.006652 & 116 & 4367 & 139792 & 1 \\
\hline 2840 & co & Eagle County & 0.997679 & 0.197172 & 0.000000 & 86 & 2461 & 139880 & 0 \\
\hline 2841 & CO & Elbert County & 0.999940 & 0.933341 & 0.037969 & 75 & 868 & 124789 & 0 \\
\hline 2842 & co & El Paso County & 0.998583 & 0.629942 & 0.122556 & 76 & 862 & 113837 & 0 \\
\hline 2843 & co & Fremont County & 0.999332 & 0.338013 & 0.357260 & 100 & 1208 & 87557 & 0 \\
\hline 2844 & co & Garfield County & 0.997095 & 0.233557 & 0.009107 & 89 & 1978 & 109238 & 1 \\
\hline 2845 & co & Gilpin County & 0.997431 & 0.138612 & 0.000000 & 25 & 1680 & 97859 & 7 \\
\hline 2846 & $\mathrm{CO}$ & Grand County & 0.989333 & 0.252687 & 0.007567 & 79 & 1412 & 117664 & 1 \\
\hline 2847 & co & Gunnison County & 0.993579 & 0.085545 & 0.016298 & 123 & 1950 & 92176 & 1 \\
\hline 2848 & $\mathrm{CO}$ & Hinsdale County & 0.995142 & 0.012610 & 0.000000 & 163 & 3044 & 127837 & 0 \\
\hline 2849 & $\mathrm{CO}$ & Huerfano County & 0.998512 & 0.630263 & 0.026779 & 31 & 720 & 85078 & 1 \\
\hline 2850 & $\mathrm{CO}$ & Jackson County & 0.995225 & 0.457151 & 0.003862 & 98 & 1001 & 110525 & 1 \\
\hline 2851 & co & Jefferson County & 0.992324 & 0.209358 & 0.003494 & 492 & 6328 & 142217 & 1 \\
\hline 2852 & $\mathrm{co}$ & Kiowa County & 0.991732 & 0.774983 & 0.002800 & 72 & 536 & 215889 & 1 \\
\hline 2853 & co & Kit Carson County & 0.999680 & 0.970127 & 0.011206 & 319 & 960 & 148570 & 0 \\
\hline 2854 & co & Lake County & 0.981746 & 0.059740 & 0.000000 & 124 & 1563 & 69466 & 0 \\
\hline 2855 & $\mathrm{CO}$ & La Plata County & 0.995446 & 0.542293 & 0.028074 & 60 & 1714 & 105269 & 0 \\
\hline 2856 & $\mathrm{co}$ & Larimer County & 0.987637 & 0.324587 & 0.220228 & 438 & 2900 & 116093 & 0 \\
\hline 2857 & $\mathrm{co}$ & Las Animas County & 0.999419 & 0.748666 & 0.019732 & 28 & 304 & 84110 & 0 \\
\hline 2858 & $\mathrm{CO}$ & Lincoln County & 0.999884 & 1.002982 & 0.001977 & 80 & 499 & 137327 & 9 \\
\hline 2859 & $\mathrm{co}$ & Logan County & 0.996567 & 0.906293 & 0.004143 & 529 & 863 & 108811 & 0 \\
\hline 2860 & Co & Mesa County & 0.996001 & 0.197305 & 0.074248 & 268 & 2554 & 102397 & 0 \\
\hline 2861 & $\mathrm{CO}$ & Mineral County & 0.997737 & 0.027722 & 0.000000 & 35 & 2108 & 105276 & 10 \\
\hline 2862 & CO & Moffat County & 0.998172 & 0.382119 & 0.004323 & 35 & 642 & 99322 & 1 \\
\hline 2863 & co & Montezuma County & 0.998458 & 0.639762 & 0.019483 & 44 & 1042 & 95102 & 1 \\
\hline
\end{tabular}




\begin{tabular}{|c|c|c|c|c|c|c|c|c|c|}
\hline $\begin{array}{l}\text { County } \\
\text { Code }\end{array}$ & State & $\begin{array}{l}\text { County } \\
\text { Name }\end{array}$ & $\begin{array}{l}\text { FRCLND } \\
\text { Absolute }\end{array}$ & $\begin{array}{l}\text { FRMFRC } \\
\text { Absolute }\end{array}$ & $\begin{array}{c}\text { DPF } \\
\text { Absolute }\end{array}$ & $\begin{array}{l}\text { ASFP } \\
\$ / \text { ha }\end{array}$ & $\begin{array}{c}\text { VFRM } \\
\text { \$/ha }\end{array}$ & $\begin{array}{l}\text { VNFRM } \\
\text { \$/person }\end{array}$ & Notes \\
\hline 2864 & $\mathrm{co}$ & Montrose County & 0.999125 & 0.311986 & 0.074717 & 304 & 2333 & 97243 & 0 \\
\hline 2865 & co & Morgan County & 0.993426 & 0.913482 & 0.032925 & 1139 & 1447 & 100230 & 0 \\
\hline 2866 & $\mathrm{CO}$ & Otero County & 0.994575 & 0.783496 & 0.005272 & 400 & 866 & 94215 & 0 \\
\hline 2867 & $\mathrm{CO}$ & Ouray County & 0.999644 & 0.343842 & 0.000000 & 62 & 2870 & 109807 & 0 \\
\hline 2868 & $\mathrm{co}$ & Park County & 0.995478 & 0.276106 & 0.000000 & 39 & 1086 & 104138 & 0 \\
\hline 2869 & $\mathrm{CO}$ & Phillips County & 0.999830 & 1.044435 & 0.027636 & 444 & 1409 & 125067 & 9 \\
\hline 2870 & co & Pitkin County & 0.997114 & 0.051637 & 0.099327 & 167 & 4752 & 219181 & 1 \\
\hline 2871 & CO & Prowers County & 0.997586 & 0.956611 & 0.000371 & 411 & 838 & 107145 & 0 \\
\hline 2872 & $\mathrm{CO}$ & Pueblo County & 0.996227 & 0.586716 & 0.051247 & 99 & 764 & 96159 & 0 \\
\hline 2873 & co & Rio Blanco County & 0.999398 & 0.265111 & 0.000000 & 68 & 1002 & 115077 & 0 \\
\hline 2874 & co & Rio Grande County & 0.999415 & 0.376009 & 0.001656 & 489 & 2516 & 95827 & 1 \\
\hline 2875 & co & Routt County & 0.997285 & 0.381333 & 0.005458 & 113 & 1400 & 136758 & 1 \\
\hline 2876 & co & Saguache County & 0.999431 & 0.227860 & 0.004558 & 253 & 1232 & 75365 & 1 \\
\hline 2877 & CO & San Juan County & 0.997780 & 0.001152 & 0.000000 & 112 & 6269 & 122019 & 2 \\
\hline 2878 & co & San Miguel County & 0.998460 & 0.243714 & 0.000000 & 54 & 1747 & 116343 & 0 \\
\hline 2879 & $\mathrm{CO}$ & Sedgwick County & 0.997520 & 0.884568 & 0.000000 & 304 & 1251 & 134482 & 0 \\
\hline 2880 & co & Summit County & 0.982106 & 0.098822 & 0.000000 & 53 & 1485 & 152329 & 0 \\
\hline 2881 & CO & Teller County & 0.996614 & 0.291720 & 0.000000 & 27 & 1316 & 102133 & 0 \\
\hline 2882 & co & Washington County & 0.998758 & 0.826492 & 0.005580 & 168 & 769 & 146477 & 0 \\
\hline 2883 & $\mathrm{Co}$ & Weld County & 0.992792 & 0.816432 & 0.063029 & 1398 & 1905 & 107518 & 0 \\
\hline 2884 & $\mathrm{CO}$ & Yuma County & 0.998703 & 0.946362 & 0.014395 & 692 & 1219 & 145448 & 0 \\
\hline 2885 & NM & Bernalillo County & 0.997767 & 0.556396 & 0.555758 & 134 & 1228 & 118653 & 0 \\
\hline 2886 & NM & Catron County & 0.999824 & 0.350315 & 0.038427 & 20 & 331 & 88837 & 1 \\
\hline 2887 & NM & Chaves County & 0.999307 & 0.800956 & 0.513579 & 120 & 402 & 96044 & 0 \\
\hline 2888 & NM & Cibola County & 0.999448 & 0.716189 & 0.000000 & 12 & 331 & 60214 & 0 \\
\hline 2889 & NM & Colfax County & 0.996979 & 0.867314 & 0.013899 & 41 & 484 & 95732 & 1 \\
\hline 2890 & NM & Curry County & 0.998779 & 1.027557 & 0.063399 & 375 & 947 & 95888 & 9 \\
\hline 2891 & NM & DeBaca County & 0.996159 & 0.902690 & 0.019522 & 46 & 287 & 87232 & 1 \\
\hline 2892 & NM & Dona Ana County & 0.998050 & 0.216027 & 0.316460 & 937 & 4625 & 77072 & 0 \\
\hline 2893 & NM & Eddy County & 0.996295 & 0.425414 & 0.071182 & 89 & 504 & 96288 & 1 \\
\hline 2894 & NM & Grant County & 0.999591 & 0.476426 & 0.000000 & 22 & 429 & 84218 & 0 \\
\hline 2895 & NM & Guadalupe County & 0.999603 & 0.790315 & 0.000000 & 26 & 238 & 68789 & 0 \\
\hline 2896 & NM & Harding County & 0.999713 & 0.948112 & 0.017313 & 27 & 296 & 88465 & 0 \\
\hline 2897 & NM & Hidalgo County & 0.999919 & 0.382427 & 0.000000 & 55 & 414 & 88641 & 0 \\
\hline 2898 & NM & Lea County & 0.999758 & 0.764469 & 0.208408 & 49 & 309 & 90951 & 0 \\
\hline 2899 & NM & Lincoln County & 0.999960 & 0.608566 & 0.116820 & 16 & 295 & 95218 & 1 \\
\hline 2900 & NM & Los Alamos County & 1.000000 & 0.000143 & 0.000000 & 5922 & 26626 & 188606 & 19 \\
\hline 2901 & NM & Luna County & 0.999937 & 0.420022 & 0.000000 & 154 & 467 & 71220 & 0 \\
\hline 2902 & NM & McKinley County & 0.998810 & 0.924485 & 0.142595 & 8 & 423 & 65483 & 1 \\
\hline 2903 & NM & Mora County & 0.998769 & 0.732403 & 0.005838 & 24 & 456 & 59855 & 0 \\
\hline 2904 & NM & Otero County & 0.999864 & 0.274922 & 0.148349 & 21 & 558 & 84712 & 1 \\
\hline 2905 & $N M$ & Quay County & 0.997615 & 0.961471 & 0.035871 & 57 & 383 & 88268 & 1 \\
\hline 2906 & NM & Rio Árriba County & 0.993475 & 0.414190 & 0.003264 & 19 & 899 & 61372 & 0 \\
\hline 2907 & NM & Roosevelt County & 0.997403 & 1.050750 & 0.261529 & 144 & 575 & 80533 & 9 \\
\hline 2908 & NM & Sandoval County & 0.998656 & 0.324387 & 0.191367 & 49 & 831 & 87767 & 1 \\
\hline 2909 & NM & San Juan County & 0.995609 & 0.537267 & 0.010437 & 67 & 826 & 80310 & 0 \\
\hline 2910 & NM & San Miguel County & 0.996074 & 0.854458 & 0.001667 & 17 & 469 & 64054 & 0 \\
\hline 2911 & NM & Santa Fe County & 0.999164 & 0.423860 & 0.121258 & 38 & 726 & 115774 & 1 \\
\hline 2912 & NM & Sierra County & 0.986765 & 0.461139 & 0.111228 & 26 & 272 & 84482 & 20 \\
\hline 2913 & NM & Socorto County & 0.999702 & 0.439120 & 0.202046 & 36 & 437 & 70441 & 0 \\
\hline 2914 & NM & Taos County & 0.999338 & 0.230105 & 0.000000 & 20 & 1206 & 70719 & 10 \\
\hline 2915 & NM & Torrance County & 0.999702 & 0.839597 & 0.000793 & 26 & 320 & 70333 & 0 \\
\hline 2916 & NM & Union County & 0.999795 & 0.964551 & 0.000660 & 114 & 332 & 114657 & 0 \\
\hline 2917 & NM & Valencia County & 0.999395 & 0.511100 & 0.513272 & 120 & 1415 & 76036 & 0 \\
\hline 2918 & $A Z$ & Apache County & 0.998798 & 0.806748 & 0.010278 & 6 & 299 & 53393 & 0 \\
\hline 2919 & $A Z$ & Cochise County & 0.992069 & 0.479043 & 0.033864 & 62 & 830 & 86812 & 1 \\
\hline 2920 & $A Z$ & Coconino County & 0.997673 & 0.502672 & 0.000000 & 6 & 450 & 86650 & 0 \\
\hline 2921 & $A Z$ & Gila County & 0.994155 & 0.377274 & 0.066308 & 10 & 707 & 80195 & 1 \\
\hline 2922 & $A Z$ & Graham County & 0.997451 & 0.623190 & 0.018394 & 47 & 636 & 67265 & 1 \\
\hline 2923 & $A Z$ & Greenlee County & 0.999235 & 0.116594 & 0.142216 & 81 & 837 & 88946 & 1 \\
\hline 2924 & $A Z$ & La Paz County & 0.996878 & 0.085438 & 0.000000 & 619 & 3841 & 81644 & 0 \\
\hline 2925 & $A Z$ & Maricopa County & 0.997744 & 0.123917 & 0.345155 & 1709 & 6802 & 122202 & 0 \\
\hline 2926 & $A Z$ & Mohave County & 0.988265 & 0.232623 & 0.053918 & 22 & 502 & 82606 & 1 \\
\hline 2927 & $A Z$ & Navajo County & 0.999356 & 1.134865 & 0.073380 & 7 & 413 & 65043 & 13 \\
\hline 2928 & $A Z$ & Pima County & 0.999735 & 0.590550 & 0.016601 & 27 & 401 & 102892 & 1 \\
\hline 2929 & $A Z$ & Pinal County & 0.999163 & 0.553554 & 0.047092 & 419 & 2461 & 77187 & 0 \\
\hline 2930 & $A Z$ & Santa Cruz County & 0.999617 & 0.421991 & 0.000000 & 35 & 1998 & 78230 & 0 \\
\hline 2931 & $A Z$ & Yavapai County & 0.999411 & 0.405617 & 0.000000 & 23 & 491 & 92664 & 0 \\
\hline 2932 & $A Z$ & Yuma County & 0.999115 & 0.064990 & 0.000000 & 4333 & 10323 & 77824 & 0 \\
\hline 2933 & UT & Beaver County & 0.999107 & 0.115999 & 0.258715 & 261 & 971 & 84902 & 0 \\
\hline
\end{tabular}




\begin{tabular}{|c|c|c|c|c|c|c|c|c|c|}
\hline $\begin{array}{l}\text { County } \\
\text { Code }\end{array}$ & State & $\begin{array}{l}\text { County } \\
\text { Name }\end{array}$ & $\begin{array}{l}\text { FRCLND } \\
\text { Absolute }\end{array}$ & $\begin{array}{l}\text { FRMFRC } \\
\text { Absolute }\end{array}$ & $\begin{array}{c}\text { DPF } \\
\text { Absolute }\end{array}$ & $\begin{array}{l}\text { ASFP } \\
\text { S/ha }\end{array}$ & $\begin{array}{l}\text { VFRM } \\
\text { S/ha }\end{array}$ & $\begin{array}{l}\text { VNFRM } \\
\text { \$/person }\end{array}$ & Notes \\
\hline 2934 & UT & Box Elder County & 0.850543 & 0.395826 & 0.210935 & 144 & 954 & 106481 & 0 \\
\hline 2935 & UT & Cache County & 0.992718 & 0.359456 & 0.476985 & 811 & 3432 & 83243 & 0 \\
\hline 2936 & UT & Carbon County & 0.995880 & 0.308427 & 0.026869 & 26 & 756 & 101612 & 1 \\
\hline 2937 & UT & Daggett County & 0.965833 & 0.049126 & 0.000000 & 149 & 1557 & 113119 & 0 \\
\hline 2938 & UT & Davis County & 0.480611 & 0.258399 & 0.060494 & 1673 & 10119 & 90720 & 0 \\
\hline 2939 & UT & Duchesne County & 0.994504 & 0.192520 & 0.208575 & 138 & 1421 & 82938 & 0 \\
\hline 2940 & UT & Emery County & 0.997828 & 0.084417 & 0.143224 & 84 & 1046 & 75420 & 0 \\
\hline 2941 & UT & Garfield County & 0.993476 & 0.041529 & 0.032468 & 136 & 2144 & 86968 & 1 \\
\hline 2942 & UT & Grand County & 0.996612 & 0.026786 & 0.069742 & 92 & 1452 & 90612 & 1 \\
\hline 2943 & UT & Iron County & 0.998907 & 0.205672 & 0.071794 & 143 & 1110 & 74261 & 0 \\
\hline 2944 & UT & Juab County & 0.995724 & 0.153254 & 0.026702 & 68 & 1027 & 72541 & 1 \\
\hline 2945 & UT & Kane County & 0.971663 & 0.082121 & 0.001103 & 32 & 956 & 78176 & 0 \\
\hline 2946 & UT & Millard County & 0.965016 & 0.114801 & 0.076239 & 304 & 1586 & 84604 & 0 \\
\hline 2947 & UT & Morgan County & 0.997277 & 0.601721 & 0.301932 & 110 & 1199 & 99857 & 0 \\
\hline 2948 & UT & Piute County & 0.989619 & 0.120656 & 0.431962 & 254 & 1701 & 75162 & 0 \\
\hline 2949 & UT & Rich County & 0.946838 & 0.749001 & 0.000000 & 79 & 670 & 103562 & 0 \\
\hline 2950 & UT & Salt Lake County & 0.912844 & 0.228124 & 0.185661 & 463 & 5559 & 104300 & 0 \\
\hline 2951 & UT & San Juan County & 0.985767 & 0.064916 & 0.036415 & 68 & 790 & 55168 & 1 \\
\hline 2952 & UT & Sanpete County & 0.990921 & 0.440233 & 0.160814 & 419 & 1431 & 72697 & 0 \\
\hline 2953 & UT & Sevier County & 0.995815 & 0.129383 & 0.191349 & 479 & 1698 & 80601 & 0 \\
\hline 2954 & UT & Summit County & 0.994156 & 0.311959 & 0.248744 & 99 & 1492 & 137394 & 0 \\
\hline 2955 & UT & Tooele County & 0.953097 & 0.098358 & 0.034099 & 81 & 681 & 101374 & 1 \\
\hline 2956 & UT & Uintah County & 0.995130 & 0.451828 & 0.127771 & 35 & 439 & 74864 & 0 \\
\hline 2957 & UT & Utah County & 0.933375 & 0.352088 & 0.210068 & 481 & 2727 & 77668 & 0 \\
\hline 2958 & UT & Wasatch County & 0.976589 & 0.184371 & 0.433816 & 137 & 3306 & 85363 & 0 \\
\hline 2959 & UT & Washington County & 0.998819 & 0.107746 & 0.031495 & 100 & 2049 & 76679 & 0 \\
\hline 2960 & UT & Wayne County & 0.997507 & 0.067045 & 0.228280 & 204 & 1409 & 68985 & 0 \\
\hline 2961 & UT & Weber County & 0.872769 & 0.696362 & 0.412186 & 296 & 2350 & 103637 & 0 \\
\hline 2962 & NV & Churchill County & 0.981228 & 0.084964 & 0.543783 & 285 & 2532 & 104219 & 0 \\
\hline 2963 & NV & Clark County & 0.977711 & 0.016216 & 0.687062 & 567 & 5162 & 126151 & 0 \\
\hline 2964 & NV & Douglas County & 0.962311 & 0.175286 & 0.267298 & 357 & 4228 & 159787 & 0 \\
\hline 2965 & NV & Elko County & 0.998693 & 0.286443 & 0.023147 & 40 & 375 & 95638 & 21 \\
\hline 2966 & NV & Esmeralda County & 0.999862 & 0.848768 & 0.000000 & 6 & 171 & 150751 & 0 \\
\hline 2967 & NV & Eureka County & 0.998976 & 0.088238 & 0.000000 & 86 & 651 & 193903 & 0 \\
\hline 2968 & NV & Humboldt County & 0.998969 & 0.119521 & 0.025716 & 153 & 652 & 116458 & 21 \\
\hline 2969 & NV & Lander County & 0.995318 & 0.140586 & 0.000000 & 40 & 616 & 108825 & 0 \\
\hline 2970 & NV & Lincoln County & 0.999741 & 0.007195 & 0.088103 & 337 & 2602 & 110274 & 21 \\
\hline 2971 & NV & Lyon County & 0.988739 & 0.148292 & 0.141365 & 473 & 3295 & 112191 & 0 \\
\hline 2972 & NV & Mineral County & 0.985170 & 0.112956 & 0.000000 & 26 & 1170 & 104890 & 0 \\
\hline 2973 & NV & Nye County & 0.999314 & 0.012087 & 0.128053 & 243 & 1411 & 102743 & 21 \\
\hline 2974 & NV & Pershing County & 0.990312 & 0.162411 & 0.000000 & 100 & 530 & 121633 & 0 \\
\hline 2975 & NV & Storey County & 0.998697 & 0.261179 & 0.000000 & 4 & 1171 & 106983 & 0 \\
\hline 2976 & NV & Washoe County & 0.968097 & 0.175064 & 0.039925 & 51 & 658 & 144452 & 21 \\
\hline 2977 & NV & White Pine County & 0.997685 & 0.040795 & 0.000000 & 93 & 859 & 110640 & 0 \\
\hline 2978 & NV & Carson City & 0.921043 & 0.058353 & 0.901479 & 350 & 4125 & 138444 & 22 \\
\hline 2979 & WA & Adams County & 0.997550 & 0.809044 & 0.035262 & 548 & 1411 & 114447 & 0 \\
\hline 2980 & WA & Asotin County & 0.992540 & 0.674588 & 0.041655 & 65 & 828 & 104456 & 1 \\
\hline 2981 & WA & Benton County & 0.967576 & 0.587499 & 0.010974 & 825 & 2773 & 117393 & 0 \\
\hline 2982 & WA & Chelan County & 0.975872 & 0.059943 & 0.000497 & 3351 & 9475 & 112361 & 1 \\
\hline 2983 & WA & Clallam County & 0.652118 & 0.021715 & 0.361574 & 728 & 10352 & 111094 & 0 \\
\hline 2984 & WA & Clark County & 0.956765 & 0.206463 & 0.373985 & 1233 & 11616 & 113722 & 0 \\
\hline 2985 & WA & Columbia County & 0.994604 & 0.548398 & 0.000000 & 159 & 1749 & 121423 & 0 \\
\hline 2986 & WA & Cowlitz County & 0.976259 & 0.048956 & 0.172934 & 1184 & 7374 & 109509 & 0 \\
\hline 2987 & WA & Douglas County & 0.984766 & 0.787897 & 0.000692 & 294 & 1415 & 106630 & 1 \\
\hline 2988 & WA & Ferry County & 0.976310 & 0.530352 & 0.000000 & 15 & 841 & 82735 & 0 \\
\hline 2989 & WA & Franklin County & 0.981667 & 0.842956 & 0.037386 & 883 & 27.14 & 99796 & 0 \\
\hline 2990 & WA & Garfield County & 0.989359 & 0.715725 & 0.004078 & 141 & 1080 & 162442 & 1 \\
\hline 2991 & WA & Grant County & 0.958803 & 0.634045 & 0.030083 & 1097 & 2821 & 105059 & 0 \\
\hline 2992 & WA & Grays Harbor County & 0.861847 & 0.036463 & 0.639554 & 1026 & 7649 & 103400 & 0 \\
\hline 2993 & WA & Island County & 0.403164 & 0.146264 & 0.723597 & 1171 & 12816 & 105018 & 0 \\
\hline 2994 & WA & Jefferson County & 0.830484 & 0.008295 & 0.477198 & 863 & 8532 & 109590 & 0 \\
\hline 2995 & WA & King County & 0.921679 & 0.031079 & 0.478072 & 4940 & 25276 & 161060 & 0 \\
\hline 2996 & WA & Kitsap County & 0.699657 & 0.040649 & 0.007467 & 2538 & 24020 & 115205 & 0 \\
\hline 2997 & WA & Kittitas County & 0.984606 & 0.241695 & 0.064546 & 489 & 2645 & 99519 & 0 \\
\hline 2998 & WA & Klickitat County & 0.983294 & 0.575473 & 0.065735 & 122 & 1135 & 106380 & 0 \\
\hline 2999 & WA & Lewis County & 0.988250 & 0.072851 & 0.431173 & 1358 & 6194 & 100961 & 0 \\
\hline 3000 & WA & Lincoln County & 0.987820 & 0.990935 & 0.000925 & 138 & 1342 & 149823 & 1 \\
\hline 3001 & WA & Mason County & 0.914392 & 0.017826 & 0.189327 & 270 & 7417 & 95787 & 1 \\
\hline 3002 & WA & Okanogan County & 0.991142 & 0.382927 & 0.004366 & 265 & 1617 & 101307 & 1 \\
\hline 3003 & WA & Pacific County & 0.796486 & 0.052326 & 0.309700 & 960 & 5466 & 102736 & 0 \\
\hline
\end{tabular}




\begin{tabular}{|c|c|c|}
\hline $\begin{array}{l}\text { County } \\
\text { Code }\end{array}$ & State & $\begin{array}{c}\text { County } \\
\text { Name }\end{array}$ \\
\hline 3004 & WA & Pend Oreille County \\
\hline 3005 & WA & Pierce County \\
\hline 3006 & WA & San Juan County \\
\hline 3007 & WA & Skagit County \\
\hline 3008 & WA & Skamania County \\
\hline 3009 & WA & Snohomish County \\
\hline 3010 & WA & Spokane County \\
\hline 3011 & WA & Stevens County \\
\hline 3012 & WA & Thurston County \\
\hline 3013 & WA & Wahkiakum County \\
\hline 3014 & WA & Walla Walla County \\
\hline 3015 & WA & Whatcom County \\
\hline 3016 & WA & Whitman County \\
\hline 3017 & WA & Yakima County \\
\hline 3018 & OR & Baker County \\
\hline 3019 & OR & Benton County \\
\hline 3020 & OR & Clackamas County \\
\hline 3021 & OR & Clatsop County \\
\hline 3022 & OR & Columbia County \\
\hline 3023 & OR & Coos County \\
\hline 3024 & OR & Crook County \\
\hline 3025 & OR & Curry County \\
\hline 3026 & OR & Deschutes County \\
\hline 3027 & OR & Douglas County \\
\hline 3028 & OR & Gilliam County \\
\hline 3029 & OR & Grant County \\
\hline 3030 & OR & Harney County \\
\hline 3031 & OR & Hood River County \\
\hline 3032 & OR & Jackson County \\
\hline 3033 & OR & Jefferson County \\
\hline 3034 & OR & Josephine County \\
\hline 3035 & OR & Klamath County \\
\hline 3036 & OR & Lake County \\
\hline 3037 & OR & Lane County \\
\hline 3038 & OR & Lincoln County \\
\hline 3039 & OR & Linn County \\
\hline 3040 & OR & Malheur County \\
\hline 3041 & OR & Marion County \\
\hline 3042 & OR & Morrow County \\
\hline 3043 & OR & Multnomah County \\
\hline 3044 & OR & Polk County \\
\hline 3045 & OR & Sherman County \\
\hline 3046 & OR & Tillamook County \\
\hline 3047 & OR & Umatilla County \\
\hline 3048 & OR & Union County \\
\hline 3049 & OR & Wallowa County \\
\hline 3050 & OR & Wasco County \\
\hline 3051 & OR & Washington County \\
\hline 3052 & OR & Wheeler County \\
\hline 3053 & OR & Yamhill County \\
\hline 3054 & $\mathrm{CA}$ & Alameda County \\
\hline 3055 & CA & Alpine County \\
\hline 3056 & $\mathrm{CA}$ & Amador County \\
\hline 3057 & $\mathrm{CA}$ & Butte County \\
\hline 3058 & $\mathrm{CA}$ & Calaveras County \\
\hline 3059 & CA & Colusa County \\
\hline 3060 & $\mathrm{CA}$ & Contra Costa County \\
\hline 3061 & $\mathrm{CA}$ & Del Norte County \\
\hline 3062 & $\mathrm{CA}$ & El Dorado County \\
\hline 3063 & $\mathrm{CA}$ & Fresno County \\
\hline 3064 & CA & Glenn County \\
\hline 3065 & CA & Humboldt County \\
\hline 3066 & $\mathrm{CA}$ & Imperial County \\
\hline 3067 & $\mathrm{CA}$ & Inyo County \\
\hline 3068 & $\mathrm{CA}$ & Kern County \\
\hline 3069 & $\mathrm{CA}$ & Kings County \\
\hline 3070 & $\mathrm{CA}$ & Lake County \\
\hline 3071 & $\mathrm{CA}$ & Lassen County \\
\hline 3072 & CA & Los Angeles County \\
\hline 3073 & $\mathrm{CA}$ & Madera County \\
\hline
\end{tabular}

FRCLND FRMFRC DPF ASFP VFRM VNFRM Absolute Absolute Absolute \$/ha \$/ha \$/person Notes

$\begin{array}{lllrrrr}0.982556 & 0.061765 & 0.170802 & 124 & 2967 & 88085 & 0 \\ 0.927427 & 0.054786 & 0.257821 & 3579 & 13954 & 109685 & 0 \\ 0.281669 & 0.183355 & 0.064236 & 142 & 9046 & 148658 & 1 \\ 0.903564 & 0.082906 & 0.332488 & 3716 & 10188 & 111439 & 0 \\ 0.983714 & 0.003814 & 0.000000 & 612 & 8919 & 92298 & 0 \\ 0.951593 & 0.055432 & 0.566523 & 3267 & 15897 & 125609 & 0 \\ 0.990447 & 0.554349 & 0.113574 & 292 & 3009 & 110843 & 0 \\ 0.975466 & 0.344425 & 0.237458 & 106 & 1743 & 90686 & 0 \\ 0.939744 & 0.128707 & 0.333681 & 3202 & 11840 & 117258 & 0 \\ 0.921708 & 0.074566 & 0.776003 & 981 & 6983 & 110119 & 0 \\ 0.977879 & 0.873859 & 0.013660 & 687 & 2077 & 111338 & 0 \\ 0.846788 & 0.087067 & 0.685751 & 4429 & 12517 & 108385 & 0 \\ 0.991629 & 1.016123 & 0.001933 & 275 & 2284 & 105913 & 13 \\ 0.996368 & 0.596456 & 0.112248 & 1039 & 2508 & 104131 & 0 \\ 0.993422 & 0.416934 & 0.083468 & 118 & 1015 & 99363 & 0 \\ 0.996303 & 0.274431 & 0.123283 & 775 & 5409 & 111487 & 0 \\ 0.994236 & 0.124485 & 0.043667 & 2510 & 13926 & 135071 & 0 \\ 0.762640 & 0.046725 & 0.480708 & 655 & 4679 & 115022 & 0 \\ 0.954087 & 0.170910 & 0.185436 & 422 & 5899 & 104937 & 0 \\ 0.886016 & 0.170716 & 0.292840 & 389 & 3374 & 100880 & 0 \\ 0.997311 & 0.469273 & 0.009385 & 78 & 704 & 105079 & 1 \\ 0.818365 & 0.071408 & 0.015396 & 347 & 3333 & 102492 & 0 \\ 0.98006 & 0.072206 & 0.12206 & 290 & 4741 & 113905 & 0\end{array}$

$\begin{array}{lllllll}0.988006 & 0.072206 & 0.122066 & 290 & 4741 & 113905 & 0\end{array}$

$\begin{array}{llllrrr}0.984647 & 0.994457 & 0.003806 & 56 & 507 & 143802 & 1\end{array}$

$\begin{array}{lll}0.999843 & 0.398282 & 0.003629\end{array}$

$0.990988 \quad 0.224678 \quad 0.001186$

$\begin{array}{lll}0.978990 & 0.081360 & 0.001199\end{array}$

$\begin{array}{llll}0.994084 & 0.147114 & 0.022408\end{array}$

$\begin{array}{lll}0.994209 & 0.465846 & 0.001551\end{array}$

$\begin{array}{lll}0.998756 & 0.029779 & 0.474065\end{array}$

$\begin{array}{llll}0.968779 & 0.189287 & 0.121985\end{array}$

$0.973359 \quad 0.159974 \quad 0.000137$

$\begin{array}{lll}0.964464 & 0.083070 & 0.101132\end{array}$

$0.820551 \quad 0.054694 \quad 0.056990$

$0.992256 \quad 0.259435 \quad 0.099150$

$0.995694 \quad 0.208346 \quad 0.055264$

$\begin{array}{llll}0.991459 & 0.398819 & 0.105344\end{array}$

$\begin{array}{lll}0.992274 & 0.860095 & 0.001400\end{array}$

$0.934649 \quad 0.112338 \quad 0.001704$

$\begin{array}{llll}0.995809 & 0.353956 & 0.159264\end{array}$

$\begin{array}{llll}0.990381 & 0.925268 & 0.003200\end{array}$

$\begin{array}{lll}0.826954 & 0.056079 & 0.909728\end{array}$

$\begin{array}{llll}0.995049 & 0.712687 & 0.002951\end{array}$

$\begin{array}{llll}0.999157 & 0.363118 & 0.000065\end{array}$

$\begin{array}{llll}0.997983 & 0.344898 & 0.009187\end{array}$

$\begin{array}{llll}0.994040 & 0.756559 & 0.002703\end{array}$

$\begin{array}{llll}0.996377 & 0.301837 & 0.081889\end{array}$

$\begin{array}{lll}0.999683 & 0.663384 & 0.013724\end{array}$

$\begin{array}{llll}0.996093 & 0.392560 & 0.122395\end{array}$

$\begin{array}{llll}0.898010 & 0.606540 & 0.023127\end{array}$

$\begin{array}{llll}0.993848 & 0.010086 & 0.000000\end{array}$

$\begin{array}{lll}0.980645 & 0.622879 & 0.000000\end{array}$

$\begin{array}{llll}0.977572 & 0.431076 & 0.010599\end{array}$

$\begin{array}{llll}0.983859 & 0.376887 & 0.022254\end{array}$

$0.995204 \quad 0.611320 \quad 0.001915$

$\begin{array}{llll}0.897914 & 0.353670 & 0.030475\end{array}$

$\begin{array}{llll}0.819529 & 0.019524 & 0.230491\end{array}$

$\begin{array}{llll}0.955446 & 0.093146 & 0.034544\end{array}$

$\begin{array}{llll}0.990913 & 0.465003 & 0.070692\end{array}$

$\begin{array}{llll}0.990686 & 0.563167 & 0.156677\end{array}$

$\begin{array}{llll}0.881623 & 0.261422 & 0.497351\end{array}$

$\begin{array}{llll}0.931498 & 0.199423 & 0.008049\end{array}$

$\begin{array}{llll}0.996512 & 0.037951 & 0.000000\end{array}$

$\begin{array}{llll}0.997497 & 0.544950 & 0.046348\end{array}$

$\begin{array}{llll}0.998494 & 0.872432 & 0.277335\end{array}$

$\begin{array}{llll}0.946507 & 0.203783 & 0.030778\end{array}$

$\begin{array}{llll}0.965445 & 0.167135 & 0.020525\end{array}$

$\begin{array}{llll}0.854314 & 0.070647 & 0.032256\end{array}$

$\begin{array}{llll}0.993051 & 0.547617 & 0.080855\end{array}$

$\begin{array}{rrrr}39 & 575 & 108358 & 1 \\ 60 & 686 & 110112 & 0 \\ 4989 & 17051 & 111223 & 1 \\ 487 & 4667 & 105540 & 0 \\ 198 & 932 & 94506 & 1 \\ 973 & 9910 & 95773 & 0 \\ 292 & 1812 & 96843 & 0 \\ 108 & 862 & 111900 & 0 \\ 717 & 5920 & 106346 & 0 \\ 416 & 4550 & 107653 & 1 \\ 906 & 4744 & 95834 & 0 \\ 374 & 1350 & 98374 & 0 \\ 2558 & 8698 & 108520 & 0 \\ 208 & 980 & 127797 & 1 \\ 3053 & 13791 & 130147 & 1 \\ 966 & 5254 & 99817 & 0 \\ 104 & 812 & 166993 & 1 \\ 3977 & 8748 & 101374 & 0 \\ 315 & 1377 & 102065 & 0 \\ 242 & 1440 & 99119 & 0 \\ 102 & 857 & 118267 & 1 \\ 104 & 1016 & 111765 & 1 \\ 2254 & 12354 & 135003 & 0 \\ 22 & 523 & 139061 & 0 \\ 1699 . & 7736 & 103596 & 0 \\ 369 & 6259 & 150013 & 1 \\ 92 & 4007 & 155784 & 7 \\ 237 & 2999 & 101442 & 0 \\ 997 & 6301 & 101943 & 0 \\ 149 & 2624 & 92657 & 1 \\ 943 & 4514 & 115219 & 1 \\ 818 & 8050 & 172872 & 1 \\ 4118 & 9769 & 78908 & 0 \\ 231 & 9276 & 130384 & 1 \\ 2898 & 7953 & 110843 & 0 \\ 899 & 5127 & 106028 & 0 \\ 256 & 2369 & 109306 & 0 \\ 3492 & 6090 & 98367 & 0 \\ 60 & 3256 & 122249 & 0 \\ 1163 & 3171 & 107565 & 0 \\ 1853 & 4560 & 94195 & 0 \\ 483 & 5460 & 104930 & 1 \\ 163 & 1319 & 86589 & 1 \\ 2698 & 16573 & 140788 & 0 \\ 1556 & 5622 & 94127 & 0 \\ & & & \end{array}$




\begin{tabular}{|c|c|c|c|c|c|c|c|c|c|}
\hline $\begin{array}{l}\text { County } \\
\text { Code }\end{array}$ & State & $\begin{array}{l}\text { County } \\
\text { Name }\end{array}$ & $\begin{array}{l}\text { FRCLND } \\
\text { Absolute }\end{array}$ & $\begin{array}{l}\text { FRMFRC } \\
\text { Absolute }\end{array}$ & $\begin{array}{c}\text { DPF } \\
\text { Absolute }\end{array}$ & $\begin{array}{l}\text { ASFP } \\
\text { \$/ha }\end{array}$ & $\begin{array}{l}\text { VFRM } \\
\text { S/ha }\end{array}$ & $\begin{array}{l}\text { VNFRM } \\
\text { S/person }\end{array}$ & Notes \\
\hline $\begin{array}{l}3074 \\
3075\end{array}$ & $\begin{array}{l}C A \\
C A\end{array}$ & $\begin{array}{l}\text { Marin County } \\
\text { Mariposa County }\end{array}$ & $\begin{array}{l}0.627585 \\
0.992017\end{array}$ & $\begin{array}{l}0.507667 \\
0.221945\end{array}$ & $\begin{array}{l}0.665291 \\
0.000000\end{array}$ & $\begin{array}{r}617 \\
84\end{array}$ & $\begin{array}{l}6688 \\
2094\end{array}$ & $\begin{array}{l}254273 \\
109536\end{array}$ & $\begin{array}{l}0 \\
0\end{array}$ \\
\hline $\begin{array}{l}3075 \\
3076\end{array}$ & $\mathrm{CA}$ & Mendocino County & 0.904804 & 0.322857 & 0.044349 & 228 & 2938 & 109177 & $\begin{array}{l}0 \\
1\end{array}$ \\
\hline 3077 & $\mathrm{CA}$ & Merced County & 0.978130 & 0.792902 & 0.311481 & 2291 & 6302 & 94561 & 0 \\
\hline 3078 & $\mathrm{CA}$ & Modoc County & 0.938325 & 0.272095 & 0.005604 & 211 & 1635 & 96681 & 1 \\
\hline 3079 & $\mathrm{CA}$ & Mono County & 0.972091 & 0.053013 & 0.000000 & 178 & 1734 & 127350 & 0 \\
\hline 3080 & $\mathrm{CA}$ & Monterey County & 0.880893 & 0.645696 & 0.006188 & 2165 & 4936 & 131840 & 0 \\
\hline 3081 & $\mathrm{CA}$ & Napa County & 0.956263 & 0.487676 & 0.002677 & 1291 & 13483 & 153535 & 1 \\
\hline 3082 & $\mathrm{CA}$ & Nevada County & 0.982680 & 0.118243 & 0.361924 & 155 & 8734 & 121403 & 1 \\
\hline 3083 & $\mathrm{CA}$ & Orange County & 0.833079 & 0.120183 & 0.000000 & 7581 & 31190 & 165266 & 0 \\
\hline 3084 & $\mathrm{CA}$ & Placer County & 0.936127 & 0.153226 & 0.036849 & 641 & 10491 & 136602 & 1 \\
\hline 3085 & $\mathrm{CA}$ & Plumas County & 0.977126 & 0.073118 & 0.000000 & 137 & 2154 & 110904 & 0 \\
\hline 3086 & $\mathrm{CA}$ & Riverside County & 0.986903 & 0.091824 & 0.284468 & 4941 & 15561 & 116397 & 0 \\
\hline 3087 & $\mathrm{CA}$ & Sacramento County & 0.969906 & 0.613297 & 0.163469 & 1366 & 7351 & 128244 & 0 \\
\hline 3088 & $\mathrm{CA}$ & San Benito County & 0.998795 & 0.674963 & 0.018677 & 446 & 3147 & 109753 & 0 \\
\hline 3089 & $\mathrm{CA}$ & San Bernardino County & 0.997781 & 0.100242 & 0.689341 & 1090 & 1894 & 111074 & 0 \\
\hline 3090 & $\mathrm{CA}$ & San Diego County & 0.928977 & 0.192451 & 0.031484 & 2369 & 13637 & 132673 & 0 \\
\hline 3091 & $\mathrm{CA}$ & San Francisco County & 0.201392 & 0.000234 & 0.000000 & 260519 & 2750264 & 193253 & 7 \\
\hline 3092 & $\mathrm{CA}$ & San Joaquin County & 0.981118 & 0.875034 & 0.197842 & 2475 & 10704 & 104666 & 0 \\
\hline 3093 & $\mathrm{CA}$ & San Luis Obispo County & 0.913922 & 0.626230 & 0.005475 & 345 & 3985 & 116641 & 0 \\
\hline 3094 & $\mathrm{CA}$ & San Mateo County & 0.606032 & 0.199763 & 0.000000 & 4308 & 18726 & 195197 & 0 \\
\hline 3095 & $\mathrm{CA}$ & Santa Barbara County & 0.722634 & 0.477564 & 0.021669 & 1154 & 6175 & 148347 & 0 \\
\hline 3096 & $\mathrm{CA}$ & Santa Clara County & 0.989797 & 0.414643 & 0.015019 & 902 & 5861 & 170637 & 0 \\
\hline 3097 & $\mathrm{CA}$ & Santa Cruz County & 0.733546 & 0.185432 & 0.002722 & 11296 & 24407 & 149180 & 1 \\
\hline 3098 & $\mathrm{CA}$ & Shasta County & 0.983899 & 0.160179 & 0.048316 & 211 & 2689 & 110247 & 0 \\
\hline 3099 & $\mathrm{CA}$ & Sierra County & 0.991067 & 0.090866 & 0.000000 & 85 & 2300 & 114799 & 0 \\
\hline 3100 & $C A$ & Siskiyou County & 0.990460 & 0.160902 & 0.045550 & 195 & 2638 & 104280 & 0 \\
\hline 3101 & $\mathrm{CA}$ & Solano County & 0.913234 & 0.642096 & 0.044857 & 822 & 5754 & 116960 & 0 \\
\hline 3102 & $\mathrm{CA}$ & Sonoma County & 0.891344 & 0.512617 & 0.235019 & 1342 & 12133 & 149383 & 0 \\
\hline 3103 & $\mathrm{CA}$ & Stanislaus County & 0.986638 & 0.794180 & 0.287131 & 2918 & 9730 & 103210 & 0 \\
\hline 3104 & $\mathrm{CA}$ & Sutter County & 0.989878 & 0.824827 & 0.003487 & 1651 & 9461 & 107890 & 0 \\
\hline 3105 & CA & Tehama County & 0.996200 & 0.538395 & 0.100357 & 231 & 2301 & 82924 & 0 \\
\hline 3106 & $\mathrm{CA}$ & Trinity County & 0.990983 & 0.057058 & 0.191245 & 37 & 895 & 93565 & 1 \\
\hline 3107 & $\mathrm{CA}$ & Tulare County & 0.996883 & 0.438618 & 0.288025 & 2530 & 8046 & 99634 & 0 \\
\hline 3108 & $\mathrm{CA}$ & Tuolumne County & 0.982909 & 0.096120 & 0.026905 & 220 & 2188 & 100406 & 1 \\
\hline & & Ventura County & 0.835891 & 0.271368 & 0.001479 & 5147 & 17519 & 145082 & 1 \\
\hline & & Yolo County & 0.989805 & 0.800826 & 0.002811 & 1115 & 5551 & 133615 & 1 \\
\hline 3111 & & Yuba County & 0.979641 & 0.581876 & 0.029790 & 1142 & 6226 & 84089 & 0 \\
\hline
\end{tabular}

Notes:

1. See note explanation $A$.

2. See note explanations $B, C, D, E$.

3. See note explanations $B, C, D, E, K$.

4. See note explanations $C, D, L$.

5. See note explanations $B, C, D, E, L$.

6. See note explanations $A, B$.

7. See note explanation $C$.

8. See note explanations $B, C$.

9. See note explanation $\mathrm{F}$.

10. See note explanation $E$.

11. See note explanations $C, D, E$.

12. See note explanation $B$.

13. See note explanations $A, F$.

14. See note explanations A, C, D.

15. See note explanations $B, E$.

16. See note explanations $B, G$.

17. See note explanations $B, H$.

18. See note explanation $D$.

19. See note explanations $C, E, M$.

20. See note explanations $A, C, N$.

21. See note explanations $A, J$.

22. See note explanations $A, I$.

23. See note explanation $O$. 
24. See note explanations $A, O$.

25. See note explanations $B, C, D, E, O$.

Note explanations:

A. The amount of dairy sales for this county were not disclosed because of Bureau of Census confidentiality requirements. The dairy sales for this county were estimated by distributing the state's total amount of undisclosed dairy sales equally amongst the state's dairy farms that resided in counties with undisclosed dairy sales.

B. The amount of farm land for this county was not disclosed because of Bureau of Census confidentiality requirements. The farm land for this county was estimated by distributing the state's total amount of undisclosed farm land equally amongst the state's farms that resided in counties with undisclosed farm land.

C. The value of farm land and buildings for this county were not disclosed because of Bureau of Census confidentiality requirements. The value of farm land and buildings for this county were estimated by distributing the state's total amount of undisclosed farm land and building value equally amongst the state's farms that resided in counties with undisclosed farm land and building values.

D. The value of farm machinery for this county was not disclosed because of Bureau of Census confidentiality requirements. The value of farm machinery for this county was estimated by distributing the state's total amount of undisclosed farm machinery value equally amongst the state's farms that resided in counties with undisclosed farm machinery values.

E. The amount of farm sales for this county were not disclosed because of Bureau of Census confidentiality requirements. The farm sales for this county were estimated by distributing the state's total amount of undisclosed farm sales equally amongst the state's farms that resided in counties with undisclosed farm sales.

F. The value of FRMFCD was truncated to 1.000000 . The resulting excess acreage was considered insignificant.

G. The value of FRMFCD was truncated to 1.000000 . The resulting excess estimated undisclosed acreage, 121579 acres, was added to Kleberg County.

H. The estimated undisclosed acreage includes 121579 acres from El Paso County.

I. Estimated undisclosed dairy sales reduced to equal the same average value per farm as Douglas County. The excess undisclosed dairy sales were added to the other undisclosed county estimates.

J. Estimated undisclosed dairy sales include the excess estimated undisclosed amount from Carson City.

$\mathrm{K}$. Estimated undisclosed farm land and building and machinery values set so that farm land and building and machinery values per farm land area equal those of the average value of the surrounding counties, Franklin, Herkimer, and St. Lawrence. The excess undisclosed estimate farm land and building and machinery values were apportioned among Kings, Queens, and Richmond counties so that those county's undisclosed estimates would all have equal farm land and building and machinery values per farmland area.

L. Estimated undisclosed farm land and building and machinery values include excess undisclosed estimates from Hamilton County.

M. Estimated undisclosed farm land and building value per farm land were set equal to the average value of the surrounding counties, Sandoval, Santa Fe, and Rio Arriba. The excess estimated undisclosed farm land and building value was added to the Sierra County undisclosed estimate.

N. Estimated undisclosed farm land and building value includes excess undisclosed estimate from Los Alamos County.

O. One or more counties reported per capita income figures cojointly with this county. The per capita income for all such cojoint counties was set to be equal. The list of counties reporting cojointly is as follows:

Yellowstone National Park included with Park County; Independent city of Charlottesville included with Albemarle County; Independent cities of Clifton Forge and Covington included with Alleghany County; Independent cities of Staunton and Waynesboro included with Augusta County; Independent city of Bedford included with Bedford County; Independent city of Lynchburg included with Campbell County; Independent city of Galax included with Carroll County; 
Independent cities of Colonial Heights and Petersburg included with Dinwiddie County; Independent cities of Fairfax and Falls Church included with Fairfax County;

Independent city of Winchester included with Frederick County;

Independent city of Emporia included with Greensville County;

Independent city of South Boston included with Halifax County;

Independent city of Martinsville included with Henry County;

Independent city of Williamsburg included with James City County;

Independent city of Radford included with Montgomery County;

Independent city of Danville included with Pittsylvania County;

Independent city of Hopewell included with Prince George County;

Independent cities of Manassas and Manassas Park included with Prince William County;

Independent city of Salem included with Roanoke County;

Independent cities of Buena Vista and Lexington included with Rockbridge County;

Independent city of Harrisonburg included with Rockingham County;

Independent city of Franklin included with Southampton County;

Independent city of Fredericksburg included with Spotsylvania County;

Independent city of Bristol included with Washington County;

Independent city of Norton included with Wise County;

Independent city of Poquoson included with York County; and

Menominee County included with Shawano County. 


\section{APPENDIX D — STATISTICAL ABSTRACTS}

This appendix contains the following values and source information for the parameters used in estimating the value of nonfarm assets (VNFRM, \$ / person).

1993 \& 1994 Statistical Abstract of the U.S.

Reproducible Tangible Wealth (1990):

$2.5652 E+13 \$$

Urban and Built-Up Land (1987) :

77187303 acres

Urban and Built-Up Land (1992) :

91815303 acres

Urban and Built-Up Land (1990 Straight Line approx.) :

85964103 acres

Median Housing Value (1990):

$79100 \$$

Total Farm Assets (1990) :

$1.0036 E+12 \$$

Farm Household Possessions (1990) :

$4.6400 E+10 \$$

Population (1990) :

248709873 persons

\section{NUREG/CR-4551 Volume 2, Revision 1, Part 7}

Typical Suburban Lot Size :

0.2 acres

Land - Fraction of Housing Value :

$20 \%$

Average non-farm value for the U.S. (1990) :

126632 \$/person

1994 County and City Data Book

U.S. Per Capita Income (1990):

18696 \$/person 
No. 754. Gross and Net Stock of Fixed Reproduclble Tangible Wealth in Curront and Constant (1987) Dollars

(In blllions of dollars. As of December 31)

\begin{tabular}{|c|c|c|c|c|c|c|c|c|c|c|c|c|}
\hline ITEM & 1970 & 1980 & 1981 & 1982 & 1983 & 1984 & 1985 & 1986 & 1987 & 1988 & 1989 & 1990 \\
\hline \multicolumn{13}{|l|}{ CURRENT DOLLARS } \\
\hline Gross stock, total & 4,428 & 14,306 & 15,670 & 16,527 & 17,277 & 18,259 & 19,330 & 20,503 & 21,774 & 22,966 & 24,361 & 25,652 \\
\hline Private & 2,689 & 9,364 & 10,387 & 11,005 & 11,477 & 12,082 & 12,747 & 13,536 & 14,388 & 15,078 & 16,022 & 16,894 \\
\hline Nonresidential equipment & 679 & 2,389 & 2,723 & 2,939 & 3,067 & 3,214 & 3,374 & 3,599 & 3,775 & 4,010 & 4,257 & 4,543 \\
\hline Nonresidentlal structures & 790 & 2,683 & 3,032 & 3,247 & 3,376 & 3,573 & 3,783 & 3,951 & 4.156 & 4,478 & 4,755 & 4,964 \\
\hline ResIdentlal & 1,219 & 4,292 & 4,632 & 4,818 & 5,034 & 5,296 & 5,590 & 5,986 & 6,457 & 6,590 & 7,010 & 7,387 \\
\hline Government & 1,060 & 2,979 & 3,141 & 3,237 & 3,368 & 3,568 & 3,772 & 3,916 & 4,083 & 4,306 & 4,516 & 4,713 \\
\hline Equipment & 274 & 488 & 543 & 583 & 621 & 657 & 695 & 732 & 764 & 814 & 873 & 949 \\
\hline Structures & 785 & 2,491 & 2,599 & 2,654 & 2,748 & 2,911 & 3,077 & 3,184 & 3,319 & 3,492 & 3,643 & 3,764 \\
\hline Federal & 424 & 885 & 968 & 1,018 & 1,067 & 1,119 & 1,176 & 1,227 & 1,269 & 1,325 & 1,394 & 1,469 \\
\hline Millta & 276 & 493 & 535 & 566 & 601 & 638 & 678 & 715 & 738 & 776 & 818 & 873 \\
\hline State a & 636 & 2,094 & 2,173 & 2,219 & 2,301 & 2,449 & 2,596 & 2,690 & 2,814 & 2,981 & 3,122 & 3,244 \\
\hline Consumer durable goods & 680 & 1,963 & 2,142 & 2,285 & 2,433 & 2,608 & 2,811 & 3.051 & 3,303 & 3,582 & 3,823 & 4,045 \\
\hline Net stock, total & 2,708 & 8,619 & 9,374 & 9,792 & 10,172 & 10,734 & 11,367 & 12,063 & 12,803 & 13,458 & 14,245 & 14,936 \\
\hline Private & 1,674 & 5,814 & 6,413 & 6,737 & 6,987 & 7,348 & 7,752 & 8,224 & 8,729 & 9,108 & 9,650 & 10,130 \\
\hline Government & 662 & 1,790 & 1,875 & 1,921 & 1,991 & 2,105 & 2,224 & 2,311 & 2,414 & 2,542 & 2,665 & 2,775 \\
\hline Consumer durable goods & 372 & 1,014 & 1,086 & 1,134 & 1,194 & 1,281 & 1,391 & 1,527 & 1,660 & 1,808 & 1,930 & 2,031 \\
\hline \multicolumn{13}{|l|}{ CONSTAR } \\
\hline Gross stock & 12,476 & 17,469 & 17,963 & 18,370 & 18,845 & 19,441 & 20,091 & 20,753 & 21,400 & 22,062 & 22,717 & 23,339 \\
\hline Private & 8,131 & 11,585 & 11,936 & 12,217 & 12,523 & 12,909 & 13,322 & 13,728 & 14,112 & 14,504 & 14.886 & 15,245 \\
\hline Nontesident & 1,839 & 3,009 & 3,126 & 3,206 & 3,285 & 3,396 & 3,518 & 3,633 & 3,737 & 3,855 & 3,979 & 4,093 \\
\hline Nonsosic & 2,411 & 3,255 & 3,384 & 3,5 & 3,606 & 3,7 & 3,870 & 3,9 & 4,088 & 4,191 & 4,293 & 4,398 \\
\hline Reslden & 3,881 & 5,321 & 5,427 & 5,50 & 5,632 & 5,783 & 5,934 & 6,112 & 6,286 & 6.458 & 6,614 & 6,754 \\
\hline Governm & 3,001 & 3,544 & 3,603 & 3,648 & 3,705 & 3,770 & 3,849 & 3,934 & 4,026 & 4,115 & 4,204 & 4,307 \\
\hline Equlpn & 662 & 625 & 630 & 634 & 647 & 670 & 698 & 730 & 768 & 802 & 837 & 879 \\
\hline Structu & 2,339 & 2,918 & 2,972 & 3,014 & 3,058 & 3,100 & 3,152 & 3,204 & 3,259 & 3,313 & 3,367 & 3.428 \\
\hline Feder & 1,141 & 1,122 & 1,138 & 1,14 & 1,156 & 1,17 & 1.204 & 1,2 & 1,266 & 1,2 & 1,319 & 1,351 \\
\hline Milite & 723 & 641 & 643 & 64 & 652 & 668 & 691 & 716 & 743 & of & 786 & 813 \\
\hline State & 1,860 & 2, & 2,465 & 2 & 2,549 & 2,5 & 2,645 & 2,7 & 2,760 & 2,822 & 2,885 & 2,956 \\
\hline Consumer dural & 1,344 & 2,3 & 2,424 & 2,50 & 2,617 & 2,762 & 2,920 & 3.091 & 3,262 & 3,444 & 3,627 & 3,787 \\
\hline Net-stock, total & 7,691 & 10,524 & 10,755 & 10,902 & 11,112 & 11,441 & 11,823 & 12,214 & 12,577 & 12,942 & 13,293 & 13,598 \\
\hline Privat & 5,074 & 7,198 & 7,384 & 7,499 & 7,642 & 7,866 & 8,112 & 8,346 & 8,558 & 8,774 & 8,980 & 9,157 \\
\hline Cournm & 1,884 & 2,123 & 2,148 & 2,166 & 2,191 & 2,223 & 2,269 & 2,320 & 2,380 & 2,430 & 2,482 & 2,537 \\
\hline Consumer d & 733 & 1,203 & 1,223 & 1,236 & 1,278 & 1,352 & 1,442 & 1,547 & 1,639 & 1,738 & 1,831 & 1,903 \\
\hline
\end{tabular}

Source: U.S. Bureau of Economlc Analysls, Survey of Current Business,

$$
\text { nuary and August } 1992 .
$$


No. 359. Land Cover/Use, by State: 1987

[In thousands of acres. Excludes Alaska and District of Columbia]

\begin{tabular}{|c|c|c|c|c|c|c|c|c|c|c|}
\hline REGION, & & & & & & Rural land & & & Rural land & \\
\hline $\begin{array}{l}\text { AND } \\
\text { STATE }\end{array}$ & $\begin{array}{l}\text { surface } \\
\text { area II }\end{array}$ & land & Total & Developed 12 & Total & $\begin{array}{l}\text { Crop } \\
\text { land }\end{array}$ & $\begin{array}{l}\text { Pasture } \\
\text { land }\end{array}$ & $\begin{array}{c}\text { Range- } \\
\text { band }\end{array}$ & $\begin{array}{c}\text { Forest } \\
\text { land }\end{array}$ & $\begin{array}{c}\text { Minor } \\
\text { cover/uses }\end{array}$ \\
\hline United States & $1,937,726$ & 404,069 & $1,484,156$ & 77,305 & $1,406,851$ & 422,416 & 129,021 & 401,685 & 393,904 & 59,826 \\
\hline Northeast & 108,080 & 2,392 & 100,778 & 9,611 & 91,166 & 14,532 & 7,669 & 0 & 64,246 & 4,720 \\
\hline New England & 42,670 & 1,332 & 38,609 & 3,005 & 35,604 & 2,311 & 1,248 & 0 & 30,307 & 1,739 \\
\hline Maine & 21,290 & 161 & 19,517 & 508 & 19,009 & 943 & 419 & 0 & 16,933 & 714 \\
\hline New Hampshire & 5,938 & 729 & 4,971 & 372 & 4,599 & 163 & 115 & 0 & 4,052 & 269 \\
\hline Vermont & 6,153 & 335 & 5,556 & 208 & 5,348 & 653 & 388 & 0 & 4.184 & 122 \\
\hline Massachusetts & 5,302 & 89 & 4,849 & 1.063 & 3,786 & 291 & 179 & 0 & 2,937 & 379 \\
\hline Rhode Island & 776 & 4 & 661 & 161 & 500 & 22 & 37 & 0 & 404 & 37 \\
\hline Connecticut & 3,212 & 14 & 3,056 & 693 & 2,362 & 239 & 110 & 0 & 1.797 & 218 \\
\hline Middie Attantic & 65,410 & 1,060 & 62,168 & 6,606 & 55,562 & 12,221 & 6,422 & 0 & 33,939 & 2,981 \\
\hline New York & 31,429 & 234 & 29,782 & 2,485 & 27,297 & 5,774 & 3.686 & 0 & 16,650 & 1,187 \\
\hline New Jersey & 4,984 & 148 & 4,563 & 1,325 & 3,239 & 673 & 229 & 0 & 1,890 & 447 \\
\hline Pennsylvania & 28,997 & 677 & 27,823 & 2,796 & 25,027 & 5,774 & 2,507 & 0 & 15,398 & 1,348 \\
\hline Midwest & 490,474 & 17,883 & 460,128 & 23,789 & 436,339 & 233,455 & 40,718 & 71,859 & 71,637 & 18,670 \\
\hline East North Central & 159,066 & 6,269 & 148,602 & 92,358 & 136,233 & 72,743 & 12,982 & 0 & 42,482 & 8,027 \\
\hline Ohio & 26,451 & 347 & 25,686 & 2,925 & 22,762 & 12,537 & 2,444 & 0 & 6,426 & 1.354 \\
\hline Indiana & 23,159 & 487 & 22,302 & 1,780 & 20,522 & 13,930 & 2,073 & 0 & 3,698 & 821 \\
\hline Illinois & 36,061 & 492 & 34,792 & 2,792 & 32,000 & 25,121 & 2,689 & 0 & 3,447 & 744 \\
\hline Miehigan & 37,457 & 3,130 & 33,051 & 2,921 & 30,130 & 9,484 & 2,735 & 0 & 15,483 & 2,429 \\
\hline Wisconsin & 35,938 & 1,813 & 32,770 & 1,951 & 30,820 & 11.671 & 3,041 & 0 & 13,428 & 2,680 \\
\hline West North Central & 331,408 & 11,615 & 311,527 & 11,421 & 300,105 & 160,713 & 27,736 & 71,859 & 29,155 & 10,643 \\
\hline MInnesota & 54,017 & 3,390 & 47,077 & 2,136 & 44,941 & 22,990 & 3,425 & 157 & 13,952 & 4,417 \\
\hline lowa & 36,016 & 172 & 35,387 & 1,688 & 33,699 & 27.031 & 3,866 & 0 & 1,841 & 961 \\
\hline Missouri & 44,605 & 2,060 & 41,655 & 2,165 & 39,491 & 15,090 & 12,606 & 56 & 10,959 & 781 \\
\hline North Dakota & 45,250 & 1,882 & 42,255 & 1,242 & 41,013 & 28.064 & 1,206 & 9,933 & 428 & 1,382 \\
\hline South Dakota & 49,354 & 2,873 & 45.467 & 1.064 & 44,403 & 17,819 & 2,354 & 22,152 & 565 & 1,513 \\
\hline Nebraska & 49,507 & 652 & 48,218 & 1,250 & 46,967 & 20.601 & 1,957 & 22,900 & 728 & 782 \\
\hline Kansas & 52,658 & 587 & 51,467 & 1,876 & 49,592 & 29.119 & 2,324 & 16,660 & 681 & 808 \\
\hline South & 575,044 & 26,391 & 527,041 & 30.657 & 496,384 & 107,532 & 67,937 & 113,837 & 189,507 & 17,571 \\
\hline South Attantic & 178,469 & 12,757 & 156,196 & 13,346 & 142,849 & 26.495 & 16,165 & 3,592 & 88,408 & 8,190 \\
\hline Delaware & 1,309 & 33 & 1,213 & 165 & 1.048 & 521 & 30 & 0 & 357 & 141 \\
\hline Maryland & 6.695 & 159 & 6,048 & 936 & 5.111 & 1.795 & 514 & 0 & 2,415 & 388 \\
\hline Virginla & 26,091 & 2,368 & 22,812 & 1,663 & 21,150 & 3,309 & 3,315 & 0 & 13,622 & 904 \\
\hline West Virginia & 15,508 & 1,116 & 14,227 & 532 & 13,695 & 1,053 & 1,892 & 0 & 10,466 & 284 \\
\hline North Carolina & 33,708 & 2,309 & 28,622 & 2,487 & 26.135 & 6,548 & 1,992 & 0 & 16,528 & 1,057 \\
\hline South Carolina & 19,912 & 1,340 & 17,785 & 1,422 & 16,363 & 3,371 & 1,177 & 0 & 11,073 & 742 \\
\hline Georgla & 37,702 & 2,062 & 34,664 & 2,375 & 32,289 & 6,307 & 3,040 & 0 & 21,860 & 1,083 \\
\hline Flonda & 37,545 & 3,369 & 30,825 & 3,766 & 27,059 & 3,592 & 4,205 & 3,592 & 12,088 & 3,583 \\
\hline East South Central & 116,446 & 5,116 & 108,068 & 5,705 & 102,363 & 22,870 & 18,493 & 96 & 58,115 & 2,789 \\
\hline Kentucky & 25,862 & 1,169 & 24,023 & 1,224 & 22,799 & 5,818 & 5,955 & 0 & 10,054 & 972 \\
\hline Tennessee & 26,972 & 1,369 & 24,759 & 1,669 & 23,090 & 5,765 & 5,019 & 0 & 11,601 & 706 \\
\hline Alabama & 33,091 & 904 & 31,230 & 1,640 & 29,591 & 4,210 & 3,595 & 96 & 21.017 & 673 \\
\hline Mississippl & 30,521 & 1,674 & 28,056 & 1,172 & 26,884 & 7,078 & 3,924 & 0 & 15,443 & 439 \\
\hline West South Central & 280,129 & 8,518 & 262,778 & 11,606 & 251,172 & 58,167 & 33,279 & 110,149 & 42,984 & 6,593 \\
\hline Arkansas & 34,040 & 3,129 & 29,904 & 1,232 & 28,672 & 8,182 & 5,678 & 164 & 14,268 & 380 \\
\hline Loulsiana & 30,561 & 1,174 & 26,472 & 1,455 & 25,016 & 6.484 & 2,276 & 234 & 12,736 & 3,286 \\
\hline Okfahoma & 44,772 & 1.176 & 42,431 & 1,716 & 40,715 & 11,557 & 7,590 & 14,546 & 6,505 & 517 \\
\hline Texas & 170,756 & 3,040 & 163,971 & 7,203 & 156,768 & 31,944 & 17,735 & 95,204 & 9,476 & 2,410 \\
\hline West & 764,128 & 357,403 & 395,209 & 13,247 & 382,962 & 66,896 & 12,697 & 215,989 & 68,514 & 18,865 \\
\hline Mountain & 552,680 & 266,171 & 280,033 & 5,964 & 274,069 & 44,235 & 7,828 & 182,653 & 27,532 & 11,822 \\
\hline Montana & 94,109 & 27,074 & 65,682 & 999 & 64,682 & 17,881 & 3,169 & 36,769 & 5,253 & 1,611 \\
\hline Idaho & 53,481 & 33,190 & 19,628 & 477 & 19,152 & 6,532 & 1,354 & 6,596 & 4,071 & 600 \\
\hline Wyoming & 62,598 & 29,457 & 32,576 & 501 & 32,075 & 2,362 & 928 & 26,784 & 984 & 1,017 \\
\hline Colorado & 66,618 & 23,833 & 42,320 & 1,375 & 40,945 & 10,967 & 1,266 & 23,427 & 4,079 & 1,207 \\
\hline New Mexico & 77,819 & 26,423 & 51,144 & 698 & 50,445 & 2,297 & 186 & 40,782 & 4,685 & 2,496 \\
\hline Arizona & 72,960 & 30,647 & 41,994 & 1,116 & 40,878 & 1,306 & 81 & 31,867 & 4,912 & 2,712 \\
\hline Utan & 54,336 & 35,476 & 16,440 & 465 & 15,975 & 2,002 & 563 & 8,507 & 3,194 & 1,711 \\
\hline Nevada & 70,759 & 60,071 & 10,250 & 333 & 9,916 & 889 & 282 & 7,921 & 356 & 469 \\
\hline Pacific & 211,448 & 91,232 & 116,176 & 7,283 & 108,893 & 22,662 & 4,869 & 33,337 & 40,982 & 7,043 \\
\hline Washington & 43,608 & 12,471 & 29,947 & 1,564 & 28,383 & 7,758 & 1,421 & 5,574 & 12,634 & 997 \\
\hline Oregon & 62,127 & 32,305 & 28,918 & 941 & 27,977 & 4,348 & 1,916 & 9,152 & 11,857 & 705 \\
\hline Califoma & 101,572 & 46,014 & 53,654 & 4,621 & 49,033 & 10,209 & 1,501 & 17,719 & 15,073 & 4,531 \\
\hline Hawaii & 4,141 & 443 & 3,657 & 157 & 3,500 & 348 & 31 & 891 & 1,419 & 811 \\
\hline
\end{tabular}

Source: U.S. Dept. of Agricutture, Soil Conservaton Service, and lowa

State Unversity, Statistcal Laboratory.

Statistucal Bulletin No. 790, Summary Report, 1987 National Resources Inventory, December 1989 


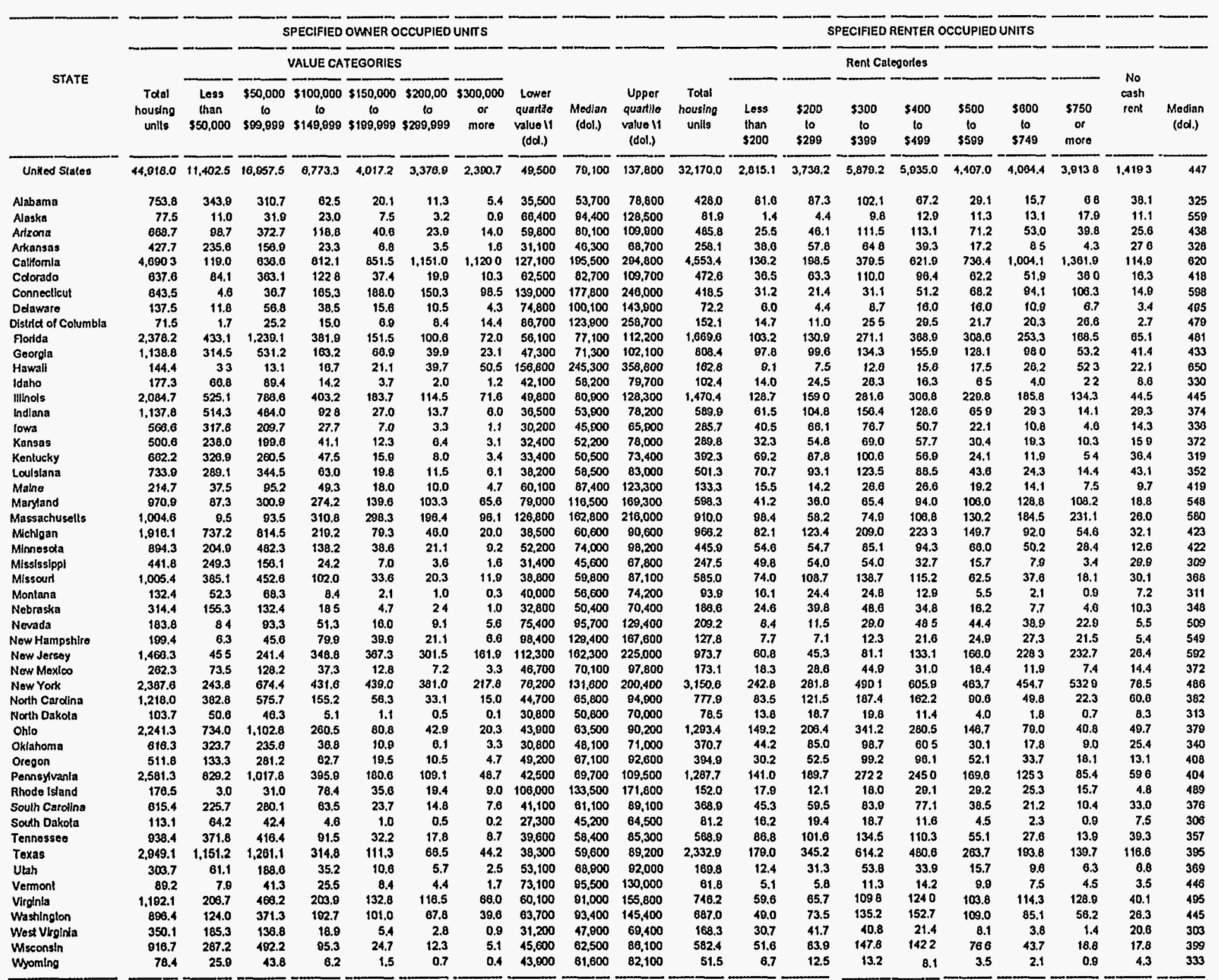

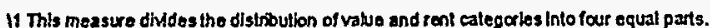
The lower quartile value is the value that deatines the upper fimil of the lowest ono-quartex of the cases.

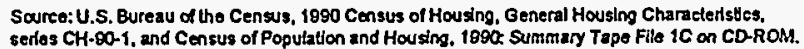


No. 1104. Balance Sheet of the Farming Sector

In Current and Constant (1987) Dollars

\section{In billions of dollars, except as indicated. As of December 31.}

Includes farm operalor households.

See Historical Statistics, Colonlal Times to 1970

serles K 204-219, for data betore revislons]

CURRENT DOLLARS
Assets
Physical assets:
Real estate
Nonreal estate
Llvestock and poultry II
Machinery, motor vehicles
Crops stored
Household furnishings, equipment
Purchased Inputs
Financial assets
Investment In cooperatlves
Other financlal assets
Clalms
Debt
Real estate debt
Nonfeal estate debt
Equity

Farm debt/asset rato (percent)

\begin{tabular}{|c|c|c|c|c|c|c|c|c|c|c|c|}
\hline 1970 & 1975 & 1980 & 1981 & 1982 & 1983 & 1984 & 1985 & 1986 & 1987 & 1988 & 1989 \\
\hline 324.3 & 579.5 & $1,089.0$ & t.088.9 & $1,056.2$ & $1,063.6$ & 975.7 & 892.8 & 847.7 & 911.3 & 951.5 & 985.8 \\
\hline 224.5 & 421.0 & 850.1 & 851.7 & 819.1 & 829.3 & 735.0 & 657.0 & 613.0 & 658.6 & 682.2 & 703.9 \\
\hline 76.6 & 127.2 & 199.6 & 196.3 & 194.4 & 189.6 & 193.0 & 186.5 & 181.0 & 196.1 & 212.1 & 223.6 \\
\hline 23.7 & 29.4 & 60.6 & 53.5 & 53.0 & 49.5 & 49.5 & 46.3 & 47.8 & 58.0 & 62.2 & 66.2 \\
\hline 34.4 & 63.1 & 86.9 & 92.5 & 92.6 & 92.1 & 91.1 & 88.3 & 861 & 84.5 & 86.1 & 89.2 \\
\hline 8.5 & 20.5 & 32.7 & 29.5 & 25.8 & 23.6 & 26.1 & 22.9 & 16.3 & 17.5 & 23.3 & 23.4 \\
\hline to.o & 14.2 & 19.4 & 20.8 & 23.0 & 24.4 & 24.3 & 27.8 & 28.7 & 32.9 & 37.0 & 42.2 \\
\hline (NA) & (NA) & (NA) & (NA) & (NA) & (NA) & 2.0 & 1.2 & 2.1 & 3.2 & 3.5 & 2.6 \\
\hline 23.2 & 31.4 & 39.3 & $40.9^{\circ}$ & 42.8 & 44.6 & 47.7 & 49.3 & 53.8 & 56.7 & 57.2 & 58.3 \\
\hline 7.2 & 13.0 & 19.3 & 20.6 & 21.9 & 22.8 & 24.3 & 24.3 & 24.4 & 25.3 & 25.1 & 26.3 \\
\hline 16.0 & 18.4 & 20.0 & 20.3 & 20.9 & 21.8 & 23.4 & 25.0 & 29.4 & 31.4 & 32.1 & 32.0 \\
\hline 324.3 & 579.5 & $1,089.0$ & $1,088.9$ & $1,056.2$ & $1,063.6$ & 975.7 & 892.8 & 847.7 & 911.3 & 951.5 & 985.8 \\
\hline 52.8 & 91.5 & 178.7 & 195.4 & 203.1 & 206.5 & 204.3 & 187.9 & 166.6 & 153.7 & 148.5 & 146.0 \\
\hline 30.5 & 49.9 & 97.5 & 107.2 & 111.3 & 113.7 & 112.3 & 105.7 & 95.9 & 87.7 & 83.0 & 80.5 \\
\hline 22.3 & 41.6 & 81.2 & 88.2 & 91.8 & 92.7 & 92.0 & 82.2 & 70.8 & 66.0 & 65.6 & 65.5 \\
\hline 271.5 & 488.0 & 910.3 & 893.5 & 853.1 & 857.1 & 771.4 & 704.9 & 681.0 & 757.6 & 802.9 & 839.8 \\
\hline 16.3 & 15.8 & 16.4 & 17.9 & 19.2 & 19.4 & 20.9 & 21.0 & 19.7 & 16.9 & 15.6 & 14.8 \\
\hline 923.9 & $1,177.7$ & $1,518.8$ & $1,380.1$ & $1,260.4$ & $1,219.7$ & $1,072.2$ & 945.8 & 874.8 & 911.3 & 915.8 & 908.6 \\
\hline 150.3 & 185.9 & 249.3 & 247.7 & 242.4 & 236.8 & 224.5 & 199.0 & 171.9 & 153.7 & 142.9 & 134.6 \\
\hline 773.6 & 991.8 & $1,269.6$ & $1,132,4$ & $1,018.0$ & $98 \dot{.9}$ & 847.7 & 746.7 & 702.8 & 757.6 & 772.8 & 774.0 \\
\hline
\end{tabular}

CONSTANT (1987) DOLLARS

Assets
Dobt
Equity

NA Not avallable.

.

2 Constant dollar fligures are based on

gross domestle product implicti price deflators for year.

Source: U.S. Dept. of Agricuthure, Economle Research Service,

Economic Indkators of the Farm Sector:

Natlonal Financial Summary, 1991. 


\section{APPENDIX E - OTHER CENSUS INFORMATION}

This appendix contains additional census information that can be found on the PL 94-171 CD-ROMs. Included in this appendix is APPENDLX A - Area Classifications, APPENDIX C - Accuracy of the Data, APPENDLX D - Collection and Processing Procedures, and the PL 94-171 database Data Dictionary.

Census of Population and Housing, 1990: Public Law (P.L.) 94-171 Data on CD-ROM (Name of State) [machine-readable data files] / prepared by the Bureau of the Census. --Washington: The Bureau [producer and distributor], 1991.

\section{APPENDIX A.}

\section{Area Classifications}

\section{CONTENTS}

Alaska Native Regional Corporation (See American Indian and Alaska Native Area)

Alaska Native Village Statistical Area (See American Indian and Alaska Native Area)

American Indian and Alaska Native Area................... A-1

American Indian Reservation (See American Indian and Alaska

Native Area, see County Subdivision)

American Indian Reservation and Trust Land (See American Indian and Alaska Native Area)

Area Measurement............................... A

Assessment District (See County Subdivision)

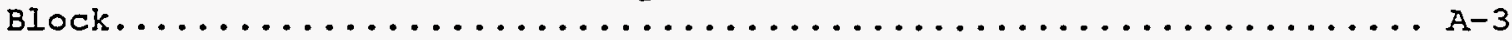

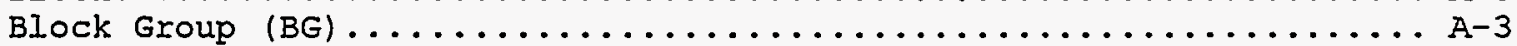

Block Numbering Area (BNA) (See Census Tract and Block Numbering Area)

Borough (See County Subdivision, see Place) Borough and Census Area (Alaska) (See County) Boundary Changes............................. Census Area (Alaska) (See County) Census Block (See Block)

Census Code (See Geographic Code)

Census County Division (See County Subdivision)

Census Designated Place (CDP) (See Place)

Census Geographic Code (See Geographic Code)

Census Subarea (Alaska) (See County Subdivision)

Census Tract and Block Numbering Area................... A-4 City (See Place)

Congressional District............................ A-5

Consolidated City (See Place)

County...................................

County subdivision.............................. $A-5$

Crews of Vessels (See Area Measurement, see Block, see

Census Tract and Block Numbering Area)

Division (See County Subdivision)

Election District (See County Subdivision, see Voting District)

Federal Information Processing Standards (FIPS)

Code (See Geographic Code)

Geographic Block Group (See Block Group)

Geographic Code................................. A-6

Geographic Presentation........................... A 6

Gore (See County Subdivision) 
Grant (See County Subdivision)

Hierarchical Presentation (See Geographic Presentation)

Historic Areas of Oklahoma (See American Indian and

Alaska Native Area, Tribal Jurisdiction Statistical Area

Incorporated Place (See Place)

Independent City (See County)

Internal Point..............................

Inventory Presentation (See Geographic Presentation)

Land Area (See Area Measurement)

Latitude (See Internal Point)

Longitude (See Internal Point)

Magisterial District (See County Subdivision)

Minor Civil Division (See County Subdivision)

Parish (Louisiana) (See County)

Parish Governing Authority District (See County Subdivision)

Place....................................

Plantation (See County Subdivision)

Precinct (See County Subdivision, see Voting District)

Purchase (See County Subdivision)

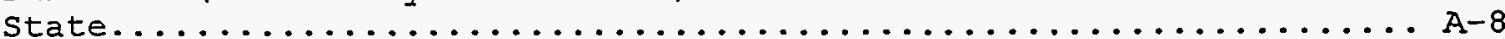

Supervisors' District (See County Subdivision)

Tabulation Block Group (See Block Group)

TIGER..................................

Town (See County Subdivision, see Place)

Township (See County Subdivision)

Tract (See Census Tract and Block Numbering Area)

Tribal Designated Statistical Area (TDSA) (See American

Indian and Alaska Native Area)

Tribal Jurisdiction Statistical Area (TJSA) (See American

Indian and Alaska Native Area)

Trust Land (See American Indian and Alaska Native Area)

United states.................................. A-8

United States Postal Service (USPS) Code (See Geographic

Code)

Unorganized Territory (unorg.) (See County Subdivision)

Village (see Place)

Voting District (VTD) $\ldots \ldots \ldots \ldots \ldots \ldots \ldots \ldots \ldots \ldots \ldots \ldots \ldots \ldots \ldots \ldots$

Water Area (See Area Measurement)

\section{AMERICAN INDIAN AND AI.ASKA NATIVE AREA}

\section{Alaska Native Regional Corporation}

Alaska Native Regional Corporations (ANRC's) are corporate entities established under the Alaska Native Claims Settlement Act of 1972, Public Law 92-203, as amended by Public Law 94-204, to conduct both business and nonprofit affairs of Alaska Natives. Alaska is divided into 12 ANRC's that cover the entire state, except for the Annette Islands Reserve. The boundaries of the 12 ANRC's were established by the Department of the Interior, in cooperation with Alaska Natives. Each ANRC was designed to include, as far as practicable, Alaska Natives with a common heritage and common interests. The ANRC

boundaries for the 1990 census were identified by the Bureau of Land Management. A 13th region was established for Alaska Natives who are not permanent residents and who chose not to enroll in one of the 12 ANRC's; no census products are prepared for the 13th region. ANRC's were first identified for the 1980 census.

Each ANRC is assigned a two-digit census code ranging from 07 through 84. These census codes are assigned in alphabetical order of the 
ANRC's.

Alaska Native Village Statistical Area

Alaska Native villages (ANV's) constitute tribes, bands, clans, groups, villages, communities, or associations in Alaska that are recognized pursuant to the Alaska Native Claims Settlement Act of 1972, Public Law 92-203. Because ANV's do not have legally designated boundaries, the Census Bureau has established Alaska Native village statistical areas (ANVSA's) for statistical purposes. For the 1990 census, the Census Bureau cooperated with officials of the nonprofit corporation within each participating Alaska Native Regional Corporation (ANRC), as well as other knowledgeable officials, to delineate boundaries that encompass the settled area associated with each ANV. ANVSA's are located within ANRC's and do not cross ANRC boundaries. ANVSA's for the 1990 census replace the ANV's that the Census Bureau recognized for the 1980 census.

Each ANVSA is assigned a four-digit census code ranging from 6001 through 8989. Each ANVSA also is assigned a five-digit FIPS code. Both the census and FIPS codes are assigned in alphabetical order of ANVSA's.

American Indian Reservation and Trust Iand

American Indian Reservation--Federal American Indian reservations are areas with boundaries established by treaty, statute, and/or executive or court order, and recognized by the Federal Government as territory in which American Indian tribes have jurisdiction. State reservations are lands held in trust by state governments for the use and benefit of a given tribe. The reservations and their boundaries were identified for the 1990 census by the Bureau of Indian Affairs (BIA), Department of Interior (for Federal reservations), and state governments (for state reservations). The names of American Indian reservations recognized by State governments, but not by the Federal Government, are followed by "(State)." Areas composed of reservation lands that are administered jointly and/or are claimed by two reservations, as identified by the BIA, are called "joint areas," and are treated as separate American Indian reservations for census purposes.

Eederal reservations may cross state boundaries, and Federal and state reservations may cross county, county subdivision, and place boundaries. For reservations that cross state boundaries, only the portion of the reservations in a given state are shown in the data products for that state; the entire reservations are shown in data products for the United states.

Each American Indian reservation is assigned a four-digit census code ranging from 0001 through 4989. These census codes are assigned in alphabetical order of American Indian reservations nationwide, except that joint areas appear at the end of the code range. Each American Indian reservation also is assigned a five-digit FIPS code; because the FIPS codes are assigned in alphabetical sequence of American Indian reservations within each state, the FIPS code is different in each state for reservations in more than one state.

Trust Land--Trust lands are property associated with a particular American Indian reservation or tribe, held in trust by the Federal Government. Trust lands may be held in trust either for a tribe (tribal trust land) or for an individual member of a tribe (individual trust land). Trust lands recognized for the 1990 census comprise all tribal trust lands and inhabited individual trust lands located outside of a reservation boundary. As with other American Indian areas, trust lands may be 
located in more than one state. Only the trust lands in a given state are shown in the data products for that state; all trust lands associated with a reservation or tribe are shown in data products for the United States. The Census Bureau first reported data for tribal trust lands for the 1980 census.

Trust lands are assigned a four-digit census code and a five-digit FIPS code, the same as that for the reservation with which they are associated. Trust lands not associated with a reservation are presented by tribal name, interspersed alphabetically among the reservations.

Tribal Designated Statistical Area (TDSA)

Tribal designated statistical areas (TDSA's) are areas, delineated outside Oklahoma by federally- and state-recognized tribes without a land base or associated trust lands, to provide statistical areas for which the Census Bureau tabulates data. TDSA's represent areas generally containing the American Indian population over which federally-recognized tribes have jurisdiction and areas in which state tribes provide benefits and services to their members. The names of TDSA's delineated by State-recognized tribes are followed by "(State)." The Census Bureau did not recognize TDSA's before the 1990 census.

Each TDSA is assigned a four-digit census code ranging from 9001 through 9589. The census codes are assigned in alphabetical order of TDSA's nationwide. Each TDSA also is assigned a five-digit FIPS code in alphabetical order within state.

Tribal Jurisdiction Statistical Area (TJSA)

Tribal jurisdiction statistical areas (TJSA's) are areas, delineated by federally-recognized tribes in Oklahoma without a reservation, for which the Census Bureau tabulates data. TJSA's represent areas generally containing the American Indian population over which one or more tribal governments have jurisdiction; if tribal officials delineated adjacent TJSA's so that they include some duplicate territory, the overlap area is called a "joint use area," which is treated as a separate TJSA for census purposes.

TJSA's replace the "Historic Areas of Oklahoma (excluding urbanized areas)" shown in 1980 census data products. The Historic Areas of Oklahoma comprised the territory located within reservations that had legally established boundaries from 1900 to 1907; these reservations were dissolved during the 2- to 3-year period preceding the statehood of Oklahoma in 1907. The Historic Areas of Oklahoma (excluding urbanized areas) were identified only for the 1980 census.

Each TJSA is assigned a four-digit census code ranging from 5001 through 5989. The census codes are assigned in alphabetical order of TJSA's, except that joint areas appear at the end of the code range. Each TJSA also is assigned a five-digit FIPS code in alphabetical order within Oklahoma.

\section{AREA MEASUREMENT}

Area measurements provide the size, in square kilometers (also in square miles in printed reports), recorded for each geographic entity for which the Census Bureau tabulates data in general-purpose data products (except crews-of-vessels entities and ZIP Codes). (Square kilometers may be divided by 2.59 to convert an area measurement to square miles.) Area was calculated from the specific set of boundaries recorded for the entity in the Census Bureau's geographic data base 
(see "TIGER"). On machine-readable files, area measurements are shown to three decimal places; the decimal point is implied. In printed reports and listings, area measurements are shown to one decimal.

The Census Bureau provides area measurements for both land and inland water. "Inland water" consists of any lake, reservoir, pond, or similar body of water that is recorded in the Census Bureau's geographic data base. It also includes any river, creek, canal, stream, or similar feature that is recorded in that data base as a twodimensional feature (rather than as a single line). The portions of the oceans and related large embayments (such as the Chesapeake Bay and Puget Sound), the Gulf of Mexico, and the Caribbean Sea that belong to the United States and its possessions are considered to be "coastal" and "territorial" waters; the Great Lakes are treated as a separate water entity. Rivers and bays that empty into these bodies of water are treated as "inland water" from the point beyond which they are narrower than one nautical mile across. Identification of land and inland, coastal, and territorial waters is for statistical purposes, and does not necessarily reflect legal definitions thereof. By definition, census blocks do not include inland water within their boundaries; therefore, the water area of a block is always zero. Land area measurements may disagree with the information displayed on census maps and in the TIGER file because, for area measurement purposes, features identified as "intermittent water" and "glacier" are reported as land area. For this reason, it may not be possible to derive the land area for an entity by summing the land area of its component census blocks. Crews-of-vessels entities (see "Census Tract and Block Numbering Area" and "Block")

do not encompass territory and therefore have no area measurements. 2IP Codes do not have specific boundaries, and therefore, also do not have area measurements.

The accuracy of any area measurement figure is limited by the inaccuracy inherent in (1) the location and shape of the various boundary features in the data base, and (2) rounding affecting the last digit in all operations that compute and/or sum the area measurements.

\section{BLOCK}

Census blocks are small areas bounded on all sides by visible features such as streets, roads, streams, and railroad tracks, and by invisible boundaries such as city, town, township, and county limits, property lines, and short, imaginary extensions of streets and roads.

Tabulation blocks, used in census data products, are in most cases the same as collection blocks, used in the census enumeration. In some cases, collection blocks have been "split" into two or more parts required for data tabulations. Tabulation blocks do not cross the boundaries of counties, county subdivisions, places, census tracts or block numbering areas, American Indian and Alaska Native areas, congressional districts, voting districts, urban or rural areas, or urbanized areas. The 1990 census is the first for which the entire United States and its possessions are block-numbered.

Blocks are numbered uniquely within each census tract or BNA. A block is identified by a three-digit number, sometimes with a single alphabetical suffix. Block numbers with suffixes generally represent collection blocks that were "split" in order to identify separate geographic entities that divide the original block. For example, when a city limit runs through data collection block 101, the data for the portion inside the city is tabulated in block $101 \mathrm{~A}$ and the portion outside, in block 101B. A block number with the suffix "Z" represents a "crews-of-vessels" entity for which the Census 
Bureau tabulates data, but that does not represent a true geographic area; such a block is shown on census maps associated with an anchor symbol and a census tract or block numbering area with a .99 suffix.

BLOCK GROUP (BG)

Geographic Block Group

A geographic block group (BG) is a cluster of blocks having the same first digit of their three-digit identifying numbers within a census tract or block numbering area (BNA). For example, BG 3 within a census tract or BNA includes all blocks numbered between 301 and 397. In most cases, the numbering involves substantially fewer than 97 blocks. Geographic BG's never cross census tract or BNA boundaries, but may cross the boundaries of county subdivisions, places, American Indian and Alaska Native areas, urbanized areas, voting districts, and congressional districts. BG's generally contain between 250 and 550 housing units, with the ideal size being 400 housing units.

Tabulation Block Group

In the data tabulations, a geographic BG may be split to present data for every unique combination of county subdivision, place, American Indian and Alaska Native area, urbanized area, voting district, urban/rural, and congressional district shown in the data product. BG's are used in tabulating decennial census data nationwide in the 1990 census, in all block-numbered areas in the 1980 census, and in Tape Address Register (TAR) areas in the 1970 census. For purposes of data presentation, BG's are a substitute for the enumeration districts (ED's) used for reporting data in many parts of the United States for the 1970 and 1980 censuses, and in all areas for pre-1970 censuses.

\section{BOUNDARY CHANGES}

The boundaries of some counties, county subdivisions, American Indian and Alaska Native areas, and many incorporated places, changed between those reported for the 1980 census and January 1, 1990. Boundary changes to legal entities result from:

1. Annexations to or detachments from legally established governmental units.

2. Mergers or consolidations of two or more governmental units.

3. Establishment of new governmental units.

4. Disincorporations or disorganizations of existing governmental units.

5. Changes in treaties and Executive Orders.

The historical counts shown for counties, county subdivisions, and places are not updated for such changes, and thus reflect the population and housing units in the area as delineated at each census. Information on boundary changes reported between the 1980 and 1990 censuses for counties, county subdivisions, and incorporated places is presented in the "User Notes" section of the technical documentation of summary Tape Files 1 and 3, and in the $1990 \mathrm{CPH}-2$, Population and Housing Unit Counts printed reports. For information on boundary changes for such areas in the decade preceding other decennial censuses, see the Number of Inhabitants reports for each census. Boundary changes are not reported for some 
areas, such as census designated places and block groups.

CENSUS TRACT AND BIOCK NUMBERING AREA

Block Numbering Area (BNA)

Block numbering areas (BNA's) are small statistical subdivisions of a county for grouping and numbering blocks in nonmetropolitan counties where local census statistical areas committees have not established census tracts. State agencies and the Census Bureau delineated BNA's for the 1990 census, using guidelines similar to those for the delineation of census tracts. BNA's do not cross county boundaries.

BNA's are identified by a four-digit basic number and may have a two-digit suffix; for example, 9901.07. The decimal point separating the four-digit basic BNA number from the two-digit suffix is shown in printed reports, in microfiche, and on census maps; in machine-readable files, the decimal point is implied. Many BNA's do not have a suffix; in such cases, the suffix field is left blank in all data products. BNA numbers range from 9501 through 9989.99, and are unique within a county (numbers in the range of 0001 through 9499.99 denote a census tract). The suffix .99 identifies a BNA that was populated entirely by persons aboard one or more civilian or military ships. A "crews-of-vessels" BNA appears on census maps only as an anchor symbol with its BNA number (and block numbers on maps showing block numbers); the BNA relates to the ships associated with the onshore BNA's having the same four-digit basic number. Suffixes in the range .80 through .98 usually identify BNA's that either were revised or were created during the 1990 census data collection activities. Some of these revisions produced BNA's that have extremely small land area and may have little or no population or housing. For data analysis, such a BNA can be summarized with an adjacent BNA.

\section{Census Tract}

Census tracts are small, relatively permanent statistical subdivisions of a county. Census tracts are delineated for all metropolitan areas (MA's) and other densely populated counties by local census statistical areas committees following Census Bureau guidelines (more than 3,000 census tracts have been established in 221 counties outside MA's). Six States (California, Connecticut, Delaware, Hawaii, New Jersey, and Rhode Island) and the District of Columbia are covered entirely by census tracts. Census tracts usually have between 2,500 and 8,000 persons and, when first delineated, are designed to be

homogeneous with respect to population characteristics, economic status, and living conditions. Census tracts do not cross county boundaries. The spatial size of census tracts varies widely depending on the density of settlement. Census tract boundaries are delineated with the intention of being maintained over a long time so that statistical comparisons can be made from census to census. However, physical changes in street patterns caused by highway construction, new development, etc., may require occasional revisions; census tracts occasionally are split due to large population growth, or combined as a result of substantial population decline. Census tracts are referred to as "tracts" in all 1990 data products.

Census tracts are identified by a four-digit basic number and may have a two-digit suffix; for example, 6059.02. The decimal point separating the four-digit basic tract number from the two-digit suffix is shown in printed reports, in microfiche, and on census maps; in machine-readable files, the decimal point is implied. Many census tracts do not have a suffix; in such cases, the suffix field is left blank in all data products. Leading zeros in a census tract number (for example, 002502) are shown only on machine-readable files. 
Census tract numbers range from 0001 through 9499.99 and are unique within a county (numbers in the range of 9501 through 9989.99 denote a block numbering area). The suffix.99 identifies a census tract that was populated entirely by persons aboard one or more civilian or military ships. A "crews-of-vessels" census tract appears on census maps only as an anchor symbol with its census tract number (and block numbers on maps showing block numbers). These census tracts relate to the ships associated with the onshore census tract having the same four-digit basic number. Suffixes in the range .80 through .98 usually identify census tracts that either were revised or were created during the 1990 census data collection activities. Some of these revisions may have resulted in census tracts that have extremely small land area and may have little or no population or housing. For data analysis, such a census tract can be summarized with an adjacent census tract.

\section{CONGRESSIONAI DISTRICT}

Congressional districts (CD's) are the 435 areas from which persons are elected to the U.S. House of Representatives. After the apportionment of congressional seats among the states, based on census population counts, each state is responsible for establishing $\mathrm{CD}$ 's for the purpose of electing representatives. Each $\mathrm{CD}$ is to be as equal in population to all other $\mathrm{CD}^{\prime} \mathrm{s}$ in the state as practicable, based on the decennial census counts.

The CD's that were in effect on January 1, 1990 were those of the 101st Congress. Data on the 101st Congress appear in an early 1990 census data product (Summary Tape File 1A). The CD's of the 101st Congress are the same as those in effect for the 102nd Congress. CD's of the 103rd Congress, reflecting redistricting based on the 1990 census, are summarized in later 1990 data products (STF's 1D and 3D, and 1990 $\mathrm{CPH}-4$, Population and Housing Characteristics for Congressional Districts of the lo3rd Congress printed reports).

\section{COUNTY}

The primary political divisions of most states are termed "counties." In Louisiana, these divisions are known as "parishes." In Alaska, which has no counties, the county equivalents are the organized "boroughs" and the "census areas" that are delineated for statistical purposes by the state of Alaska and the Census Bureau. In four states (Maryland, Missouri, Nevada, and Virginia), there are one or more cities that are independent of any county organization and thus constitute primary divisions of their states. These cities are known as "independent cities" and are treated as equivalent to counties for statistical purposes. That part of Yellowstone National Park in Montana is treated as a county equivalent. The District of Columbia has no primary divisions, and the entire area is considered equivalent to a county for statistical purposes.

Each county and county equivalent is assigned a three-digit FIPS code that is unique within state. These codes are assigned in alphabetical order of county or county equivalent within state, except for the independent cities, which follow the listing of counties.

\section{COUNTY SUBDIVISION}

County subdivisions are the primary subdivisions of counties and their equivalents for the reporting of decennial census data. They include census county divisions, census subareas, minor civil 
divisions, and unorganized territories.

Each county subdivision is assigned a three-digit census code in alphabetical order within county and a five-digit FIPS code in alphabetical order within state.

Census County Division

Census county divisions (CCD's) are subdivisions of a county that were delineated by the Census Bureau, in cooperation with state officials and local census statistical areas committees, for statistical purposes. CCD's were established in 21 states where there are no legally established minor civil divisions (MCD's), where the MCD's do not have governmental or administrative purposes, where the boundaries of the MCD's change frequently, and/or where the MCD's are not generally known to the public. CCD's have no legal functions, and are not governmental units.

The boundaries of CCD's usually are delineated to follow visible features, and in most cases coincide with census tract or block numbering area boundaries. The name of each CCD is based on a place, county, or well-known local name that identifies its location. CCD's have been established in the following 21 states: Alabama, Arizona, California, Colorado, Delaware, Florida, Georgia, Hawaii, Idaho, Kentucky, Montana, Nevada, New Mexico, Oklahoma, Oregon, South Carolina, Tennessee, Texas, Utah, Washington, and Wyoming. For the 1980 census, the county subdivisions recognized for Nevada were MCD's.

Census Subarea (Alaska)

Census subareas are statistical subdivisions of boroughs and census areas (county equivalents) in Alaska. Census subareas were delineated cooperatively by the state of Alaska and the Census Bureau. The census subareas, identified first in 1980, replaced the various types of subdivisions used in the 1970 census.

\section{Minor Civil Division}

Minor civil divisions (MCD's) are the primary political or administrative divisions of a county. MCD's represent many different kinds of legal entities with a wide variety of governmental and/or administrative functions. MCD's are variously designated as American Indian reservations, assessment districts, boroughs, election districts, gores, grants, magisterial districts, parish governing authority districts, plantations, precincts, purchases, supervisors' districts, towns, and townships. In some states, all or some incorporated places are not located in any MCD and thus serve as MCD's in their own right. In other states, incorporated places are subordinate to (part of) the MCD's in which they are located, or the pattern is mixed--some incorporated places are independent of MCD's and others are subordinate to one or more MCD's.

The Census Bureau recognizes MCD's in the following 28 states: Arkansas, Connecticut, Illinois, Indiana, Iowa, Kansas, Louisiana, Maine, Maryland, Massachusetts, Michigan, Minnesota, Mississippi, Missouri, Nebraska, New Hampshire, New Jersey, New York, North Carolina, North Dakota, Ohio, Pennsylvania, Rhode Island, South Dakota, Vermont, Virginia, West Virginia, and Wisconsin. The District of Columbia has no primary divisions, and the entire area is considered equivalent to an MCD for statistical purposes.

Unorganized Territory (unorg.) 
In nine States (Arkansas, Iowa, Kansas, Louisiana, Maine, Minnesota, North Carolina, North Dakota, and South Dakota), some counties contain territory that is not included in an MCD recognized by the Census Bureau. Each separate area of unorganized territory in these states is recognized as one or more separate county subdivisions for census purposes. Each unorganized territory is given a descriptive name, followed by the designation "unorg."

\section{GEOGRAPHIC CODE}

Geographic codes are shown primarily on machinereadable data products, such as computer tape and compact disc-read only memory (CD-ROM), but also appear on other products such as microfiche; they also are shown on some census maps. Codes are identified as "census codes" only if there is also a Federal Information Processing Standards (FIPS) code for the same geographic entity. A code that is not identified as either "census" or "FIPS" is usually a census code for which there is no FIPS equivalent, or for which the Census Bureau does not use the FIPS code. The exceptions, which use only the FIPS code in census products, are county, congressional district, and metropolitan area (that is, metropolitan statistical area, consolidated metropolitan statistical area, and primary metropolitan statistical area).

\section{Census Code}

Census codes are assigned for a variety of geographic entities, including American Indian and Alaska Native area, census division, census region, county subdivision, place, state, urbanized area, and voting district. The structure, format, and meaning of census codes appear in the 1990 census Geographic Identification Code Scheme; in the technical documentation for summary tape files, CD-ROM's, and microfiche; and in the data dictionaries of summary tape files and CD-ROM's.

\section{Federal Information Processing Standards (FIPS) Code}

Federal Information Processing Standards (FIPS) codes are assigned for a variety of geographic entities, including American Indian and Alaska Native area, congressional district, county, county subdivision, metropolitan area, place, and state. The structure, format, and meaning of FIPS codes used in the census are shown in the 1990 census Geographic Identification Code Scheme; in the technical documentation for summary tape files, $C D-R O M^{\prime} s$, and microfiche; and in the data dictionaries of summary tape files and CD-ROM's.

The objective of the FIPS codes is to improve the use of data resources of the Federal Government and avoid unnecessary duplication and incompatibilities in the collection, processing, and dissemination of data. More information about FIPS and FIPS code documentation is available from the National Technical Information Service, Springfield, VA 22161 .

United States Postal Service (USPS) Code

United States Postal Service (USPS) codes for States are used in all 1990 data products. The codes are two-character alphabetic abbreviations. These codes are the same as the FIPS two-character alphabetic abbreviations.

\section{GEOGRAPHIC PRESENTATION}

Hierarchical Presentation 
A hierarchical geographic presentation shows the geographic entities in a superior/subordinate structure in census products. This structure is derived from the legal, administrative, or areal relationships of the entities. The hierarchical structure is depicted in report tables by means of indentation, and is explained for machine-readable media in the discussion of file structure in the technical documentation. An example of hierarchical presentation is the "standard census geographic hierarchy": block, within block group, within census tract or block numbering area, within place, within county subdivision, within county, within state, within division, within region, within the United States. Graphically, this is shown as:

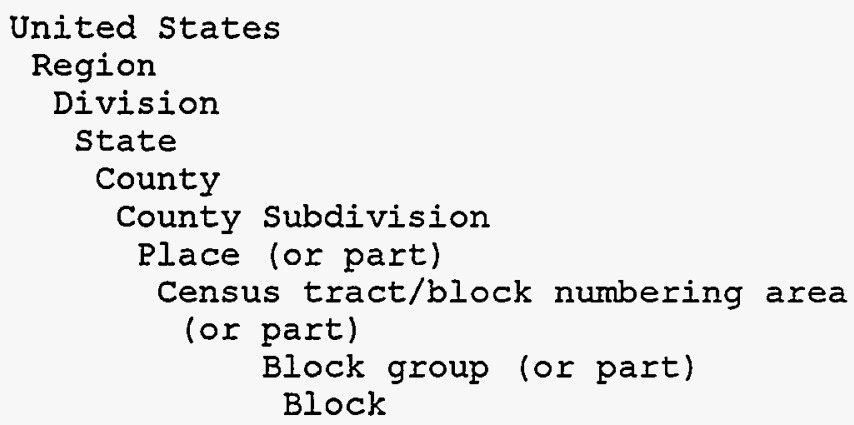

\section{Inventory Presentation}

An inventory presentation of geographic entities is one in which all entities of the same type are shown in alphabetical or code sequence, without reference to their hierarchical relationships. Generally, an inventory presentation shows totals for entities that may be split in a hierarchical presentation, such as place, census tract/block numbering area, or block group. An example of a series of inventory presentations is: state, followed by all the counties in that state, followed by all the places in that state. Graphically, this is shown as:

State

County "A"
County "B"
County " $\mathrm{C}$ "
Place " $\mathrm{X}$ "
Place "Y"
Place " $\mathrm{Z}$ "

INTERNAI POINT

An internal point is a set of geographic coordinates (latitude and longitude) that is located within a specified geographic entity. A single point is identified for each entity; for many entities, this point represents the approximate geographic center of that entity. If the shape of the entity caused this point to be located outside the boundaries of the entity, it is relocated from the center so that it is within the entity. If the internal point for a block falls in a water area, it is relocated to a land area within the block. On machine-readable products, internal points are shown to six decimal places; the decimal point is implied.

PIACE

Places, for the reporting of decennial census data, include census designated places and incorporated places. Each place is assigned a 
four-digit census code that is unique within State. Each place is also assigned a five-digit FIPS code that is unique within state. Both the census and FIPS codes are assigned based on alphabetical order within state. Consolidated cities (see below) are assigned a one-character alphabetical census code that is unique nationwide and a five-digit FIPS code that is unique within state.

Census Designated Place (CDP)

Census designated places ( $\mathrm{CDP}^{\prime} \mathrm{s}$ ) are delineated for the decennial census as the statistical counterparts of incorporated places. CDR's comprise densely settled concentrations of population that are identifiable by name, but are not legally incorporated places. Their boundaries, which usually coincide with visible features or the boundary of an adjacent incorporated place, have no legal status, nor do these places have officials elected to serve traditional municipal functions. CDP boundaries may change with changes in the settlement pattern; a CDP with the same name as in previous censuses does not necessarily have the same boundaries.

Beginning with the 1950 census, the Census Bureau, in cooperation with State agencies and local census statistical areas committees, has identified and delineated boundaries for CDP's. For the 1990 census, the name of each such place is followed by "CDP." For the 1980 census, "(CDP)" was used; for 1970, 1960, and 1950 censuses, these places were identified by "(U)," meaning "unincorporated place."

To qualify as a CDP for the 1990 census, an unincorporated community must have met the following criteria:

1. In all States except Alaska and Hawaii, the Census Bureau uses three population size criteria to designate a CDP. These criteria are:

a. 1,000 or more persons if outside the boundaries of an urbanized area (UA) delineated for the 1980 census or a subsequent special census.

b. 2,500 or more persons if inside the boundaries of a UA delineated for the 1980 census or a subsequent special census.

c. 250 or more persons if outside the boundaries of a UA delineated for the 1980 census or a subsequent special census, and within the official boundaries of an American Indian reservation recognized for the 1990 census.

2. In Alaska, 25 or more persons if outside a UA, and 2,500 or more persons if inside a UA delineated for the 1980 census or a subsequent special census.

3. In Hawaii, 300 or more persons, regardless of whether the community is inside or outside a UA.

For the 1990 census, CDP's qualified on the basis of the population counts prepared for the 1990 Postcensus Local Review Program. Because these counts were subject to change, a few CDP's may have final population counts lower than the minimums shown above.

Hawaii is the only state with no incorporated places recognized by the Bureau of the Census. All places shown for Hawaii in the data products are CDP's. By agreement with the state of Hawaii, the Census Bureau 
does not show data separately for the city of Honolulu, which is coextensive with Honolulu County.

Consolidated City

A consolidated government is a unit of local government for which the functions of an incorporated place and its county or minor civil division (MCD) have merged. The legal aspects of this action may result in both the primary incorporated place and the county or MCD continuing to exist as legal entities, even though the county or MCD performs few or no governmental functions and has few or no elected officials. Where this occurs, and where one or more other incorporated places in the county or MCD continue to function as separate governments, even though they have been included in the consolidated government, the primary incorporated place is referred to as a "consolidated city."

The data presentation for consolidated cities varies depending upon the geographic presentation. In hierarchical presentations, consolidated cities are not shown. These presentations include the semi-independent places and the "consolidated city (remainder)." Where the consolidated city is coextensive with a county or county subdivision, the data shown for those areas in hierarchical presentations are equivalent to those for the consolidated government.

For inventory geographic presentations, the consolidated city appears at the end of the listing of places. The data for the consolidated city include places that are part of the consolidated city. The "consolidated city (remainder)" is the portion of the consolidated government minus the semi-independent places, and is shown in alphabetical sequence with other places.

In summary presentations by size of place, the consolidated city is not included. The places semi-independent of consolidated cities are categorized by their size, as is the "consolidated city (remainder)."

Each consolidated city is assigned a one-character alphabetic census code. Each consolidated city also is assigned a five-digit FIPS code that is unique within state. The semi-independent places and the "consolidated city (remainder)" are assigned a four-digit census code and a five-digit FIPS place code that are unique within state. Both the census and FIPS codes are assigned based on alphabetical order within state.

\section{Incorporated Place}

Incorporated places recognized in 1990 census data products are those reported to the Census Bureau as legally in existence on January 1 , 1990 under the laws of their respective states as cities, boroughs, towns, and villages, with the following exceptions: the towns in the New England States, New York, and Wisconsin, and the boroughs in New York are recognized as minor civil divisions for census purposes; the boroughs in Alaska are county equivalents.

STATE

States are the primary governmental divisions of the United States. The District of Columbia is treated as a statistical equivalent of a state for census purposes.

Each State and the District of Columbia is assigned a two-digit numeric Federal Information Processing Standards (FIPS) code in alphabetical order by state name. Each state and the District of Columbia also is 
assigned a two-digit census code. This code is assigned on the basis of the geographic sequence of each state within each census division; the first digit of the code is the code for the respective division. Each state and equivalent area also is assigned the two-letter FIPS/United States Postal Service (USPS) code.

\section{TIGER}

TIGER is an acronym for the new digital (computer-readable) geographic data base that automates the mapping and related geographic activities required to support the Census Bureau's census and survey programs. The Census Bureau developed the Topologically Integrated Geographic Encoding and Referencing (TIGER) System to automate the geographic support processes needed to meet the major geographic needs of the 1990 census: producing the cartographic products to support data collection and map publication, providing the geographic structure for tabulation and publication of the collected data, assigning residential and employer addresses to their geographic location and relating those locations to the Census Bureau's geographic units, and so forth. The content of the TIGER data base is made available to the public through a variety of "TIGER Extract" files that may be obtained from the Data User Services Division, U.S. Bureau of the Census, Washington, DC 20233.

\section{UNITED STATES}

The United States comprises the 50 states and the District of Columbia.

\section{VOTING DISTRICT (VTD)}

A voting district (VTD) is any of a variety of types of areas (for example, election districts, precincts, wards, legislative districts) established by state and local governments for purposes of elections. For census purposes, each State participating in Phase 2 of the 1990 Census Redistricting Data Program outlined the boundaries of VTD's around groups of whole census blocks on census maps. The entities identified as VTD's are not necessarily those legally or currently established. Also, to meet the "whole block" criterion, a state may have had to adjust VTD boundaries to nearby block boundaries. Therefore, the VTD's shown on the 1990 census tapes, listings, and maps may not represent the actual VTD's in effect at the time of the census. In the 1980 census, VTD's were referred to as "election precincts."

Each VTD is assigned a four-character alphanumeric code that is unique within each county. The code "Zzzz" is assigned to nonparticipating areas; the Census Bureau reports data for areas coded "zzzz." 
Census of Population and Housing, 1990: Public Law (P.L.) 94-171 Data on CD-ROM (Name of State) [machine-readable data files] / prepared by the Bureau of the Census. --Washington: The Bureau [producer and distributor], 1991.

\section{APPENDIX C.}

ACCURACY OF THE DATA

\section{CONTENTS}

Confidentiality of the Data........................... Editing of Unacceptable Data........................... Sources of Error.................................

\section{CONEIDENTIALITY OF THE DATA}

To maintain confidentiality required by law (Title 13, U.S. Code), the Bureau of the Census applies a confidentiality edit to assure published data do not disclose information about specific individuals, households, and housing units. The result is that a small amount of uncertainty is introduced into some of the census characteristics to prevent identification of specific individuals, households, or housing units. The edit is controlled so that the counts of total persons, totals by race and American Indian tribe, Hispanic origin, and age 18 years and over are not affected by the confidentiality edit and are published as collected. In addition, total counts for housing units by tenure are not affected by this edit.

The confidentiality edit is conducted by selecting a sample of census households from the 100-percent data internal census files and interchanging its data with other households that have identical characteristics on a set of selected key variables but are in different geographic locations within the same state. To provide more protection for "small areas," a higher sampling rate was used for these areas. The net result of this procedure is that the data user's ability to obtain census data, particularly for small areas and subpopulation groups, has been significantly enhanced.

\section{EDITING OF UNACCEPTABLE DATA}

The objective of the processing operation is to produce a set of data that describes the population as accurately and clearly as possible. To meet this objective, questionnaires were edited during field data collection operations for consistency, completeness, and acceptability. Questionnaires were also reviewed by census clerks for omissions, certain inconsistencies, and population coverage. For example, write-in entries such as "Don't know" or "NA" were considered unacceptable. For some district offices, the initial edit was automated; however, for the majority of the district offices, it was performed by clerks. As a result of this operation, a telephone or personal visit followup was made to obtain missing information. Potential coverage errors were included in the followup, as well as a sample of questionnaires with omissions or inconsistencies.

Subsequent to field operations, remaining incomplete or inconsistent information on the questionnaires was assigned using imputation procedures during the final automated edit of the collected data. Allocations, or computer assignments of acceptable codes in place of unacceptable entries or blanks, are needed most often when an entry for a given item is lacking or when the information reported for a person or housing unit on that item is inconsistent with other information for 
that same person or housing unit. As in previous censuses, the general procedure for changing unacceptable entries was to assign an entry for a person or housing unit that was consistent with entries for persons or housing units with similar characteristics. The assignment of acceptable codes in place of blanks or unacceptable entries enhances the usefulness of the data.

Another way in which corrections were made during the computer editing process was through substitution; that is, the assignment of a full set of characteristics for a person or housing unit. When there was an indication that a housing unit was occupied, but the questionnaire contained no information for the people within the household, or the occupants were not listed on the questionnaire, a previously accepted household was selected as a substitute, and the full set of characteristics for the substitute was duplicated. The assignment of the full set of housing characteristics occurred when there was no housing information available. If the housing unit was determined to be occupied, the housing characteristics were assigned from a previously processed occupied unit. If the housing unit was vacant, the housing characteristics were assigned from a previously processed vacant unit.

\section{SOURCES OF ERROR}

In any large-scale statistical operation, such as the 1990 decennial census, human- and machine-related errors occur. These errors are commonly referred to as nonsampling errors. Such errors include not enumerating every household or every person in the population, not obtaining all required information from the respondents, obtaining incorrect or inconsistent information, and recording information incorrectly. In addition, errors can occur during the field review of the enumerators' work, during clerical handling of the census questionnaires, or during the electronic processing of the questionnaires.

To reduce various types of nonsampling errors, a number of techniques were implemented during the planning, development of the mailing address list, data collection, and data processing activities. Quality assurance methods were used throughout the data collection and processing phases of the census to improve the quality of the data. A reinterview program was designed to minimize the errors in the data collection phase for enumerator-filled questionnaires.

Several coverage improvement programs were implemented during the development of the census address list and census enumeration and processing to minimize undercoverage of the population and housing units. These programs were developed based on experience from the 1980 decennial census and results from the 1990 decennial census testing cycle. In developing and updating the census address list, the census Bureau used a variety of specialized procedures in different parts of the country.

- In urban areas, the Census Bureau purchased address lists, had the United States Postal Service (USPS) review and update this list, and conducted a dependent canvass and update operation. Prior to mailout, local officials were given the opportunity to examine block counts of address listings (local review) and identify possible errors, and the USPS conducted a final review.

- In more rural parts of the country, the Census Bureau created the address list through a listing operation. The USPS reviewed and updated this list, and the Census Bureau reconciled usPS corrections through a dependent recanvass. Prior to mailout, the UsPS conducted a final review, and local officials participated in this review. 
Coverage improvement programs continued during and after mailout. The Census Bureau (rather than the USPS) delivered census questionnaires in certain rural areas and in inner city public housing developments. Computer and clerical edits and telephone and personal visit followups also contributed to improved coverage.

If the 1990 census is not subject to count adjustment, the population counts shown in 100-percent data products will be tabulated from the entries for persons on all questionnaires. These counts will not be subject to sampling error. If count adjustment is done, a discussion of the count adjustment methodology and the appropriate methods for

calculating sampling errors of adjusted counts can be found in appendix H. (Housing unit counts will not be subject to count adjustment.) 
Census of Population and Housing, 1990: Public Law (P.L.) 94-171 Data on CD-ROM (Name of state) [machine-readable data files] / prepared by the Bureau of the Census. --Washington: The Bureau [producer and distributor], 1991.

APRENDIX D.

COLLECTION AND PROCESSING PROCEDURES

CONTENTS

ENUMERATION AND RESIDENCE RULES................... D

Enumeration Rules

Residence Rules

Persons in the Armed Forces

Persons on Maritime Ships

Persons Away at School

Persons in Institutions

Persons Away From Their Usual Residence on Census Data

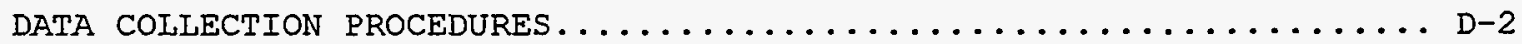

Enumeration of Housing Units

Eollowup

Special Enumeration Procedures

Shelter and Street Night (S-Night)

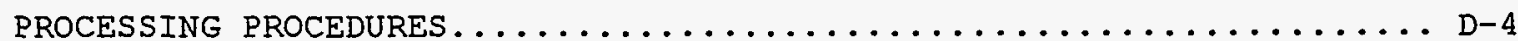

\section{ENUMERATION AND RESIDENCE RULES}

In accordance with census practice dating back to the first United States census in 1790, each person was to be enumerated as an inhabitant of his or her "usual residence" in the 1990 census. Usual residence is the place where the person lives and sleeps most of the time or considers to be his or her usual residence. This place is not necessarily the same as the person's legal residence or voting residence. In the vast majority of cases, however, the use of these different bases of classification would produce substantially the same statistics, although there might be appreciable differences for a few areas.

The implementation of this practice has resulted in the establishment of rules for certain categories of persons whose usual place of residence is not immediately apparent. Furthermore, this practice means that persons were not always counted as residents of the place where they happened to be staying on Census Day (April 1, 1990).

Enumeration Rules

Each person whose usual residence was in the United States was to be included in the census, without regard to the person's legal status or citizenship. In a departure from earlier censuses, foreign diplomatic personnel participated voluntarily in the census, regardless of their residence on or off the premises of an embassy. As in previous censuses, persons in the United states specifically excluded from the census were foreign travelers who had not established a residence.

Americans with a usual residence outside the United States were not enumerated in the 1990 census. United States military and Federal civilian employees, and their dependents overseas, are included in the population counts for States for purposes of Congressional apportionment, but are excluded from all other tabulations for states 
and their subdivisions. The counts of United States military and Federal civilian employees, and their dependents, were obtained from administrative records maintained by Federal departments and agencies. Other Americans living overseas, such as employees of international agencies and private businesses, and students, were not enumerated, nor were their counts obtained from administrative sources. On the other hand, Americans overseas temporarily were to be enumerated at their usual residence in the United States.

\section{Residence Rules}

Each person included in the census was to be counted at his or her usual residence--the place where he or she lives and sleeps most of the time or the place where the person considers to be his or her usual home. If a person had no usual residence, the person was to be counted where he or she happened to be staying on April 1, 1990.

Persons temporarily away from their usual residence, whether in the United states or overseas, on a vacation or on a business trip, were counted at their usual residence. Persons who occupied more than one residence during the year were counted at the one they considered to be their usual residence. Persons who moved on or near Census Day were counted at the place they considered to be their usual residence.

Persons in the Armed Forces--Members of the Armed Forces were counted as residents of the area in which the installation was located, either on the installation or in the surrounding community. Family members of Armed Forces personnel were counted where they were living on Census Day (for example, with the Armed Forces person or at another location).

Each Navy ship not deployed to the 6 th or the 7 th Fleets was attributed to the municipality that the Department of the Navy designated as its homeport. If the homeport included more than one municipality, ships berthed there on Census Day were assigned by the Bureau of the Census to the municipality in which the land immediately adjacent to the dock or pier was actually located. Ships attributed to the homeport, but not physically present and not deployed to the 6th or the 7 th Fleets, were assigned to the municipality named on the Department of the Navy's homeport list.

Personnel assigned to each Navy ship were given the opportunity to report a residence off the ship. Those who did report an off-ship residence in the communities surrounding the homeport were counted there; those who did not were counted as residents of the ship. Personnel on Navy ships deployed to the 6th or 7th Fleets on Census Day were considered to be part of the overseas population.

Persons on Maritime Ships--Persons on maritime ships who did not report a usual residence elsewhere were counted as residents of the ship. Those counted on the ship were attributed to:

1. The port where the ship was docked in the United States.

2. The port of departure if between United States ports or between a United States and a foreign port.

If the ship was docked at a foreign port, or between foreign ports, persons counted on the ship were considered to be part of the overseas population.

Persons Away at School--College students were counted as residents of the area in which they were living while attending college, as they have been since the 1950 census. Children in boarding schools below the college level 
were counted at their parental home.

Persons in Institutions--Persons under formally authorized, supervised care or custody, such as in Federal or State prisons; local jails; Federal detention centers; juvenile institutions; nursing, convalescent, and rest homes for the aged and dependent; or homes, schools, hospitals, or wards for the physically handicapped, mentally retarded, or mentally ill, were counted at these places.

Persons Away From Their Usual Residence on Census Day--Migrant agricultural workers who did not report a usual residence elsewhere were counted as residents of the place where they were on Census Day. Persons in worker camps who did not report a usual residence elsewhere were counted as residents of the camp where they were on Census Day.

In some parts of the country, natural disasters displaced significant numbers of households from their usual place of residence. If these persons reported a destroyed or damaged residence as their usual residence, they were counted at that location.

Persons away from their usual residence were counted by means of interviews with other members of their families, resident managers, or neighbors.

\section{DATA COLLECTION PROCEDURES}

The 1990 census was conducted primarily through self-enumeration. Census questionnaires were delivered 1 week before Census Day (April 1, 1990). The questionnaire packet included general information about the 1990 census and an instruction guide explaining how to complete the questionnaire. Spanish-language questionnaires and instruction guides were available on request. Instruction guides also were available in 32 other languages.

\section{Enumeration of Housing Units}

Each housing unit in the country received one of two versions of the census questionnaire:

1. A short-form questionnaire which contained a limited number of basic population and housing questions; these questions were asked of all persons and housing units and are often referred to as 100percent questions.

2. A long-form questionnaire which contained the 100-percent items and a number of additional questions; a sampling procedure was used to determine those housing units that were to receive the long-form questionnaire.

Three sampling rates were employed. For slightly more than one-half of the country, one in every six housing units (about 17 percent) received the long-form or sample questionnaire. In functioning local governmental units (counties and incorporated places, and in some parts of the country, towns and townships) estimated to have fewer than 2,500 inhabitants, every other housing unit (50 percent) received the sample questionnaire in order to enhance the reliability of the sample data for these small areas. For census tracts and block numbering areas having more than 2,000 housing units in the Census Bureau's address files, one in every eight housing units (about 13 percent) received a sample questionnaire, providing reliable statistics for these areas while permitting the Census Bureau to stay within a limit of 17.7 million sample questionnaires, or a one-in-six sample, nationwide. 
The mail-out/mail-back procedure was used mainly in cities, suburban areas, towns, and rural areas where mailing addresses consisted of a house number and street name. In these areas, the Census Bureau developed mailing lists that included about 88.4 million addresses. The questionnaires were delivered through the mail and respondents were to return them by mail.

The update/leave/mail-back method was used mainly in densely populated rural areas where it was difficult to develop mailing lists because mailing addresses were not house number and street name type. The Census Bureau compiled lists of housing units in advance of the census. The enumerators delivered the questionnaires, asked respondents to return them by mail, and added housing units not on the mailing lists. This method was used mainly in the South, and also included some high-rise, low-income urban areas. A variation of this method was used in urban areas having large numbers of boarded-up buildings. About 11 million housing units were enumerated using this method.

The list/enumerate method (formerly called conventional or door-to-door enumeration) was used mainly in very remote and sparsely-settled areas. The United States Postal Service delivered unaddressed short-form questionnaires before Census Day. Starting a week before Census Day, enumerators canvassed these areas, checked that all housing units received a questionnaire, created a list of all housing units, completed long-form questionnaires, and picked up the completed short-form questionnaires. This method was used mainly in the west and Northeast to enumerate an estimated 6.5 million housing units.

\section{Followup}

Nonresponse Followup--In areas where respondents were to mail back their questionnaires, an enumerator visited each address from which a questionnaire was not received.

Coverage and Edit-Failure Followup--In the mail-back areas, some households returned a questionnaire that did not meet specific quality standards because of incomplete or inconsistent information, or the respondent had indicated difficulty in deciding who was to be listed on the questionnaire. These households were contacted by telephone or by personal visit to obtain the missing information, or to clarify who was to be enumerated in the household. In areas where an enumerator picked up the questionnaires, the enumerator checked the respondent-filled questionnaire for completeness and consistency.

\section{Special Enumeration Procedures}

Special procedures and questionnaires were used for the enumeration of persons in group quarters, such as college dormitories, nursing homes, prisons, military barracks, and ships. The questionnaires (Individual Census Reports, Military Census Reports, and Shipboard Census Reports) included the 100-percent population questions, but did not include any housing questions. In all group quarters, all persons were asked the basic population questions; in most group quarters, additional questions were asked of a sample (one-in-six) of persons.

Shelter and Street Night (S-Night)

The Census Bureau collected data for various components of the homeless population at different stages in the 1990 census. "Shelter and Street Night" (S-Night) was a special census operation to count the population in four types of locations where homeless people are found. On the evening of March 20, 1990, and during the early morning hours of March 21, 1990, enumerators counted persons in pre-identified 
locations:

1. Emergency shelters for the homeless population (public and private; permanent and temporary).

2. Shelters with temporary lodging for runaway youths.

3. Shelters for abused women and their children.

4. Open locations in streets or other places not intended for habitation.

Emergency shelters include all hotels and motels costing $\$ 12$ or less (excluding taxes) per night regardless of whether persons living there considered themselves to be homeless, hotels and motels (regardless of cost) used entirely to shelter homeless persons, and pre-identified rooms in hotels and motels used for homeless persons and families. Enumeration in shelters usually occurred from 6 p.m. to midnight; street enumeration, from $2 \mathrm{a} . \mathrm{m}$. to $4 \mathrm{a} . \mathrm{m} . ;$ abandoned and boarded-up buildings from $4 \mathrm{a} . \mathrm{m}$. to $8 \mathrm{a} . \mathrm{m}$.; and shelters for abused women, from 6 p.m. on March 20 to noon on March 21.

Other components, which some consider as part of the homeless population, were enumerated as part of regular census operations. These include persons doubled up with other families, as well as persons with no other usual home living in transient sites, such as commercial campgrounds, maternity homes for unwed mothers, and drug/alcohol abuse detoxification centers. In institutions, such as local jails and mental hospitals, the Census Bureau does not know who has a usual home elsewhere; therefore, even though some are literally homeless, these persons cannot be identified separately as a component of the homeless population.

There is no generally agreed-upon definition of "the homeless," and there are limitations in the census count that prevent obtaining a total count of the homeless population under any definition. As such, the Census Bureau does not have a definition and will not provide a total count of "the homeless." Rather, the Census Bureau will provide counts and characteristics of persons found at the time of the census in selected types of living arrangements. These selected components can be used as building blocks to construct a count of homeless persons appropriate to particular purposes as long as the data limitations are taken into account.

In preparation for "Shelter-and-Street-Night" enumeration, the regional census centers (RCC's) mailed a certified letter (Form D-33 (I)) to the highest elected official of each active functioning government of the United States (more than 39,000 ) requesting them to identify:

1. All shelters with sleeping facilities (permanent and temporary, such as church basements, armories, public buildings, and so forth, that could be open on March 20).

2. Hotels and motels used to house homeless persons and families.

3. A list of outdoor locations where homeless persons tend to be at night.

4. Places such as bus or train stations, subway stations, airports, hospital emergency rooms, and so forth, where homeless persons seek shelter at night. 
5. The specific addresses of abandoned or boarded-up buildings where homeless persons were thought to stay at night.

The letter from the RCC's to the governmental units emphasized the importance of listing night-time congregating sites. The list of shelters was expanded using information from administrative records and informed local sources. The street sites were limited to the list provided by the jurisdictions. All governmental units were eligible for "Shelter and Street Night." For cities with 50,000 or more persons, the Census Bureau took additional steps to update the list of shelter and street locations if the local jurisdiction did not respond to the certified letter. Smaller cities and rural areas participated if the local jurisdiction provided the Census Bureau a list of shelters or open public places to visit or if shelters were identified through our inventory development, local knowledge update, or during the special Place Prelist operation.

The Census Bureau encouraged persons familiar with homeless persons and the homeless themselves to apply as enumerators. This recruiting effort was particularly successful in larger cities.

For shelters, both long- and short-form Individual Census Reports (ICR's) were distributed. For street enumeration, only short-form ICR's were used. Persons in shelters and at street locations were asked the basic population questions. Additional questions about social and economic characteristics were asked of a sample of persons in shelters only.

Enumerators were instructed not to ask who was homeless; rather, they were told to count all persons (including children) staying overnight at the shelters, and everyone they saw on the street except the police, other persons in uniform, and persons engaged in employment or obvious money-making activities other than begging and panhandling.

At both shelter and street sites, persons found sleeping were not awakened to answer questions. Rather, the enumerator answered the sex and race questions by observation and estimated the person's age to the best of his or her ability. In shelters, administrative records and information from the shelter operator were used, when available, for persons who were already asleep.

Less than one percent of shelters refused to participate in the census count at first. By the end of the census period, most of those eventualiy cooperated and the number of refusals had been reduced to a few. For the final refusals, head counts and population characteristics were obtained by enumerators standing outside such shelters and counting people as they left in the morning.

The "street" count was restricted to persons who were visible when the enumerator came to the open, public locations that had been identified by local jurisdictions. Homeless persons who were well hidden, moving about, or in locations other than those identified by the local governments were likely missed. The number missed will never be known and there is no basis to make an estimate of the number missed from census data. The count of persons in open, public places was affected by many factors, including the extra efforts made to encourage people to go to shelters for "Shelter and Street Night," the weather (which was unusually cold in many parts of the country), the presence of the media, and distrust of the census. Expectations of the number of homeless persons on the street cannot be based on the number seen during the day because the night-time situation is normally very different as more homeless persons are in shelters or very well hidden. 
For both "Shelter-and-Street-Night" locations, the Census Bureau assumed that the usual home of those enumerated was in the block where they were found (shelter or street).

The "Shelter-and-Street-Night" operation replaced and expanded the 1980 Mission Night (M-Night) and Casual Count operations. These two operations were aimed at counting the population who reported having no usual residence. M-Night was conducted a week after Census Day, in April 1980. Enumerators visited hotels, motels, and similar places costing $\$ 4$ or less each night; missions, flophouses, local jails and similar places at which the average length of stay was 30 days or less; and nonshelter locations, such as bus depots, train stations, and all night movie theaters. Questions were asked of everyone, regardless of age. Enumerators conducted M-Night up to midnight on April 8, 1980 and returned the next morning to collect any forms completed after midnight.

The Casual Count operation was conducted in May 1980 at additional nonshelter locations, such as street corners, pool halls, welfare and employment offices. This operation lasted for approximately 2 weeks. Casual count was conducted during the day only in selected large central cities. Only persons who appeared to be at least 15 years of age were asked if they had been previously enumerated. Casual Count was actually a coverage-improvement operation. It was not specifically an operation to count homeless persons living in the streets. Persons were excluded if they said they had a usual home outside the city because it was not cost effective to check through individual questionnaires in another city to try to find the person.

\section{PROCESSING PROCEDURES}

Respondents returned many census questionnaires by mail to one of over 344 census district offices or to one of six processing offices. In these offices, the questionnaires were "checked in" and edited for completeness and consistency of the responses. After this initial processing had been performed, all questionnaires were sent to the processing offices.

In the processing offices, the household questionnaires were microfilmed and processed by the Film Optical Sensing Device for Input to Computers (FOSDIC). For most items on the questionnaire, the information supplied by the respondent was indicated by filling circles in predesignated positions. FOSDIC electronically "read" these filled circles from the microfilm copy of the questionnaire and transferred the information to computer disk. The computer tape did not include individual names, addresses, or handwritten responses.

The data processing was performed in several stages. All questionnaires were microfilmed, "read" by FOSDIC, and transferred to computer disk. Selected written entries in the race question on both the short and long forms were keyed from the microfilm and coded using the data base developed from the 1980 census and subsequent content and operational tests. Keying other written entries on the long forms occurred in the seven processing offices.

The information (for example, income dollar amounts or homeowner shelter costs) on these keyed files was merged with the FOSDIC data or processed further through one of three automated coding programs. The codes for industry, occupation, migration, place-of-work, ancestry, language, relationship, race, and Hispanic origin were merged with the FOSDIC data for editing, weighting, and tabulating operations at Census Bureau headquarters. All responses to the questions on Individual 
Census Reports (ICR's), Military Census Reports (MCR's), and Shipboard Census Reports (SCR's) were keyed, not processed by microfilm or FOSDIC. 
Census of Population and Housing, 1990: Public Law (P.I.) 94-171 Data on CD-ROM (Name of State) [machine-readable data files] / prepared by the Bureau of the Census. --Washington: The Bureau [producer and distributor], 1991.

DATA DICTIONARY

\section{CONTENTS}

Identification Section

Footnote section

Table (Matrix) Section

IDENTIFICATION SECTION

Field name

Data dictionary reference name

\section{Field} size
Starting position
Data type

Record Codes

File Identification(1)

State/US Abbreviation

$\begin{array}{llll}\text { AK } & \text { Alaska } & \text { ID } & \text { Idaho } \\ \text { AL } & \text { Alabama } & \text { IL } & \text { Illinois } \\ \text { AR Arkansas } & \text { IN } & \text { Indiana } \\ \text { AZ Arizona } & \text { KS } & \text { Kansas } \\ \text { CA } & \text { California } & \text { KY } & \text { Kentucky } \\ \text { CO Colorado } & \text { IA } & \text { Louisiana } \\ \text { CT } & \text { Connecticut } & \text { MA } & \text { Massachusetts } \\ \text { DC District of } & \text { MD } & \text { Maryland } \\ & \text { Columbia } & \text { ME } & \text { Maine } \\ \text { DE Delaware } & \text { MI } & \text { Michigan } \\ \text { FL } & \text { Florida } & \text { MN } & \text { Minnesota } \\ \text { GA Georgia } & \text { MO } & \text { Missouri } \\ \text { HI Hawaii } & \text { MS } & \text { Mississippi } \\ \text { IA Iowa } & \text { MT } & \text { Montana }\end{array}$

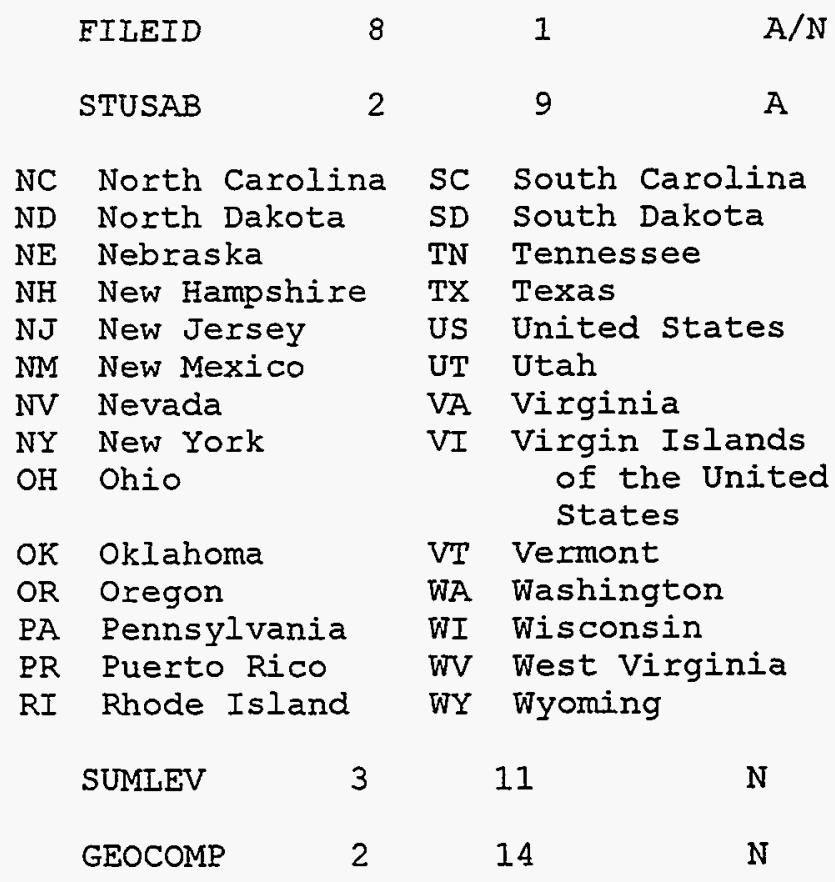

Geographic Component(3)

01 Urban

02 Urban-in urbanized area

03 Urban-in urbanized area-in urbanized area central place

04 Urban-in urbanized area-not in urbanized area central place

05 Urban-not in urbanized area

06 Urban-not in urbanized area-place [10,000 or more persons]

07 Urban-not in urbanized area-place [2,500 to 9,999 persons]

08 Rural

09 Rural-place [1,000 to 2,499 population, not in an extended city]

10 Rural-place [0 to 999 population, not in an extended city]

11 Rural-not in place [or rural part of extended city]

12 Rural-farm

13 Urban portion of extended city

14 Rural portion of extended city

20 In metropolitan statistical area/consolidated metropolitan statistical area 
21 In metropolitan statistical area/consolidated metropolitan statistical area-urban

22 In metropolitan statistical area/consolidated metropolitan statistical area-rural

23 In metropolitan statistical area/consolidated metropolitan statistical area-in metropolitan statistical area/primary metropolitan statistical area central city

24 In metropolitan statistical area/consolidated metropolitan statistical area-not in metropolitan statistical area/primary metropolitan statistical area central city

25 In metropolitan statistical area/consolidated metropolitan statistical area-not in metropolitan statistical area/primary metropolitan statistical area central city-urban

26 In metropolitan statistical area/consolidated metropolitan statistical area-not in metropolitan statistical area/primary metropolitan statistical area central city-urban-in urbanized area

In metropolitan statistical area/consolidated metropolitan statistical area-not in metropolitan statistical area/primary metropolitan statistical area central city-urban-not in urbanized area

28 In metropolitan statistical area/consolidated metropolitan statistical area-not in metropolitan statistical area/primary metropolitan statistical area central city-rural

30 Not in metropolitan statistical area/consolidated metropolitan statistical area

31 Not in metropolitan statistical area/consolidated metropolitan statistical areaurban

32 Not in metropolitan statistical area/consolidated metropolitan statistical areaurban-in urbanized area

33 Not in metropolitan statistical area/consolidated metropolitan statistical areaurban-not in urbanized area

34 Not in metropolitan statistical area/consolidated metropolitan statistical areaurban-not in urbanized area-place $[10,000$ or more population]

35 Not in metropolitan statistical area/consolidated metropolitan statistical areaurban-not in urbanized area-place [2,500 - 9,999 population]

36 Not in metropolitan statistical area/consolidated metropolitan statistical arearural

40 American Indian reservation and trust land [American Indian reservations (AIR codes 0001-4989) including any trust lands]

42 Tribal Jurisdiction Statistical Area [Oklahoma only]

43 Tribal Designated Statistical Area

44 Alaska Native village statistical area [Alaska only]

Characteristic Iteration(4)

CHARITER

316

N

000 Not a characteristic iteration

Logical Record Number(5)

LOGRECNU

$6 \quad 19$

$\mathrm{N}$ 
Logical Record Part Number(6)

Total Number of Parts in Record(7)

Geographic Area Codes

\section{LOGRECPN}

PARTREC
4

4

2

33

$\mathrm{A} / \mathrm{N}$

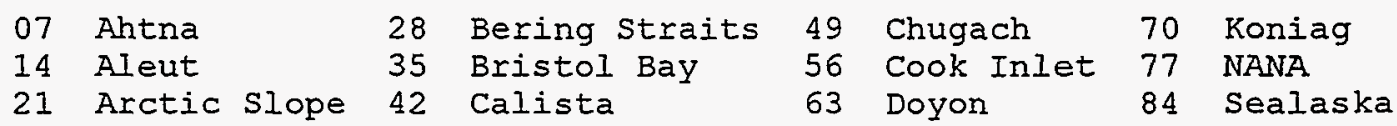

American Indian/Alaska Native Area (Census) (9)
AIANACE

AIANAFP

AIANACC
4

35

$A / N$

American Indian/Alaska Native Area (EIPS) (10)

American Indian/Alaska Native Area class Code

D1 Federally recognized American Indian reservation that does not serve as a minor civil division (MCD) equivalent

D2 Federally recognized American Indian reservation that also serves as an $\mathrm{MCD}$ equivalent

D3 American Indian tribal government that holds property in trust "trust land" for a tribe or individual member(s) of the tribe, and the trust land(s) is not associated with a specific American Indian reservation

D4 State-recognized American Indian reservation that does not serve as an MCD equivalent

D5 State-recognized American Indian reservation that also serves as an MCD equivalent

D6 Tribal designated statistical area (TDSA); tribal jurisdiction statistical area (TJSA) (TJSAs occur only in oklahoma)

El Alaska Native village statistical area (ANVSA) that does not coincide with or approximate an incorporated place or a census designated place (CDP)

E2 ANVSA that coincides with or approximates a CDP

E6 ANVSA that coincides with or approximates an incorporated place

American Indian Reservation Trust Iand

Indicator Code

ARTII

1

46

$\mathrm{A} / \mathrm{N}$

1 American Indian reservation; including Tribal Juxisdiction statistical Area (TJSA) and Tribal Designated Statistical Area (TDSA)

2 Off-reservation trust land

9 Not an American Indian area

Block (8)

Block Group (8)

Census Tract/Block Numbering Area(8)

Congressional District

(101st Congress) (8)

$$
\text { BLCK }
$$

BLCKGR

TRACTBNA

CONGDIS
4

1

6

2

58

$A / N$

01-45 The actual congressional district number 
00 Applies to states whose representative is elected ' at Iarge" '; i.e., the state has only one representative in the United States House of Representatives

98 Applies to areas that have a nonvoting delegate in the United States House of Representatives

99 Applies to areas that have no representation in the United states House of Representatives

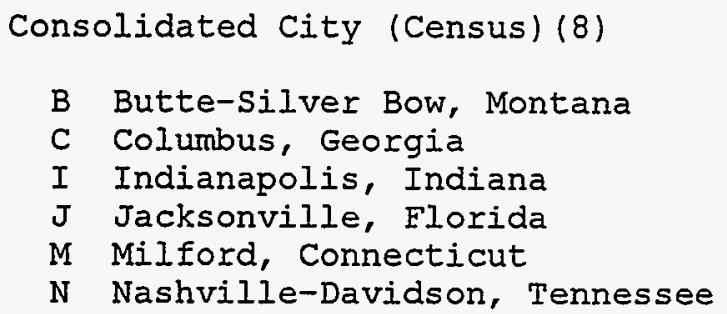

$\begin{array}{ll}00 & \text { Not in universe } \\ 01 & 0 \\ 02 & 1-24 \\ 03 & 25-99 \\ 04 & 100-199 \\ 05 & 200-249 \\ 06 & 250-299 \\ 07 & 300-499 \\ 08 & 500-999 \\ 09 & 1,000-1,499 \\ 10 & 1,500-1,999 \\ 11 & 2,000-2,499\end{array}$

$$
\begin{array}{ll}
12 & 2,500-4,999 \\
13 & 5,000-9,999 \\
14 & 10,000-19,999 \\
15 & 20,000-24,999 \\
16 & 25,000-49,999 \\
17 & 50,000-99,999 \\
18 & 100,000-249,999 \\
19 & 250,000-499,999 \\
20 & 500,000-999,999 \\
21 & 1,000,000-2,499,999 \\
22 & 2,500,000-4,999,999 \\
23 & 5,000,000 \text { or more }
\end{array}
$$

Consolidated Metropolitan Statistical Area (9)

\begin{tabular}{cl} 
& \multicolumn{1}{c}{ CNSA } \\
& \multicolumn{1}{c}{ CNTY } \\
CNTYSC \\
code & \multicolumn{1}{c}{$l$} \\
12 & $2,500-4,999$ \\
13 & $5,000-9,999$ \\
14 & $10,000-19,999$ \\
15 & $20,000-24,999$ \\
16 & $25,000-49,999$ \\
17 & $50,000-99,999$ \\
18 & $100,000-249,999$ \\
19 & $250,000-499,999$ \\
20 & $500,000-999,999$ \\
21 & $1,000,000-2,499,999$ \\
22 & $2,500,000-4,999,999$ \\
23 & $5,000,000$ or more
\end{tabular}

County (9)

County Population Size Code
County Subdivision (Census) (9)

County Subdivision (FIPS) (10)

County Subdivision Class Code

$\begin{array}{llll}\text { COUSUBCE } & 3 & 77 & \mathrm{~A} / \mathrm{N} \\ \text { COUSUBEP } & 5 & 80 & \mathrm{~A} / \mathrm{N} \\ \text { COUSUBCC } & 2 & 85 & \mathrm{~A} / \mathrm{N}\end{array}$


C2 Incorporated place that also serves as a minor civil division (MCD) equivalent because, although the place is coextensive with an MCD, the Census Bureau, in agreement with state officials, does not recognize that MCD for presenting census data because the MCD cannot provide governmental services (Ohio only)

C5 Incorporated place that also serves as an MCD equivalent because it is not part of an MCD or a county subdivision classified as 25

C7 Incorporated place that also serves as a county equivalent and an MCD equivalent; generally referred to as an 'independent city'"

D2 Federally recognized American Indian reservation that also serves as an MCD equivalent

D5 State-recognized American Indian reservation that also serves as an MCD equivalent

T1 Governmentally active minor civil division (MCD) that is not coextensive with an incorporated place

T5 Governmentally active MCD that is coextensive with an incorporated place

Z1 MCD that is governmentally inactive or cannot provide general-purpose governmental services

Z3 Unorganized territory identified by the Census Bureau as an MCD equivalent for presenting census data

Z5 Census county division (CCD); census subarea (CSA) (Alaska only); census subdistrict (Virgin Islands only)

Z7 Incorporated place that the Census Bureau treats as a minor civil division (MCD) equivalent because it is not in any MCD or is coextensive with a legally established but nonfunctioning MCD that the Census Bureau does not recognize for data presentation, AND is located in a state or county whose MCDs cannot provide governmental services (Iowa and Nebraska only)

County Subdivision Population Size Code

COUSUBSC

2

87

$\mathrm{A} / \mathrm{N}$

00 Not in universe

010

$02 \quad 1-24$

$03 \quad 25-99$

$04 \quad 100-199$

$05 \quad 200-249$

$06 \quad 250-299$

$07 \quad 300-499$

$08 \quad 500-999$

$091,000-1,499$

$101,500-1,999$

$112,000-2,499$
12

13

14

15

16

17

18

19

20

21

22

23
$2,500-4,999$

$5,000-9,999$

$10,000-19,999$

$20,000-24,999$

$25,000-49,999$

$50,000-99,999$

$100,000-249,999$

$250,000-499,999$

$500,000-999,999$

$1,000,000-2,499,999$

$2,500,000-4,999,999$

$5,000,000$ or more

Division (8)

DIVIS

1

89

$\mathrm{A} / \mathrm{N}$

0 Not in a division

1 New England

4 West North Central

5 South Atlantic

Middle Atlantic

6 East South Central

7 West South Central

8 Mountain

9 Pacific

3 East North Central

Extended City Indicator (8)

EXTCITIN

1

90

$\mathrm{A} / \mathrm{N}$

1 Extended city

9 Not an extended city

Internal Use Code(11)

INTUC

15

91

$\mathrm{A} / \mathrm{N}$ 
Metropolitan Statistical Area/ Consolidated Metropolitan Statistical

Area (9)

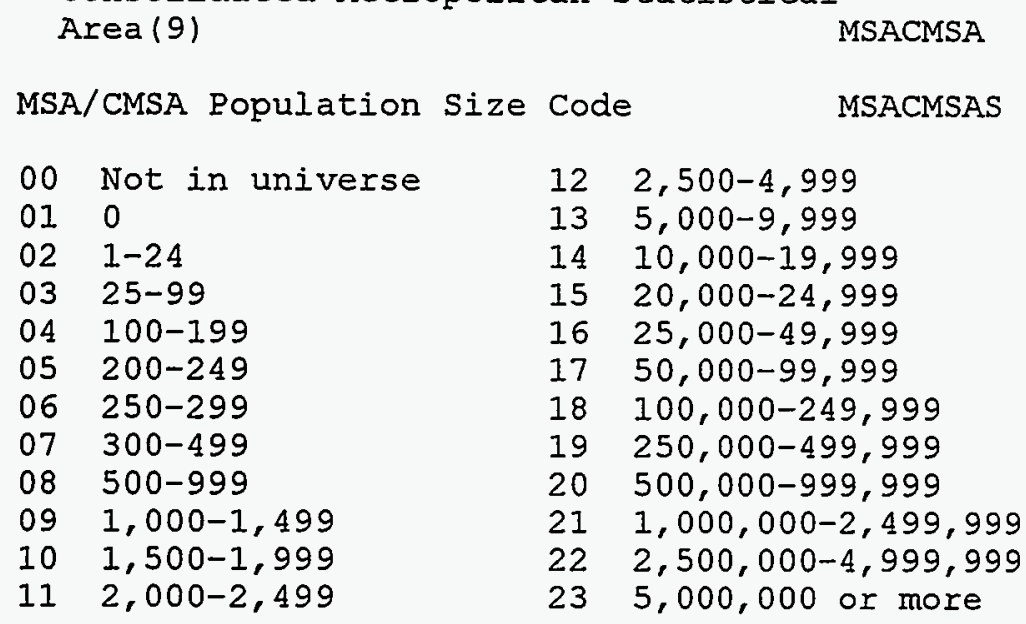

MSA/CMSA Population Size Code $\quad$ MSACMSAS $22110 \quad$ A/N

$\begin{array}{lllll}\text { Place (Census) }(9) & \text { PIACECE } & 4 & 112 & \mathrm{~A} / \mathrm{N} \\ \text { Place (FIPS) (10) } & \text { PI.ACEFP } & 5 & 116 & \mathrm{~A} / \mathrm{N} \\ \text { Place Class Code } & \text { PIACECC } & 2 & 121 & \mathrm{~A} / \mathrm{N}\end{array}$

Cl Incorporated place that is governmentally active, is not related to an Alaska Native village statistical area (ANVSA), and does not serve as a minor civil division (MCD) equivalent

C2 Incorporated place that also serves as a minor civil division (MCD) equivalent because, although the place is coextensive with an MCD, the Census Bureau, in agreement with State officials, does not recognize that MCD for presenting census data because the MCD cannot provide governmental services (Iowa and Ohio only)

C5 Incorporated place that also serves as an MCD equivalent because it is not part of an MCD

C6 Incorporated place that coincides with or approximates an ANVSA

C7 Incorporated place that also serves as a county equivalent; generally referred to as an "independent city" "

C8 The portion ('remainder' ') of a consolidated city that excludes the incorporated place(s) within that jurisdiction

C9 Incorporated place whose government is operationally inactive and is not included in any other $C$ subclass

M2 Military or Coast Guard installation (or part of an installation) that serves as a census designated place (CDP)

U1 CDP with a name that is commonly recognized for the populated area

U2 CDP with a name that is not commonly recognized for the populated area (e.g., a combination of the names of two or three commonly recognized communities, or a name. that identifies the location of the CDP in relation to an adjacent incorporated place)

U9 CDP that coincides with or approximates an ANVSA.

Note: In Ohio, a multi-county place that has a different MCD relationship in each county is assigned only a single class code of $\mathrm{Cl}$, C2, or C5.

$\begin{array}{lllll}\text { Place Description Code } & \text { PLACEDC } & 1 & 123 & \mathrm{~A} / \mathrm{N}\end{array}$

1 Incorporated central city of a metropolitan statistical area/primary metropolitan

statistical area (MSA/PMSA), but not a 
central place of an urbanized area (UA)

Incorporated central place of an urbanized area (UA), but not a central city of a metropolitan statistical area/primary metropolitan statistical area (MSA/PMSA)

Incorporated central city of a metropolitan statistical area/primary metropolitan statistical area (MSA/PMSA) and a central place of an urbanized area (UA)

Consolidated city or an incorporated place that is not a central city of a metropolitan statistical area/primary metropolitan statistical area (MSA/PMSA) and not a central place of an urbanized area (UA)

Incorporated place, which is the central place of an urbanized area (UA), but only part of which is the central city of a metropolitan statistical area/primary metropolitan statistical area (MSA/PMSA)

Incorporated place, which is not a central place of an urbanized area (UA), but

part of which is the central city of a metropolitan statistical area/primary metropolitan statistical area (MSA/PMSA)

Census designated place (CDP) that is a central place of an urbanized area (UA), but not a central city of a metropolitan statistical area/primary metropolitan statistical area (MSA/PMSA)

Census designated place (CDP) that is a central city of a metropolitan statistical area/primary metropolitan statistical area (MSA/PMSA), but not a central place of an urbanized area (UA)

Census designated place (CDP) that is a central city of a metropolitan statistical area/primary metropolitan statistical area (MSA/PMSA) and a central place of an urbanized area (UA) Census designated place (CDP) that is in a 1980 or special census urbanized area (UA) and is not a central city or a central place; these CDP's must have a 1990 population of at least 300 in Hawaii and the Virgin Islands of the United States, 1,000 in Puerto Rico, and 2,500 elsewhere Census designated place (CDP) not classified elsewhere; these CDP's must meet the following minimum population requirements: 300 in Hawaii and the Virgin Islands of the United States 25 in Alaska 1,000 in all other states and Puerto Rico

Zona urbana that is a central place of an urbanized area (UA) in Puerto Rico, but not a central city of a metropolitan statistical area/primary metropolitan statistical area (MSA/PMSA)

zona urbana that is a central city of a metropolitan statistical area/primary metropolitan statistical area (MSA/PMSA) in Puerto Rico, but not a central place of an urbanized area (UA)

zona urbana that is a central city of a metropolitan statistical area/primary metropolitan statistical area (MSA/PMSA) and a central place of an urbanized area (UA)

zona urbana in Puerto Rico that is not a central city or a central place 
I Census designated place (CDP) entirely within an American Indian reservation and entirely outside of a 1980 or special

census urbanized area (UA); these CDP's must have a 1990 population of at least

25 in Alaska and 250 elsewhere

\begin{tabular}{|c|c|c|c|}
\hline & e Population Siz & de & PLACESC \\
\hline 00 & Not in universe & 12 & $2,500-4,999$ \\
\hline 01 & 0 & 13 & $5,000-9,999$ \\
\hline 02 & $1-24$ & 14 & $10,000-19,999$ \\
\hline 3 & $25-99$ & 15 & $20,000-24,999$ \\
\hline 04 & $100-199$ & 16 & $25,000-49,999$ \\
\hline 05 & $200-249$ & 17 & $50,000-99,999$ \\
\hline 06 & $250-299$ & 18 & $100,000-249,999$ \\
\hline 07 & $300-499$ & 19 & $250,000-499,999$ \\
\hline 08 & $500-999$ & 20 & $500,000-999,999$ \\
\hline 09 & $1,000-1,499$ & 21 & $1,000,000-2,499,999$ \\
\hline 10 & $1,500-1,999$ & 22 & $2,500,000-4,999,999$ \\
\hline 11 & $2,000-2,499$ & 23 & $5,000,000$ or more \\
\hline
\end{tabular}

Primary Metropolitan Statistical Area (9)

Region (8)

PMSA

2 Midwest

1 Northeast

State (Census) (8)

06 Puerto Rico

07 Virgin Islands of the United States

11 Maine

12 New Hampshire

13 Vermont

14 Massachusetts

15 Rhode Island

16 Connecticut

21 New York

22 New Jersey

23 Pennsylvania

31 Ohio

32 Indiana

33 Illinois

34 Michigan

35 Wisconsin

41 Minnesota

42 Iowa

43 Missouri

44 North Dakota

45 South Dakota

46 Nebraska

47 Kansas

51 Delaware

52 Maryland

53 District of Columbia $\begin{array}{rrrr} & \text { REG } & & 1 \\ 3 \text { South } & 4 & \text { West }\end{array}$

STATECE

2

131

A/N

Virginia

West Virginia

North Carolina

South Carolina

Georgia

Florida

Kentucky

Tennessee

Alabama

Mississippi

Arkansas

Iouisiana

Oklahoma

Texas

Montana

Idaho

Wyoming

Colorado

New Mexico

Arizona

Utah

Nevada

Washington

Oregon

California

Alaska

Hawaii
126

130

A.N

$\mathrm{A} / \mathrm{N}$

$130 \mathrm{~A} / \mathrm{N}$

state (FIPS) (8)

01 Alabama

17 Illinois

\section{STATEFP}

31 Nebraska
2

133

$\mathrm{A} / \mathrm{N}$ 


$\begin{array}{llllll}02 & \text { Alaska } & 18 & \text { Indiana } & 32 & \text { Nevada } \\ 04 & \text { Arizona } & 19 & \text { Iowa } & 33 & \text { New Hampshire } \\ 05 & \text { Arkansas } & 20 & \text { Kansas } & 34 & \text { New Jersey } \\ 06 & \text { California } & 21 & \text { Kentucky } & 35 & \text { New Mexico } \\ 08 & \text { Colorado } & 22 & \text { Louisiana } & 36 & \text { New York } \\ 09 & \text { Connecticut } & 23 & \text { Maine } & 37 & \text { North Carolina } \\ 10 & \text { Delaware } & 24 & \text { Maryland } & 38 & \text { North Dakota } \\ 11 & \text { District of } & 25 & \text { Massachusetts } & 39 & \text { Ohio } \\ & \text { Columbia } & 26 & \text { Michigan } & 40 & \text { Oklahoma } \\ 12 & \text { Elorida } & 27 & \text { Minnesota } & 41 & \text { Oregon } \\ 13 & \text { Georgia } & 28 & \text { Mississippi } & 42 & \text { Pennsylvania } \\ 15 & \text { Hawaii } & 29 & \text { Missouri } & 44 & \text { Rhode Island } \\ 16 & \text { Idaho } & 30 & \text { Montana } & 45 & \text { South Carolina }\end{array}$

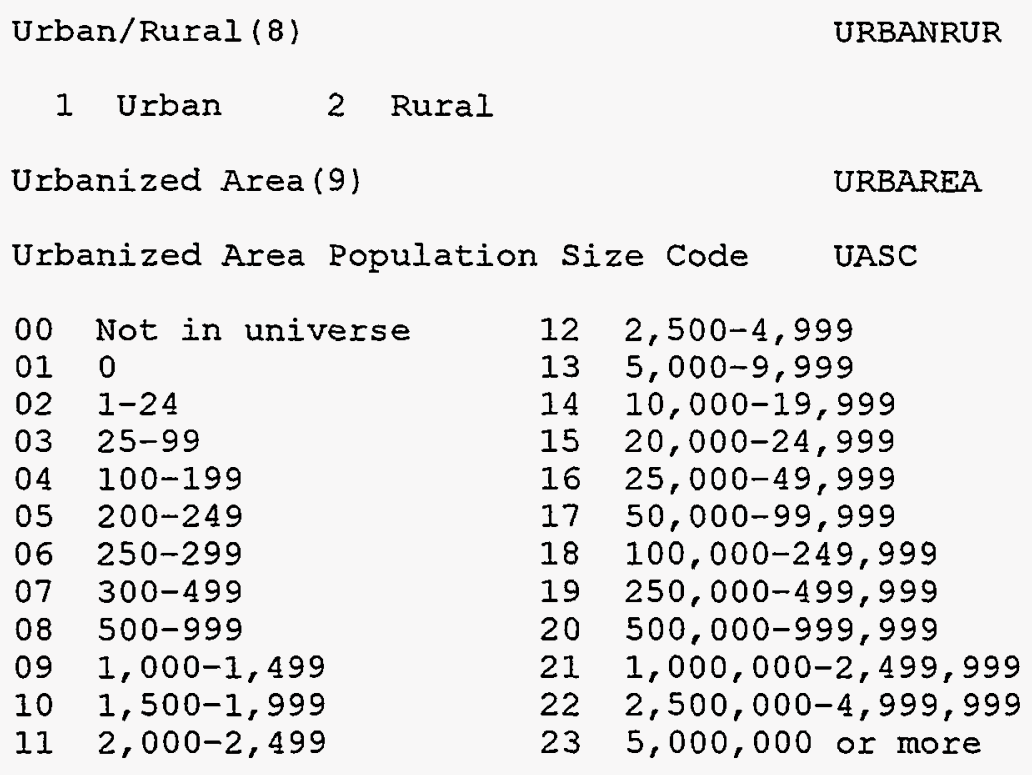

Special Area Code [1]

Special Area Code [2]

Special Area Code [3]

Special Area Code [4]

Special Area Code [5]

Special Area Code [6]

Special Area Code [7]

Special Area Code [8]

Special Area Code [9]

Special Area Code [10]

Area Characteristics

Area (land) (12)

Area (water) (13)
SAC1

SAC2

SAC3

SAC4

SAC5

SAC 6

SAC7

SAC 8

SAC 9

SAC10

AREAI_AND

10

172

$A / N$

AREAWAT

10

182

$\mathrm{A} / \mathrm{N}$ 
Area Name/PSAD Term/ Part

Indicator (14)

Functional status Code

ANPSADPI

66

192

$A / N$

EUNCSTAT

1

258

$\mathrm{A} / \mathrm{N}$

A Active governmental unit, except American Indian reservations and Alaska Native village statistical areas. An active governmental unit has the legal capacity to have officers, to raise revenue, and to conduct governmental activities under state laws, and currently is doing so.

B Functioning governmental unit providing no substantive governmental services. These areas may be coextensive with and administered by officials of another governmental unit (such as towns in Connecticut that are coextensive with cities), or the number of officials and/or the functions they perform are so minimal and/or vestigial that the Census Bureau does not recognize them as governmental units for census purposes (such as townships in Iowa).

F False entity. In order to maintain complete coverage of every state at the county level and of every county at the county subdivision level, we create false entities at these levels for any place that is independent of a county or independent of a minor civil division (MCD). This code also is used for place records that represent the remainder of a consolidated city or the remainder of a county subdivision.

I Inactive governmental unit. An inactive governmental unit has the legal capacity to be active, but currently has no legal officers, raises no revenues, and conducts no activities.

$\mathrm{N}$ Nonfunctioning governmental unit. A nonfunctioning governmental unit has legally established boundaries, but has no legal capacity to conduct governmental activity.

$R$ An American Indian reservation, an American Indian tribe whose name is associated with trust lands, or an Alaska Native village statistical area.

S Statistical entity, except Alaska Native village statistical areas and tribal jurisdiction statistical areas. A statistical entity has no governmental status and is defined by or in cooperation with the Census Bureau or other Eederal agency. This category includes census regions, census divisions, census county divisions (CCDs), census designated places (CDPs), metropolitan areas (MSA/CMSA/PMSAs), urbanized areas (UAs), unorganized territories (UTs), tribal designated statistical areas (TDSAs), census areas and census subareas in Alaska, and comunidades and zonas urbanas in Puerto Rico.

T Tribal jurisdiction statistical area. A tribal jurisdiction statistical area (TJSA) is not a legally defined governmental unit, but is recognized as a statistical area for the 1990 census. These areas exist only in the state of oklahoma and were not recognized separately in 1980.

Geographic Change User Note Indicator GCUNI $\quad 1 \quad 259 \quad$ A/N

0 No geographic change note for the area within the summary level

1 See User Notes for a geographic change note

Housing Unit Count (1008)(15)

HU100

9

260

$\mathrm{A} / \mathrm{N}$

Internal point (latitude) (16)

Internal Point (longitude) (17)

Part Flag

$\begin{array}{llll}\text { INTPTLAT } & 9 & 269 & \mathrm{~A} / \mathrm{N} \\ \text { INTPTLNG } & 10 & 278 & \mathrm{~A} / \mathrm{N} \\ \text { PARTFLAG } & 1 & 288 & \mathrm{~A} / \mathrm{N}\end{array}$


0 Not a part 1 Part

Political/statistical Area Description code

PSADC

2

289

$\mathrm{A} / \mathrm{N}$

01 State or state equivalent - no status is appended to the name of the entity in census publications and related data products.

Borough - county equivalent in Alaska; "Borough" is appended to the name of the entity in census publications and related data products.

05 Census area - county equivalent in Alaska; 'Census Area' is appended to the name of the entity in census publications and related data products.

County - 'County' is appended to the name of the entity in census publications and related data products.

08 Independent city - county equivalent in Maryland, Missouri, and Virginia; "city"' is appended to the name of the entity in census publications and related data products.

09 Independent city - county equivalent in Nevada; no status is appended to the name of the entity in census publications and related data products.

10 Island - county equivalent in Virgin Islands of the United states; 'Island' is appended to the name of the entity in census publications and related data products.

13 Municipio - county equivalent in Puerto Rico; "Municipio" is appended to the name of the entity in census publications and related data products.

14 (none) - county equivalent; used at county level for the District of Columbia and Yellowstone National Park (Montana). No status is appended to the name of the entity in census publications and related data products.

15 Parish - county equivalent in Louisiana; 'Parish' is appended to the name of the entity in census publications and related data products.

19 American Indian reservation - minor civil division (MCD) in Maine and New York; "Reservation" is appended to the name of the entity in census publications and related data products.

20 Barrio - minor civil division (MCD) in Puerto Rico; "barrio" is appended to the name of the entity in census publications and related data products.

21 Borough - minor civil division (MCD) in New York; MCD equivalent in New Jersey and Pennsylvania; 'borough' ' is

appended to the name of the entity in census publications and related data products.

Census county division (CCD) - minor civil division (MCD) equivalent in 21 states; "division" is appended to the name of the entity in census publications and related data products.

Census subarea - minor civil division (MCD) equivalent in Alaska; "census subarea" is appended to the name of the entity in census publications and related data products.

Census subdistrict - minor civil division (MCD) equivalent in the Virgin Islands of the United States; ' 'subdistrict' ' is appended to the name of the entity in census publications and related data products.

City - minor civil division (MCD) equivalent in 20 states; "city'" is appended to the name of the entity in census publications and related data products.

District (magisterial, road) - minor civil division (MCD) in Pennsylvania, Virginia, and West Virginia; "district' ' is appended tothe name of the entity in census publications and 
related data products.trict (assessment, election, magisterial, supervisors', or parish governing authority) - minor civil division (MCD) in Louisiana, Maryland, Mississippi, and West Virginia; no status is appended to the name of the entity in census publications and related data products.

Election precinct - minor civil division (MCD) in Illinois and Nebraska; "precinct' is appended to the name of the entity in census publications and related data products. Nebraska; no status is appended to the name of the entity in census publications and related data products.

Gore - minor civil division (MCD) in Maine and Vermont; "gore' is appended to the name of the entity in census publications and related data products.

Grant - minor civil division (MCD) in New Hampshire and Vermont; "grant"' is appended to the name of the entity in census publications and related data products.

Independent city - minor civil division (MCD) equivalent in Maryland, Missouri, and Virginia; "city"' is appended to the name of the entity in census publications and related data products.

Independent city - minor civil division (MCD) equivalent in Nevada; no status is appended to the name of the entity in census publications and related data products.

Location - minor civil division (MCD) in New Hampshire; "location'" is appended to the name of the entity in census publications and related data products.

(none) - minor civil division (MCD) equivalent for District of Columbia and Arlington County, Virginia; no status is appended to the name of the entity in census publications and related data products.

39 Plantation - minor civil division (MCD) in Maine; "plantation" ' is appended to the name of the entity in census publications and related data products.

Plantation - minor civil division (MCD) in Maine; no status is appended to the name of the entity in census publications and related data products.

Barrio-pueblo - minor civil division (MCD) in Puerto Rico; 'barriopueblo' is appended to the name of the entity in census publications and related data products.

Purchase - minor civil division (MCD) in New Hampshire; "purchase'" is appended to the name of the entity in census publications and related data products.

43 Town - minor civil division (MCD) in 8 states; MCD equivalent in New Jersey, Pennsylvania, and South Dakota; "town"' is appended to the name of the entity in census publications and related data products.

44 Township - minor civil division (MCD) in 16 states; 'township'" is appended to the name of the entity in census publications and related data products.

45 Township - minor civil division (MCD) in Kansas, Nebraska, and North Carolina; no status is appended to the name of the entity in census publications and related data products.

Unorganized territory - minor civil division (MCD) in 9 states; "unorg." is appended to the name of the entity in census publications and related data products.

Village - minor civil division (MCD) equivalent in New Jersey, Ohio, South Dakota, and Wisconsin; 'village'" is appended to the name of the entity in census publications and related data products.

51 Subbarrio - sub-minor civil division (sub-MCD) in Puerto Rico; "subbarrio" is appended to the name of the entity in census publications and related data products. 
Comunidad - place (census designated place) in Puerto Rico;

"comunidad" is appended to the name of the entity in

census publications and related data products.

Borough - place in Connecticut, New Jersey, and Pennsylvania;

'borough'' is appended to the name of the entity in census publications and related data products.

Census designated place - place; 'CDP'' is appended to the name of the entity in census publications and related data products.

City - place; "city" is appended to the name of the entity in census publications and related data products.

City - place; used for some cities that have a unique description, no description, or a description included with their name, as follows:

Anaconda-Deer Lodge County (Montana): incorporated municipality;

Butte-Silver Bow (remainder) (Montana): (none);

Carson City, Nevada: (none);

Columbus city (remainder) (Georgia): (none);

Indianapolis city (remainder) (Indiana): (none);

Jacksonville city (remainder) (Florida): (none);

Lexington-Fayette (Kentucky): Urban County Government;

Lynchburg-Moore County (Tennessee): (none);

Milford city (remainder) (Connecticut): (none);

Nashville-Davidson (remainder) (Tennessee): 'Metropolitan Government of

Nashville and Davidson County' ';

No status is appended to the name of the entity in census

publications and related data products.

Town - place; place in 30 states and the Virgin Islands of the United States; "town" is appended to the name of the entity in census publications and related data products.

61 Village - place; place in 19 states; "village'" is appended to the name of the entity in census publications and related data products.

Zona urbana - place (census designated place) in Puerto Rico; 'zona urbana' is appended to the name of the entity in census publications and related data products.

Consolidated city in Connecticut, Florida, Georgia, and Indiana "city" is appended to the name of the entity in census publications and related data products.

Consolidated city - used for some consolidated cities that have unique descriptions or no descriptions, as follows:

Butte-Silver Bow (Montana): (none);

Nashville-Davidson (Tennessee): "Metropolitan Government of Nashville and Davidson County' ';

No status is appended to the name of the entity in census

publications and related data products.

68 Census region - no status is appended to the name of the entity in census publications and related data products.

69 Census division - no status is appended to the name of the entity in census publications and related data products.

71 Consolidated metropolitan statistical area (CMSA) - 'CMSA' is appended to the name of the entity in census publications and related data products.

72 Metropolitan statistical area (MSA) - 'MSA'' is appended to the name of the entity in census publications and related data products.

Primary metropolitan statistical area (PMSA) - 'PMSA'' is appended to the name of the entity in census publications and related data products.

Urbanized area (UA) - no status is appended to the name of the entity in census publications and related data products. 
77 Alaska Native Regional Corporation - no status is appended to the name of the entity in census publications and related data products.

79 Alaska Native village statistical area - no status is appended to the name of the entity in census publications and related data products.

80 Tribal designated statistical area - American Indian reservation equivalent for non-land-based tribes outside of Oklahoma; 'TDSA'" is appended to the name of the entity in census publications and related data products.

81 Colony - American Indian reservation; "Colony"' is appended to the name of the entity in census publications and related data products.

82 Community - American Indian reservation; 'Comunity' is appended to the name of the entity in census publications and related data products.

83 Joint area - American Indian reservation equivalent; "joint area'" is appended to the name of the entity in census publications and related data products.

84 Pueblo - American Indian reservation; "Pueblo'" is appended to the name of the entity in census publications and related data products.

85 Rancheria - American Indian reservation; "Rancheria' is appended to the name of the entity in census publications and related data products.

86 Reservation - American Indian reservation; "Reservation' is appended to the name of the entity in census publications and related data products.

87 Reserve - American Indian reservation; 'Reserve' ' is appended to the name of the entity in census publications and related data products.

88 Tribal jurisdiction statistical area - American Indian reservation equivalent representing historic tribal areas in Oklahoma; 'TJSA'" is appended to the name of the entity in census publications and related data products.

89 Trust lands - American Indian reservation equivalent; no status is appended to the name of the entity in census publications and related data products.

90 (none) - American Indian reservation; no status is appended to the name of the entity in census publications and related data products.

Population Count (1008)(18)

POP100

9

291

$\mathrm{A} / \mathrm{N}$

Special Flag

SPFLAG

1

300

$\mathrm{A} / \mathrm{N}$

\section{FOOTNOTE SECTION}

1 A unique identifier for each file series. Dress rehearsal files have a ' $D$ '' in the character following the last character in the file identification code for the equivalent 1990 decennial census file; for example, PL94171D is the identifier for the dress rehearsal file for the P.I. 94-171 data. File identification code for the P.I. 94-171 file is PL94171. File identification codes for summary tape files are STFnn where $\mathrm{nn}=$ file number; for example, STFIA is the identification for Summary Tape File 1A. File identification codes for subject summary tape files are SSTFnn where $\mathrm{nn}=$ the number of the subject report. File identification codes for special project files are identified by SPmm where mmm = a three digit special project number. See How to Use This File for further

information. 
2. Identifies the geographic level for which the data matrices on the summary tape file have been summarized. The summary level sequence chart describes the hierarchical arrangement of the specified geographic areas with other geographic areas, if any. The summary level must be used in combination with the geographic area codes to identify a specific geographic area (for example, summary level 050 and a specific county code must be used together to locate the data for a particular county). See How to Use This File for further information.

3. Indicates an iteration (repetition), for the specified summary level, of the data matrices on the summary tape file for the geographic components listed in the Geographic Component field. See How To Use This File for further information.

4. Indicates an iteration (repetition), for the specified summary level, of the data matrices on the summary tape file for a population or housing characteristic. Only matrices containing a " $B$ " in the prefix have characteristic iterations. See How to Use This File for further information.

5. The logical record is the complete record [identification and set of tables (matrices)] for a geographic entity defined by the summary level, but exclusive of the characteristic iteration. A logical record may have one or more parts (or segments). Each logical record has an assigned sequential integer number within the file. See How to Use This File for further information.

6. Within the logical record, each part is identified uniquely in terms of its sequence. See How to Use This File for further information.

7. This field identifies how many parts (or segments) comprise the entire logical record. See How to Use This File for further information.

8. See appendix A, Area Classifications, for definition of this field if it is applicable to this file.

9. See the publication, Geographic Identification Code Scheme, for codes in this field and related teminology if it is applicable to this file. See appendix $A$, Area Classifications, for definition of this field if it is applicable to this file.

10. See the publication, Geographic Identification Code Scheme, for FIPS 55 codes in this field and related terminology if it is applicable to this file. See appendix A, Area Classifications, for definition of this field if it is applicable to this file.

11. Codes in unspecified arrangement for Census Bureau use.

12. Iand area measurement in thousandths (.001) of a square kilometer. The accuracy of the area measurement is limited by the inaccuracy inherent in 1) the mapping of the various boundary features in the TIGER File and 2) rounding affecting the last digit in all operations that compute and/or sum the area measurements. Iand area includes intermittent water and glaciers, which appear on census maps and in the TIGER File as hydrographic features. An area of .0005 square kilometer is rounded to .001 ; an area smaller than .0005 is rounded to .000 . The decimal point is implied on the file. Square miles can be derived by dividing square kilometers by 2.59 . See appendix A, Area Classifications, for definition of this field if it is applicable to this file.

13. Water area measurement in thousandths (.001) of a square kilometer. Water area is excluded from census blocks by definition, so the water area 
for a block always will be zero. The accuracy of the area measurement is limited by the inaccuracy inherent in 1) the mapping of the various boundary features in the TIGER File and 2) rounding affecting the last digit in all operations that compute and/or sum the area measurements. Water area excludes intermittent water and glaciers, which are treated as land even though they appear on census maps and in the TIGER File as hydrographic features. An area of .0005 square kilometer is rounded to .001 ; an area smaller than .0005 is rounded to .000 . The decimal point is implied on the file. Square miles can be derived by dividing square kilometers by 2.59. See appendix A, Area Classifications, for definition of this field if it is applicable to this file.

14. Name of the lowest-level entity represented by the summary level. In addition to the name of the entity, the name field contains the political/statistical area description (PSAD) when appropriate, and sometimes contains the state abbreviation.

For legal entities, the name is the one reported to the Bureau of the Census in the Boundary and Annexation Survey and by other appropriate sources; for statistical entities, the name is determined by the office of Management and Budget for metropolitan areas and, for other areas, the name is determined by the Bureau of the Census, usually in cooperation with local officials.

When the summary level represents only part of the area specified in the name, the name usually will have ' (pt.)' appended to the name/code terminology to designate that this entry for the entity represents only a part of the total entity.

15. The total number of housing units enumerated in the specified summary level as determined in the 100-percent processing.

16. Latitude in degrees, to six decimal places, of a point within the geographic area represented by the summary level. The decimal point is implied on the file. The character immediately preceding the first digit of the latitude of an internal point identifies the direction (hemisphere): a plus sign (t) indicates the Northern Hemisphere; a minus sign $(-)$

indicates the Southern Hemisphere. See appendix A, Area Classifications, for definition of this field if it is applicable to this file.

17. Longitude in degrees, to six decimal places, of a point with the geographic area represented by the summary level. The decimal point is implied on the file. The character immediately preceding the first digit of the longitude of an internal point identifies the direction

(hemisphere): a plus sign ( + ) indicates the Eastern Hemisphere; a minus sign (-) indicates the Western Hemisphere. A point on the 180th meridian is assigned to the Western Hemisphere $(-18000000)$. See appendix A, Area Classifications, for definition of this field if it is applicable to this file.

18. The total number of persons enumerated in the specified summary level as determined in the 100-percent processing. 


\section{APPENDIX F — POPULATION TABLES}

This appendix contains the population tables described in section 5.3.2 External Verification and Validation. There are three tables for each licensee. The first table is the SECPOP90 estimated population. The second table is the licensee's reported population. And the third table is a comparison of the SECPOP90 estimated population as a percent of the licensee reported population. 


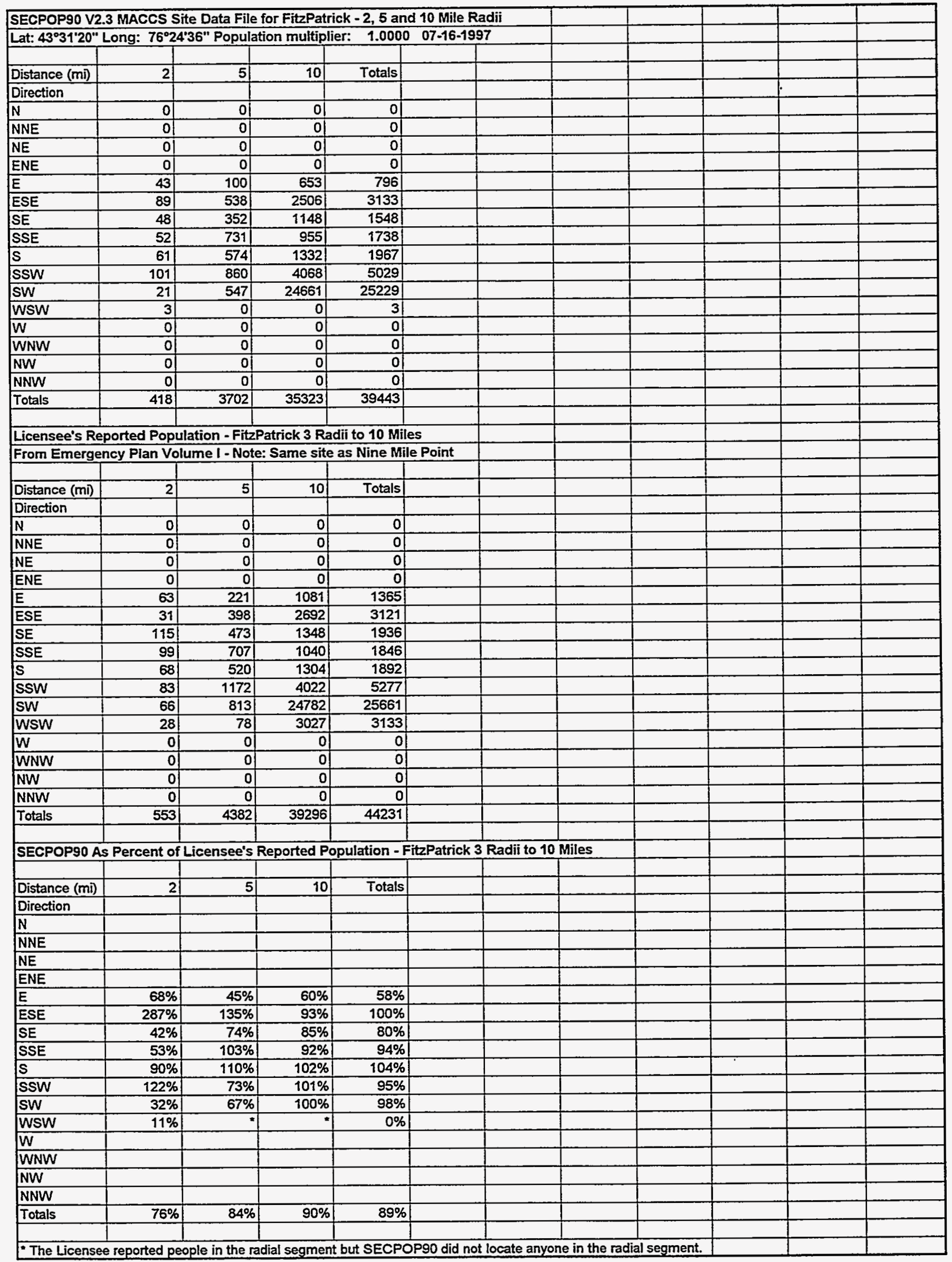




\begin{tabular}{|c|c|c|c|c|c|c|c|c|c|c|c|c|c|c|c|}
\hline \multicolumn{16}{|c|}{ SECPOP90 V2.3 MACCS Site Data File for Millstone 1 - 14 Radii to 50 Miles } \\
\hline \multicolumn{16}{|c|}{ Lat: $41^{\circ} 18^{\prime} 35^{\prime \prime}$ Long: $72^{\circ} 10^{\circ} 10^{\prime \prime}$ Population multiplier: $1.0000 \quad 07-16-1997$} \\
\hline & & & & & & & & & & & & & & & \\
\hline Distance (mi) & 1 & 2 & 3 & 4 & 5 & 6) & 71 & 8 & 9 & 10 & 20 & 30 & 40 & 50 & Totals \\
\hline \multicolumn{16}{|l|}{ Direction } \\
\hline $\mathbf{N}$ & 164 & 707 & 822 & 538 & 145 & 168 & 2901 & 417 & 337 & 1616 & 12718 & 30268 & 31037 & 18263 & 97490 \\
\hline NNE & 64 & 313 & 1436 & 1781 & 2348 & 1958 & 1506 & 938 & 1952 & 3374 & 45291 & 24461 & 31663 & 30642 & 147727 \\
\hline NE & 95 & 582 & 726 & 3211 & 11195 & 6546 & 6024 & 9720 & 1937 & 2416 & 9897 & 6095 & 18734 & 204209 & 281387 \\
\hline ENE & 154 & 357 & 373 & 498र्वा & 1621 & 7200 & 5057 & 2281 & 1184 & 2161 & 33806 & 15981 & 41417 & 96617 & 213194 \\
\hline $\bar{E}$ & 0 & 604 & 403 & 1790 & 198 & 541 & 0 & $11 \mid$ & 1255 & 1199 & $7933 \mid$ & 6689 & 6584 & 0 & 27207 \\
\hline ESE & 0 & 42 & 200 & 0 & 0 & 0 & 0 & 17! & 287 & 24 & 11 & 0 & 836 & 0 & 1407 \\
\hline$\overline{S E}$ & 0 & 0 & 0 & 0 & 0 & 0 & 0 & 0) & 0 & 01 & 45 & 1409 & 0 & 0 & 1454 \\
\hline SSE & 0 & 0 & 0 & 0 & 0 & of & 이 & 0 & 0 & of & of & 1707 & 0 & 0 & 1707 \\
\hline S & 0 & 0 & 0 & 0 & 01 & 0 & 0 & 0 & 0 & 01 & 2488 & 9558 & \begin{tabular}{l|l}
0 \\
\end{tabular} & 0 & 12146 \\
\hline SSW & 0 & 0 & 0 & of & DI & 0 & 0 & 0 & 0 & 0 & 2797 & 11999 & 19528 & 179 & 34503 \\
\hline SW & 0 & 0 & 8 & 0 & 01 & 0 & 0 & of & of & 0 & 4499 & 12703 & $253 \pi$ & 67416 & 110003 \\
\hline WSW & of & 52 & 300 & 66 & 82 & 383 & 494 & 92 & 0 & 53 & 1832 & o & of & 26797 & 30151 \\
\hline W & 0 & 224 & 1167 & 1039 & 395 & 754 & 508 & 812 & 713 & 866 & 28340 & 36820 & 204426 & 260338 & 536402 \\
\hline WNW & 0 & 541 & 912 & 1375 & 243 & 281 & 6811 & 1084 & 571 & 328 & 12126 & 24691 & 138491 & 258079 & 439503 \\
\hline NW & 0 & 657 & 1699 & 736 & 1511 & 495 & 28 & 81 & 71 & 212 & 5862 & 23702 & 217352 & 360336 & 612742 \\
\hline NNW & 0 & 404 & 405 & 1079 & 772 & 665 & 306 & 1058 & 233 & 343 & 9180 & 19906 & 90957 & 108664 & 233972 \\
\hline Totals & 477 & 4483 & 8451 & 16600 & 18510 & 18991 & 14894 & 16511 & 8640 & 12592 & 176815 & 225089 & 826402 & 1431540 & 2780995 \\
\hline & & & & & & & & & & & & & & & \\
\hline \multicolumn{16}{|c|}{ Llcensee's Reported Population - Millstone - 14 Radii to 50 Miles } \\
\hline \multicolumn{16}{|c|}{\begin{tabular}{|l|l|l|} 
Unit 3's UFSAR Rev. 6, Tables 2.1-4 \& 2.1-10 & \\
\end{tabular}} \\
\hline & & & & & & & & & & & & & & & \\
\hline Distance (m) & 1 & 2 & 3 & $4 \mid$ & 5 & 61 & 7 & 8 & 9 & 10 & 20 & 30 & 40 & 50 & Totals \\
\hline \multicolumn{16}{|l|}{ Direction } \\
\hline N & 16 & 722 & 866 & 784 & 116 & 213 & 542 & 209 & 536 & 1717 & 22283 & 26357 & 32610 & \begin{tabular}{|c|}
18658 \\
\end{tabular} & 105629 \\
\hline NNE & 13 & 359 & 1146 & 1978 & 1861 & 1622 & 1656 & 2242 & 2192 & 3142 & 34824 & 23730 & 27465 & 35598 & 137838 \\
\hline $\mathrm{NE}$ & 165 & 455 & 839 & 3888 & 10584 & 7752 & 8164 & 8129 & 911 & 1961 & 9444 & 91334 & 29987 & 199334 & 292947 \\
\hline ENE & 22 & 455 & 292 & 4953 & 971 & 7186 & 3748 & 3047 & 1008 & 2662 & 23914 & 16498 & 43001 & 628307 & 736074 \\
\hline$E$ & 0 & 636 & 413 & 1804 & 193 & 552 & 0 & 63 & 1434 & 904 & 10712 & 7992 & 10920 & 0 & 35623 \\
\hline ESE & 0 & 143 & 36 & 0 & of & 0 & 0 & 01 & 115 & 214 & 0 & 0 & 836 & 0 & 1344 \\
\hline SE & 0 & 0 & of & of & 0 & 0 & of & 이 & 0 & 0 & 01 & 807 & 0 & 0 & 807 \\
\hline SSE & 0 & 0 & of & D & of & 0 & of & 0 & of & of & of & 2420 & of & 01 & 2420 \\
\hline$s$ & 0 & 0 & 0 & 0 & 01 & 0 & 0 & 0 & of & o & 의 & 15155 & 0 & 0 & 15155 \\
\hline SSW & 0 & 0 & 0 & 0 & 0 & o| & 0 & of & 0 & of & 2443 & 12569 & 14807 & 4498 & 34317 \\
\hline SW & 0 & 0 & 14 & of & \begin{tabular}{l|l}
0 \\
\end{tabular} & 0 & 0 & 0 & 이 & 0 & 0 & 22980 & 8252 & 143933 & 175179 \\
\hline WSW & 0 & 0 & 489 & 91 & 86 & 312 & 472 & 158 & 0 & 74 & 2471 & 0 & 0 & 20389 & 24542 \\
\hline$W$ & 0 & 178 & 1061 & 1014 & 440 & 763 & 475 & 562 & 881 & 408 & 27956 & 34384 & 184723 & 267465 & 520310 \\
\hline WNW & 0 & 476 & 1165 & 1964 & 346 & 239 & 211 & 1654 & 509 & 417 & 12474 & 27895 & 148259 & 259824 & 455433 \\
\hline NW & 0 & 634 & 87 & 1192 & 1140 & 644 & 599 & 101 & 209 & 811 & 6215 & 31331 & 191757 & 365578 & 600364 \\
\hline NNW & 148 & 314 & 892 & 522 & 646 & 918 & 221 & 429 & 456 & 314 & 8809 & 17850 & 115424 & 78820 & 225763 \\
\hline Totals & 364 & 4372 & 8086 & 18200 & 16383 & 20201 & 16098 & 16594 & 8251 & 11894 & 161545 & 251302 & 808051 & 2022404 & 3363745 \\
\hline & & & & & & & & & & & & & & & \\
\hline SECPOP90 & Percent & of Licens & e's Rep & rted Pop & Ilation - & illstone & 14 Radi & to $50 \mathrm{Mil}$ & iles & & & & & & \\
\hline & & & & & & & & & & & & & & & \\
\hline Distance (mi) & 1 & 2 & 3 & 4 & 5 & 6 & 7 & 8 & 9 & 10 & 201 & 30 & 40 & 50 & Totals \\
\hline Direction & & & & & & & & & & & & & & & \\
\hline $\mathbf{N}$ & $1025 \%$ & \begin{tabular}{l|l|}
$98 \%$ \\
\end{tabular} & $95 \%$ & $69 \%$ & $125 \%$ & $79 \%$ & $54 \%$ & $200 \%$ & $63 \%$ & $94 \%$ & $57 \%$ & $115 \%$ & $95 \%$ & $98 \%$ & $92 \%$ \\
\hline NNE & $492 \%$ & $87 \%$ & $125 \%$ & $90 \%$ & $126 \%$ & $121 \%$ & $90 \%$ & $42 \%$ & $89 \%$ & $107 \%$ & $130 \%$ & $103 \%$ & $115 \%$ & $86 \%$ & $107 \%$ \\
\hline \begin{tabular}{|l} 
NE \\
\end{tabular} & $58 \%$ & $128 \%$ & $87 \%$ & $83 \%$ & $106 \%$ & $84 \%$ & $74 \%$ & $120 \%$ & $213 \%$ & $123 \%$ & $105 \%$ & $54 \%$ & $62 \%$ & $102 \%$ & $96 \%$ \\
\hline ENE & $700 \%$ & $78 \%$ & $128 \%$ & $100 \%$ & $167 \%$ & $100 \%$ & $135 \%$ & $75 \%$ & $117 \%$ & $89 \%$ & $141 \%$ & $97 \%$ & $96 \%$ & $15 \%$ & $29 \%$ \\
\hline$E$ & & $95 \%$ & $98 \%$ & $99 \%$ & $103 \%$ & $98 \%$ & & $17 \%$ & $88 \%$ & $133 \%$ & $74 \%$ & $84 \%$ & $60 \%$ & & $76 \%$ \\
\hline ESE & & $20 \%$ & $556 \%$ & & & & & न & $250 \%$ & $11 \%$ & $=1$ & & $100 \%$ & & $105 \%$ \\
\hline SE & & & & & & & & & & & $\infty$ & $175 \%$ & & & $180 \%$ \\
\hline SSE & & & & & & & & & & & & $71 \%$ & & & $71 \%$ \\
\hline $\mathbf{S}$ & & & & & & & & & & & -1 & $64 \%$ & & & $80 \%$ \\
\hline SSW & & & & & & & & & & & $114 \%$ & $95 \%$ & $132 \%$ & $4 \%$ & $101 \%$ \\
\hline SW & & & $57 \%$ & & & & & & & & -1 & $55 \%$ & $308 \%$ & $47 \%$ & $63 \%$ \\
\hline WSW & & -1 & $61 \%$ & $73 \%$ & $95 \%$ & $123 \%$ & $105 \%$ & $58 \%$ & & $72 \%$ & $74 \%$ & & & $131 \%$ & $123 \%$ \\
\hline$W$ & & $126 \%$ & $110 \%$ & $102 \%$ & $90 \%$ & $99 \%$ & $107 \%$ & $144 \%$ & $81 \%$ & $212 \%$ & $101 \%$ & $107 \%$ & $111 \%$ & $97 \%$ & $103 \%$ \\
\hline WNW & & $114 \%$ & $78 \%$ & $70 \%$ & $70 \%$ & $118 \%$ & $323 \%$ & $66 \%$ & $132 \%$ & $79 \%$ & $97 \%$ & $89 \%$ & $93 \%$ & $99 \%$ & $97 \%$ \\
\hline NW & & $104 \%$ & $195 \%$ & $62 \%$ & $133 \%$ & $77 \%$ & $5 \%$ & $80 \%$ & $34 \%$ & $252 \%$ & $94 \%$ & $76 \%$ & $113 \%$ & $99 \%$ & $102 \%$ \\
\hline NNW & & $129 \%$ & $45 \%$ & $207 \%$ & $120 \%$ & $72 \%$ & $138 \%$ & $247 \%$ & $51 \%$ & $109 \%$ & $104 \%$ & $112 \%$ & $79 \%$ & $138 \%$ & $104 \%$ \\
\hline Totals & $131 \%$ & $103 \%$ & $105 \%$ & 91\% & $113 \%$ & $94 \%$ & $93 \%$ & $99 \%$ & $105 \%$ & $105 \%$ & $109 \%$ & $90 \%$ & $102 \%$ & $71 \%$ & $83 \%$ \\
\hline & & & & & & & & & & & & & & & \\
\hline 110 & & Tade & $\pi D$ & P90 & $\$ 1000$ & te in & false & & & & & & & & \\
\hline - The Uleens & otreport & anyone in the & fal segm & nt but SECPC & P9o boate & Eople in th & radial seg & & & & & & & & \\
\hline
\end{tabular}




\begin{tabular}{|c|c|c|c|c|c|c|c|c|c|c|c|}
\hline SECPOP9O V2 & .3 MACCS Sit & te Data File f & for Monticell & 0-10 Radii & to 50 Miles & & & & & & \\
\hline Lat: $45^{\circ} 20^{\prime} 0^{\prime \prime}$ & Long: $93^{\circ} 50^{\prime} 5$ & 54" Populatio & ion multiplie & r: 1.0000 & $07-16-1997$ & & & & & & \\
\hline & & & & & & & & & & & \\
\hline Distance (mi) & $1 \mid$ & 2 & 3 & 4 & 5 & 10 & 20 & 30 & 40 & 50 & Totals \\
\hline Direction & & & & & & & & & & & \\
\hline$N$ & 0 & 이 & 0 & 60 & 100 & 425 & 1367 & 4283 & 2064 & 1164 & 9463 \\
\hline NNE & 01 & 11 & 9 & 42 & 211 & 668 & 881 & 3355 & 5442 & 2009 & 12628 \\
\hline $\mathrm{NE}$ & 0 & of & 15 & 0 & 101 & 879 & 4062 & 7256 & 3213 & 9224 & 24750 \\
\hline ENE & 0 & 0 & 201 & 39 & 226 & 1164 & 4279 & 5382 & 13283 & 10565 & 34958 \\
\hline $\mathrm{E}$ & 이 & 393 & 83 & 18 & 1090 & 3292 & 13358 & 19180 & 16810 & 29826 & 84050 \\
\hline ESE & 0 & 81 & 78 & 165 & 591 & 2114 & 11312 & 122391 & 206414 & 234781 & 577927 \\
\hline SE & 이 & 895 & 1285 & 1688 & 53 & 908 & 9984 & 107073 & 536360 & 528544 & 1186790 \\
\hline SSE & 0 & 158 & 83 & 868 & 538 & 250 & 6708 & 30162 & 95122 & 74342 & 208231 \\
\hline$s$ & 3 & 0 & $11 \mid$ & 이 & 43 & 2588 & 9870 & 9961 & 12857 & 7499 & 42832 \\
\hline SSW & 28 & of & 195 & 28 & 47 & 1052 & 3646 & 6017 & 5579 & 8768 & 25360 \\
\hline SW & 0 & 126 & 0 & 30 & 113 & 850 & 3183 & 5977 & 7473 & 10468 & 28220 \\
\hline WSW & 0 & 0 & 108 & 6 & 303 & 657 & 4710 & 2077 & 8988 & 4231 & 21080 \\
\hline $\bar{W}$ & 0 & 177 & 33 & 40 & 13 & 725 & 2412 & 3963 & 3964 & 5510 & 16837 \\
\hline WNW & 0 & 56 & 11 & 59 & 89 & 560 & 3734 & 8466 & 7767 & 5689 & 26431 \\
\hline NW & 0 & 0 & 이 & 16 & 14 & 342 & 5983 & 81395 & 7072 & 4659 & 99481 \\
\hline NNW & 0 & 0 & 01 & 21 & 674 & 830 & 2294 & 3018 & 3921 & 7076 & 17834 \\
\hline Totals & 31 & 1897 & 1931 & 3080 & 4206 & 17304 & 87783 & 419956 & 936329 & 944355 & 2416872 \\
\hline & & & & & & & & & & & \\
\hline Licensee's $\mathbf{R e}$ & ported Popula & ation - Montic & icello $10 \mathrm{Rac}$ & iii to 50 Mile & & & & & & & \\
\hline Data provided & by project ma & lanager & & & & & & & & & \\
\hline & & & & & & & & & & & \\
\hline Distance $(\mathrm{mi})$ & 1 & 2 & 3 & $4 \mid$ & 5 & 10 & 20 & 30 & 40 & 50 & Totals \\
\hline Direction & & & & & & & & & & & \\
\hline $\mathbf{N}$ & $4 \mid$ & 58 & 96 & 134 & 171 & 1421 & 5071 & 6872 & 5590 & 5036 & 24453 \\
\hline NNE & 8 & 58 & 96 & 134 & 171 & 1421 & 4510 & 3275 & 4315 & 4845 & 18833 \\
\hline $\mathrm{NE}$ & 0 & 54 & 96 & 134 & 171 & 1367 & 5629 & 5277 & 6916 & 5460 & 25104 \\
\hline ENE & 0 & 46 & 95 & 136 & 171 & 1421 & 8975 & 12184 & 8069 & 11932 & 43029 \\
\hline$E$ & 이 & 50 & 96 & 134 & 171 & 1421 & 17947 & 54892 & 73132 & 33812 & 181655 \\
\hline ESE & 0 & 50 & 96 & 134 & 170 & 1441 & 43619 & 114477 & 190714 & 299783 & 650484 \\
\hline SE & 0 & 54 & 96 & 137 & 171 & 1381 & 69505 & 171280 & 239887 & 233190 & 715701 \\
\hline SSE & 6 & 54 & 96 & 137 & 171 & 1140 & 41813 & 157183 & 91658 & 36340 & 328598 \\
\hline $\mathrm{s}$ & $6 \mid$ & 54 & 96 & 137 & 171 & 1339 & 5737 & 19948 & 18844 & 15140 & 61472 \\
\hline SSW & 13 & 54 & 96 & 137 & 171 & 1436 & 5737 & 9211 & 9382 & 9304 & 35541 \\
\hline SW & 13 & 54 & 96 & 137 & 171 & 1311 & 5778 & 7744 & 6310 & 7761 & 29375 \\
\hline WSW & 13 & 54 & 96 & 137 & 171 & 1360 & 5400 & 4054 & 4531 & 6223 & 22039 \\
\hline$w$ & 6 & 54 & 96 & 137 & 171 & 1360 & 5090 & 7206 & 8345 & 10319 & 32784 \\
\hline \begin{tabular}{|l} 
WNW \\
\end{tabular} & 이 & 26 & 96 & 137 & 171 & 1448 & 5131 & 8386 & 11759 & 15300 & 42454 \\
\hline \begin{tabular}{|l}
$\mathrm{NW}$ \\
\end{tabular} & 0 & 42 & 95 & 133 & 171 & 1421 & 5393 & 8124 & 11461 & 8351 & 35191 \\
\hline NNW & 0 & 54 & 96 & 134 & 171 & 1421 & 5109 & 7422 & 7582 & 4511 & 26500 \\
\hline Totals & 69 & 816 & 1534 & 2169 & 2735 & 22109 & 240444 & 597535 & 698495 & 707307 & 2273213 \\
\hline & & & & & & & & & & & \\
\hline SECPOP9O A & 5 Percent of $\mathrm{Li}$ & icensee's Re & eported Pop & ulation - Mo & onticello $10 \mathrm{~F}$ & Radii to $50 \mathrm{~N}$ & Miles & & & & \\
\hline & & & & & & & & & & & \\
\hline Distance (mi) & 1 & 2 & 3 & 4 & 5 & 10 & 20 & 30 & 40 & 50 & Totals \\
\hline Direction & & & & & & & & & & & \\
\hline$N$ & -1 & . & 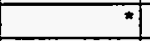 & $45 \%$ & $58 \%$ & $30 \%$ & $27 \%$ & $62 \%$ & $37 \%$ & $23 \%$ & $39 \%$ \\
\hline NNE & *1 & $19 \%$ & $9 \%$ & $31 \%$ & $123 \%$ & $47 \%$ & $20 \%$ & $102 \%$ & $126 \%$ & $41 \%$ & $67 \%$ \\
\hline NE & & 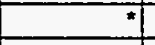 & $16 \%$ & 7 & $59 \%$ & $64 \%$ & $72 \%$ & $138 \%$ & $46 \%$ & $169 \%$ & $99 \%$ \\
\hline ENE & & 1 & $21 \%$ & $29 \%$ & $132 \%$ & $82 \%$ & $48 \%$ & $44 \%$ & $165 \%$ & $89 \%$ & $81 \%$ \\
\hline$E$ & & $786 \%$ & $86 \%$ & $13 \%$ & $637 \%$ & $232 \%$ & $74 \%$ & $35 \%$ & $23 \%$ & $88 \%$ & $46 \%$ \\
\hline ESE & & $162 \%$ & $81 \%$ & $123 \%$ & $348 \%$ & $147 \%$ & $26 \%$ & $107 \%$ & $108 \%$ & $78 \%$ & $89 \%$ \\
\hline$\overline{S E}$ & & $1657 \%$ & $1339 \%$ & $1232 \%$ & $31 \%$ & $66 \%$ & $14 \%$ & $63 \%$ & $224 \%$ & $227 \%$ & $166 \%$ \\
\hline SSE & \pm & $293 \%$ & $86 \%$ & $634 \%$ & $315 \%$ & $22 \%$ & $16 \%$ & $19 \%$ & $104 \%$ & $205 \%$ & $63 \%$ \\
\hline$s$ & $50 \%$ & 1 & $11 \%$ & \pm & $25 \%$ & $193 \%$ & $172 \%$ & $50 \%$ & $68 \%$ & $50 \%$ & $70 \%$ \\
\hline SSW & $215 \%$ & 1 & $203 \%$ & $20 \%$ & $27 \%$ & $73 \%$ & $64 \%$ & $65 \%$ & $59 \%$ & $94 \%$ & $71 \%$ \\
\hline SW & $=1$ & $233 \%$ & - & $22 \%$ & $66 \%$ & $65 \%$ & $55 \%$ & $77 \%$ & $118 \%$ & $135 \%$ & $96 \%$ \\
\hline WSW & -1 & 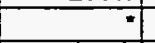 & $113 \%$ & $4 \%$ & $177 \%$ & $48 \%$ & $87 \%$ & $51 \%$ & $198 \%$ & $68 \%$ & $96 \%$ \\
\hline $\bar{W}$ & *1 & $328 \%$ & $34 \%$ & $29 \%$ & $8 \%$ & $53 \%$ & $47 \%$ & $55 \%$ & $48 \%$ & $53 \%$ & $51 \%$ \\
\hline WNW & & $215 \%$ & $11 \%$ & $43 \%$ & $52 \%$ & $39 \%$ & $73 \%$ & $101 \%$ & $66 \%$ & $37 \%$ & $62 \%$ \\
\hline NW & & $*$ & -1 & $12 \%$ & $8 \%$ & $24 \%$ & $111 \%$ & $1002 \%$ & $62 \%$ & $56 \%$ & $283 \%$ \\
\hline NNW & & $*$ & . & $16 \%$ & $394 \%$ & $58 \%$ & $45 \%$ & $41 \%$ & $52 \%$ & $157 \%$ & $67 \%$ \\
\hline Totals & $45 \%$ & $232 \%$ & $126 \%$ & $142 \%$ & $154 \%$ & $78 \%$ & $37 \%$ & $70 \%$ & $134 \%$ & $134 \%$ & $106 \%$ \\
\hline & & & & & & & & & & & \\
\hline The Lic & Ortod nean & $\mathrm{ad}$ & conment & $\overline{O E}$ & 10 & 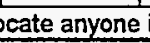 & $\mathrm{di}$ & & & & \\
\hline
\end{tabular}




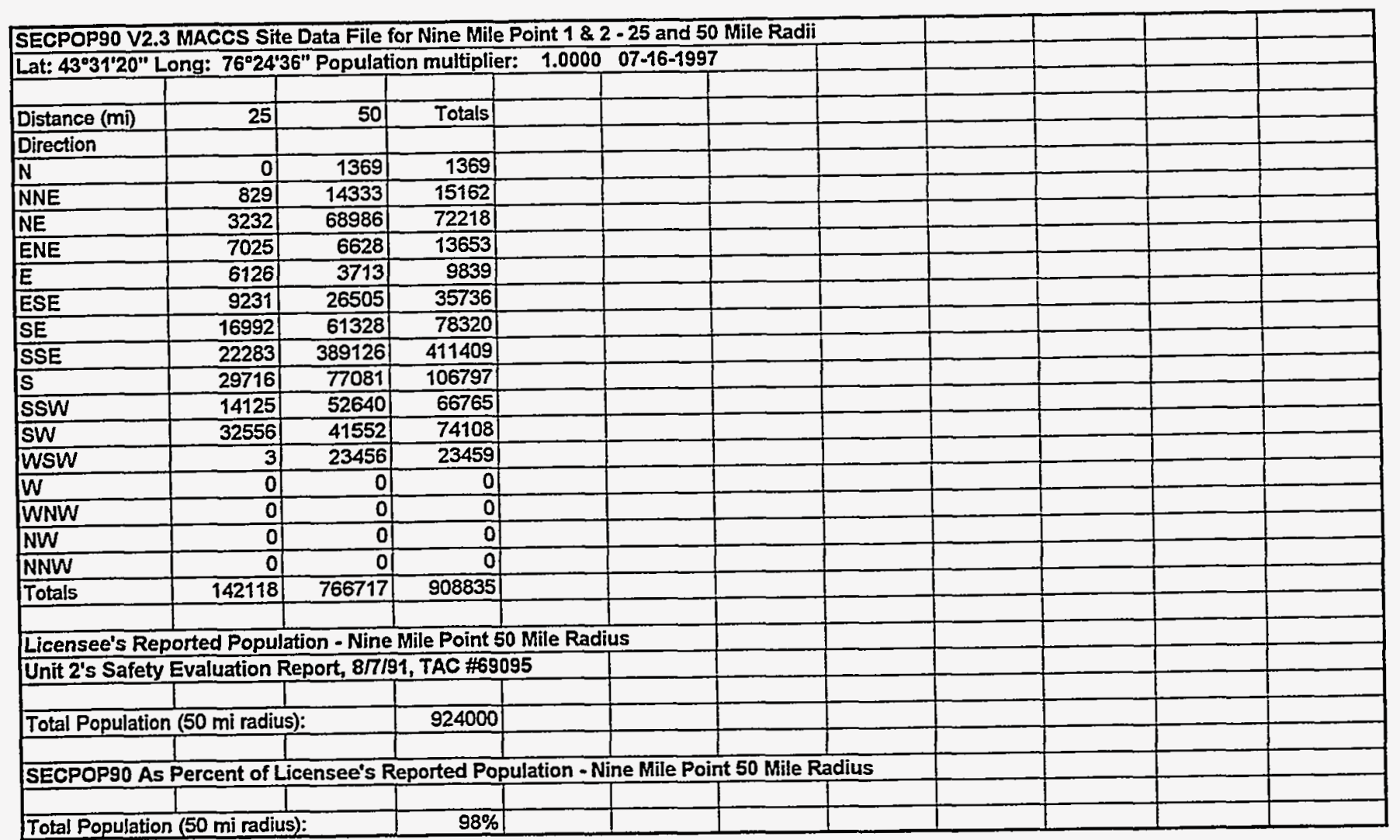




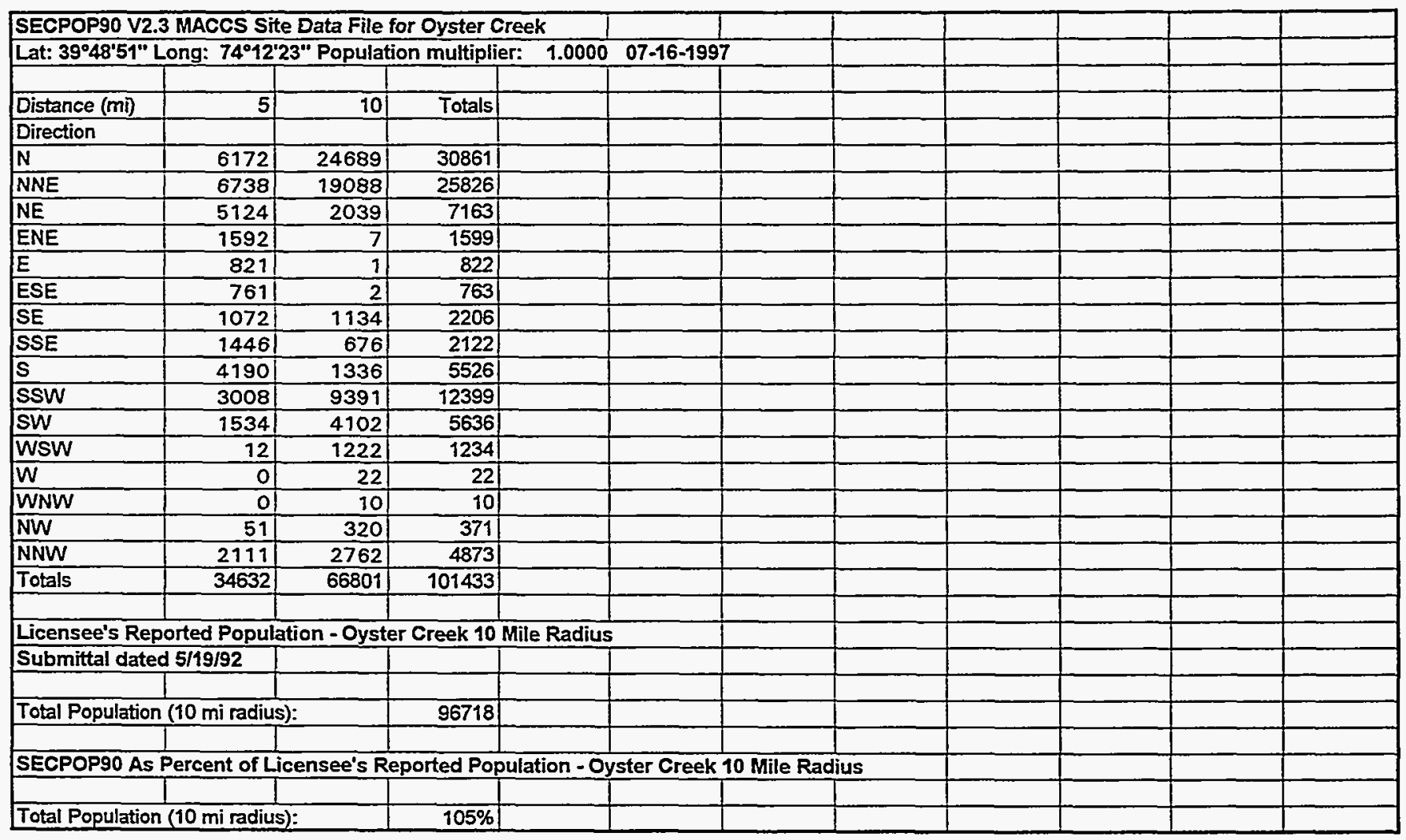




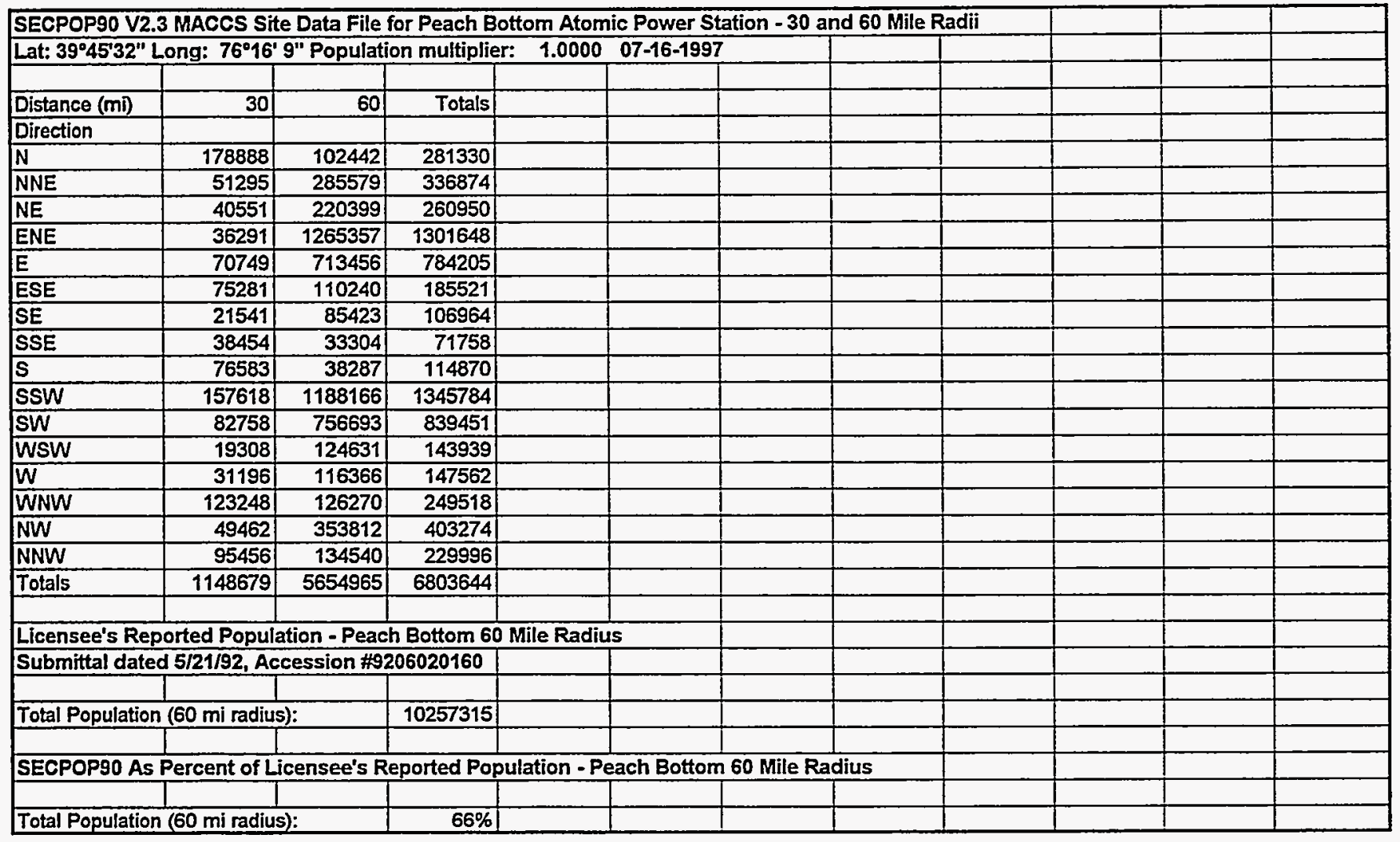




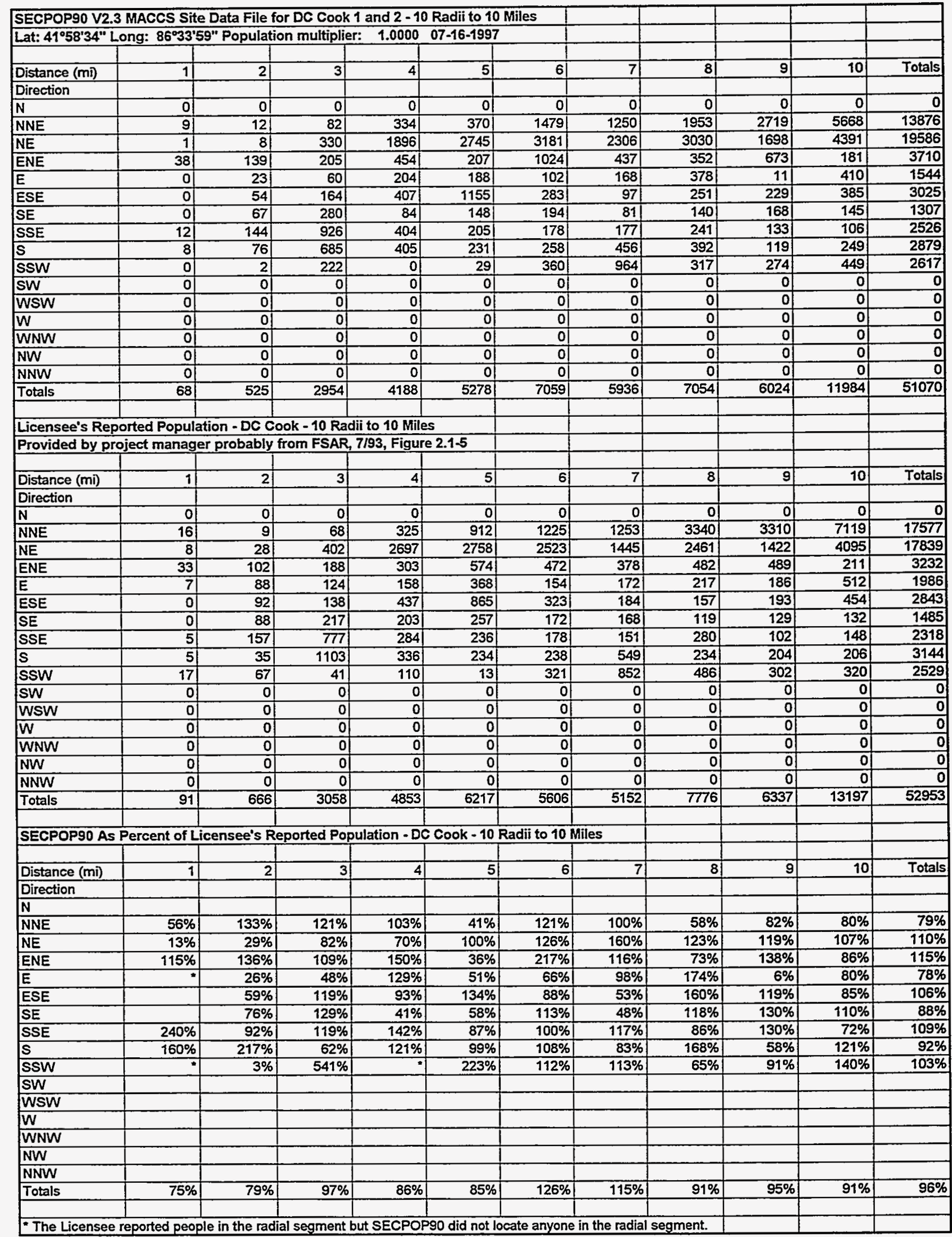




\begin{tabular}{|c|c|c|c|c|c|c|c|c|c|c|c|}
\hline \multicolumn{12}{|c|}{ SECPOP90 V2.3 MACCS Site Data File for Diablo Canyon Units 1 and 2 - 10 Radii to 50 Miles } \\
\hline \multicolumn{12}{|c|}{\begin{tabular}{|l|l|l|} 
Lat: $35^{\circ} 12^{\prime} 42^{\prime \prime}$ Long: $120^{\circ} 51^{\prime} 16^{\prime \prime}$ Population multiplier: $1.0000 \quad 07-16-1997$ & \\
\end{tabular}} \\
\hline & & & & & & & & & & & \\
\hline Distance (mi) & 1 & 2 & 3 & 4 & 5 & 10 & 20 & 30 & 40 & 50 & Totals \\
\hline \multicolumn{12}{|l|}{ Direction } \\
\hline $\mathbf{N}$ & of & 0 & 0 & 0 & 0 & 10136 & 12774 & 565 & 2366 & (333) & 26174 \\
\hline NNE & 0 & 0 & 13 & of & 이 & 4968 & 1990 & 34237 & 16989 & 114 & 58311 \\
\hline NE & 0 & 0 & 0 & 이 & 0 & 228 & 8909 & 6648 & 1005 & 839 & 17629 \\
\hline ENE & 0 & 요 & of & of & of & 5848 & 39875 & 374 & 5 & 126 & 46228 \\
\hline E & of & 인 & 0 & 0 & 의 & 564 & 2992 & 164 & 16 & 513 & 4249 \\
\hline ESE & 0 & 0 & of & 0 & 의 & 1182 & 43961 & 11012 & 1195 & 184 & 57534 \\
\hline$S E$ & 0 & o & 0 & 0 & 0 & 0 & 913 & 46494 & 52600 & 3491 & 103498 \\
\hline SSE & 01 & of & 0 & of & of & 0 & 이 & 2 & 9846 & 45564 & 55412 \\
\hline 5 & 0 & of & of & 0 & 이 & 0 & of & 0 & 0 & of & 0 \\
\hline SSW & 0 & 0) & 0 & 의 & 0 & 0 & 이 & 0 & \begin{tabular}{l|l}
0 \\
\end{tabular} & 0 & $\overline{0}$ \\
\hline SW & 0 & of & of & of & 0 & D & 이 & of & 0 & 0 & 0 \\
\hline WSW & 0 & of & 0 & 0 & a & o & 이 & 이 & 0 & 0 & 0 \\
\hline$W$ & 0 & 이 & of & 0 & 0 & o & 0 & 0 & 0 & 0 & $\overline{0}$ \\
\hline WNW & 0 & of & 이 & of & 0 & 0 & of & 0) & 0 & of & 0 \\
\hline NW & 0 & 4 & 01 & 이 & 0 & 0 & 아 & 의 & 44 & 22 & 70 \\
\hline NNW & 0 & 0 & of & 0 & of & 4 & 49 & 5783 & 579 & 492 & 6907 \\
\hline Totals & 0 & 4 & 13 & 0 & 의 & 22930 & 111463 & 105279 & 84645 & 51678 & 376012 \\
\hline & & & & & & & & & & & \\
\hline \multicolumn{12}{|c|}{ Licensee's Reported Population - Diablo Canyon 10 Radii to 50 Miles } \\
\hline \multicolumn{12}{|c|}{\begin{tabular}{|l|l|l|l|l|l|} 
FSAR update & & & & & \\
\end{tabular}} \\
\hline & & & & & & & & & & & \\
\hline Distance (mi) & 1. & 2 & 3 & 4 & 5 & 10 & 20 & 30 & $40 \mid$ & 50 & Totals \\
\hline \multicolumn{12}{|l|}{ Direction } \\
\hline $\mathbf{N}$ & 0 & 0| & 0 & 0 & of & 12335 & 1013 & 7263 & 1022 & 341 & 21974 \\
\hline NNE & of & 이 & 0] & o| & 4 & 1494 & 11700 & 1482 & 6062 & 1516 & 22258 \\
\hline $\mathrm{NE}$ & 0 & 0 & 0) & 0 & 可 & 204 & 3461 & 29332 & 10405 & 1087 & 44489 \\
\hline ENE & of & 0 & 0 & 이 & 3 & 4915 & 3043 & 3521 & 185 & 1200 & 12857 \\
\hline$E$ & of & 0 & 0 & 이 & 3 & 1969 & 42238 & 1346 & 150 & 1199 & 46905 \\
\hline ESE & 이 & 0 & 0 & 0 & 3 & 1265 & 7674 & 323 & 81 & 0 & 9346 \\
\hline SE & 0 & 0 & 0 & 0 & 0 & 0 & 42339 & 13026 & 1362 & 908 & 57635 \\
\hline SSE & 0 & 0 & 0 & 0 & 0 & 0 & 1822 & 50837 & 47360 & 2456 & 102475 \\
\hline$s$ & 0 & 0 & 0 & \begin{tabular}{l|l}
0 \\
\end{tabular} & 의 & 0 & o & o & 7010 & 49738 & 56748 \\
\hline SSW & 0 & 0 & 0 & 0 & 0) & D & of & 이 & 0 & 0 & 0 \\
\hline SW & 의 & 0 & 0 & 0 & 의 & 익 & 의 & 0 & of & 0 & 0 \\
\hline WSW & 0 & 0 & 0 & 01 & 이 & 이 & 아 & 0 & \begin{tabular}{l|l}
0 \\
\end{tabular} & 0 & 0 \\
\hline $\bar{W}$ & 0 & 0 & 0 & 이 & 이 & 이 & of & 0 & 0 & 0 & 0 \\
\hline WNW & 0 & 0 & 0 & 0 & of & of & of & 0 & 0 & 0 & 은 \\
\hline NW & 0 & 0] & 0 & \begin{tabular}{l|l}
0 \\
\end{tabular} & 이 & 0 & of & 이 & 0 & 0 & 0 \\
\hline NNW & 0 & 4 & \begin{tabular}{l|l}
0 \\
\end{tabular} & 0 & 이 & of & 0 & 이 & 0 . & 0 & 4 \\
\hline Totals & 0 & 4 & of & 0 & 13 & 22182 & 113290 & 107130 & 73637 & 58445 & 374701 \\
\hline & & & & & & & & & & & \\
\hline \multicolumn{12}{|c|}{ SECPOP90 As Percent of Licensee's Reported Population - Diablo Canyon 10 Radii to 50 Miles } \\
\hline & & & & & & & & & & & \\
\hline Distance (mi) & 1 & 2 & 3) & 4 & 5 & 10! & 20 & 30 & 40 & 50 & Totals \\
\hline \multicolumn{12}{|l|}{ Direction } \\
\hline$N$ & & & & & 1 & $82 \%$ & $1261 \%$ & $8 \%$ & $232 \%$ & $98 \%$ & $119 \%$ \\
\hline NNE & & & *\# & & 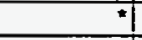 & $333 \%$ & $17 \%$ & $2310 \%$ & $280 \%$ & $8 \%$ & $252 \%$ \\
\hline NE & & & & & T & $112 \%$ & $257 \%$ & $23 \%$ & $10 \%$ & $77 \%$ & $40 \%$ \\
\hline ENE & & & & & 1 & $119 \%$ & $1310 \%$ & $11 \%$ & $3 \%$ & $11 \%$ & $359 \%$ \\
\hline E & & & & & * & $29 \%$ & $7 \%$ & $12 \%$ & $11 \%$ & $43 \%$ & $\mathbf{9 \%}$ \\
\hline ESE & & & & & 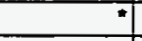 & $93 \%$ & $573 \%$ & $3409 \%$ & $1475 \%$ & 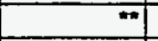 & $616 \%$ \\
\hline SE & & & & & & & $2 \%$ & $357 \%$ & $3862 \%$ & $384 \%$ & $180 \%$ \\
\hline SSE & & & & & & & -1 & $0 \%$ & $21 \%$ & $1855 \%$ & $54 \%$ \\
\hline $\mathbf{s}$ & & & & & & & & & 1 & . & \\
\hline SSW & & & & & & & & & & & \\
\hline SW & & & & & & & & & & & \\
\hline WSW & & & & & & & & & & & \\
\hline $\bar{w}$ & & & & & & & & & & & \\
\hline WNW & & & & & & & & & & & \\
\hline NW & & $\cdots$ & & & & & & & $=1$ & $*=$ & 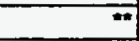 \\
\hline NNW & & $\because$ & & & & $\#$ & 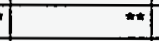 & $n$ & *1 & $*+$ & $172675 \%$ \\
\hline Totals & & $100 \%$ & $=$ & & -1 & $103 \%$ & $98 \%$ & $98 \%$ & $115 \%$ & $88 \%$ & $100 \%$ \\
\hline & & & & & & & & & & & \\
\hline e Licens & onte & le in the radia & ial se & ou & 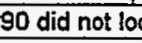 & ocate & $\underline{e}$ & & & & \\
\hline The License & $\operatorname{did} n$ & $t$ anyo & segn & ment b & log & cated people & 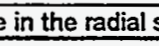 & & & & \\
\hline
\end{tabular}




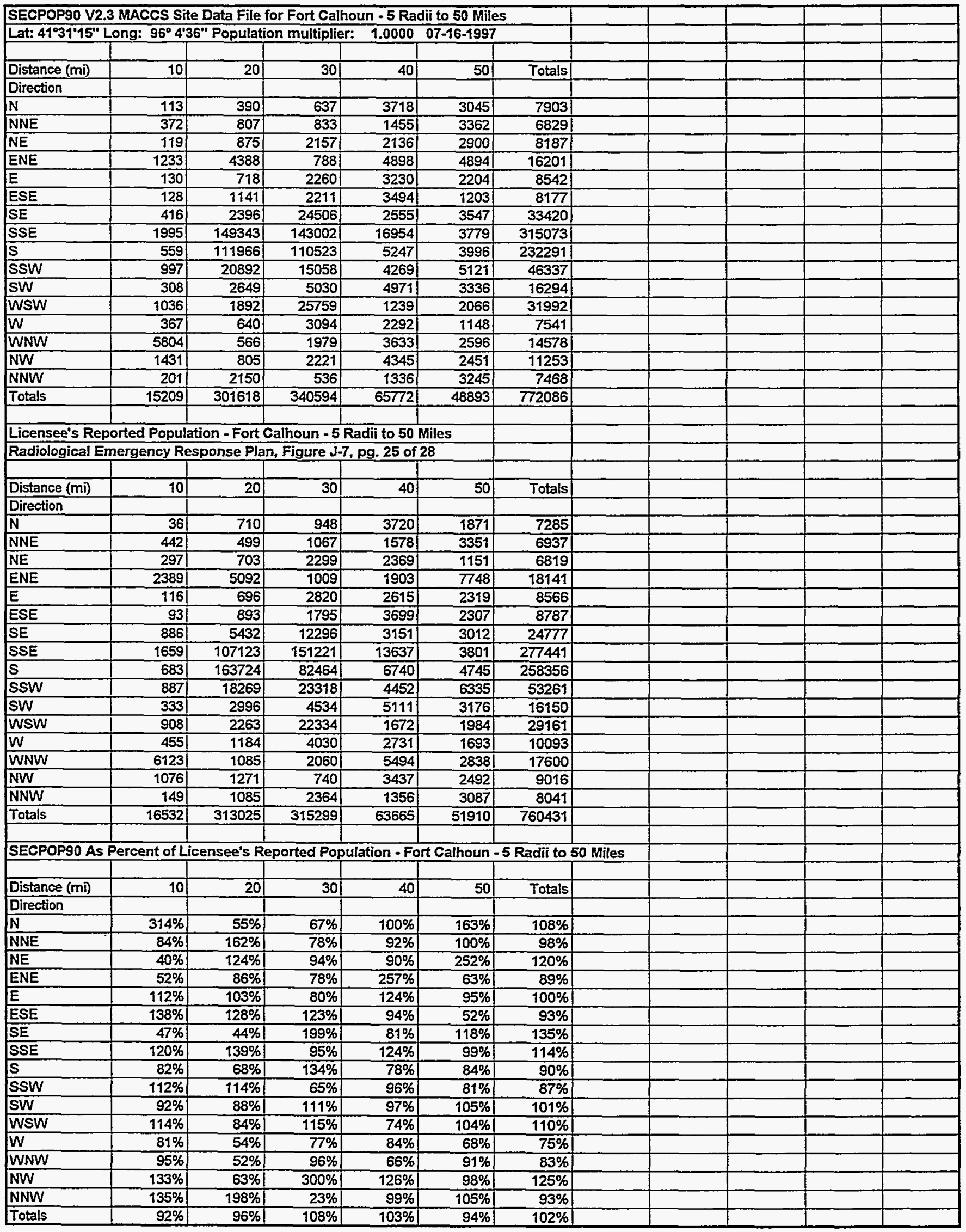




\begin{tabular}{|c|c|c|c|c|c|c|c|c|c|c|c|}
\hline \multicolumn{12}{|c|}{ SECPOP9O V2.3 MACCS Site Data File for Prairie Island 1 and 2 - 10 Radii to 10 Miles } \\
\hline \multicolumn{12}{|c|}{ Lat: $44^{\circ} 37^{\prime} 19^{\prime \prime}$ Long: $92^{\circ} 37^{\prime} 59^{\prime \prime}$ Population multiplier: $1.0000 \quad 07-16-1997$} \\
\hline & & & & & & & & & & & \\
\hline Distance (mi) & 1 & 2 & 3 & 4 & 5 & 6 & 7 & 8 & 9 & 10 & Totals \\
\hline \multicolumn{12}{|l|}{ Direction } \\
\hline $\mathrm{N}$ & 0 & 16 & 31 & 47 & 0 & 28 & 23 & 83 & 78 & 97 & 403 \\
\hline NNE & o & 22 & 50 & 24 & 35 & 28 & 90 & 181 & 151 & 249 & 830 \\
\hline NE & 0 & 14 & 184 & 15 & 23 & 104 & 182 & 130 & 120 & 171 & 943 \\
\hline ENE & 0 & 11 & 14 & 0 & 22 & 37 & 81 & 96 & 80 & 127 & 468 \\
\hline$E$ & 0 & 0 & 6 & of & 84 & 53 & 60 & 71 & 67 & 43 & 384 \\
\hline ESE & 0 & 0 & 0 & 155 & 168 & 258 & 414 & 179 & 260 & 593 & 2027 \\
\hline$\overline{S E}$ & 0 & 0 & 0 & 2 & 195 & 1198 & 6981 & 3328 & 293 & 139 & 12136 \\
\hline SSE & 14 & 0 & 0 & \begin{tabular}{|l|}
733 \\
\end{tabular} & 608 & 482 & 205 & 104 & 27 & 95 & 2268 \\
\hline $\mathbf{S}$ & 0 & 0 & 40 & 66 & 747 & 37 & 55 & 50 & 24 & 25 & 1044 \\
\hline SSW & 0 & 0 & 35 & 65 & 23 & 16 & 50 & 121 & 38 & 165 & 513 \\
\hline SW & 0 & 01 & 30 & 0 & 44 & 67 & 27 & 107 & 22 & 83 & 380 \\
\hline WSW & 0 & 10 & 33 & o & 36 & 0 & 88 & 26 & 47 & 80 & 320 \\
\hline $\mathrm{W}$ & 8 & 32 & 0 & 13 & 89 & 50 & 217 & 24 & 112 & 148 & 693 \\
\hline WNW & 113 & 5 & 0 & \begin{tabular}{l|l}
60 & \\
\end{tabular} & 0 & 145 & 442 & 232 & 432 & 116 & 1545 \\
\hline NW & 0 & 6 & 7 & 81 & 10 & 9 & 0 & 82 & 328 & 16 & 466 \\
\hline NNW & 21 & 0 & 0 & 9 & 31 & 8 & 4 & 0 & 372 & 104 & 549 \\
\hline Totals & 156 & 116 & 430 & 1197 & 2115 & 2520 & 8919 & 4814 & 2451 & 2251 & 24969 \\
\hline & & & & & & & & & & & \\
\hline \multicolumn{12}{|c|}{ Licensee's Reported Population - Prairie Island - 10 Radii to 10 Miles } \\
\hline \multicolumn{12}{|c|}{ Evacuation time estimates, 12/92, Table 3.1} \\
\hline & & & & & & & & & & & \\
\hline Distance (mi) & 1 & 2 & 3 & 4 & 5 & 6 & 7 & 8 & 9 & 10 & Totals \\
\hline \multicolumn{12}{|l|}{ Direction } \\
\hline $\mathbf{N}$ & 0. & 21 & 61 & 25 & 26 & 27 & 45 & 71 & 114 & 80 & 470 \\
\hline NNE & 0 & 54 & 50 & 43 & 48 & 64 & 76 & 124 & 149 & 137 & 745 \\
\hline NE & 0 & 20 & 31 & 16 & 29 & 86 & 154 & 153 & 186 & 198 & 873 \\
\hline ENE & 0 & 11 & 63 & 29 & 37 & 29 & 74 & 73 & 91 & 83 & 490 \\
\hline$E$ & 0 & 2 & 42 & 49 & 42 & 60 & 41 & 61 & 68 & 53 & 418 \\
\hline ESE & 0 & 0 & 1 & 56 & 196 & 205 & 733 & 299 & 599 & 429 & 2518 \\
\hline $\mathrm{SE}$ & 0 & 2 & of & 2 & 317 & 1343 & 6724 & 3299 & 220 & 83 & 11990 \\
\hline SSE & 7 & 11 & 4 & 928 & 730 & 158 & 86 & 183 & 57 & 64 & 2228 \\
\hline s & 0 & 4 & 13 & 244 & 436 & 44 & 34 & 50 & 53 & 53 & 931 \\
\hline SSW & 0 & 6ा & 31 & 70 & 13 & 15 & 52 & 66 & 98 & 139 & 490 \\
\hline SW & 0 & 6) & 301 & $17 \mid$ & 14| & 34 & 100 & 58 & 84 & 66 & 409 \\
\hline WSW & o & 0 & 35 & 34 & 29 & 33 & 46 & 25 & 50 & 58 & 310 \\
\hline$W$ & 8 & 10 & 3 & 26 & 55 & 133 & 70 & 100 & 128 & 130 & 663 \\
\hline WNW & 129 & 54 & 10 & 12 & 32 & 265 & 311 & 437 & 451 & 99 & 1800 \\
\hline NW & 22 & 6 & 6 & 2 & 9 & 21 & 1 & 126 & 151 & 163 & 507 \\
\hline NNW & 21 & 0 & o & 4 & 13 & 21 & 86 & 112 & 108 & 135 & 500 \\
\hline Totals & 187 & 207 & 380 & 1557 & 2026 & 2538 & 8633 & 5237 & 2607 & 1970 & 25342 \\
\hline & & & & & & & & & & & \\
\hline \multicolumn{12}{|c|}{ SECPOP90 As Percent of Licensee's Reported Population - Prairie Island - 10 Radii to 10 Miles } \\
\hline & & & & & & & & & & & \\
\hline Distance (mi) & 1 & 2 & 3 & 4 & 5 & 6 & 7 & 81 & 9 & 10 & Totals \\
\hline \multicolumn{12}{|l|}{ Direction } \\
\hline $\mathrm{N}$ & & $76 \%$ & $51 \%$ & $188 \%$ & 7 & $104 \%$ & $51 \%$ & $117 \%$ & $68 \%$ & $121 \%$ & $86 \%$ \\
\hline NNE & & $41 \%$ & $100 \%$ & $56 \%$ & $73 \%$ & $44 \%$ & $118 \%$ & $146 \%$ & $101 \%$ & $182 \%$ & $111 \%$ \\
\hline $\mathrm{NE}$ & & $70 \%$ & $594 \%$ & $94 \%$ & $79 \%$ & $121 \%$ & $118 \%$ & $85 \%$ & $65 \%$ & $86 \%$ & $108 \%$ \\
\hline ENE & & $100 \%$ & $22 \%$ & . & $59 \%$ & $128 \%$ & $109 \%$ & $132 \%$ & $88 \%$ & $153 \%$ & $96 \%$ \\
\hline $\mathrm{E}$ & & . & $14 \%$ & $\Rightarrow$ & $200 \%$ & $88 \%$ & $146 \%$ & $116 \%$ & $99 \%$ & $81 \%$ & $92 \%$ \\
\hline ESE & & & $*$ & $277 \%$ & $86 \%$ & $126 \%$ & $56 \%$ & $60 \%$ & $43 \%$ & $138 \%$ & $81 \%$ \\
\hline SE & & 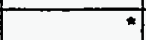 & & $100 \%$ & $62 \%$ & $89 \%$ & $104 \%$ & $101 \%$ & $133 \%$ & $167 \%$ & $101 \%$ \\
\hline SSE & $200 \%$ & 1 & -1 & $79 \%$ & $83 \%$ & $305 \%$ & $238 \%$ & $57 \%$ & $47 \%$ & $148 \%$ & $102 \%$ \\
\hline S & & $*$ & $308 \%$ & $27 \%$ & $171 \%$ & $84 \%$ & $162 \%$ & $100 \%$ & $45 \%$ & $47 \%$ & $112 \%$ \\
\hline SSW & &. & $113 \%$ & $93 \%$ & $177 \%$ & $107 \%$ & $96 \%$ & $183 \%$ & $39 \%$ & $119 \%$ & $105 \%$ \\
\hline SW & & -1 & $100 \%$ &. & $314 \%$ & $197 \%$ & $27 \%$ & $184 \%$ & $26 \%$ & $126 \%$ & $93 \%$ \\
\hline WSW & & $=1$ & $94 \%$ & .1 & $124 \%$ & $*$ & $191 \%$ & $104 \%$ & $94 \%$ & $138 \%$ & $103 \%$ \\
\hline $\mathrm{W}$ & $100 \%$ & $320 \%$ & 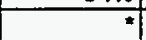 & $50 \%$ & $162 \%$ & $38 \%$ & $310 \%$ & $24 \%$ & $88 \%$ & $114 \%$ & $105 \%$ \\
\hline WNW & $88 \%$ & $9 \%$ & .1 & $500 \%$ & 1 & $55 \%$ & $142 \%$ & $53 \%$ & $96 \%$ & $117 \%$ & $86 \%$ \\
\hline NW & . & $100 \%$ & $117 \%$ & $400 \%$ & $111 \%$ & $43 \%$ &. & $65 \%$ & $217 \%$ & $10 \%$ & $92 \%$ \\
\hline NNW & $100 \%$ & & & $225 \%$ & $238 \%$ & $38 \%$ & $5 \%$ & 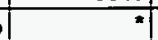 & $344 \%$ & $77 \%$ & $110 \%$ \\
\hline Totals & $83 \%$ & $56 \%$ & $113 \%$ & $77 \%$ & $104 \%$ & $99 \%$ & $103 \%$ & $92 \%$ & $94 \%$ & $114 \%$ & $99 \%$ \\
\hline & & & & & & & & & & & \\
\hline & & & & & & & & & & & \\
\hline The Licen & d not repor & one in $t$ & lial se & but $\mathbf{S}$ & P90 loc & 0 & & & & & \\
\hline
\end{tabular}




\begin{tabular}{|c|c|c|c|c|c|c|c|c|c|c|c|}
\hline \multicolumn{12}{|c|}{ SECPOP90 V2.3 MACCS Site Data File for Turkey Point Units 3 and 4 - 10 Radii to 50 Miles } \\
\hline \multicolumn{12}{|c|}{ Lat: $25^{\circ} 26^{\prime} 6^{\prime \prime}$ Long: $80^{\circ} 19^{\prime} 53^{\prime \prime}$ Population multiplier: $1.0000 \quad 07-16-1997$} \\
\hline & & & & & & & & & & & \\
\hline Distance (mi) & 1 & & 3 & 4) & 5 & 10 & 20 & 30 & 40 & 50 & Totals \\
\hline \multicolumn{12}{|l|}{ Direction } \\
\hline $\mathbf{N}$ & 이 & & 0 & 0 & 0] & 11435 & 200878 & 413887 & 325282 & 326227 & 1277709 \\
\hline NNE & 01 & & 0 & 可 & 0 & of & 10185 & 429275 & 352455 & 195887 & 987802 \\
\hline NE & 0 & & 0 & of & 0 & 이 & 0 & 0 & 0 & 0 & 0 \\
\hline ENE & 0 & & 0 & of & 이 & 0 & 0 & 0 & 0 & of & 0 \\
\hline$E$ & 0 & & 0 & 0 & 0 & 6 & of & 0 & 0 & 0 & 6 \\
\hline ESE & 0 & & 0| & 0 & 0 & 7 & 0 & 0 & 0) & ot & $\overline{7}$ \\
\hline SE & 0) & & 0 & 이 & 0 & 이 & 0 & 0 & 0 & 01 & 0 \\
\hline SSE & 0 & & 0] & 0) & 01 & 1265 & 0 & 0 & 0 & 0 & 1265 \\
\hline S & 0 & & 0 & 0 & 0 & 0 & 1805 & 228 & 0 & 0 & 2033 \\
\hline SSW & 의 & & 0 & o & 3 & of & 255 & 9939 & 6949 & 1628 & 18774 \\
\hline SW & 0 & & 0 & 0 & 0 & o) & 0 & 0 & 0 & 12 & 12 \\
\hline WSW & 0 & & 0 & 0 & 0 & 57 & 0 & 3256 & 58 & 195 & 3566 \\
\hline$w$ & 0 & & 0 & 0 & 0 & 6407 & 3982 & 0 & 0 & 0 & 10389 \\
\hline WNW & of & & 0 & 0 & 0 & 36153 & 13184 & 384 & 0 & 21 & 49746 \\
\hline NW & of & & 0 & 0 & of & 25338 & 8204 & 2 & 94 & 18 & 33656 \\
\hline NNW & 0 & 26 & 인 & 0 & 0 & 14956 & 153775 & 48654 & 236 & 222 & 217869 \\
\hline Totals & 0 & 30 & 0 & 0 & 3 & 95624 & 392268 & 905625 & 685074 & 524210 & 2602834 \\
\hline \multirow{2}{*}{\multicolumn{12}{|c|}{ Licensee's Reported Population - Turkey Point 10 Radii to 50 Miles }} \\
\hline & & & & & & & & & & & \\
\hline \multicolumn{12}{|c|}{\begin{tabular}{|l|l|l} 
FSAR Section 2.4, Tables 2.4-1 \& 2.4-3 & & \\
\end{tabular}} \\
\hline & & & & & & & & & & & \\
\hline Distance (mi) & 1 & & 3 & 4 & 5 & 10 & 20 & 30 & $40 \mid$ & 50 & Totals \\
\hline \multicolumn{12}{|l|}{ Direction } \\
\hline $\mathbf{N}$ & 의 & & 0 & 0 & of & 15799 & 213226 & 430335 & 350347 & 320863 & 1330570 \\
\hline NNE & 이 & & 이 & of & 0 & of & 9746 & 429713 & 349676 & 183681 & 972816 \\
\hline NE & 0 & 인 & 이 & 0 & 0 & 0 & 0 & 0 & 의 & 01 & 0 \\
\hline ENE & 0 & & 익 & 이 & 0) & 0 & 0) & of & 의 & 0 & $\underline{0}$ \\
\hline$E$ & 0 & & 0 & of & of & 0 & 0 & 0 & 0 & 0 & $\overline{0}$ \\
\hline ESE & of & & 0 & 0 & 0 & 0 & 0 & 0 & 0 & 0 & 0 \\
\hline$\overline{S E}$ & of & & 0 & 0 & 0 & 0 & 0 & 0 & 0 & 0 & 0 \\
\hline SSE & 0 & & 0) & 요 & 0 & 1427 & 0 & 0 & 0| & 0 & 1427 \\
\hline $\mathbf{s}$ & 0 & & 이 & 0| & o) & 0 & 1223 & 333 & 0 & 0 & 1556 \\
\hline SSW & a) & & 이 & of & 0 & 0 & 726 & 9826 & 6876 & 1591 & 19019 \\
\hline SW & of & 0 & 이 & of & 0 & 0 & 0 & of & 0 & 45 & 45 \\
\hline WSW & 0 & 0 & 이 & 0 & 0 & 0 & 0 & 0 & 58 & 190 & 248 \\
\hline $\mathbf{W}$ & 0 & & 0 & 이 & 0 & 10641 & 521 & 이 & 0 & 0 & 11162 \\
\hline WNW & 0 & & 이 & 0 & 이 & 37006 & 15205 & 0 & 0 & 23 & 52234 \\
\hline NW & 0 & & of & 0 & 0 & 24813 & 8699 & 0 & 0 & 0 & 33512 \\
\hline NNW & 0 & & 0 & 0| & 0 & 15993 & 142481 & 32254 & 218 & 0 & 190946 \\
\hline Totals & 0 & 0 & 0 & 0 & 0 & 105679 & 391827 & 902461 & 707175 & 506393 & 2613535 \\
\hline & & & & & & & & & & & \\
\hline \multicolumn{12}{|c|}{ SECPOP90 As Percent of Licensee's Reported Population - Turkey Point 10 Radii to 50 Miles } \\
\hline & & & & & & & & & & & \\
\hline$\overline{D i s t a n c e ~(m i) ~}$ & 1 & 2 & 3 & 4 & 5 & 10 & 20 & 30 & 40 & 50 & Totals \\
\hline \multicolumn{12}{|l|}{ Direction } \\
\hline $\mathbf{N}$ & & & & & & $72 \%$ & $94 \%$ & $96 \%$ & $93 \%$ & $102 \%$ & $96 \%$ \\
\hline NNE & & & & & & & $105 \%$ & $100 \%$ & $101 \%$ & $107 \%$ & $102 \%$ \\
\hline \multicolumn{12}{|l|}{$\mathrm{NE}$} \\
\hline ENE & & & & & & & & & & & \\
\hline$E$ & & & & & & .1 & & & & & . \\
\hline ESE & & & & & & .1 & & & & & \\
\hline$\overline{S E}$ & & & & & & & & & & & \\
\hline SSE & & & & & & $89 \%$ & & & & & $89 \%$ \\
\hline S & & & & & & & $148 \%$ & $68 \%$ & & & $131 \%$ \\
\hline SSW & & & & & 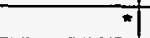 & & $35 \%$ & $101 \%$ & $101 \%$ & $102 \%$ & $99 \%$ \\
\hline SW & & & & & & & & & & $27 \%$ & $27 \%$ \\
\hline WSW & & & & & & -1 & & + & $100 \%$ & $103 \%$ & $1438 \%$ \\
\hline $\bar{W}$ & & & & & & $60 \%$ & $764 \%$ & & & & $93 \%$ \\
\hline WNW & & 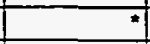 & *1 & & & $98 \%$ & $87 \%$ & . & & $91 \%$ & $95 \%$ \\
\hline NW & & & & & & $102 \%$ & $94 \%$ & 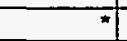 & . & 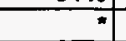 & $100 \%$ \\
\hline NNW & & न & "I & & & $94 \%$ & $108 \%$ & $151 \%$ & $108 \%$ & .1 & $114 \%$ \\
\hline Totals & & & 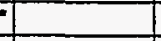 & & . & $90 \%$ & $100 \%$ & $100 \%$ & $97 \%$ & $104 \%$ & $100 \%$ \\
\hline & & & & & & & & & & & \\
\hline
\end{tabular}




\section{APPENDIX G - CONVERSION PROGRAM LISTINGS}

FILENAME: CONVERT1.BAS

OPTION EXPLICIT

DECLARE SUB Convert (Filename AS STRING)

- Skip past the DBASE III field information.

CONST CensusFileoffset $\&=2946$

- Set $C R$ and LF to be the carriage return and line feed characters.

DIM SHARED CR AS STRING * 1, LF AS STRING * 1

$\mathrm{CR}=\operatorname{CHR}(13)$

$L F=\operatorname{CHR} \$(10)$

- The structure below will hold the last read census record. The fields

- within the structure hold the information in the fields as described

' in the pb94stru.dbf file.

TYPE PL94171Record

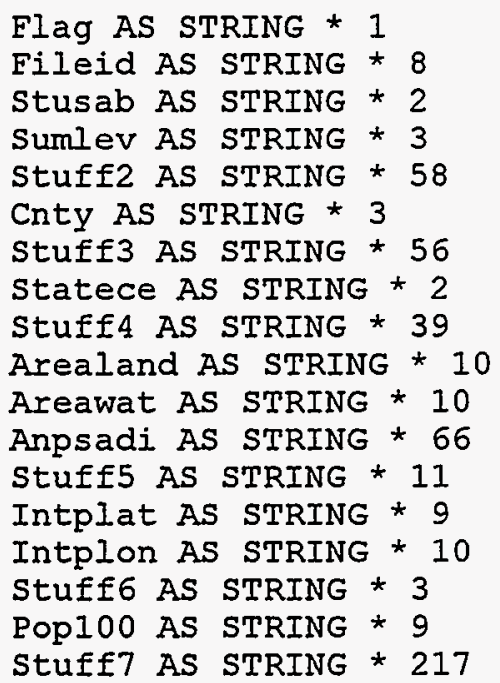

END TYPE

OPEN "d: \secpop90\census\county 1.txt" FOR BINARY AS \#I

OREN "d: \secpop90\census\block_I.bin" FOR BINARY AS \#2

DIM Dummy AS STRING

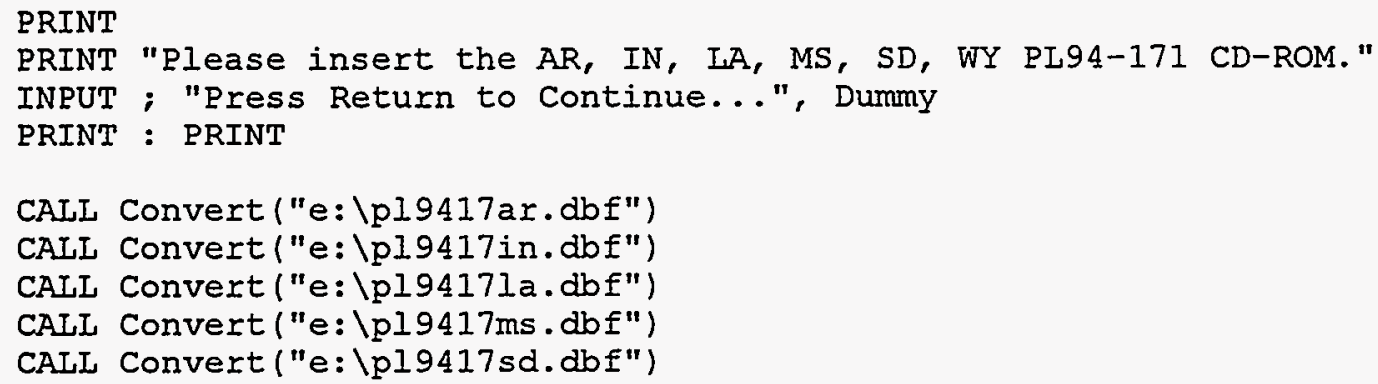


CALI Convert ("e: (pl9417wy.dbf")

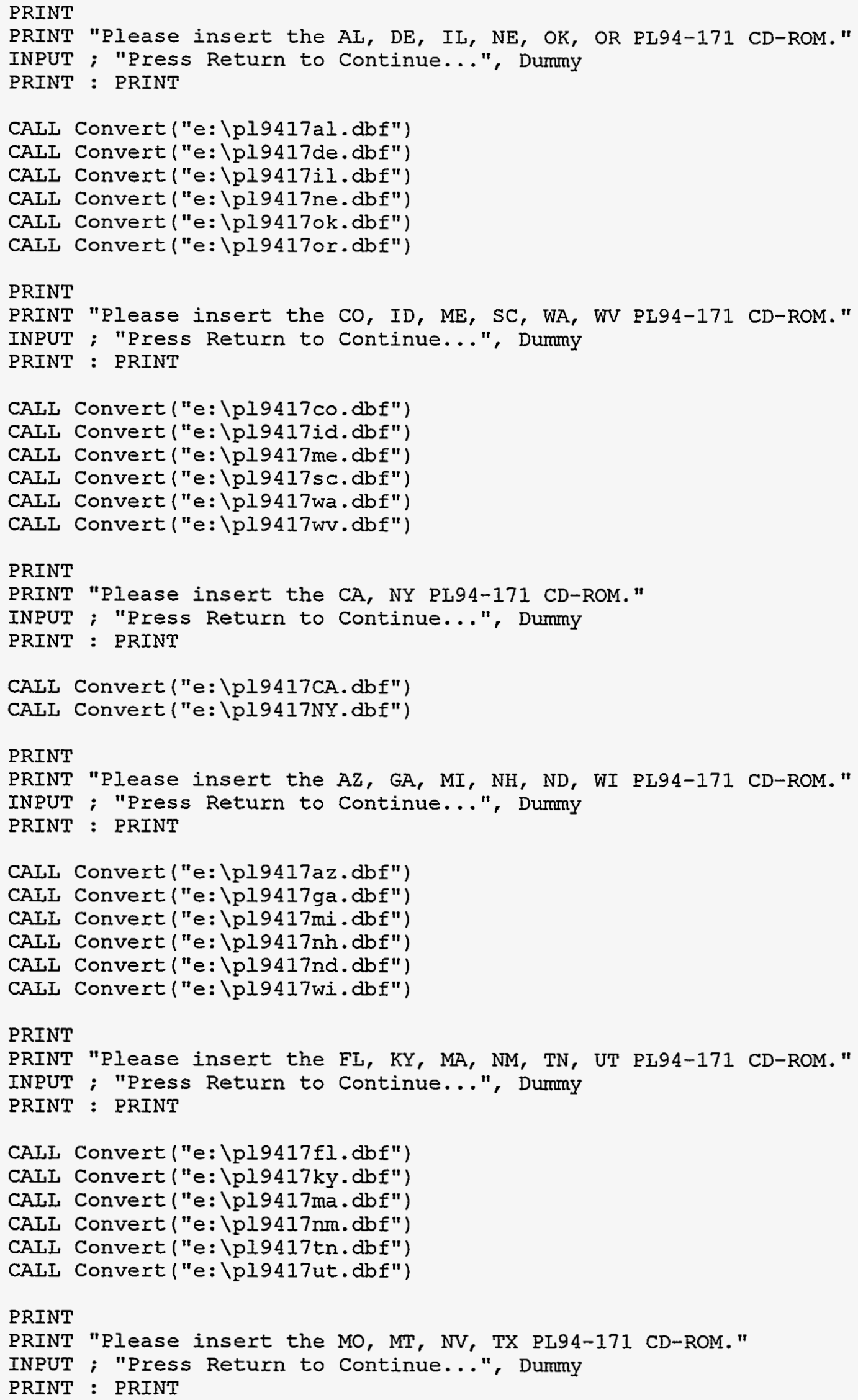

CAII Convert ("e: (pl9417az.dbf")

CAII Convert ("e: \pl9417ga.dbf")

CAII Convert ("e: $\backslash p 19417 \mathrm{mi} . \mathrm{db} f ")$

CAII Convert ("e: (pl9417nh.dbf")

CAIL Convert ("e: \pl9417nd.dbf")

CAIL Convert ("e: (pl9417wi.dbf")

PRINT

PRINT "Please insert the EI, KY, MA, NM, TN, UT PL94-171 CD-ROM." INPUT ; "Press Return to Continue...", Dummy

PRINT : PRINT

CAIL Convert ("e: (pl9417fl.dbf")

CAIL Convert ("e: $\backslash p 19417 \mathrm{ky} \cdot \mathrm{dbf} "$ )

CALL Convert ("e: \pl9417ma.dbf")

CALL Convert ("e: (pl9417nm.dbf")

CALL Convert ("e: (pl9417tn.dbf")

CALL Convert ("e: \pl9417ut.dbf")

PRINT

PRINT "Please insert the MO, MT, NV, TX PL94-171 CD-ROM."

INPUT ; "Press Return to Continue...", Dummy

PRINT : PRINT 
CALI Convert("e: (pl9417mo.dbf")

CALI Convert ("e: $\backslash p 19417 \mathrm{mt} . d \mathrm{db} ")$

CALI Convert ("e: (pl9417nv.dbf")

CAIL Convert ("e: $\backslash p 19417 t x . d b f ")$

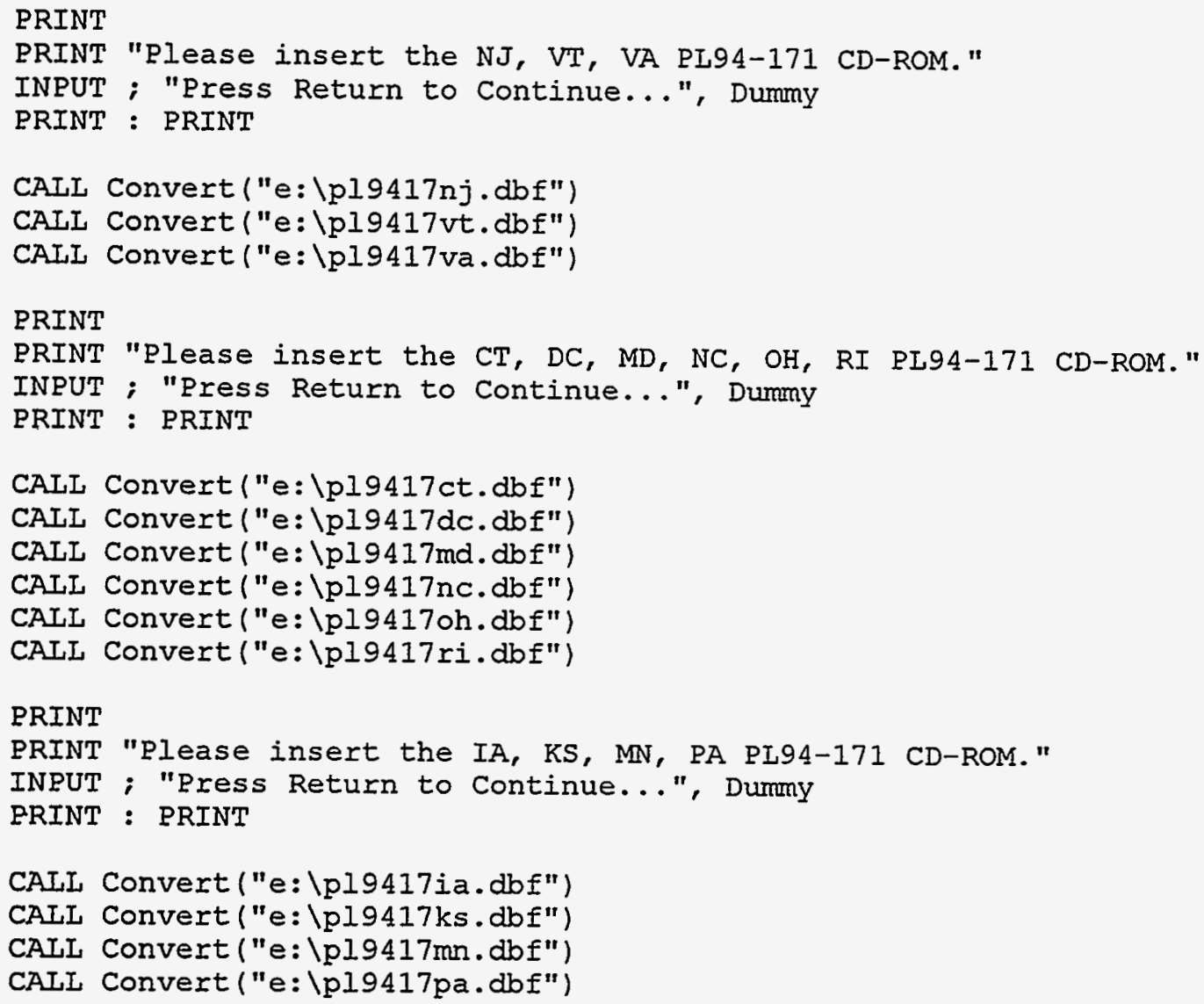

PRINT

PRINT "Please insert the IA, KS, MN, PA PI94-171 CD-ROM."

INPUT ; "Press Return to Continue...", Dummy

PRINT : PRINT

CAII Convert("e: (pl9417ia.dbf")

CALL Convert("e: (pl9417ks.dbf")

CALL Convert ("e: (pl9417mn.dbf")

CALI Convert ("e: (pl9417pa.dbf")

CLOSE \#I, \#2

END

SUB Convert (Filename AS STRING)

DIM PL94171 AS PL94171Record

DIM Longitude AS LONG, Lattitude AS LONG

DIM Population AS LONG, Area AS IONG

DIM State AS STRING * 1 , County AS INTEGER

OPEN Filename FOR BINARY AS \#3

SEEK \#3, Censusfileoffset\&

DO

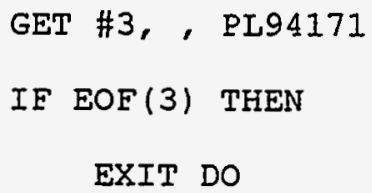




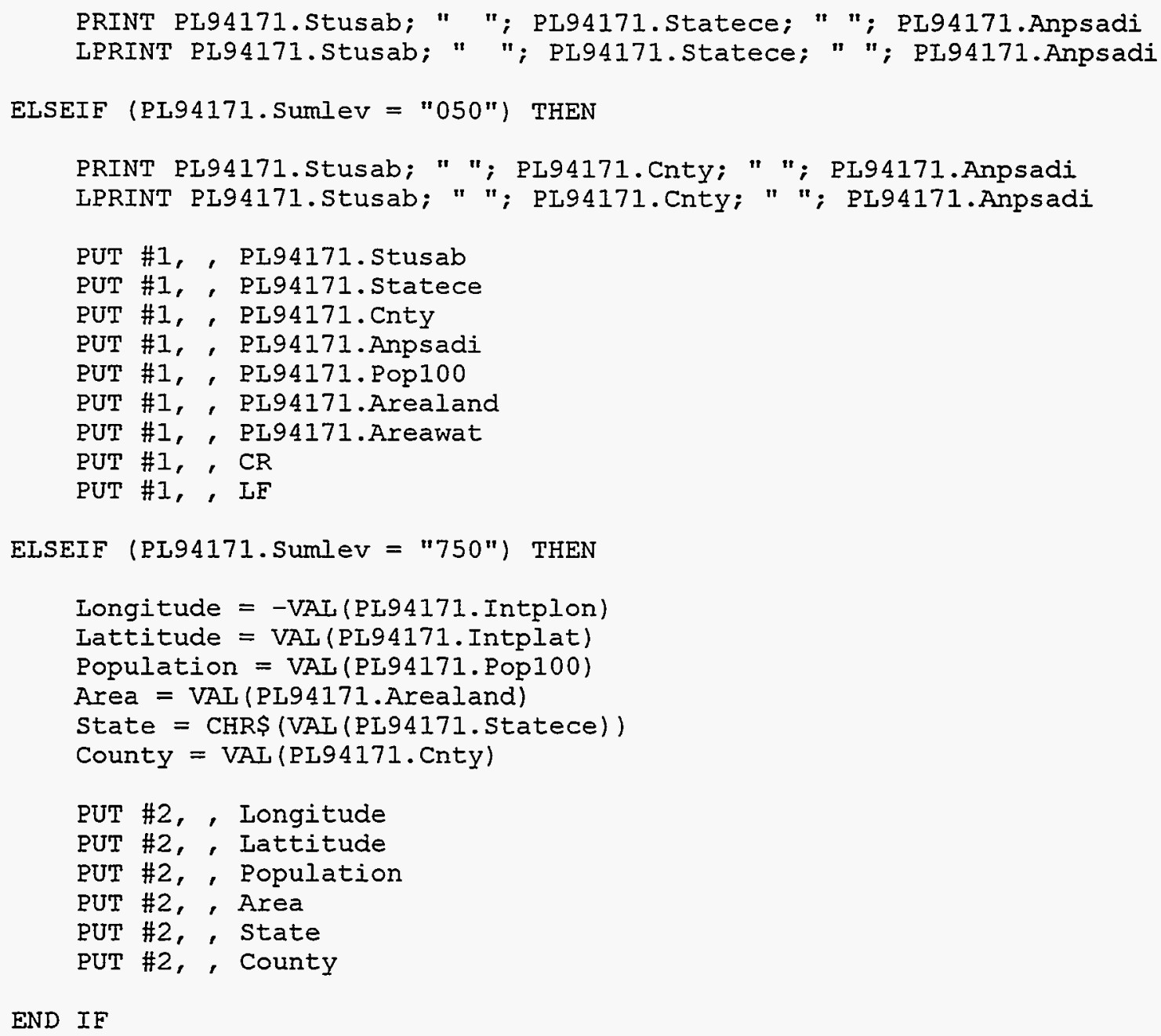


DECLARE SUB MakeCountyConvert ()

OPTION EXPLICIT

CONST MAXSTATE $=93$

CONST MAXCOUNTY $=840$

DIM Longitude AS LONG, Lattitude AS LONG

DIM Population AS LONG, Area AS LONG

DIM state AS STRING * 1, County AS INTEGER

DIM PresentState AS STRING * 1 , PresentCounty AS INTEGER

DIM Shortlongitude AS INTEGER, ShortLattitude AS INTEGER

DIM ShortPopulation AS INTEGER, LongArea AS LONG

DIM ShortCounty AS INTEGER

REDIM SHARED CountyConvert (MAXSTATE, MAXCOUNTY) AS INTEGER

CALI MakeCountyConvert

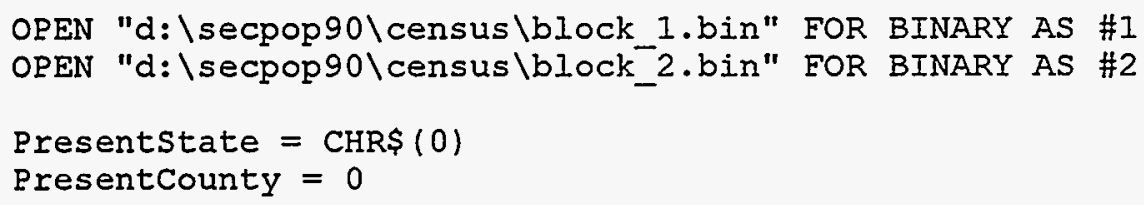

DO

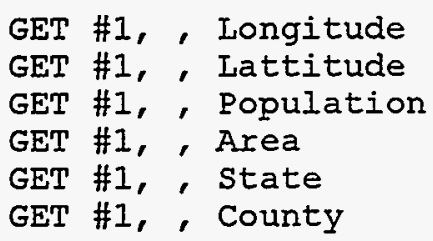

IF EOF(1) THEN

EXIT DO

ELSE

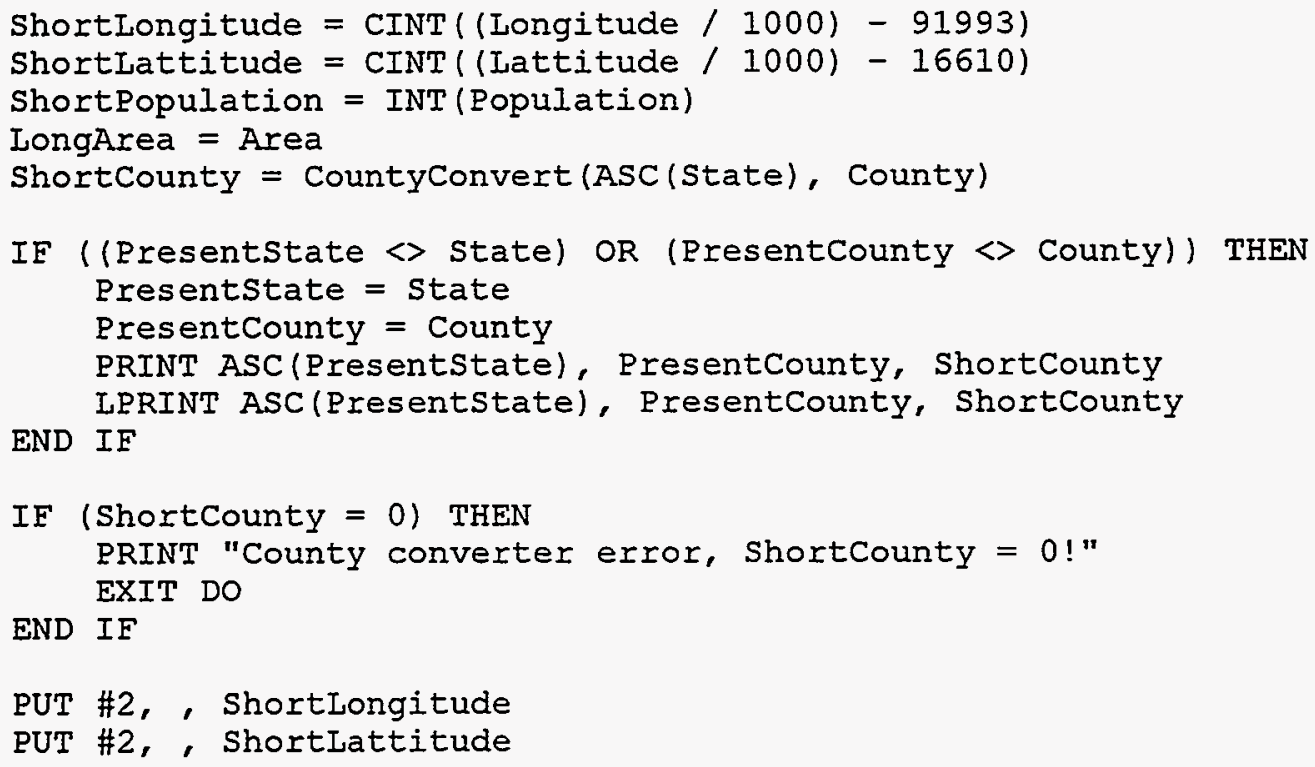


PUT \#2, , ShortPopulation

PUT \#2, , LongArea

PUT \#2, , ShortCounty

\section{END IF}

IOOP

CLOSE \#1, \#2

END

'Data for converting census/FIPS county designations to a single integer.

'The format is Census state Number, FIPS County Number, secPop90 County Number.

'There are ten counties per line except for the last Iine which only has one.

DATA 11, 1, 1, 11, 3, 2, 11, 5, 3, 11, 7, 4, 11, 9, 5, 11, 11, 6, 11, 13, 7, 11, 15, $8,11,17,9,11,19,10$

DATA 11, 21, 11, 11, 23, 12, 11, 25, 13, 11, 27, 14, 11, 29, 15, 11, 31, 16, 12, 1, $17,12,3,18,12,5,19,12,7,20$

DATA 12, 9, 21, 12, 11, 22, 12, 13, 23, 12, 15, 24, 12, 17, 25, 12, 19, 26, 13, 1, $27,13,3,28,13,5,29,13,7,30$

DATA 13, 9, 31, 13, 11, 32, 13, 13, 33, 13, 15, 34, 13, 17, 35, 13, 19, 36, 13, 21, $37,13,23,38,13,25,39,13,27,40$

DATA 14, 1, 41, 14, 3, 42, 14, 5, 43, 14, 7, 44, 14, 9, 45, 14, 11, 46, 14, 13, 47, $14,15,48,14,17,49,14,19,50$

DATA 14, 21, 51, 14, 23, 52, 14, 25, 53, 14, 27, 54, 15, 1, 55, 15, 3, 56, 15, 5, $57,15,7,58,15,9,59,16,1,60$

DATA $16,3,61,16,5,62,16,7,63,16,9,64,16,11,65,16,13,66,16,15,67$, $21,1,68,21,3,69,21,5,70$

DATA 21, 7, 71, 21, 9, 72, 21, 11, 73, 21, 13, 74, 21, 15, 75, 21, 17, 76, 21, 19, $77,21,21,78,21,23,79,21,25,80$

DATA 21, 27, 81, 21, 29, 82, 21, 31, 83, 21, 33, 84, 21, 35, 85, 21, 37, 86, 21, 39, $87,21,41,88,21,43,89,21,45,90$

DATA 21, 47, 91, 21, 49, 92, 21, 51, 93, 21, 53, 94, 21, 55, 95, 21, 57, 96, 21, 59, $97,21,61,98,21,63,99,21,65,100$

DATA 21, 67, 101, 21, 69, 102, 21, 71, 103, 21, 73, 104, 21, 75, 105, 21, 77, 106, $21,79,107,21,81,108,21,83,109,21,85,110$

DATA 21, 87, 111, 21, 89, 112, 21, 91, 113, 21, 93, 114, 21, 95, 115, 21, 97, 116, $21,99,117,21,101,118,21,103,119,21,105,120$

DATA 21, 107, 121, 21, 109, 122, 21, 111, 123, 21, 113, 124, 21, 115, 125, 21, 117, $126,21,119,127,21,121,128,21,123,129,22,1,130$

DATA $22,3,131,22,5,132,22,7,133,22,9,134,22,11,135,22,13,136,22$, $15,137,22,17,138,22,19,139,22,21,140$

DATA 22, 23, 141, 22, 25, 142, 22, 27, 143, 22, 29, 144, 22, 31, 145, 22, 33, 146, $22,35,147,22,37,148,22,39,149,22,41,150$

DATA 23, 1, 151, 23, 3, 152, 23, 5, 153, 23, 7, 154, 23, 9, 155, 23, 11, 156, 23, $13,157,23,15,158,23,17,159,23,19,160$

DATA 23, 21, 161, 23, 23, 162, 23, 25, 163, 23, 27, 164, 23, 29, 165, 23, 31, 166, $23,33,167,23,35,168,23,37,169,23,39,170$

DATA 23, 41, 171, 23, 43, 172, 23, 45, 173, 23, 47, 174, 23, 49, 175, 23, 51, 176, $23,53,177,23,55,178,23,57,179,23,59,180$

DATA 23, 61, 181, 23, 63, 182, 23, 65, 183, 23, 67, 184, 23, 69, 185, 23, 71, 186, $23,73,187,23,75,188,23,77,189,23,79,190$

DATA 23, 81, 191, 23, 83, 192, 23, 85, 193, 23, 87, 194, 23, 89, 195, 23, 91, 196, $23,93,197,23,95,198,23,97,199,23,99,200$

DATA 23, 101, 201, 23, 103, 202, 23, 105, 203, 23, 107, 204, 23, 109, 205, 23, 111, $206,23,113,207,23,115,208,23,117,209,23,119,210$

DATA 23, 121, 211, 23, 123, 212, 23, 125, 213, 23, 127, 214, 23, 129, 215, 23, 131, $216,23,133,217,31,1,218,31,3,219,31,5,220$

DATA 31, 7, 221, 31, 9, 222, 31, 11, 223, 31, 13, 224, 31, 15, 225, 31, 17, 226, 31, $19,227,31,21,228,31,23,229,31,25,230$ 
DATA 31, 27, 231, 31, 29, 232, 31, 31, 233, 31, 33, 234, 31, 35, 235, 31, 37, 236, $31,39,237,31,41,238,31,43,239,31,45,240$

DATA 31, 47, 241, 31, 49, 242, 31, 51, 243, 31, 53, 244, 31, 55, 245, 31, 57, 246, $31,59,247,31,61,248,31,63,249,31,65,250$

DATA 31, 67, 251, 31, 69, 252, 31, 71, 253, 31, 73, 254, 31, 75, 255, 31, 77, 256, $31,79,257,31,81,258,31,83,259,31,85,260$

DATA $31,87,261,31,89,262,31,91,263,31,93,264,31,95,265,31,97,266$, $31,99,267,31,101,268,31,103,269,31,105,270$

DATA 31, 107, 271, 31, 109, 272, 31, 111, 273, 31, 113, 274, 31, 115, 275, 31, 117, $276,31,119,277,31,121,278,31,123,279,31,125,280$

DATA $31,127,281,31,129,282,31,131,283,31,133,284,31,135,285,31,137$, $286,31,139,287,31,141,288,31,143,289,31,145,290$

DATA 31, 147, 291, 31, 149, 292, 31, 151, 293, 31, 153, 294, 31, 155, 295, 31, 157, $296,31,159,297,31,161,298,31,163,299,31,165,300$

DATA 31, 167, 301, 31, 169, 302, 31, 171, 303, 31, 173, 304, 31, 175, 305, 32, 1, $306,32,3,307,32,5,308,32,7,309,32,9,310$

DATA 32, 11, 311, 32, 13, 312, 32, 15, 313, 32, 17, 314, 32, 19, 315, 32, 21, 316, $32,23,317,32,25,318,32,27,319,32,29,320$

DATA $32,31,321,32,33,322,32,35,323,32,37,324,32,39,325,32,41,326$, $32,43,327,32,45,328,32,47,329,32,49,330$

DATA $32,51,331,32,53,332,32,55,333,32,57,334,32,59,335,32,61,336$, $32,63,337,32,65,338,32,67,339,32,69,340$

DATA $32,71,341,32,73,342,32,75,343,32,77,344,32,79,345,32,81,346$, $32,83,347,32,85,348,32,87,349,32,89,350$

DATA 32, 91, 351, 32, 93, 352, 32, 95, 353, 32, 97, 354, 32, 99, 355, 32, 101, 356, $32,103,357,32,105,358,32,107,359,32,109,360$

DATA 32, 111, 361, 32, 113, 362, 32, 115, 363, 32, 117, 364, 32, 119, 365, 32, 121, $366,32,123,367,32,125,368,32,127,369,32,129,370$

DATA 32, 131, 371, 32, 133, 372, 32, 135, 373, 32, 137, 374, 32, 139, 375, 32, 141, $376,32,143,377,32,145,378,32,147,379,32,149,380$

DATA 32, 151, 381, 32, 153, 382, 32, 155, 383, 32, 157, 384, 32, 159, 385, 32, 161, $386,32,163,387,32,165,388,32,167,389,32,169,390$

DATA 32, 171, 391, 32, 173, 392, 32, 175, 393, 32, 177, 394, 32, 179, 395, 32, 181, $396,32,183,397,33,1,398,33,3,399,33,5,400$

DATA 33, 7, 401, 33, 9, 402, 33, 11, 403, 33, 13, 404, 33, 15, 405, 33, 17, 406, 33, $19,407,33,21,408,33,23,409,33,25,410$

DATA 33, 27, 411, 33, 29, 412, 33, 31, 413, 33, 33, 414, 33, 35, 415, 33, 37, 416, $33,39,417,33,41,418,33,43,419,33,45,420$

DATA $33,47,421,33,49,422,33,51,423,33,53,424,33,55,425,33,57,426$, $33,59,427,33,61,428,33,63,429,33,65,430$

DATA $33,67,431,33,69,432,33,71,433,33,73,434,33,75,435,33,77,436$, $33,79,437,33,81,438,33,83,439,33,85,440$

DATA 33, 87, 441, 33, 89, 442, 33, 91, 443, 33, 93, 444, 33, 95, 445, 33, 97, 446, $33,99,447,33,101,448,33,103,449,33,105,450$

DATA 33, 107, 451, 33, 109, 452, 33, 111, 453, 33, 113, 454, 33, 115, 455, 33, 117, $456,33,119,457,33,121,458,33,123,459,33,125,460$

DATA 33, 127, 461, 33, 129, 462, 33, 131, 463, 33, 133, 464, 33, 135, 465, 33, 137, $466,33,139,467,33,141,468,33,143,469,33,145,470$

DATA 33, 147, 471, 33, 149, 472, 33, 151, 473, 33, 153, 474, 33, 155, 475, 33, 157, $476,33,159,477,33,161,478,33,163,479,33,165,480$

DATA 33, 167, 481, 33, 169, 482, 33, 171, 483, 33, 173, 484, 33, 175, 485, 33, 177, $486,33,179,487,33,181,488,33,183,489,33,185,490$

DATA 33, 187, 491, 33, 189, 492, 33, 191, 493, 33, 193, 494, 33, 195, 495, 33, 197, $496,33,199,497,33,201,498,33,203,499,34,1,500$

DATA 34, 3, 501, 34, 5, 502, 34, 7, 503, 34, 9, 504, 34, 11, 505, 34, 13, 506, 34, $15,507,34,17,508,34,19,509,34,21,510$

DATA 34, 23, 511, 34, 25, 512, 34, 27, 513, 34, 29, 514, 34, 31, 515, 34, 33, 516, $34,35,517,34,37,518,34,39,519,34,41,520$

DATA $34,43,521,34,45,522,34,47,523,34,49,524,34,51,525,34,53,526$, $34,55,527,34,57,528,34,59,529,34,61,530$

DATA $34,63,531,34,65,532,34,67,533,34,69,534,34,71,535,34,73,536$, $34,75,537,34,77,538,34,79,539,34,81,540$ 
DATA 34, 83, 541, 34, 85, 542, 34, 87, 543, 34, 89, 544, 34, 91, 545, 34, 93, 546, $34,95,547,34,97,548,34,99,549,34,101,550$

DATA 34, 103, 551, 34, 105, 552, 34, 107, 553, 34, 109, 554, 34, 111, 555, 34, 113, $556,34,115,557,34,117,558,34,119,559,34,121,560$

DATA 34, 123, 561, 34, 125, 562, 34, 127, 563, 34, 129, 564, 34, 131, 565, 34, 133, $566,34,135,567,34,137,568,34,139,569,34,141,570$

DATA 34, 143, 571, 34, 145, 572, 34, 147, 573, 34, 149, 574, 34, 151, 575, 34, 153, $576,34,155,577,34,157,578,34,159,579,34,161,580$

DATA 34, 163, 581, 34, 165, 582, 35, 1, 583, 35, 3, 584, 35, 5, 585, 35, 7, 586, 35, $9,587,35,11,588,35,13,589,35,15,590$

DATA 35, 17, 591, 35, 19, 592, 35, 21, 593, 35, 23, 594, 35, 25, 595, 35, 27, 596, $35,29,597,35,31,598,35,33,599,35,35,600$

DATA $35,37,601,35,39,602,35,41,603,35,43,604,35,45,605,35,47,606$, $35,49,607,35,51,608,35,53,609,35,55,610$

DATA 35, 57, 611, 35, 59, 612, 35, 61, 613, 35, 63, 614, 35, 65, 615, 35, 67, 616, $35,69,617,35,71,618,35,73,619,35,75,620$

DATA $35,77,621,35,78,622,35,79,623,35,81,624,35,83,625,35,85,626$, $35,87,627,35,89,628,35,91,629,35,93,630$

DATA 35, 95, 631, 35, 97, 632, 35, 99, 633, 35, 101, 634, 35, 103, 635, 35, 105,

$636,35,107,637,35,109,638,35,111,639,35,113,640$

DATA 35, 115, 641, 35, 117, 642, 35, 119, 643, 35, 121, 644, 35, 123, 645, 35, 125, $646,35,127,647,35,129,648,35,131,649,35,133,650$

DATA 35, 135, 651, 35, 137, 652, 35, 139, 653, 35, 141, 654, 41, 1, 655, 41, 3, 656, $41,5,657,41,7,658,41,9,659,41,11,660$

DATA 41, 13, 661, 41, 15, 662, 41, 17, 663, 41, 19, 664, 41, 21, 665, 41, 23, 666, $41,25,667,41,27,668,41,29,669,41,31,670$

DATA 41, 33, 671, 41, 35, 672, 41, 37, 673, 41, 39, 674, 41, 41, 675, 41, 43, 676, $41,45,677,41,47,678,41,49,679,41,51,680$

DATA 41, 53, 681, 41, 55, 682, 41, 57, 683, 41, 59, 684, 41, 61, 685, 41, 63, 686, $41,65,687,41,67,688,41,69,689,41,71,690$

DATA 41, 73, 691, 41, 75, 692, 41, 77, 693, 41, 79, 694, 41, 81, 695, 41, 83, 696, $41,85,697,41,87,698,41,89,699,41,91,700$

DATA 41, 93, 701, 41, 95, 702, 41, 97, 703, 41, 99, 704, 41, 101, 705, 41, 103, 706, $41,105,707,41,107,708,41,109,709,41,111,710$

DATA 41, 113, 711, 41, 115, 712, 41, 117, 713, 41, 119, 714, 41, 121, 715, 41, 123, $716,41,125,717,41,127,718,41,129,719,41,131,720$

DATA 41, 133, 721, 41, 135, 722, 41, 137, 723, 41, 139, 724, 41, 141, 725, 41, 143, $726,41,145,727,41,147,728,41,149,729,41,151,730$

DATA $41,153,731,41,155,732,41,157,733,41,159,734,41,161,735,41,163$, $736,41,165,737,41,167,738,41,169,739,41,171,740$

DATA 41, 173, 741, 42, 1, 742, 42, 3, 743, 42, 5, 744, 42, 7, 745, 42, 9, 746, 42, $11,747,42,13,748,42,15,749,42,17,750$

DATA $42,19,751,42,21,752,42,23,753,42,25,754,42,27,755,42,29,756$, $42,31,757,42,33,758,42,35,759,42,37,760$

DATA 42, 39, 761, 42, 41, 762, 42, 43, 763, 42, 45, 764, 42, 47, 765, 42, 49, 766, $42,51,767,42,53,768,42,55,769,42,57,770$

DATA 42, 59, 771, 42, 61, 772, 42, 63, 773, 42, 65, 774, 42, 67, 775, 42, 69, 776, $42,71,777,42,73,778,42,75,779,42,77,780$

DATA 42, 79, 781, 42, 81, 782, 42, 83, 783, 42, 85, 784, 42, 87, 785, 42, 89, 786, $42,91,787,42,93,788,42,95,789,42,97,790$

DATA 42, 99, 791, 42, 101, 792, 42, 103, 793,42, 105, 794, 42, 107, 795, 42, 109, $796,42,111,797,42,113,798,42,115,799,42,117,800$

DATA 42, 119, 801, 42, 121, 802, 42, 123, 803, 42, 125, 804, 42, 127, 805, 42, 129, $806,42,131,807,42,133,808,42,135,809,42,137,810$

DATA 42, 139, 811, 42, 141, 812, 42, 143, 813, 42, 145, 814, 42, 147, 815, 42, 149, $816,42,151,817,42,153,818,42,155,819,42,157,820$

DATA 42, 159, 821, 42, 161, 822, 42, 163, 823, 42, 165, 824, 42, 167, 825, 42, 169, $826,42,171,827,42,173,828,42,175,829,42,177,830$

DATA 42, 179, 831, 42, 181, 832, 42, 183, 833, 42, 185, 834, 42, 187, 835, 42, 189, $836,42,191,837,42,193,838,42,195,839,42,197,840$

DATA $43,1,841,43,3,842,43,5,843,43,7,844,43,9,845,43,11,846,43$, $13,847,43,15,848,43,17,849,43,19,850$ 
DATA $43,21,851,43,23,852,43,25,853,43,27,854,43,29,855,43,31,856$, $43,33,857,43,35,858,43,37,859,43,39,860$

DATA 43, 41, 861, 43, 43, 862, 43, 45, 863, 43, 47, 864, 43, 49, 865, 43, 51, 866, $43,53,867,43,55,868,43,57,869,43,59,870$

DATA 43, 61, 871, 43, 63, 872, 43, 65, 873, 43, 67, 874, 43, 69, 875, 43, 71, 876, $43,73,877,43,75,878,43,77,879,43,79,880$

DATA $43,81,881,43,83,882,43,85,883,43,87,884,43,89,885,43,91,886$, $43,93,887,43,95,888,43,97,889,43,99,890$

DATA $43,101,891,43,103,892,43,105,893,43,107,894,43,109,895,43,111$, $896,43,113,897,43,115,898,43,117,899,43,119,900$

DATA 43, 121, 901, 43, 123, 902, 43, 125, 903, 43, 127, 904, 43, 129, 905, 43, 131, $906,43,133,907,43,135,908,43,137,909,43,139,910$

DATA 43, 141, 911, 43, 143, 912, 43, 145, 913,43, 147, 914, 43, 149, 915, 43, 151, $916,43,153,917,43,155,918,43,157,919,43,159,920$

DATA 43, 161, 921, 43, 163, 922, 43, 165, 923, 43, 167, 924, 43, 169, 925, 43, 171, $926,43,173,927,43,175,928,43,177,929,43,179,930$

DATA 43, 181, 931, 43, 183, 932, 43, 185, 933, 43, 186, 934, 43, 187, 935, 43, 189, $936,43,195,937,43,197,938,43,199,939,43,201,940$

DATA $43,203,941,43,205,942,43,207,943,43,209,944,43,211,945,43,213$, $946,43,215,947,43,217,948,43,219,949,43,221,950$

DATA 43, 223, 951, 43, 225, 952, 43, 227, 953, 43, 229, 954, 43, 510, 955, 44, 1, $956,44,3,957,44,5,958,44,7,959,44,9,960$

DATA 44, 11, 961, 44, 13, 962, 44, 15,963, 44, 17, 964, 44, 19, 965, 44, 21, 966, $44,23,967,44,25,968,44,27,969,44,29,970$

DATA 44, 31, 971, 44, 33, 972, 44, 35, 973, 44, 37, 974, 44, 39, 975, 44, 41, 976, $44,43,977,44,45,978,44,47,979,44,49,980$

DATA 44, 51, 981, 44, 53, 982, 44, 55, 983, 44, 57, 984, 44, 59, 985, 44, 61, 986, $44,63,987,44,65,988,44,67,989,44,69,990$

DATA 44, 71, 991, 44, 73, 992, 44, 75, 993, 44, 77, 994, 44, 79, 995, 44, 81, 996, $44,83,997,44,85,998,44,87,999,44,89,1000$

DATA 44, 91, 1001,44, 93, 1002,44,95, 1003, 44, 97, 1004,44, 99, 1005, 44, 101, $1006,44,103,1007,44,105,1008,45,3,1009,45,5,1010$

DATA 45, 7, 1011, 45, 9, 1012, 45, 11, 1013, 45, 13, 1014, 45, 15, 1015, 45, 17, $1016,45,19,1017,45,21,1018,45,23,1019,45,25,1020$

DATA 45, 27, 1021, 45, 29, 1022, 45, 31, 1023, 45, 33, 1024, 45, 35, 1025, 45, 37, $1026,45,39,1027,45,41,1028,45,43,1029,45,45,1030$ DATA $45,47,1031,45,49,1032,45,51,1033,45,53,1034,45,55,1035,45,57$, $1036,45,59,1037,45,61,1038,45,63,1039,45,65,1040$ DATA 45, 67, 1041, 45, 69, 1042, 45, 71, 1043, 45, 73, 1044, 45, 75, 1045, 45, 77, $1046,45,79,1047,45,81,1048,45,83,1049,45,85,1050$ DATA $45,87,1051,45,89,1052,45,91,1053,45,93,1054,45,95,1055,45,97$, $1056,45,99,1057,45,101,1058,45,103,1059,45,105,1060$

DATA 45, 107, 1061, 45, 109, 1062, 45, 111, 1063, 45, 113, 1064, 45, 115, 1065, 45, $117,1066,45,119,1067,45,121,1068,45,123,1069,45,125,1070$

DATA 45, 127, 1071, 45, 129, 1072, 45, 135, 1073, 45, 137, 1074, 46, 1, 1075, 46, 3, $1076,46,5,1077,46,7,1078,46,9,1079,46,11,1080$

DATA $46,13,1081,46,15,1082,46,17,1083,46,19,1084,46,21,1085,46,23$, $1086,46,25,1087,46,27,1088,46,29,1089,46,31,1090$

DATA $46,33,1091,46,35,1092,46,37,1093,46,39,1094,46,41,1095,46,43$, $1096,46,45,1097,46,47,1098,46,49,1099,46,51,1100$ DATA $46,53,1101,46,55,1102,46,57,1103,46,59,1104,46,61,1105,46,63$, $1106,46,65,1107,46,67,1108,46,69,1109,46,71,1110$ DATA $46,73,1111,46,75,1112,46,77,1113,46,79,1114,46,81,1115,46,83$, $1116,46,85,1117,46,87,1118,46,89,1119,46,91,1120$

DATA $46,93,1121,46,95,1122,46,97,1123,46,99,1124,46,101,1125,46,103$, $1126,46,105,1127,46,107,1128,46,109,1129,46,111,1130$

DATA $46,113,1131,46,115,1132,46,117,1133,46,119,1134,46,121,1135,46$, $123,1136,46,125,1137,46,127,1138,46,129,1139,46,131,1140$

DATA $46,133,1141,46,135,1142,46,137,1143,46,139,1144,46,141,1145,46$, $143,1146,46,145,1147,46,147,1148,46,149,1149,46,151,1150$

DATA $46,153,1151,46,155,1152,46,157,1153,46,159,1154,46,161,1155,46$, $163,1156,46,165,1157,46,167,1158,46,169,1159,46,171,1160$ 
DATA 46, 173, 1161, 46, 175, 1162, 46, 177, 1163, 46, 179, 1164, 46, 181, 1165, 46, $183,1166,46,185,1167,47,1,1168,47,3,1169,47,5,1170$

DATA $47,7,1171,47,9,1172,47,11,1173,47,13,1174,47,15,1175,47,17$, $1176,47,19,1177,47,21,1178,47,23,1179,47,25,1180$

DATA $47,27,1181,47,29,1182,47,31,1183,47,33,1184,47,35,1185,47,37$, $1186,47,39,1187,47,41,1188,47,43,1189,47,45,1190$

DATA 47, 47, 1191, 47, 49, 1192, 47, 51, 1193,47, 53, 1194, 47, 55, 1195, 47, 57, $1196,47,59,1197,47,61,1198,47,63,1199,47,65,1200$

DATA $47,67,1201,47,69,1202,47,71,1203,47,73,1204,47,75,1205,47,77$, $1206,47,79,1207,47,81,1208,47,83,1209,47,85,1210$

DATA $47,87,1211,47,89,1212,47,91,1213,47,93,1214,47,95,1215,47,97$, $1216,47,99,1217,47,101,1218,47,103,1219,47,105,1220$

DATA $47,107,1221,47,109,1222,47,111,1223,47,113,1224,47,115,1225,47$, $117,1226,47,119,1227,47,121,1228,47,123,1229,47,125,1230$

DATA $47,127,1231,47,129,1232,47,131,1233,47,133,1234,47,135,1235,47$, $137,1236,47,139,1237,47,141,1238,47,143,1239,47,145,1240$

DATA $47,147,1241,47,149,1242,47,151,1243,47,153,1244,47,155,1245,47$, $157,1246,47,159,1247,47,161,1248,47,163,1249,47,165,1250$

DATA $47,167,1251,47,169,1252,47,171,1253,47,173,1254,47,175,1255,47$, $177,1256,47,179,1257,47,181,1258,47,183,1259,47,185,1260$

DATA $47,187,1261,47,189,1262,47,191,1263,47,193,1264,47,195,1265,47$, $197,1266,47,199,1267,47,201,1268,47,203,1269,47,205,1270$

DATA $47,207,1271,47,209,1272,51,1,1273,51,3,1274,51,5,1275,52,1$, $1276,52,3,1277,52,5,1278,52,9,1279,52,11,1280$

DATA $52,13,1281,52,15,1282,52,17,1283,52,19,1284,52,21,1285,52,23$, $1286,52,25,1287,52,27,1288,52,29,1289,52,31,1290$

DATA $52,33,1291,52,35,1292,52,37,1293,52,39,1294,52,41,1295,52,43$, $1296,52,45,1297,52,47,1298,52,510,1299,53,1,1300$

DATA $54,1,1301,54,3,1302,54,5,1303,54,7,1304,54,9,1305,54,11,1306$, $54,13,1307,54,15,1308,54,17,1309,54,19,1310$

DATA $54,21,1311,54,23,1312,54,25,1313,54,27,1314,54,29,1315,54,31$, $1316,54,33,1317,54,35,1318,54,36,1319,54,37,1320$

DATA $54,41,1321,54,43,1322,54,45,1323,54,47,1324,54,49,1325,54,51$, $1326,54,53,1327,54,57,1328,54,59,1329,54,61,1330$

DATA 54, 63, 1331, 54, 65, 1332, 54, 67, 1333, 54, 69, 1334, 54, 71, 1335, 54, 73, $1336,54,75,1337,54,77,1338,54,79,1339,54,81,1340$

DATA $54,83,1341,54,85,1342,54,87,1343,54,89,1344,54,91,1345,54,93$, $1346,54,95,1347,54,97,1348,54,99,1349,54,101,1350$

DATA 54, 103, 1351, 54, 105, 1352, 54, 107, 1353, 54, 109, 1354, 54, 111, 1355, 54, $113,1356,54,115,1357,54,117,1358,54,119,1359,54,121,1360$

DATA $54,125,1361,54,127,1362,54,131,1363,54,133,1364,54,135,1365,54$, $137,1366,54,139,1367,54,141,1368,54,143,1369,54,145,1370$

DATA 54, 147, 1371, 54, 149, 1372, 54, 153, 1373, 54, 155, 1374, 54, 157, 1375, 54, $159,1376,54,161,1377,54,163,1378,54,165,1379,54,167,1380$

DATA $54,169,1381,54,171,1382,54,173,1383,54,175,1384,54,177,1385,54$, $179,1386,54,181,1387,54,183,1388,54,185,1389,54,187,1390$

DATA $54,191,1391,54,193,1392,54,195,1393,54,197,1394,54,199,1395,54$, $510,1396,54,515,1397,54,520,1398,54,530,1399,54,540,1400$

DATA 54, 550, 1401, 54, 560, 1402, 54,570, 1403, 54, 580, 1404, 54, 590, 1405, 54, $595,1406,54,600,1407,54,610,1408,54,620,1409,54,630,1410$

DATA $54,640,1411,54,650,1412,54,660,1413,54,670,1414,54,678,1415,54$, $680,1416,54,683,1417,54,685,1418,54,690,1419,54,700,1420$

DATA 54, 710, 1421, 54, 720, 1422, 54,730, 1423, 54, 735, 1424, 54, 740, 1425, 54, $750,1426,54,760,1427,54,770,1428,54,775,1429,54,780,1430$

DATA $54,790,1431,54,800,1432,54,810,1433,54,820,1434,54,830,1435,54$, $840,1436,55,1,1437,55,3,1438,55,5,1439,55,7,1440$

DATA 55, 9, 1441, 55, 11, 1442, 55, 13, 1443, 55, 15, 1444, 55, 17, 1445, 55, 19, $1446,55,21,1447,55,23,1448,55,25,1449,55,27,1450$

DATA $55,29,1451,55,31,1452,55,33,1453,55,35,1454,55,37,1455,55,39$, $1456,55,41,1457,55,43,1458,55,45,1459,55,47,1460$

DATA 55, 49, 1461, 55, 51, 1462, 55, 53, 1463, 55, 55, 1464, 55, 57, 1465, 55, 59, $1466,55,61,1467,55,63,1468,55,65,1469,55,67,1470$ 
DATA 55, 69, 1471, 55, 71, 1472, 55, 73, 1473, 55, 75, 1474, 55, 77, 1475, 55, 79, $1476,55,81,1477,55,83,1478,55,85,1479,55,87,1480$ DATA 55, 89, 1481, 55, 91, 1482, 55, 93, 1483, 55, 95, 1484, 55, 97, 1485, 55, 99, $1486,55,101,1487,55,103,1488,55,105,1489,55,107,1490$

DATA 55, 109, 1491, 56, 1, 1492, 56, 3, 1493, 56, 5, 1494, 56, 7, 1495, 56, 9, 1496, $56,11,1497,56,13,1498,56,15,1499,56,17,1500$

DATA 56, 19, 1501, 56, 21, 1502, 56, 23, 1503, 56, 25, 1504, 56, 27, 1505, 56, 29, $1506,56,31,1507,56,33,1508,56,35,1509,56,37,1510$

DATA $56,39,1511,56,41,1512,56,43,1513,56,45,1514,56,47,1515,56,49$, $1516,56,51,1517,56,53,1518,56,55,1519,56,57,1520$ DATA 56, 59, 1521, 56, 61, 1522, 56, 63, 1523, 56, 65, 1524, 56, 67, 1525, 56, 69, $1526,56,71,1527,56,73,1528,56,75,1529,56,77,1530$

DATA $56,79,1531,56,81,1532,56,83,1533,56,85,1534,56,87,1535,56,89$, $1536,56,91,1537,56,93,1538,56,95,1539,56,97,1540$ DATA 56, 99, 1541, 56, 101, 1542, 56, 103, 1543, 56, 105, 1544, 56, 107, 1545, 56, $109,1546,56,111,1547,56,113,1548,56,115,1549,56,117,1550$

DATA 56, 119, 1551, 56, 121, 1552, 56, 123, 1553, 56, 125, 1554, 56, 127, 1555, 56, $129,1556,56,131,1557,56,133,1558,56,135,1559,56,137,1560$

DATA 56, 139, 1561, 56, 141, 1562, 56, 143, 1563, 56, 145, 1564, 56, 147, 1565, 56, $149,1566,56,151,1567,56,153,1568,56,155,1569,56,157,1570$

DATA $56,159,1571,56,161,1572,56,163,1573,56,165,1574,56,167,1575,56$, $169,1576,56,171,1577,56,173,1578,56,175,1579,56,177,1580$

DATA 56, 179, 1581, 56, 181, 1582, 56, 183, 1583, 56, 185, 1584, 56, 187, 1585, 56, $189,1586,56,191,1587,56,193,1588,56,195,1589,56,197,1590$

DATA 56, 199, 1591, 57, 1, 1592, 57, 3, 1593, 57, 5, 1594, 57, 7, 1595, 57, 9, 1596, $57,11,1597,57,13,1598,57,15,1599,57,17,1600$

DATA $57,19,1601,57,21,1602,57,23,1603,57,25,1604,57,27,1605,57,29$, $1606,57,31,1607,57,33,1608,57,35,1609,57,37,1610$

DATA 57, 39, 1611, 57, 41, 1612, 57, 43, 1613, 57, 45, 1614, 57, 47, 1615, 57, 49, $1616,57,51,1617,57,53,1618,57,55,1619,57,57,1620$ DATA $57,59,1621,57,61,1622,57,63,1623,57,65,1624,57,67,1625,57,69$, $1626,57,71,1627,57,73,1628,57,75,1629,57,77,1630$ DATA $57,79,1631,57,81,1632,57,83,1633,57,85,1634,57,87,1635,57,89$, $1636,57,91,1637,58,1,1638,58,3,1639,58,5,1640$

DATA $58,7,1641,58,9,1642,58,11,1643,58,13,1644,58,15,1645,58,17$, $1646,58,19,1647,58,21,1648,58,23,1649,58,25,1650$

DATA $58,27,1651,58,29,1652,58,31,1653,58,33,1654,58,35,1655,58,37$, $1656,58,39,1657,58,43,1658,58,45,1659,58,47,1660$

DATA $58,49,1661,58,51,1662,58,53,1663,58,55,1664,58,57,1665,58,59$, $1666,58,61,1667,58,63,1668,58,65,1669,58,67,1670$ DATA $58,69,1671,58,71,1672,58,73,1673,58,75,1674,58,77,1675,58,79$, $1676,58,81,1677,58,83,1678,58,85,1679,58,87,1680$

DATA $58,89,1681,58,91,1682,58,93,1683,58,95,1684,58,97,1685,58,99$, $1686,58,101,1687,58,103,1688,58,105,1689,58,107,1690$ DATA 58, 109, 1691, 58, 111, 1692, 58, 113, 1693, 58, 115, 1694, 58, 117, 1695, 58, $119,1696,58,121,1697,58,123,1698,58,125,1699,58,127,1700$

DATA $58,129,1701,58,131,1702,58,133,1703,58,135,1704,58,137,1705,58$, $139,1706,58,141,1707,58,143,1708,58,145,1709,58,147,1710$

DATA 58, 149, 1711, 58, 151, 1712, 58, 153, 1713, 58, 155, 1714, 58, 157, 1715, 58, $159,1716,58,161,1717,58,163,1718,58,165,1719,58,167,1720$

DATA 58, 169, 1721, 58, 171, 1722, 58, 173, 1723, 58, 175, 1724, 58, 177, 1725, 58, $179,1726,58,181,1727,58,183,1728,58,185,1729,58,187,1730$

DATA $58,189,1731,58,191,1732,58,193,1733,58,195,1734,58,197,1735,58$, $199,1736,58,201,1737,58,205,1738,58,207,1739,58,209,1740$

DATA $58,211,1741,58,213,1742,58,215,1743,58,217,1744,58,219,1745,58$, $221,1746,58,223,1747,58,225,1748,58,227,1749,58,229,1750$

DATA $58,231,1751,58,233,1752,58,235,1753,58,237,1754,58,239,1755,58$, $241,1756,58,243,1757,58,245,1758,58,247,1759,58,249,1760$

DATA 58, 251, 1761, 58, 253, 1762, 58, 255, 1763, 58, 257, 1764, 58, 259, 1765, 58, $261,1766,58,263,1767,58,265,1768,58,267,1769,58,269,1770$

DATA 58, 271, 1771, 58, 273, 1772, 58, 275, 1773, 58, 277, 1774, 58, 279, 1775, 58, $281,1776,58,283,1777,58,285,1778,58,287,1779,58,289,1780$ 
DATA 58, 291, 1781, 58, 293, 1782, 58, 295, 1783, 58, 297, 1784, 58, 299, 1785, 58, $301,1786,58,303,1787,58,305,1788,58,307,1789,58,309,1790$

DATA 58, 311, 1791, 58, 313, 1792, 58, 315, 1793, 58, 317, 1794, 58, 319, 1795, 58, $321,1796,59,1,1797,59,3,1798,59,5,1799,59,7,1800$

DATA $59,9,1801,59,11,1802,59,13,1803,59,15,1804,59,17,1805,59,19$, $1806,59,21,1807,59,23,1808,59,25,1809,59,27,1810$ DATA 59, 29, 1811, 59, 31, 1812, 59, 33, 1813, 59, 35, 1814, 59, 37, 1815, 59, 39, $1816,59,41,1817,59,43,1818,59,45,1819,59,47,1820$ DATA $59,49,1821,59,51,1822,59,53,1823,59,55,1824,59,57,1825,59,59$, $1826,59,61,1827,59,63,1828,59,65,1829,59,67,1830$ DATA 59, 69, 1831, 59, 71, 1832, 59, 73, 1833, 59, 75, 1834, 59, 77, 1835, 59, 79, $1836,59,81,1837,59,83,1838,59,85,1839,59,87,1840$

DATA 59, 89, 1841, 59, 91, 1842, 59, 93, 1843, 59, 95, 1844, 59, 97, 1845, 59, 99, $1846,59,101,1847,59,103,1848,59,105,1849,59,107,1850$

DATA 59, 109, 1851, 59, 111, 1852, 59, 113, 1853, 59, 115, 1854, 59, 117, 1855, 59, $119,1856,59,121,1857,59,123,1858,59,125,1859,59,127,1860$

DATA $59,129,1861,59,131,1862,59,133,1863,61,1,1864,61,3,1865,61,5$, $1866,61,7,1867,61,9,1868,61,11,1869,61,13,1870$

DATA 61, 15, 1871, 61, 17, 1872, 61, 19, 1873, 61, 21, 1874, 61, 23, 1875, 61, 25, $1876,61,27,1877,61,29,1878,61,31,1879,61,33,1880$ DATA $61,35,1881,61,37,1882,61,39,1883,61,41,1884,61,43,1885,61,45$, $1886,61,47,1887,61,49,1888,61,51,1889,61,53,1890$ DATA $61,55,1891,61,57,1892,61,59,1893,61,61,1894,61,63,1895,61,65$, $1896,61,67,1897,61,69,1898,61,71,1899,61,73,1900$ DATA 61, 75, 1901, 61, 77, 1902, 61, 79, 1903, 61, 81, 1904, 61, 83, 1905, 61, 85, $1906,61,87,1907,61,89,1908,61,91,1909,61,93,1910$ DATA 61, 95, 1911, 61, 97, 1912, 61, 99, 1913,61, 101, 1914, 61, 103, 1915, 61, $105,1916,61,107,1917,61,109,1918,61,111,1919,61,113,1920$

DATA 61, 115, 1921, 61, 117, 1922, 61, 119, 1923, 61, 121, 1924, 61, 123, 1925, 61, $125,1926,61,127,1927,61,129,1928,61,131,1929,61,133,1930$

DATA 61, 135, 1931, 61, 137, 1932, 61, 139, 1933, 61, 141, 1934, 61, 143, 1935, 61, $145,1936,61,147,1937,61,149,1938,61,151,1939,61,153,1940$

DATA 61, 155, 1941, 61, 157, 1942, 61, 159, 1943, 61, 161, 1944, 61, 163, 1945, 61, $165,1946,61,167,1947,61,169,1948,61,171,1949,61,173,1950$

DATA 61, 175, 1951, 61, 177, 1952, 61, 179, 1953, 61, 181, 1954, 61, 183, 1955, 61, $185,1956,61,187,1957,61,189,1958,61,191,1959,61,193,1960$

DATA 61, 195, 1961, 61, 197, 1962, 61, 199, 1963, 61, 201, 1964, 61, 203, 1965, 61, $205,1966,61,207,1967,61,209,1968,61,211,1969,61,213,1970$

DATA 61, 215, 1971, 61, 217, 1972, 61, 219, 1973, 61, 221, 1974, 61, 223, 1975, 61, $225,1976,61,227,1977,61,229,1978,61,231,1979,61,233,1980$

DATA 61, 235, 1981, 61, 237, 1982, 61, 239, 1983, 62, 1, 1984, 62, 3, 1985, 62, 5, $1986,62,7,1987,62,9,1988,62,11,1989,62,13,1990$

DATA $62,15,1991,62,17,1992,62,19,1993,62,21,1994,62,23,1995,62,25$, $1996,62,27,1997,62,29,1998,62,31,1999,62,33,2000$ DATA $62,35,2001,62,37,2002,62,39,2003,62,41,2004,62,43,2005,62,45$, $2006,62,47,2007,62,49,2008,62,51,2009,62,53,2010$ DATA $62,55,2011,62,57,2012,62,59,2013,62,61,2014,62,63,2015,62,65$, $2016,62,67,2017,62,69,2018,62,71,2019,62,73,2020$ DATA $62,75,2021,62,77,2022,62,79,2023,62,81,2024,62,83,2025,62,85$, $2026,62,87,2027,62,89,2028,62,91,2029,62,93,2030$

DATA 62, 95, 2031, 62, 97, 2032, 62, 99, 2033, 62, 101, 2034, 62, 103, 2035, 62, $105,2036,62,107,2037,62,109,2038,62,111,2039,62,113,2040$

DATA $62,115,2041,62,117,2042,62,119,2043,62,121,2044,62,123,2045,62$, $125,2046,62,127,2047,62,129,2048,62,131,2049,62,133,2050$

DATA $62,135,2051,62,137,2052,62,139,2053,62,141,2054,62,143,2055,62$, $145,2056,62,147,2057,62,149,2058,62,151,2059,62,153,2060$

DATA 62, 155, 2061, 62, 157, 2062, 62, 159, 2063, 62, 161, 2064, 62, 163, 2065, 62, $165,2066,62,167,2067,62,169,2068,62,171,2069,62,173,2070$

DATA $62,175,2071,62,177,2072,62,179,2073,62,181,2074,62,183,2075,62$, $185,2076,62,187,2077,62,189,2078,63,1,2079,63,3,2080$

DATA $63,5,2081,63,7,2082,63,9,2083,63,11,2084,63,13,2085,63,15$, $2086,63,17,2087,63,19,2088,63,21,2089,63,23,2090$ 
DATA 63, 25, 2091, 63, 27, 2092, 63, 29, 2093, 63, 31, 2094, 63, 33, 2095, 63, 35, $2096,63,37,2097,63,39,2098,63,41,2099,63,43,2100$ DATA 63, 45, 2101, 63, 47, 2102, 63, 49, 2103, 63, 51, 2104, 63, 53, 2105, 63, 55, $2106,63,57,2107,63,59,2108,63,61,2109,63,63,2110$ DATA $63,65,2111,63,67,2112,63,69,2113,63,71,2114,63,73,2115,63,75$, $2116,63,77,2117,63,79,2118,63,81,2119,63,83,2120$ DATA 63, 85, 2121, 63, 87, 2122, 63, 89, 2123, 63, 91, 2124, 63, 93, 2125, 63, 95, $2126,63,97,2127,63,99,2128,63,101,2129,63,103,2130$

DATA $63,105,2131,63,107,2132,63,109,2133,63,111,2134,63,113,2135,63$, $115,2136,63,117,2137,63,119,2138,63,121,2139,63,123,2140$

DATA 63, 125, 2141, 63, 127, 2142, 63, 129, 2143, 63, 131, 2144, 63, 133, 2145, 64, $1,2146,64,3,2147,64,5,2148,64,7,2149,64,9,2150$

DATA 64, 11, 2151, 64, 13, 2152, 64, 15, 2153, 64, 17, 2154, 64, 19, 2155, 64, 21, $2156,64,23,2157,64,25,2158,64,27,2159,64,29,2160$ DATA $64,31,2161,64,33,2162,64,35,2163,64,37,2164,64,39,2165,64,41$, $2166,64,43,2167,64,45,2168,64,47,2169,64,49,2170$ DATA $64,51,2171,64,53,2172,64,55,2173,64,57,2174,64,59,2175,64,61$, $2176,64,63,2177,64,65,2178,64,67,2179,64,69,2180$ DATA $64,71,2181,64,73,2182,64,75,2183,64,77,2184,64,79,2185,64,81$, $2186,64,83,2187,64,85,2188,64,87,2189,64,89,2190$ DATA $64,91,2191,64,93,2192,64,95,2193,64,97,2194,64,99,2195,64,101$, $2196,64,103,2197,64,105,2198,64,107,2199,64,109,2200$ DATA $64,111,2201,64,113,2202,64,115,2203,64,117,2204,64,119,2205,64$, $121,2206,64,123,2207,64,125,2208,64,127,2209,64,129,2210$

DATA $64,131,2211,64,133,2212,64,135,2213,64,137,2214,64,139,2215,64$, $141,2216,64,143,2217,64,145,2218,64,147,2219,64,149,2220$

DATA $64,151,2221,64,153,2222,64,155,2223,64,157,2224,64,159,2225,64$, $161,2226,64,163,2227,71,1,2228,71,3,2229,71,5,2230$

DATA 71, 7, 2231, 71, 9, 2232, 71, 11, 2233, 71, 13, 2234, 71, 15, 2235, 71, 17, $2236,71,19,2237,71,21,2238,71,23,2239,71,25,2240$

DATA $71,27,2241,71,29,2242,71,31,2243,71,33,2244,71,35,2245,71,37$, $2246,71,39,2247,71,41,2248,71,43,2249,71,45,2250$ DATA $71,47,2251,71,49,2252,71,51,2253,71,53,2254,71,55,2255,71,57$, $2256,71,59,2257,71,61,2258,71,63,2259,71,65,2260$ DATA $71,67,2261,71,69,2262,71,71,2263,71,73,2264,71,75,2265,71,77$, $2266,71,79,2267,71,81,2268,71,83,2269,71,85,2270$ DATA $71,87,2271,71,89,2272,71,91,2273,71,93,2274,71,95,2275,71,97$, $2276,71,99,2277,71,101,2278,71,103,2279,71,105,2280$

DATA $71,107,2281,71,109,2282,71,111,2283,71,113,2284,71,115,2285,71$, $117,2286,71,119,2287,71,121,2288,71,123,2289,71,125,2290$

DATA 71, 127, 2291, 71, 129, 2292, 71, 131, 2293, 71, 133, 2294, 71, 135, 2295, 71, $137,2296,71,139,2297,71,141,2298,71,143,2299,71,145,2300$

DATA 71, 147, 2301, 71, 149, 2302, 72, 1, 2303, 72, 3, 2304, 72, 5, 2305, 72, 7, $2306,72,9,2307,72,11,2308,72,13,2309,72,15,2310$

DATA 72, 17, 2311, 72, 19, 2312, 72, 21, 2313, 72, 23, 2314, 72, 25, 2315, 72, 27, $2316,72,29,2317,72,31,2318,72,33,2319,72,35,2320$

DATA $72,37,2321,72,39,2322,72,41,2323,72,43,2324,72,45,2325,72,47$, $2326,72,49,2327,72,51,2328,72,53,2329,72,55,2330$

DATA $72,57,2331,72,59,2332,72,61,2333,72,63,2334,72,65,2335,72,67$, $2336,72,69,2337,72,71,2338,72,73,2339,72,75,2340$ DATA $72,77,2341,72,79,2342,72,81,2343,72,83,2344,72,85,2345,72,87$, $2346,72,89,2347,72,91,2348,72,93,2349,72,95,2350$

DATA 72, 97, 2351, 72, 99, 2352, 72, 101, 2353, 72, 103, 2354, 72, 105, 2355, 72, $107,2356,72,109,2357,72,111,2358,72,113,2359,72,115,2360$

DATA $72,117,2361,72,119,2362,72,121,2363,72,123,2364,72,125,2365,72$, $127,2366,73,1,2367,73,3,2368,73,5,2369,73,7,2370$

DATA $73,9,2371,73,11,2372,73,13,2373,73,15,2374,73,17,2375,73,19$, $2376,73,21,2377,73,23,2378,73,25,2379,73,27,2380$

DATA $73,29,2381,73,31,2382,73,33,2383,73,35,2384,73,37,2385,73,39$, $2386,73,41,2387,73,43,2388,73,45,2389,73,47,2390$

DATA $73,49,2391,73,51,2392,73,53,2393,73,55,2394,73,57,2395,73,59$, $2396,73,61,2397,73,63,2398,73,65,2399,73,67,2400$ 
DATA 73, 69, 2401, 73, 71, 2402, 73, 73, 2403, 73, 75, 2404, 73, 77, 2405, 73, 79, $2406,73,81,2407,73,83,2408,73,85,2409,73,87,2410$

DATA $73,89,2411,73,91,2412,73,93,2413,73,95,2414,73,97,2415,73,99$, $2416,73,101,2417,73,103,2418,73,105,2419,73,107,2420$

DATA 73, 109, 2421, 73, 111, 2422, 73, 113, 2423, 73, 115, 2424, 73, 117, 2425, 73, $119,2426,73,121,2427,73,123,2428,73,125,2429,73,127,2430$

DATA 73, 129, 2431, 73, 131, 2432, 73, 133, 2433, 73, 135, 2434, 73, 137, 2435, 73, $139,2436,73,141,2437,73,143,2438,73,145,2439,73,147,2440$

DATA 73, 149, 2441, 73, 151, 2442, 73, 153, 2443, 74, 1, 2444, 74, 3, 2445, 74, 5, $2446,74,7,2447,74,9,2448,74,11,2449,74,13,2450$

DATA 74, 15, 2451, 74, 17, 2452, 74, 19, 2453, 74, 21, 2454, 74, 23, 2455, 74, 25, $2456,74,27,2457,74,29,2458,74,31,2459,74,33,2460$

DATA $74,35,2461,74,37,2462,74,39,2463,74,41,2464,74,43,2465,74,45$, $2466,74,47,2467,74,49,2468,74,51,2469,74,53,2470$

DATA $74,55,2471,74,57,2472,74,59,2473,74,61,2474,74,63,2475,74,65$, $2476,74,67,2477,74,69,2478,74,71,2479,74,73,2480$

DATA $74,75,2481,74,77,2482,74,79,2483,74,81,2484,74,83,2485,74,85$, $2486,74,87,2487,74,89,2488,74,91,2489,74,93,2490$

DATA $74,95,2491,74,97,2492,74,99,2493,74,101,2494,74,103,2495,74$, $105,2496,74,107,2497,74,109,2498,74,111,2499,74,113,2500$

DATA $74,115,2501,74,117,2502,74,119,2503,74,121,2504,74,123,2505,74$, $125,2506,74,127,2507,74,129,2508,74,131,2509,74,133,2510$

DATA 74, 135, 2511, 74, 137, 2512, 74, 139, 2513, 74, 141, 2514, 74, 143, 2515, 74, $145,2516,74,147,2517,74,149,2518,74,151,2519,74,153,2520$

DATA $74,155,2521,74,157,2522,74,159,2523,74,161,2524,74,163,2525,74$, $165,2526,74,167,2527,74,169,2528,74,171,2529,74,173,2530$

DATA 74, 175, 2531, 74, 177, 2532, 74, 179, 2533, 74, 181, 2534, 74, 183, 2535, 74, $185,2536,74,187,2537,74,189,2538,74,191,2539,74,193,2540$

DATA $74,195,2541,74,197,2542,74,199,2543,74,201,2544,74,203,2545,74$, $205,2546,74,207,2547,74,209,2548,74,211,2549,74,213,2550$

DATA $74,215,2551,74,217,2552,74,219,2553,74,221,2554,74,223,2555,74$, $225,2556,74,227,2557,74,229,2558,74,231,2559,74,233,2560$

DATA $74,235,2561,74,237,2562,74,239,2563,74,241,2564,74,243,2565,74$, $245,2566,74,247,2567,74,249,2568,74,251,2569,74,253,2570$

DATA 74, 255, 2571, 74, 257, 2572, 74, 259, 2573, 74, 261, 2574, 74, 263, 2575, 74, $265,2576,74,267,2577,74,269,2578,74,271,2579,74,273,2580$

DATA 74, 275, 2581, 74, 277, 2582, 74, 279, 2583, 74, 281, 2584, 74, 283, 2585, 74, $285,2586,74,287,2587,74,289,2588,74,291,2589,74,293,2590$

DATA 74, 295, 2591, 74, 297, 2592, 74, 299, 2593, 74, 301, 2594, 74, 303, 2595, 74, $305,2596,74,307,2597,74,309,2598,74,311,2599,74,313,2600$

DATA 74, 315, 2601, 74, 317, 2602, 74, 319, 2603, 74, 321, 2604, 74, 323, 2605, 74, $325,2606,74,327,2607,74,329,2608,74,331,2609,74,333,2610$

DATA $74,335,2611,74,337,2612,74,339,2613,74,341,2614,74,343,2615,74$, $345,2616,74,347,2617,74,349,2618,74,351,2619,74,353,2620$

DATA $74,355,2621,74,357,2622,74,359,2623,74,361,2624,74,363,2625,74$, $365,2626,74,367,2627,74,369,2628,74,371,2629,74,373,2630$

DATA $74,375,2631,74,377,2632,74,379,2633,74,381,2634,74,383,2635,74$, $385,2636,74,387,2637,74,389,2638,74,391,2639,74,393,2640$
DATA 74, 395, 2641, 74, 397, 2642, 74, 399, 2643, 74, 401, 2644, 74, 403, 2645, 74, $405,2646,74,407,2647,74,409,2648,74,411,2649,74,413,2650$

DATA $74,415,2651,74,417,2652,74,419,2653,74,421,2654,74,423,2655,74$, $425,2656,74,427,2657,74,429,2658,74,431,2659,74,433,2660$

DATA 74, 435, 2661, 74, 437, 2662, 74, 439, 2663,74,441, 2664, 74, 443, 2665, 74, $445,2666,74,447,2667,74,449,2668,74,451,2669,74,453,2670$

DATA 74, 455, 2671, 74, 457, 2672, 74, 459, 2673, 74, 461, 2674, 74, 463, 2675, 74, $465,2676,74,467,2677,74,469,2678,74,471,2679,74,473,2680$

DATA 74, 475, 2681, 74, 477, 2682, 74, 479, 2683, 74,481, 2684, 74, 483, 2685, 74, $485,2686,74,487,2687,74,489,2688,74,491,2689,74,493,2690$

DATA 74, 495, 2691, 74, 497, 2692, 74, 499, 2693, 74, 501, 2694, 74, 503, 2695, 74, $505,2696,74,507,2697,81,1,2698,81,3,2699,81,5,2700$

DATA $81,7,2701,81,9,2702,81,11,2703,81,13,2704,81,15,2705,81,17$, $2706,81,19,2707,81,21,2708,81,23,2709,81,25,2710$ 
DATA $81,27,2711,81,29,2712,81,31,2713,81,33,2714,81,35,2715,81,37$, $2716,81,39,2717,81,41,2718,81,43,2719,81,45,2720$ DATA $81,47,2721,81,49,2722,81,51,2723,81,53,2724,81,55,2725,81,57$, $2726,81,59,2727,81,61,2728,81,63,2729,81,65,2730$ DATA $81,67,2731,81,69,2732,81,71,2733,81,73,2734,81,75,2735,81,77$, $2736,81,79,2737,81,81,2738,81,83,2739,81,85,2740$ DATA $81,87,2741,81,89,2742,81,91,2743,81,93,2744,81,95,2745,81,97$, $2746,81,99,2747,81,101,2748,81,103,2749,81,105,2750$

DATA $81,107,2751,81,109,2752,81,111,2753,81,113,2754,82,1,2755,82,3$, $2756,82,5,2757,82,7,2758,82,9,2759,82,11,2760$

DATA $82,13,2761,82,15,2762,82,17,2763,82,19,2764,82,21,2765,82,23$, $2766,82,25,2767,82,27,2768,82,29,2769,82,31,2770$ DATA $82,33,2771,82,35,2772,82,37,2773,82,39,2774,82,41,2775,82,43$, $2776,82,45,2777,82,47,2778,82,49,2779,82,51,2780$ DATA $82,53,2781,82,55,2782,82,57,2783,82,59,2784,82,61,2785,82,63$, $2786,82,65,2787,82,67,2788,82,69,2789,82,71,2790$ DATA $82,73,2791,82,75,2792,82,77,2793,82,79,2794,82,81,2795,82,83$, $2796,82,85,2797,82,87,2798,83,1,2799,83,3,2800$ DATA $83,5,2801,83,7,2802,83,9,2803,83,11,2804,83,13,2805,83,15$, $2806,83,17,2807,83,19,2808,83,21,2809,83,23,2810$ DATA $83,25,2811,83,27,2812,83,29,2813,83,31,2814,83,33,2815,83,35$, $2816,83,37,2817,83,39,2818,83,41,2819,83,43,2820$ DATA $83,45,2821,84,1,2822,84,3,2823,84,5,2824,84,7,2825,84,9,2826$, $84,11,2827,84,13,2828,84,15,2829,84,17,2830$

DATA $84,19,2831,84,21,2832,84,23,2833,84,25,2834,84,27,2835,84,29$, $2836,84,31,2837,84,33,2838,84,35,2839,84,37,2840$ DATA $84,39,2841,84,41,2842,84,43,2843,84,45,2844,84,47,2845,84,49$, $2846,84,51,2847,84,53,2848,84,55,2849,84,57,2850$ DATA $84,59,2851,84,61,2852,84,63,2853,84,65,2854,84,67,2855,84,69$, $2856,84,71,2857,84,73,2858,84,75,2859,84,77,2860$ DATA $84,79,2861,84,81,2862,84,83,2863,84,85,2864,84,87,2865,84,89$, $2866,84,91,2867,84,93,2868,84,95,2869,84,97,2870$ DATA $84,99,2871,84,101,2872,84,103,2873,84,105,2874,84,107,2875,84$, $109,2876,84,111,2877,84,113,2878,84,115,2879,84,117,2880$

DATA $84,119,2881,84,121,2882,84,123,2883,84,125,2884,85,1,2885,85,3$, $2886,85,5,2887,85,6,2888,85,7,2889,85,9,2890$

DATA $85,11,2891,85,13,2892,85,15,2893,85,17,2894,85,19,2895,85,21$, $2896,85,23,2897,85,25,2898,85,27,2899,85,28,2900$ DATA $85,29,2901,85,31,2902,85,33,2903,85,35,2904,85,37,2905,85,39$, $2906,85,41,2907,85,43,2908,85,45,2909,85,47,2910$ DATA $85,49,2911,85,51,2912,85,53,2913,85,55,2914,85,57,2915,85,59$, $2916,85,61,2917,86,1,2918,86,3,2919,86,5,2920$

DATA $86,7,2921,86,9,2922,86,11,2923,86,12,2924,86,13,2925,86,15$, $2926,86,17,2927,86,19,2928,86,21,2929,86,23,2930$

DATA $86,25,2931,86,27,2932,87,1,2933,87,3,2934,87,5,2935,87,7,2936$, $87,9,2937,87,11,2938,87,13,2939,87,15,2940$

DATA 87, 17, 2941, 87, 19, 2942, 87, 21, 2943,87, 23, 2944, 87, 25, 2945, 87, 27, $2946,87,29,2947,87,31,2948,87,33,2949,87,35,2950$ DATA $87,37,2951,87,39,2952,87,41,2953,87,43,2954,87,45,2955,87,47$, $2956,87,49,2957,87,51,2958,87,53,2959,87,55,2960$

DATA $87,57,2961,88,1,2962,88,3,2963,88,5,2964,88,7,2965,88,9,2966$, $88,11,2967,88,13,2968,88,15,2969,88,17,2970$

DATA $88,19,2971,88,21,2972,88,23,2973,88,27,2974,88,29,2975,88,31$, $2976,88,33,2977,88,510,2978,91,1,2979,91,3,2980$

DATA 91, 5, 2981, 91, 7, 2982, 91, 9, 2983, 91, 11, 2984, 91, 13, 2985, 91, 15, $2986,91,17,2987,91,19,2988,91,21,2989,91,23,2990$ DATA 91, 25, 2991, 91, 27, 2992, 91, 29, 2993, 91, 31, 2994, 91, 33, 2995, 91, 35, $2996,91,37,2997,91,39,2998,91,41,2999,91,43,3000$ DATA 91, 45, 3001, 91, 47, 3002, 91, 49, 3003, 91, 51, 3004, 91, 53, 3005, 91, 55, $3006,91,57,3007,91,59,3008,91,61,3009,91,63,3010$ DATA 91, 65, 3011, 91, 67, 3012, 91, 69, 3013, 91, 71, 3014, 91, 73, 3015, 91, 75, $3016,91,77,3017,92,1,3018,92,3,3019,92,5,3020$ 
DATA 92, 7, 3021, 92, 9, 3022, 92, 11, 3023, 92, 13, 3024, 92, 15, 3025, 92, 17, $3026,92,19,3027,92,21,3028,92,23,3029,92,25,3030$

DATA $92,27,3031,92,29,3032,92,31,3033,92,33,3034,92,35,3035,92,37$, $3036,92,39,3037,92,41,3038,92,43,3039,92,45,3040$

DATA 92, 47, 3041, 92, 49, 3042, 92, 51, 3043, 92, 53, 3044, 92, 55, 3045, 92, 57, $3046,92,59,3047,92,61,3048,92,63,3049,92,65,3050$

DATA 92, 67, 3051, 92, 69, 3052, 92, 71, 3053, 93, 1, 3054, 93, 3, 3055, 93, 5, $3056,93,7,3057,93,9,3058,93,11,3059,93,13,3060$

DATA 93, 15, 3061, 93, 17, 3062, 93, 19, 3063, 93, 21, 3064, 93, 23, 3065, 93, 25, $3066,93,27,3067,93,29,3068,93,31,3069,93,33,3070$

DATA 93, 35, 3071, 93, 37, 3072, 93, 39, 3073, 93, 41, 3074, 93, 43, 3075, 93, 45, $3076,93,47,3077,93,49,3078,93,51,3079,93,53,3080$

DATA 93, 55, 3081, 93, 57, 3082,93, 59, 3083, 93, 61, 3084, 93, 63, 3085, 93, 65, $3086,93,67,3087,93,69,3088,93,71,3089,93,73,3090$

DATA 93, 75, 3091, 93, 77, 3092, 93, 79, 3093, 93, 81, 3094, 93, 83, 3095, 93, 85, $3096,93,87,3097,93,89,3098,93,91,3099,93,93,3100$

DATA 93, 95, 3101, 93, 97, 3102, 93,99, 3103, 93, 101, 3104, 93, 103, 3105, 93, $105,3106,93,107,3107,93,109,3108,93,111,3109,93,113,3110$

DATA 93, 115, 3111

SUB Makecountyconvert ()

CONST COUNTYCOUNT $=3111$

DIM i AS INTEGER, $j$ AS INTEGER

DIM State AS INTEGER, County AS INTEGER, CountyNumber AS INTEGER

FOR $i=1$ TO MAXSTATE STEP 1

FOR $j=1$ TO MAXCOUNTY STEP 1 NEXT j Countyconvert $(i, j)=0$

NEXT i

FOR $i=1$ TO COUNTYCOUNT STEP 1

READ state, county, CountyNumber NEXT i Countyconvert (State, County) = CountyNumber

END SUB 


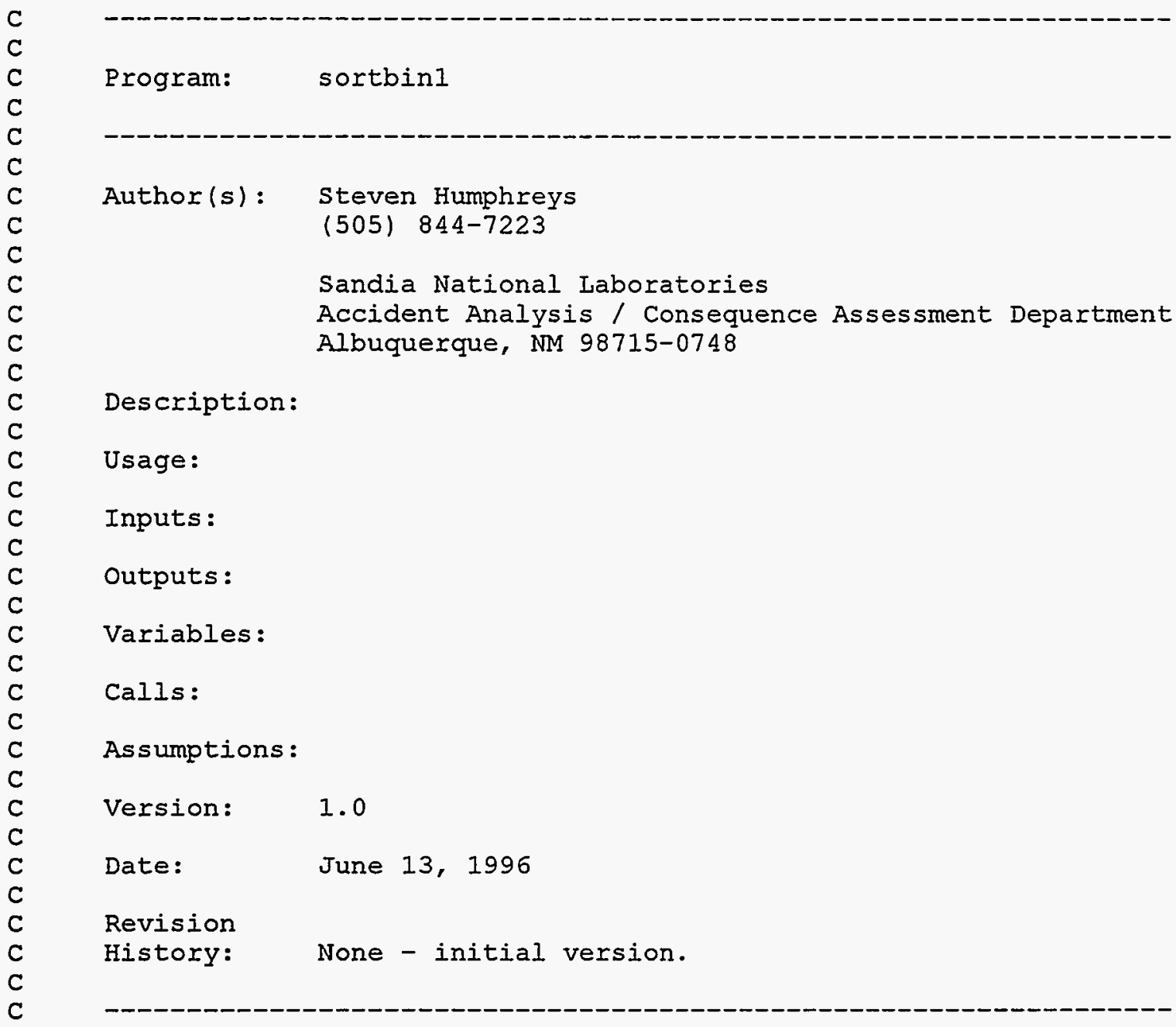

program sortbin 1

integer BINMIN, BINMAX, BINCOUNT, FIRSTUNIT, RECORDSMAX

parameter

1

BINMIN $=-26$,

BINMAX $=33$,

BINCOUNT $=30$,

FIRSTUNIT = 10,

)

RECORDSMAX $=6930787$

character * 17 FileName

integer $i, j$, Block_2Unit, Bin(BINMIN : BINMAX)

integer LongitudeBin, Area

integer * 2 Longitude, Lattitude, Population, CountyCode

$j=$ FIRSTUNIT

do $i=$ BINMIN, BINMAX, 2

$\operatorname{Bin}(i)=j$

$\operatorname{Bin}(i+1)=j$ 


$$
\begin{aligned}
& \text { write }(*, *) i, i+1, j \\
& j=j+1
\end{aligned}
$$

end do

$$
j=\text { BINMIN }
$$

do $i=$ FIRSTUNIT, BINCOUNT + FIRSTUNIT - 1, 1

write (FileName, '(a, i2.2, a)') 'sortlblock', i, '.bin' write (*,' '(2i4, $1 x, a) ')$ Bin $(j), \operatorname{Bin}(j+1)$, Filename

end do

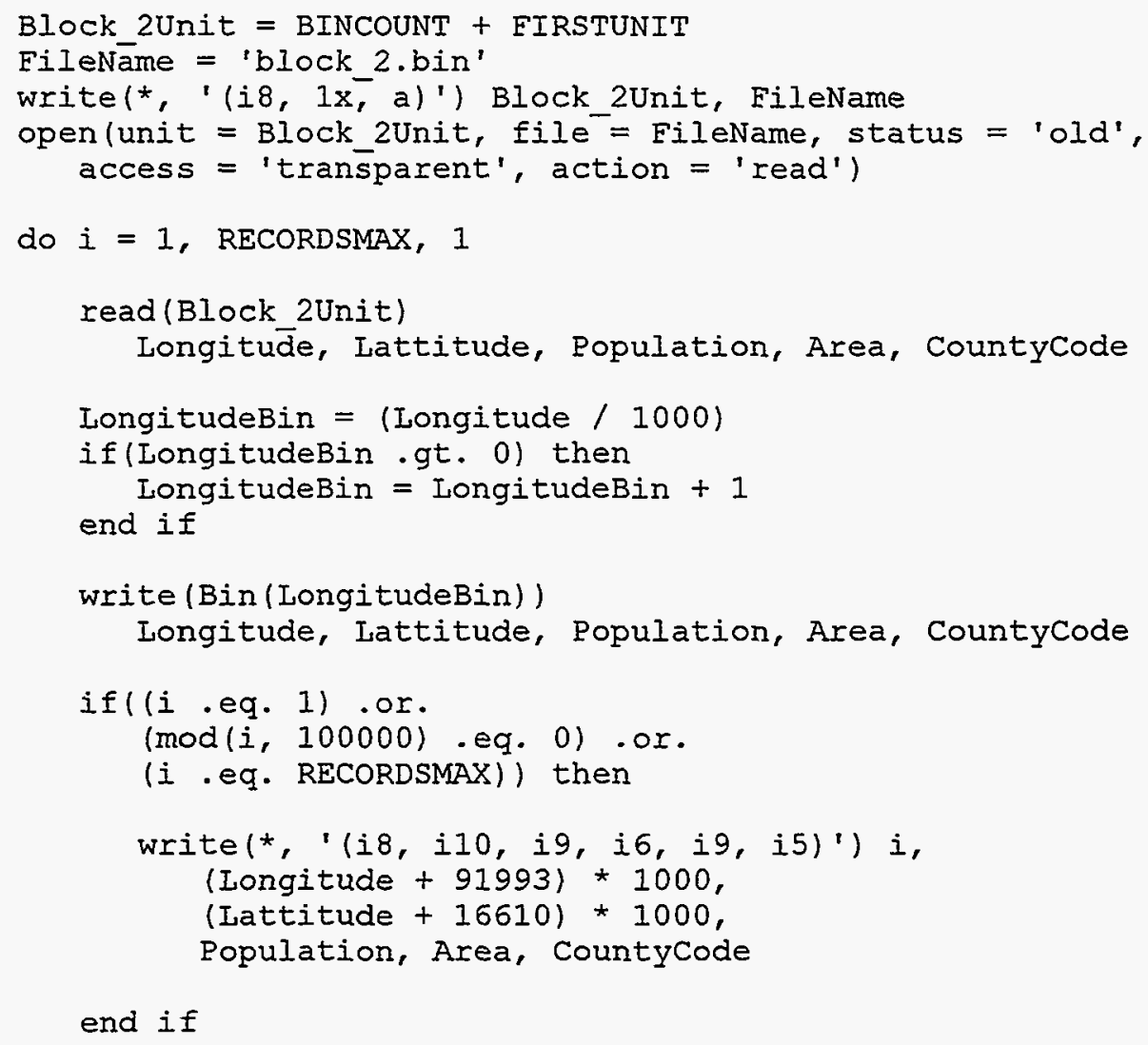


C

C

C

C

$\mathrm{C}$

C

$\mathrm{C}$

C

C

C

C

C

C

C

C

C

C

C

C

Author (s): Steven Humphreys

(505) $844-7223$

Sandia National Laboratories

Accident Analysis / Consequence Assessment Department Albuquerque, NM 98715-0748

Description:

Usage:

Inputs :

Outputs :

Variables:

Calls :

Assumptions :

Version: $\quad 1.0$

Date: June 13, 1996

Revision

History: None - initial version.

program sortbin2

integer EOF, BINCOUNT, FIRSTUNIT, RECORDSMAX

parameter

(

$\begin{array}{llr}\text { EOF } & = & -1, \\ \text { BINCOUNT } & = & 30, \\ \text { FIRSTUNIT } & = & 10, \\ \text { RECORDSMAX } & =553652\end{array}$

Define the varibles for determining the io status of the file operations we'll be performing. Note: this is not very portable code. It depends on specific io status codes to mean certain things, eg. -1 means an end of file. It also depends on the order of the most significant and least significant bytes of the two byte integer io status. Finally it uses the two integer * size functions which are not standard f77 fortran.

integer * 2 iostatus

integer * 1 iostatusByte (2)

equivalence (iostatus, iostatusByte) 


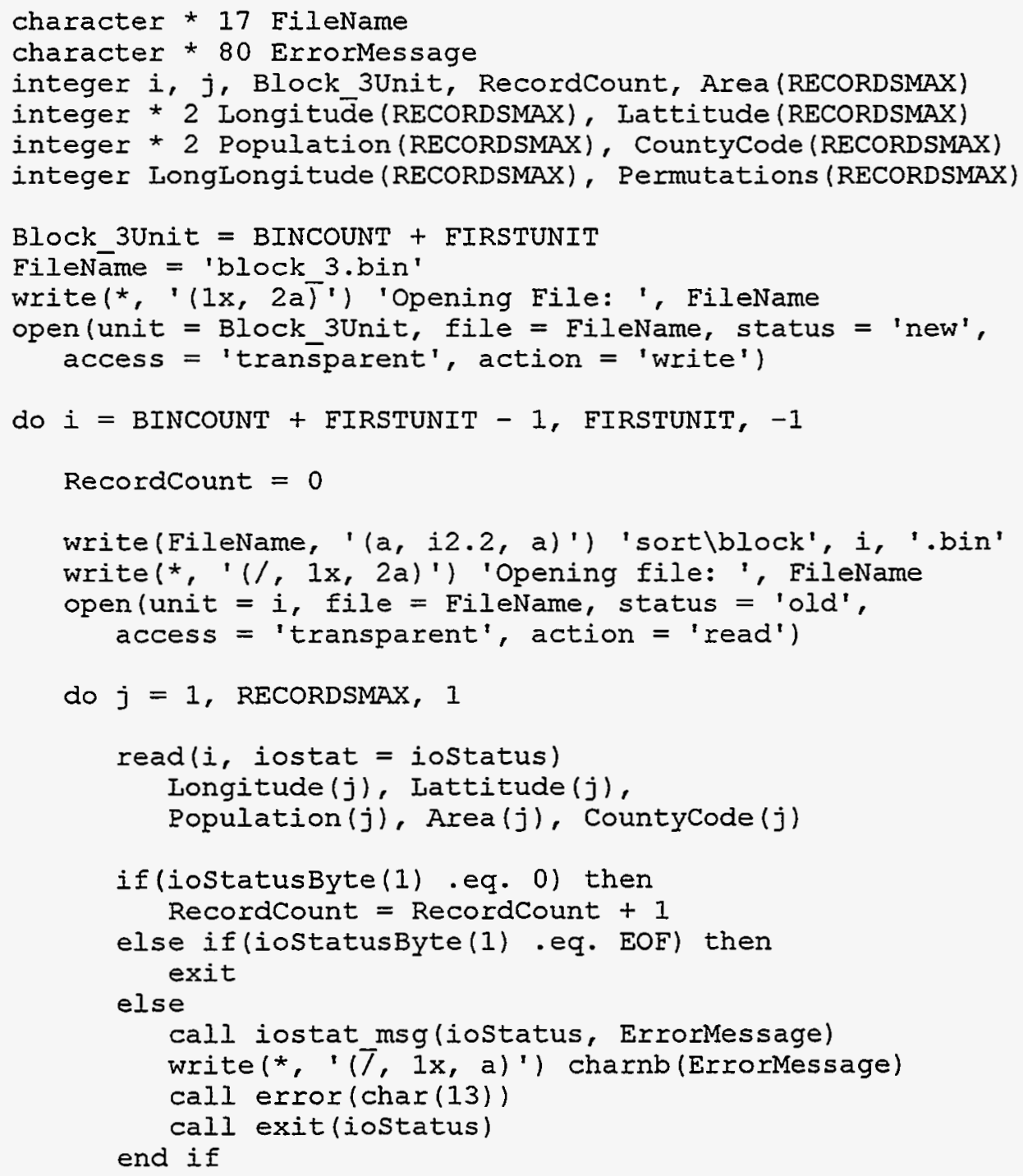


end do

call svigp(RecordCount, Longlongitude, Longlongitude, Permutations)

write(*,' '(1x, 2a)') 'Saving file: ', FileName

do $j=$ RecordCount; $1,-1$

write (Block 3Unit)

Longitude (Permutations (j)),

Lattitude (Permutations $(j)$ ),

Population (Permutations (j)),

Area (Permutations (j)),

CountyCode (Permutations (j))

if $((j$.eq. 1$)$.or. $(\bmod (j, 100000)$.eq. 0$))$ then write (*, '(i8, i10, i9, i6, i9, i5)')

Permutations $(j)$,

(Longitude (Permutations $(j))+91993) * 1000$,

(Lattitude (Permutations $(j))+16610) * 1000$,

Population (Permutations $(j))$,

Area (Permutations (j)),

end if

CountyCode (Permutations (j))

end do

end do

writel*, '(/, 1x, a)') 'Closing file: block_3.bin'

close (Block_3Unit)

end

C 
Block 4Unit $=$ FIRSTUNIT +1

Eilename $=$ 'Block $4 . \mathrm{bin}$ '

write (*, '(i8, $1 x^{-}$, a)') Block 4Unit, FileName

open (unit $=$ Block 4 Unit, file $=$ FileName, status $=$ 'new',

Duplicatecount $=0$

MaxPopulation $=0$

MaxArea $=0$

read (Block 3Unit)

PresentIongitude, PresentLattitude,

PresentPopulation, PresentArea, PresentCountycode

do $i=2$, RECORDSMAX, 1

$\&$

$\&$

read (Block 3Unit)

Iongitude,e, Lattitude, Population, Area, CountyCode

if ((PresentIongitude .eq. Longitude) . and.

(Presentlattitude .eq. Lattitude) . and.

(PresentCountycode .eq. Countycode)) then

Duplicatecount $=$ DuplicateCount +1

PresentPopulation = PresentPopulation + Population

PresentArea $=$ PresentArea + Area

else

write (Block_4Unit)

PresentIōngitude, Presentlattitude,

PresentPopulation, PresentArea, PresentCountycode

PresentLongitude $=$ Longitude

Presentlattitude $=$ Lattitude

PresentPopulation = Population

PresentArea = Area

PresentCountycode $=$ Countycode

end if

if(PresentPopulation .gt. MaxPopulation) then

MaxPopulation = PresentPopulation

end if

if(PresentArea .gt. MaxArea) then

MaxArea $=$ PresentArea

end if

if $((\bmod (i, 100000)$.eq. 0) .or. (i .eq. RECORDSMAX)) then

write (*, ' $\left.(1 \mathrm{x}, 2(\mathrm{a}, \mathrm{i} 8))^{\prime}\right)$

'Record =', i,

' Duplicate Count =', Duplicatecount write (*, '(1x, 2(a, i8))')

'Max Population $=$ ', MaxPopulation,

- Max Area = ', MaxArea

end if

end do

write (Block 4Unit) 
\& PresentIongitude, Presentlattitude,

\& PresentPopulation, PresentArea, PresentCountyCode

close (Block_3Unit)

close (Block_4Unit)

end

C 


\section{APPENDIX H - TEST PROGRAM LISTINGS}

FIIEHAME: TEST1.BAS

\section{OPTION EXPLICIT}

DIM Longitude AS LONG, Iattitude AS LONG

DIM Population AS LONG, Area AS LONG

DIM state AS STRING * 1 , County AS INTEGER

DIM Presentstate AS STRING * 1, PresentCounty AS INTEGER

DIM statePopulation AS LONG, CountyPopulation AS LONG

DIM stateArea AS LONG, CountyArea AS LONG

OPEN "block 1.bin" FOR BINARY AS \#1

OPEN "testoutt1.txt" FOR OUTPUT AS \#2 LEN = 32767

Presentstate $=\operatorname{CHR} \$(0)$

PresentCounty $=0$

statepopulation $=0$

Countypopulation $=0$

stateArea $=0$

CountyArea $=0$

DO

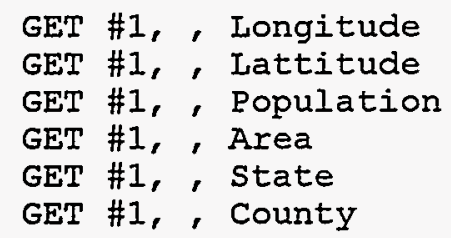

IF (EOF (1)) THEN

EXIT DO

ELSEIE ((Presentstate $<>$ State) AND (ASC (PresentState) $<>0)$ ) THEN

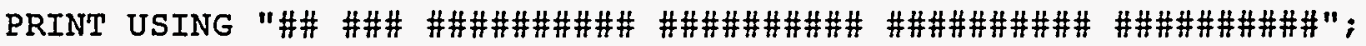
ASC (Presentstate); PresentCounty; CountyPopulation; CountyArea; StatePopulation; stateArea

PRINT \#2, USING "\#\# \#\#\# \#\#\#\#\#\#\#\#\# \#\#\#\#\#\#\#\#\#\#\#\#\#\#\#\#\#\#\#\#\#\#\#\#\#"; ASC(PresentState); PresentCounty; CountyPopulation; CountyArea; StatePopulation; StateArea

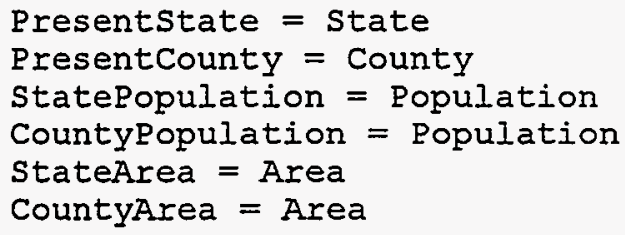

ELSEIF ((PresentCounty $<>$ County) AND (PresentCounty $<>0)$ ) THEN

PRINT USING "\#\# \#\#\# \#\#\#\#\#\#\#\#\# \#\#\#\#\#\#\#\#"; ASC(PresentState); PresentCounty; CountyPopulation; CountyArea

PRINT \#2, USING "\#\# \#\#\# \#\#\#\#\#\#\#\#\# \#\#\#\#\#\#\#\#\#"; ASC(Presentstate); PresentCounty; CountyPopulation; CountyArea

Presentstate = state 
Presentcounty = County

StatePopulation = statePopulation + Population

CountyPopulation = Population

StateArea = stateArea + Area

CountyArea $=$ Area

ELSE

Presentstate $=$ state

Presentcounty $=$ county

StatePopulation = StatePopulation + Population

CountyPopulation = CountyPopulation + Population

StateArea = stateArea + Area

CountyArea $=$ CountyArea + Area

END IF

LOOP

END 
OPTION EXPLICIT

CONST MAXRECORDS $=6930787$

DIM Longitude AS LONG, Lattitude AS LONG

DIM Population AS LONG, Area AS LONG

DIM state AS STRING * 1, County AS INTEGER

DIM ShortLongitude AS INTEGER, ShortLattitude AS INTEGER

DIM ShortPopulation AS INTEGER, LongArea AS IONG

DIM Countycode AS INTEGER

DIM RecordNumber AS LONG

OPEN "block 1.bin" FOR BINARY AS \#1

OPEN "block-2.bin" FOR BINARY AS \#2

GET \#1, , Longitude

GET \#1, , Lattitude

GET \#1, , Population

GET \#I, , Area

GET \#I, , State

GET \#I, , County

GET \#2, , ShortLongitude

GET \#2, , Shortlattitude

GET \#2, , ShortPopulation

GET \#2, , LongArea

GET \#2, , Countycode

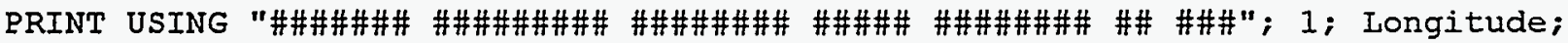
Lattitude; Population; Area; ASC(State); County

PRINT USING "\#\#\#\#\#\# \#\#\#\#\#\#\#\# \#\#\#\#\#\#\# \#\#\#\#\# \#\#\#\#\#\#\#\#\#\#\#\#"; 1;

((ShortLongitude + 91993) * 1000); ((ShortLattitude + 16610\&) * 1000);

ShortPopulation; LongArea; CountyCode

PRINT

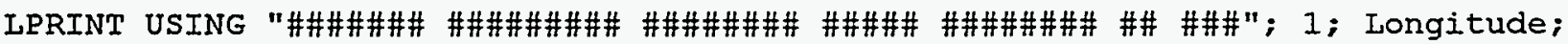

Iattitude; Population; Area; AsC(State); County

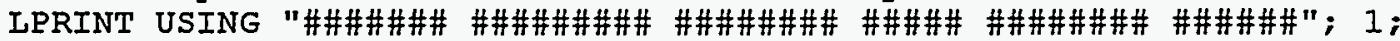

((ShortLongitude + 91993) * 1000); ((ShortLattitude + 16610\&) * 1000);

ShortPopulation; LongArea; CountyCode LPRINT

FOR RecordNumber $=100000$ TO MAXRECORDS STEP 100000

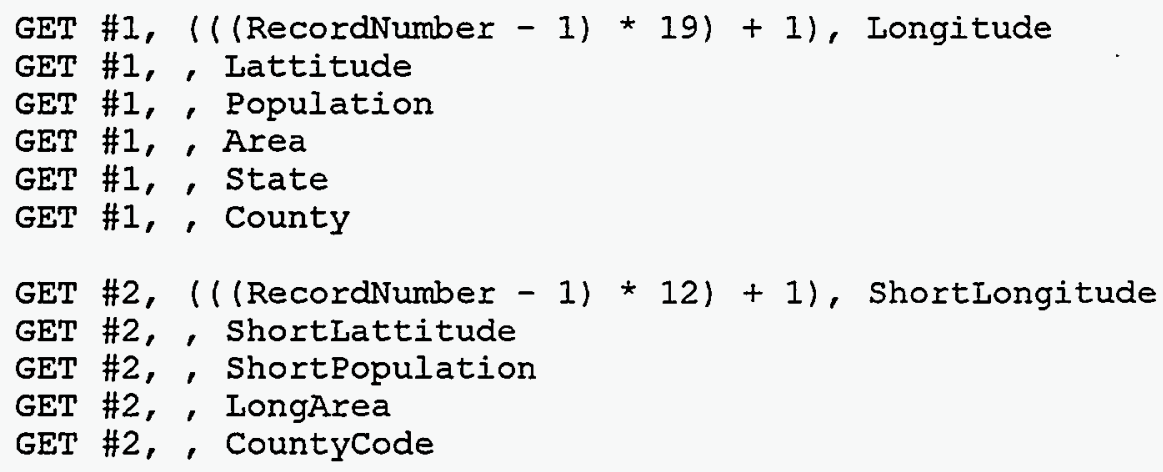




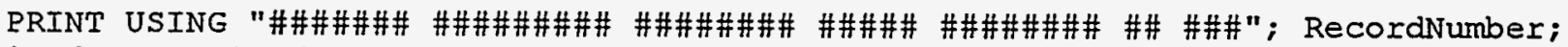
Longitude; Lattitude; Population; Area; ASC(State); County

PRINT USING "\#\#\#\#\#\# \#\#\#\#\#\#\#\# \#\#\#\#\#\#\# \#\#\#\#\# \#\#\#\#\#\#\# \#\#\#\#\#"; RecordNumber;

((ShortIongitude + 91993) * 1000); ((ShortLattitude + 16610\&)* 1000);

ShortPopulation; LongArea; Countycode PRINT

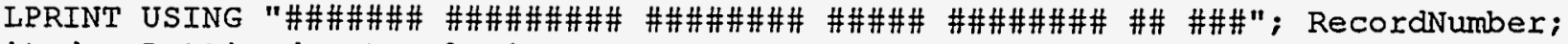
Longitude; Lattitude; Population; Area; ASC(State); County

IPRINT USING "\#\#\#\#\#\#\#\#\#\#\#\#\#\# \#\#\#\#\#\#\# \#\#\#\# \#\#\#\#\#\#\# \#\#\#\#\#"; RecordNumber; ((Shortlongitude + 91993) * 1000); ((ShortLattitude + 16610\&) * 1000);

ShortPopulation; LongArea; CountyCode LPRINT

NEXT RecordNumber

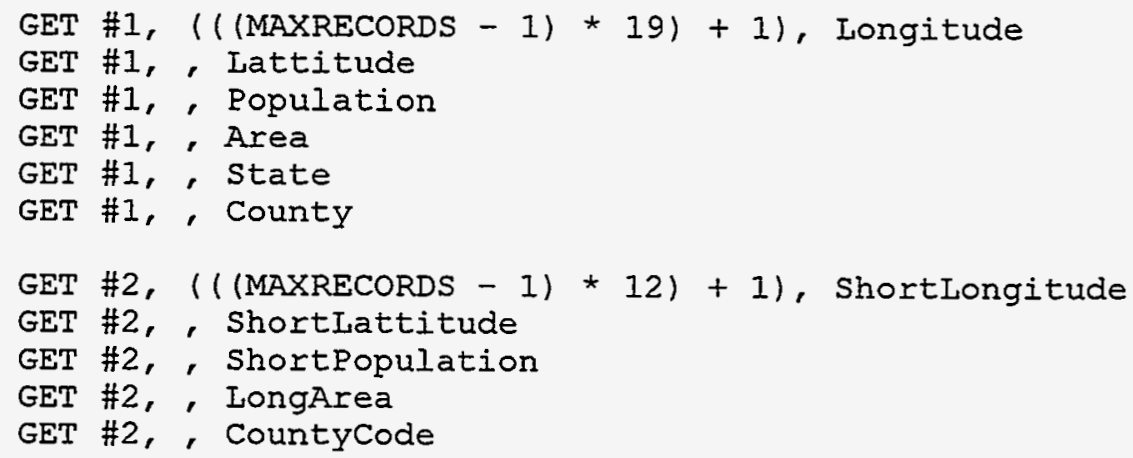

PRINT USING "\#\#\#\#\#\# \#\#\#\#\#\#\#\# \#\#\#\#\#\#\# \#\#\#\#\#\#\#\#\#\#\#\# \#\# \#\#\#"; MAXRECORDS; Longitude; Lattitude; Population; Area; ASC(State); County PRINT USING "\#\#\#\#\#\#\# \#\#\#\#\#\#\#\# \#\#\#\#\#\#\# \#\#\#\#\# \#\#\#\#\#\#\# \#\#\#\#\#\#; MAXRECORDS; $(($ ShortLongitude +91993$) * 1000) ;(($ ShortIattitude $+16610 \&) * 1000)$; ShortPopulation; LongArea; Countycode PRINT

LPRINT USING "\#\#\#\#\#\#\# \#\#\#\#\#\#\#\# \#\#\#\#\#\#\#\# \#\#\#\#\# \#\#\#\#\#\#\#\#\#\#\#\#"; MAXRECORDS; Longitude; Lattitude; Population; Area; ASC(State); County IPRINT USING "\#\#\#\#\#\# \#\#\#\#\#\#\#\# \#\#\#\#\#\#\# \#\#\#\#\# \#\#\#\#\#\#\# \#\#\#\#\#\#"; MAXRECORDS; $(($ Shortlongitude + 91993) * 1000); ((ShortLattitude + 16610\&) * 1000); ShortPopulation; LongArea; CountyCode IPRINT

END 


\section{OPTION EXPLICIT}

DIM Longitude AS INTEGER, Lattitude AS INTEGER

DIM Population AS INTEGER, Area AS LONG

DIM CountyCode AS INTEGER

OPEN "block_3.bin" FOR BINARY A.S \#1

DO

$$
\begin{aligned}
& \text { GET \#I, , Longitude } \\
& \text { GET \#I, , Lattitude } \\
& \text { GET \#I, , Population } \\
& \text { GET \#I, , Area } \\
& \text { GET \#I, } \text { CountyCode }
\end{aligned}
$$

IF (EOF (I) OR (INKEYS <> " ")) THEN

EXIT DO

ELSE

PRINT USING "\#\#\#\#\#\#\#\# \#\#\#\#\#\#\# \#\#\#\#\# \#\#\#\#\#\#\#\#\#\#\#\#"; ((Longitude + 91993)

* 1000); ((Lattitude + 16610\&) * 1000); Population; Area; CountyCode

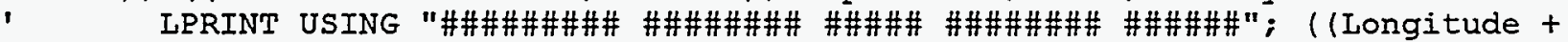

91993) * 1000); ((Lattitude + 16610\&) * 1000); Population; Area; CountyCode

END IF

LOOP

END 
FIIFNAME: TEST4.BAS

OPTION EXPLICIT

CONST MAXCOUNTY $=3111$

CONST MAXRECORDS $=6660337$

DIM i AS LONG

DIM Iongitude AS INTEGER, Lattitude AS INTEGER

DIM Population AS INTEGER, Area AS LONG, County AS INTEGER

DIM countyPopulation (MAXCOUNTY) AS LONG, CountyArea (MAXCOUNTY) AS LONG

OPEN "block 4.bin" FOR BINARY AS \#1

OPEN "testoūt4.txt" FOR OUTPUT AS \#2 LEN = 32767

FOR $i=1$ TO MAXCOUNTY STEP 1

CountyPopulation (i) $=0$

CountyArea $(i)=0$

NEXT i

FOR $i=1$ TO MAXRECORDS STEP 1

GET \#1, , Longitude

GET \#1, , Lattitude

GET \#I, , Population

GET \#1, Area

GET \#1, , County

CountyPopulation (County) = CountyPopulation (County) + Population

CountyArea (County) = CountyArea (County) + Area

IE $((i$ MOD 100000) $=0)$ THEN

END IF

PRINT $i$, Longitude, Lattitude, Population, Area, County

NEXT i

FOR $i=1$ TO MAXCOUNTY STEP 1

PRINT USING "\#\#\#\# \#\#\#\#\#\#\#\# \#\#\#\#\#\#\#\#"; $i$; CountyPopulation(i); CountyArea(i)

PRINT \#2, USING "\#\#\#\# \#\#\#\#\#\#\#\# \#\#\#\#\#\#\#\#"; i; CountyPopulation(i); CountyArea $(i)$

NEXT $i$

END 
if (NewLongitude > OldLongitude) then

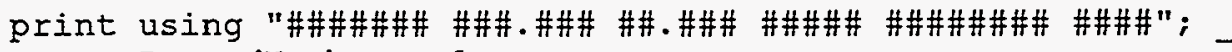
RecordNumber - I;

((OldLongitude $+9 \overline{1} 993) / 1000)$;

((OldLattitude + 16610\&) / 1000);

oldPopulation; OldArea; OldCountycōde

print "This record is out of order!!!"

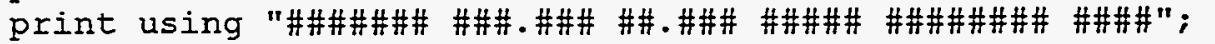

RecordNumber;

((NewLongitude $\left.\left.{ }^{-}+91993\right) / 1000\right)$;

((NewLattitude $+16610 \AA) / 1000) ;$

NewPopulation; NewArea; NewCountycōde

if (HardCopy) then

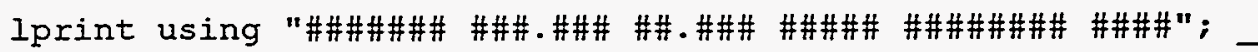
RecordNumber - 1;

((OldLongitude $+9 \overline{1} 993) / 1000)$ :

((OldLattitude + 16610\&)/ 1000);

OldPopulation; OldArea; OldCountycōde

lprint "This record is out of order!!!"

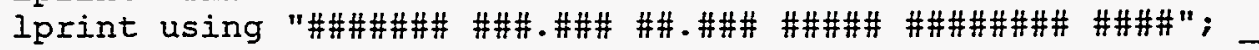
RecordNumber;

((NewLongitude $\left.\left.{ }^{-}+91993\right) / 1000\right)$;

((NewLattitude $+16610 \&) / 1000) ;$

NewPopulation; NewArea; NewCountyCode

end if

end if

OldLongitude $=$ NewLongitude

OldLattitude = NewLattitude

OldPopulation = NewPopulation

OldArea = NewArea

OldCountyCode $=$ NewCountyCode

loop

end 
option explicit

dim HardCopy as integer

dim RecordNumber as long

dim NewLongitude as integer, NewLattitude as integer

dim NewPopulation as integer, NewArea as long

dim NewCountyCode as integer

dim oldLongitude as integer, OldLattitude as integer

dim OldPopulation as integer, OldArea as long

dim oldCountycode as integer

HardCopy $=1$

RecordNumber $=0 \&$

OldLongitude $=32767$

OldLattitude $=0$

OldPopulation $=0$

OldArea $=0 \&$

OldCountyCode $=0$

open "c: \secpop90\census \census90.dat" for binary as \#1

do

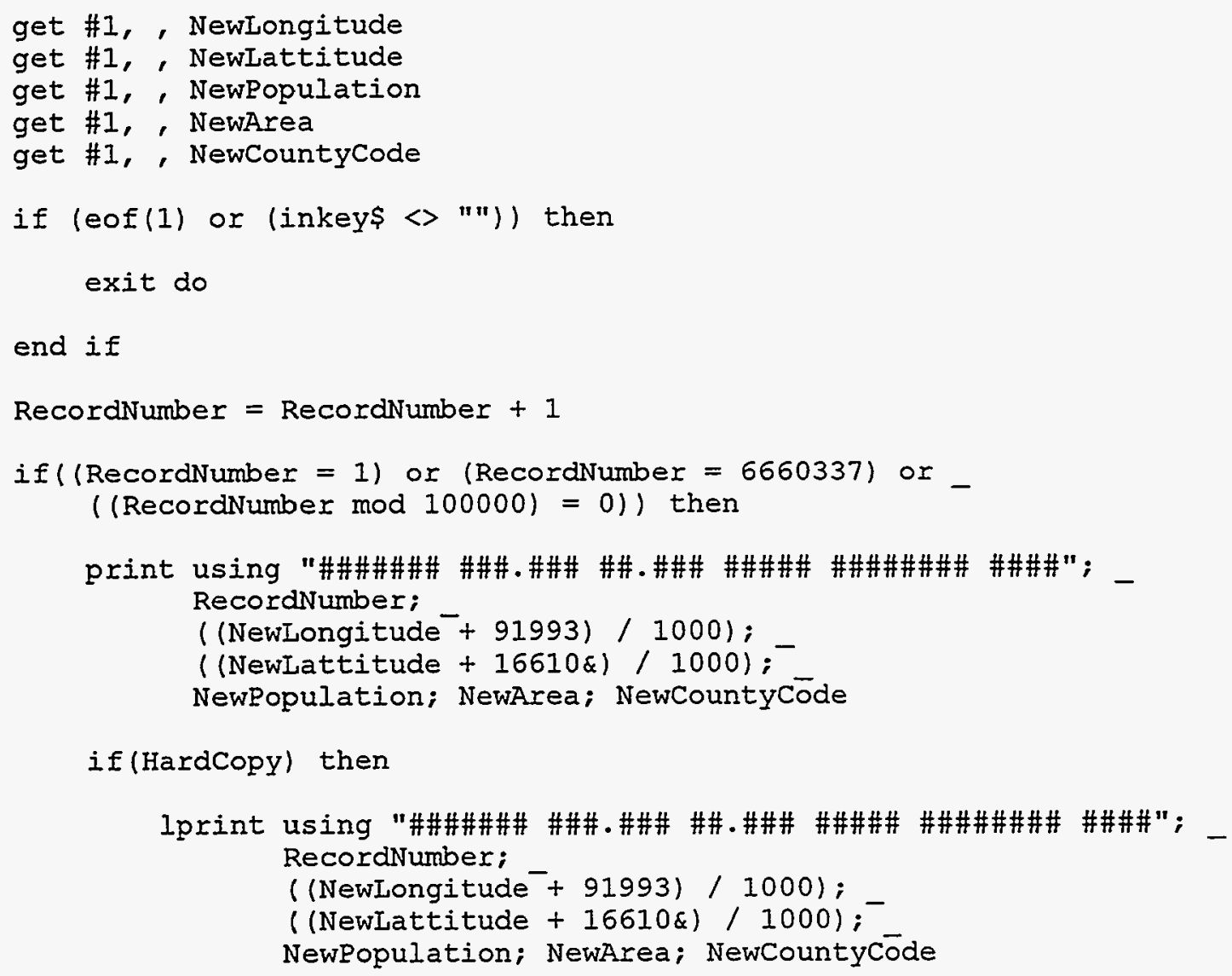

end if

end if 
if $(($ RecordNumber $\Rightarrow 1396205 \&)$ and (RecordNumber $<=1396215 \&)$ ) then

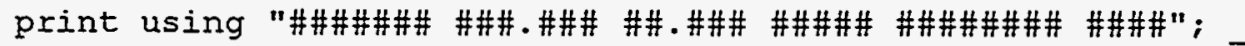
RecordNumber;

((NewLongitude + +91993) / 1000);

( (NewLattitude + 16610\&) / 1000);

NewPopulation; NewArea; NewCountyCōde

if (HardCopy) then

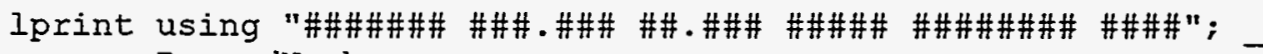
RecordNumber;

((NewLongitude $\left.\left.{ }^{-}+91993\right) / 1000\right)$;

((NewLattitude + 16610\&) / 1000);

NewPopulation; NewArea; NewCountycōde

end if

end if

if $(($ RecordNumber $\Rightarrow 1675345 \&)$ and (RecordNumber $<=1675355 \&))$ then

print using "\#\#\#\#\#\# \#\#\#.\#\#\# \#\#.\#\#\#\#\#\#\#\#\#\#\#\#\#\#\#\#\#\#\#"; -

RecordNumber;

((NewLongitude $\left.\left.{ }^{-}+91993\right) / 1000\right)$ :

( (NewLattitude $+16610 \&) / 1000) ;$

NewPopulation; NewArea; NewCountycōde

if (HardCopy) then

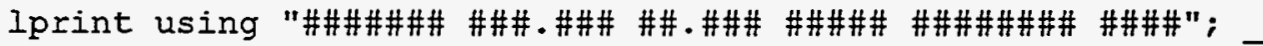
RecordNumber;

((NewLongitude $\left.\left.{ }^{-}+91993\right) / 1000\right)$;

( (NewLattitude $+16610 \&) / 1000) ;$

NewPopulation; NewArea; NewCountycōde

end if

end if

if $(($ RecordNumber $=>1774660 \&)$ and (RecordNumber $s=1774670 \&))$ then

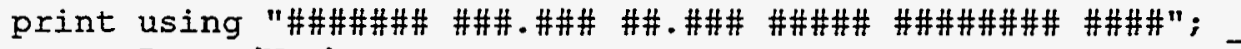

RecordNumber;

((NewLongitude + 91993) / 1000);

$(($ NewLattitude $+16610 \&) / 1000) ;$

NewPopulation; NewArea; NewCountycöde

if(HardCopy) then

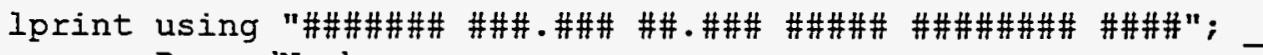
RecordNumber;

((NewLongitude $\left.\left.{ }^{-}+91993\right) / 1000\right)$;

$(($ NewLattitude $+16610 \&) / 1000) ;$

NewPopulation; NewArea; NewCountycōde

end if

end if

OldLongitude $=$ NewLongitude

OldLattitude $=$ NewLattitude 
OldPopulation = NewPopulation

OldArea = NewArea

OldCountyCode $=$ NewCountyCode

loop

end 
FILENAME: FIX2.BAS

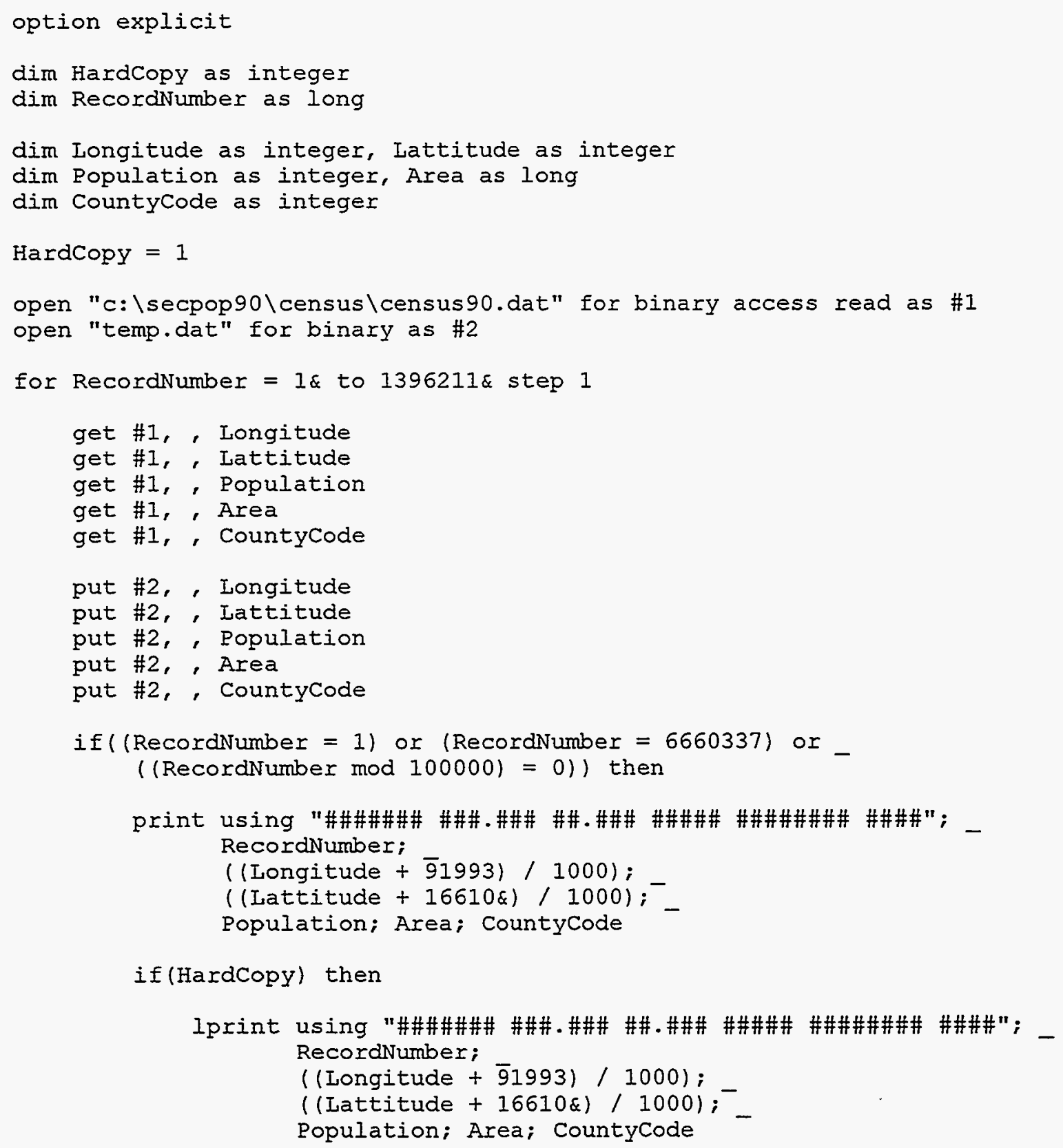

end if

end if

next RecordNumber

for RecordNumber $=1396212 \&$ to $1675348 \&$ step 1

get \#1, , Longitude
get \#1, , Lattitude
get \#1, , Population
get \#1, , Area
get \#1, , CountyCode

next RecordNumber 
for RecordNumber $=1675349 \&$ to $1774662 \&$ step 1

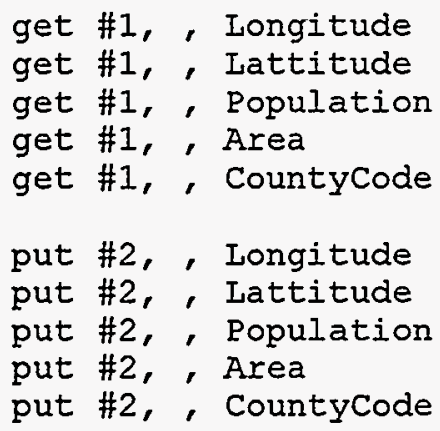

end if

end if

next RecordNumber

close (1)

open "c: \secpop90\census\census90.dat" for binary access read as \#1

for RecordNumber $=1 \&$ to 1396211 \& step 1

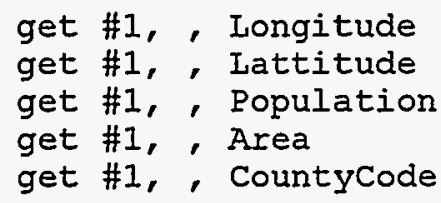

next RecordNumber

for RecordNumber $=1396212 \&$ to $1675348 \&$ step 1

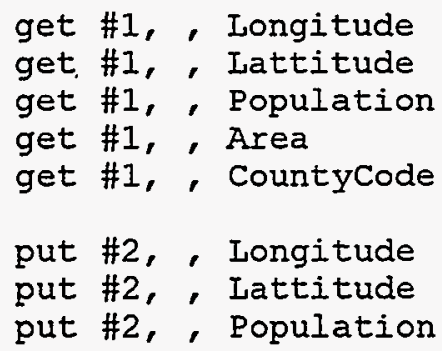


put \#2, Area

put \#2, , CountyCode

if $(($ RecordNumber $=1)$ or (RecordNumber $=6660337)$ or -

$(($ RecordNumber mod 100000) $=0))$ then

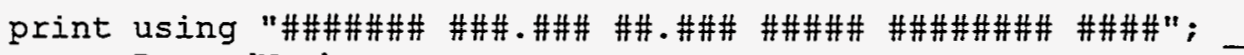

RecordNumber;

((Iongitude $+\overline{9} 1993) / 1000)$;

((Iattitude + 16610\&) / 1000);

Population; Area; CountyCode

if (HardCopy) then

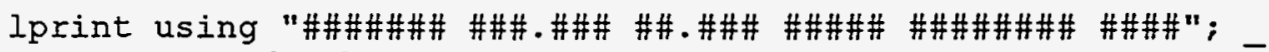
RecordNumber;

((Longitude $+\overline{9} 1993) / 1000)$;

((Lattitude $+16610 \&) / 1000) i^{-}$

Population; Area; CountyCode

end if

end if

next RecordNumber

for RecordNumber $=1675349 \&$ to $1774662 \&$ step 1

get \#I, , Longitude

get \#1, , Lattitude

get \#1, , Population

get \#1, , Area

get \#1, , CountyCode

next RecordNumber

for RecordNumber $=1774663 \&$ to $6660337 \&$ step $I$

get \#1, , Longitude

get \#1, , Lattitude

get \#1, , Population

get \#1, , Area

get \#1, CountyCode

put \#2, , Longitude

put \#2, , Lattitude

put \#2, , Population

put \#2, Area

put \#2, , CountyCode

if $(($ RecordNumber $=1)$ or (RecordNumber $=6660337)$ or _

$(($ RecordNumber mod 100000) $=0))$ then

print using "\#\#\#\#\#\#\# \#\#\#.\#\#\# \#\#.\#\#\# \#\#\#\#\# \#\#\#\#\#\#\#\#\#\#\#"; RecordNumber;

((Longitude $+\overline{9} 1993) / 1000)$ :

((Lattitude + 16610\&)/1000);

Population; Area; CountyCode

if (HardCopy) then

lprint using "\#\#\#\#\#\#\# \#\#\#.\#\#\# \#\#.\#\#\# \#\#\#\#\#\#\#\#\#\#\#\#\#\#\#"; RecordNumber; 
((Longitude + 91993) / 1000);

((Lattitude + 16610\&) / 1000) $i^{-}$

Population; Area; CountyCode

end if

end if

next RecordNumber

close (1)

close (2)

end 


\title{
APPENDIX I - SECPOP9O PROGRAM LISTINGS
}

FIIENAME: CALCCODE.BAS

\author{
OPTION EXPLICIT
}

'\$INCLUDE: 'SECPOP90.BI'

- THIS SUBROUTINE ALLOCATES THE 1990 CENSUS DATA TO A MACCS GRID FOR MULTIPLE SITES BASED ON INTERACTIVE COMMUNICATION WITH THE USER. EACH SITE LOCATION IS DEFINED BY THE NUMBER OF DEGREES, MINUTES, AND SECONDS OE LATITUDE AND LONGITUDE. THE MACCS GRID IS BASED ON 16 RADIAL DIRECTIONS COUNTED CLOCKWISE EROM DUE NORTH WITH ' DIRECTION 1 HAVING DUE NORTH AS ITS AXIS OF SYMMETRY.

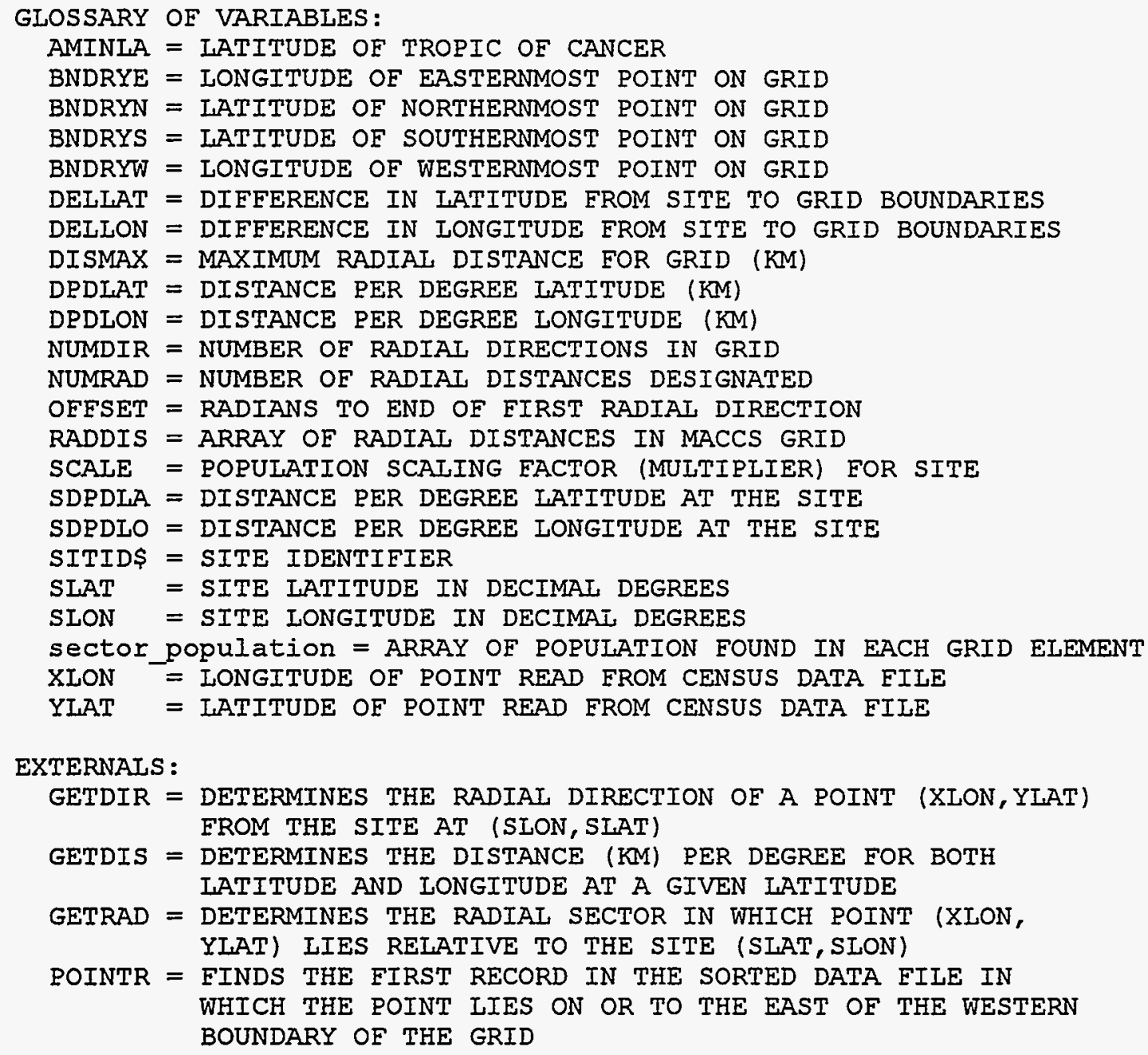




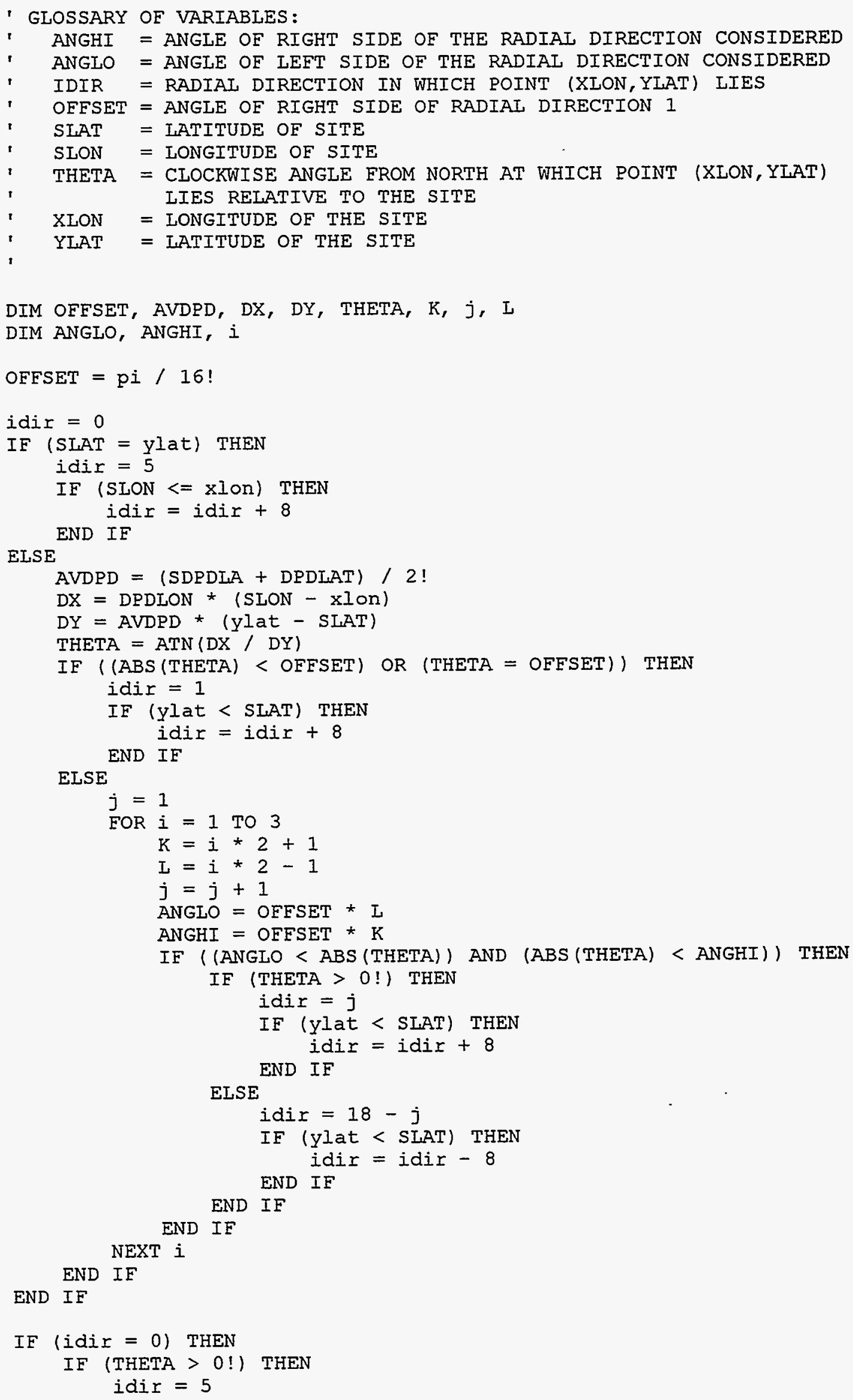


END IF

IF (XION > SLON) THEN idix $=$ idir +8

END IE

ELSE

idir $=13$

IF ( $x$ lon < SLON) THEN idir $=$ idir -8

END IF

END IF

END SUB

SUB GETDIS ( $Y$ AS SINGLE)

- THIS SUBROUTINE RETURNS THE DISTANCE PER DEGREE LATITUDE AND

- DISTANCE PER DEGREE LONGITUDE AT THE GEODETIC LATITUDE (SLAT)

- GLOSSARY OF VARIABLES:

COSGC $=$ COSINE OF GEOCENTRIC ANGLE

COS2GC = SQUARE OF COSGC

DGTORD = CONVERSION FACTOR FOR DEGREES TO RADIANS

DPDLAT = DISTANCE PER DEGREE LATITUDE AT SLAT (KM)

DPDLON = DISTANCE PER DEGREE LONGITUDE AT SION (KM)

GDRLA = GEODETIC LATITUDE IN RADIANS

QLAT = GEODETIC LATITUDE OF POINT CONSIDERED

RE2 = SQUARE OF EQUATORIAI RADIUS OF EARTH

RGD = RADIUS OF GEODETIC IATITUDE CIRCIE (KM)

RLONG = RADIUS OF LONGITUDE "CIRCLE" (KM)

RP2 = SQUARE OF POLAR RADIUS OF EARTH

RPROD = PRODUCT OF EQUATORIAI AND POLAR RADII

SINGC = SINE OF GEOCENTRIC ANGLE

SIN2GC $=$ SQUARE OF SINGC

THGC = GEOCENTRIC ANGLE CORRESPONDING TO THE GEODETIC IATITUDE

DIM RE, RE2, RP, RP2, RRATIO, RPROD, GDRLA, THGC, SINGC, SIN2GC, COSGC, COS2GC DIM RGC, RGD, RLONG

- DEFINE CONSTANTS

$\mathrm{RE}=6378.137$

$\mathrm{RE} 2=\mathrm{RE} \wedge 2$ !

$\mathrm{RP}=6356.7523142 \#$

$\mathrm{RP} 2=\mathrm{RP} \wedge 2$ !

RRATIO $=\mathrm{RP} 2 / \mathrm{RE} 2$

$\mathrm{RPROD}=\mathrm{RE} * \mathrm{RP}$

- FIND THE GEODETIC LATITUDE IN RADIANS

GDRLA $=Y *$ DGTORD

- FIND THE GEODETIC IATITUDE IN THE MASTER COORDINATE SYSTEM

THGC $=$ ATN $($ RRATIO * TAN $(G D R I A))$

SINGC $=$ SIN (THGC)

$\operatorname{SIN} 2 \mathrm{GC}=\operatorname{SINGC} \wedge 2$ !

$\operatorname{cosGC}=\cos ($ THGC $)$

$\cos 2 \mathrm{GC}=\cos \mathrm{GC} \wedge 2$ !

- FIND THE GEOCENTRIC RADIUS (KM) 
$\mathrm{RGC}=\mathrm{RPROD} / \mathrm{SQR}(\mathrm{RE} 2 * \mathrm{SIN} 2 \mathrm{GC}+\mathrm{RP} 2 * \operatorname{COS} 2 \mathrm{GC})$

' FIND THE RADIUS OF THE IONGITUDE "CIRCIE"

RIONG $=$ RGC $* \operatorname{COSGC}$

- FIND THE GEODETIC RADIUS

RGD $=$ RIONG * $(T A N(T H G C) / \operatorname{SIN}(G D R L A))$

'EIND THE DISTANCE PER DEGREE LATITUDE AND IONGITUDE (KM)

DPDIAT $=$ RGD * DGTORD

DPDLON $=$ RIONG $\star$ DGTORD

END SUB

SUB GETRAD (ylat, NUMRAD AS INTEGER, irad AS INTEGER)

' THIS SUBROUTINE GETS THE RADIAI SPATIAI ELEMENT IN WHICH THE

' POINT (XLON, YLAT) LIES

GLOSSARY OF VARIABLES:

AVDPD = AVERAGE DISTANCE PER DEGREE IATITUDE BETWEEN THE SITE AND A POINT AT LATITUDE YLAT

DELX = DIFFERENCE IN LONGITUDE BETWEEN A POINT AT (XLON, YLAT) AND THE SITE

DELY = DIFFERENCE IN IATITUDE BETWEEN A POINT AT (XLON, YLAT) AND THE SITE

DISMAX = RADIUS OE THE EUII MACCS GRID

DISTST = DISTANCE FROM POINT (XION, YLAT) TO THE SITE

IRAD = THE RADIAI SECTOR OF THE MACCS GRID IN WHICH POINT (XION, YLAT) IIES

RADDIS = ARRAY OF RADIAI DISTANCES OE THE MACCS GRID BEING USED

SIAT = ILATITUDE OF THE SITE

SLON = LONGITUDE OE THE SITE

DIM DELX, AVDPD, DELY, DISTST, JRAD

DELX $=$ ABS $($ SLON $-X I O N) *$ DPDION

AVDPD $=$ (SDPDLA + DPDLAT) $/ 2$ !

DELY = ABS (SIAT - Ylat) * AVDPD

DISTST $=$ DIST $($ DEIX, DELY $)$

irad $=0$

IF (DISTST $<=$ DISMAX) THEN

IF (DISTST $<=$ RADDIS(1)) THEN

irad $=1$

ELSEIF (NUMRAD $>=2$ ) THEN

FOR JRAD $=2$ TO NUMRAD

IF ((DISTST > RADDIS (JRAD - 1)) AND (DISTST <= RADDIS (JRAD))) THEN irad $=$ JRAD

END IF

EXIT FOR

NEXT JRAD

END IF

END IF

END SUB 
SUB pointr (BYVAL BNDRYW, census file AS LONG, ylat, ipop AS INTEGER, area AS LONG, county_code AS INTEGER, ibyte AS IONG)

- THIS SUBROUTINE FINDS THE FIRST RECORD IN THE CENSUS DATA FILE

- WHICH IS AT THE WESTERN BOUNDARY OF THE GRID

GLOSSARY OF VARIABLES:

ALAT = POINT LATITUDE IN DECIMAL NOTATION

ALON = POINT LONGITUDE IN DECIMAI NOTATION

BNDRYW = LONGITUDE OF WESTERN BOUNDARY OF GRID

ILAT = CODED INTEGER REPRESENTATION OF IAATITUDE OF POINT IN CENSUS DATA FILE

ILOM = CODED INTEGER REPRESENTATION OF LONGITUDE OF POINT IN CENSUS DATA FILE

ILONW = CODED INTEGER REPRESENTATION OF WESTERN BOUNDARY LONGITUDE OF GRID

XLON = LONGITUDE OF FIRST CENSUS DATA POINT LOCATED ON OR WITHIN THE MACCS GRID

YLAT = LATITUDE OF FIRST CENSUS DATA POINT LOCATED ON OR WITHIN THE MACCS GRID

CONST NUMREC $=$ number_of_records

' ROUTINE TO READ FROM RANDOM ACCESS BINARY FILE *

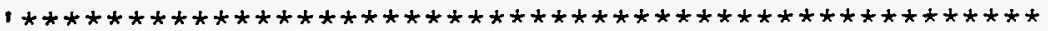

DIM MIDREC AS LONG, NBYTE AS LONG

DIM IBEG AS LONG, IEND AS LONG

DIM ILONW AS LONG, ilon, A\$, JLON, ilat, CEND\$

ILONW = BNDRYW * 1000 - longitude_offset

GET \#census file, 1, rec

ilon $=\operatorname{CVI}(\bar{M} I D \$($ rec $, 1,2))$

IF (iION > ILONW) THEN

IBEG $=1$

IEND $=$ NUMREC

ibyte $=1$

ELSE

ibyte $=1$

GOTO process

END IF

ReadRecord:

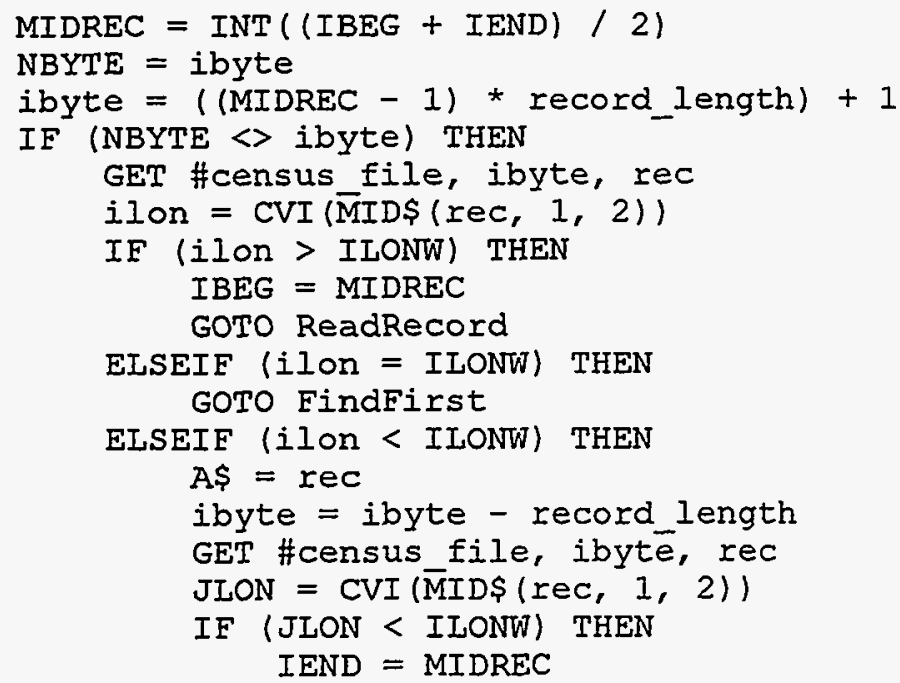


GOTO ReadRecord

ELSEIF (JLON = ILONW) THEN

GOTO FindEirst

ELSEIE (JLON > ILONW) THEN

ibyte = ibyte + record_length

rec $=A S$

GOTO process

ELSE

END IE

END IF

MSGBOX "Site not within Continental US.", MB OK, "Error" EXIT SUB

END IE

- Find the first point inside the western boundary

FindFirst:

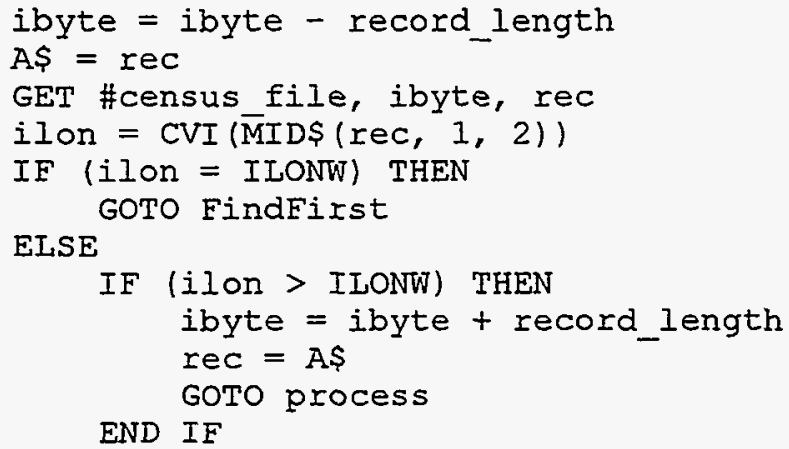

END IF

' When find the first point process the information

process :

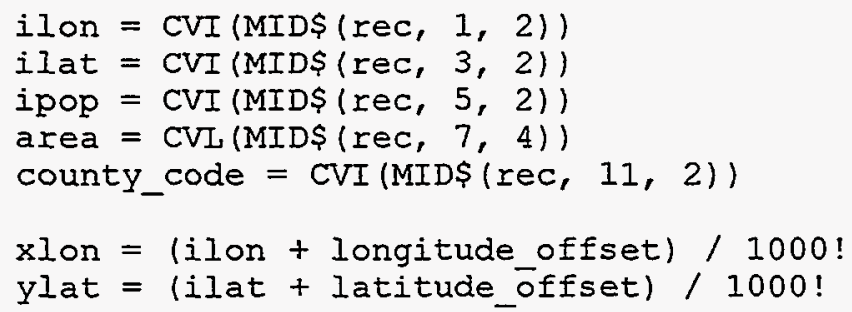

END SUB

SUB popcalc ()

DIM tempstring AS STRING

DIM census file AS LONG, county file A.S LONG, debug file AS LONG

DIM AS, NUM̄IIN AS INTEGER, INUM AS INTEGER, BS, JBEEG AS INTEGER

DIM JEND AS INTEGER, NLEFT AS INTEGER, C\$

DIM L1\$, L2\$, L3\$, L4\$, L5\$, L6\$, L7\$, LA1 AS INTEGER, LA2 AS INTEGER

DIM IA3 AS INTEGER, LO1 AS INTEGER, LO2 AS INTEGER, LO3 AS INTEGER

DIM DS, DELLON, DELLAT, ilon AS INTEGER, ilat AS INTEGER

DIM NP1, NP2, NP3, NP4, NP5, NP6, NP7, NP8, CENDS

DIM ILIN AS INTEGER, i AS INTEGER, j AS INTEGER, $m$ AS INTEGER

DIM events open AS INTEGER, average counter AS INTEGER

DIM start file pos AS LONG, end file pos AS LONG

DIM num récords AS LONG, num pröcessed AS LONG, census records AS LONG

DIM hours AS INTEGER, minutes AS INTEGER, seconds AS SINNGLE

DIM time_begin AS LONG, time_end AS LONG, total_time AS IONG 
'Open census data file, located at the position specified

'by the user in the setup form

tempstring $=$ frmSetup.txtData_Path.text + "\CENSUS90.DAT"

census file $=$ FREEFILE

OREN tempstring FOR BINARY ACCESS READ AS \#census_file LEN = 32767

'Initialize the county data arrays to their startup values

'These arrays contain various types of county economic

'data and state abreviations.

frmCalculate.lblstatus.caption = "Reading county information."

ipop $=0$

tempstring $=$ frmsetup.txtData_Path.text + "\COUNTY90.DAT"

county_file = FREEFILE

OPEN tempstring FOR INPUT ACCESS READ AS \#county_file

' debug file = FREEFILE

'OREN "temp.txt" FOR OUTPUT ACCESS WRITE AS \#debug_file

'Read in the header line. County_state $(0)$ is used as a dummy variable.

IINE INPUT \#county_file, county_state(0)

'Read in the rest of the file.

FOR $i=1$ TO number of counties STEP 1

INPUT \#county_fille, county_code

county_state $(\bar{i})=$ INPUTS(2, \#county_file)

county name $(i)=\operatorname{INPUT}(4$, \#county $\bar{f}$ ile $)$

county_name $(i)=$ INPUT (max county name_length, \#county_file)

INPUT \#county_file, county_E $\overline{\text { frclnd }}(\bar{i})$

INPUT \#county_file, county_frmfrc(i)

INPUT \#county file, county dpf(i)

INPUT \#county_file, county_asfp(i)

INPUT \#county_file, county_vfrm(i)

INPUT \#county_file, county_vnfrm(i)

'Skip over the notes.

LINE INPUT \#county_file, county state(0)

'PRINT \#debug_file, USING "\#\#\#\#\#\#\#\#\#"; county_code;

'PRINT \#debug_file, USING "\& "; county_state $(\bar{i})$;

'PRINT \#debug_file, USING "\& "; county_name(i);

'PRINT \#debug_file, USING "\#.\#\#\#\#\# "; county_frclnd(i);

'PRINT \#debug_file, USING "\#.\#\#\#\#\# "; county_frmfrc(i);

'PRINT \#debug file, USING "\#.\#\#\#\#\# "; county dpf(i);

'PRINT \#debug_file, USING "\#\#\#\#\#"; county_asfp(i);

'PRINT \#debug_file, USING "\#\#\#\#\#\# "; county_vfrm(i);

NEXT i

'PRINT \#debug_file, USING "\#\#\#\#\#\#\#"; county_vnfrm(i)

CLOSE (county file)

'CLOSE (debug_file)

'set degrees to radians conversion

DGTORD $=$ pi $/ 180$ !

'Set the latitude of the tropic of cancer

AMINLA $=23.45$ 
'Initialize RADDIS array to the values in the radial distance array so

'that the values can be changed locally without affecting other modules.

FOR $i=1$ TO number of radii

IF frmProblem Data-optKilometers.value = TRUE THEN

ELSE RADDIS $(i)^{-}=$radial_distance $(i)$

END IF

RADDIS $(i)=$ radial_distance $(i)$ * miles_to_kilometers

NEXT i

'Initialize the population and land fraction sector arrays and economics

'region arrays to zero.

FOR $i=1$ TO number_of_segments STEP 1

FOR $j=1$ TO number of radii STEP 1

sector_population $(\bar{i}, j)=0$

sector_area $(i, j)=0$

NEXT $j$

sector_frclnd $(i, j)=0$

NEXT i

FOR $i=0$ TO number econ_regions

econ data (i).region_àrea $=0$

econ_data(i).region_frmfrc $=0$

econ_data(i). region_dp $f=0$

econ_data(i).region_asfp $=0$

econ_data(i).region_vfrm $=0$

NEXT i

econ_data(i).region_vnfrm $=0$

'Set local constants for number of raddii and distance of farthest radii.

NUMRAD = number of radii

DISMAX $=$ RADDIS (NUMMRAD)

Processite:

'Reading interactive input.

SITIDS = frmSite Data.txtSite Name.text

LIS = frmSite Dā́ta.txtLatitudē Degrees.text

L2S = frmsite-Data.txtLatitude-Minutes.text

I $3 \$$ = frmsite Data.txtLatitude Seconds.text

I $4 \$$ = frmsite-Data.txtLongitude Degrees.text

I5\$ = frmsite Data.txtIongitude Minutes.text

L6\$ = frmsite Data.txtLongitude-seconds.text

I7S = frmProbIem_Data.txtPopulaEtion_multiplier.text

'Convert interactive input strings into numeric values.

$\operatorname{LA} I=\operatorname{INT}(\operatorname{VAL}(\mathrm{I} 1 \$))$

IAA2 = INT (VAL (I2\$))

$\operatorname{LA3}=\operatorname{INT}(\operatorname{VAL}(\mathrm{I} 3 \$))$

LO1 $=\operatorname{INT}(\operatorname{VAL}(\mathrm{L} 4 \$))$

$\mathrm{LO2}=\operatorname{INT}(\operatorname{VAL}(\mathrm{L} 5 \$))$

LO3 $=$ INT $(\operatorname{VAL}($ L6\$) $)$

SCALE $=\operatorname{VAL}(L 7 \$)$

'Convert Degree/Minute/Second designation to decimal degrees.

SLAT $=$ VAL $(I 1 \$)+\operatorname{VAL}(I 2 \$) / 60 !+\operatorname{VAL}(L 3 \$) / 3600 !$

SLON $=\operatorname{VAL}(\mathrm{L} 4 \$)+\operatorname{VAL}(\mathrm{L} 5 \$) / 60 !+\operatorname{VAL}(\mathrm{L} 6 \$) / 3600 !$ 
'Find the longitude-latitude boundaries of grid.

'Set distance per degree latitude for site.

CALL GETDIS (SLAT)

SDPDLA = DPDLAT

'Set eastern and western boundaries. These boundaries determine which

'records are searched in the census file.

DELLON $=$ DISMAX $/$ DPDLON

BNDRYW $=$ INT $(($ SLON + DELION $) * 1000+.5) / 1000$

BNDRYE $=$ INT $(($ SLON - DELLON $) * 1000+.5) / 1000$

'Find distance change per degree latitude at some minimun latitude

' (AMINLA).

CALI GETDIS (AMINLA)

'Set northern and southern boundaries. These boundaries do not affect

'which records are searched, but do determine whether or not a point

'is inside of the circle being analyzed.

DELIAAT = DISMAX / SDPDLA

BNDRYN $=$ INT $(($ SLAT + DELLAT $) * 1000+.5) / 1000$

DELLAT = DISMAX / DPDLAT

BNDRYS $=$ INT $(($ SLAT - DELLAT $) * 1000+.5) / 1000$

'Find the last record in the file with the longitude within the

'longitudinal boundaries of the grid. This record is used to

'estimate the run time for the problem.

xlon $=0$ !

ylat $=0 !$

area $=0 \&$

county_code $=0$

CALL pöintr(BYVAL BNDRYE, census_file, ylat, ipop, area, county_code, ibyte)

end file_pos = ibyte

IF (xlon $=0$ !) THEN

END IF end_file_pos = LOF (census_file)

frmCalculate.lblstatus.caption = "Searching for first census record."

ipop $=0$

'Find the first record in the file with the longitude within the

'longitudinal boundaries of the grid.

ipop $=0$

xlon $=0$ !

ylat $=0$ !

area $=0 \&$

county_code $=0$

CALL pöintr(BYVAI BNDRYW, census_file, ylat, ipop, area, county_code, ibyte)

IF (xlon $=0$ !) THEN

END IF GOTO finishup

start_file_pos = ibyte

'Determine number of records to be scanned in census file (this

'number is used in determining estimated run time for a problem).

num_records $=($ (end_file_pos - start_file_pos) $/$ record_length) 
'Verify that the starting position is within the specified

'longitude and latitude. If so, read in and process the data

'from the first specified record in the census database.

idir $=0$

irad $=0$

IF ( $(x l o n<=$ BNDRYW) AND (xlon >= BNDRYE) ) THEN

IF ((ylat <= BNDRYN) AND (ylat >= BNDRYS)) THEN

'Determine the distance between the specified site and the first

'location in the census database.

CALL GETDIS (ylat)

CALI GETRAD(ylat, NUMRAD, irad)

'If the point is outside of the outermost radius of the circle,

'read in and process the next record ...

IF (irad $=0$ ) THEN

GOTO Continue1

ELSE

'Otherwise, determine in which sector the block lies.

CALI GETDIR(ylat, idir)

'Set the population of the sector to the population of the 'block.

sector population(idir, irad) = ipop * SCALE

sector area (idir, irad) = area

sector_frclnd(idir, irad) = area * county_frclnd(county_code)

'Set the regional economic data by setting each of the

'appropriate elements in the user defined data type.

econ data (region index(irad, idir - 1)) .region area = area econ_data (region_index(irad, idir - 1)).region_frmfrc = area *

county_frmfrc(county_code) econ_data(region_index(irad, idir - 1)).region_dpf = area * county_dpf(county_code) county_asfp (county_code) econ_data(region_index(irad, idir - 1)).region_asfp = area * county_vfrm (county_cōde) econ data(region index(irad, idir - 1)).region_vfrm = area * econ_data (region_index(irad, idir - 1)).region_vnfrm = area * county_vnfrm(county_code)

'Set number of census records processed thus far.

census_records $=1$

END IF

END IF

END IF 


\section{Continuel:}

'Update labels on calculate form, and begin timer to estimate

'the run time of the calculation

frmcalculate.lblstatus.caption = "Processing census records." time_begin = TIMER

'Read each subsequent record in the data file until the first record

'with a longitude outside of the longitudinal boundaries

readit :

'If end of file is reached, finish the economic calculations and exit.

IF (EOF(census file)) THEN

END IF GOTO finishup

'Change to get statement with binary file, and Go to the next record.

ibyte $=$ ibyte + record_length

'Read the data from that record, split it into its constituent pieces, 'and convert those peices from file format to program format. This 'portion of code is done by the pointr function for the first record.

GET \#census file, ibyte, rec

ilon $=\operatorname{CVI}(\bar{M} I D$ (rec, 1,2$))$

ilat $=\operatorname{CVI}(\operatorname{MID} S($ rec, 3, 2))

ipop $=\operatorname{CVI}(\operatorname{MID} \$($ rec $, 5,2))$

area $=\operatorname{CVI}(\operatorname{MID} \$($ rec, 7, 4))

county_code $=\operatorname{CVI}(\operatorname{MID} \$($ rec $, 11,2))$

xlon $=$ (ilon + longitude_offset) / 1000!

ylat $=$ (ilat + latitude_offset) / 1000!

'Check and see if the point is within the lat and Iong boundaries of 'the circle.

IE ( $x$ lon $<=$ BNDRYW) AND ( $x$ lon >= BNDRYE)) THEN

IF ( (ylat <= BNDRYN) AND (ylat >= BNDRYS)) THEN

'if so, then determine the distance between the point and the

'center of the circles.

idir $=0$

irad $=0$

CALL GETDIS (ylat)

CAII GETRAD (ylat, NUMRAD, irad)

'If the point lies outside of the farthest radii, proceed to the 'next point.

IF (irad $=0)$ THEN
GOTO Continue2

ELSE

'Determine which sector the point lies in.

CALI GETDIR(ylat, idir)

'Add the population of the block to the total population of 'the sector in which it lies. 
(ipop * SCALE)

sector population(idir, irad) = sector_population(idir, irad) +

sector_area (idir, irad) = sector_area(idir, irad) + area

sector frclnd(idir, irad) = sector_frclnd(idir, irad) + (area * county_frclind (county_cöde))

'Increment the regional economic data by incrementing each of

'the appropriate elements in the user defined data type.

econ data (region index(irad, idir - 1)).region_area =

econ_data (region_index (irad, idi $\bar{r}$ - l)).region area t area

econ data(region index(irad, idir - 1)) . region_frmfrc =

econ data (region_index(irad, idi $\bar{r}-1)$ ). region_frmfrc + (area $\bar{k}$

county Ermfrc(county_code))

econ data(region index(irad, idir - 1)).region_dpf =

econ_data (region_index(irad, idi $\bar{r}-1)$ ).region_dp + (area *

county dpf (count $\bar{y}_{-}$code))

econ data(region index(irad, idir - 1)).region_asfp =

econ_data (region index (irad, idir - 1)) . region_asfp + (area *

county_asfp (county_code))

econ data (region_index(irad, idir - 1)).region_vfrm =

econ data (region_index(irad, idis - 1)) .region_vfrm + (area *

county_vfrm(county_code))

econ data (region index (irad, idir - 1)).region_vnfrm = econ_data (region_index(irad, idi $\bar{r}-1)$ ).region_vnfrm + (area *

county_vnfrm (couñty_code))

'Increment the number of records processed.

census_records $=$ census_records +1

IF (census_records $<=1000$ ) THEN

ylat; ipop; idir; irad

'PRINT \#debug_file, USING "\#\#\#.\#\#\#\#\#.\#\#\#\#\#\#\#\#\#\#\#"; xlon; END IE

IE census_records MOD $100=0$ THEN

'Process any user events that may have occured while

calculating. At this point in time only the close

calculation button is allowed.

events_open = DOEVENTS ()

'If the user has selected close from the calculate

'form, then close the census file and exit the

'subroutine.

IF (leave calculation) THEN

CLOSE \#census file

leave calculation = EAISE

END IF EXIT $\bar{S} U B$

'Update the number of census records displayed

'status line.

frmcalculate. Iblstatus. caption =

LTRIMS (STRS (census_records)) $\mp$

"census records processed." 


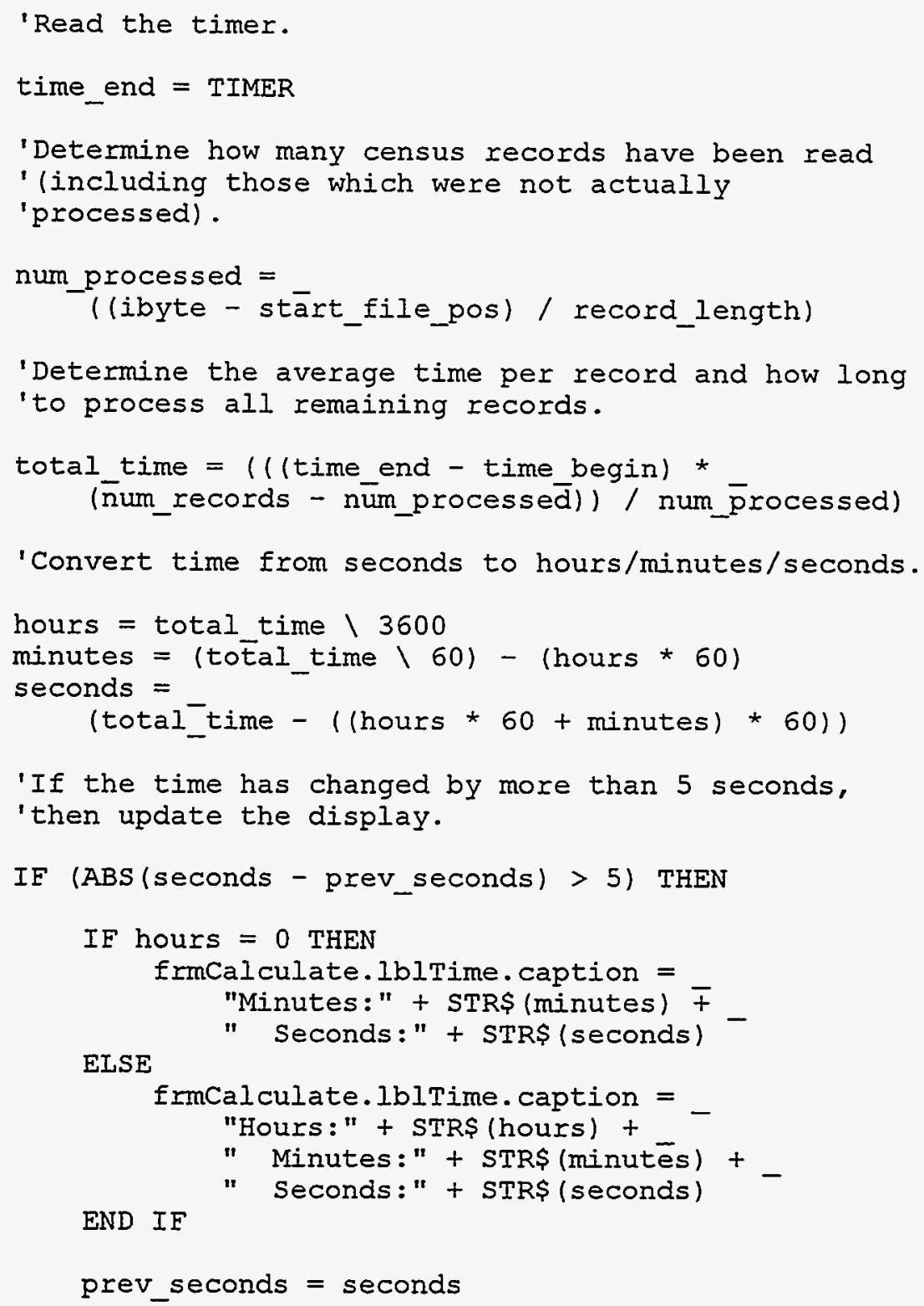


'Close census data file, and update display labels.

CLOSE \#census file

'CLOSE \#debug_file

frmCalculate.Iblstatus. caption = "Calculating economic data."

'Determine the land fraction values for each sector.

FOR $i=1$ TO number_of_segments STEP 1

FOR $j=1$ TO number_of_radii STEP 1

IF (sector_area $(i, j)<0$ ) THEN

$\operatorname{sector} \operatorname{frclnd}(i, j)=\operatorname{sector} \operatorname{frclnd}(i, j) / \operatorname{sector} a r e a(i, j)$

EISE

sector_frclnd $(i, j)=0$

END IF

NEXT j

NEXT $i$

'Determine the economic values for each region.

FOR $i=1$ TO number_econ_regions STEP 1

IF (econ_data(i).region_area $<>0$ ) THEN

econ_data (i).region_frmfrc = econ_data (i).region_frmfrc /

econ_data (i).regīon area econ_data (i).region_dpf = econ_data(i).region_dpf /

econ data (i). regín area econ_data(i).region_asfp = econ_data(i).region_asfp /

econ data (i). region area econ_data(i).region_vfrm = econ_data(i).region_vfrm /

econ_data (i).region_area econ_data(i).region_vnfrm = econ_data(i).region_vnfrm /

econ_data (i).region_area

EISE

$$
\begin{aligned}
& \text { econ_data }(i) \text {. region_frmfrc }=0 \\
& \text { econ_data (i). region_dpf }=0 \\
& \text { econ_data (i). region_asfp }=0 \\
& \text { econ_data(i).region_vfrm }=0 \\
& \text { econ_data(i).region_vnfrm }=0
\end{aligned}
$$

END IE

NEXT i

'Update the user display for the final time letting the user know 'That we are finished and the total number of census records 'processed.

frmCalculate. lblstatus. caption = "Finished, " + ITRIMS (STRS (census_records)) + " processed." 
'CLOSE (debug_file)

END SUB

SUB popdensity (exceeded AS INTEGER)

'Define local variables

DIM DELLON, DELIAT, ilon AS INTEGER, ilat AS INTEGER, i AS INTEGER

DIM IIIN AS INTEGER, open_windows AS INTEGER

DIM census file AS IONG

DIM tempstring AS STRING

on local error goto popdensity_error

'Initilize population array.

for $i=1$ to numrad step 1

population(i) $=0$

next $i$

'Retrieve path for census database from setup, and open database for

'input.

tempstring = frmsetup.txtData Path.text + "\CENSUS90.DAT"

OPEN tempstring FOR BINARY ACCEESS READ AS \#2 LEN = 32767

DGTORD $=p i / 180$ !

'Set the latitude of the tropic of cancer

AMINLA $=23.45$

'Set dismax to the farthest distance, which is the radii provided by

'the user.

DISMAX $=$ RADDIS (NUMRAD)

'Find the longitude-latitude boundaries of the grid

CALL GETDIS (SLAT)

SDPDLA $=$ DPDI.AT

'Find number of degrees longitude traversed

DELLON = DISMAX / DPDLON

'Find the eastern and Western boundries of this section

BNDRYW $=$ INT $(($ SLON + DELLON $) * 1000+.5) / 1000$

BNDRYE $=$ INT $(($ SLON - DELLON $) * 1000+.5) / 1000$

'Find distance change per degree latitude at some minimum latitude

' (AMINLA).

CALI GETDIS (AMINLA)

'Find number of degrees latitude traversed northward

DELLAT = DISMAX / SDPDLA 


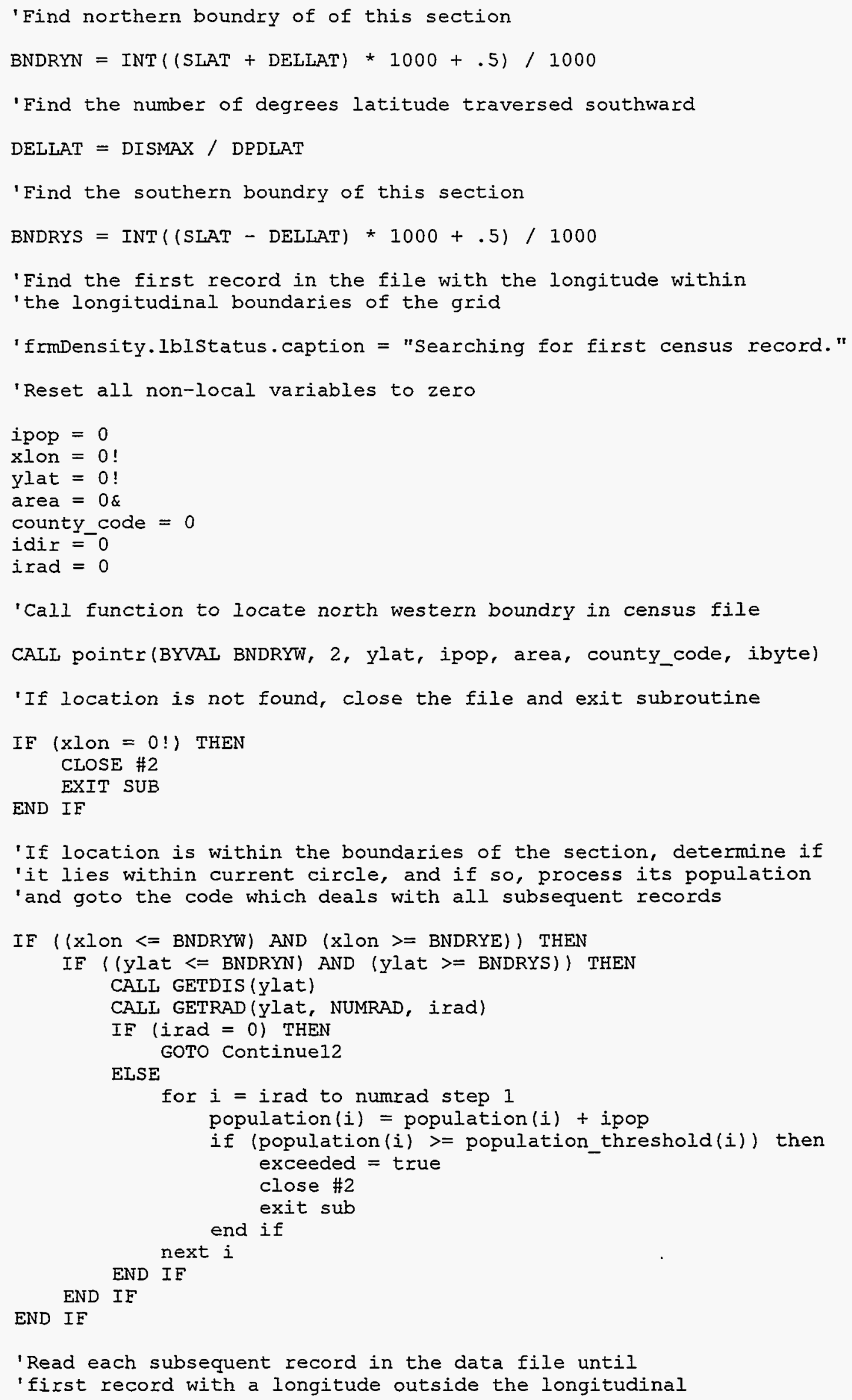

'Call function to locate north western boundry in census file

CALI pointr(BYVAL BNDRYW, 2, ylat, ipop, area, county_code, ibyte)

'If location is not found, close the file and exit subroutine

IF (xION $=0 !)$ THEN

'If location is within the boundaries of the section, determine if

'it lies within current circle, and if so, process its population

'and goto the code which deals with all subsequent records

IF ( $x$ lon <= BNDRYW) AND (Xlon >= BNDRYE)) THEN

IF (ylat <= BNDRYN) AND (ylat >= BNDRYS)) THEN

CAII, GETDIS (ylat)

CALI GETRAD (ylat, NUMRAD, irad)

IF (irad $=0$ ) THEN

ELSE

GOTO Continue12

for $i=$ irad to numrad step 1 population $(i)=$ population $(i)+$ ipop

if (population(i) $>=$ population_threshold(i)) then exceeded $=$ true close \#2 exit sub end if

END IE

END IF

'Read each subsequent record in the data file until

'first record with a longitude outside the longitudinal 
'boundaries

Continue12:

'frmDensity.Iblstatus.caption = "Processing census records."

ReadIt2:

'If end of file is reached, close file and exit subroutine

IF (EOF (2)) THEN

CLOSE \#2

EXIT SUB

END IE

'Jump seven bytes forward, to the next postion in the census file

ibyte $=$ ibyte + record_length

'Take from the current position (ibyte) in the file

'one record, which will be cut into its various pieces

GET \#2, ibyte, rec

'Parse the record into longitude, latitude, population, and region

ilon $=$ CVI (MIDS (rec, 1, 2))

ilat $=$ CVI (MID\$ $($ rec, 3, 2))

ipop $=$ CVI (MIDS (rec, 5, 2))

area $=$ CVL $(\operatorname{MID} \$($ rec, 7, 4))

county_code $=\operatorname{CVI}(\operatorname{MID} \$($ rec, 11,2$))$

'Convert Iongitude from file format into standard decimal degrees

xlon $=($ ilon + longitude_offset $) / 1000$ !

'Convert latitude from file format into standard decimal degrees

ylat $=($ ilat + latitude_offset $) / 1000 !$

'If latitude and longitude are within this section, verify that

'they are actually within current circle, and if so add its

'population to the correct sector

IF ( $(x l o n<=$ BNDRYW) AND ( $x$ lon $>=$ BNDRYE)) THEN

IE ( (ylat $<=$ BNDRYN) AND (ylat >= BNDRYS)) THEN

idir $=0$

irad $=0$

GETDIS (ylat)

CAII GETRAD (ylat, NUMRAD, irad)

IF (irad = 0) THEN

GOTO ReadIt2

ELSE

for $i=i r a d$ to numrad step 1

population (i) = population (i) + ipop

if (population(i) $>=$ population_threshold(i)) then exceeded = true

close \#2

exit sub

end if

next $i$

END IF

END IE 
'If the end of file has been reached, exit the subroutine

ELSEIF (EOF(2)) THEN

CLOSE \#2

EXIT SUB

'If longitude is outside of the specified segment, exit the

'subroutine.

ELSE CIOSE \#2

EXIT SUB

END IF

'If longitude is still within bounds, read in the next record

GOTO ReadIt2

popdensity_error:

msgbox "Popdensity error" + chr\$(13) + error\$(err) + chr\$(13) + -

"At line \#" + str\$(erl)

close \#2

exit sub

END SUB 


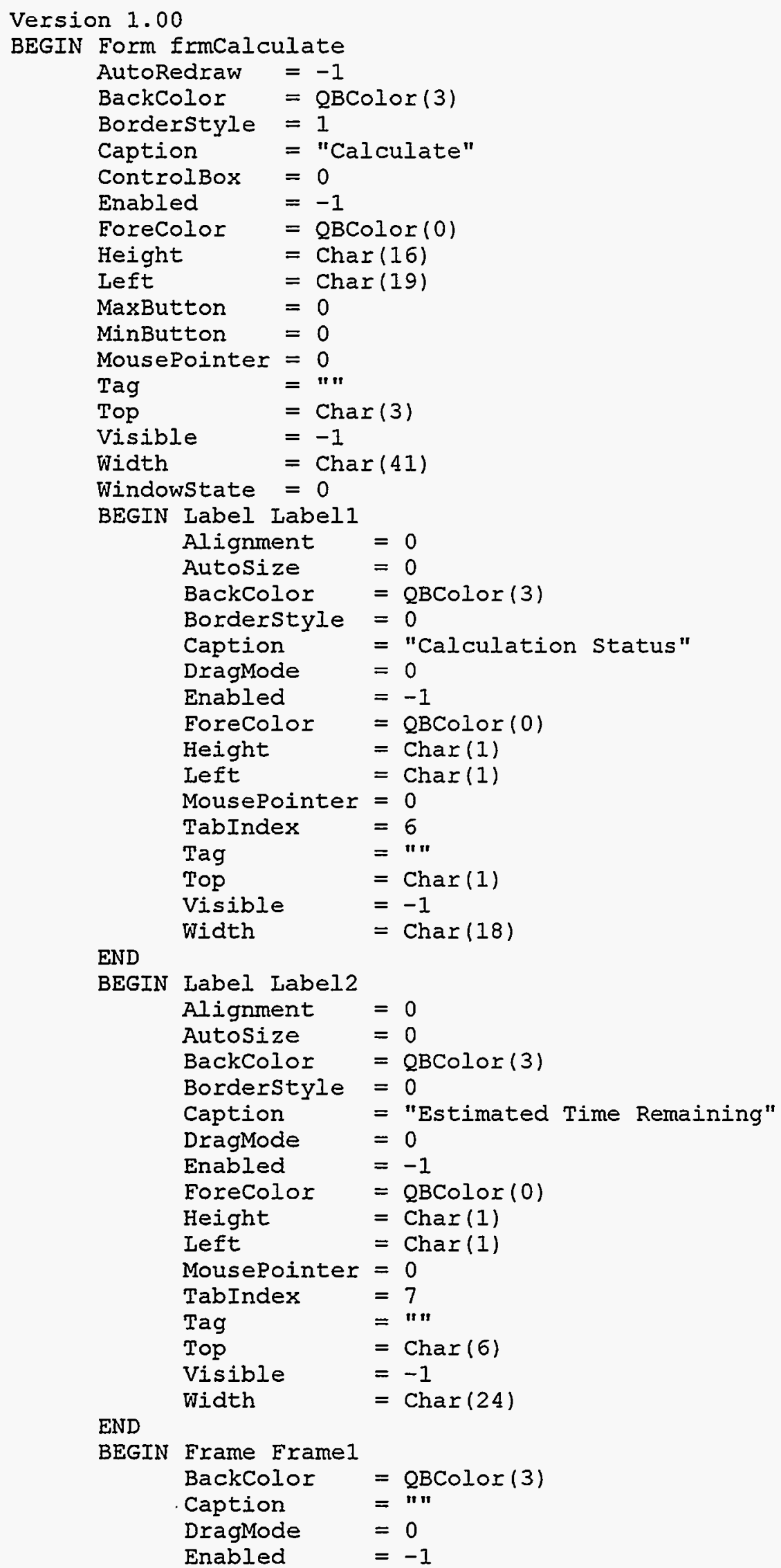




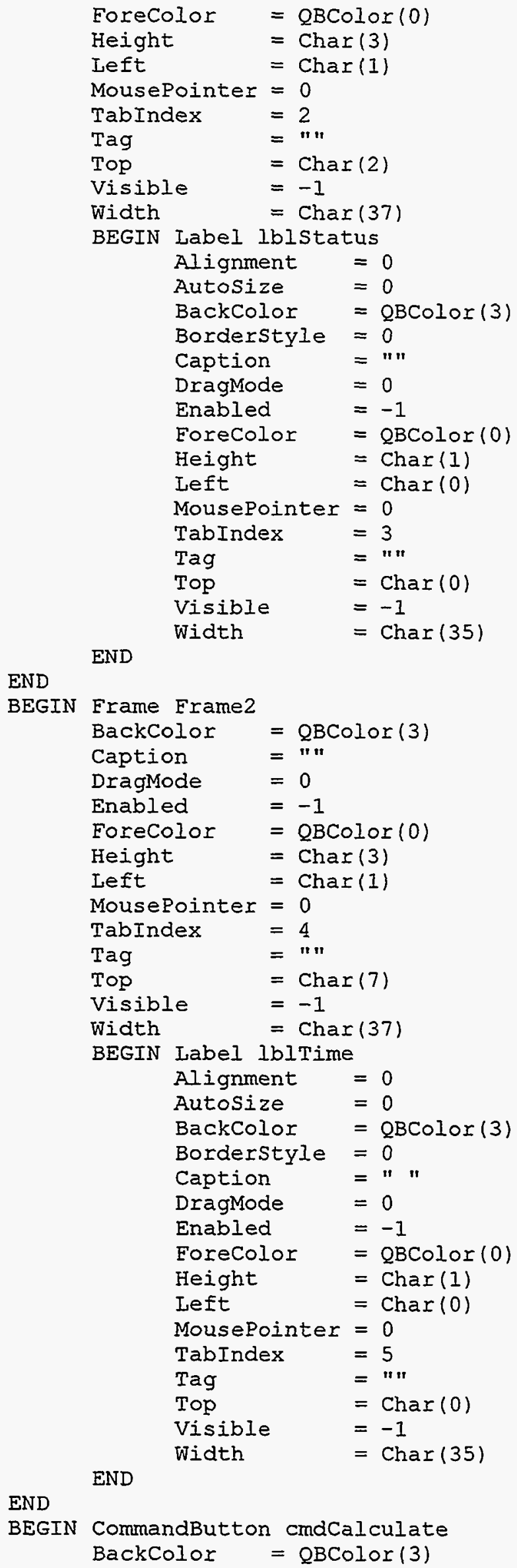




$\begin{array}{ll}\text { Cancel } & =0 \\ \text { Caption } & =\text { "\&Calculate" } \\ \text { Default } & =-1 \\ \text { DragMode } & =0 \\ \text { Enabled } & =-1 \\ \text { Height } & =\operatorname{Char}(3) \\ \text { Left } & =\text { Char (1) } \\ \text { MousePointer } & =0 \\ \text { TabIndex } & =0 \\ \text { Tabstop } & =-1 \\ \text { Tag } & =" 1 \\ \text { Top } & =\text { Char (11) } \\ \text { Visible } & =-1 \\ \text { Width } & =\text { Char (16) }\end{array}$

END

BEGIN CommandButton cmdClose

BackColor = QBColor (3)

Cancel $=-1$

Caption $=$ "C\&lose"

Default $\quad=0$

DragMode $\quad=0$

Enabled $\quad=-1$

Height $=\operatorname{Char}(3)$

Left $=$ Char (22)

MousePointer $=0$

TabIndex $=1$

Tabstop $\quad=-1$

Tag $=$ " "

Top $=\operatorname{Char}(11)$

Visible $\quad=-1$

END

Width $\quad=\operatorname{Char}(16)$

END

OPTION EXPLICIT

'\$INCLUDE: 'secpop90.bi'

'This form handles much of the overhead associated

'with regional calculations, including the user

'interface, reporting, pausing, and canceling

SUB cmdCalculate_Click ()

'This subroutine drives the function that performs site calculations

'It calls the popcalc function and keeps track of the amount of

'time spent on a site calculation.

CONST seconds_in_a_day $=86400$

DIM time begin AS SINGLE, time end AS SINGLE, total time AS SINGIE

DIM hours AS INTEGER, minutes $\bar{A} S$ INTEGER, seconds As SINGLE.

'Disable calculate button on form and reset the leave_calculations

'flag.

cmaCalculate. Enabled = EAISE

leave_calculation = EAISE

'Read the timer.

time_begin $=$ TIMER

'Call function to perform the actual calculation. 
CALL popcalc

'If the form is still visible, ie it wasn't closed before the

'calculation was complete, then perform the following actions.

IF (frmcalculate.Visible) THEN

'Read the timer.

time_end $=$ TIMER

'Convert the time into standard format.

IF time_end < time_begin THEN

ELSE

totāl_time $=$ time_end - time_begin + seconds_in_a_day

END IF

total_time $=$ time_end - time_begin

hours $=$ total time $\backslash 3600$

minutes $=($ total_time $\backslash 60)-($ hours $* 60)$

seconds $=($ total_time $-(($ hours $* 60+$ minutes $) * 60))$

'Display time to user.

frmCalculate.lblTime.Caption = "Total processing time $="$ + STRS(hours) + ":"

+ LTRIMS (STR\$ (minutes)) + ":" + FORMAT\$ (seconds, "0.00")

'Enable calculate button.

cmdCalculate. Enabled $=$ TRUE

END IF

END SUB

SUB cmaClose_Click ()

'This procedure allows the user to exit a calculation

'before it has been completed, and it allows the

'user to close the calculate window when a

'calculation is finished.

DIM reply AS INTEGER

'If a calculation is not in progress then unload the calculate form.

IF frmCalculate.cmdCalculate. Enabled = TRUE THEN

UNLOAD frmcalculate

ELSE

'If the calculation is in progress ask the user if they really

'want to leave.

reply = MSGBOX ("Do you really want to stop the present calculation?", MB_YESNO + MB_DEFBUTTON1, "Close")

IF (reply = IDYES) THEN

UNLOAD frmCalculate 


\section{leave_calculation $=$ TRUE}

END IF .

END IF

END SUB

SUB Form_Load ()

'Initialize status and time lables.

frmCalculate.1blstatus.Caption = "Press Calculate to start." frmCalculate.lblTime. Caption $=" "$

'Enable the Calculate button and reset the leave calculation flag.

frmCalculate. cmdCalculate. Enabled = TRUE

leave_calculation $=$ FALSE

END SUB 


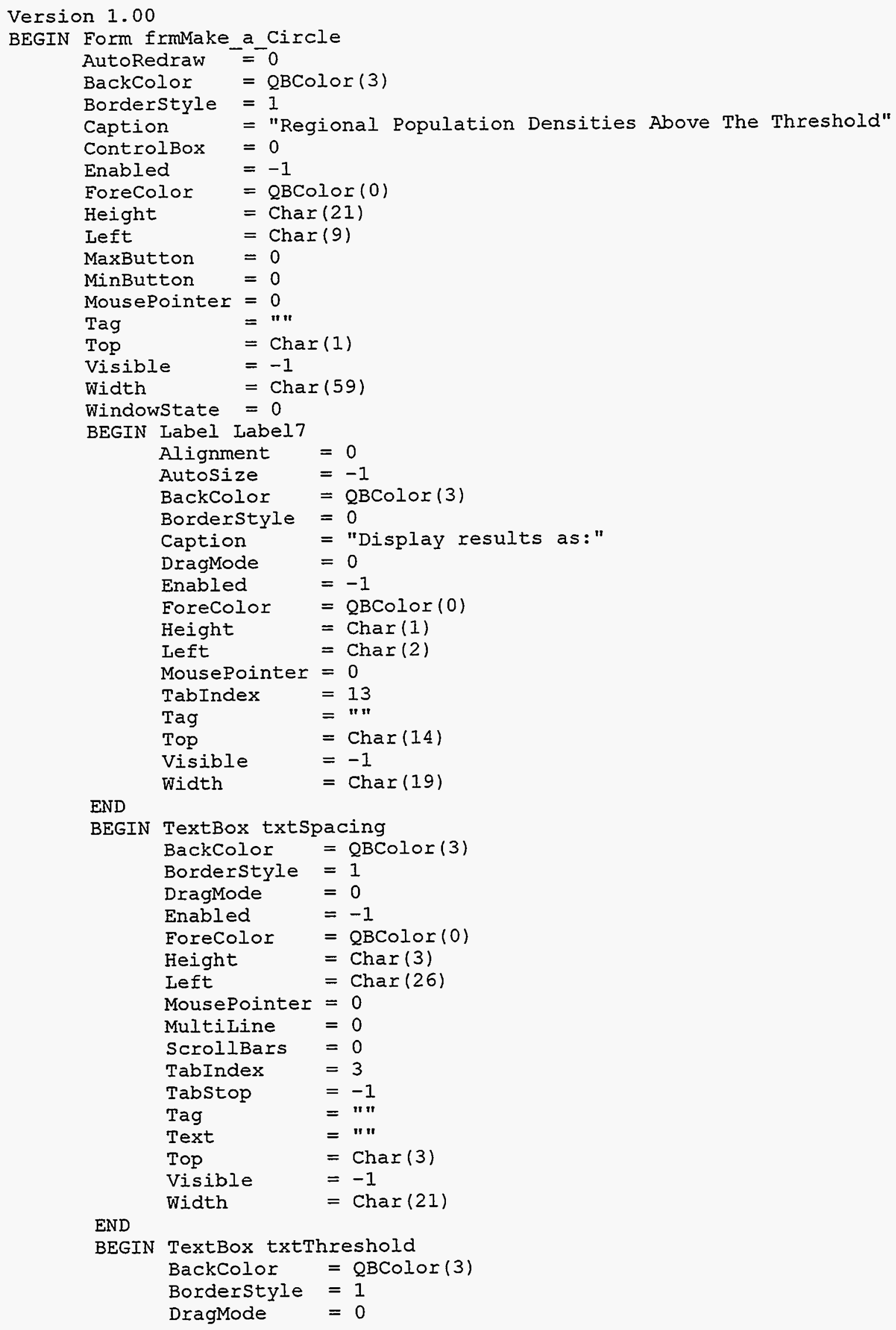




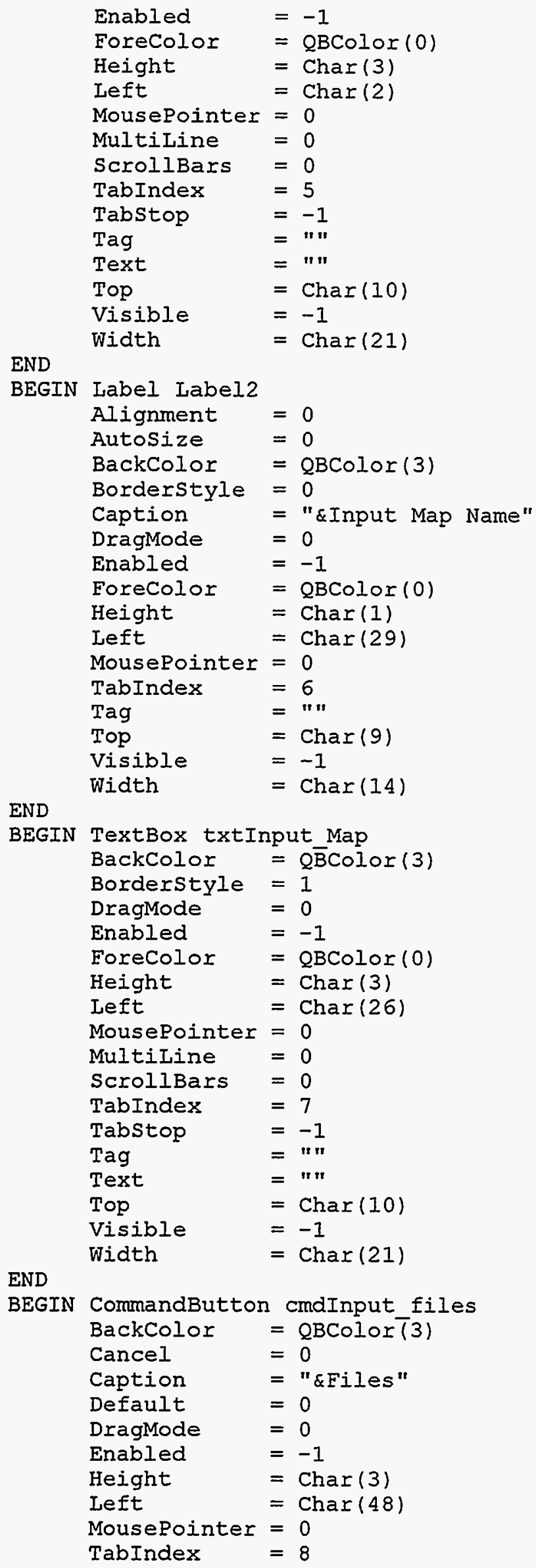




\begin{tabular}{|c|c|c|}
\hline & Tabstop & $=-1$ \\
\hline & Tag & $=" 1 "$ \\
\hline & Top & $=\operatorname{Char}(10)$ \\
\hline & Visible & $=-1$ \\
\hline & Width & $=\operatorname{Char}(7)$ \\
\hline END & & \\
\hline BEGIN & OptionButton & optPoints \\
\hline & BackColor & $=\mathrm{QBColor}(3)$ \\
\hline & Caption & $=" \&$ Points" \\
\hline & DragMode & $=0$ \\
\hline & Enabled & $=-1$ \\
\hline & ForeColor & $=Q B C o l o r(0)$ \\
\hline & Height & $=\operatorname{Char}(1)$ \\
\hline & Left & $=\operatorname{Char}(36)$ \\
\hline & MousePointer $=$ & $=0$ \\
\hline & TabIndex $\quad=$ & $=10$ \\
\hline & Tabstop & $=0$ \\
\hline & Tag & $=" n$ \\
\hline & Top & $=\operatorname{Char}(14)$ \\
\hline & Value & $=0$ \\
\hline & Visible & $=-1$ \\
\hline & width & $=\operatorname{Char}(10)$ \\
\hline END & & \\
\hline BEGIN & OptionButton & optcircles \\
\hline & BackColor = & $=$ QBColor $(3)$ \\
\hline & Caption & $=" \&$ Circles" \\
\hline & DragMode & $=0$ \\
\hline & Enabled & $=-1$ \\
\hline & ForeColor & $=\mathrm{QBColor}(0)$ \\
\hline & Height & $=\operatorname{Char}(1)$ \\
\hline & Left & $=\operatorname{Char}(23)$ \\
\hline & MousePointer & $=0$ \\
\hline & TabIndex & $=9$ \\
\hline & Tabstop & $=-1$ \\
\hline & Tag & $=" n$ \\
\hline & Top & $=\operatorname{Char}(14)$ \\
\hline & Value & $=-1$ \\
\hline & Visible & $=-1$ \\
\hline & Width & $=\operatorname{Char}(12)$ \\
\hline END & & \\
\hline BEGIN & CommandButton & n cmdCalculate_Regional \\
\hline & BackColor & $=$ oBColor $(3)$ \\
\hline & Cancel & $=0$ \\
\hline & Caption & $=$ "C\&alculate" \\
\hline & Default & $=0$ \\
\hline & DragMode & $=0$ \\
\hline & Enabled & $=-1$ \\
\hline & Height & $=\operatorname{Char}(3)$ \\
\hline & Left & $=\operatorname{Char}(10)$ \\
\hline & MousePointer & $=0$ \\
\hline & TabIndex & $=11$ \\
\hline & Tabstop & $=-1$ \\
\hline & Tag & $=" n$ \\
\hline & Top & $=\operatorname{Char}(16)$ \\
\hline & Visible & $=-1$ \\
\hline & Width & $=\operatorname{Char}(17)$ \\
\hline END & & \\
\hline BEGIN & CommandButton & n cmdClose \\
\hline & BackColor & $=$ QBColor $(3)$ \\
\hline & Cancel & $=-1$ \\
\hline & Caption & $=$ "C\&lose" \\
\hline & Default & $=0$ \\
\hline & DragMode & $=0$ \\
\hline
\end{tabular}




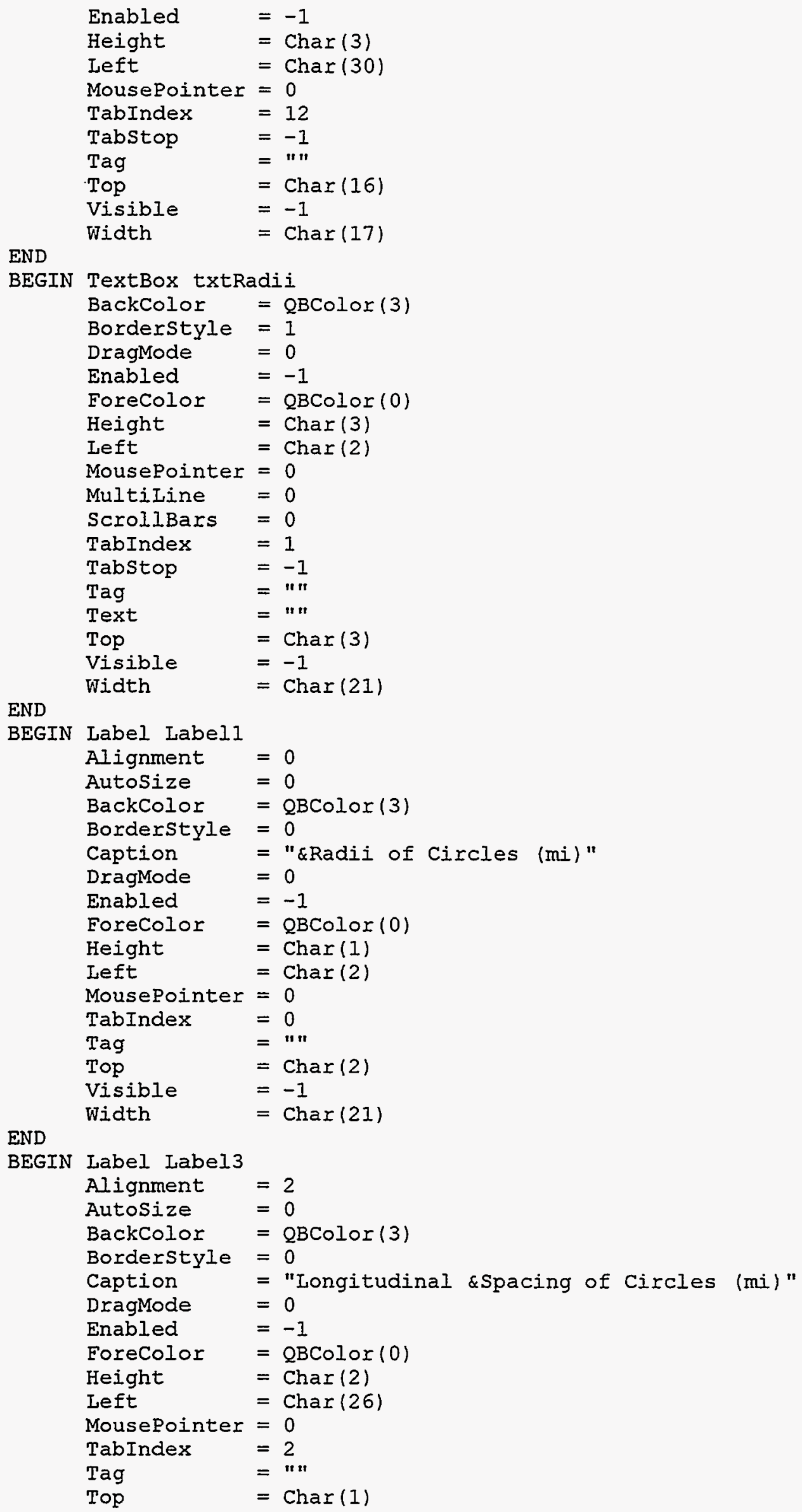


Visible $\quad=-1$

Width $\quad=\operatorname{Char}(20)$

END

BEGIN Iabel Iabel4

$\begin{array}{ll}\text { Alignment } & =2 \\ \text { Autosize } & =0\end{array}$

BackColor $=$ QBColor $(3)$

Borderstyle $=0$

Caption = "Population \&Density Threshold (People/Square mi)"

DragMode $\quad=0$

Enabled $\quad=-1$

Forecolor $=$ QBColor $(0)$

Height $=\operatorname{Char}(3)$

Left = Char (3)

MousePointer $=0$

TabIndex $=4$

Tag = " "

Top $\quad=\operatorname{Char}(7)$

Visible $\quad=-1$

END

Width $\quad=\operatorname{Char}(18)$

END

OPTION EXPLICIT

'\$INCLUDE: 'secpop90.bi'

'This form deals with much of the overhead associated with regional

'calculations, including the user interface parsing the data into a

'usable format, and reporting the status of the calculation.

SUB cmaCalculate_Regional_Click ()

'Declare a few local variables. Most of these variables are required

'to put the data in a format acceptable to mapplan.

DIM tempstring AS STRING, tempchar AS STRING

DIM orig input AS STRING

DIM number of circle segments AS INTEGER, i AS INTEGER

DIM $x$ int $\bar{A} S$ INTEGER, $y$ int AS INTEGER, spacing AS DOUBIE

DIM rötation increment $\bar{A} S$ DOUBLE, radii AS DOUBLE, $x$ AS DOUBLE

DIM $Y$ radius AS DOUBLE, $y$ space AS DOUBLE

DIM y $\overline{0}$ AS DOUBLE, y2 AS DOUBLE

DIM $x$ radius AS DOUBLE, $x$ space AS DOUBLE

DIM $x \overline{0}$ AS DOUBLE, $x I$ AS DOUBLE, $x 2$ AS DOUBIE

DIM xmin AS INTEGER, ymin AS INTEGER

DIM xmax AS INTEGER, ymax AS INTEGER

DIM two AS INTEGER, max points AS INTEGER

DIM last $x$ AS INTEGER, Iast $y$ AS INTEGER

DIM map_name AS STRING, lin $\bar{k}$ field AS STRING, offset AS INTEGER

DIM inpūt map AS STRING, output_map AS STRING

DIM output_database AS STRING

DIM min longitude AS DOUBLE, min latitude AS DOUBLE

DIM max longitude AS DOUBLE, $\max$ latitude AS DOUBLE

DIM Ionḡitude factor AS DOUBLE, Iatitude factor AS DOUBLE

DIM number_of layers AS INTEGER

DIM number of map records AS LONG, number of data records AS LONG

DIM bytes in map header AS INTEGER, bytes in data header AS INTEGER

DIM bytes in map_record AS INTEGER, bytes_in_data_record AS INTEGER

DIM midl $\bar{A} S \bar{S} T R I \bar{N} G$ * 18 , layerno AS STRING $\overline{\text { S }}$ - 3

DIM object type AS STRING * 1

DIM stp_ $x \bar{A} S$ STRING * 5, stp_y AS STRING * 5, no_elm AS STRING * 3

DIM pos_off AS STRING * 8, nop AS STRING * 5

DIM object_xmin AS STRING * 5 
DIM object ymin AS STRING * 5, object xmax AS STRING * 5

DIM object_ymax AS STRING * 5, deleted A.S STRING * 1

DIM mid2 A S STRING * 8 , density AS STRING * 1

DIM vector offset AS LONG

DIM threshold AS SINGLE

DIM exceeded AS INTEGER

DIM total_circles AS LONG, number_of circles AS LONG

DIM rows A.S LONG, rowO AS LONG, rOWI AS LONG

DIM $m$ AS DOUBLE, previous AS DOUBLE

DIM time_begin AS SINGLE, time end AS SINGLE, total time AS SINGLE

DIM hours $\bar{S}$ AS INTEGER, minutes $\bar{A} S$ INTEGER, seconds A $\bar{S}$ SINGLE

DIM reply as integer

'Check for valid input from the user.

SELECT CASE verify_input (3)

CASE 0

frmMake_a_circle.txtRadii.SETFOCUS

EXIT SUB

CASE -1

frmMake a circle.txtspacing. SETFOCUS

EXIT SU $\bar{B}$

CASE -2

frmMake a_circle.txtThreshold.SETFOCUS EXIT SU $\bar{B}$

CASE - 3

frmMake_a_circle.txtinput_map.SETFOCUS

END SELECT EXIT SUB

'start timer.

time_begin = TIMER

'Put filenames into an acceptable format.

'Remove extension from input map name, if necessary. This section

'allows the user to enter file names on frmmake a circle with or

'without extension. If an extension is present, it is temporarily

'removed from the file name, so that secpop can append the necessary

'extensions for the various map files. The filename and extension

'offered by the user are preserved.

tempstring $=" " n$

FOR $i=1$ TO (LEN (frmMake a circle.txtinput map.text))

tempchar = MIDS (frmMak̄e_a_circle.txtinpüt_map.text, $i, 1)$

IF tempchar $=$ ". "THEN EXIT FOR

ELSE END IF

tempstring $=$ tempstring + tempchar

NEXT i

orig_input $=$ frmMake_a_circle.txtinput_map.text

frmMake_a_circle.txtīnput map.text = tēmpstring

'Set up final error checking to catch file names that are too long

'and anything else that may have slipped by.

ON LOCAL ERROR GOTO regional_file_error

'Read in the user input. Convert miles to kilometers.

spacing $=$ VAL (frmMake_a_circle.txtspacing.text $)$ * miles_to_kilometers 
threshold = VAI (frmMake_a_circle.txtThreshold.text) /

(miles_to_kilometers $\bar{\wedge} 2$ )

'Set the radii to be equal to 1 mile increments out to 20 miles

'and 10 mile increments after that. 170 miles is the largest

'possible radii. Convert the miles to kilometers.

radii $=\operatorname{val}($ txtRadii.text)

for $i=1$ to max number of radii step 1

if (regional radii(i) < radii) then else raddis $(i \overline{)}=$ regional_radii(i) * miles_to_kilometers

raddis $(i)=$ radii * miles to kilometers

numrad $=i$ exit for

next $i$

end if

radii $=$ radii * miles_to_kilometers

'Intialize popultion threshold array.

for $i=1$ to numrad step 1

population threshold $(i)=$ fix(threshold * pi *

next $i$

$$
(\operatorname{raddis}(i) \wedge 2))+1
$$

input_map = frmmake_a_circle.txtinput_map.text

output_map = "mapplān Tmap_out"

output_database = "mapplañ \db_out"

'Copy the inputmap. dbf and . vtr files to the output map files,

'and supress the dos messages by dumping them into a temp file.

call copy file (input map + ".dbf ", output map + ".dbf")

call copy_file (input map + "spc ", output_map + ".spc")

call copy file (input map + ".vtr ", output map + "vtr")

call copy file ("mappIan\database.hdr", output_database + ".dbf")

Open the input map .cfg file and read in the data from it.

OPEN input map + ".cfg"

FOR INPUT ACCESS REA $\bar{D}$ LOCK READ WRITE AS \#30 LEN = 1024

INPUT \#30, xmin, ymin, xmax, ymax, two, max_points

IINE INPUT \#30, map name

IINE INPUT \#30, Iin $\bar{k}$ field

INPUT \#30, min_longit̄ude, min_latitude, max_longitude, max_latitude

INPUT \#30, longitude factor, Iatitude factor

CLOSE \#30

'Open the input map. Iyr file, and read in the data from it.

OPEN input map + ".IYr"

FOR INPUT ACCESS READ LOCK READ WRITE AS \#30 IEN $=1024$

$i=1$

'Read in information on each layer of the map until end of file is

'reached.

WHILE (NOT EOF(30))

$i=i+1$

INPUT \#30, layer_number(i), data_flag(i), display_flag(i)

INPUT \#30, active layer(i), layer name(i)

INPUT \#30, pts_cōor(i), pts_type(i), pts_size(i), pts_mode(i) 


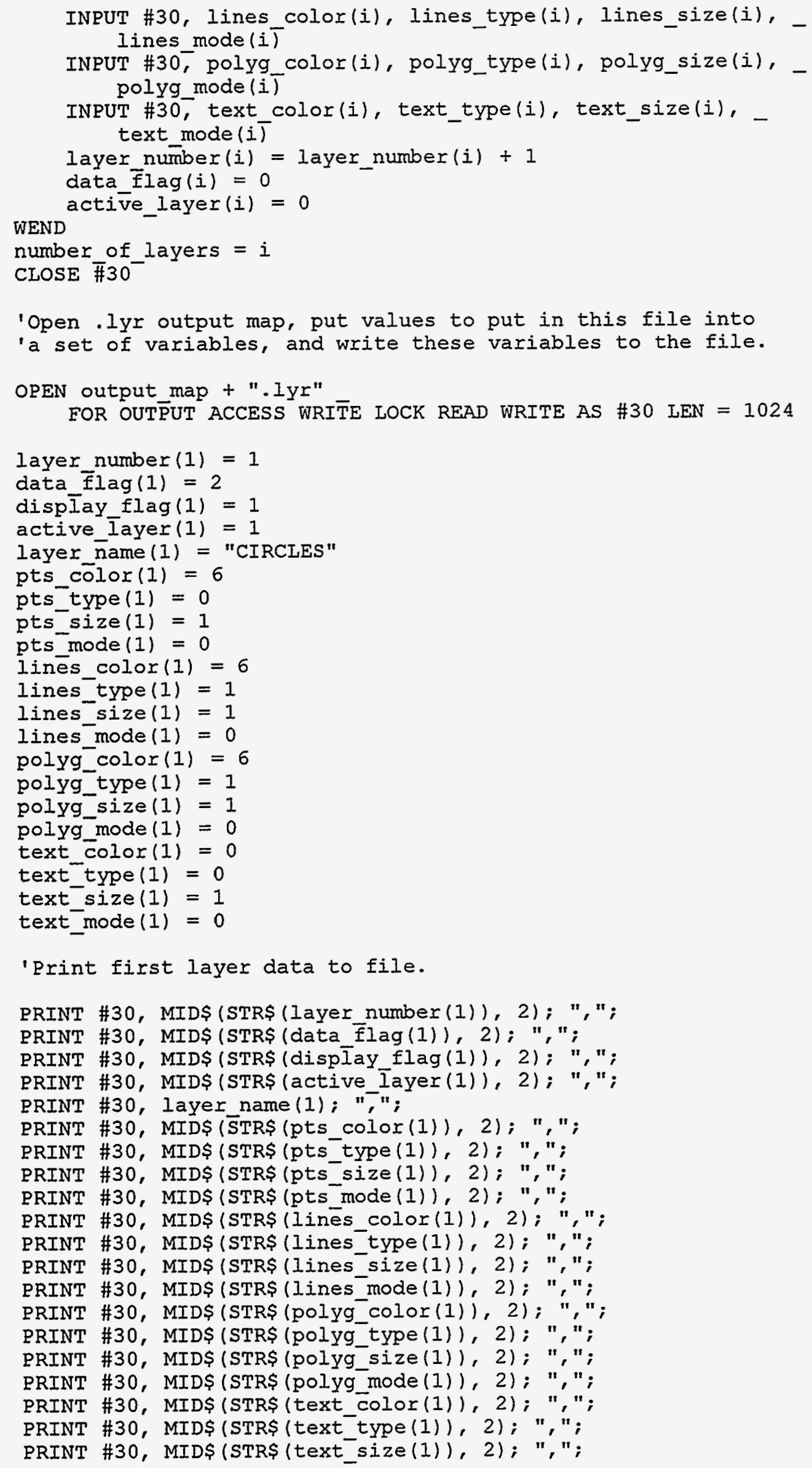


PRINT \#30, MIDS(STRȘ(text_mode (1)), 2)

'Print all other layers to file.

FOR $i=2$ TO number of layers STEP 1

PRINT \#30, MIDŞ (STRŞ (layer number (i)), 2); ",";

PRINT \#30, MIDS(STRS(data $\bar{f}$ lag(i)), 2); ", ";

PRINT \#30, MIDS(STRS (display flag(i)), 2); ",";

PRINT \#30, MIDS(STRS (active_Iayer(i)), 2); ",";

PRINT \#30, layer name (i); ",";

PRINT \#30, MIDS(STRS(pts_color (i)), 2); ", ";

PRINT \#30, MIDS(STRS (pts_type (i)), 2); ", ";

PRINT \#30, MIDS (STRS (pts_size(i)), 2); ", ";

PRINT \#30, MIDS (STRS (pts mode (i)), 2); ", ";

PRINT \#30, MIDS(STRS(Iines_color (i)), 2); ", ";

PRINT \#30, MIDS (STRS(Iines_type (i)), 2); ",";

PRINT \#30, MIDS(STRS(Iines size (i)), 2); ", ";

PRINT \#30, MIDS (STRS (Iines_mode(i)), 2); ",";

PRINT \#30, MIDS(STRS(polyg_color $(i)), 2)$; ", ";

PRINT \#30, MIDS(STRS (polyg_type (i)), 2); ",";

PRINT \#30, MIDS(STRS(polyg_size(i)), 2); ",";

PRINT \#30, MIDS(STRS(polyg_mode (i)), 2); ", ";

PRINT \#30, MIDS(STRS(text color(i)), 2); ",";

PRINT \#30, MIDS (STRS (text type (i)), 2); " ";

PRINT \#30, MIDS(STRS(text size(i)), 2); ",";

NEXT $i$

PRINT \#30, MIDS (STRS (text_mode (i)), 2)

CLOSE \#30

'Place degrees to radians conversion factor into DGTORD (DeGrees TO 'RaDians).

$\mathrm{DGTORD}=\mathrm{pi} / 180 !$

'Set Distance per degree longitude/latitude at average latitude.

CALI GETDIS (CSNG((min_latitude + max_latitude) / 2\#))

'Set up variables used to determine how far apart vertically (rows, 'row*) the circles are, and how far apart horizontally (*_radius, '*_space) these same circles are.

$\mathrm{x}$ radius $=(($ radii $/$ DPDLON $) /$ longitude factor $) * 1000000 \#$

Y_radius $=($ (radii $/$ DPDLAT $) /$ latitude_factor $) * 1000000 \#$

$\mathrm{x}$ space $=(($ spacing $/$ DPDLON $) /$ longitude factor $) * 1000000 \#$

Y_space $=($ (spacing $/$ DPDLAT $) /$ latitude_factor $) * 1000000 \# / 2 \#$

rows $=-\operatorname{INT}\left(-\left(y \max +y_{\text {_radius }}-y_{\text {min }}+1 !\right) / y_{\text {space }}\right)$

row0 $=-\operatorname{INT}(-(\mathrm{xmax}+\mathrm{x}$ radius - xmin $+1 !) / \mathrm{x}$-space $)$

row $1=-\operatorname{INT}\left(-\left(x \max +x_{\text {_radius }}-\operatorname{xmin}-\left(x_{-} \operatorname{space} / 2 !\right)+1 !\right) /\right.$ $x$ space)

'Determine total number of circles.

total circles $=$

$\operatorname{INT}(($ rows $/ \overline{2} !)+.75)$ * row $0+\operatorname{INT}(($ rows $/ 2 !))$ * row 1

'Open all output map files.

OPEN output map + ".dbf"

FOR BINĀRY ACCESS READ WRITE LOCK READ WRITE AS \#30

OPEN output map + ".vtr"

FOR BINĀRY ACCESS READ WRITE LOCK READ WRITE AS \#31 


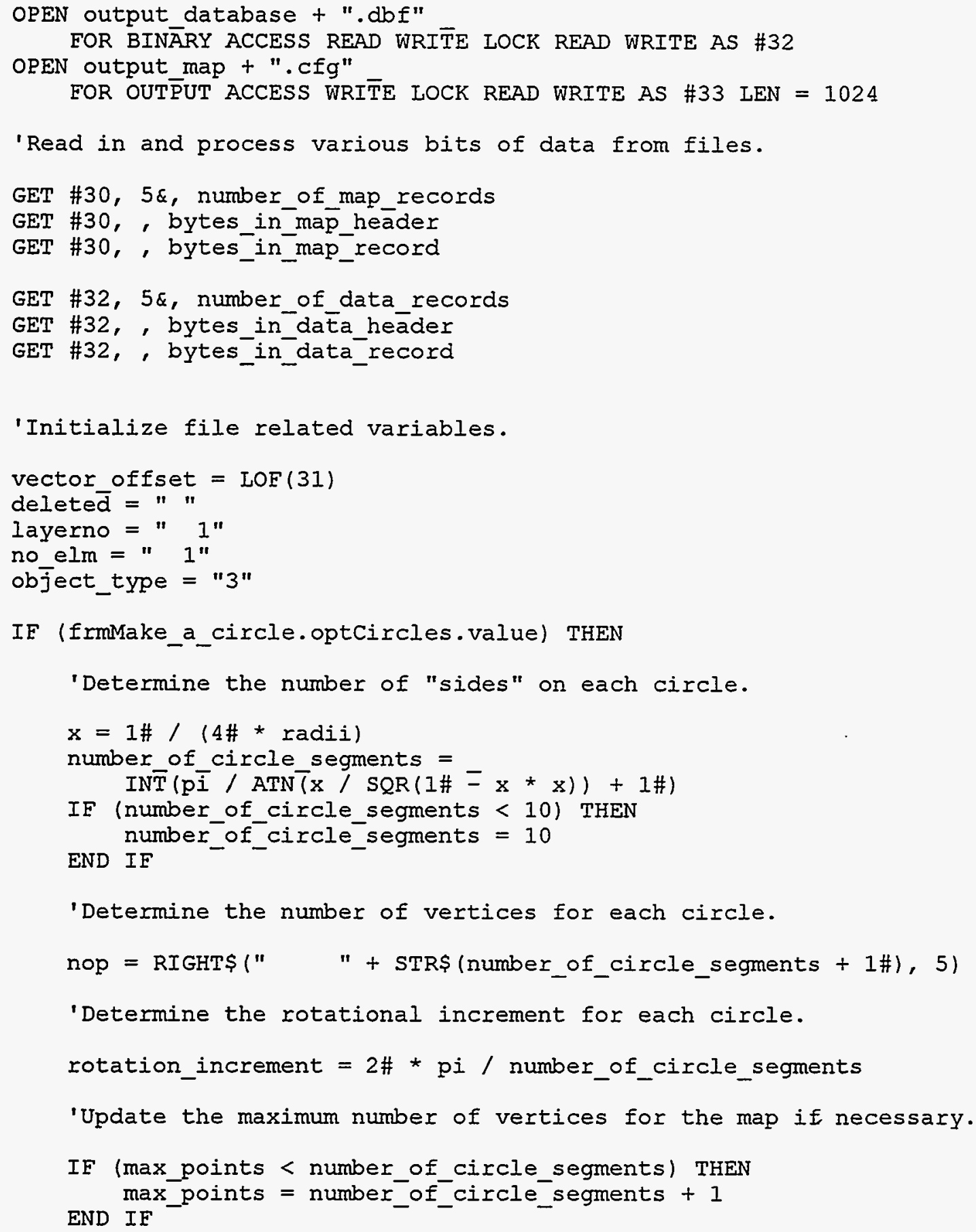

ELSE

"If the user has selected "points" from the circle form, set "number of vertices to 3 , and ignore all of the circle

'variables. This will give the user a triangle, which is the

'smallest item that mapplan can process.

nop $=" 3 "$

END IF

'Print data to the . cfg map file.

PRINT \#33, MID\$(STRS (xmin), 2); ymin; xmax; ymax; two; max_points 


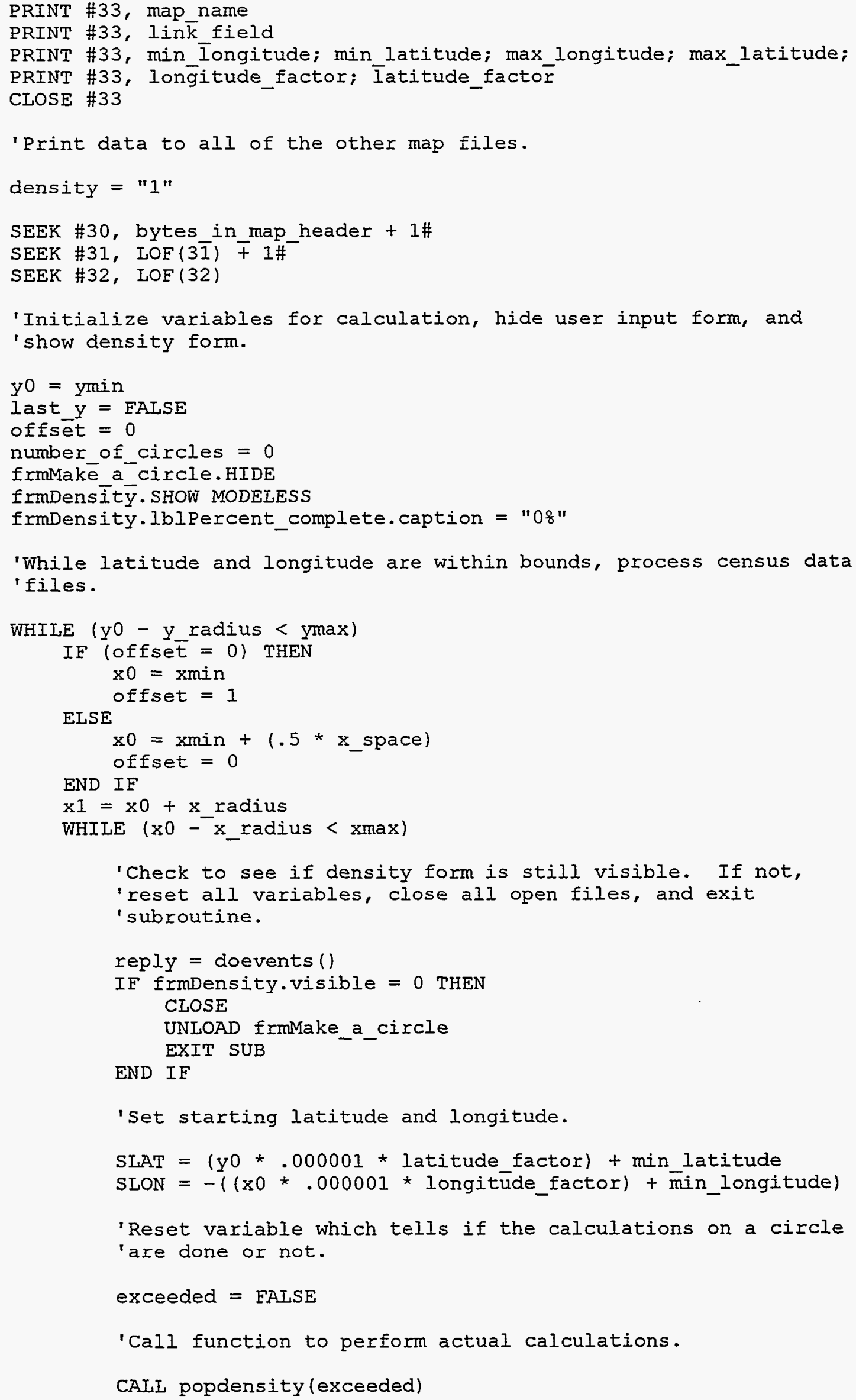


'If the number of people in the circle are greater than the 'threshold, then plot the circle in the map file, in a

'manner understood by mapplan, and increment counters.

IF (exceeded) THEN

number of map_records $=$ number of map records +1

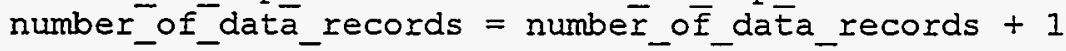

midl = LEFTS $($ "POP" + MIDS (STRS (number_of data_records), 2) +

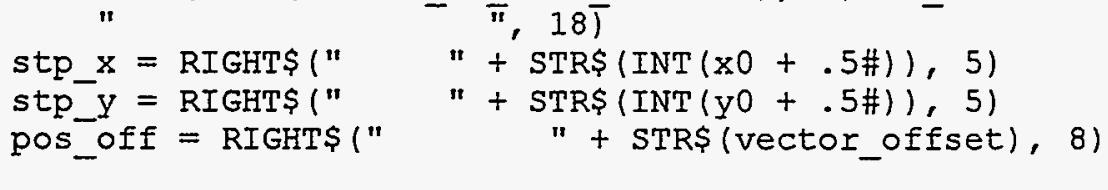

IF (INT ( $\mathrm{x} 0-\mathrm{x}$ radius $+.5 \#)>=\mathrm{xmin}$ ) THEN

object_xmin = RIGHT\$(" " +

ELSE

STR $\left.\$\left(I N T\left(x 0-x_{\text {radius }}+.5 \overline{\#}\right)\right), 5\right)$

END IF

object_xmin $=$ RIGHT\$(" "+ STR\$ $(x \min ), 5)$

IF (INT (yO - y_radius $+.5 \#)>=$ ymin) THEN

object ymin = RIGHTS (" "+ STRS(INT (YO - Y_radius + .5\#)), 5)

ELSE

object_ymin = RIGHT\$(" "+ STRS (ymin), 5)

END IF

IF (INT ( $x 0+x_{-}$radius $\left.+.5 \#\right)<=x \operatorname{xmax}$ ) THEN

object $x \max =$ RIGHT\$(" "+

ELSE

$\mathrm{ST} \overline{\mathrm{R}}$ (INT $\left.\left(\mathrm{X}_{0}+\mathrm{x}_{\text {_radius }}+.5 \overline{\#}\right)\right), 5$ )

object_xmax $=\operatorname{RIGHT}(" \quad$ " $+\operatorname{STR} \$(x \max ), 5)$

END IF

IF (INT ( $\mathrm{y} 0+\mathrm{y}$ radius $+.5 \#)<=$ ymax) THEN

object ymax $=$ RIGHT $(" \quad "+$

ELSE

STRS(INT ( YO $^{\circ}$ y_radius $\left.\left.\left.+.5 \overline{\#}\right)\right), 5\right)$

END IF

object_ymax $=$ RIGHT\$(" " $+\operatorname{STR} \$(y \max ), 5)$

'Write data to output file.

PUT \#30, deleted

PUT \#30, , midl

PUT \#30, , layerno

PUT \#30, , object_type

PUT \#30, , stp_x

PUT \#30, stp- sy

PUT \#30, , no elm

PUT \#30, , pos_off

PUT \#30, , nop

PUT \#30, , object_xmin

PUT \#30, , object_ymin

PUT \#30, , object_xmax

PUT \#30, , object_ymax

$\operatorname{mid} 2=\operatorname{LEFT} \$(\operatorname{mid} 1,8)$

PUT \#32, , deleted

PUT \#32, , mid2

PUT \#32, , density 
'Plot the actual points for the circle, and place them

'into the vector file.

IF (frmMake a circle.optcircles.value) THEN

$$
\begin{aligned}
& x \text { int }=\operatorname{INT}(x 1+.5 \#) \\
& y \text { int }=\operatorname{INT}\left(\mathrm{y}_{0}+.5 \#\right)
\end{aligned}
$$

PUT \#31, , x int

PUT \#31, $y$ int

vector_offset $=$ vector_offset $+4 \#$

FOR $i=1$ TO number of circle segments STEP $I$

$\mathrm{x} 2=\mathrm{x}$ radius ${ }^{-} \cos (i *$ rotation_increment $)+\mathrm{x} 0$

$\mathrm{y} 2=y_{\text {_radius }}$ * SIN $(i$ * rotation increment $)+\mathrm{y} 0$

IF $\left(x^{-}<=x \operatorname{xin}\right)$ THEN $x$ int $=\operatorname{INT}(x \min +.1)$

IF $(x 2>=x \operatorname{xax})$ THEN $y$ int $=\operatorname{INT}(y m i n+.1)$

IF $(y 2<=y \min )$ THEN $x_{-}$int $=$INT $(x \min -.1)$

IF $\left(y^{2}>=y \operatorname{ymax}\right)$ THEN $y_{-}$int $=\operatorname{INT}(y \min -.1)$

$x$ int $=\operatorname{INT}(x 2+.5 \#)$

$y_{\text {int }}=\operatorname{INT}\left(\mathrm{y}^{-}+.5 \#\right)$

vector_offset = vector_offset $+4 \#$

PUT \#3I, , $x_{-}$int

NEXT i

PUT \#31, $y_{-}^{-}$int

ELSE

'Draw very small triangles instead of circles if

'that is what the user has requested. This method

'is much faster than plotting circles.

$x 2=(x 0-5 \#)$

$y^{2}=\left(y_{0}-4.33 \#\right)$

IF $(x 2<=x \min )$ THEN $x$ int $=\operatorname{INT}(x \min +.1)$

IF $(x 2>=x \max )$ THEN $y_{\text {_int }}=\operatorname{INT}(y \min +.1)$

IF $(y 2<=y \min )$ THEN $\mathrm{x}$ int $=$ INT $(x \min -.1)$

IF $(\mathrm{y} 2>=\mathrm{ymax})$ THEN $\mathrm{y}_{-}$int $=\operatorname{INT}(\mathrm{ymin}-.1)$

$x_{\text {int }}=\operatorname{INT}(\mathrm{x} 2+.5 \#)$

$y$ int $=$ INT $\left(y^{2}+.5 \#\right)$

PUT \#31, $x$ int

PUT \#31, y_int

$\mathrm{y} 2=\left(\mathrm{y}^{0}+4.33 \#\right)$

IF $(x 2>=x \max )$ THEN $y_{\text {int }}=\operatorname{INT}(y \min +.1)$

IF $\left(y^{2}>=\right.$ ymax $)$ THEN $y_{-}$int $=$INT $(y \min -.1)$

$y$ int $=\operatorname{INT}\left(\mathrm{y}^{2}+.5 \#\right)$

$\mathrm{x}$-int $=\operatorname{INT}(\mathrm{x} 0+.5 \mathrm{H})$

PUT \#31, $x$ int

PUT \#31, y_int

$\mathrm{x} 2=(\mathrm{x} 0+5 \#)$

$y^{2}=(y 0-4.33 \#)$

IF $(x 2<=x \operatorname{xin})$ THEN $x$ int $=\operatorname{INT}(x \min +.1)$.

IF $(x 2>=x \max )$ THEN $y^{-i n t}=\operatorname{INT}(y m i n+.1)$

IF $(y 2<=y$ min) THEN $x$ int $=$ INT $(x m i n-.1)$

IF ( $y 2>=y \max$ ) THEN $y_{-}$int $=$INT (ymin - .I)

$x$ int $=\operatorname{INT}(x 2+.5 \#)$

$y_{\text {int }}=$ INT $\left(\mathrm{y}^{2}+.5 \#\right)$

PUT \#31, $x_{\text {_int }}$

PUT \#31, $y_{-}$int

END IF

vector_offset $=$ vector_offset $+12 \#$

END IF 


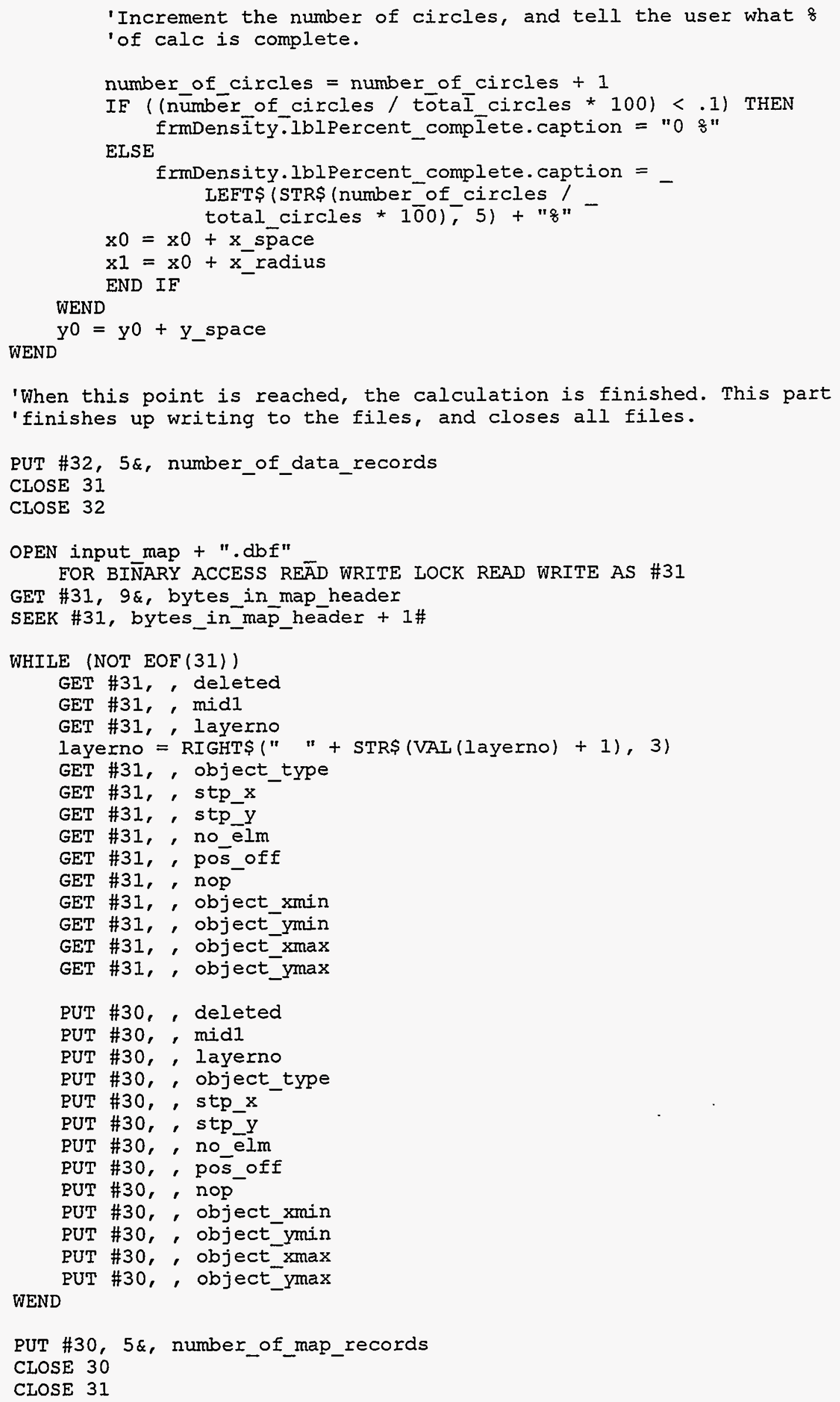

'When this point is reached, the calculation is finished. This part 'finishes up writing to the files, and closes all files.

PUT \#32, 5\&, number_of_data_records

CLOSE 31

CLOSE 32

OPEN input map + ".dbf"

FOR BINAARY ACCESS REĀD WRITE LOCK READ WRITE AS \#31

GET \#31, 9\&, bytes in map header

SEEK \#31, bytes_in_map_header + 1\#

WHILE (NOT EOE (31))

GET \#31, , deleted

GET \#31, , midl

GET \#31, , layerno

layerno = RIGHTS(" " + STRS(VAI (layerno) + 1), 3)

GET \#31, , object_type

GET \#31, , stp_x

GET \#31, , stp_y

GET \#3I, no elm

GET \#31, , pos_off

GET \#31, , nop

GET \#31, , object xmin

GET \#31, , object_ymin

GET \#31, , object_xmax

GET \#31, , object_ymax

PUT \#30, deleted

PUT \#30, , mid1

PUT \#30, , layerno

PUT \#30, , object type

PUT \#30, , stp_x

PUT \#30, , stp_y

PUT \#30, no elm

PUT \#30, , pos_off

PUT \#30, , nop

PUT \#30, , object xmin

PUT \#30, , object ymin

PUT \#30, , object xmax

WEND

PUT \#30, , object_ymax

PUT \#30, 5\&, number_of_map_records

CIOSE 30

CLOSE 31 


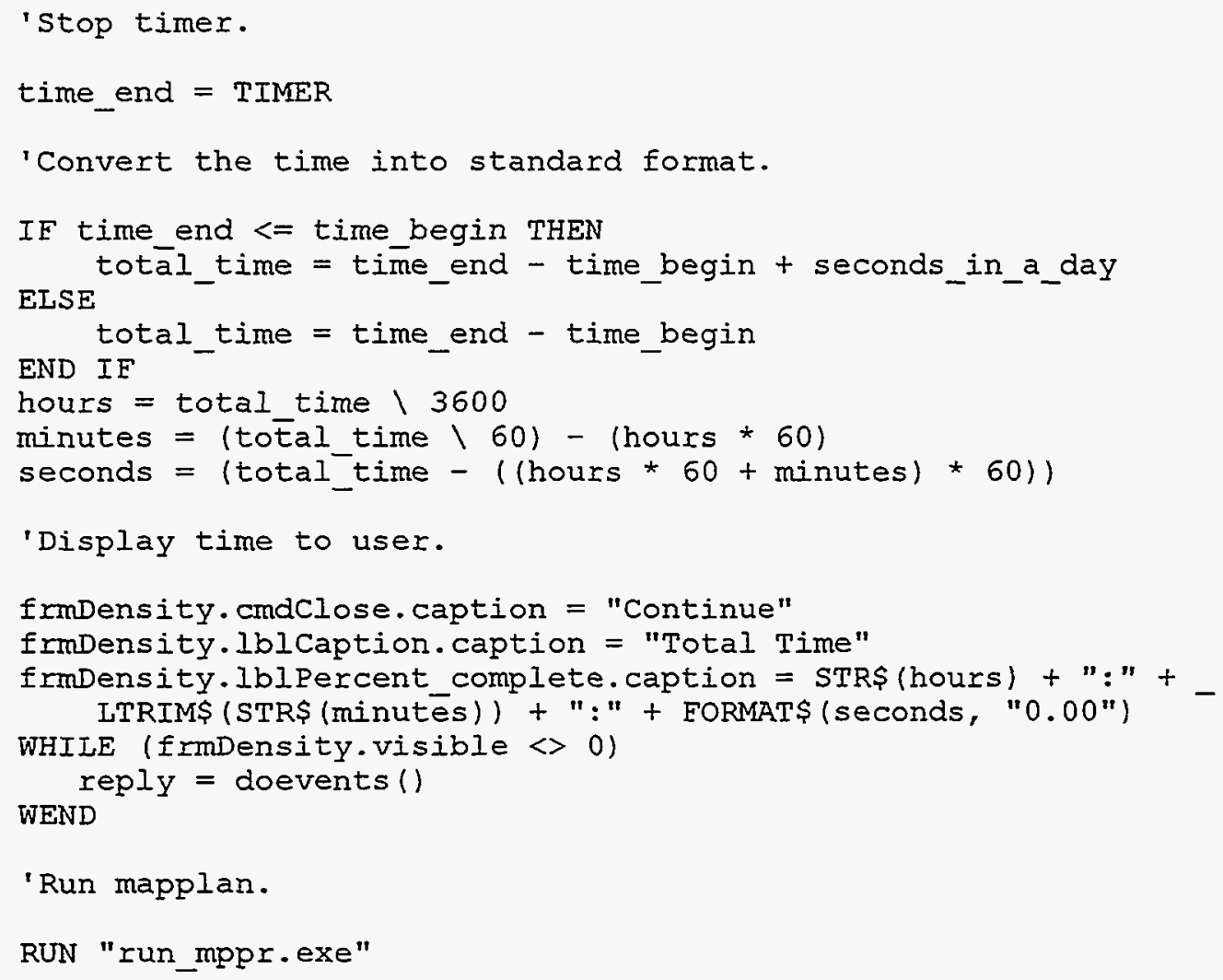

'Run mapplan.

RUN "run_mppr.exe"

EXIT SUB

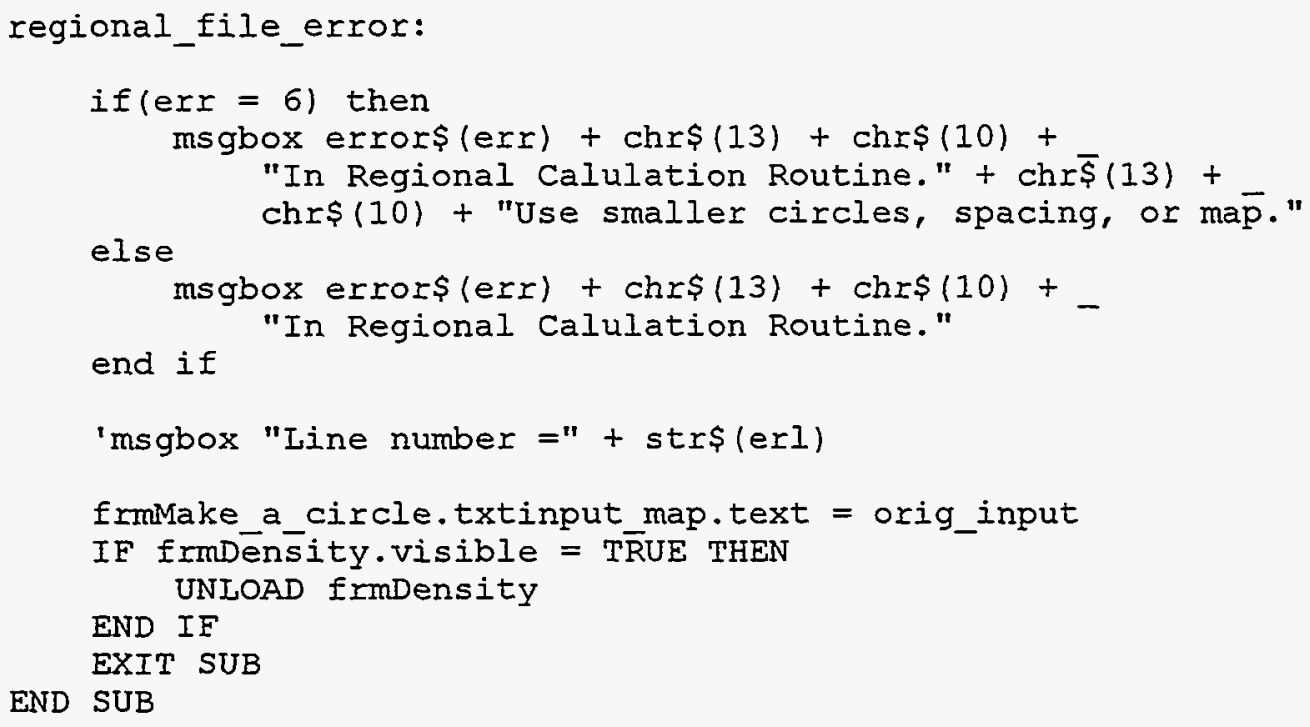


SUB cmdInput_files_Click ()

'This routine allows the user to select an input map file for regional

'calculations.

DIM filenum AS INTEGER, forecolor AS INTEGER, backcolor AS INTEGER

DIM Flags AS INTEGER, Cancel AS INTEGER

DIM filename AS STRING

DIM pathname AS STRING

DIM Defaultext AS STRING, DialogTitle AS STRING

'Initialize file dialogue box.

DefaultExt $=" *$.CFG"

DialogTitle = "Input Map File"

backcolor $=$ WHITE

forecolor $=$ BLACK

pathname = "mapplan\maps"

'Prompt user for file name (from dialogue box).

CALI Fileopen(filename, pathname, DefaultExt, DialogTitle, forecolor, backcolor, Flags, Cancel)

'If user did not choose cancel in dialogue box...

IF NOT Cancel THEN

'If filename is not in current directory, append path name

'to it.

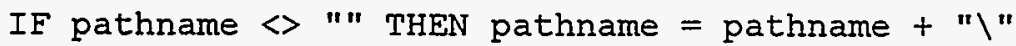

filename $=$ pathname + filename

frmMake_a_circle.txtinput_map.text $=$ filename

'set focus on next field in regional form.

frmMake_a_circle.cmdCalculate_regional.SETFOCUS

ELSE

frmMake_a_circle.txtinput_map.SETFOCUS

END IF

END SUB

SUB optCircles_Click ()

ErmMake_a_circle.optCircles.value = TRUE

frmMake_a_circle.cmdCalculate_regional.SETFocus

END SUB

SUB optPoints_Click ()

frmMake_a_circle.optPoints.value = TRUE

frmMake_a_circle.cmdCalculate_regional.SETFOCUS

END SUB 


\section{OPTION EXPLICIT}

'SINCIUDE: 'secpop90.bi'

Visual Basic for MS-DOS Common Dialog Toolkit

The Common Dialog Toolkit (CMNDLG.BAS and CMNDLGF.FRM)

provides support for the following dialogs:

Fileopen

Filesave

Fileprint

Support for each dialog is provided via procedures with these same names that create the corresponding dialog and return user input to your program. These procedures only provide the user interface and return user input.

They do not actually carry out the corresponding actions

such as opening the file. Detailed descriptions of

these procedures are contained in the comment headers above each.

All common dialogs are created from the same form (CMNDLGF.FRM).

The necessary controls for each dialog are children of

a container picture box for the dialog. Thus the

form (CMNDLGF. FRM) contains a picture box with

appropriate controls for common dialog listed above.

When a particular common dialog is created and displayed,

the container picture box for that dialog is made visible

(thus all controls on that picture box become visible)

and the form is centered and sized to match the

container picture box.

To use these common dialogs in your programs, include

CMNDLG.BAS and CMNDLGF. FRM in your program or use the

supplied Iibrary (CMNDLG.LIB, CMNDLGA. LIB - AltMath version

for Professional Edition only) and Quick library (CMNDIG.QLB)

and call the appropriate procedure to invoke the dialog

you need.

Copyright (C) 1982-1992 Microsoft Corporation

You have a royalty-free right to use, modify, reproduce

and distribute the sample applications and toolkits provided with

Visual Basic for MS-DOS (and/or any modified version)

in any way you find useful, provided that you agree that

Microsoft has no warranty, obligations or liability for

any of the sample applications or toolkits.

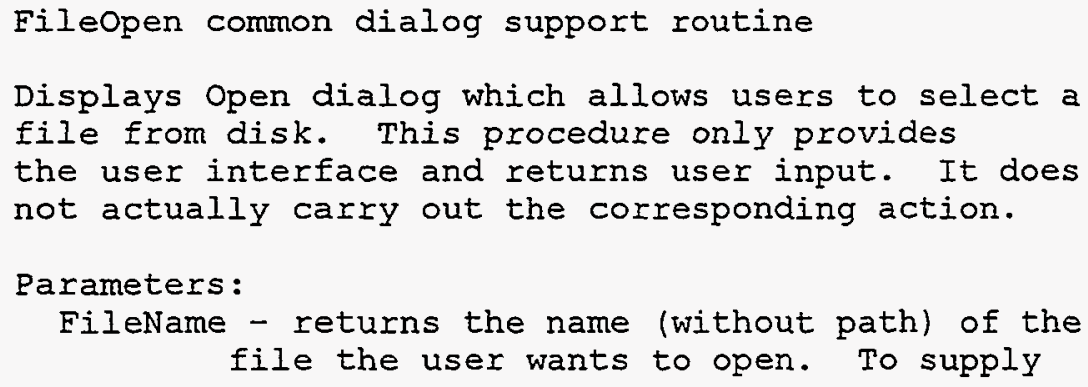


default filename in dialog, assign default

to FileName then pass it to this procedure.

PathName - returns the path (without filename) of

the file the user wants to open. To supply

default path in dialog, assign default to

PathName then pass it to this procedure.

Note, only pass a valid drive and path. Do

not include a filename or file pattern.

DefaultExt - sets the default search pattern for the

File listbox. Default pattern when DefaultExt

is null is "*.*". To specify a different

search pattern (i.e. "*.BAS"), assign new

value to Defaultext then pass it to this

procedure.

DialogTitle - sets the dialog title. Default title

when DialogTitle is null is "Open". To

specify a different title (i.e. "Open My File"), assign new value to DialogTitle then pass it to this procedure.

ForeColor - sets the dialog foreground color. Does not affect SCREEN. Controlpanel color settings.

BackColor - sets the dialog background color. Does not affect SCREEN. Controlpanel color settings.

Flags - unused. Use this to customize dialog action if needed.

Cancel - returns whether or not user pressed the dialog's Cancel button. True $(-1)$ means the user cancelled the dialog.

SUB Fileopen (FileName AS STRING, PathName AS STRING,

DefaultExt AS STRING, DialogTitle AS STRING, ForeCōlor AS INTEGER, -

BackColor AS INTEGER, Flags AS INTEGER, Cancel AS INTEGER)

- Set up error handling for option validation.

ON LOCAI ERROR GOTO FileOpenError

- Set form caption.

IF DialogTitle $=" "$ THEN

ELSE

frmCmnDlg. Caption $=$ "Open"

END IF

frmCmnDlg. Caption $=$ DialogTitle

- Determine search pattern for file listbox.

IF DefaultExt $<>~ "$ "THEN

ELSE

fxmCmnDlg.filopenList.Pattern $=$ DefaultExt

END IF

fxmCmndg. filopenList.Pattern $=" *$ *"

- Determine default path.

IF PathName <> " THEN

- Set drive and path for file-system controls.

- Set Directory listbox path. If PathName is different

- than current path, PathChange event will be triggered

- which updates Drive Iistbox drive and File listbox path.

END IF

frmCmnDlg.diropenList. Path = PathName

- Display current path to the user.

frmCmnDlg. lblopenPath. Caption $=$ frmCmnDlg. filopenList. Path

- Determine default filename to display in edit field.

IF FileName $<$ " "THEN

ELSE

frmCmnDlg.txtopenFile.Text = UCASES (FileName) 
END IF

frmCmnDlg.txtopenFile.Text $=$ frmCmnDlg.filopenList.Pattern

- Set default and cancel command buttons.

frmcmnDlg. cmdopenoK. Default = TRUE

frmCmnDlg. cmopenCancel. Cancel $=$ TRUE

- Size and position Open/Save container.

frmCmnDlg.pctFileopen. Borderstyle $=0$

frmCmnDlg.pctFileOpen.visible = TRUE

' Size and center dialog.

frmCmnDlg.MOVE frmCmnDlg.Left, frmCmnDlg.Top, frmCmnDlg.pctFileopen.Width +2 , frmCminlg.pctFileopen. Height +2

fmCmnDlg.MOVE (SCREEN.Width - fmCmnDlg.Width) \2, ((SCREEN.Height frmCmnDlg.Height) (2) - 2

- Set dialog colors.

frmcmndlg. ForeColor = ForeColor

frmCmndig.BackColor = BackColor

frmCmnDlg.pctFileopen. ForeColor = Forecolor

frmCmnDlg.pctFileopen. BackColor = BackColor

frmCmnDlg.lblopenfile. ForeColor = ForeColor

frmCmnDlg.lblopentile.BackColor = BackColor

frmCmnDlg.txtopenfile. ForeColor = ForeColor

frmCmnDlg.txtopenFile.BackColor = BackColor

frmCmnDlg.lblopenPath. ForeColor = ForeColor

frmcmnDlg.lblopenPath. BackColor = BackColor

frmCmnDlg. filopentist. ForeColor = ForeColor

frmCmnDlg.filopenList. BackColor = BackColor

frmCmnDlg.drvopenIist. ForeColor = ForeColor

frmcmndlg.drvopenIist. BackColor = BackColor

frmcrnolg.diropenList. ForeColor = ForeColor

frmCmnDlg.diropenList. BackColor = BackColor

frmCmnDlg.cmolopenoK. BackColor = BackColor

frmCinnDlg.cmdopenCancel. BackColor = BackColor

- Display dialog modally.

frmcmnDlg. SHOW 1

- Determine if user canceled dialog.

IF frmcmnDlg. cmdopenCancel.Tag $<$ "FAISE" THEN

Cancel = TRUE

' If not, return FileName and PathName.

ELSE

Cancel = EAISE

FileName $=$ frmCmnDlg.txtopenFile. Text

PathName $=$ frmCmnDlg.filopentist.Path

END IE

ErmCmnDlg. cmaopenCancel. Tag = " "

' Hide or unload dialog and return control to user's program.

' (Hide if user chose to preload form for performance.)

IE LEFT\$(frmCmnDIg.Tag, 1) = "H" THEN

frmCmnDlg.pctFileopen.visible = EALSE

EISE frmCmnDlg. HIDE

END IF

UNLOAD frmCmnDlg

EXIT SUB

- Option error handling routine. 
- Ignore errors here and let dialog's controls

' handle the errors.

FileopenError:

SELECT CASE ERR

CASE 7:

- Out of memory.

MSGBOX "Out of memory. Can't load dialog.", 0, "Fileopen"

Cancel $=$ TRUE

CASE ELSE

EXIT SUB

END SELECT

END SUB

RESUME NEXT

- FilePrint common dialog support routine

Displays Print dialog which allows users to select

- Print destination (PRINTER.PrintTarget) and the

number of copies to print. This procedure only provides

the user interface and returns user input. It does

- not actually carry out the corresponding action.

Parameters :

Copies - returns the number of copies ( 1 to 99) the user wants

to print. To supply default number of copies

in dialog, assign default to Copies then

pass it to this procedure (default when copies

is 0 is 1 ).

Forecolor - sets the dialog foreground color. Does not affect SCREEN. Controlpanel color settings.

BackColor - sets the dialog background color. Does not affect SCREEN. Controlpanel color settings.

Cancel - returns whether or not user pressed the dialog's Cancel button. True $(-1)$ means the user cancelled the dialog.

SUB FilePrint (Copies AS INTEGER, ForeColor AS INTEGER, BackColor AS INTEGER, Cancel AS INTEGER)

DIM I AS INTEGER

ON LOCAI ERROR GOTO FilePrintError

frmCmnDlg.Caption = "Print" ' Set form caption.

- Determine default number of copies.

IF Copies $=0$ THEN

ELSE

frmCmnDlg.txtPrintCopies. Text $=$ "1"

END IF

frmCmnDlg.txtPrintCopies. Text $=$ STRS (Copies)

- Set default and cancel command buttons.

frmCmnDlg.cmdPrintoK. Default = TRUE

frmCmnDlg. cmaPrintCancel. Cancel $=$ TRUE

- Size and position Print container.

frmCmnDlg.pctFilePrint. Borderstyle $=0$

frmCmnDlg.pctFilePrint.visible = TRUE

- Size and center dialog.

frmCmnDlg.MOVE frmCmnDlg.Left, frmCmnDlg.Top, frmCmnDlg.pctFilePrint.Width +2 , frmCmnDlg.pctFilePrint. Height +2

frmCmnDlg.MOVE (SCREEN.Width - EmMCmnDlg.Width) \2, ((SCREEN.Height frmCmnDlg. Height) \2) - 2 
- Set dialog colors.

frmcmndig. ForeColor = ForeColor

frmCmnDlg.BackColor = BackColor

frmCmnDlg.pctFilePrint. ForeColor = ForeColor

frmCmnDlg.pctEilePrint.BackColor = BackColor

frmcmnDlg. IblPrintcopies. ForeColor = Forecolor

frmCmnDlg.lblerintCopies.BackColor = BackColor

frmCmnDlg.txtPrintCopies. ForeColor = ForeColor

frmCmnDlg.txterintCopies.BackColor = BackColor

frmCmnDlg.txtPrintFile. ForeColor = ForeColor

frmCmnDlg.txtPrintFile.BackColor = BackColor

frmcmnDlg. fraPrintTarget. ForeColor = ForeColor

frmCmnDlg. fraPrintTarget.BackColor = BackColor

FOR is $=0$ TO 3

frmCmnDlg.optPrintTarget (if). ForeColor = ForeColor

frmCmnDlg.optPrintTarget $(i \delta)$. BackColor = BackColor

NEXT is

FOR is $=0$ TO 1

frmCmnDlg.optPrintAppend (if). ForeColor = ForeColor

frmCmnDlg.optPrintAppend (i 8$)$. BackColor = BackColor

NEXT is

frmCmnDIg.lblprintAppend.ForeColor = ForeColor

frmCmnDlg.IblPrintAppend.BackColor = BackColor

frmCmnDlg. cmdPrintoK. BackColor = BackColor

frmCmnDlg.cmdPrintCancel.BackColor = BackColor

- Display dialog modally.

frmCmnDlg. SHOW 1

- Determine if user canceled dialog.

IF frmCmnDlg.cmdPrintCancel.Tag $\gg$ "EALSE" THEN

Cancel = TRUE

- If not, return number of copies to print.

ELSE

Cancel = EALSE

IF VAI (ErmCmnDlg.txtPrintCopies.Text) > 99 THEN Copies $=99$

ELSEIF VAL (ErmCmnDIg.txtPrintCopies.Text) $<1$ THEN

ELSE Copies $=1$

END IF

Copies = VAI (IrmCmnDlg.txtPrintCopies.Text)

END IF

frmCmnlg. cmdPrintCancel. Tag = " "

- Hide or unload dialog and return control to user's program.

- (Hide if user chose to preload form for performance.)

IF LEFTS (frmCmnDlg.Tag, 1) = "H" THEN

frmCmnDlg.pctFilePrint.visible = FALSE

frmCmnDlg.HIDE

ELSE

UNLOAD frmCmnDlg

END IF

EXIT SUB

- Error handling routine.

FilePrinterror:

SELECT CASE ERR

CASE 7 :

- Out of memory.

MSGBOX "Out of memory. Can't load dialog.", 0, "FindPrint" Cancel $=$ TRUE 


\section{EXIT SUB \\ CASE ELSE \\ RESUME NEXT \\ END SELECT}

END SUB

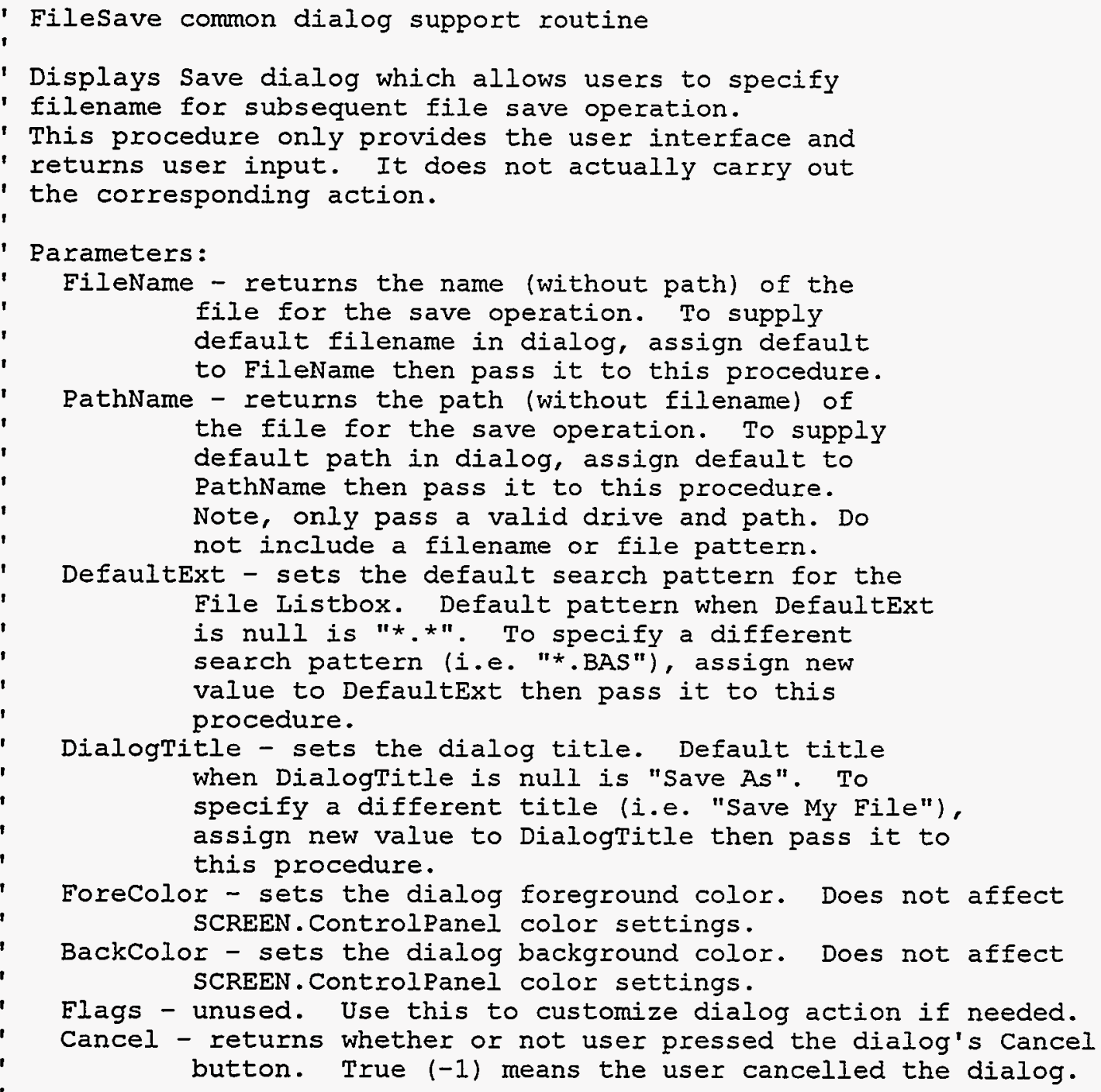

SUB Filesave (FileName AS STRING, PathName AS STRING, DefaultExt AS STRING, DialogTitle AS STRING, ForeColor AS INTEGER, BackColor AS INTEGER, Flags AS INTEGER, Cancel AS INTEGER)

' Set up error handling for option validation.

ON LOCAI ERROR GOTO FilesaveError

- Set form caption.

IF DialogTitle = " "THEN

ELSE

ErmCmnDlg. Caption = "Save As"

END IE

frmCmnDlg.Tag = frmCmnDlg. Tag + "SAVE" ' Set form tag for common

unload procedure.

- Determine search pattern for file listbox.

IF DefaultExt $<>"$ THEN

frmCmnDlg. filopenList.Pattern = DefaultExt

ELSE 
END IF

frmCrndlg. filopenIist.Pattern $=" * * "$

- Determine default path.

IF PathName <> " "THEN

- If the path ends with a backslash, remove it.

IF RIGHTS(PathName, 1) = "\" THEN

PathName = IEFTS(PathName, LEN (PathName) - 1)

END IF

' Set drive and path for file-system controls.

- Set File listbox path. If PathName is different

- than current path, Pathchange event will be triggered

- which updates Drive listbox drive and Directory Iistbox path.

frmCmnDlg.filopenList.Path = PathName

END IF

- Display current path to the user.

frmCmnDlg.lblopenPath. Caption = frmCmnDlg.filopenlist.Path

- Determine default filename to display in edit field.

IF FileName $<>$ " THEN

frmCmnDlg.txtopenFile.Text = UCASES (FileName)

ELSE

frmCmnDlg.txtopenFile.Text = frmCmnDlg.filopenList.Pattern

END IF

- Set default and cancel command buttons.

frmCmnDlg.cmdopenoK. Default = TRUE

frmcmnDlg. cmopencancel. Cancel = TRUE

- Size and position Open/Save container.

frmCmnDlg.pctFileopen. Borderstyle $=0$

frmCmnDlg.pctFileopen. visible = TRUE

- Size and center dialog.

frmCmnDlg.MOVE frmCmnDlg. Left, frmCmnDlg.Top, frmCmnDlg.pctFileopen.Width + 2, frmCmnDlg.pctFileopen. Height +2

frmCmnDIg.MOVE (SCREEN.Width - frmCmnDlg.Width) \2, ((SCREEN.Height -

frmCmnDlg.Height) (2)-2

- Set dialog colors.

frmcmnDlg. ForeColor = ForeColor

frmCmnDlg.BackColor = BackColor

frmCmnDlg.pctFileopen. ForeColor = ForeColor

frmCmnDlg.pctFileopen.BackColor = BackColor

frmcmndlg.Iblopentile. ForeColor = ForeColor

frmCmnDlg.lblopenFile.BackColor = BackColor

frmCmnDlg.txtopenFile. ForeColor = ForeColor

frmCmnDlg.txtopenFile.BackColor = BackColor

frmCmnDlg.lblopenPath. ForeColor = ForeColor

frmCmnDlg.lblopenPath. BackColor = BackColor

frmcmnDlg.filopentist. ForeColor = ForeColor

frmCmnDlg.filopenlist.BackColor = BackColor

frmCmnDlg. drvopenList. ForeColor = ForeColor

frmCmnlg.drvopenlist.BackColor = BackColor

frmCmnDlg.diropenList. ForeColor = ForeColor

frmCmnDlg.diropentist.BackColor = BackColor

frmCmnDlg.cmdopenoK. BackColor = BackColor

frmCmnDlg. cmdopenCancel. BackColor = BackColor

- Display dialog modally.

frmCmnDlg. SHOW 1 
- Determine if user canceled dialog.

IF frmCmnDlg.cmdopenCancel.Tag <> "FAISE" THEN

Cancel = TRUE

- If not, return FileName and PathName. ELSE

Cancel $=$ FAISE

FileName $=$ frmCmnDlg.txtopenFile.Text

PathName $=$ frmCmnDlg. filopenList.Path

END IF

frmCmnDlg.cmdOpenCancel.Tag $=" "$

- Hide or unload dialog and return control to user's program.

' (Hide if user chose to preload form for performance.)

IF LEET\$ (frmCmnDlg.Tag, 1) = "H" THEN

frmCmnDlg.pctFileopen.visible = FALSE

frmCmnDlg. HIDE

ELSE

frmCmnDlg.Tag $=$ "H" $\quad$ ' Reset tag.

END IE

UNLOAD frmCmnDlg

EXIT SUB

- Option error handling routine.

- Ignore errors here and let dialog's controls

- handle the errors.

FilesaveError:

SELECT CASE ERR

CASE 7:

MSGBOX "Out of memory. Can't load dialog.", 0, "Filesave"

Cancel $=$ TRUE

CASE ELSE

EXIT SUB

END SELECT

END SUB 
Version 1.00

BEGIN Form frmcinndig

AutoRedraw $=0$

Borderstyle $=1$

Caption = "Common Dialog"

ControlBox $=-1$

Enabled $=-1$

ForeColor $=$ QBColor $(0)$

Height $=$ Char (19)

Left $=\operatorname{Char}(12)$

MaxButton $=0$

MinButton $\quad=0$

MousePointer $=0$

Tag $=" "$

Top $=\operatorname{Char}(3)$

Visible $\quad=-1$

Width $=\operatorname{Char}(64)$

Windowstate $=0$

BEGIN PictureBox pctFilePrint

AutoRedraw $=0$

BackColor = QBColor (7)

Borderstyle $=1$

DragMode $=0$

Enabled $\quad=-1$

Forecolor $=$ oBColor $(0)$

Height $=$ Char (10)

Left $=$ Char (0)

MousePointer $=0$

TabIndex $=9$

Tabstop $\quad=0$

Tag = "

Top $=\operatorname{Char}(0)$

Visible $\quad=0$

Width $\quad=\operatorname{Char}(60)$

BEGIN Erame fraPrintTarget

$$
\begin{array}{ll}
\text { BackColor } & =\text { QBColor }(7) \\
\text { Caption } & =\text { "Print Target" } \\
\text { DragMode } & =0 \\
\text { Enabled } & =-1 \\
\text { EoreColor } & =\text { QBColor }(0) \\
\text { Height } & =\operatorname{Char}(8) \\
\text { Left } & =\text { Char }(1) \\
\text { MousePointer } & =0 \\
\text { TabIndex } & =10 \\
\text { Tag } & =" \text { "Char (1) } \\
\text { Top } & =-1 \\
\text { Visible } & =\text { Char (43) } \\
\text { Width } & \text { Bidt }
\end{array}
$$

BEGIN OptionButton optPrintTarget

BackColor $=$ QBColor (7)

Caption = "IPT\&I"

DragMode $\quad=0$

Enabled $\quad=-1$

Forecolor $=$ QBColor $(0)$

Height $=\operatorname{Char}(1)$

Index $=0$

Left $=\operatorname{Char}(1)$

MousePointer $=0$

TabIndex $=11$ 


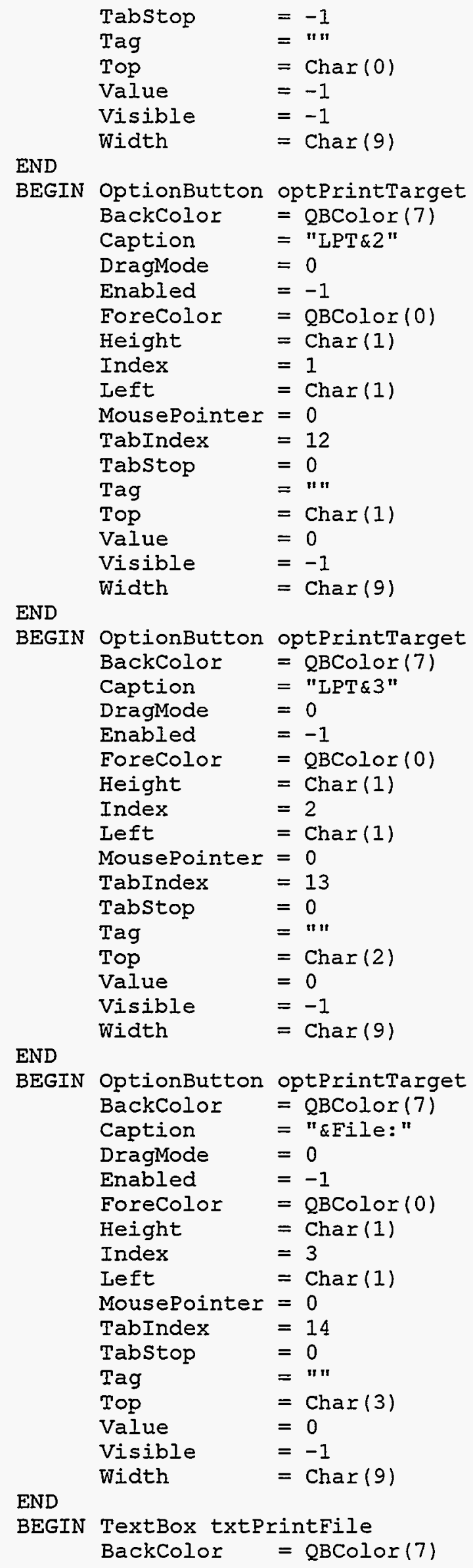




$\begin{array}{ll}\text { BorderStyle } & =1 \\ \text { DragMode } & =0 \\ \text { Enabled } & =0 \\ \text { ForeColor } & =\text { QBColor }(0) \\ \text { Height } & =\text { Char (3) } \\ \text { Left } & =\text { Char (11) } \\ \text { MousePointer } & =0 \\ \text { MultiLine } & =0 \\ \text { ScrollBars } & =0 \\ \text { TabIndex } & =15 \\ \text { Tabstop } & =-1 \\ \text { Tag } & =" " \\ \text { Text } & =" " \\ \text { Top } & =\text { Char (2) } \\ \text { Visible } & =-1 \\ \text { Width } & =\text { Char (29) }\end{array}$

END

BEGIN PictureBox pctPrintAppend AutoRedraw =

BackColor $=$ QBColor (7)

Borderstyle $=0$

DragMode $\quad=0$

Enabled $\quad=0$

ForeColor $=$ QBColor $(0)$

Height $=\operatorname{Char}(1)$

Left $=$ Char (0)

MousePointer $=0$

TabIndex $=16$

Tabstop $\quad=0$

Tag $=" n$

Top $=\operatorname{Char}(5)$

Visible $\quad=-1$

width $\quad=\operatorname{Char}(41)$

BEGIN Iabel 1blPrintAppend

Alignment $=0$

Autosize $=0$

BackColor $=$ QBColor (7)

Borderstyle $=0$

Caption = "If file exists:"

DragMode $\quad=0$

Enabled $\quad=-1$

ForeColor $=$ QBColor $(0)$

Height $=\operatorname{Char}(1)$

Left $=\operatorname{Char(1)}$

MousePointer $=0$

TabIndex $=17$

Tag = " "

Top $=\operatorname{Char}(0)$

Visible $\quad=-1$

END

Width $\quad=\operatorname{Char}(16)$

BEGIN OptionButton optPrintAppend

BackColor = QBColor (7)

Caption = "A\&ppend"

DragMode $\quad=0$

Enabled $\quad=-1$

ForeColor $=$ oBColor $(0)$

Height $=\operatorname{Char}(1)$

Index $\quad=0$

Left $=\operatorname{Char}(17)$

MousePointer $=0$

TabIndex $=18$

Tabstop $\quad=0$ 


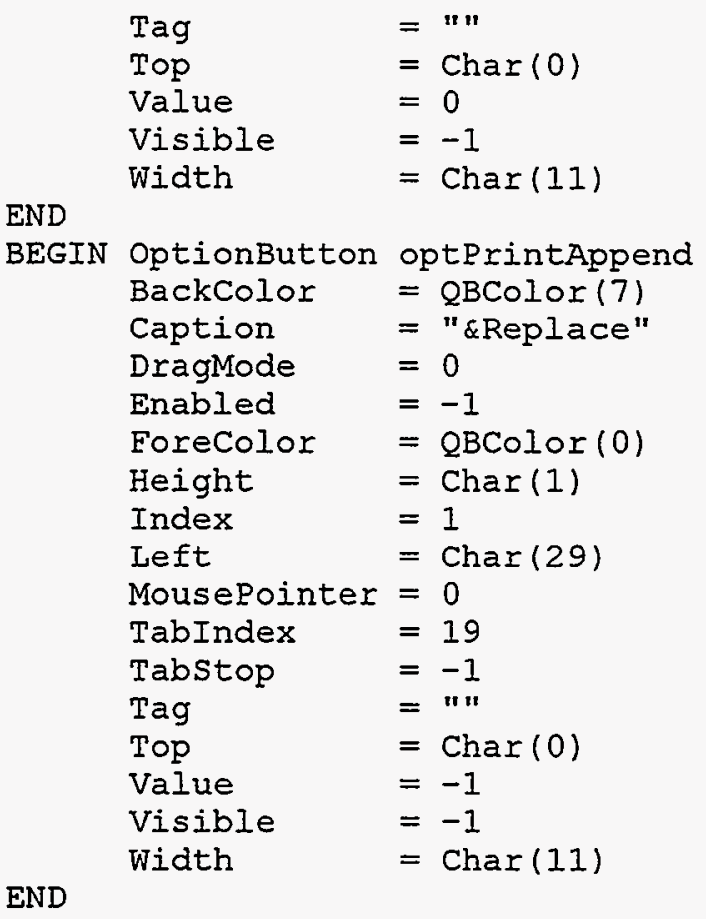

END

END

BEGIN CommandButton cmdPrintoK

BackColor = QBColor (7)

Cancel $=0$

Caption $\quad=$ "OK"

Default $\quad=0$

DragMode $\quad=0$

Enabled $\quad=-1$

Height $=\operatorname{Char}(3)$

Left $=$ Char (46)

MousePointer $=0$

TabIndex $\quad=20$

Tabstop $\quad=-1$

Tag $="$ "

Top $=\operatorname{Char}(1)$

Visible $\quad=-1$

Width $=\operatorname{Char}(13)$

END

BEGIN CommandButton cmdPrintcancel

BackColor $=$ QBColor (7)

Cancel $=0$

Caption = "Cancel"

Default $\quad=0$

DragMode $\quad=0$

Enabled $\quad=-1$

Height $=$ Char (3)

Left $\quad=$ Char (46)

MousePointer $=0$

TabIndex $\quad=21$

Tabstop $\quad=-1$

Tag = ""

Top $=\operatorname{Char}(4)$

Visible $\quad=-1$

END

Width $=\operatorname{Char}(13)$

BEGIN Iabel IblPrintCopies

Alignment $=0$

Autosize $=0$ 


$$
\begin{array}{ll}
\text { BackColor } & =\text { QBColor }(7) \\
\text { Borderstyle } & =0 \\
\text { Caption } & =\text { "dCopies:" } \\
\text { DragMode } & =0 \\
\text { Enabled } & =-1 \\
\text { ForeColor } & =\text { QBColor }(0) \\
\text { Height } & =\text { Char }(1) \\
\text { Left } & =\text { Char }(46) \\
\text { MousePointer } & =0 \\
\text { TabIndex } & =22 \\
\text { Tag } & =" " \\
\text { Top } & =\text { Char }(8) \\
\text { Visible } & =-1 \\
\text { Width } & =\text { Char }(7)
\end{array}
$$

END

BEGIN TextBox txtPrintCopies

$$
\begin{array}{ll}
\text { BackColor } & =\text { QBColor (7) } \\
\text { Borderstyle } & =1 \\
\text { DragMode } & =0 \\
\text { Enabled } & =-1 \\
\text { EoreColor } & =\text { QBColor (0) } \\
\text { Height } & =\text { Char (1) } \\
\text { Left } & =\text { Char (54) } \\
\text { MousePointer } & =0 \\
\text { Multiline } & =0 \\
\text { ScrollBars } & =0 \\
\text { TabIndex } & =23 \\
\text { Tabstop } & =-1 \\
\text { Tag } & =" " \\
\text { Text } & =" \\
\text { Top } & =\text { Char (8) } \\
\text { Visible } & =-1 \\
\text { Width } & =\text { Char (5) }
\end{array}
$$

END

END

BEGIN PictureBox pctFileopen

$$
\begin{aligned}
& \text { AutoRedraw }=0 \\
& \text { BackColor }=\text { QBColor (7) } \\
& \text { Borderstyle }=1 \\
& \text { DragMode }=0 \\
& \text { Enabled } \quad=-1 \\
& \text { Forecolor }=\text { QBColor }(0) \\
& \text { Height }=\operatorname{Char}(17) \\
& \text { Left }=\operatorname{Char}(0) \\
& \text { MousePointer }=0 \\
& \text { TabIndex } \quad=0 \\
& \text { Tabstop }=0 \\
& \text { Tag = " } \\
& \text { Top }=\operatorname{Char}(0) \\
& \text { Visible } \quad=0 \\
& \text { Width }=\operatorname{Char}(50) \\
& \text { BEGIN Label IbIOpenFile } \\
& \text { Alignment }=0 \\
& \text { Autosize } \quad=0 \\
& \text { BackColor }=\text { QBColor (7) } \\
& \text { Borderstyle }=0 \\
& \text { Caption = "File \&Name:" } \\
& \text { Dragmode } \quad=0 \\
& \text { Enabled } \quad=-1 \\
& \text { Forecolor }=\text { QBColor }(0) \\
& \text { Height }=\operatorname{Char}(1) \\
& \text { Left }=\operatorname{Char}(1) \\
& \text { MousePointer }=0
\end{aligned}
$$




\begin{tabular}{|c|c|c|}
\hline & $\begin{array}{l}\text { TabIndex } \\
\text { Tag } \\
\text { Top } \\
\text { Visible } \\
\text { Width }\end{array}$ & $\begin{array}{l}=1 \\
=n " \\
=\operatorname{Char}(2) \\
=-1 \\
=\operatorname{Char}(11)\end{array}$ \\
\hline \multicolumn{3}{|l|}{ END } \\
\hline BEGIN & $\begin{array}{l}\text { TextBox txtop } \\
\text { BackColor } \\
\text { BorderStyle } \\
\text { DragMode } \\
\text { Enabled } \\
\text { ForeColor } \\
\text { Height } \\
\text { Left } \\
\text { MousePointer } \\
\text { Multiline } \\
\text { ScrollBars } \\
\text { TabIndex } \\
\text { TabStop } \\
\text { Tag } \\
\text { Text } \\
\text { Top } \\
\text { Visible } \\
\text { Width }\end{array}$ & $\begin{array}{l}\text { denFile } \\
=\text { QBColor (7) } \\
=1 \\
=0 \\
=-1 \\
=\text { QBColor }(0) \\
=\text { Char }(3) \\
=\text { Char }(12) \\
=0 \\
=0 \\
=0 \\
=2 \\
=-1 \\
=" 1 \\
=" 1 \\
=\text { Char }(1) \\
=-1 \\
=\text { Char (23) }\end{array}$ \\
\hline \multicolumn{3}{|l|}{ END } \\
\hline BEGIN & $\begin{array}{l}\text { CommandButton } \\
\text { BackColor } \\
\text { Cancel } \\
\text { Caption } \\
\text { Default } \\
\text { DragMode } \\
\text { Enabled } \\
\text { Height } \\
\text { Left } \\
\text { MousePointer } \\
\text { TabIndex } \\
\text { TabStop } \\
\text { Tag } \\
\text { Top } \\
\text { Visible } \\
\text { Width }\end{array}$ & $\begin{array}{l}\text { CmdopenoK } \\
=\text { QBColor (7) } \\
=0 \\
=\text { "OK" } \\
=0 \\
=0 \\
=-1 \\
=\text { Char (3) } \\
=\text { Char (37) } \\
=0 \\
=7 \\
=-1 \\
=" " \\
=\text { Char }(1) \\
=-1 \\
=\text { Char }(12)\end{array}$ \\
\hline \multicolumn{3}{|l|}{ END } \\
\hline BEGIN & $\begin{array}{l}\text { CommandButton } \\
\text { BackColor } \\
\text { Cancel } \\
\text { Caption } \\
\text { Default } \\
\text { DragMode } \\
\text { Enabled } \\
\text { Height } \\
\text { Left } \\
\text { MousePointer } \\
\text { TabIndex } \\
\text { Tabstop } \\
\text { Tag } \\
\text { Top } \\
\text { Visible } \\
\text { Width }\end{array}$ & $\begin{array}{l}\text { CmdOpenCancel } \\
=\text { QBColor (7) } \\
=0 \\
=\text { "Cancel" } \\
=0 \\
=0 \\
=-1 \\
=\text { Char (3) } \\
=\text { Char (37) } \\
=0 \\
=8 \\
=-1 \\
=" 11 \\
=\text { Char }(4) \\
=-1 \\
=\text { Char (12) }\end{array}$ \\
\hline \multicolumn{3}{|l|}{ END } \\
\hline BEGIN & $\begin{array}{l}\text { DrivelistBox } \\
\text { BackColor } \\
\text { DragMode } \\
\text { Enabled }\end{array}$ & $\begin{array}{l}\text { drvopenList } \\
=\text { QBColor }(7) \\
=0 \\
=-1\end{array}$ \\
\hline
\end{tabular}




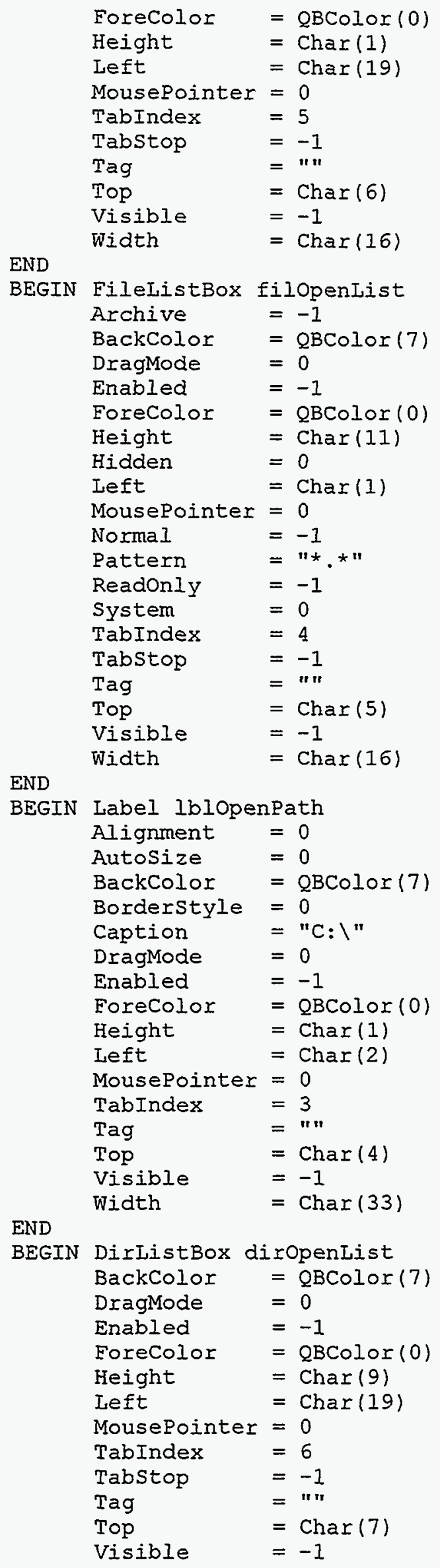


END Width $=\operatorname{Char}(16)$

END

END

\section{OPTION EXPLICIT}

'\$INCLUDE: 'secpop90.bi'

- The Common Dialog Toolkit (CMNDLG.BAS and CMNDLGF.FRM)

provides support for the following dialogs:

Fileopen

Filesave

Eileprint

Support for each dialog is provided via procedures with

these same names that create the corresponding dialog

and return user input to your program. These procedures

only provide the user interface and return user input.

- They do not actually carry out the corresponding actions

- such as opening the file. Detailed descriptions of

- these procedures are contained in the comment headers

' above each.

- All common dialogs are created from the same form (CMNDLGF.FRM).

The necessary controls for each dialog are children of

a container picture box for the dialog. Thus the

form (CMNDLGE.FRM) contains a picture box with

appropriate controls for common dialog listed above.

- When a particular common dialog is created and displayed,

- the container picture box for that dialog is made visible

' (thus all controls on that picture box become visible)

and the form is centered and sized to match the

container picture box.

To use these common dialogs in your programs, include

CMNDLG.BAS and CMNDLGF. FRM in your program or use the

supplied library (CMNDLG.IIB, CMNDLGA.IIB - AltMath version

- for Professional Edition only) and Quick library (CMNDIG.QIB)

and call the appropriate procedure to invoke the dialog

you need.

Copyright (C) 1982-1992 Microsoft Corporation

You have a royalty-free right to use, modify, reproduce

and distribute the sample applications and toolkits provided with

Visual Basic for MS-DOS (and/or any modified version)

in any way you find useful, provided that you agree that

Microsoft has no warranty, obligations or liability for

any of the sample applications or toolkits.

- Click event procedure for Open/Save dialog Cancel button.

- Makes dialog invisible to return control to Fileopen or Filesave

- procedure (dialog was shown modally).

SUB cmdopenCancel_Click ()

txtopenFile.SĒTFOCUS

Visible = FALSE

END SUB 
- Click event procedure for Open/Save dialog OK button.

- Determines whether user has selected a file or whether

- path and pattern need to be changed.

SUB cmopenOK_CIick ()

DIM OIdPattern AS STRING

- Set up error handling for directory/drive change errors.

ON LOCAI ERROR GOTO OKErrOY

cmdOpenOK. SETEOCUS

- Set focus to button, so focus can be

reset to edit field if needed.

- Update Directory listbox path if user single

- clicked or used arrow keys in Directory listbox

- (only double click automatically changes path).

diropentist.Path $=$ diropenList.Iist(diropenList.ListIndex)

- If edit field filename does not match File listbox filename

- then assign edit field value to File listbox filename

- and let it determine if path or pattern need to be

- changed.

IF filopenList.FileName $<$ txtopenFile.Text THEN

OldPattern\$ = filopenList.Pattern ' Save old pattern.

- Let File listbox control determine if path

- or pattern or filename needs to be updated.

- Pathchange event will be triggered if path needs

- updating, Patternchange event will be triggered if

- pattern needs updating, and DblClick event will

- be triggered if a valid filename has been given.

filopenList.FileName = txtopenFile.Text

- If a valid filename was not given (dialog is

- still visible to user after Dblclick event),

' then update the edit field appropriately.

IE Visible = TRUE THEN

' If no pattern change was indicated then either

- a new filename was specified for save dialog

' or file was not found for open dialog.

IF (INSTR(txtopenFile.Text, "*) + INSTR(txtopenFile.Text, "?") < I)

THEN

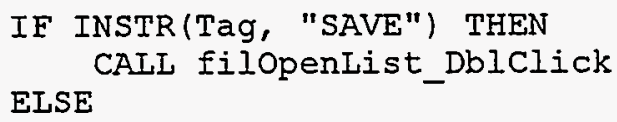

MSGBOX "File not found", 0, Caption

filopenist.Pattern = OldPattern\$ " Restore old File listbox

search pattern.

END IE

txtOpenFile. SETEOCUS

- Pattern change was indicated so just update

- textbox with pattern.

ELSE

txtopenFile.Text $=$ filopenList.Pattern

txtopenFile.SETFOCUS

END IF

END IF

- File has been selected by user so close dialog

- and return control to Fileopen or Filesave routine.

ELSE

CAIL filopenList_DblClick 
END IE

OKErrorReturn:

EXIT SUB

- Drive/Path error handling routine.

OKError:

MSGBOX ERRORS, 0, Caption

fixed.

txtOpenFile.SETEOCUS

- Display error message.

' Set focus to edit field so error can be

RESUME OKErrorReturn

- Exit procedure.

END SUB

- Click event procedure for Print dialog Cancel button.

- Makes dialog invisible to return control to Fileprint

- procedure (dialog was shown modally).

SUB cmdPrintCancel Click ()

Visible $=$ FALSE

END SUB

- Click event procedure for Print dialog oK button.

- sets print destination (PRINTER.PrintTarget) and

- makes dialog invisible to return control to Fileprint

' procedure (dialog was shown modally).

SUB cmdPrintoK_Click ()

- Set up êrror handling for print to file errors.

ON LOCAI ERROR GOTO PrintError

' set print target

IF optPrintTarget(0).Value THEN

PRINTER.PrintTarget = "IPT1:" "Use Basic LPTI device (colon specifies

this).

ELSEIF optPrintTarget (I). Value THEN

PRINTER. PrintTarget = "LPT2:"

- Use Basic LPT2 device lcolon specifies

this).

ELSEIF optPrintTarget(2).Value THEN

PRINTER. PrintTarget = "LPT3" "No Basic IPT3 device, treat as a

normal file open.

ELSE

- Print target is a file.

PRINTER.PrintTarget = txtPrintFile. Text

' If user specified "Replace" instead of "Append"

' option, delete existing file.

END IF

IF optPrintAppend(1).Value THEN KILL txtPrintFile.Text

Visible = FALSE

cmdPrintCancel. Tag = "FALSE"

EXIT SUB

- Print to file error handling routine.

- Ignores File Not Found error that occurs when

- deleting a file that does not exist (when user

- chooses "Replace" option).

Printerror:

END SUB

RESUME NEXT

- Change event procedure for Open/Save dialog Directory Iistbox.

- Updates the File listbox path to reflect

' the directory change. 


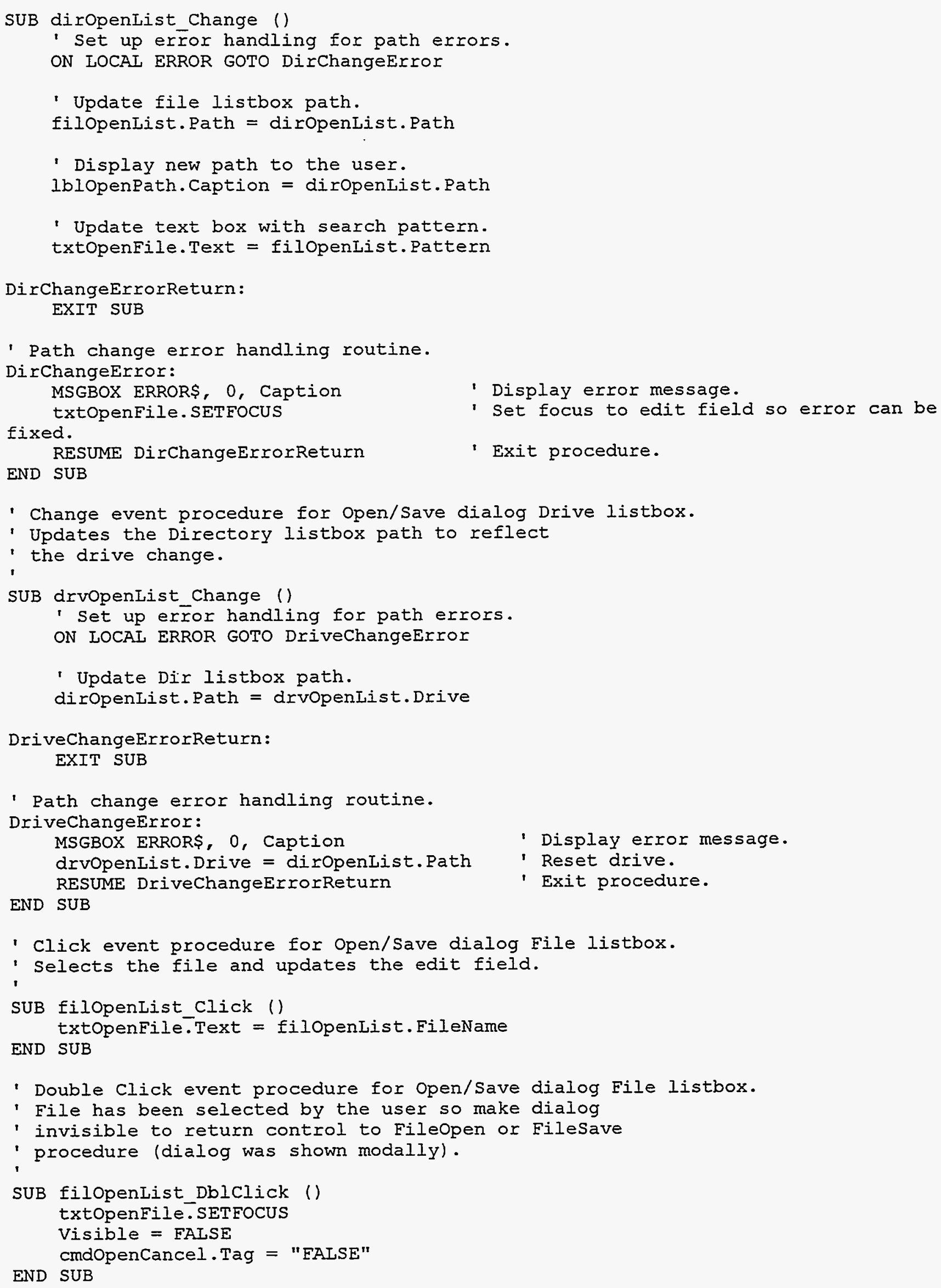


- PathChange event procedure for Open/Save dialog File listbox.

- Updates the Drive Iistbox drive and Directory

- listbox path to reflect the change.

SUB filopenList Pathchange ()

- Set up error handling for path errors.

ON LOCAI ERROR GOTO FileChangeError

- Update drive and path.

drvopenlist. Drive = filopenList. Path

diropenList.Path $=$ filopenList.Path

FileChangeErrorReturn:

EXIT SUB

- Path change error handling routine.

FilechangeError: MSGBOX ERROR\$, 0, Caption ' Display error message.

drvopenList.Drive = diropenList.Path ' Reset drive.

filopenList.Path = diropenList.Path ' Reset path.

END SUB RESUME FileChangeErrorReturn

- Exit procedure.

- PatternChange event procedure for Open/Save dialog File listbox.

- Uppercases search pattern for subsequent display

' in edit field.

SUB filopenList Patternchange ()

filopenList.Pattern = UCASE\$ (filopenList.Pattern)

END SUB

- Click event procedure for Print dialog PrintTarget option buttons (control array)

; Handles print target selection.

SUB optPrintTarget Click (Index AS INTEGER)

- If file is chosen as print target, enable

' filename edit field and append/replace options.

IF Index $=3$ THEN

txtPrintFile. Enabled $=$ TRUE

pctPrintappend. Enabled = TRUE

- If LPT1, LPT2, LPT3 is chosen as print target,

- disable filename edit field and append/replace options.

ELSE

txtPrintFile.Enabled = FALSE

pctPrintAppend. Enabled = FAISE

END IF

END SUB 


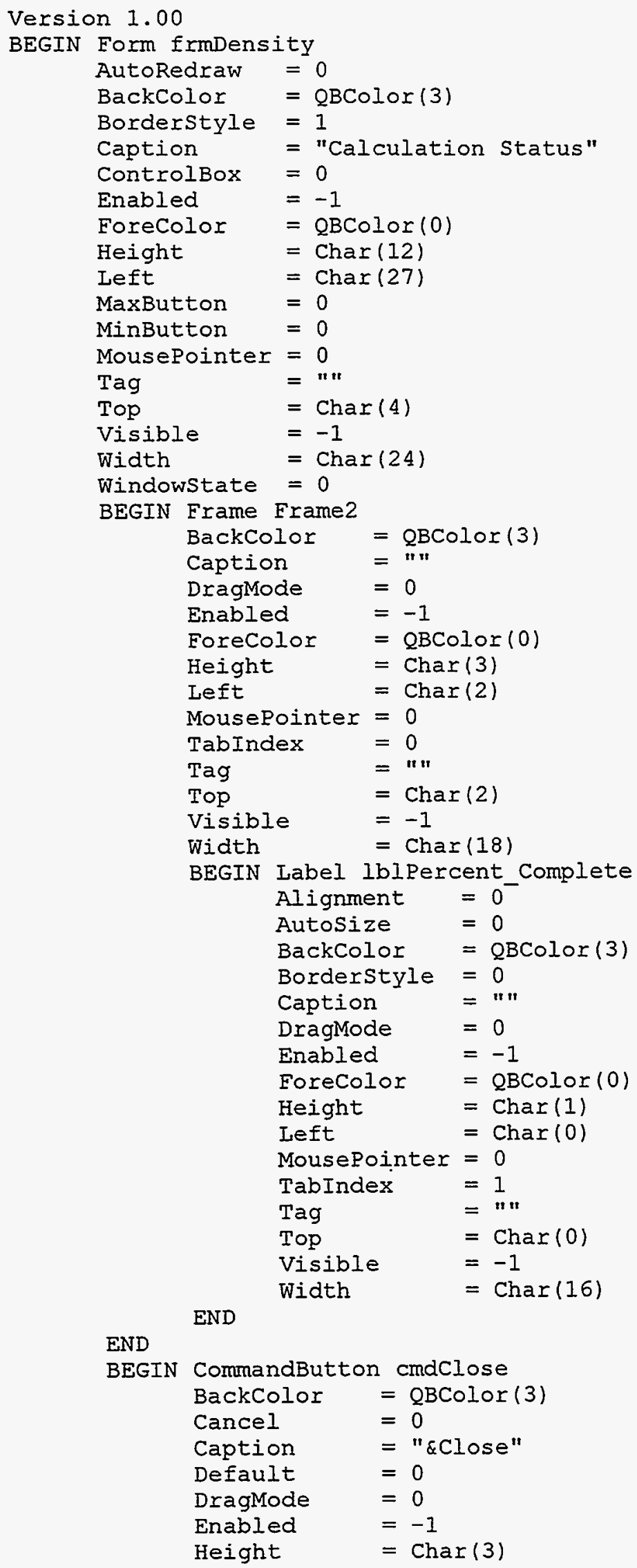




$$
\begin{array}{ll}
\text { Left } & =\operatorname{Char}(2) \\
\text { MousePointer } & =0 \\
\text { TabIndex } & =2 \\
\text { Tabstop } & =-1 \\
\text { Tag } & =" " \\
\text { Top } & =\operatorname{Char}(6) \\
\text { Visible } & =-1 \\
\text { Width } & =\operatorname{Char}(18)
\end{array}
$$

END

BEGIN Label lblCaption

$$
\begin{array}{ll}
\text { Alignment } & =0 \\
\text { AutoSize } & =0 \\
\text { BackColor } & =\text { QBColor (3) } \\
\text { BorderStyle } & =0 \\
\text { Caption } & =\text { "Percent Complete" } \\
\text { DragMode } & =0 \\
\text { Enabled } & =-1 \\
\text { ForeColor } & =\text { QBColor }(0) \\
\text { Height } & =\text { Char (1) } \\
\text { Left } & =\text { Char (3) } \\
\text { MousePointer } & =0 \\
\text { TabIndex } & =3 \\
\text { Tag } & =" " \\
\text { Top } & =\text { Char (1) } \\
\text { Visible } & =-1 \\
\text { Width } & =\text { Char (16) }
\end{array}
$$

END

END

OPTION EXPIICIT

'\$INCLUDE: 'secpop90.bi'

'This form allows the user to monitor the status

'of a calculation, and allows the user to terminate

'the calculation before it has been completed.

SUB cmaclose_Click ()

'This proceedure allows the user to end a regional

calculation.

DIM reply AS INTEGER

'If a calculation is not in progress then unload the calculate form.

IF frmDensity. cmdClose.caption = "Continue" THEN

unload frmDensity

ELSE

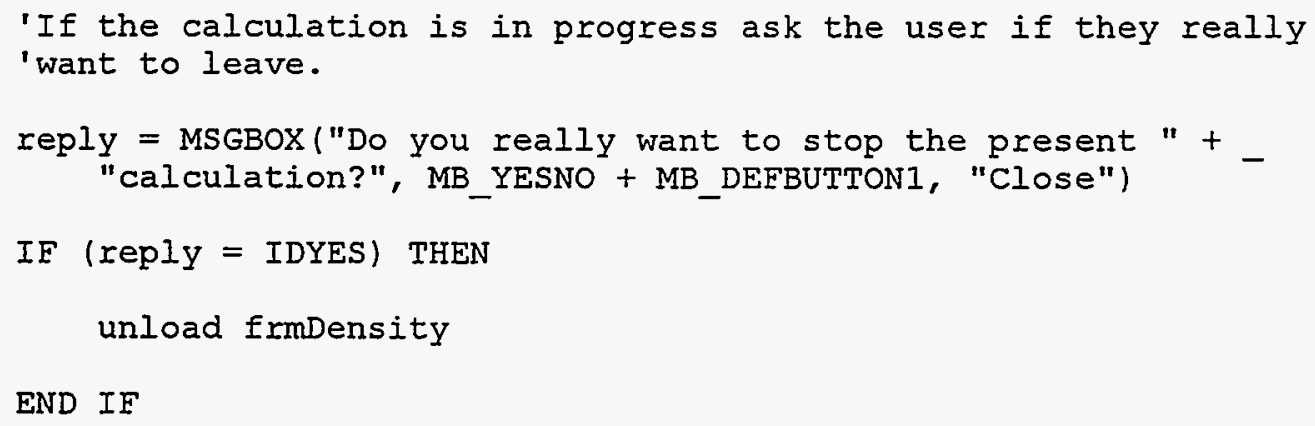


END IF

END SUB 


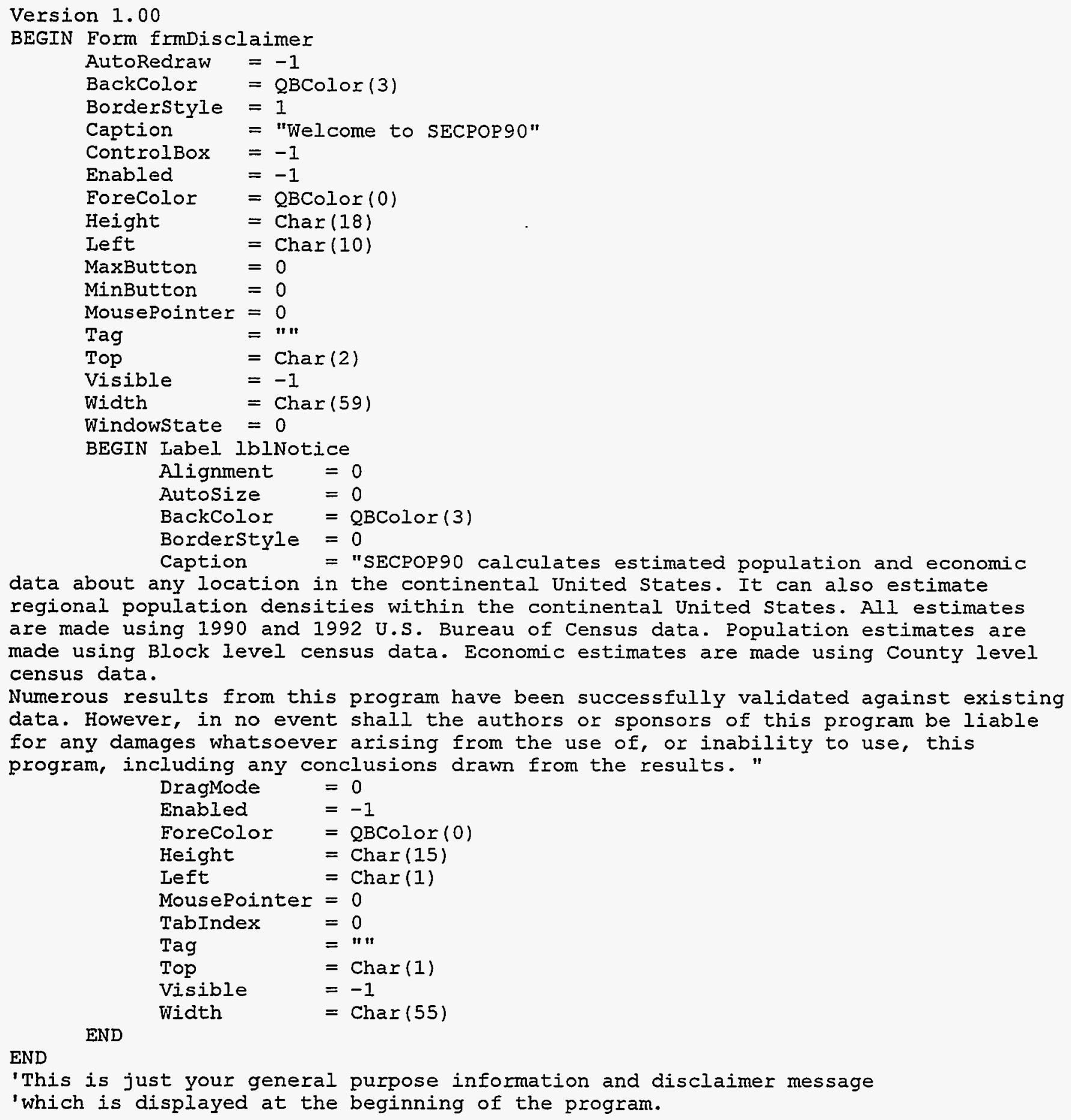

SUB Form_KeyPress (KeyAscii AS INTEGER)

UNLOAD frmDisclaimer

END SUB

SUB Form_LostFocus ()

UNLOAD frmDisclaimer 
END SUB

SUB lblNotice_Click ()

UNLOAD frmDisclaimer

END SUB 
Version 1.00

BEGIN MDIForm frmMain

$$
\begin{array}{ll}
\text { AutoRedraw } & =-1 \\
\text { BackColor } & =Q B C o l o r(1) \\
\text { Borderstyle } & =3
\end{array}
$$

$2.3^{\prime \prime}$

Caption $=$ "SECPOP90 - SECtor POPulation and Economic Estimator - Version

$$
\begin{aligned}
& \text { ControlBox }=0 \\
& \text { Enabled } \quad=-1 \\
& \text { ForeColor = QBColor(11) } \\
& \text { Height }=\text { Char (25) } \\
& \text { Left }=\operatorname{Char}(0) \\
& \text { MousePointer }=0 \\
& \text { Tag = "" } \\
& \text { Top }=\operatorname{Char}(0) \\
& \text { Visible } \quad=-1 \\
& \text { Width }=\operatorname{Char}(80)
\end{aligned}
$$

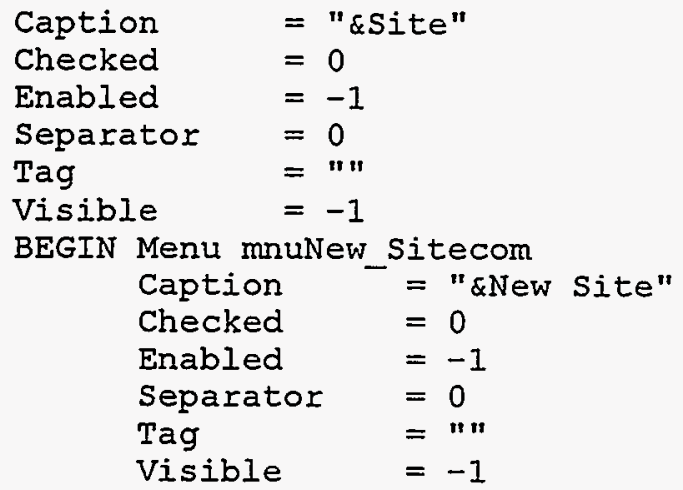

END

BEGIN Menu mnuSave_as_Sitecom

$$
\begin{array}{ll}
\text { Caption } & =\text { "Save Site \&As" } \\
\text { Checked } & =0 \\
\text { Enabled } & =0 \\
\text { Separator } & =0 \\
\text { Tag } & =" \| \\
\text { Visible } & =-1
\end{array}
$$

END

END

BEGIN Menu mnuProblemName

$$
\begin{array}{ll}
\text { Caption } & =\text { "\&Problem" } \\
\text { Checked } & =0
\end{array}
$$




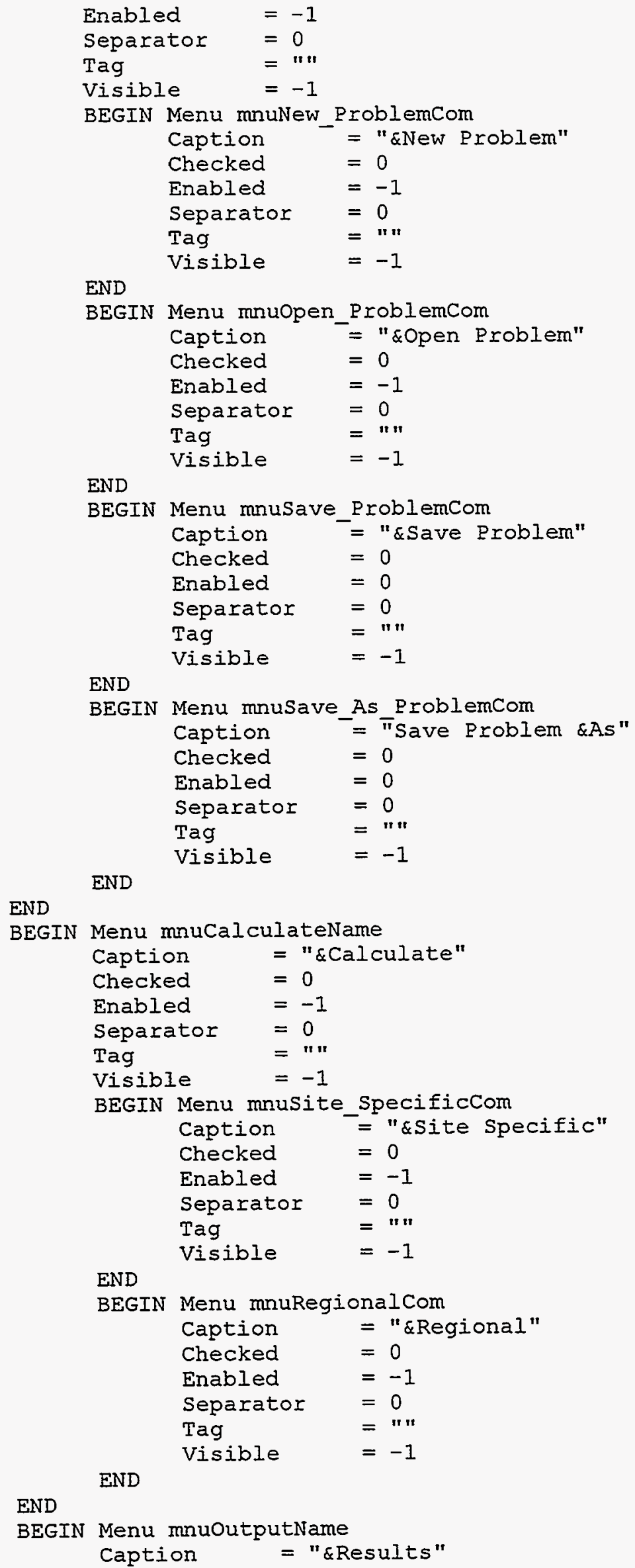

END

BEGIN Menu mnusave As Problemcom

$$
\begin{array}{ll}
\text { Checked } & =0 \\
\text { Enabled } & =0 \\
\text { Separator } & =0 \\
\text { Tag } & =" n \\
\text { Visible } & =-1
\end{array}
$$$$
\text { Caption } \quad=\text { "Save Problem \&As" }
$$

END

END

BEGIN Menu mnuCalculateName 


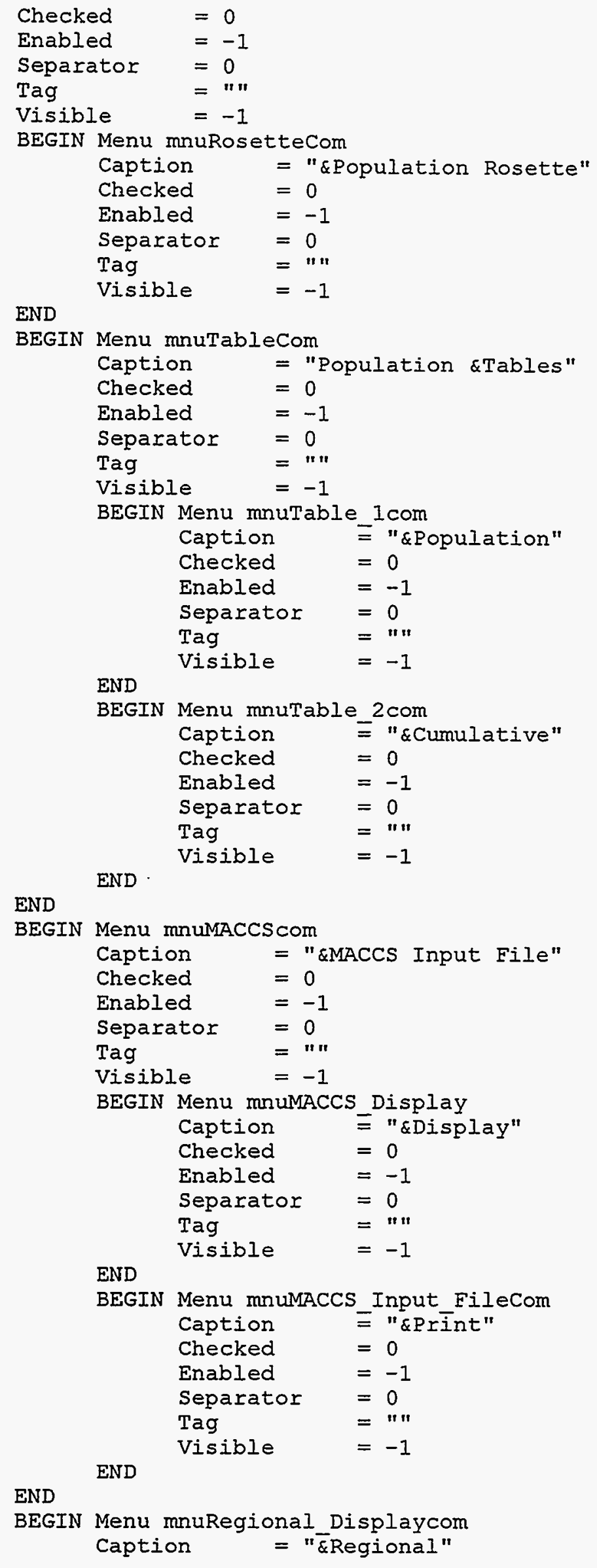

END

BEGIN Menu mnuTablecom

$$
\begin{array}{ll}
\text { Caption } & =\text { "Population \&Tables" } \\
\text { Checked } & =0 \\
\text { Enabled } & =-1 \\
\text { Separator } & =0 \\
\text { Tag } & =" 1 \\
\text { Visible } & =-1 \\
\text { BEGIN Menu mnuTable Icom } \\
\begin{array}{ll}
\text { Caption } & =\text { "\&opulation" } \\
\text { Checked } & =0 \\
\text { Enabled } & =-1 \\
\text { Separator } & =0 \\
\text { Tag } & =" 1 \\
\text { Visible } & =-1
\end{array}
\end{array}
$$

END

BEGIN Menu mnuTable 2 com

$$
\begin{array}{ll}
\text { Caption } & \equiv \text { "\&Cumulative" } \\
\text { Checked } & =0 \\
\text { Enabled } & =-1 \\
\text { Separator } & =0 \\
\text { Tag } & =" 1 \\
\text { Visible } & =-1
\end{array}
$$

END

BEGIN Menu mnUMACCScom

$$
\begin{aligned}
& \text { Caption = "\&MACCS Input File" } \\
& \text { Checked } \quad=0 \\
& \text { Enabled } \quad=-1 \\
& \text { Separator }=0 \\
& \text { Tag }=" " \\
& \text { Visible } \quad=-1 \\
& \text { BEGIN Menu mnuMACCS_Display } \\
& \text { Caption } \quad \text { "\&Display" } \\
& \text { Checked } \quad=0 \\
& \text { Enabled } \quad=-1 \\
& \text { Separator }=0 \\
& \text { Tag }=" 1 \\
& \text { Visible } \quad=-1
\end{aligned}
$$

$\begin{array}{ll}\text { Caption } & \equiv \text { "\&Print" } \\ \text { Checked } & =0 \\ \text { Enabled } & =-1 \\ \text { Separator } & =0 \\ \text { Tag } & =" 1 \\ \text { Visible } & =-1\end{array}$

END

BEGIN Menu mnuRegional Displaycom 


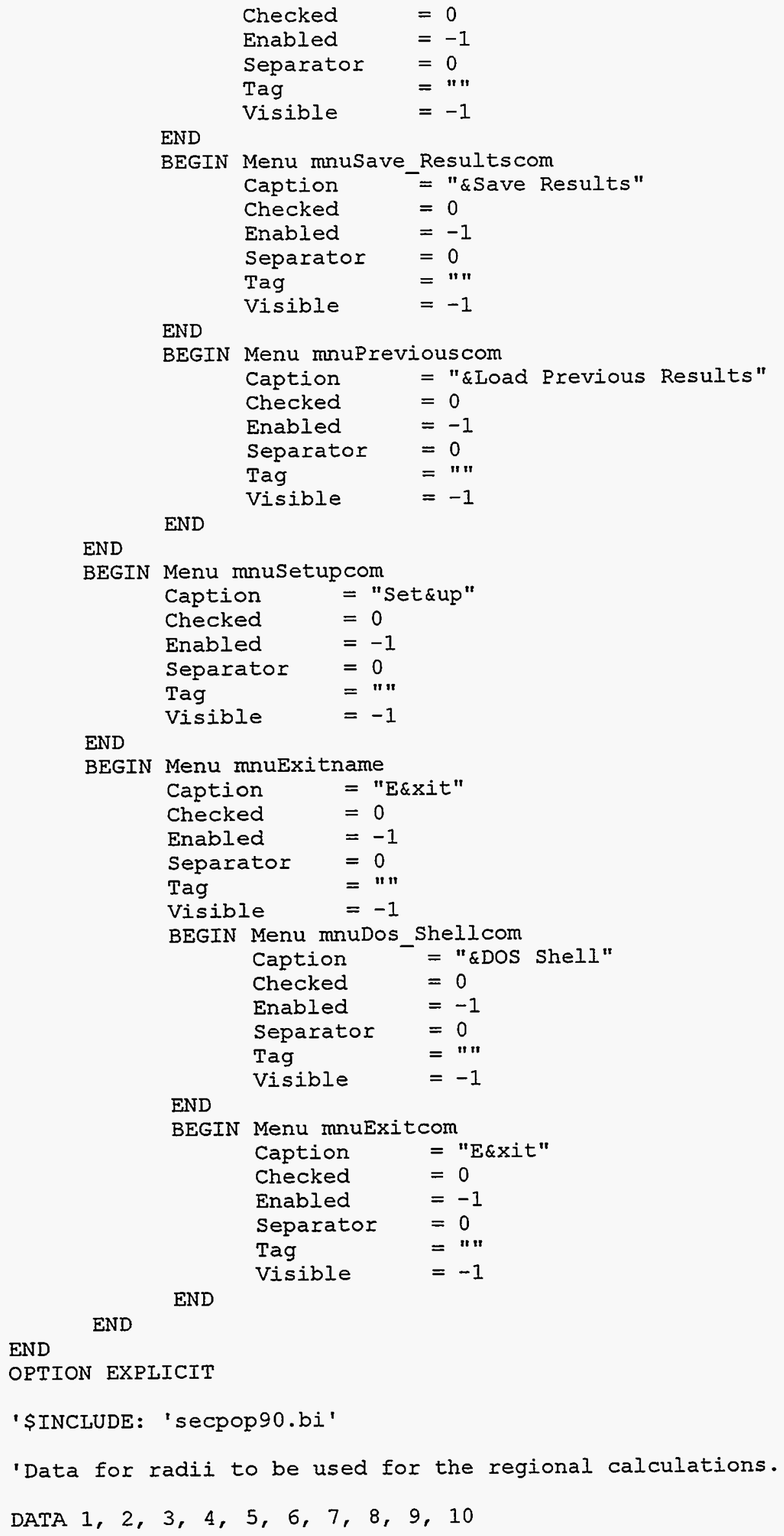


DATA 11, 12, 13, 14, 15, 16, 17, 18, 19, 20

DATA $30,40,50,60,70,80,90$

DATA $100,110,120,130,140,150,160,170$

'Data for forms which display the compass points.

DATA "N", "NNE", "NE", "ENE", "E", "ESE", "SE", "SSE", "S", "SSW", "SW"

DATA "WSW", "W", "WNW", "NW", "NNW"

SUB Form_Load ()

'Define varibles local to this event procedure.

DIM i AS INTEGER, filenum AS INTEGER, tempstring AS STRING

'set screen attributes for main program screen.

SCREEN. ControlPanel (DESKTOP PATTERN) = KEY SPACE

SCREEN. ControlPanel (ACTIVE_BORDER BACKCOLOR) = BLUE

SCREEN. ControlPanel (ACTIVE_BORDER_FORECOIOR) = BRIGHT_WHITE

SCREEN. ControlPanel (TITLEBĀR BACKC̄OLOR) = MAGENTA

SCREEN. ControlPanel (TITLEBAR FORECOLOR) = BRIGHT_WHITE

SCREEN. ControlPanel (MENU BACK̄COLOR) = WHITE

SCREEN. ControlPanel (MENU ${ }^{-}$FORECOLOR) $=$BLACK

SCREEN. ControlPanEl (SCROILBAR BACKCOLOR) = CYAN

SCREEN. ControlPanel (SCROLIBAR FORECOLOR) = BRIGHT CYAN

SCREEN.ControlPanel (ACTIVE_WIN̄DOW_SHADOW) = FALSE

'Initialize to zero the radial distance array.

FOR $i=1$ TO max_number_of_radii

NEXT i

radial distance (i) $\equiv 0^{-}$

'Reset the number of radii to 0

number_of_radii $=0$

'Initialize the radii used for regional calculations.

restore

for $i=1$ To max number of radii

read regiona $\bar{I}_{\text {radii }}(i)$

next $i$

'Read in the direction names (compass points).

FOR $i=1$ TO number of segments READ directions $(i)$

NEXT $i$

'Set up error handler for errors in the configuration

'file, and load in the various parameters from the

'setup file into the setup form. These parameters

'are set by the user in the exit menu under setup.

'Because input will not interact directly with forms,

' tempstring is used as a buffer for file data.

'Load in the froms that we need to keep permanently in memory.

LOAD frmProblem Data

LOAD frmsite DaẼa

LOAD frmRegiōn 
LOAD frmsetup

frmDisclaimer. SHOW MODELESS

ON LOCAL ERROR GOTO configerr

'Check and see if config file exists.

IF LEN(DIRS("SECPOP90.CFG")) $<0$ THEN

'Open file and read in the data from it.

filenum = FREEEILE

OPEN "SECPOP90.CFG" FOR INPUT AS filenum

INPUT \#filenum, tempstring

frmsetup.txtsite_Path.text = tempstring

INPUT \#filenum, tempstring

frmsetup.txtProblem_Path.text = tempstring

INPUT \#filenum, tempstring

frmsetup.txtoutput_path.text = tempstring

INPUT \#filenum, tempstring

frmsetup.txtData_path.text = tempstring

INPUT \#filenum, Eempstring

IF (tempstring = "MACCS") THEN

ELSE

frmsetup. optMACCS. value = TRUE

END IF

CLOSE \#filenum

'else if config file doesn't exist use defaults.

ELSE

'Defaults set in form.

END IF

'Turn off error handler.

ON IOCAI ERROR GOTO 0

EXIT SUB

configerr:

'If config file format is screwed up use defaults.

CLOSE filenum

EXIT SUB

END SUB

SUB mnuDOS_Shellcom_Click ()

'Remove all forms from the screen.

UNLOAD frmDisclaimer

SCREEN. HIDE

CLS

LOCATE 1, 1

'Place shell message on screen. 
SHELI "ECHO Type EXIT to return to SECPOP90"

LOCATE 1,1

'Run the DOS shell.

SHELI

'Redisplay screen.

SCREEN . SHOW

END SUB

SUB mnuExitCom_Click ()

DIM reply AS INTEGER

'Ask the user if they really want to exit.

reply = MSGBOX("Do you really want to exit?", MB_YESNO + MB_DEFBUTTON1, "Exit")

IF (reply = IDYES) THEN

END

END IF

END SUB

SUB mnuMACCS_Display_Click ()

'This function creates a temporary file, and then

'writes into that file the information for the user

'to view on the screen. This file is then sent to a

'text form, where the user can view it and scroll

'the various parts of the data.

DIM filenum AS INTEGER

UNLOAD frmDisclaimer

' Create temporary file, and tell the print MACCs

'input_file function to send the info to this file.

PRINTER.PrintTarget $=" \$ \$ T E M P \$ . \$ \$ \$ "$

'Write the actual data to file.

CALL print_MACCS_input_file

'Reset to printer the destination of info generated

'by the print_MACCS_input function.

PRINTER.PrintTarget $=" "$

filenum $=$ FREEFILE

'Display information in text form.

OPEN "\$\$TEMP\$\$.\$\$" FOR INPUT AS filenum

frmoutput.txtoutput.text $=" "$

frmoutput.txtOutput.text $=$ INPUT\$(LOF (filenum), filenum)

frmoutput. SHOW MODAI 
'Close and delete temporary file.

CIOSE filenum

KILL "\$\$TEMP\$ . \$\$\$"

END SUB

SUB mnuMACCS_Input_FileCom_Click ()

'This function prints the data in MACCS site File format.

These variables are used to set up the print dialog.

DIM ForeColor AS INTEGER, BackColor AS INTEGER

DIM Copies AS INTEGER, Cancel AS INTEGER, i AS INTEGER

DIM site_name AS STRING, site_remarks AS STRING

UNLOAD frmDisclaimer

'Set colors for Dialog box

BackColor $=$ WHITE

ForeColor $=$ BIACK

'Call the standard print dialog.

CAIL FilePrint(Copies, ForeColor, BackColor, Cancel)

'If user did not select cancel in the print dialog then print.

IF NOT Cancel THEN

'set up error handling for print errors.

on local error goto print_error

'If the user is printing to a printer adjust the margins so that

'there are no extra line feeds after the lines that have 80

'columns otherwise prepend the output path to the printer

'target.

if ( (printer.PrintTarget $=$ "LPTl:") or

(printer.PrintTarget = "IPT2:") or

(printer.PrintTarget $=$ "LPT3:")) then

width printer.PrintTarget, 81

else

printer.PrintTarget $=$ frmsetup.txtoutput_path.text +

"\" + printer.PrintTarget

end if

'Print however many copies specefied, each followed by a form feed.

FOR $i=1$ TO Copies

CALL print_MACCS_input_file

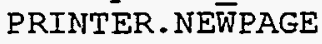

NEXT i

PRINTER. ENDDOC 
END IF

EXIT SUB

print_error:

'Deal with errors such as printer is off line or file not found.

MSGBOX "Device error - check printer or other print device."

EXIT SUB

END SUB

SUB mnuNew_ProblemCom_Click ()

'This function asks the user if they want to review

'previously entered data or create a new problem.

DIM i AS INTEGER, repIY AS INTEGER

UNLOAD frmDisclaimer

'If no problem has already been loaded, drop user

'directly into the new problem form.

IF frmProblem_Data.caption $=" "$ THEN

CAII new_problem

'Otherwise ask user if they want to review the old

'data. If they do, then show it, otherwise call

'the new problem form.

ELSE

reply = MSGBOX ("Do you wish to review the previous problem data?",

MB_YESNOCANCEL, "NeW Problem")

IF reply = IDYES THEN

fImProblem Data. SHOW MODAL

ELSEIF repIY = IDNO THEN

END IF

CALL new problem

END IF

END SUB

SUB mnuNew_SiteCom_click ()

'This function asks the user if they want to review

'previous data or create a new site.

DIM reply AS INTEGER

UNLOAD frmDisclaimer

'If no data has been entered, go directly to new site form.

IF frmsite_Data.caption $="$ "THEN

CAIL new_site 
ELSE

'If data has been entered, ask the user if they want to see it.

'If yes, then show old data, otherwise go to new site form.

reply = MSGBOX ("Do you wish to review the previous site data?", MB_YESNOCANCEL, "NeW Site")

IF reply = IDYES THEN

frmsite Data.SHOW MODAL

ELSEIF repl $\bar{y}=$ IDNO THEN

END IF

CALL new site

END IF

END SUB

SUB mnuopen_ProblemCom_Click ()

'This subroutine allows the user to look at a previously

'created problem data or review problem data that has already

'been loaded.

DIM reply AS INTEGER

UNLOAD frmDisclaimer

'If data has not yet been entered, go directly to the

'open problem function.

IF frmProblem_Data.caption = " THEN

CALI open_problem

'Otherwise, ask the user if they want to review the

'previous problem data. If so, show it to them, otherwise

call open problem.

ELSE

reply = MSGBOX("Do you wish to review the previous problem data?", MB YESNOCANCEL, "Open Problem File")

IF reply = IDYES THEN

frmProblem Data. SHOW MODAI

EISEIF reply =- IDNO THEN

END IF

CALI open problem

END IF

END SUB

SUB mnuOpen_SiteCom_Click ()

'This subroutine allows the user to look at a previously

'created site data or review site data that has already

'been loaded.

DIM reply AS INTEGER

UNLOAD frmDisclaimer

'If data has not yet been entered, go directly to the 
'open site function.

IE frmsite Data. caption = " "THEN

CALI open_site

'Otherwise, ask the user if they want to review the

'previous site data. If so, show it to them, otherwise

'call open site.

ELSE

reply = MSGBOX ("Do you wish to review the previous site data?",

MB_YESNOCANCEL, "Open Site Eile")

IF reply = IDYES THEN

frmsite Data.SHOW MODAI

ELSEIF reply $=$ IDNO THEN

END IF

CALL open site

END IE

END SUB

SUB mnuPreviouscom_Click ()

'Load in previously saved output data so that the user

'can view it again, in any of the standard forms.

DIM tempinteger AS INTEGER, tempsingle AS SINGLE

DIM filenum AS INTEGER, i AS INTEGER, j AS INTEGER, $k$ AS INTEGER

DIM tempstring AS STRING

DIM Forecolor AS INTEGER, BackColor AS INTEGER

DIM Elags AS INTEGER, Cancel AS INTEGER

DIM EileName AS STRING

STATIC PathName AS STRING

DIM Defaultext AS STRING, DialogTitle AS STRING

UNLOAD frmDisclaimer

'Set defaults for the open file dialog box.

DefaultExt $=" *$ TXT"

DialogTitle = "Load Previous Data"

BackColor = WHITE

ForeColor = BLACK

PathName $=$ frmsetup.txtoutput_path.text

'set up error checking.

ON LOCAL ERROR GOTO previous_error

'Prompt the user for a filename, and if they do not

'cancel the operation, open the file using the

'first available file handle.

CALL Fileopen(FileName, PathName, DefaultExt, DialogTitle, ForeColor, BackColor, Elags, Cancel)

'If the user did not select cancel from the previous dialogue box,

'then load the file.

IF NOT Cancel THEN 
'Determine first available file handle.

filenum = FREEFILE

IF PathName $<>"$ " THEN FileName = PathName + "\" + FileName

OPEN FileName FOR INPUT AS filenum

'Read in the header information.

tempstring = input $\$(40, \#$ filenum)

IINE INPUT \#filenum, tempstring

frmSite_data.txtSite_name.Text $=$ tempstring

tempstring $=$ input $\$(6$, \#filenum)

tempstring = input $(2$, \#filenum)

frmsite data.txtlatitude degrees. Text = ltrims (tempstring)

tempstrīng = input $(1$, \#正ilenum)

tempstring = input $\$(2$, \#filenum)

frmsite data.txtlatitude minutes. Text = Itrims (tempstring)

tempstrīng = input $(1, \# \bar{f} i l e n u m)$

tempstring = input $(2$, \#filenum)

frmsite_data.txtLatitude_seconds.Text = Itrim\$(tempstring)

tempstring $=$ input $\$(9$, \#ilenum)

tempstring = input\$(3, \#filenum)

frmsite data.txtLongitude degrees. Text = Itrim\$ (tempstring)

tempstring = input $(1, \#$ fílenum)

tempstring = input\$ $(2$, \#ilenum)

frmsite data.txtLongitude minutes. Text = ltrims (tempstring)

tempstring = input $\$(1$, \#立lenum)

tempstring = input\$ $(2, \#$ filenum)

frmsite_data.txtLongitude_seconds.Text = Itrims (tempstring)

tempstring $=$ input $\$(26$, \#ilenum)

input \#filenum, tempsingle

IINE INPUT \#filenum, tempstring

frmproblem_data.txtPopulation_multiplier.Text = str\$(tempsingle)

'Pull in various numbers and strings from the file,

'discarding extraneous labels and information.

INPUT \#filenum, tempsingle

number_of_radii = tempsingle

'Discard next 5 lines from file

FOR $i=1$ TO 5 STEP 1

NEXT i

LINE INPUT \#filenum, tempstring

'Determine number of economic regions.

INPUT \#filenum, tempsingle

number_econ regions = tempsingle

LINE INNPUT \#filenum, tempstring

'Fill number of radii array from file data.

IINE INPUT \#filenum, tempstring

FOR $i=1$ TO number_of radii

INPUT \#filenum, tempsingle

radial_distance $(i)=$ tempsingle 
NEXT i

'Fill total population array for each segment and radii.

LINE INPUT \#filenum, tempstring

FOR $i=1$ TO number_of_segments

EOR $j=1$ TO number_of_radii INPUT \#filenum, tempsingle NEXT $j$ sector_population $(i, j)=$ tempsingle

NEXT i

'Fill fraction land array for each segment and radii.

LINE INPUT \#filenum, tempstring

FOR $i=1$ TO number_of_segments

FOR $j=1$ TO number of radii INPUT \#filenum, tempsingle NEXT $j$ sector_frclnd $(i, j)=$ tempsingle

NEXT i

'Fill region index array.

IINE INPUT \#filenum, tempstring

FOR $i=0$ TO (number_of_segments - 1)

FOR $j=1$ TO number of radii tempstring $=\operatorname{IN} \bar{P} U T \bar{S}(2$, \#filenum)

IE VAL (tempstring) $=0$ THEN

tempstring $=\operatorname{INPUTS}(2, \#$ \#ilenum)

END IF NEXT $j$

region index $(j, i)=\operatorname{VAI}$ (tempstring)

NEXT $i$

IINE INPUT \#filenum, tempstring

'suck in and throw away the dumy watershed indices.

LINE INPUT \#filenum, tempstring

FOR $i=1$ TO number_of_segments NEXT $i$

IINE INPUT \#filenum, tempstring

'suck in and throw away the dummy crop season and share info.

LINE INPUT \#filenum, tempstring

LINE INPUT \#filenum, tempstring

'suck in and throw away the dumy watershed definition.

IINE INPUT \#filenum, tempstring

IINE INPUT \#filenum, tempstring

' Fill regional data array structs.

LINE INPUT \#filenum, tempstring

FOR $i=1$ TO number_econ_regions INPUT \#filenum, tempsingle tempstring $=\operatorname{INPUT} \$(10$, \#filenum) INPUT \#filenum, tempsingle 


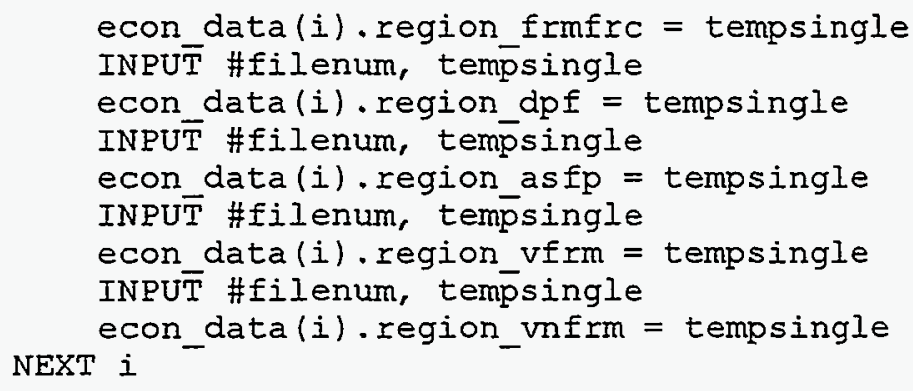

END IF

'Update site and problem form captions and remarks.

frmsite data. Caption = filename

frmsite data.txtSite remarks. Text = " "

frmProbIem data. Captīon = filename

frmProblem_data.txtProblem_remarks.Text = " "

'Update problem form radial distances, units, and number of regions.

frmproblem data.txtRadial distancel. Text = str\$(radial_distance (1))

frmProblem data.txtRadial distance 2. Text $=$ str $\$($ radial distance $(2))$

frmProblem data.txtRadial_distance 3. Text $=$ str\$(radial_distance (3))

frmProblem_Data.optKilometers.value = TRUE

frmProblem_Data.lblNumber_of_regions.Caption $=$ str\$ (number_econ_regions)

EXIT SUB

previous_error:

'If there is an exror, identify type if possible,

'inform the user of the problem, and leave subroutine.

IF $(E R R=62)$ THEN

MSGBOX "Error: Attempt has been made to read past the end of file. Data

file has been ccorrupted."

ELSEIF (ERR = 53) OR (ERR = 75) OR (ERR = 76) OR (ERR = 55) THEN

MSGBOX "Invalid filename or path, or file access denied"

ELSEIF $(E R R=61)$ OR $(E R R=68)$ OR $(E R R=71)$ OR $(E R R=72)$ THEN

MSGBOX "The disk drive is not ready, or a file error has occured"

ELSE MSGBOX Errors (err)

END IF

EXIT SUB

END SUB

SUB mnuRegionalcom_Click ()

'Call the forms and functions which handle the

'regional calculations

UNLOAD frmDisclaimer

lload form which prompts user for certain input

'necessary for regional calculations

LOAD frmMake a Circle

frmMake_a_Ci $\bar{r} c \bar{l} e$. SHOW MODAI 
END SUB

SUB mnuRegional_Displaycom_Click ()

UNLOAD ErmDisclaimer

'Display regional calculation results.

RUN "run mppr.exe"

END SUB

SUB mnuRosetteCom_Click ()

UNLOAD frmDisclaimer

'Draw the Rosette graph.

CAII Rosette

END SUB

SUB mnuSave_As_ProblemCom_Click ()

'This event procedure calls the save as problem subroutine

'which saves the problem data with a new file name.

UNLOAD ErmDisclaimer

CALI save_as_problem

END SUB

SUB mnuSave_As_SiteCom_Click ()

'This subroutine calls the save as site subroutine which saves

'the site data with a new file name.

UNLOAD frmDisclaimer

CALL save_as_site

END SUB

SUB mnusave_ProblemCom_Click ()

This subroutine calls the save problem subroutine

'which saves the problem data.

UNLOAD frmDisclaimer

CALL save_problem

END SUB

SUB mnusave_Resultscom_Click ()

'This event proceedure saves the results so that they are not lost when

'the program is exited. For this function to work, a problem must be

'loaded, along with the data that corresponds to that problem

DIM filenum AS INTEGER 
DIM ForeColor AS INTEGER, BackColor AS INTEGER

DIM Elags AS INTEGER, Cancel AS INTEGER

DIM EileName AS STRING, PathName AS STRING

DIM DefaultExt AS STRING, DialogTitle AS STRING

UNLOAD frmDisclaimer

'Initialize dialoge box which asks for file name

PathName $=$ frmsetup.txtoutput_path.text

DefaultExt $=" *$.TXT"

DialogTitle = "Save Data"

'If problem is not loaded, tell user and exit subroutine

IF frmProblem Data.txtradial distancel.text = " "THEN MSGBOX "Data is not yet àvailable - Site file has not been loaded"

END IE EXIT SUB

'Prompt user for filename

CALL FileSave(FileName, PathName, DefaultExt, DialogTitle, 7, 1, Flags, Cancel)

'If user does not select cancel from dialoge box, proceed

IF NOT Cancel THEN

'If file is not in current directory, append path to file

IF PathName $<$ " "THEN FileName = PathName + "\" + FileName

'Enable local error checking

ON IOCAI ERROR GOTO save_results_error

'If file already exists, delete it (to prevent appending to it)

IF (IEN(DIRS(FileName)) $>$ 0) THEN KILL FileName

'Reset printer object so that it points to the specified file

PRINTER.PrintTarget = FileName

'Call print function to print maccs data to the file

CAII print_MACCS_input_file

'Reset printer object so that it points to the printer

PRINTER.PrintTarget $=" "$

END IF

'Turn off local error checking

ON LOCAI ERROR GOTO 0

EXIT SUB

save_results_error:

'If any kind of error occurs, inform the user and exit subroutine 
MSGBOX "A file error has occured. Please check all filenames and devices"

EXIT SUB

END SUB

SUB mnuSave_SiteCom_Click ()

'This subroutine calls the save site subroutine

'which saves the site data.

UNLOAD frmDisclaimer

CALI save_site

END SUB

SUB mnusetupcom_Click ()

'This subroutine shows the setup form. This

' form allows the user to set up paths and file

'saving formats.

UNLOAD frmDisclaimer

frmsetup.SHOW MODAI

END SUB

SUB mnuSite_specificCom_Click ()

UNLOAD frmDisclaimer

'This subroutine checks to see if there is enough data to peform

'site calculations, and if so, calls the form to do the actual math.

'For further documentation, see frmcalculate.

'Verify that there are radial distances and coordinates.

IF ((number_of_radii $=0$ ) OR (VAL(frmProblem_Data.txtPopulation_multiplier.text) =0)) THEN

MSGBOX "Unable to calculate populations." + CHRS(KEY RETURN) + "Problem Data form is incomplete.", MB_OK, "Calculation Error"

ELSEIF ((VAL (ExmSite Data.txtLongitude Degrees.text) $=0$ ) OR

(VAL (frmSite Data.txtIatitude Degrees.text) =0)) THEN

MSGBOXX "Unable to calculate populations." + CHR\$(KEY_RETURN) + " Site Data

form is incomplete.", MB_OK, "Calculation Error"

ELSE

LOAD frmCalculate

frmCalculate. SHOW MODAL

END IF

END SUB

SUB mnuTable_lcom_Click ()

'This event proceedure allows the user to view data

' in tabular format by showing the first table

' form. This form must be loaded and unloaded each

'time that it is displayed so that the values

'displayed will be updated. For further documentation

'see frmTable 1 . 
UNLOAD frmDisclaimer

table type $=1$

LOAD frmTable 1

frmTable_1.SHŌ MODAI

END SUB

SUB mnuTable_2com_Click ()

'This event proceedure allows the user to view data

'in tabular format by showing the second table

'form. This form must be loaded and unloaded each

'time that it is displayed so that the values

'displayed will be updated. For further documentation

'see frmTable_1.

UNLOAD frmDisclaimer

table type $=2$

LOAD frmTable 1

frmTable_1.SHŌW MODAL

END SUB 
option explicit

'\$include: 'secpop90.bi'

'This module contains the print_MACCs input_file subroutine which is 'used to either print or save cálculation results in a format

' compatible with the MACCS requirements for a site input file or as a 'quotation mark and comma delimited file suitable for import into most 'spreadsheets.

sub print_MACCS_input_file ()

'This routine prints SECPOP90 output in MACCS compatible form or in 'a quotation mark comma delimited form. It can be used to print to

'a printer or a file.

dim quote as string, comma as string

dim blank as string, degree as string

dim $i$ as integer, $j$ as integer

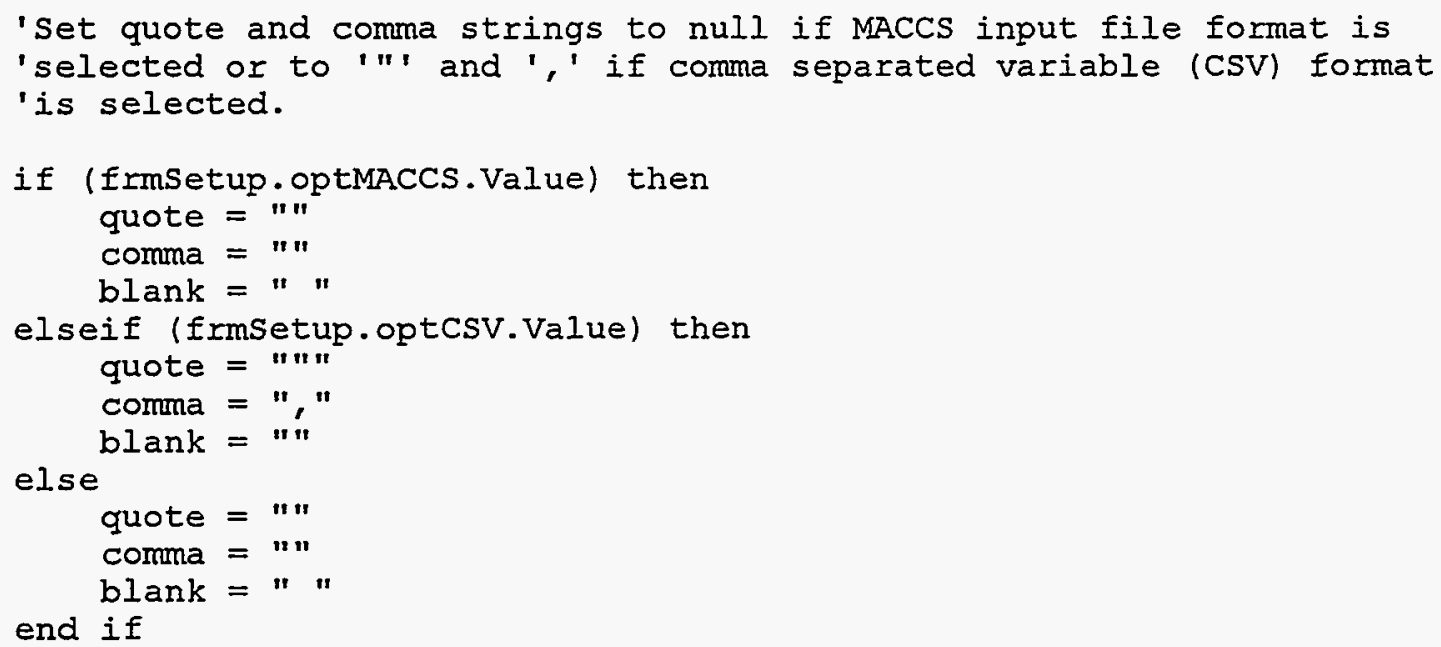

'Set degree character.

degree $=\operatorname{chr} \$(248)$

'Enable local error checking for things like printer off line, etc.

on local error goto print_maccs_error

'Initialize RADDIS array to Kilometers.

for $i=1$ to number_of_radii step 1

if (frmProblem_Data.optKilometers.value = TRUE) then

else raddis $(i) \equiv$ radial_distance $(i)$

raddis $(i)=$ radial_distance $(i) *$ miles_to_kilometers 
end if

next i

'Print (or save) results.

'Print the first line.

printer.print quote; blank:

printer.print "SECPOP90 V2.3 ";

if (frmsetup.optMACCS.Value) then printer.print "MACCS ";

elseif (frmsetup.optCSV.Value) then

else printer.print "CSV";

printer.print "MACCS ";

end if

printer.print "Site Data File for";

printer.print quote; comma; blank;

printer.print quote;

printer.print ltrims (rtrims (frmsite_data.txtSite_name.Text));

printer.print quote

'Print the second line.

printer.print quote; blank;

printer.print "Lat:";

printer.print quote; comma; blank;

printer.print using "\#";

val (frmsite_data.txtLaẼitude_degrees.Text);

printer.print comma;

printer.print quote;

printer.print degree;

printer.print quote; comma;

printer.print using "\#\#";

val (frmsite data. txtLaEitude minutes. Text);

printer.print comma;

printer.print quote;

printer.print "r";

printer.print quote; comma;

printer.print using "\#\#";

val (frmsite_data.txtLatitude_seconds.Text);

printer.print comma;

printer.print quote;

printer.print "i";

printer.print quote; comma; blank;

printer.print quote;

printer.print "Long:";

printer.print quote; comma; blank;

printer.print using "\#\#";

val (frmsite_data.txtLonḡitude_degrees.Text); 


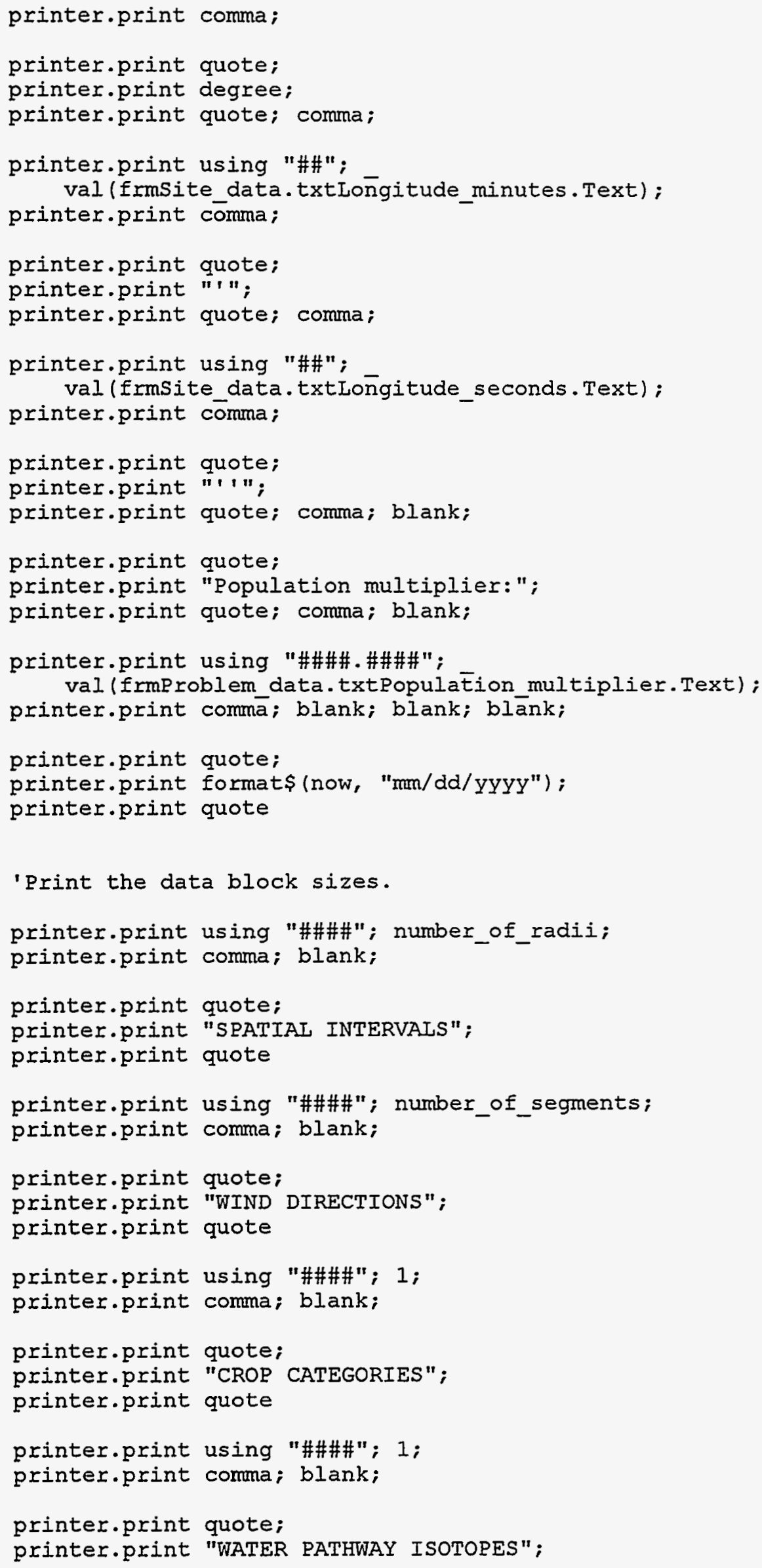




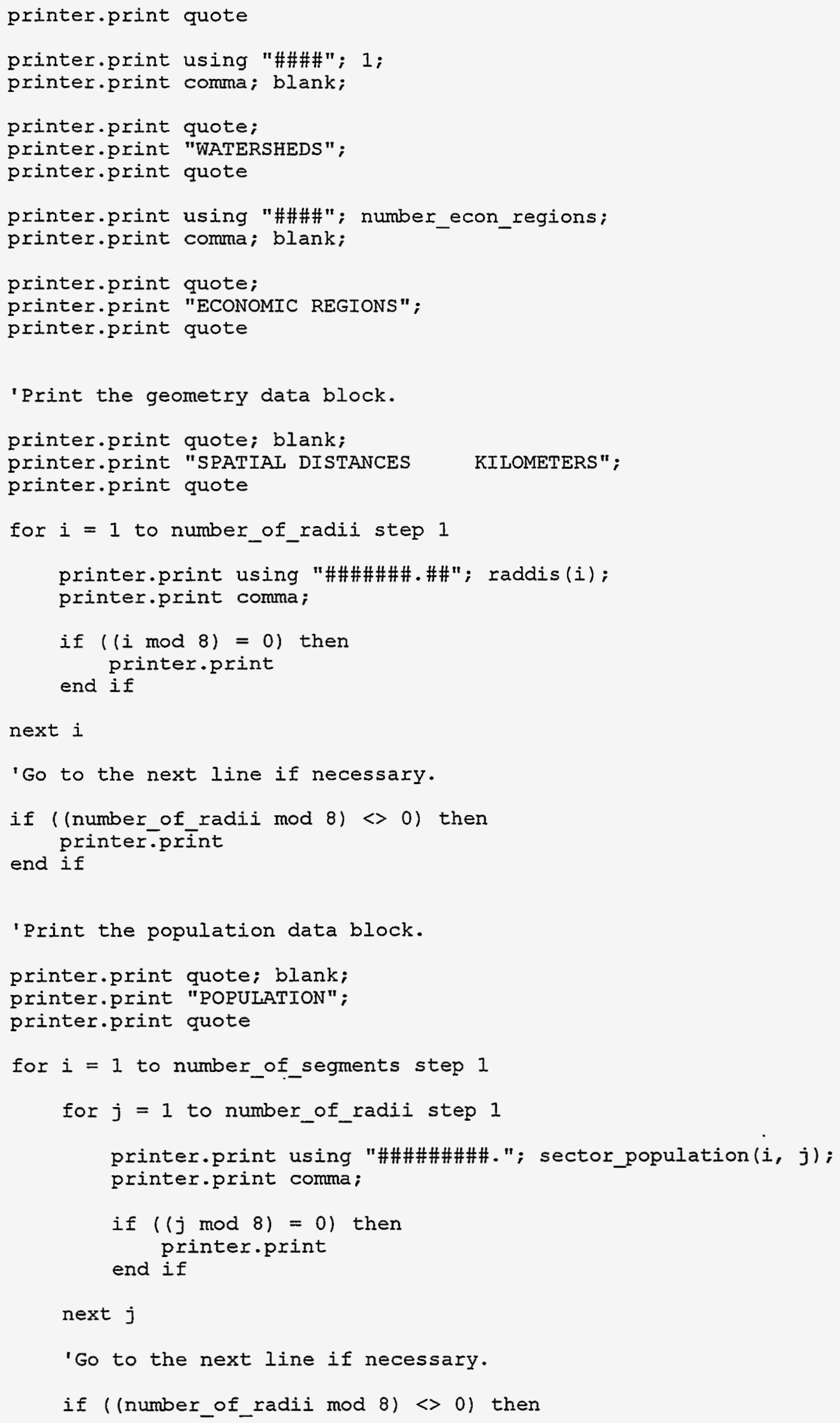


printer.print

end if

next $i$

'Print the land fraction block.

printer.print quote; blank;

printer.print "IAND ERACTION";

printer.print quote

for $i=1$ to number_of_segments step $I$

for $j=1$ to number_of_radii step 1

printer.print using "\#\#.\#\#"; sector_frclnd(i, $j)$; printer.print comma;

if $((j \bmod 16)=0)$ then

end if printer.print

next j

'Go to the next line if necessary.

if ( (number_of radii mod 16$) \ll 0$ ) then end if printer-print

next $i$

'Print the region identification block.

printer.print quote; blank;

printer.print "REGION INDEX";

printer.print quote

for $i=0$ to (number_of_segments - 1) step 1

for $j=1$ to number_of_radii step 1

printer.print using "\#\#"; region_index(j, i);

printer.print comma;

next $j$

printer.print

next $i$

'Print the (dummy) watershed identification block.

printer.print quote; blank;

printer.print "WATERSHED INDEX" ;

printer.print quote

for $i=1$ to number_of_segments step 1

for $j=1$ to number_of_radii step 1 


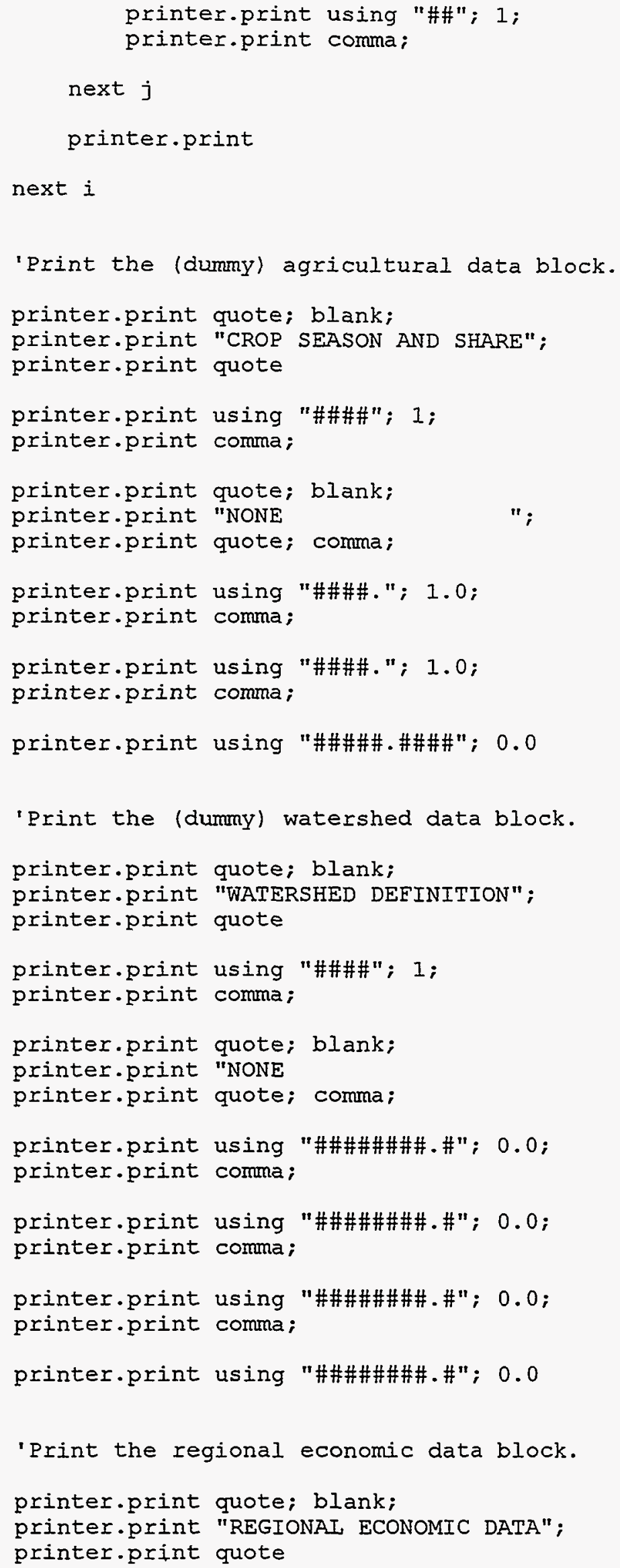




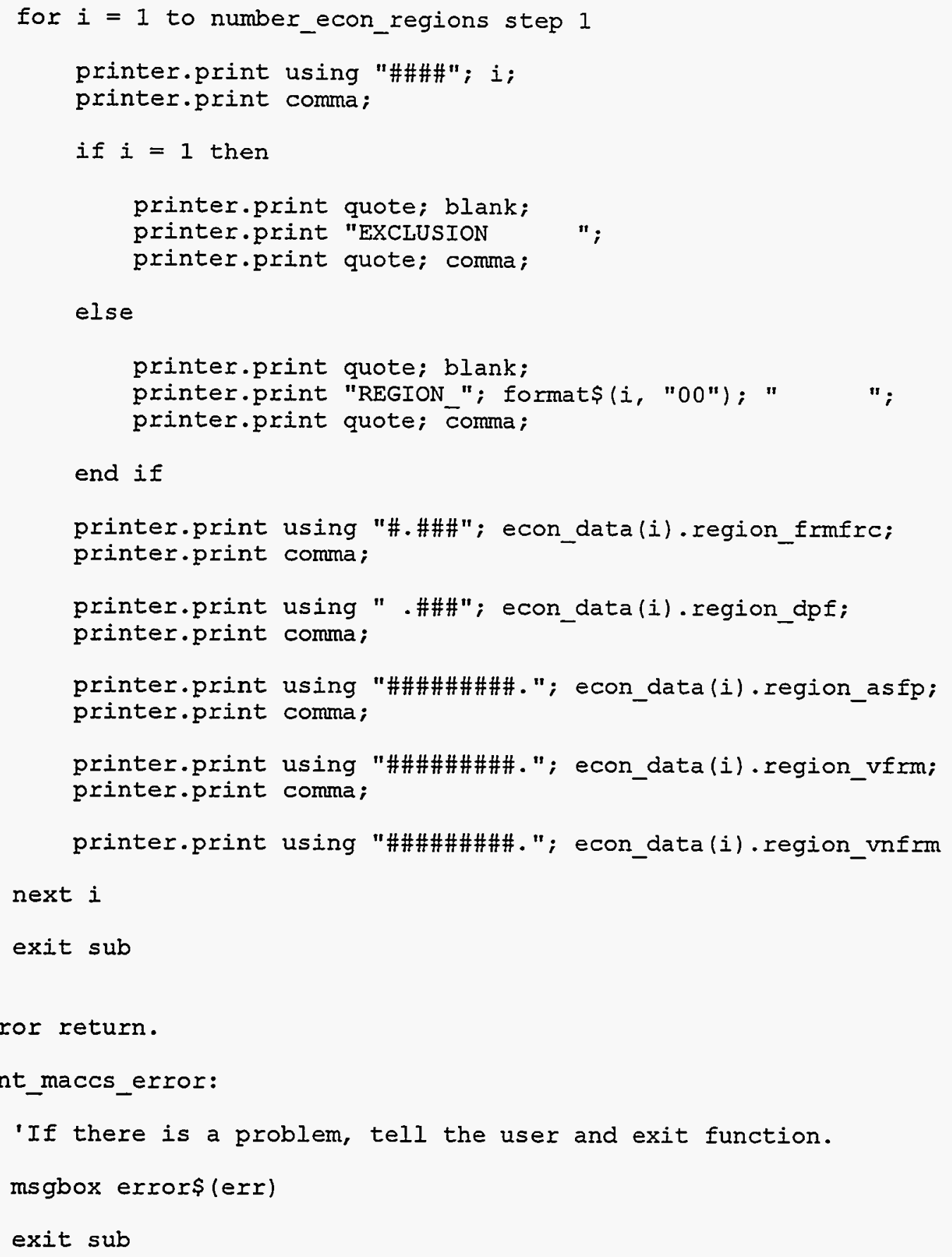




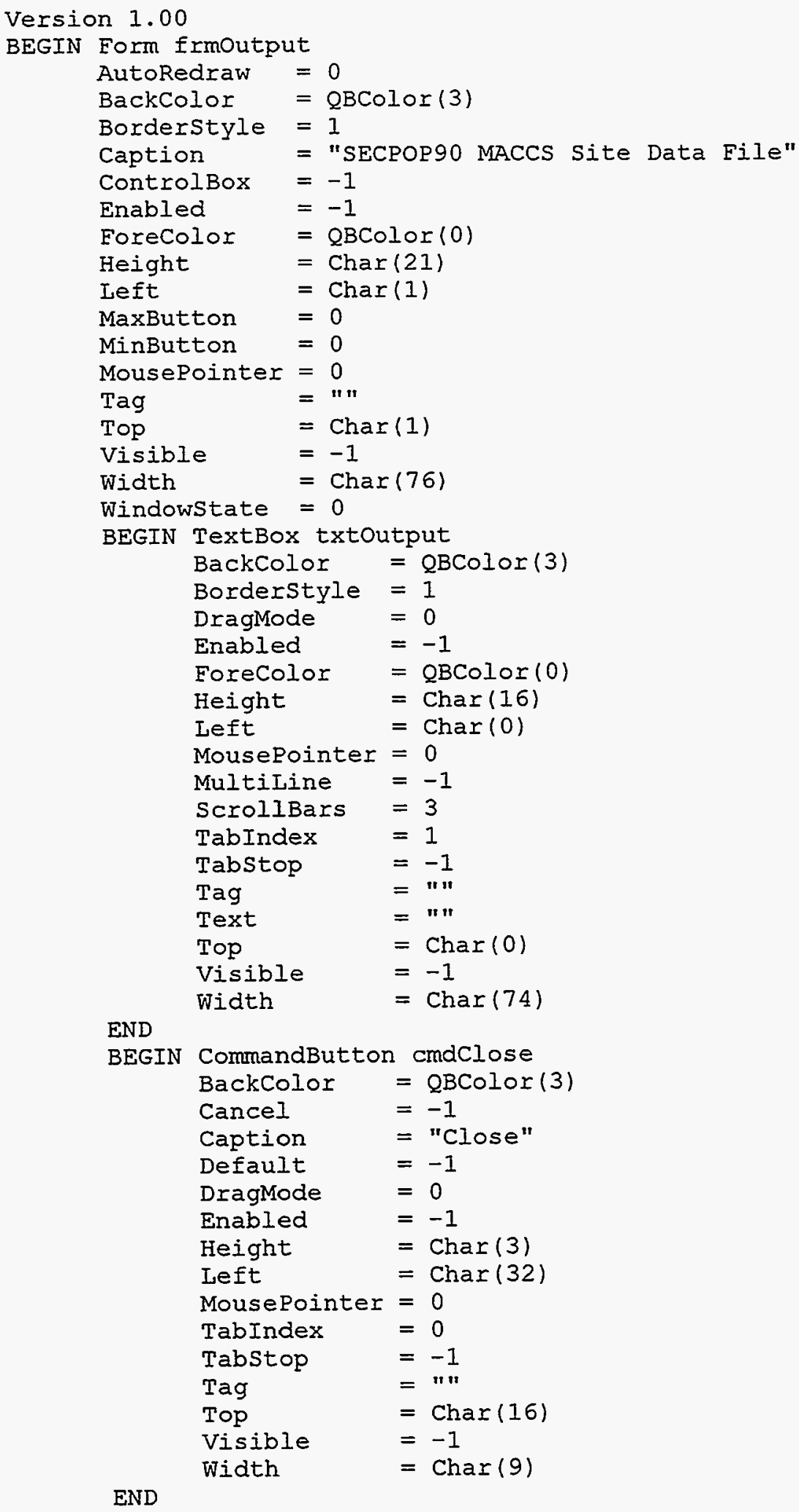

END

END

\section{OPTION EXPLICIT}

'Output (in maccs format) is displayed on this form. 
'it is basically just a text viewer with a close box

'attatched to allow the user to leave.

SUB cmaClose_Click ()

'This command allows the user to close the output

' text box.

frmoutput.HIDE

END SUB 


\section{OPTION EXPIICIT}

'\$INCLUDE: 'secpop90.bi'

'This module contains procedures for dealing with

'various problem file releted items, such as

'loading and saving problems, and creating new problems.

SUB browse_site_file ()

'this procedure allows the user to view and select previousiy created

'site files when creating a new problem.

DIM ForeColor AS INTEGER, BackColor AS INTEGER

DIM Elags AS INTEGER, Cancel AS INTEGER

DIM filename AS STRING

STATIC Pathname AS STRING

DIM DefaulText AS STRING, DialogTitle AS STRING

Initialize file dialogue box.

Defaultext $=" *$.SIT"

DialogTitle = "Browse Site"

BackColor $=$ WHITE

EoreColor $=$ BIACK

Pathname $=$ frmsetup.txtSite_Path.text

'Display file choices to user, and let them select one.

CAII Fileopen(filename, Pathname, DefaulText, DialogTitle, ForeColor, BackColor, Flags, Cancel)

'If user did not select cancel from file dialogue box, then proceed.

IF NOT Cancel THEN

'If file is not in current directory, append pathname.

filename = Pathname + "\" filename

'Put site file name into text box on problem form.

frmProblem_data.txtsite_file_name.text = filename

'Mark file as modified.

frmMain.mnusave ProblemCom. enabled = TRUE

frmMain.mnusave As Problemcom.enabled = TRUE

IF (INSTR (frmProblem data.caption, " (Modified)") = 0) THEN END IF frmproblem data. caption = frmproblem data.caption + " (Modified)"

'Call procedure to open site file and read in values.

open_site_from_problem (filename)

'If cancel was selected in file dialogue box, reset focus to

'site file name. 
END IF

frmProblem_data.txtsite_file_name.SETFOCUS

END SUB

SUB default_regions ()

DIM h AS INTEGER, i AS INTEGER, j AS INTEGER, $k$ AS INTEGER

DIM Im AS INTEGER, I AS INTEGER

DIM num sectors AS INTEGER

DIM dividend AS INTEGER, remainder AS INTEGER

num_sectors $=(($ (number_of_radii -1$) *$ number_of_segments $)+1)$ $k=2$

frmProblem_data.lblNumber_of_regions.tag = STRS (number_of_radii)

FOR $i=0$ TO (number_of segments)

NEXT $i$ region index $(1, i \overline{)}=1$

IF (num_sectors $<=$ max_econ_regions) THEN

number_econ_regions = num_sectors

FOR $i=0$ TO (number of segments)

FOR $j=2$ TO (ñumber of radii)

region_index $(j, i \bar{j}=\mathrm{k}$

NEXT j

$k=k \mp 1$

NEXT i

ELSE

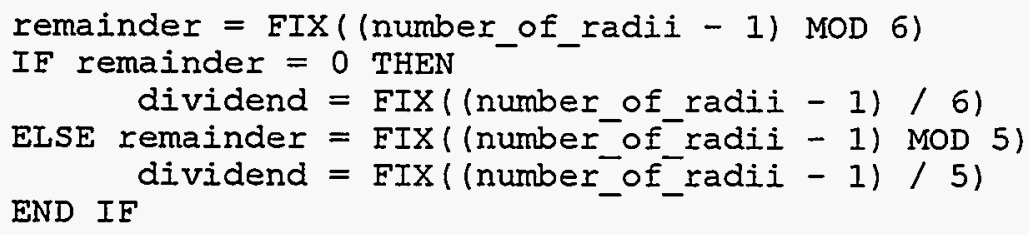

FOR $h=0$ TO (number_of_segments)

$m=2$

IF (remainder > dividend) THEN

FOR $\mathrm{m}=2$ TO (remainder +1 )

NEXT II

region index $(m, h)=k$

END IF

$k=k+1$

FOR $i=1$ TO 5

FOR $j=m$ TO $(m+$ dividend -1$)$

NEXT $j$

region_index $(j, h)=k$

$m=j$

$\mathrm{k}=\mathrm{k}+1$

NEXT $i$

IF remainder $<=$ dividend THEN

FOR $i=m$ TO (number of radii)

region index $(i,-h)^{-}=k$

NEXT i

$k=k+1$

END IF

NEXT $h$

number_econ_regions $=97$ 
END IE

frmproblem_data.IblNumber_of_regions.caption = STR\$ (number_econ_regions)

END SUB

SUB new_problem ()

'This procedure initializes a new problem to default settings

'and opens the problem form.

DIM I AS INTEGER, j AS INTEGER

'Initialize settings to default.

frmproblem data.IblNumber_of_regions. caption $=" \|$

FOR $i=0$ TO number of seḡmeñts - 1

FOR $j=2$ TO max number of radii

NEXT j region index $(j, i \bar{j}=0$

NEXT i

frmMain.musave Problemcom. enabled = EALSE

frmmain.mnusave As ProblemCom. enabled = FAISE

frmProblem data.caption = CURDIRS + "\" + "NEW PROB.PRB"

frmProblem_data.txtsite_file_name.text = (frmsētup.txtsite_Path.text + "\*.SIT")

frmproblem data.vsbRadius_Number.value $=1$

frmProblem data.txtRadial Distancel.text = " "

frmProblem data.txtRadial Distance 2 . text $=" n$

frmproblem data.txtRadial Distance 3 . text $=n n$

frmproblem data. txtPopulation multiplier.text $=" 1.0 "$

frmProblem_data.txtProblem_Remarks.text = " "

FOR $i=0$ TO max number_of_radii STEP 1

NEXT i radial distance $(i \overline{)}=0$

number_of radii $=0$

number ecōn regions $=0$

$x$ position $\equiv 0$

$y$ position $=0$

'Show new problem form

frmProblem_data.SHOW MODAL

END SUB

SUB open_problem ()

open a previously created problem from file.

DIM site file name AS STRING, longitude AS STRING, latitude AS STRING, units of measure AS STRING

DIM site_name AS STRING, site_remarks AS STRING, problem_remarks AS STRING,

dummy AS STRING

DIM longitude degrees AS INTEGER, longitude minutes AS INTEGER,

longitude seconds $\bar{A} \bar{S}$ INTEGER

AS INTEGER

DIM-latitude_degrees AS INTEGER, latitude_minutes AS INTEGER, latitude_seconds

DIM i AS INTEGER, j AS INTEGER

DIM ForeColor AS INTEGER, BackColor AS INTEGER 
DIM Flags AS INTEGER, Cancel AS INTEGER

DIM filename AS STRING

STATIC Pathname AS STRING

DIM DefaulText AS STRING, DialogTitle AS STRING

'Define as constants the file handle numbers for problem and site files,

'and the file buffer size.

CoNST problem_file $=10$, site_file $=20$, file_buffer $=1024$

'Initialize file dialog box.

DefaulText $=" * . P R B "$

DialogTitle = "Problem Open"

Pathname $=$ frmsetup.txtProblem path.text

BackColor = WHITE

EoreColor = BLACK

'Enable local error checking for file errors.

ON LOCAL ERROR GOTO open_prob_error

'Open file dialog box.

CALL Fileopen(filename, Pathname, DefaulText, DialogTitle, Forecolor, BackColor, Flags, Cancel)

'If user does not select cancel from file dialogue box.

IF NOT Cancel THEN

'if filename is not in current directory, add path.

IF Pathname $<$ " "THEN filename = Pathname + "\" + filename

'Reset the table position variables.

$x$ position $=0$

y_position $=0$

'Open filename and read problem information into temporary variables.

file buffer

OPEN filename FOR INPUT ACCESS READ LOCK WRITE AS \#problem file LEN =

IINE INPUT \#problem file, dummy

IINE INPUT \#problem file, dummy

IINE INPUT \#problem_file, site_file_name

IINE INPUT \#problem-file, dummy

INPUT \#problem file, number of radii

IINE INPUT \#prōblem file, dưmmy

IINE INPUT \#problem_file, units_of_measure

IINE INPUT \#problem file, dummy

FOR $i=1$ TO number of radii STEP 1

NEXT i

INPUT \#problem_fíile, radial_distance (i)

FOR $i=$ number_of_radii +1 TO max_number_of_radii STEP 1

NEXT $i$

radial_distānce $(i)=0 \#$

IINE INPUT \#problem file, dummy

INPUT \#problem file, Population multiplier

IINE INPUT \#prōblem file, dummy

INPUT \#problem file, number econ regions

LINE INPUT \#problem_file, dümy 
FOR $i=0$ TO number_of_segments -1

FOR $j=1$ TO number of radii NEXT $j$

INPUT \#problem_file, region_index $(j, i)$

NEXT i

IINE INPUT \#problem file, dummy

LINE INPUT \#problem_file, problem_remarks

CLOSE problem_file

'Transfer information from temporary variables to correct

' form locations.

frmProblem data. caption = filename

frmProblem_data.txtsite_file_name.text = site_file_name

IF units of measure $=$ " $\overline{\mathrm{K}}$ ilometers" THEN

frmproblem data.optKilometers.value $=$ TRUE

frmProblem_data.optKilometers.tag = "Previously_True"

EISE

frmProblem data.optmiles.value = TRUE

END IF

frmProblem_data.optmiles.tag = "Previously_True"

ErmProblem_data.vsbRadius_Number.value $=1$

frmProblem_data.txtRadial_Distancel.text = RIGHT\$ $" ~ "+$

FORMAT\$(radial distance (1), "0.0000;0.0000; "), 9)

frmproblem_data.txtRadial_Distance2.text = RIGHT\$(" $"+$

FORMAT\$(radial_distance (2), "0.0000;0.0000; "), 9)

frmproblem data.txtRadial_Distance3.text = RIGHT\$(" " +

FORMAT\$(radial_distance $(3), " 0.0000 ; 0.0000 ; \quad "$ ), 9)

frmProblem_data.txtPopulation multiplier.text $=$ RIGHT\$(" $"+$

FORMAT\$(Population mulËiplier, "0.0000;0.0000; "), 9)

frmPrō̄lem_data.txtProblem_Remarks.text = problem_remarks

'If a site file is listed, call function to read in site data.

IF site_file name $<>"$ "THEN

END IF open_site_from_problem (site_file_name)

'Set the number of economic regions to new value, and re-paint

'data form.

frmProblem_data.lblNumber_of_regions.tag = STRS(number_of_radii)

frmProblem_data.IblNumber_of_regions.caption = STRS (number $\bar{r}_{\text {_econ_regions) }}$

CALI paint_region $(0,0)$

'If problem data form is not visible, show it.

IF (frmproblem data.visible = FALSE) THEN

END IF frmProblem data. SHOW MODAL

END IF

EXIT SUB

open_prob_error:

'On error, inform the user, and exit subroutine.

MSGBOX ERRORS (ERR), MB_OK, "Error"

'MSGBOX "File error - check spelling of file names."

EXIT SUB 
END SUB

SUB open_site_from_problem (file_name AS STRING)

'Open site from problem and fill site form with data from file.

DIM site name AS STRING, site_remarks AS STRING, dummy AS STRING

DIM longitude degrees AS INTEGER, longitude_minutes AS INTEGER, longitude seconds $A \bar{S}$ INTEGER

AS INTEGER

DIM latitude_degrees AS INTEGER, latitude minutes AS INTEGER, latitude seconds

'Declare as constant the file handle number for site file and the

'file buffer size.

CoNST site_file $=20$, file_buffer $=1024$

'Enable local error checking.

ON LOCAI ERROR GOTO Open_site_problem_file_error

'Open site file named on problem form, and read input into

'temporary variables.

file_buffer

OPEN file name FOR INPUT ACCESS READ LOCK WRITE AS \#site file LEN =

IINE INPUT \#site file, dummy

IINE INPUT \#site file, site name

LINE INPUT \#site-file, dummy

LINE INPUT \#site file, dummy

INPUT \#site file, longitude degrees

INPUT \#site_file, longitude_minutes

INPUT \#site file, longitude_seconds

LINE INPUT \#site file, dummy

INPUT \#site file, latitude degrees

INPUT \#site-file, latitude minutes

INPUT \#site-file, latitude_seconds

LINE INPUT \#site file, duminy

IINE INPUT \#site_file, site_remarks

CLOSE site_file

'Transfer values from temp variables to site form.

frmsite_Data.caption = file_name

frmsite Data.txtSite Name.text = site name

frmSite Data.txtIongitude_Degrees.tex $\bar{t}$ = STRS (longitude_degrees)

frmSite_Data.txtIongitude_Minutes.text = STRS (longitude_minutes)

frmsite Data.txtIongitude-seconds.text $=$ STR\$ (Iongitude seconds)

frmsite Data.txtLatitude Degrees.text = STR\$ (latitude dégrees)

frmsite-Data.txtLatitude Minutes. text = STRS (latitude minutes)

frmsite_Data.txtLatitude_Seconds.text = STRS (latitude_seconds)

frmsite_Data.txtSite_Remārks.text = site_remarks

EXIT SUB

open_site_problem_file_error:

'If there is a problem, tell the user, and return to the form where

'a correction can be made.

IF frmProblem data.visible = FALSE THEN

frmProbIem data. SHOW MODELESS

MSGBOX "Error: Unable to Open Site File." 
frmProblem_data.txtSite_file_name.SETFOCUS

ELSE

frmProblem_data.HIDE

END IF

MSGBOX "Unable to Open Site File.", MB OK, "File Error"

EXIT SUB

frmProblem_data.txtsite_file_name.SETFOCCUS

END SUB

SUB paint_region ( $x$ _offset AS INTEGER, y_offset AS INTEGER)

DIM i AS INTEGER, $j$ AS INTEGER, $k$ AS INTEGER, $x$ AS INTEGER, y AS INTEGER

frmRegion.Iblnum_regions.caption = STRS (number_econ_regions)

frmRegion.lblnum_radii.caption = STRS (number_of́radii)

IF frmproblem data.optmiles.value = TRUE THEN

frmRegion.lblunits.caption = "Miles"

ELSE frmRegion.lblunits.caption = "Kilometers"

END IF

$x$ position $=x_{\text {_position }}+x_{\text {_offset }}$

y_position $=$ y_position + y_offset

IF (number of radii < 8) THEN

$x$ position $=0$

$I \bar{F}(x$ offset $<>0)$ THEN

EXIT SUB

END IF

ELSEIF ( $x$ position $<0$ ) THEN

$x$ position $=0$

EX̄XT SUB

ELSEIE ( (x position +7 ) > number_of_radii) THEN

$x$ position $=x$ position -1

END IE

EX̄XT SUB

FOR $i=1$ TO 7

$\mathrm{x}=\mathrm{x}$ position $+\mathrm{i}$

IF radial distance $(x)=0$ THEN

frmRegion.lblRdist $(i-1)$. caption $=" n$

ELSE frmRegion.lblRdist $(i-1)$. caption = LEFT\$(STRS(radial_distance $(x)$ ),

4)

END IF

NEXT i

$k=0$

FOR $i=0$ TO 6

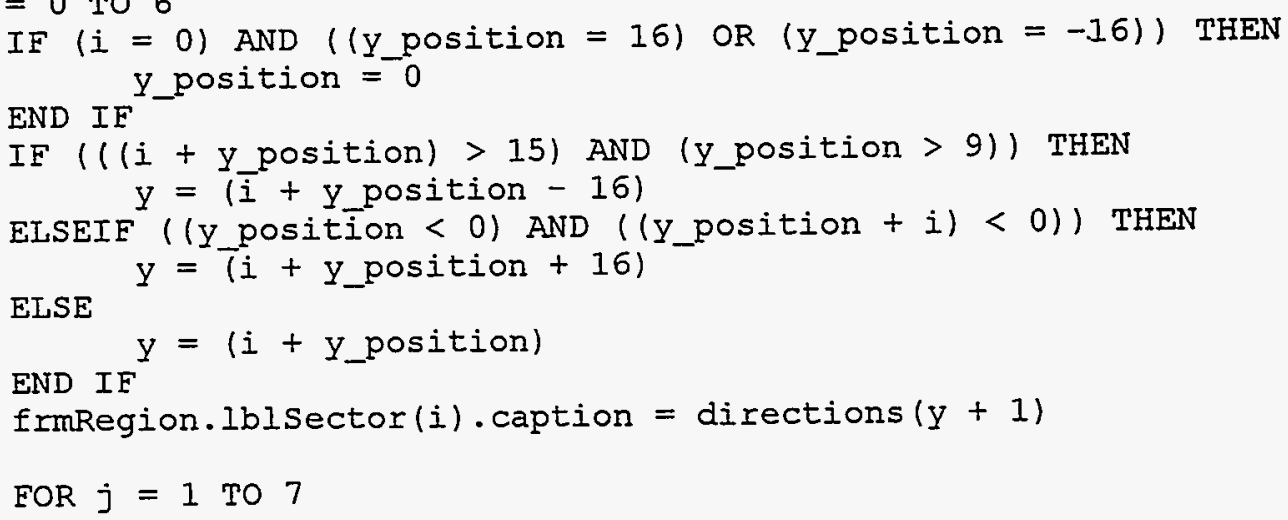




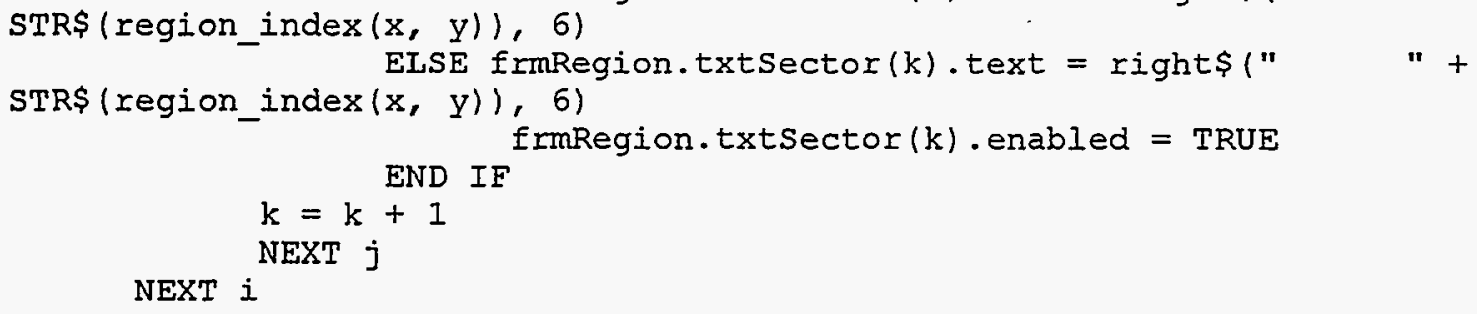

END SUB

SUB print_problem ()

'Print the data on a problem form.

DIM ForeColor AS INTEGER, BackColor AS INTEGER

DIM Copies AS INTEGER, Cancel AS INTEGER, i AS INTEGER, j AS INTEGER

DIM file name AS STRING, date AS STRING, latitude AS STRING, longitude AS

STRING, site_fille_name AS STRING, problem_remarks AS STRING

'Define as constants the margines for printing.

CONST left_margin $=10$

CONST text_width $=40$

BackColor $=$ WHITE

ForeColor $=$ BLACK

'Enable local error checking.

ON LOCAL ERROR GOTO problem_print_error

'Call printer dialogue box.

CALL FilePrint(Copies, ForeColor, BackColor, Cancel)

'If cancel is not selected from the printer dialogue box then proceed.

IF NOT Cancel THEN

'Execute once for each copy desired by the user.

FOR $i=1$ TO Copies

'Send data to printer.

printer.PRINT TAB(left margin); "File Name:";

file_name = frmproblem_data.caption

CALI print text(file_name, text_width, left_margin +

LEN ("Population Multiplier: "))

printer.PRINT

printer.PRINT TAB (left margin); "Site File Name:";

site file name = frmproblem data.txtsite file name.text

CALL print_text(site_file_name, text_width, lēeft_margin +

LEN ("Population Multiplier: "))

printer. PRINT 
printer.PRINT TAB (left margin); "Number of Radii:";

printer.PRINT USING "\#\#"; TAB(left_margin + IEN("Population

Multiplier: ")); number of radii

printe $\bar{r} \cdot \mathrm{P} \overline{\mathrm{R}} \mathrm{INT}$

printer.PRINT TAB (left margin); "Radial Distances:";

FOR $j=1$ TO number of radii STEP 1 printer.PRINT USING "\#\#\#.\#\#\#"; TAB(left_margin +

LEN("Population Multiplier: ")) ; radial_distance(j)

NEXT $j$

printer. PRINT

printer.PRINT TAB(left margin); "Population Multiplier Factor:";

printer.PRINT USING "\#\#\#.\#\#\#"; TAB(left_margin + LEN("Population

Multiplier: ")); VAI (frmproblem_data.txtpopulation_multiplier.text)

printer.PRINT

printer.PRINT TAB(left_margin); "Problem Remarks:";

problem remarks = frmproblem data.txtProblem Remarks.text

IEN ("Population Multiplier: "))

CAIL print_text (problem_remariks, text_width, left_margin +

NEXT i

printer. NEWPAGE

'Tell printer that it is at the end of the document. printer. ENDDOC

END IF

EXIT SUB

problem_print_error:

'If an error ocurrs, the user is notified, and the sub routine exited.

MSGBOX "Device error: Check printer or other print device."

EXIT SUB

END SUB

SUB save_as_problem ()

'Allow the user to specify a new name for a file that is being saved.

DIM problem name AS STRING, problem remarks AS STRING, dumy AS STRING, caption AS STRING

DIM longitude_degrees AS INTEGER, longitude_minutes AS INTEGER,

longitude seconds $A \bar{S}$ INTEGER

DIM latitude degrees AS INTEGER, latitude_minutes AS INTEGER, latitude_seconds

AS INTEGER

DIM ForeColor AS INTEGER, BackColor AS INTEGER

DIM Flags AS INTEGER, Cancel AS INTEGER

DIM filename AS STRING, Pathname AS STRING

DIM DefaulText AS STRING, DialogTitle AS STRING

DIM temp AS INTEGER

CONST problem file $=10$, file_buffer $=1024$

'Verify that the user has entered valid input into all necessary fields

'Do not save until all input is correct.

temp = verify_input (1)

IF temp $=0$ THEN

frmproblem data.txtSite_file_name.SETFOCUS

EXIT SUB

ELSEIE temp $=-1$ THEN 
frmProblem_data.txtPopulation_multiplier.SETFOCUS

EXIT SUB

ELSEIE temp $=-2$ THEN

frmProblem_data.txtRadial_Distancel.SETFOCUS

EXIT SUB

ELSEIF temp $=-3$ THEN

frmProblem_data.txtRadial_Distance2.SETFOCUS

EXIT SUB

ELSEIF temp $=-4$ THEN

MSGBOX "Setting Economic regions to default values"

END IF

CALI default_regions

'Break filename into two pieces - path and filename.

caption = frmproblem_data. caption

CALI parse_path_and_file(caption, Pathname, filename)

'Initialize file dialogue box.

DefaulText $=" *$. PRB"

Pathname = frmSetup.txtProblem path.text

DialogTitle = "Save As Problem"

BackColor $=$ WHITE

ForeColor = BLACK

'Open file dialogue box.

BackColor, Flags, Cancel)

CALL FileSave(filename, Pathname, DefaulText, DialogTitle, ForeColor,

'If the user did not select cancel in the file dialogue box

'then proceed.

IF NOT Cancel THEN

'If the file is not in the current directory, add the path to it.

IF Pathname $<" ~ "$ THEN filename = Pathname $+" \backslash "+$ filename

frmproblem_data.caption = filename

'Call the function to save the problem.

CALI save_problem

END IF

END SUB

SUB save_problem ()

'Save problem data into previously named file.

DIM caption AS STRING, i AS INTEGER, j AS INTEGER

DIM filename AS STRING, Pathname AS STRING, tempstring AS STRING

DIM temp AS INTEGER, filenum AS INTEGER

CONST problem file $=10$, file_buffer $=1024$

'Remove the path from the filename.

caption = frmproblem data. caption

CAII parse_path_and_file(caption, Pathname, filename) 
'If it is a never-before-named problem, then call save as.

IF filename $=$ "NEW_PROB.PRB" THEN

CALL save_as_problem

EISE

'Verify that all of the data inputed by the user is correct. If not, 'go back and demand a correction.

temp = verify_input(1)

IF temp $=0$ THEN frmProblem_data.txtsite_file_name.SETFOCUS EXIT SUB

ELSEIF temp $=-1$ THEN frmProblem_data.txtPopulation_multiplier.SETFOCUS EXIT SUB

ELSEIF temp $=-2$ THEN frmProblem_data.txtRadial_Distancel.SETFOCUS EXIT SUB

ELSEIF temp $=-3$ THEN frmProblem data.txtRadial Distance2.SETFOCUS EXIT SUB

EISEIE temp $=-4$ THEN MSGBOX "Setting Economic regions to default values"

END IF CALI default_regions

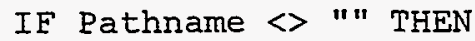
END IE

filename $=$ Pathname $+" \backslash "+$ filename

'Enable local error correction.

ON IOCAL ERROR GOTO prOb_save_error

'Open output file, and write information to it.

OPEN filename FOR OUTPUT ACCESS WRITE LOCK READ WRITE AS \#problem_file LEN = file_buffer

PRINT \#problem file, "SECPOP90 V2.3"

PRINT \#problem file, "Site File: "

PRINT \#problem_file, frmProblem_data.txtsite_file_name.text

PRINT \#problem-file, "Number of Radii:"

PRINT \#problem-file, USING "\#\#"; number of radii

PRINT \#problem file, "Units of Measure:"

IF frmProblem data.optkilometers.value = TRUE THEN

PRINT \#problem file, "Kilometers"

ELSE PRINT \#problem fīle, "Miles"

END IF

PRINT \#problem file, "Radial Distances:"

FOR $i=1$ TO number of radii STEP 1 PRINT \#probIem file, USING "\#\#\#.\#\#\#"; radial_distance(i)

NEXT i

PRINT \#problem file, "Population Multiplier:"

PRINT \#problem file, USING "\#\#\#.\#\#\#";

VAI (frmProblem data.txtPopūlation multiplier.text)

PRINT \#problem file, "Number of Economic regions:"

PRINT \#problem_file, USING "\#\#"; number_econ_regions

PRINT \#problem file, "Economic regions:"

FOR $i=0$ TO (ñumber_of_segments - 1) 


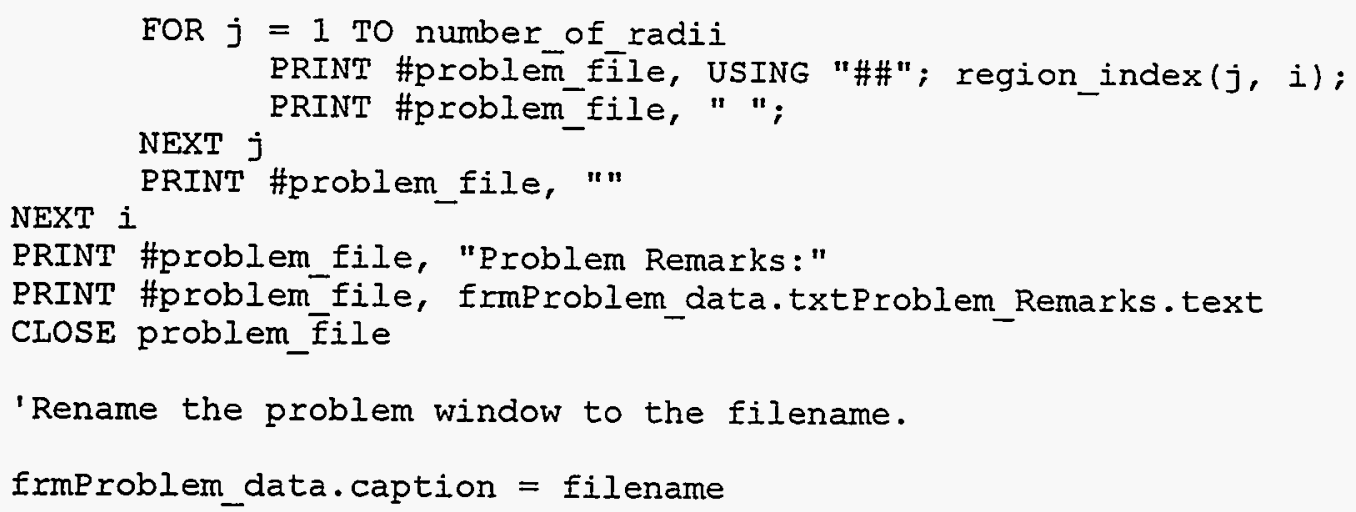

END SUB 


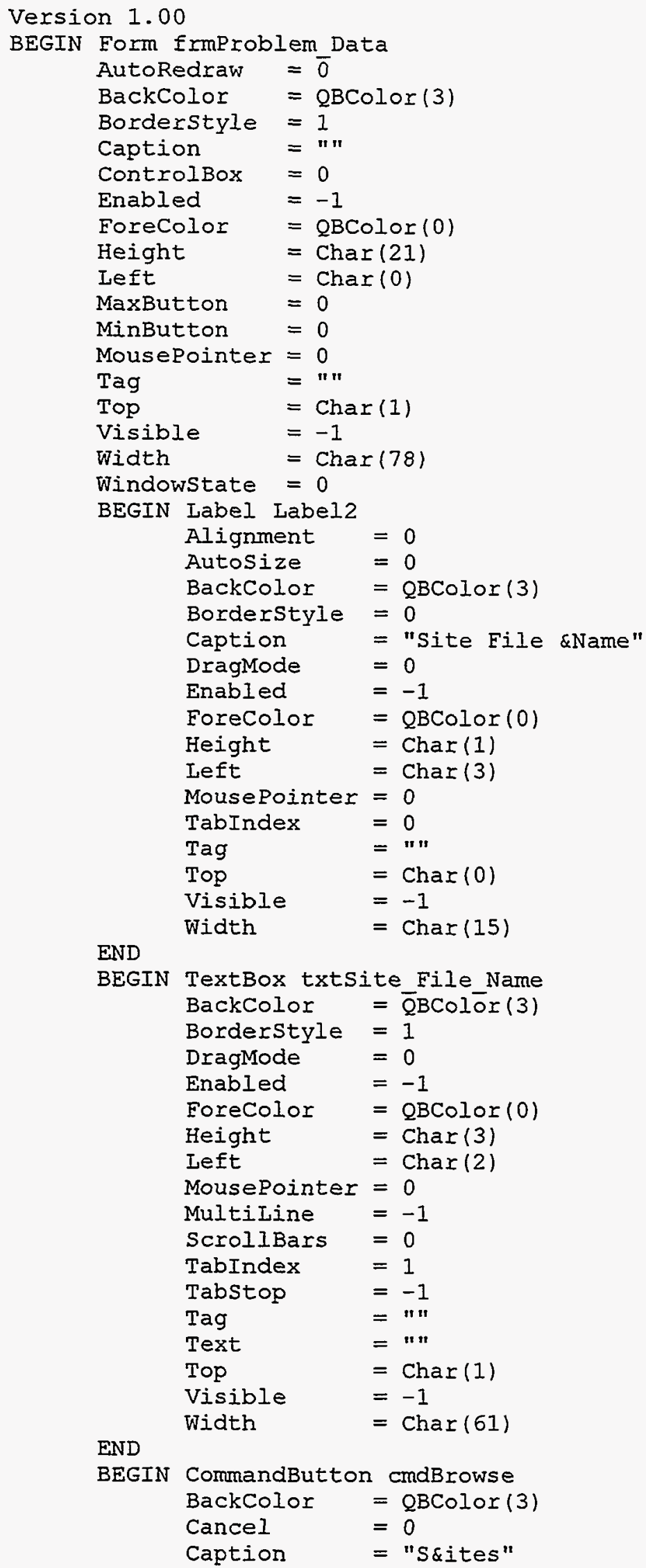




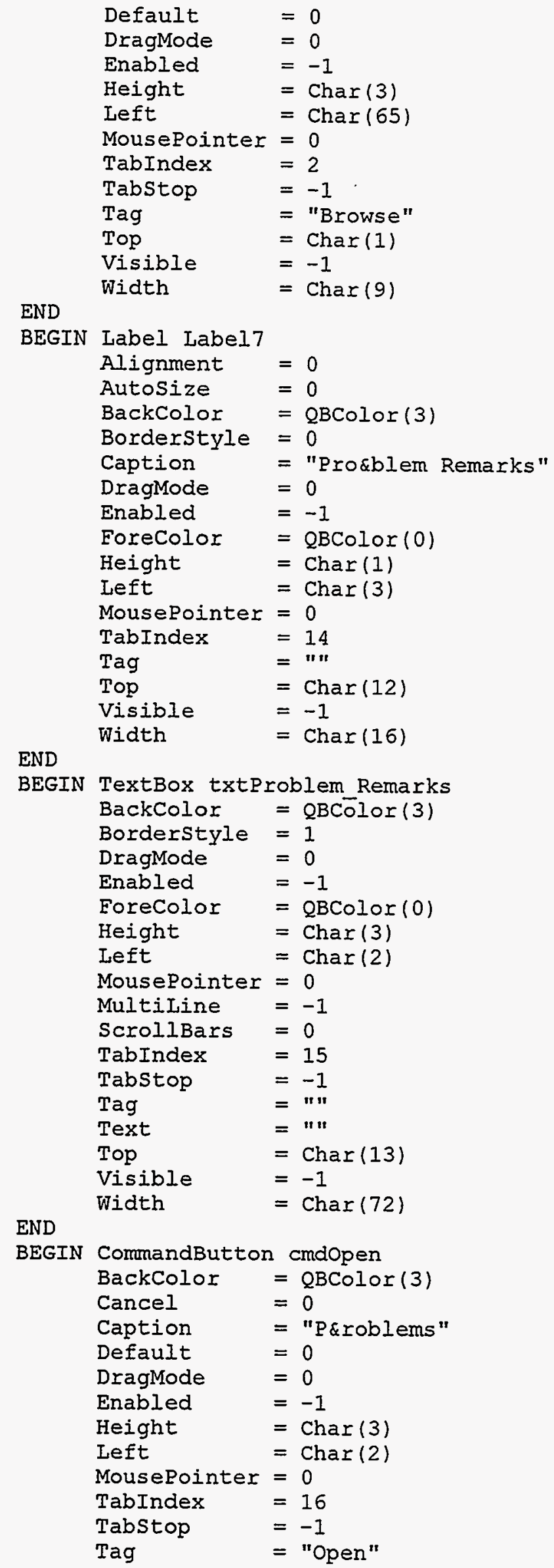


$\begin{array}{ll}\text { Top } & =\operatorname{Char}(16) \\ \text { Visible } & =-1 \\ \text { Width } & =\operatorname{Char}(11)\end{array}$

END

BEGIN CommandButton cmdSave

BackColor = QBColor (3)

Cancel $=0$

Caption $=$ "\&Save"

Default $\quad=0$

DragMode $\quad=0$

Enabled $\quad=-1$

Height = Char (3)

Left = Char(32)

MousePointer $=0$

TabIndex $=18$

Tabstop $=-1$

Tag $=" "$

Top $=\operatorname{Char}(16)$

Visible $\quad=-1$

Width $=\operatorname{Char}(11)$

END

BEGIN CommandButton cmdSave As

BackColor = QBColor $(3)$

Cancel $=0$

Caption = "Save \&As"

Default $\quad=0$

DragMode $\quad=0$

Enabled $\quad=-1$

Height $=\operatorname{Char(3)}$

Left $=\operatorname{Char}(47)$

MousePointer $=0$

TabIndex $=19$

Tabstop $\quad=-1$

Tag = " "

Top $=\operatorname{Char}(16)$

Visible $=-1$

Width $\quad=\operatorname{Char}(11)$

END

BEGIN CommandButton cmdPrint

BackColor = QBColor (3)

Cancel $=0$

Caption = "\&Print"

Default $\quad=0$

DragMode $\quad=0$

Enabled $\quad=-1$

Height $=\operatorname{Char}(3)$

Left $=\operatorname{Char}(62)$

MousePointer $=0$

TabIndex $\quad=20$

Tabstop $=-1$

Tag $=" n$

Top $=\operatorname{Char}(16)$

Visible $\quad=-1$

Width $\quad=\operatorname{Char}(11)$

END

BEGIN Label Label5

$\begin{array}{ll}\text { Alignment } & =2 \\ \text { AutoSize } & =0 \\ \text { BackColor } & =\text { QBColor }(3) \\ \text { Borderstyle } & =0 \\ \text { Caption } & =\text { "Radial \&Distances" } \\ \text { DragMode } & =0 \\ \text { Enabled } & =-1\end{array}$




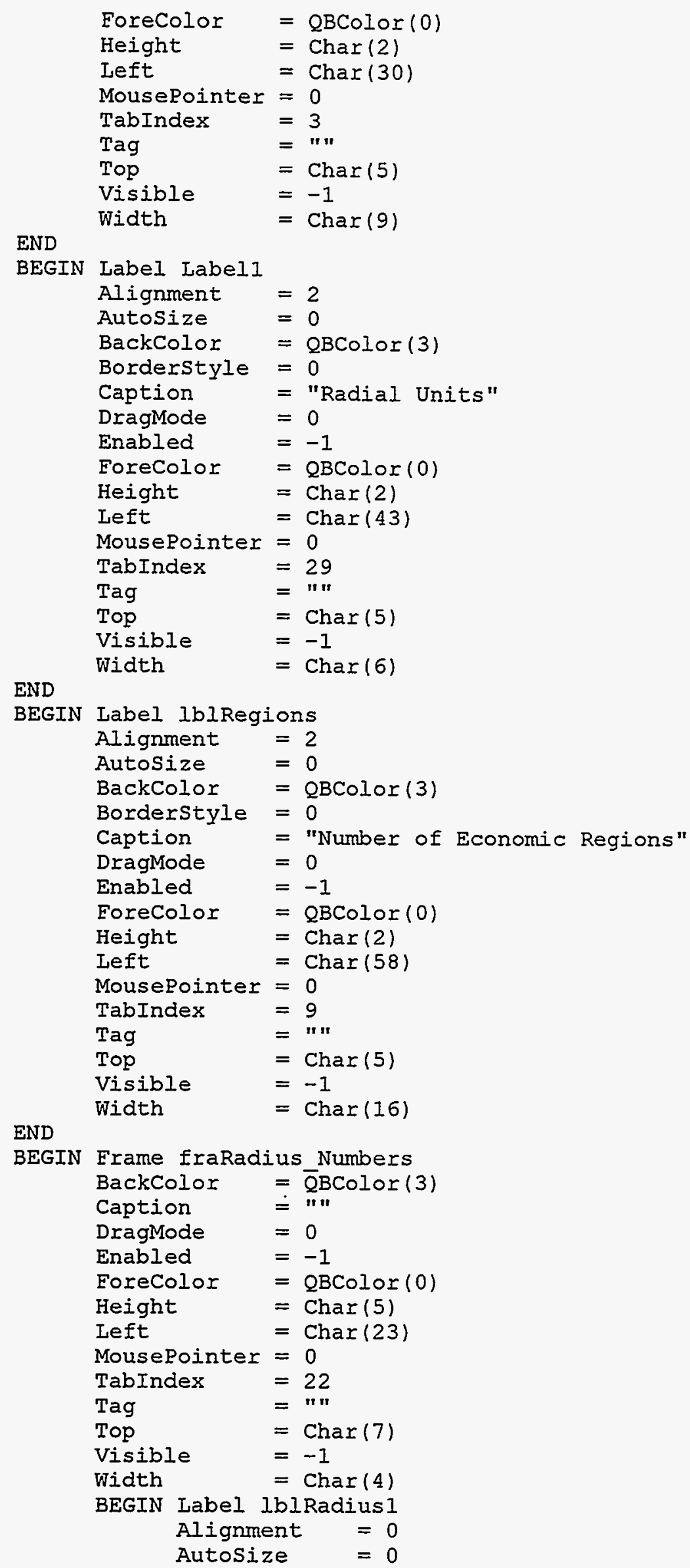




$$
\begin{array}{ll}
\text { BackColor } & =\text { QBColor (3) } \\
\text { Borderstyle } & =0 \\
\text { Caption } & =" I " \\
\text { DragMode } & =0 \\
\text { Enabled } & =-1 \\
\text { ForeColor } & =\text { QBColor }(0) \\
\text { Height } & =\text { Char }(1) \\
\text { Left } & =\text { Char }(0) \\
\text { MousePointer } & =0 \\
\text { TabIndex } & =23 \\
\text { Tag } & =" " \\
\text { Top } & =\text { Char }(0) \\
\text { Visible } & =-1 \\
\text { Width } & =\text { Char (2) }
\end{array}
$$

END

BEGIN Label 1blRadius 3

$$
\begin{array}{ll}
\text { Alignment } & =0 \\
\text { AutoSize } & =0 \\
\text { BackColor } & =\text { OBColor (3) } \\
\text { BorderStyle } & =0 \\
\text { Caption } & =" 3 " \\
\text { DragMode } & =0 \\
\text { Enabled } & =-1 \\
\text { ForeColor } & =\text { QBColor (0) } \\
\text { Height } & =\text { Char (1) } \\
\text { Left } & =\text { Char (0) } \\
\text { MousePointer } & =0 \\
\text { TabIndex } & =25 \\
\text { Tag } & =" \text { " } \\
\text { Top } & =\text { Char (2) } \\
\text { Visible } & =-1 \\
\text { Width } & =\text { Char (2) }
\end{array}
$$

END

BEGIN Label IblRadius2

$$
\begin{array}{ll}
\text { Alignment } & =0 \\
\text { Autosize } & =0 \\
\text { BackColor } & =\text { QBColor (3) } \\
\text { Borderstyle } & =0 \\
\text { Caption } & =" 2 " \\
\text { DragMode } & =0 \\
\text { Enabled } & =-1 \\
\text { ForeColor } & =\text { QBColor (0) } \\
\text { Height } & =\text { Char (1) } \\
\text { Left } & =\text { Char (0) } \\
\text { MousePointer } & =0 \\
\text { TabIndex } & =24 \\
\text { Tag } & =" \\
\text { Top } & =\text { Char (1) } \\
\text { Visible } & =-1 \\
\text { Width } & =\text { Char (2) }
\end{array}
$$

END

BEGIN Frame fraRadial Distances

$$
\begin{array}{ll}
\text { BackColor } & =\overline{Q B C o l o r}(3) \\
\text { Caption } & =" 1 \\
\text { DragMode } & =0 \\
\text { Enabled } & =-1 \\
\text { ForeColor } & =Q B C o l o r(0) \\
\text { Height } & =\operatorname{Char}(5) \\
\text { Left } & =\operatorname{Char}(28) \\
\text { MousePointer } & =0 \\
\text { TabIndex } & =26 \\
\text { Tag } & =" 1
\end{array}
$$




\begin{tabular}{|c|c|c|c|}
\hline & $\begin{array}{l}\text { Top } \\
\text { Visib } \\
\text { Width } \\
\text { BEGIN }\end{array}$ & $\begin{array}{l}\quad=\text { Cha } \\
\text { le } \quad-1 \\
\quad=\text { Cha } \\
\text { TextBox txtR } \\
\text { BackColor } \\
\text { Borderstyle } \\
\text { DragMode } \\
\text { Enabled } \\
\text { ForeColor } \\
\text { Height } \\
\text { Left } \\
\text { MousePointer } \\
\text { Multiline } \\
\text { ScrollBars } \\
\text { TabIndex } \\
\text { Tabstop } \\
\text { Tag } \\
\text { Text } \\
\text { Top } \\
\text { Visible } \\
\text { Width }\end{array}$ & $\begin{array}{l}r(7) \\
r(12) \\
\text { adial Distancel } \\
=\text { QBColor (3) } \\
=0 \\
=0 \\
=-1 \\
=\text { QBColor }(0) \\
=\text { Char }(1) \\
=\text { Char }(0) \\
=0 \\
=-1 \\
=0 \\
=4 \\
=-1 \\
=" n \\
=" n \\
=\text { Char }(0) \\
=-1 \\
=\text { Char }(10)\end{array}$ \\
\hline & $\begin{array}{l}\text { END } \\
\text { BEGIN }\end{array}$ & $\begin{array}{l}\text { TextBox txtRe } \\
\text { BackColor } \\
\text { Borderstyle } \\
\text { DragMode } \\
\text { Enabled } \\
\text { ForeColor } \\
\text { Height } \\
\text { Left } \\
\text { MousePointer } \\
\text { Multiline } \\
\text { ScrollBars } \\
\text { TabIndex } \\
\text { Tabstop } \\
\text { Tag } \\
\text { Text } \\
\text { Top } \\
\text { Visible } \\
\text { Width }\end{array}$ & $\begin{array}{l}\text { adial Distance2 } \\
=Q B \overline{C o l o r}(3) \\
=0 \\
=0 \\
=-1 \\
=\text { QBColor }(0) \\
=\operatorname{Char}(1) \\
=\operatorname{Char}(0) \\
=0 \\
=-1 \\
=0 \\
=5 \\
=-1 \\
=" " \\
=" " \\
=\operatorname{Char}(1) \\
=-1 \\
=\operatorname{Char}(10)\end{array}$ \\
\hline & $\begin{array}{l}\text { END } \\
\text { BEGIN }\end{array}$ & $\begin{array}{l}\text { TextBox txtR } \\
\text { BackColor } \\
\text { Borderstyle } \\
\text { DragMode } \\
\text { Enabled } \\
\text { EoreColor } \\
\text { Height } \\
\text { Left } \\
\text { MousePointer } \\
\text { Multiline } \\
\text { ScrollBars } \\
\text { TabIndex } \\
\text { Tabstop } \\
\text { Tag } \\
\text { Text } \\
\text { Top } \\
\text { Visible } \\
\text { Width }\end{array}$ & $\begin{array}{l}\text { adial Distance3 } \\
=\text { QBColor (3) } \\
=0 \\
=0 \\
=-1 \\
=\text { QBColor }(0) \\
=\text { Char (1) } \\
=\operatorname{Char}(0) \\
=0 \\
=-1 \\
=0 \\
=6 \\
=-1 \\
=" " \\
=" " \\
=\text { Char (2) } \\
=-1 \\
=\text { Char (10) }\end{array}$ \\
\hline \multicolumn{4}{|l|}{ END } \\
\hline & $\begin{array}{l}\text { Eram } \\
\text { Back }\end{array}$ & $\begin{array}{l}\text { Trame1 } \\
\text { Lor }\end{array}$ & Lor \\
\hline
\end{tabular}




$$
\begin{aligned}
& \text { Caption } \quad=" \text { " } \\
& \text { Enabled } \\
& \text { ForeColor }=Q B C o l o r(0) \\
& \text { Height }=\operatorname{Char}(4) \\
& \text { Left }=\operatorname{Char}(41) \\
& \text { MousePointer }=0 \\
& \text { TabIndex }=28 \\
& \text { Tag = " } \\
& \text { Top }=\operatorname{Char}(7) \\
& \text { Visible } \quad=-1 \\
& \text { Width }=\operatorname{Char}(10) \\
& \text { BEGIN OptionButton optKilometers } \\
& \text { BackColor = oBColor (3) } \\
& \text { Caption } \quad=\text { "\&KM" } \\
& \text { DragMode } \quad=0 \\
& \text { Enabled } \quad=-1 \\
& \text { Forecolor }=\text { QBColor }(0) \\
& \text { Height }=\operatorname{Char}(1) \\
& \text { Left = Char (1) } \\
& \text { MousePointer }=0 \\
& \text { TabIndex }=7 \\
& \text { Tabstop }=0 \\
& \text { Tag }=\text { " " } \\
& \text { Top }=\operatorname{Char}(0) \\
& \text { Value } \quad=0 \\
& \text { Visible } \quad=-1 \\
& \text { BackColor = oBColor }(3) \\
& \text { Cancel }=0 \\
& \text { Caption = "Edit Regions" } \\
& \text { Default } \quad=0 \\
& \text { DragMode } \quad=0 \\
& \text { Enabled } \quad=-1 \\
& \text { Height }=\operatorname{Char}(3) \\
& \text { Ieft }=\operatorname{Char}(58) \\
& \text { MousePointer }=0 \\
& \text { TabIndex } \quad=11 \\
& \text { Tabstop } \quad=-1 \\
& \text { Tag }=" n \\
& \text { Top }=\operatorname{Char}(10) \\
& \text { Visible } \quad=-1 \\
& \text { Width } \quad=\operatorname{Char}(16)
\end{aligned}
$$

$\begin{array}{ll}\text { BackColor } & =\text { QBColor (3) } \\ \text { Caption } & =" \text { "MI" } \\ \text { DragMode } & =0 \\ \text { Enabled } & =-1 \\ \text { EoreColor } & =\text { QBColor (0) } \\ \text { Height } & =\text { Char }(1) \\ \text { Left } & =\text { Char (1) } \\ \text { MousePointer } & =0 \\ \text { TabIndex } & =8 \\ \text { TabStop } & =-1 \\ \text { Tag } & =" 1 \\ \text { Top } & =\text { Char (1) } \\ \text { Value } & =-1 \\ \text { Visible } & =-1 \\ \text { Width } & =\text { Char (7) }\end{array}$

END 
END

BEGIN Label Label4

Alignment $=2$

Autosize $=0$

BackColor = QBColor (3)

Borderstyle $=0$

Caption $=$ "Radius Number"

DragMode $\quad=0$

Enabled $\quad=-1$

Forecolor $=$ QBColor $(0)$

Height $=$ Char (2)

Left $=$ Char (22)

MousePointer $=0$

TabIndex $=21$

Tag = " "

Top $=\operatorname{Char}(5)$

Visible $\quad=-1$

END

Width $\quad=\operatorname{Char}(\sigma)$

BEGIN Label IblNumber of regions

Alignment $=\overline{1}$

Autosize $=0$

BackColor = QBColor (3)

Borderstyle $=1$

Caption $="$ "

DragMode $\quad=0$

Enabled $\quad=-1$

Forecolor $=$ QBColor $(0)$

Height $=$ Char (3)

Left $=$ Char(58)

MousePointer $=0$

TabIndex $=10$

Tag = " "

Top $=$ Char (7)

Visible $\quad=-1$

END

Width $=\operatorname{Char}(16)$

BEGIN VScrollBar vsbRadius Number

Attached $=0$

DragMode $\quad=0$

Enabled $\quad=-1$

Height $=\operatorname{Char}(3)$

IargeChange $=1$

Left $=\operatorname{Char}(39)$

$\operatorname{Max} \quad=48$

Min $=1$

MousePointer $=0$

SmallChange $=1$

TabIndex $\quad=27$

Tabstop $\quad=0$

Tag $=" n$

Top $=\operatorname{Char}(8)$

Value $\quad=1$

Visible $\quad=-1$

Width $\quad=\operatorname{Char}(1)$

END

BEGIN Label Label 6

Alignment $=2$

Autosize $=0$

BackColor $=$ QBColor (3)

Borderstyle $=0$

Caption $=$ "P\&opulation Multiplier"

Dragmode $\quad=0$ 


$$
\begin{array}{ll}
\text { Enabled } & =-1 \\
\text { ForeColor } & =\text { QBColor (0) } \\
\text { Height } & =\text { Char (2) } \\
\text { Ieft } & =\text { Char (4) } \\
\text { MousePointer } & =0 \\
\text { TabIndex } & =12 \\
\text { Tag } & =" " \\
\text { Top } & =\text { Char (5) } \\
\text { Visible } & =-1 \\
\text { Width } & =\operatorname{Char}(11)
\end{array}
$$

END

BEGIN TextBox txtPopulation multiplier

BackColor $=$ QBColor $(3)$

Borderstyle $=1$

DragMode $=0$

Enabled $\quad=-1$

Forecolor $=$ eBColor $(0)$

Height $=$ Char (3)

Left $=\operatorname{Char}(3)$

MousePointer $=0$

Multiline $=0$

ScrollBars $=0$

TabIndex $\quad=13$

Tabstop $\quad=-1$

Tag $=" n$

Text $\quad=" n$

Top $\quad=\operatorname{Char}(7)$

Visible $\quad=-1$

END

Width $\quad=\operatorname{Char}(12)$

BEGIN CommandButton cmdClose

$\begin{array}{ll}\text { BackColor } & =\text { QColor (3) } \\ \text { Cancel } & =-1 \\ \text { Caption } & =\text { "\&Close" } \\ \text { Default } & =0 \\ \text { DragMode } & =0 \\ \text { Enabled } & =-1 \\ \text { Height } & =\text { Char (3) } \\ \text { Left } & =\text { Char (17) } \\ \text { MousePointer } & =0 \\ \text { TabIndex } & =17 \\ \text { Tabstop } & =-1 \\ \text { Tag } & =\text { "Close" } \\ \text { Top } & =\text { Char (16) } \\ \text { Visible } & =-1 \\ \text { Width } & =\text { Char (11) }\end{array}$

END

END

OPTION EXPIICIT

'\$INCLUDE: 'secpop90.bi'

'This form allows the user to create, edit, and save different problems SUB cmdBrowse_click ()

'Call the function which allows the user to see which

'site files are available. For more info, see browse_site_file.

CALI browse_site_file

END SUB 
SUB cmaClose_Click ()

'This routine allows the user to close the problem

'window without saving.

DIM temp AS INTEGER, filenum AS INTEGER

DIM tempstring AS STRING

'Verify that all of the data entered by the user

is valid.

temp = verify_input (I)

'Remove the problem data form from the screen.

frmProblem_data.HIDE

END SUB

SUB cmdEdit_regions_Click ()

IF (number of radii < 2) THEN

edited."

MSGBOX "Ērror: At least two radii must be specified before regions are

EXIT SUB

ELSEIF (INSTR (frmProblem data.caption, " (Modified)") = 0) THEN

END IF

frmproblem_data. caption = frmProblem_data. caption + " (Modified)"

IF (number_econ_regions $=0$ ) OR (number_econ_regions > max_econ_regions) OR

(number_econ_regions <min_econ_regions) THEN

END IF

CALL default regions

$x$ position $=0$

Y_position $=0$

CAIL paint_region $(0,0)$

frmRegion. SHOW MODAI

END SUB

SUB cmdOpen_Click ()

'This routine allows the user to open a previously

'defined problem from the problem form. For more

' info see the open_problem procedure

CALL open_problem

END SUB

SUB cmaprint_Click ()

'This routine allows the user to print the data in

'a problem file. For more information

'see the print_problem procedure.

CALI print_problem

END SUB

SUB cmdSave_As_Click () 
'This routine allows the user to save a file by a

'new name. For more information, see the

'save_as_problem procedure.

CALL save_as_problem

END SUB

SUB cmaSave_Click ()

This routine allows the user to save data entered

'in a problem form. For more information,

'see the save_problem procedure.

CAIL save problem

END SUB

SUB Form_Unload (Cancel AS INTEGER)

'This routine allows the user to close the problem

'window without saving.

DIM temp AS INTEGER, filenum AS INTEGER

DIM tempstring AS STRING

'Verify that all of the data entered by the user

'is valid.

temp = verify input (1)

'Remove the problem data form from the screen.

frmProblem data. HIDE

LOAD frmProblem data

ExmProblem data. SHOW MODAL

END SUB

SUB optKilometers_GotFocus ()

'This event procedure allows the user to convert miles to kilometers if

'necessary, and it allows the user to specify which unit measures

'should be in.

DIM i AS INTEGER, j AS INTEGER, reply AS INTEGER, tempstring AS STRING, tempchar AS STRING

'If user had not previously specified kilometers, ask if a conversion

'is necessary.

IF (optkilometers.tag $<$ "Previously_True") THEN reply = MSGBOX ("Do you wish to convert any existing radial" + CHR\$ (13) + " distances from Miles to Kilometers?", MB_YESNOCANCEL + MB_DEEBUTTON2, "Conversion") IF reply = IDYES THEN

FOR $i=1$ TO number of radii

IF (radial distancē $(i)<.125$ ) AND (radial distance (i) $<0$ ) THEN MSGBOX "Error: unable to convert to kiIometers distances of less

than .125 miles."

optmiles. value $=$ TRUE

optmiles.SETFOCUS

EXIT SUB 
ELSEIF radial_distance $(i)>6213$ THEN

than 6213 miles."

MSGBOX "Errror: unable to convert to kilometers distances of more

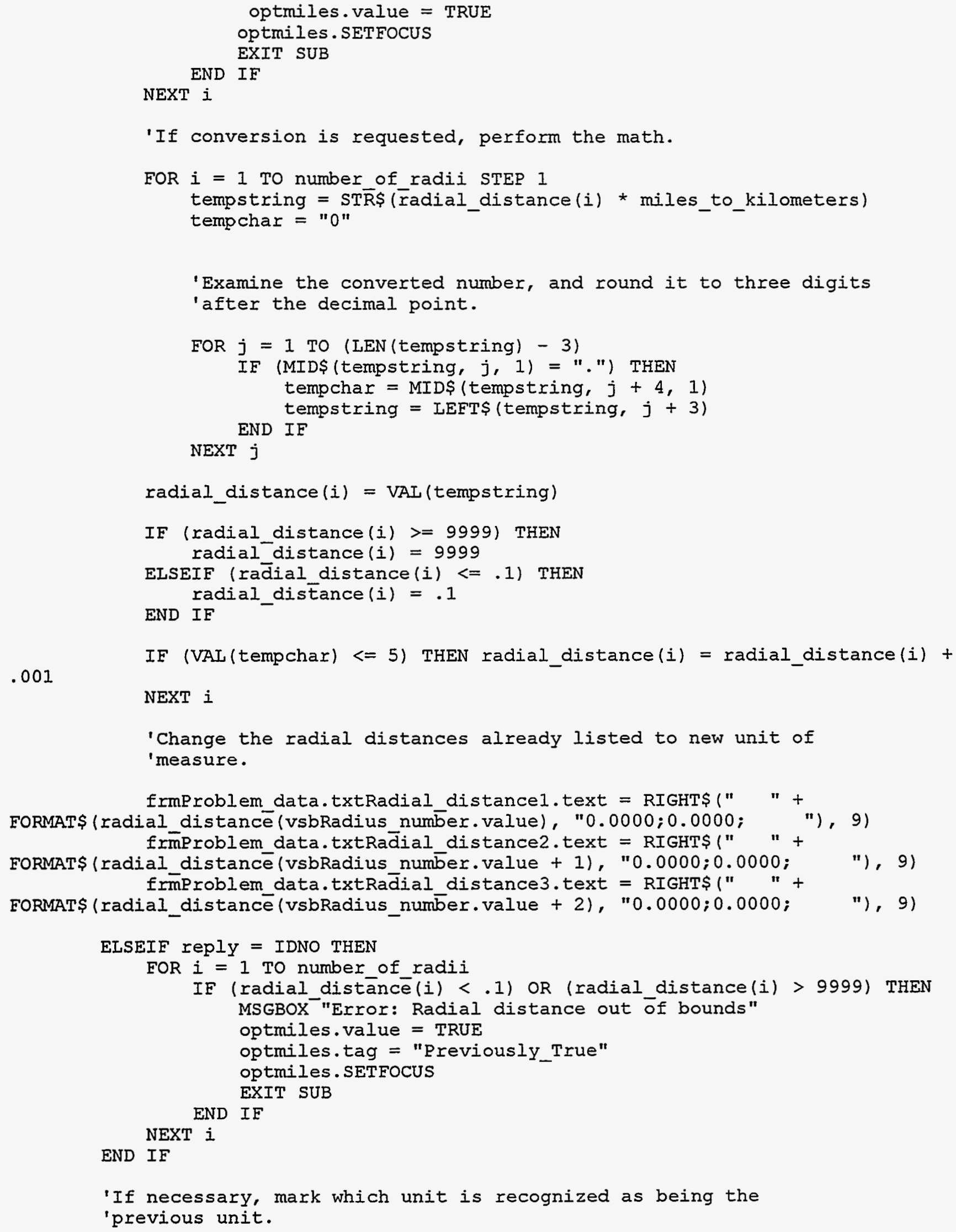


IF reply $<>$ IDCANCEL THEN

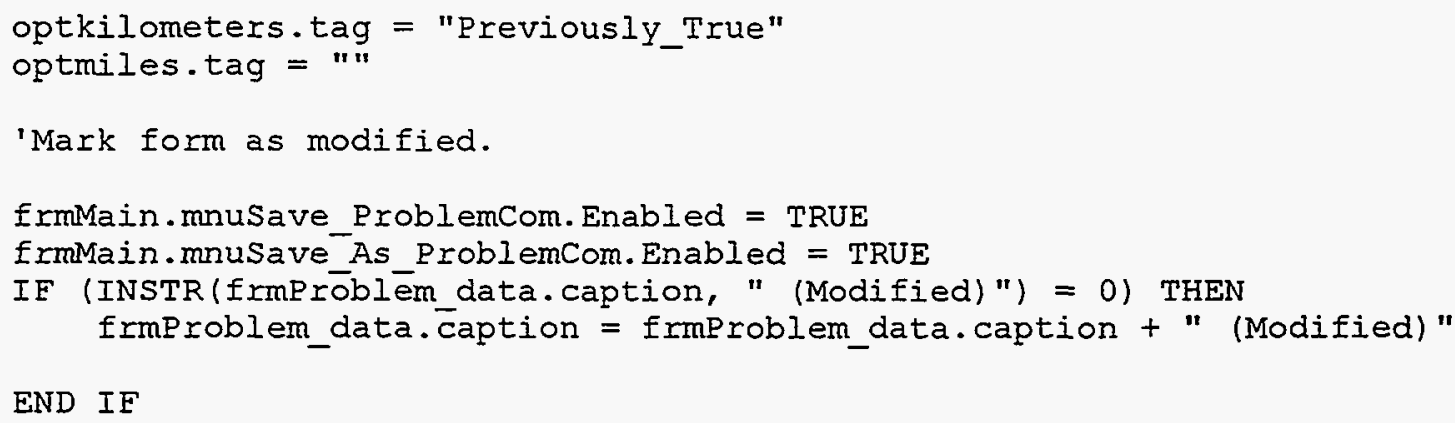

'Allow the user to convert kilometers to miles or enter data in miles.

DIM i AS INTEGER, j AS INTEGER, reply AS INTEGER, tempstring AS STRING, tempchar AS STRING

'If user has changed units then proceed.

IF (optmiles.tag $<>$ "Previously_True") THEN reply = MSGBOX ("Do you wish to convert any existing radial" + CHRS(13) + " distances from Kilometers to Miles?", MB_YESNOCANCEL + MB_DEFBUTTON2, "Conversion")

'If user wishes to convert numbers to new units, then proceed.

IF reply = IDYES THEN

FOR $i=1$ TO number_of radii

IF (radial distāncé $(i)<=.2$ ) AND (radial distance $(i) \ll 0$ ) THEN

.2 kilometers." MSGBOX "Error: unable to convert to miles distances of less than

optkilometers.value = TRUE optkilometers.SETEOCUS EXIT SUB

EISEIF radial distance (i) > 9999 THEN

9999 kilometers." MSGBOX "EFrror: unable to convert to miles distances of more than

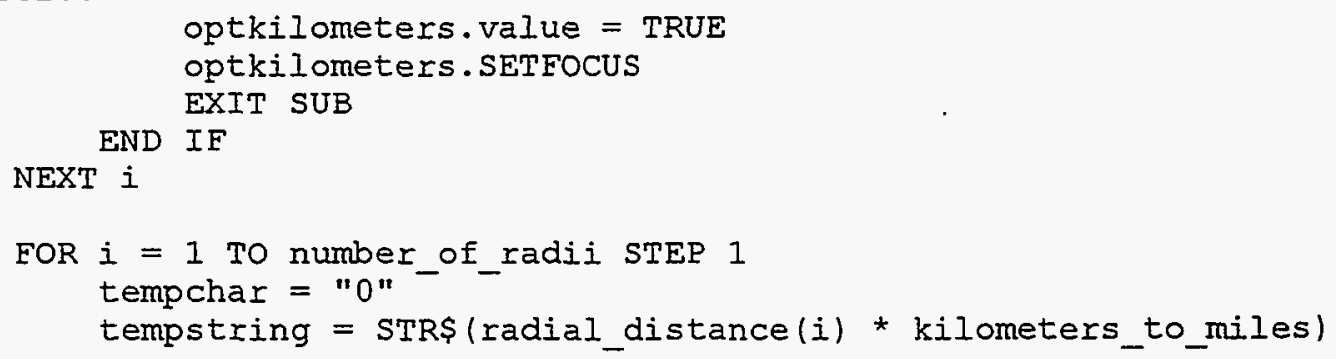


'Analyze converted number and round it to three decimal 'places.

FOR $j=1$ TO (LEN (tempstring) - 3)

IF (MIDS(tempstring, $j, 1)=" . "$ ) THEN tempchar $=$ MIDS (tempstring, $j+4,1$ ) END IF

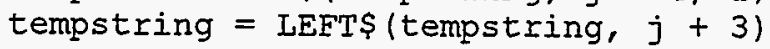

IF VAI(tempchar) > 4 THEN radial_distance(i) = radial_distance(i) +

NEXT $i$

'Write new radial distances to problem form.

frmProblem_data.txtRadial_distancel.text $=$ RIGHTS(" " + FORMAT\$ (radial_distance (vsbRadius number.value), "0.0000;0.0000;, "), 9)

frmproblem_data.txtRadial distance2.text = RIGHT\$(" " +

FORMAT\$ (radial_distancēe (vsbRadius_number.value + 1), "0.0000;0.0000; "), 9)

frmProblem_data.txtRadial distance 3 .text = RIGHT\$ $"$ " +

FORMAT\$ (radial_distancé (vsbRadius_number.value + 2), "0.0000;0.0000; "), 9)

ELSEIF reply = IDNO THEN

FOR $i=1$ TO number_of_radii

IF (radial distancé $(i)<.07$ ) OR (radial distance $(i)>6213$ ) THEN MSGBOX "Error: Radial distance out o $\overline{\mathrm{f}}$ bounds" optkilometers $\cdot$ value $=$ TRUE optkilometers.tag = "Previously_True" optkilometers.SETFOCUS EXIT SUB

NEXT $i$

END IF

END IF

'If user has not cancled, update which unit was previously used.

IF reply $<$ IDCANCEL THEN

optmiles.tag = "Previously_True"

optkilometers.tag $=" "$

'Mark form as modified.

frmMain.mnuSave ProblemCom. Enabled = TRUE

frmMain.mnuSave_As_ProblemCom.Enabled = TRUE

IF (INSTR (frmproblem_data.caption, " (Modified)") =0) THEN

frmProblem_data.Caption = frmProblem_data.caption + " (Modified)" END IF

ELSE

'If cancel is selected, leav values as they were.

optkilometers.value $=$ TRUE

optkilometers. SETFOCUS 
END IF

END IF

END SUB

SUB txtPopulation multiplier_KeyPress (KeyAscii AS INTEGER)

'This routine marks a problem form as modifed if keys are pressed in

the population multiplier field.

frmMain.mnuSave_ProblemCom. Enabled = TRUE

frmmain.mnusave_As_ProblemCom.Enabled = TRUE

IE (INSTR (frmProblem data.caption, " (Modified)") = 0) THEN

frmproblem_data.Caption = EmProblem_data.caption + " (Modified)"

END IF

END SUB

SUB txtProblem_Remarks_KeyPress (KeyAscii AS INTEGER)

'This routine marks a problem as modified if keys are pressed, and

'deals with the enter key being pressed.

'If enter key is pressed, display message.

IF KeyAscii = KEY_RETURN THEN

'Erase keystroke.

KeyAscii $=0$

MSGBOX "Sorry, no hard returns allowed." + CHRS(13) + "Words will wrap automatically.", MB OK, "Error"

'Mark problem form as modified.

ELSE

frmMain.mnusave ProblemCom. Enabled = TRUE

ErmMain.mnusave As ProblemCom. Enabled = TRUE

IF (INSTR (frmproblem data.caption, " (Modified)") = 0) THEN

END IF frmProblem_data.caption = frmproblem_data.caption + " (Modified)"

END IF

END SUB

SUB txtProblem_Title_KeyPress (KeyAscii AS INTEGER)

This routine marks the problem form as modified if a key is pressed

'within the title field. It also deals with the user pressing enter.

'If the user presses the enter key, display warning message.

IE KeyAscii = KEY_RETURN THEN

'Erase keystroke.

KeyAscii $=0$ 
MSGBOX "Sorry, no hard returns allowed." + CHR\$(13) + "Words will wrap automatically.", MB_OK, "Error"

ELSE

'Mark problem form as modified.

frmMain.mnuSave ProblemCom. Enabled = TRUE

frmMain.mnusave_As_ProblemCom. Enabled = TRUE

IF (INSTR (frmProblem_data.caption, " (Modified)") =0) THEN

END IF

ErmProblem_data. caption = frmProblem_data.caption + " (Modified)"

END IF

END SUB

SUB txtRadial_Distancel_KeyDown (KeyCode AS INTEGER, Shift AS INTEGER)

'This routine allows the user to scroll up and down on the list of

'radial distances.

'If up arrow on keyboard has been pressed then scroll up one entry.

IF KeyCode $=$ KEY_UP THEN

IE vsbRadius number.value $>1$ THEN

CALL txt $\bar{R}$ adial Distancel LostFocus

ELSE

vsbRadius_numbēr.value =-vsbRadius_number.value -1

END IF

CALI txtRadial_Distancel_LostFocus

'If down arrow has been pressed, scroll down one position.

ELSEIE KeyCode = KEY_DOWN THEN

txtRadial_distance2.SETEOCUS

END IF

END SUB

SUB txtRadial_Distancel_KeyPress (KeyAscii AS INTEGER)

'This routine marks the problem form as modified if a key is pressed

'in the distancel field.

frmMain.mnusave ProblemCom. Enabled = TRUE

frmMain.mnuSave_As_Problemcom. Enabled = TRUE

IF (INSTR(frmProblem data.caption, " (Modified)") = 0) THEN

END IF

frmProblem_data. caption = frmProblem_data.caption + " (Modified)"

IF KeyAscii = KEY RETURN THEN

txtRadial distance2. SETFOCUS

END IF

END SUB

SUB txtRadial_Distancel_LostFocus () 
'This routine sorts the entries in the radial distance list so that they 'appear in ascending order, and updates the list

DIM temp AS DOUBLE, units AS INTEGER

DIM zero found AS INTEGER, radius zero AS INTEGER

DIM radiüs edited AS INTEGER, i AS INTEGER, $j$ AS INTEGER

DIM tempchār AS STRING, tempstring AS STRING

'Record the value of the newly edited radius into the radial

'distance array.

radius_edited $=$ VAL (IblRadiusl.caption)

radial_distance (radius_edited) = VAI (txtRadial_distancel.text)

'Examine the Raddius, and round it to three digits after the decimal.

tempstring = STRS(radial_distance (radius_edited))

FOR $j=1$ TO (LEN (tempstring) - 3)

IF (MIDS (tempstring, $j, 1$ ) = ".") THEN

tempchar $=\operatorname{MIDS}$ (tempstring, $j+4,1$ )

END IF

tempstring $=$ LEFTS(tempstring, $j+3$ )

NEXT J

radial_distance (radius_edited) = VAL (tempstring)

'Check to see if the radius is within acceptable limits for maccs

'compatability.

IF (radial_distance (radius_edited) $=0$ ) THEN

radius zero $=\mathrm{TRUE}$

ELSEIF TYPËOF screen.activecontrol Is OptionButton THEN

ELSEIF (optmiles.value = TRUE) THEN

IF (radial distance (radius edited) > 6213) THEN

Miles."

MSGBOX "Error: this prögram can't process radii greater than 6213

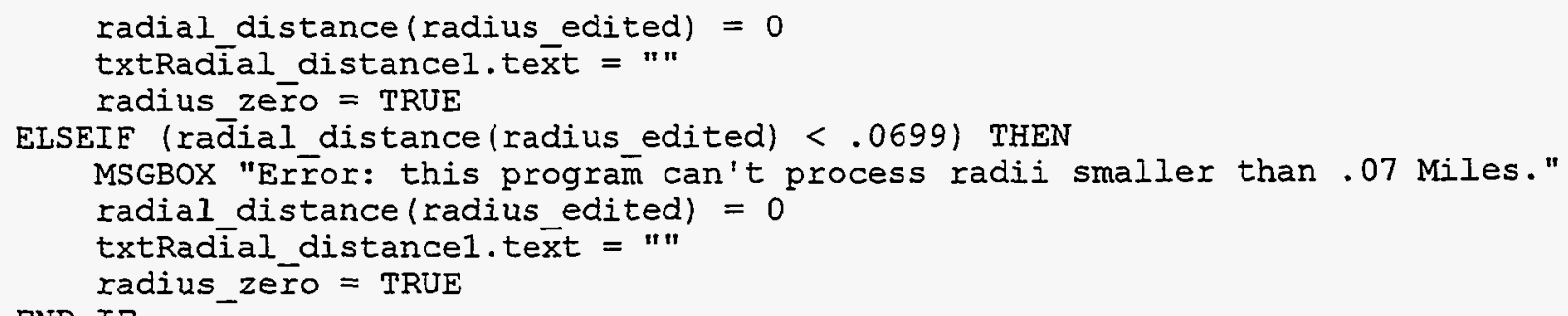

'If radius is not equal to zero, check to see if it is equal to

'any previous radial distance. If it is, set it equal to zero. 
IF NOT radius zero THEN

FOR $i=1$ TO radius edited -1 STEP 1

IF radial_distañce (radius_edited) = radial_distance(i) THEN radia $\bar{l}$ distance (radius edited) $=0$

END IF radius zero $=$ TRUE EXIT FOR

NEXT i

END IE

'If distance is still not equal to zero, check to see if it is equal

'to any following radial distance. If it is, set it equal to zero.

IF NOT radius zero THEN

FOR $i=$ rădius edited +1 TO max number of radii STEP 1

IF radial distance (radius editted) $=$ radial distance (i) THEN radiaI distance $(i)=\overline{0}$

radius zero $=$ TRUE

END IF EXIT FŌR

END IF

NEXT i

'Use standard sorting algorithm to put radii in ascending order.

FOR $i=1$ TO max number of radii - I STEP 1

EOR $j=i+\overline{1}$ TO max number of radii STEP 1 IF (radial_distance $(j)<\overline{0}$ ) THEN

= 0)) THEN

IF ( $(r \bar{a} d i a l$ distance $(i)>$ radial_distance $(j))$ OR (radial_distance (i)

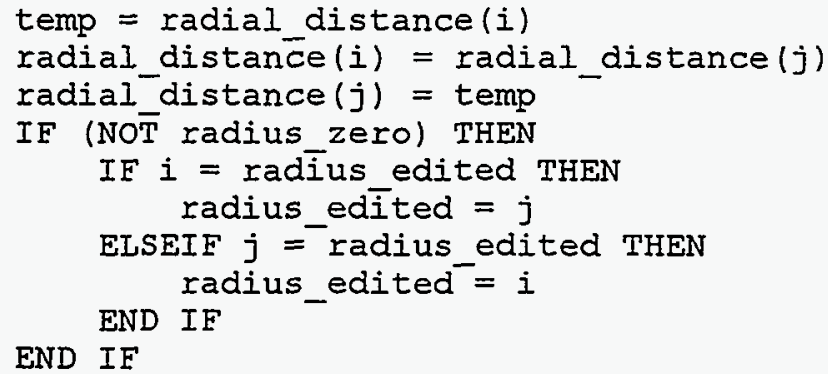

'Determine where list of radii ends.

FOR $i=1$ TO max number_of_radii STEP 1

IF radial_distance $(\bar{i}) \equiv 0$ THEN

END IF EXIT FOR

NEXT $i$

'Determine number of radii.

number_of_radii $=i-1$

IF radius edited $>$ max number of radii - 2 THEN

END IF radius_edited $=\max \bar{x}_{-}$number $\bar{x}_{-}$of $\bar{f}_{\text {radii }}-2$

'Update user display (problem form). 
lblRadiusl. caption = RIGHTS (STRS (radius_edited), 2)

txtRadial distancel.text = RIGHT\$(" "

FORMATS (radiaI distance (radius edited), "0.0000;0.0000;

lblRadius2. caption = RIGHTŞ (STRS (radius_edited +1), 2)

txtRadial distance2.text = RIGHTS(" "+ FORMATS(radial_distance(radius_edited

+ I), "0.0000;0.0000; "), 9)

lblRadius3. caption = RIGHTS (STRȘ (radius_edited +2$), 2$ )

txtRadial_distance3.text = RIGHT\$(" "- FORMAT\$(radial_distance(radius_edited

$+2), " 0.0000 \overline{;} 0.0000$; "), 9)

vsbRadius_number.value = radius_edited

IF (VAI (frmproblem_data.IblNumber_of_regions.tag) $<$ number_of_radii) THEN

CAII default régions

CALL paint region $(0,0)$

END IF

END SUB

SUB txtRadial_Distance2_KeyDown (KeyCode AS INTEGER, Shift AS INTEGER)

'If an arrow key is pressed, then call the distance 1 function

'to scroll radial distances up or down.

IF KeyCode $=$ KEY UP THEN

txtRadial distancel. SETFOCUS

ELSEIF KeyCode = KEY DOWN THEN

END IF

txtRadial distance 3 . sETEOCUS

END SUB

SUB txtRadial Distance2 KeyPress (KeyAscii AS INTEGER)

'This routine marks the problem form as modified, and goes to the next

'radii if enter is pressed.

frmmain.mnusave ProblemCom. Enabled = TRUE

ErmMain.mnusave_As ProblemCom. Enabled = TRUE

IF (INSTR (frmproblem data.caption, " (Modified)") =0) THEN

frmProblem data. Caption = Emproblem data.caption + " (Modified)"

END IF

IF KeyAscii = KEY RETURN THEN

KeyAscii = 0

txtRadial distance 3. SETFOCUS

END IE

END SUB

SUB txtRadial_Distance2_LostFocus ()

'See radial_Distancel_lostfocus for documentation of this routine.

DIM temp AS DOUBIE

DIM zero found AS INTEGER, radius zero AS INTEGER

DIM radiūs edited AS INTEGER, i A $\bar{S}$ INTEGER, $j$ AS INTEGER

DIM tempst̄ing AS STRING, tempchar AS STRING

radius edited $=$ VAI (lblRadius 2 . caption)

radiaI_distance (radius_edited) = VAI (txtRadial_distance2.text) 


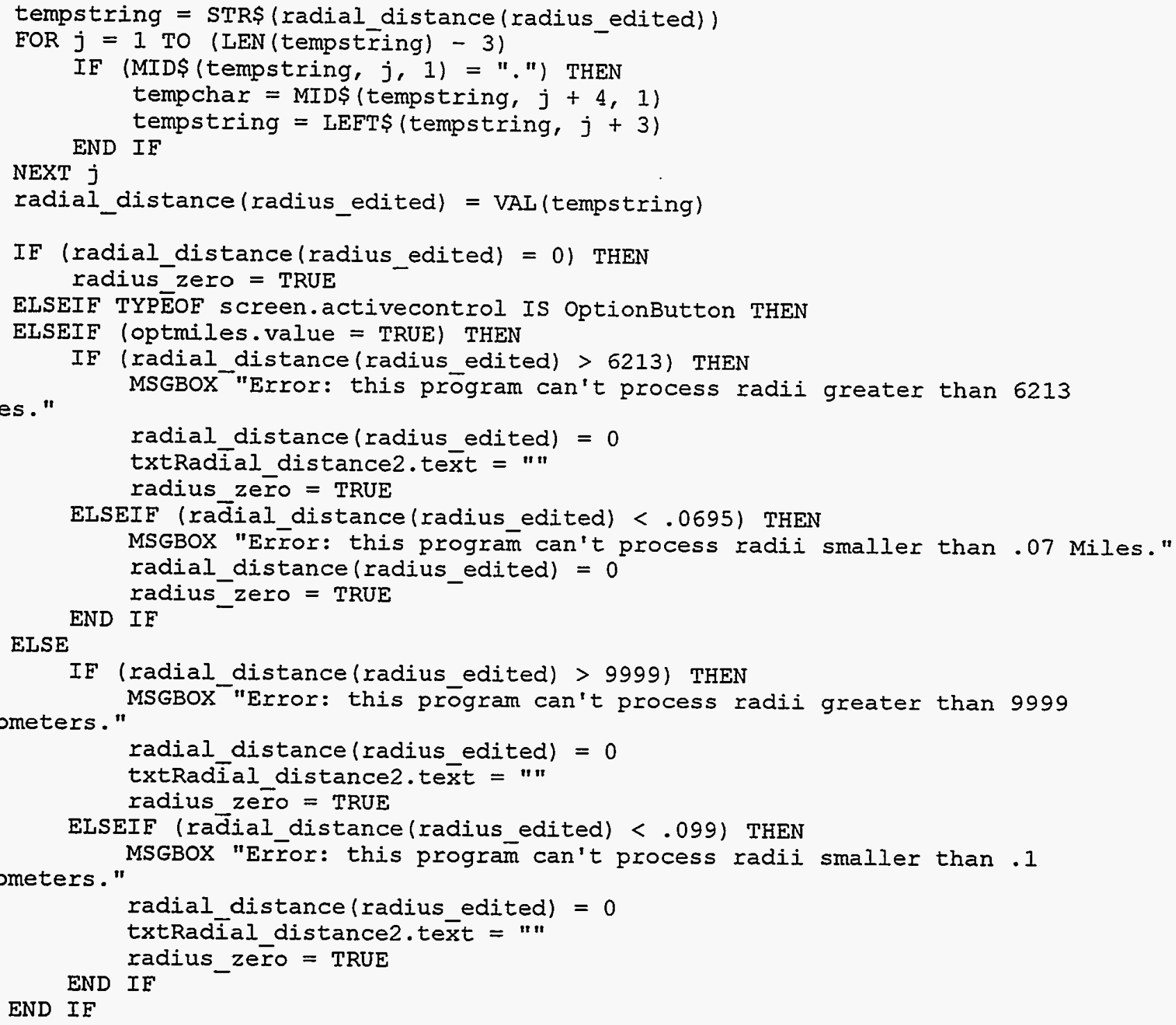


IF (radial_distance $(j)<0$ ) THEN

IF (rādial_distance $(i)>$ radial_distance $(j)$ ) OR (radial_distance(i)

(0)) THEN

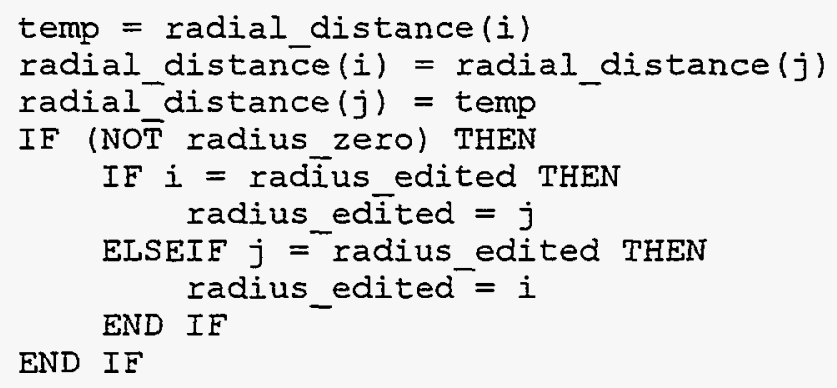

END SUB

SUB txtRadial_Distance3_KeyDown (KeyCode AS INTEGER, shift AS INTEGER)

'See txtRadial_distancel_keydown for documentation of this routine.

IF KeyCode $=$ KEY UP THEN

txtRadial distance2.SETFOCUS

EISEIF Keycode $=$ KEY_DOWN THEN 
IF VsbRadius_number.value < max_number_of_radii - 2 THEN CALI txt $\bar{R}$ adial Distance 3 Los̄tFocus ELSE vsbRadius number.value $=$-vsbRadius number. value +1

END IF

CALL txtRadial_Distance3_LostFocus

END IE

END SUB

SUB txtRadial_Distance3_KeyPress (KeyAscii AS INTEGER)

'See frmtxtRadial_distancel_keypress for documentation of this function.

frmMain.mnuSave_ProblemCom.Enabled = TRUE

frmMain.musave_As_ProblemCom.Enabled = TRUE

IF (INSTR(EmProblem_data.caption, " (Modified)") =0) THEN

END IF

frmproblem_data. caption = frmProblem_data.caption + " (Modified)"

IF KeyAsCii = KEY_RETURN THEN

CAIL txtRadiā Distance 3 LostFocus

IF vsbRadius_number.value $<$ max number of radii - 2 THEN END IE vsbRadius number.value = vsb̄Radius_number.value +1

END IF

END SUB

SUB txtRadial_Distance 3 _LostFocus ()

'See txtRadial_distancel_lostfocus for documentation of this function.

DIM temp AS DOUBLE

DIM zero found AS INTEGER, radius zero AS INTEGER

DIM radiūs_edited AS INTEGER, i AS INTEGER, j AS INTEGER

DIM tempstr̄ing AS STRING, tempchar AS STRING

radius edited $=\operatorname{VAL}$ ( 1 blRadius 3 . caption)

radial_distance (radius_edited) = VAI (txtRadial_distance3.text)

tempstring = STR\$ (radial_distance (radius_edited))

FOR $j=1$ TO (IEN (tempstring) - 3)

IF (MIDS(tempstring, $j, 1$ ) = ". ") THEN

tempchar $=$ MID\$ (tempstring, $j+4,1$ )

tempstring $=$ LEFT\$(tempstring, $j+3$ )

END IF

NEXT j

radial_distance (radius_edited) = VAI (tempstring)

IE (radial distance (radius edited) =0) THEN

radius zero $=$ TRUE

ELSEIF TYPËOF screen.activecontrol IS OptionButton THEN

ELSEIF (optmiles.value = TRUE) THEN

IF (radial distance (radius edited) > 6213) THEN

Miles."

MSGBOX "Error: this prögram can't process radii greater than 6213

radial_distance (radius_edited) $=0$

radius zero $=$ TRUE

ELSEIF (rāial_distance (radius_edited) < .0695) THEN

MSGBOX "Err̆or: this program can't process radii smaller than .07 Miles."

radial_distance (radius_edited) $=0$ 


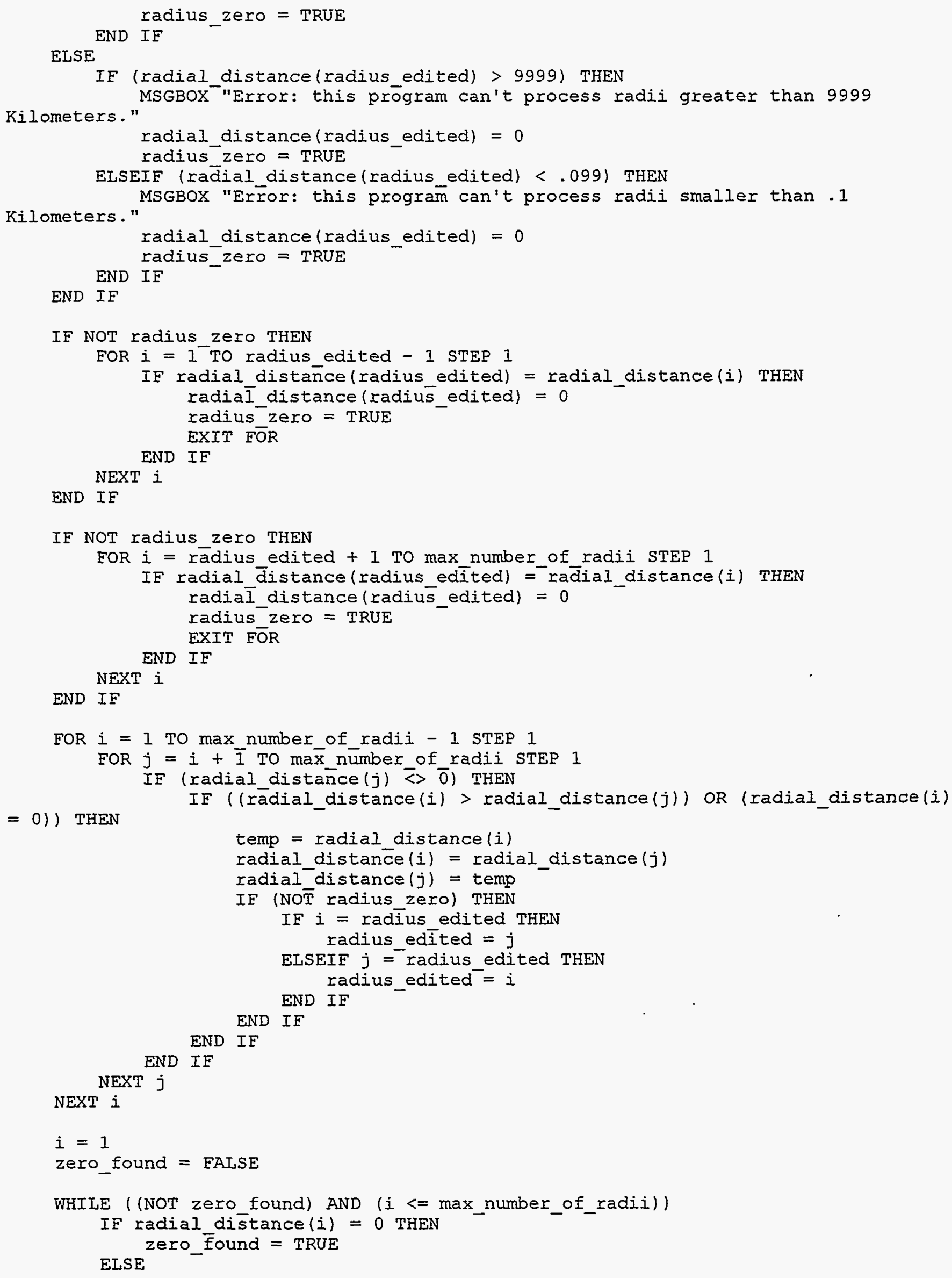




$$
i=i+1
$$

WEND

END IF

number_of_radi $i=i-1$

IF radius_edited $<3$ THEN

END IF radius_edited $=3$

IF number of radii $<=\max$ number of radii THEN IblRadius 1 . caption $=\overline{R I G H T} \$(\bar{S} T R \bar{S}$ (radius edited -2$), 2$ )
txtRadial distancel. text $=$ RIGHTS(" " ${ }_{+}^{-}$

FORMATS (radial_distance (radius_edited - 2), "0.0000;0.0000; IblRadius2. caption = RI GHTS (STRS (radius_edited - 1), 2) txtRadial distance 2 . text $=$ RIGHT $\left("{ }^{-}+\right.$

FORMAT\$ (radial_distance (radius edited - 1 ), "0.0000;0.0000; lblRadius3. caption = RI GHT\$ (STRS (radius_edited), 2) txtRadial_distance 3 . text = RIGHTS $("$ " +

FORMAT\$ (radial_distance (radius_edited), "0.0000;0.0000; "), 9) ELSE vsbRadius_number.value = radius_edited -2 lblRadius 1. caption = RIGHT\$ (STRS (radius_edited - 3), 2)
txtRadial_distancel.text = RIGHT (" "-

FORMAT (radial distance (radius_edited - 3), $0.0000 ; 0.0000$; lblRadius2. caption $=$ RIGHTS (STRS (radius_edited -2$), 2$ ) txtRadial distance 2 . text $=$ RIGHTS $\left("\right.$ " ${ }^{-}+$

FORMAT (radial_distance (radius edited - 2), "0.0000;0.0000; lblRadius 3 .caption = RT GHTS (STRS(radius_edited - 1), 2) txtRadial distance 3 . text $=$ RIGHT $("$ " +

FORMAT\$ (radial_distance (radius_edited - 1), "0.0000;0.0000;

END IF vsbRadius_number.value $=$ radius_edited -3

IF (VAL (frmProblem_data.lblNumber_of_regions.tag) <> number_of_radii) THEN CALI default_régions

END IF CALL paint_rēgion $(0,0)$

\section{END SUB}

SUB txtSite_File_Name_KeyPress (KeyAscii AS INTEGER)

'This routine marks the problem form as modified, and if the 'enter key is pressed, it attempts to open the site file and read in 'the appropriate site data.

DIM site_file_name AS STRING

frmMain.mnuSave ProblemCom. Enabled = TRUE

ErmMain.mnuSave_As_ProblemCom.Enabled = TRUE

IF (INSTR(frmProblem_data.caption, " (Modified)") =0) THEN END IF frmProblem_data.čption = frmProblem_data.caption + " (Modified)"

IF KeYASCII $=$ KEY_RETURN THEN KeyAscii $=0$ site file name = txtSite file Name.text

END IF 'open_sitēfrom_problem (site_file_name) 


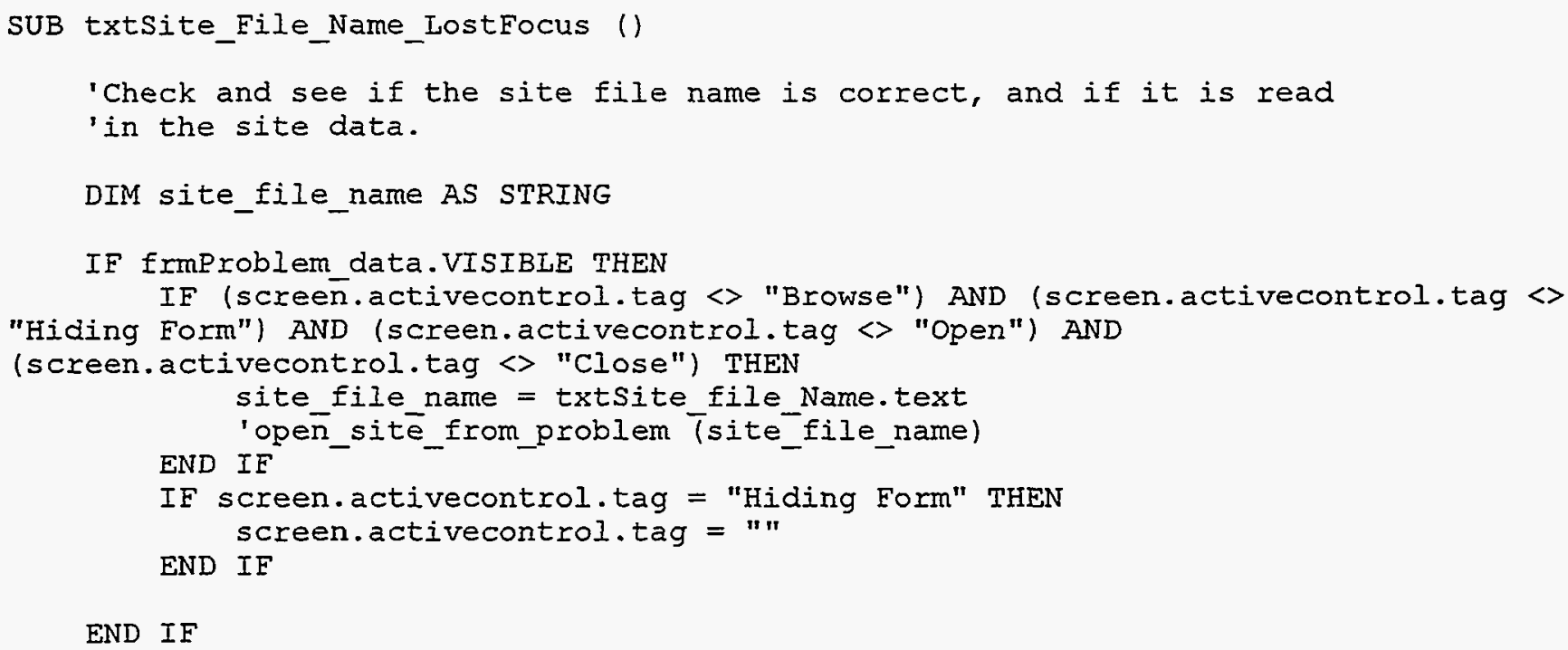

END IF

END SUB

SUB vsbRadius_Number_Change ()

'This function updates the problem form when a user has changed it.

'It simply puts the correct values for radii back in the correct places.

'It is called whenever the scroll bar on the radii box is moved.

DIM radius number(3) AS STRING

DIM i AS IÑTEGER

IF (vsbRadius number.value $<=\max$ number of radii - 2) THEN radius number (1) = RIGHTS(STR $\overline{\$}$ (vsbRadius_number.value), 2) radius_number (2) = RIGHT\$ (STRS (vsbRadius_number.value + 1), 2) radius_number (3) = RIGHTS (STRS (vsbRadius_number.value +2 ), 2 )

IblRadius 1 . caption = radius number $(1)$ txtRadial distancel.text = RIGHT\$(" "+ FORMATŞ (radial_dis̄tance (vsbRadius_number.value), "0.0000;0.0000; "), 9)

IblRadius 2 . caption = radius number $(2)$ txtRadial distance 2 . text = RIGHT\$(" " +

FORMAT\$(radial_distance (vsbRadius_number.value + 1), "0.0000;0.0000; "), 9)

IblRadius 3 . caption $=$ radius number $(3)$

txtRadial distance 3 .text = RIGHT\$(" "+

FORMAT\$(radial_distance (vsbRadius_number.value + 2), "0.0000;0.0000; "), 9)

END IF

END SUB 


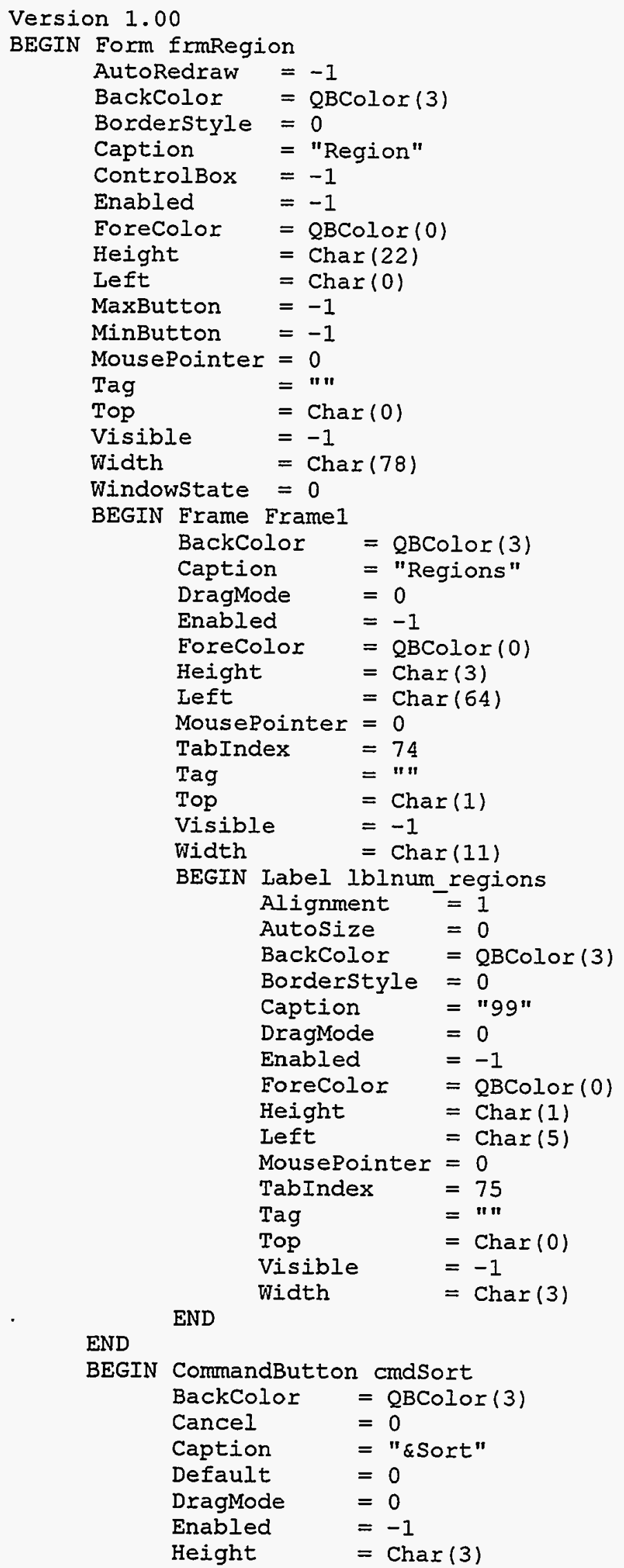




\begin{tabular}{|c|c|c|}
\hline & Left & $=\operatorname{Char}(64)$ \\
\hline & MousePointer $=$ & $=0$ \\
\hline & TabIndex $\quad=$ & $=4$ \\
\hline & Tabstop & $=-1$ \\
\hline & $\mathrm{Tag}$ & $=" n$ \\
\hline & Top & $=\operatorname{Char}(10)$ \\
\hline & Visible & $=-1$ \\
\hline & Width & $=\operatorname{Char}(11)$ \\
\hline END & & \\
\hline BEGIN & CommandButton & cmdDefault \\
\hline & BackColor & $=$ QBColor $(3)$ \\
\hline & Cancel & $=0$ \\
\hline & Caption & $=$ "De\&fault" \\
\hline & Default & $=0$ \\
\hline & DragMode & $=0$ \\
\hline & Enabled & $=-1$ \\
\hline & Height & $=\operatorname{Char}(3)$ \\
\hline & Left & $=\operatorname{Char}(64)$ \\
\hline & MousePointer = & $=0$ \\
\hline & TabIndex & $=5$ \\
\hline & Tabstop & $=-1$ \\
\hline & Tag & $=n n$ \\
\hline & Top & $=\operatorname{char}(13)$ \\
\hline & Visible & $=-1$ \\
\hline & width & $=\operatorname{Char}(11)$ \\
\hline END & & \\
\hline BEGIN & CommandButton & o cmaclear \\
\hline & BackColor & $=$ QBColor $(3)$ \\
\hline & Cancel & $=0$ \\
\hline & Caption & $=$ "Clea\&r" \\
\hline & Default & $=0$ \\
\hline & DragMode & $=0$ \\
\hline & Enabled & $=-1$ \\
\hline & Height & $=\operatorname{Char}(3)$ \\
\hline & Left & $=\operatorname{Char}(64)$ \\
\hline & MousePointer & $=0$ \\
\hline & TabIndex & $=6$ \\
\hline & Tabstop & $=-1$ \\
\hline & Tag & $=" 1$ \\
\hline & Top & $=\operatorname{Char}(16)$ \\
\hline & Visible & $=-1$ \\
\hline & Width & $=\operatorname{Char}(11)$ \\
\hline END & & \\
\hline BEGIN & CommandButton & n cmaclose \\
\hline & BackColor & $=Q B C o l o r(3)$ \\
\hline & Cancel & $=0$ \\
\hline & Caption & $=$ "\&Close" \\
\hline & Default & $=0$ \\
\hline & DragMode & $=0$ \\
\hline & Enabled & $=-1$ \\
\hline & Height & $=\operatorname{Char}(3)$ \\
\hline & Left & $=\operatorname{Char}(64)$ \\
\hline & MousePointer & $=0$ \\
\hline & TabIndex & $=7$ \\
\hline & Tabstop & $=-1$ \\
\hline & $\operatorname{Tag}$ & $=n n$ \\
\hline & Top & $=\operatorname{Char}(19)$ \\
\hline & Visible & $=-1$ \\
\hline & Width & $=\operatorname{Char}(11)$ \\
\hline END & & \\
\hline BEGIN & Erame Erame2 & \\
\hline & $\begin{array}{l}\text { BackColor } \\
\text { Caption }\end{array}$ & $\begin{array}{l}=\text { QBColor (3) } \\
=\text { "Radii" }\end{array}$ \\
\hline
\end{tabular}




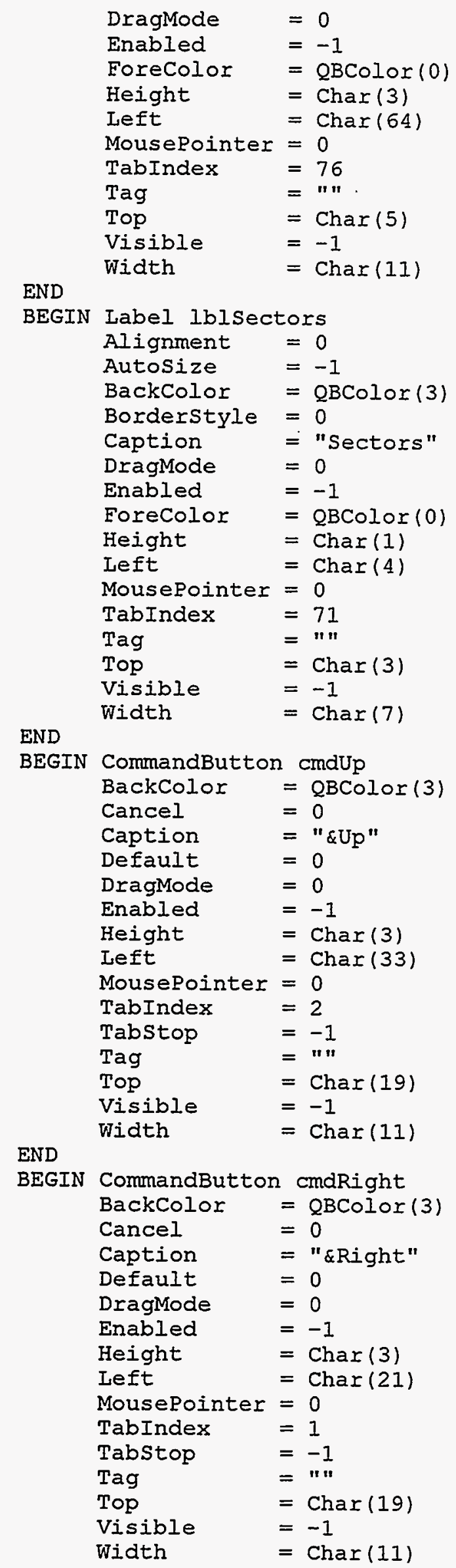


END

BEGIN CommandButton cmdDown

$\begin{array}{ll}\text { BackColor } & =\text { QBColor (3) } \\ \text { Cancel } & =0 \\ \text { Caption } & =" \text { \&Down" } \\ \text { Default } & =0 \\ \text { DragMode } & =0 \\ \text { Enabled } & =-1 \\ \text { Height } & =\text { Char (3) } \\ \text { Left } & =\text { Char (45) } \\ \text { MousePointer } & =0 \\ \text { TabIndex } & =3 \\ \text { TabStop } & =-1 \\ \text { Tag } & =" 1 \\ \text { Top } & =\text { Char (19) } \\ \text { Visible } & =-1 \\ \text { Width } & =\text { Char (11) }\end{array}$

END

$=\operatorname{Char}(11)$

BEGIN CommandButton cmaleft

$\begin{array}{ll}\text { BackColor } & =\text { QBColor (3) } \\ \text { Cancel } & =0 \\ \text { Caption } & =\text { "\&Left" } \\ \text { Default } & =0 \\ \text { DragMode } & =0 \\ \text { Enabled } & =-1 \\ \text { Height } & =\text { Char (3) } \\ \text { Left } & =\text { Char (9) } \\ \text { MousePointer } & =0 \\ \text { TabIndex } & =0 \\ \text { Tabstop } & =-1 \\ \text { Tag } & =" 1 \\ \text { Top } & =C h a r(19) \\ \text { Visible } & =-1 \\ \text { Width } & =\text { Char (11) }\end{array}$

END

Width $\quad=\operatorname{Char}(11)$

BEGIN Label lblRadial Distances

$\begin{array}{ll}\text { Alignment } & =\overline{0} \\ \text { AutoSize } & =-1 \\ \text { BackColor } & =0 B \text { Color (3) } \\ \text { Borderstyle } & =0 \\ \text { Caption } & =" \text { Radial Dist } \\ \text { DragMode } & =0 \\ \text { Enabled } & =-1 \\ \text { ForeColor } & =0 \text { Color }(0) \\ \text { Height } & =\text { Char }(1) \\ \text { Left } & =\text { Char }(24) \\ \text { MousePointer } & =0 \\ \text { TabIndex } & =72 \\ \text { Tag } & =" * \\ \text { Top } & =\text { Char }(1) \\ \text { Visible } & =-1 \\ \text { Width } & =\text { Char (20) }\end{array}$

END

BEGIN Label IblUnits

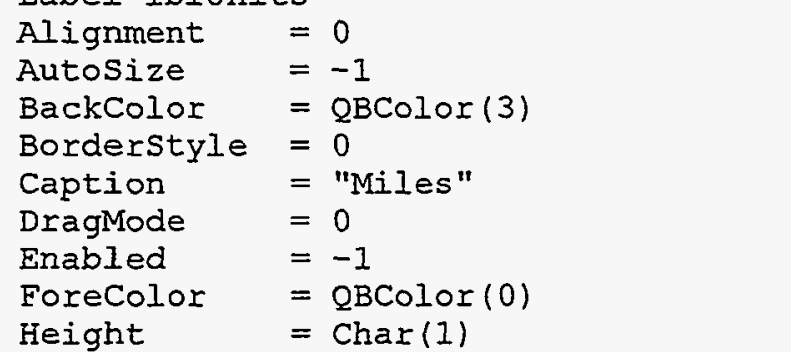




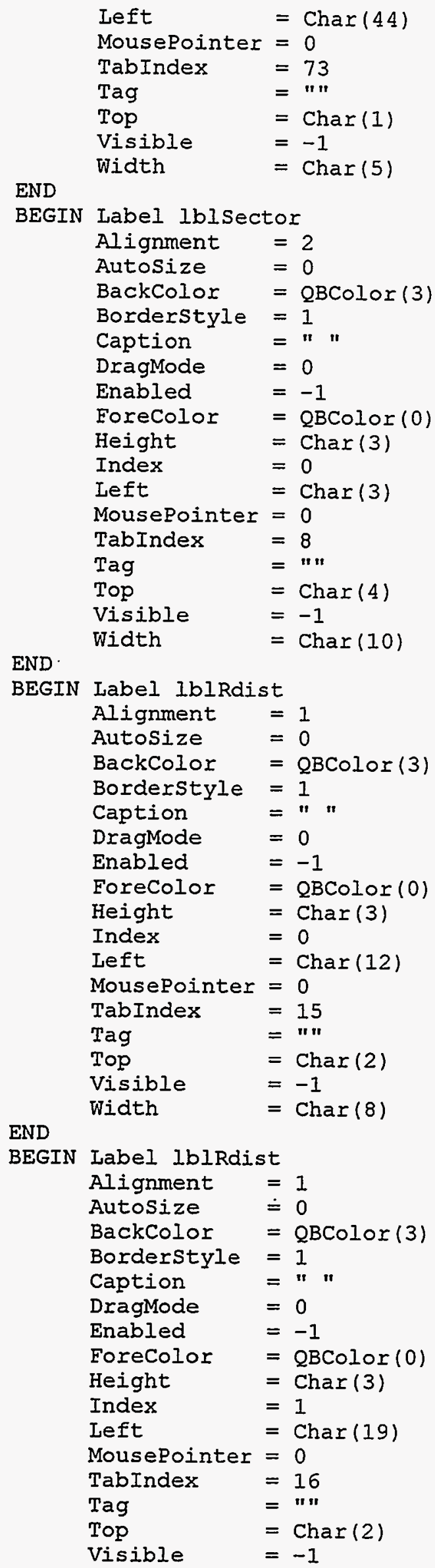




\begin{tabular}{|c|c|c|}
\hline & width & $=\operatorname{Char}(8)$ \\
\hline \multicolumn{3}{|l|}{ END } \\
\hline \multirow[t]{18}{*}{ BEGIN } & \multicolumn{2}{|l|}{ Label IblRdist } \\
\hline & Alignment & $=1$ \\
\hline & Autosize & $=0$ \\
\hline & BackColor & $=Q B C o l o r(3)$ \\
\hline & Borderstyle $=$ & $=1$ \\
\hline & Caption & $=" n$ \\
\hline & DragMode & $=0$ \\
\hline & Enabled & $=-1$ \\
\hline & Eorecolor & $=\mathrm{QBColor}(0)$ \\
\hline & Height & $=\operatorname{Char}(3)$ \\
\hline & Index & $=2$ \\
\hline & Left & $=\operatorname{Char}(26)$ \\
\hline & MousePointer $=$ & $=0$ \\
\hline & TabIndex & $\begin{array}{l}=17 \\
=n "\end{array}$ \\
\hline & Tag & $=" 1$ \\
\hline & Top & $=\operatorname{Char}(2)$ \\
\hline & Visible & $=-1$ \\
\hline & width & $=\operatorname{Char}(8)$ \\
\hline \multicolumn{3}{|l|}{ END } \\
\hline \multirow[t]{18}{*}{ BEGIN } & \multicolumn{2}{|l|}{ Label IblRdist } \\
\hline & Alignment & $=1$ \\
\hline & Autósize & $=0$ \\
\hline & BackColor & $=\mathrm{QBColor}(3)$ \\
\hline & Borderstyle & $=1$ \\
\hline & Caption & $=" n$ \\
\hline & DragMode & $=0$ \\
\hline & Enabled & $=-I$ \\
\hline & ForeColor & $=$ QBColor $(0)$ \\
\hline & Height & $=$ Char $(3)$ \\
\hline & Index & $=3$ \\
\hline & Left & $=\operatorname{Char}(33)$ \\
\hline & MousePointer & $=0$ \\
\hline & TabIndex & $=18$ \\
\hline & Tag & $=" n$ \\
\hline & Top & $=\operatorname{Char}(2)$ \\
\hline & Visible & $=-1$ \\
\hline & Width & $=\operatorname{Char}(8)$ \\
\hline \multicolumn{3}{|l|}{ END } \\
\hline \multirow[t]{18}{*}{ BEGIN } & \multicolumn{2}{|l|}{ Label lbIRdist } \\
\hline & Alignment & $=1$ \\
\hline & Autosize & $=0$ \\
\hline & BackColor & $=Q B C o l o r(3)$ \\
\hline & Borderstyle & $=1$ \\
\hline & Caption & $=" \|$ \\
\hline & DragMode & $=0$ \\
\hline & Enabled & $=-1$ \\
\hline & ForeColor & $=Q B \operatorname{Color}(0)$ \\
\hline & Height & $=\operatorname{Char}(3)$ \\
\hline & Index & $=4$ \\
\hline & Left & $=\operatorname{Char}(40)$ \\
\hline & MousePointer & $=0$ \\
\hline & TabIndex & $=19$ \\
\hline & Tag & $=n n$ \\
\hline & Top & $=\operatorname{char}(2)$ \\
\hline & Visible & $=-1$ \\
\hline & Width & $=\operatorname{Char}(8)$ \\
\hline \multicolumn{3}{|c|}{ END } \\
\hline \multirow[t]{3}{*}{ BEGIN } & Label lblRdis & st \\
\hline & Alignment & $\begin{array}{l}=1 \\
=0\end{array}$ \\
\hline & $\begin{array}{l}\text { AutoSize } \\
\text { BackColor }\end{array}$ & $\begin{array}{l}=0 \\
=Q B C o l o r(3)\end{array}$ \\
\hline
\end{tabular}




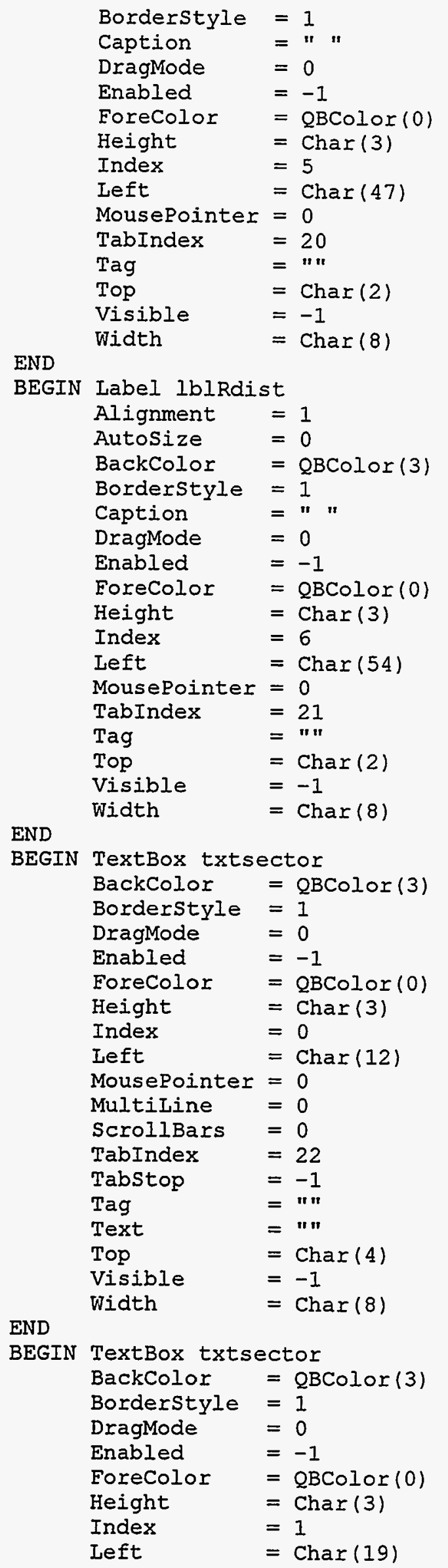




\begin{tabular}{|c|c|c|}
\hline \\
\hline & MousePointer $=$ & $=0$ \\
\hline & Multiline & $=0$ \\
\hline & ScrollBars & $=0$ \\
\hline & TabIndex & $=23$ \\
\hline & Tabstop & $=-1$ \\
\hline & Tag & $=n n$ \\
\hline & Text & $=" n$ \\
\hline & Top & $=\operatorname{Char}(4)$ \\
\hline & Visible & $=-1$ \\
\hline & Width & $=\operatorname{Char}(8)$ \\
\hline \multicolumn{3}{|l|}{ END } \\
\hline \multirow[t]{2}{*}{ BEGIN } & \multicolumn{2}{|c|}{ TextBox txtsector } \\
\hline & $\begin{array}{ll}\text { BackColor } & = \\
\text { Borderstyle } & = \\
\text { DragMode } & = \\
\text { Enabled } & = \\
\text { ForeColor } & = \\
\text { Height } & = \\
\text { Index } & = \\
\text { Left } & = \\
\text { MousePointer } & = \\
\text { Multiline } & = \\
\text { ScrollBars } & = \\
\text { TabIndex } & = \\
\text { Tabstop } & = \\
\text { Tag } & = \\
\text { Text } & = \\
\text { Top } & = \\
\text { Wisible } & =\end{array}$ & $\begin{array}{l}=Q B \operatorname{Color}(3) \\
=1 \\
=0 \\
=-1 \\
=\text { QBColor }(0) \\
=\text { Char }(3) \\
=2 \\
=\operatorname{Char}(26) \\
=0 \\
=0 \\
=0 \\
=24 \\
=-1 \\
=" 1 \\
=" 1 " \\
=\text { Char }(4) \\
=-1 \\
=\text { Char }(8)\end{array}$ \\
\hline \multicolumn{3}{|l|}{ END } \\
\hline BEGIN & $\begin{array}{ll}\text { TextBox txtsec } & = \\
\text { BackColor } & = \\
\text { Borderstyle } & = \\
\text { DragMode } & = \\
\text { Enabled } & = \\
\text { ForeColor } & = \\
\text { Height } & = \\
\text { Index } & = \\
\text { Meft } & = \\
\text { Multiline } & = \\
\text { ScroliBars } & = \\
\text { TabIndex } & = \\
\text { Tabstop } & = \\
\text { Tag } & = \\
\text { Top } & \text { Visible } \\
\text { Width } & =\end{array}$ & $\begin{array}{l}\text { ctor } \\
=\text { QBColor }(3) \\
=1 \\
=0 \\
=-1 \\
=\text { QBColor }(0) \\
=\text { Char }(3) \\
=3 \\
=\text { Char }(33) \\
=0 \\
=0 \\
=0 \\
=25 \\
=-1 \\
=" " \\
=" n \\
=\text { Char }(4) \\
=-1 \\
=\text { Char }(8)\end{array}$ \\
\hline \multicolumn{3}{|l|}{ END } \\
\hline BEGIN & $\begin{array}{l}\text { TextBox txtse } \\
\text { BackColor } \\
\text { BorderStyle } \\
\text { DragMode } \\
\text { Enabled } \\
\text { ForeColor } \\
\text { Height } \\
\text { Index } \\
\text { Ieft } \\
\text { MousePointer = } \\
\text { Multiline } \\
\text { ScrollBars }\end{array}$ & $\begin{array}{l}\text { ector } \\
=Q B C o l o r(3) \\
=1 \\
=0 \\
=-1 \\
=Q B \operatorname{Color}(0) \\
=\operatorname{Char}(3) \\
=4 \\
=C h a r(40) \\
=0 \\
=0 \\
=0\end{array}$ \\
\hline
\end{tabular}




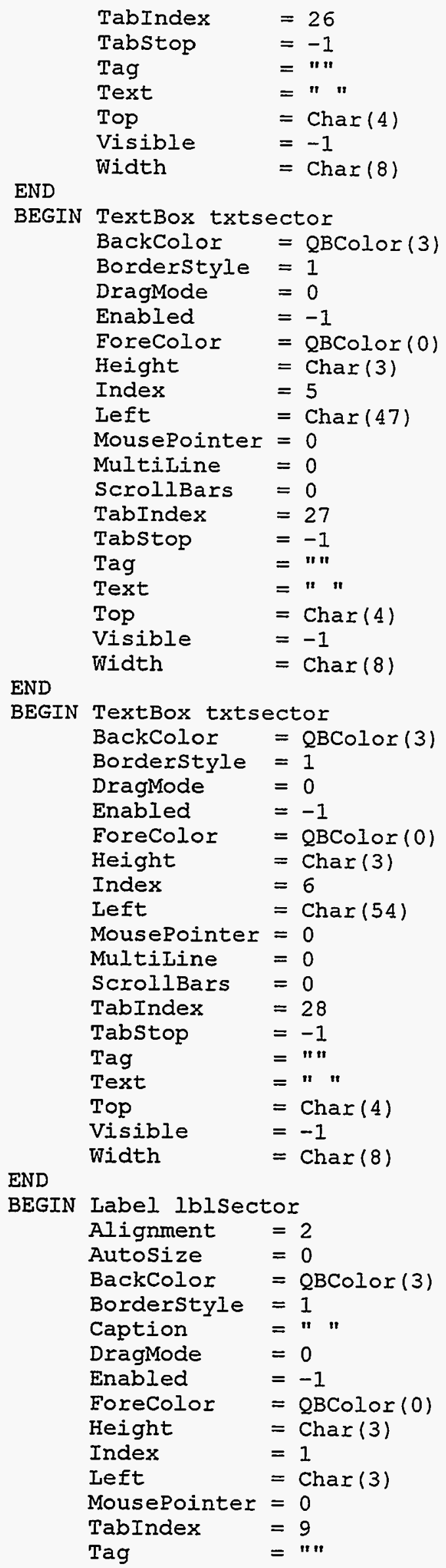




$$
\begin{array}{ll}
\text { Top } & =\operatorname{Char}(6) \\
\text { Visible } & =-1 \\
\text { Width } & =\operatorname{Char}(10)
\end{array}
$$

END

BEGIN Label Iblsector

$$
\begin{array}{ll}
\text { Alignment } & =2 \\
\text { Autosize } & =0 \\
\text { BackColor } & =\text { QBColor (3) } \\
\text { Borderstyle } & =1 \\
\text { Caption } & =" " \\
\text { DragMode } & =0 \\
\text { Enabled } & =-1 \\
\text { EoreColor } & =\text { QBColor (0) } \\
\text { Height } & =\text { Char }(3) \\
\text { Index } & =2 \\
\text { Left } & =\text { Char (3) } \\
\text { MousePointer } & =0 \\
\text { TabIndex } & =10 \\
\text { Tag } & =" 1 \\
\text { Top } & =\text { Char }(8) \\
\text { Visible } & =-1 \\
\text { Width } & =\text { Char }(10)
\end{array}
$$

END

BEGIN Label Iblsector

$$
\begin{array}{ll}
\text { Alignment } & =2 \\
\text { AutoSize } & =0 \\
\text { BackColor } & =\text { QBColor (3) } \\
\text { Borderstyle } & =1 \\
\text { Caption } & =" " \\
\text { DragMode } & =0 \\
\text { Enabled } & =-1 \\
\text { ForeColor } & =\text { QBColor (0) } \\
\text { Height } & =\text { Char (3) } \\
\text { Index } & =3 \\
\text { Left } & =\text { Char (3) } \\
\text { MousePointer } & =0 \\
\text { TabIndex } & =11 \\
\text { Tag } & =" " \\
\text { Top } & =\text { Char }(10) \\
\text { Visible } & =-1 \\
\text { Width } & =\operatorname{Char}(10)
\end{array}
$$

END

BEGIN Label Iblsector

$$
\begin{array}{ll}
\text { Alignment } & =2 \\
\text { Autosize } & =0 \\
\text { BackColor } & =\text { QBColor (3) } \\
\text { Borderstyle } & =1 \\
\text { Caption } & =" " \\
\text { DragMode } & =0 \\
\text { Enabled } & =-1 \\
\text { ForeColor } & =\text { QBColor (0) } \\
\text { Height } & =\text { Char (3) } \\
\text { Index } & =4 \\
\text { Left } & =\text { Char (3) } \\
\text { MousePointer } & =0 \\
\text { TabIndex } & =12 \\
\text { Tag } & =" 1 \\
\text { Top } & =\text { Char (12) } \\
\text { Visible } & =-1 \\
\text { Width } & =\text { Char (10) }
\end{array}
$$

BEGIN Label 1blsector

$$
\text { Alignment }=2
$$




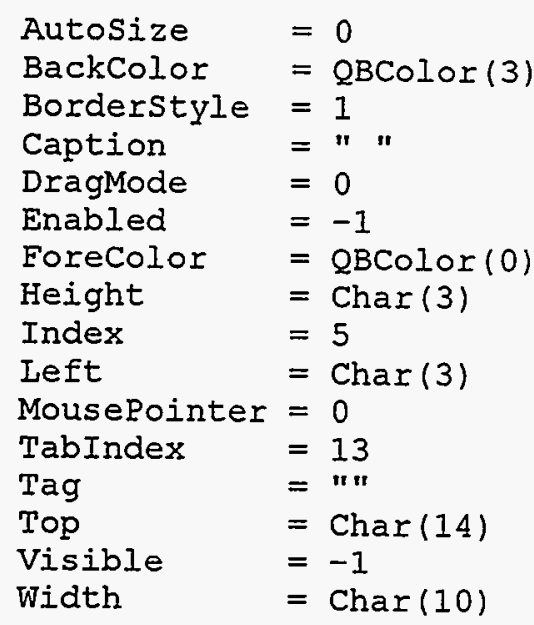

END

BEGIN Label lblsector

$\begin{array}{ll}\text { Alignment } & =2 \\ \text { AutoSize } & =0 \\ \text { BackColor } & =\text { QBColor }(3) \\ \text { Borderstyle } & =1 \\ \text { Caption } & =" ~ \\ \text { DragMode } & =0 \\ \text { Enabled } & =-1 \\ \text { ForeColor } & =\text { QBColor }(0) \\ \text { Height } & =\text { Char }(3) \\ \text { Index } & =6 \\ \text { Left } & =\text { Char }(3) \\ \text { MousePointer } & =0 \\ \text { TabIndex } & =14 \\ \text { Tag } & =" 1 \\ \text { Top } & =\text { Char }(16) \\ \text { Visible } & =-1 \\ \text { Width } & =\text { Char }(10) \\ & \end{array}$

END

BEGIN TextBox txtsector

BackColor = QBColor (3)

Borderstyle $=1$

DragMode $=0$

Enabled $\quad=-1$

ForeColor $=$ QBColor $(0)$

Height $=$ Char (3)

Index $\quad=7$

Left $=$ Char(12)

MousePointer $=0$

Multiline $=0$

ScrollBars $=0$

TabIndex $\quad=29$

Tabstop $\quad=-1$

Tag $=" 1 "$

Text $="$ "

Top $=\operatorname{Char}(6)$

Visible $\quad=-1$

END

Width $=\operatorname{Char}(8)$

BEGIN TextBox txtsector

$\begin{array}{ll}\text { BackColor } & =\text { QBColor }(3) \\ \text { BorderStyle } & =1 \\ \text { DragMode } & =0 \\ \text { Enabled } & =-1 \\ \text { ForeColor } & =\text { QBColor }(0) \\ \text { Height } & =\text { Char }(3)\end{array}$




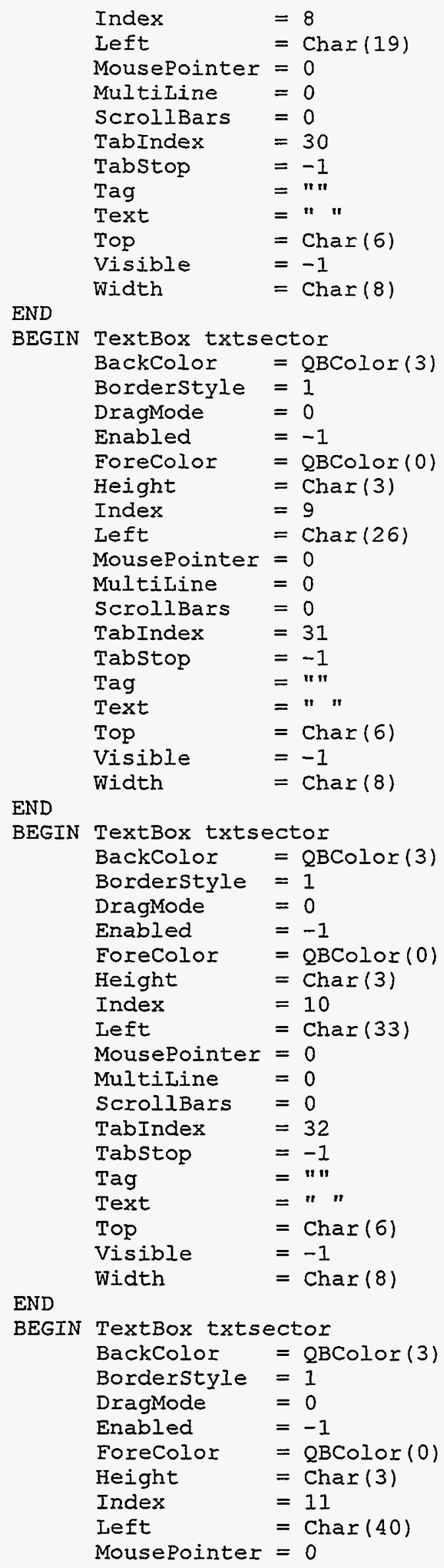




\begin{tabular}{|c|c|c|}
\hline & & \\
\hline & Multiline & $=0$ \\
\hline & ScrollBars & $=0$ \\
\hline & TabIndex & $=33$ \\
\hline & Tabstop & $=-1$ \\
\hline & Tag & $=" n$ \\
\hline & Text & $=" 11$ \\
\hline & Top & $=\operatorname{Char}(6)$ \\
\hline & Visible & $=-1$ \\
\hline & Width & $=\operatorname{Char}(8)$ \\
\hline END & & \\
\hline BEGIN & TextBox txts & ector \\
\hline & BackColor & $=$ QBColor $(3)$ \\
\hline & Borderstyle & $=1$ \\
\hline & DragMode & $=0$ \\
\hline & Enabled & $=-1$ \\
\hline & Forecolor & $=$ QBColor $(0)$ \\
\hline & Height & $=\operatorname{Char}(3)$ \\
\hline & Index & $=12$ \\
\hline & Left & $=$ Char (47) \\
\hline & MousePointer & $=0$ \\
\hline & MultiLine & $=0$ \\
\hline & ScrollBars & $=0$ \\
\hline & TabIndex & $=34$ \\
\hline & Tabstop & $=-1$ \\
\hline & Tag & $=" n$ \\
\hline & Text & $=" 1$ \\
\hline & Top & $=\operatorname{Char}(6)$ \\
\hline & Visible & $=-1$ \\
\hline & Width & $=\operatorname{Char}(8)$ \\
\hline END & & \\
\hline BEGIN & TextBox txts & ector \\
\hline & BackColor & $=$ QBColor $(3)$ \\
\hline & Borderstyle & $=I$ \\
\hline & DragMode & $=0$ \\
\hline & Enab́led & $=-1$ \\
\hline & ForeColor & $=$ QBColor $(0)$ \\
\hline & Height & $=\operatorname{Char}(3)$ \\
\hline & Index & $=13$ \\
\hline & Left & $=\operatorname{Char}(54)$ \\
\hline & MousePointer & $=0$ \\
\hline & MultiLine & $=0$ \\
\hline & ScrollBars & $=0$ \\
\hline & TabIndex & $=35$ \\
\hline & Tabstop & $=-1$ \\
\hline & Tag & $=" n$ \\
\hline & Text & $=" n$ \\
\hline & Top & $=\operatorname{Char}(6)$ \\
\hline & Visible & $=-1$ \\
\hline & width & $=\operatorname{Char}(8)$ \\
\hline END & & \\
\hline BEGIN & TextBox txts & ector \\
\hline & BackColor & $=$ QBColor (3) \\
\hline & Borderstyle & $=1$ \\
\hline & DragMode & $=0$ \\
\hline & Enabled & $=-1$ \\
\hline & ForeColor & $=$ QBColor $(0)$ \\
\hline & Height & $=\operatorname{Char}(3)$ \\
\hline & Index & $=14$ \\
\hline & Left & $=\operatorname{Char}(12)$ \\
\hline & MousePointer & $=0$ \\
\hline & Multiline & $=0$ \\
\hline & scrollBars & $=0$ \\
\hline & TabIndex & $=36$ \\
\hline
\end{tabular}




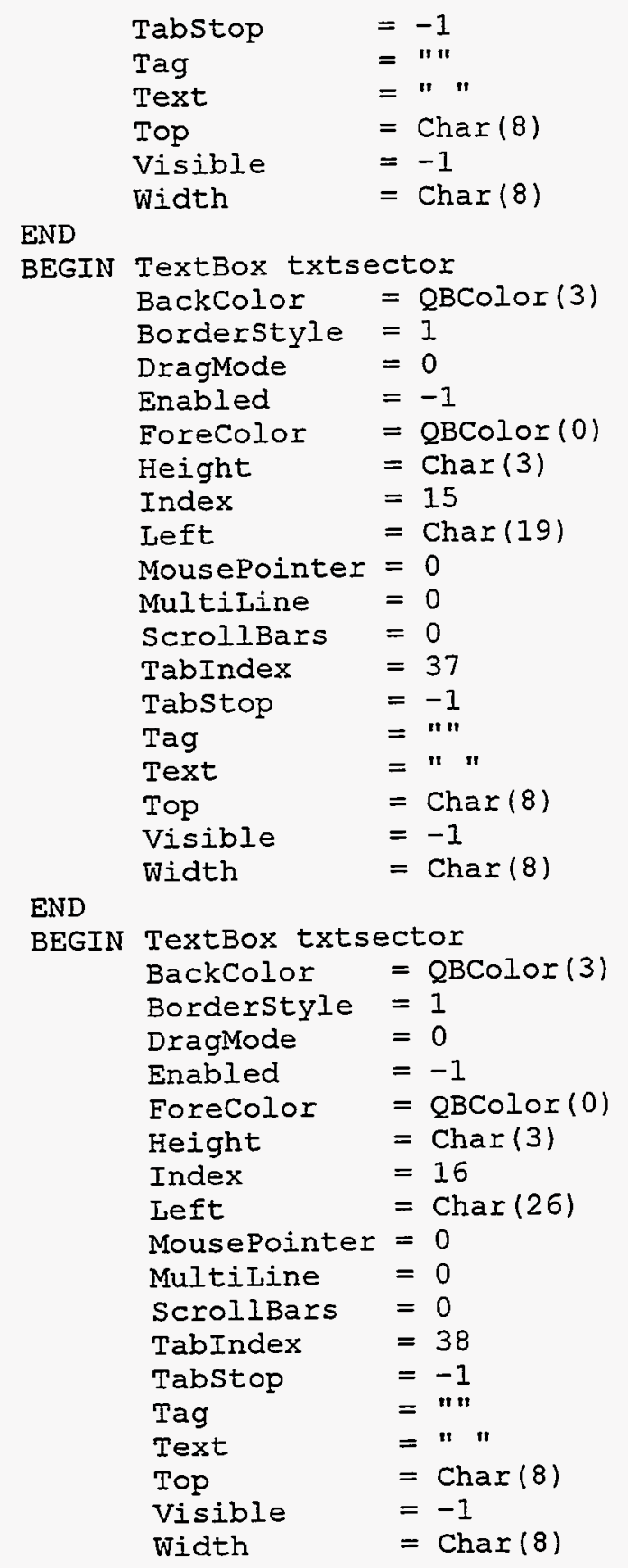

END

BEGIN TextBox txtsector

$\begin{array}{ll}\text { BackColor } & =\text { QBColor (3) } \\ \text { Borderstyle } & =1 \\ \text { DragMode } & =0 \\ \text { Enabled } & =-1 \\ \text { EoreColor } & =\text { QBColor (0) } \\ \text { Height } & =\text { Char (3) } \\ \text { Index } & =17 \\ \text { Left } & =\text { Char (33) } \\ \text { MousePointer } & =0 \\ \text { MultiLine } & =0 \\ \text { ScrollBars } & =0 \\ \text { TabIndex } & =39 \\ \text { Tabstop } & =-1 \\ \text { Tag } & =" " \\ \text { Text } & ="\end{array}$




\begin{tabular}{|c|c|c|}
\hline & $\begin{array}{l}\text { Top } \\
\text { Visible } \\
\text { Width }\end{array}$ & $\begin{array}{l}=\operatorname{Char}(8) \\
=-1 \\
=\operatorname{Char}(8)\end{array}$ \\
\hline \multicolumn{3}{|l|}{ END } \\
\hline BEGIN & $\begin{array}{l}\text { TextBox txts } \\
\text { BackColor } \\
\text { Borderstyle } \\
\text { DragMode } \\
\text { Enabled } \\
\text { ForeColor } \\
\text { Height } \\
\text { Index } \\
\text { Left } \\
\text { MousePointer } \\
\text { MultiLine } \\
\text { ScrollBars } \\
\text { TabIndex } \\
\text { Tabstop } \\
\text { Tag } \\
\text { Text } \\
\text { Top } \\
\text { Visible } \\
\text { Width }\end{array}$ & $\begin{array}{l}\text { ector } \\
=\text { QBColor (3) } \\
=1 \\
=0 \\
=-1 \\
=\text { QBColor (0) } \\
=\text { Char }(3) \\
=18 \\
=\operatorname{Char}(40) \\
=0 \\
=0 \\
=0 \\
=40 \\
=-1 \\
=" 1 \\
=" n \\
=\text { Char }(8) \\
=-1 \\
=\text { Char }(8)\end{array}$ \\
\hline \multicolumn{3}{|l|}{ END } \\
\hline BEGIN & $\begin{array}{l}\text { TextBox txts } \\
\text { BackColor } \\
\text { Borderstyle } \\
\text { DragMode } \\
\text { Enabled } \\
\text { ForeColor } \\
\text { Height } \\
\text { Index } \\
\text { Left } \\
\text { MousePointer } \\
\text { Multiline } \\
\text { ScrollBars } \\
\text { TabIndex } \\
\text { Tabstop } \\
\text { Tag } \\
\text { Text } \\
\text { Top } \\
\text { Visible } \\
\text { Width }\end{array}$ & $\begin{array}{l}\text { ctor } \\
=\text { QBColor (3) } \\
=1 \\
=0 \\
=-1 \\
=\text { QBColor (0) } \\
=\text { Char (3) } \\
=19 \\
=\text { Char }(47) \\
=0 \\
=0 \\
=0 \\
=41 \\
=-1 \\
=" n \\
=" n \\
=\text { Char (8) } \\
=-1 \\
=\text { Char (8) }\end{array}$ \\
\hline \multicolumn{3}{|l|}{ END } \\
\hline BEGIN & $\begin{array}{l}\text { TextBox txts } \\
\text { BackColor } \\
\text { Borderstyle } \\
\text { DragMode } \\
\text { Enabled } \\
\text { EoreColor } \\
\text { Height } \\
\text { Index } \\
\text { Left } \\
\text { MousePointer } \\
\text { Multiline } \\
\text { ScrollBars } \\
\text { TabIndex } \\
\text { Tabstop } \\
\text { Tag } \\
\text { Text } \\
\text { Top } \\
\text { Visible } \\
\text { Width }\end{array}$ & $\begin{array}{l}\text { ctor } \\
=Q B C o l o r(3) \\
=1 \\
=0 \\
=-1 \\
=Q B C o l o r(0) \\
=\text { Char }(3) \\
=20 \\
=\text { Char }(54) \\
=0 \\
=0 \\
=0 \\
=42 \\
=-1 \\
=" " \\
=" " \\
=\text { Char (8) } \\
=-1 \\
=\text { Char (8) }\end{array}$ \\
\hline
\end{tabular}


END

BEGIN TextBox txtsector

$\begin{array}{ll}\text { BackColor } & =Q B C o l o r(3) \\ \text { Borderstyle } & =1 \\ \text { DragMode } & =0 \\ \text { Enabled } & =-1 \\ \text { ForeColor } & =\text { QBColor (0) } \\ \text { Height } & =\text { Char (3) } \\ \text { Index } & =21 \\ \text { Left } & =\text { Char (12) } \\ \text { MousePointer } & =0 \\ \text { Multiline } & =0 \\ \text { ScrollBars } & =0 \\ \text { TabIndex } & =43 \\ \text { TabStop } & =-1 \\ \text { Tag } & =" " \\ \text { Text } & =" \text { Char (10) } \\ \text { Top } & =-1 \\ \text { Visible } & =\text { Char (8) } \\ \text { Width } & \end{array}$

END

BEGIN TextBox txtsector

BackColor = QBColor (3)

Borderstyle $=1$

DragMode $=0$

Enabled $\quad=-1$

ForeColor $=$ QBColor $(0)$

Height $=$ Char (3)

Index $\quad=22$

Left $=$ Char (19)

MousePointer $=0$

Multiline $=0$

ScrollBars $=0$

TabIndex $\quad=44$

Tabstop $\quad=-1$

Tag $=" u$

Text = " "

Top $=\operatorname{Char}(10)$

Visible $\quad=-1$

Width $\quad=\operatorname{Char}(8)$

END

BEGIN TextBox txtsector

$\begin{array}{ll}\text { BackColor } & =Q B C o l o r(3) \\ \text { Borderstyle } & =1 \\ \text { DragMode } & =0 \\ \text { Enabled } & =-1 \\ \text { ForeColor } & =Q B C o l o r(0) \\ \text { Height } & =\text { Char (3) } \\ \text { Index } & =23 \\ \text { Ieft } & =\text { Char (26) } \\ \text { MousePointer } & =0 \\ \text { Multiline } & =0 \\ \text { ScrollBars } & =0 \\ \text { TabIndex } & =45 \\ \text { Tabstop } & =-1 \\ \text { Tag } & =" " \\ \text { Text } & =" \text { "Char (10) } \\ \text { Top } & =-1 \\ \text { Visible } & =\text { Char (8) } \\ \text { Width } & \end{array}$

END

BEGIN TextBox txtsector

BackColor = QBColor (3) 


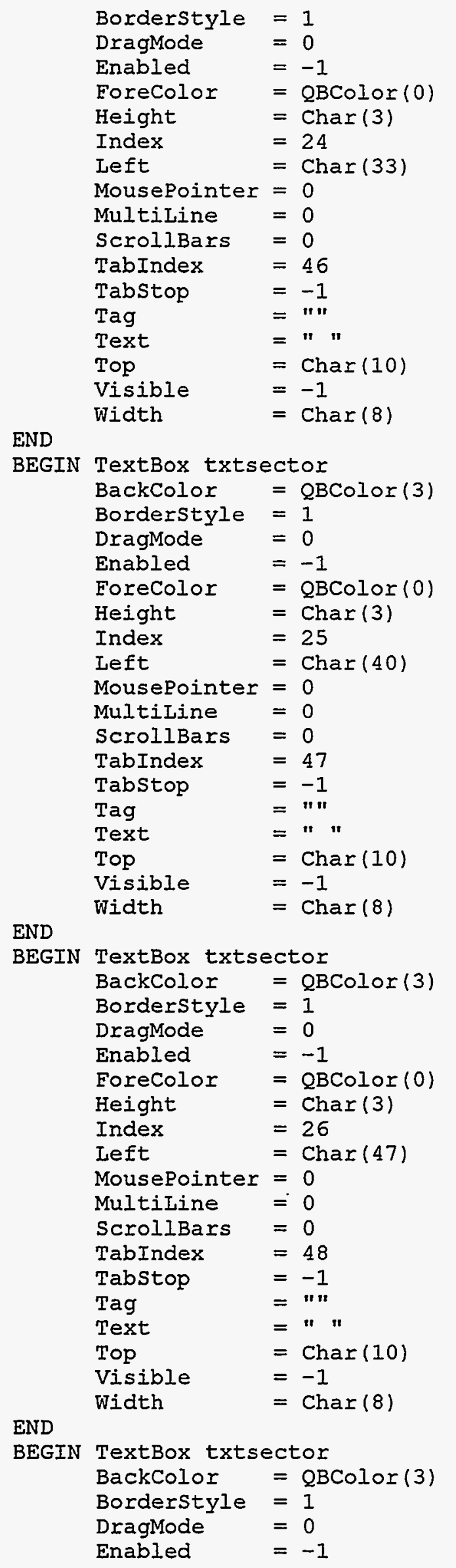




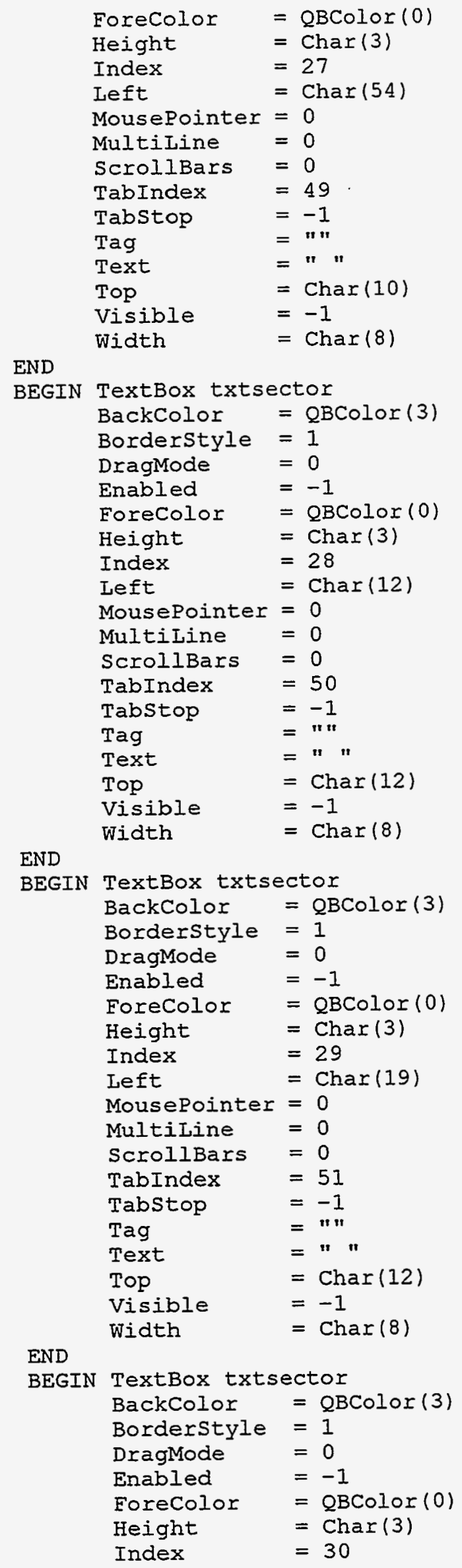




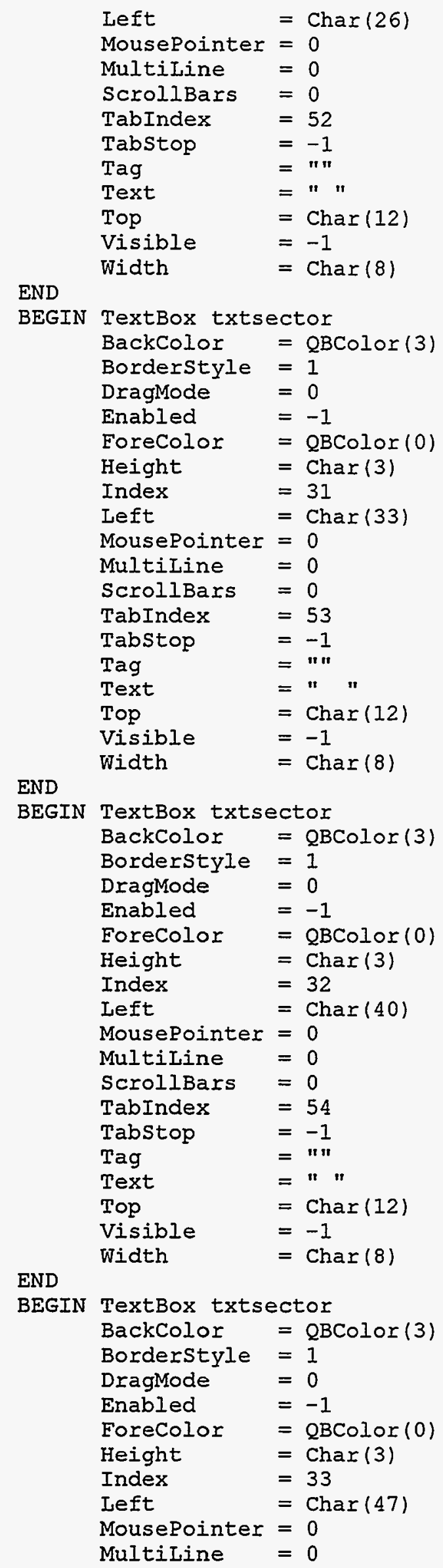




$\begin{array}{ll}\text { ScrollBars } & =0 \\ \text { TabIndex } & =55 \\ \text { Tabstop } & =-1 \\ \text { Tag } & =" " \\ \text { Text } & =" " \\ \text { Top } & =\text { Char }(12) \\ \text { Visible } & =-1 \\ \text { Width } & =\text { Char }(8)\end{array}$

END

BEGIN TextBox txtsector

$\begin{array}{ll}\text { BackColor } & =\text { QBColor (3) } \\ \text { Borderstyle } & =1 \\ \text { DragMode } & =0 \\ \text { Enabled } & =-1 \\ \text { ForeColor } & =\text { QBColor (0) } \\ \text { Height } & =\text { Char (3) } \\ \text { Index } & =34 \\ \text { Left } & =\text { Char (54) } \\ \text { MousePointer } & =0 \\ \text { Multiline } & =0 \\ \text { ScrollBars } & =0 \\ \text { TabIndex } & =56 \\ \text { Tabstop } & =-1 \\ \text { Tag } & =" 1 \\ \text { Text } & =\text { Char (12) } \\ \text { Top } & =-1 \\ \text { Visible } & =\text { Char (8) } \\ \text { Width } & \end{array}$

END

BEGIN TextBox txtsector

BackColor = oBColor (3)

Borderstyle $=1$

DragMode $=0$

Enabled $\quad=-1$

EoreColor = oBColor $(0)$

Height $=$ Char (3)

Index $\quad=35$

Left $=$ Char (12)

Mousepointer $=0$

Multiline $=0$

ScrollBars $=0$

TabIndex $\quad=57$

Tabstop $=-1$

Tag $=" n$

Text = " "

Top $=\operatorname{Char}(14)$

Visible $\quad=-1$

width $=\operatorname{Char}(8)$

END

BEGIN TextBox txtsector

$\begin{array}{ll}\text { BackColor } & =\text { QBColor (3) } \\ \text { Borderstyle } & =1 \\ \text { DragMode } & =0 \\ \text { Enabled } & =-1 \\ \text { EoreColor } & =\text { BColor (0) } \\ \text { Height } & =\text { Char (3) } \\ \text { Index } & =36 \\ \text { Left } & =\text { Char (19) } \\ \text { MousePointer } & =0 \\ \text { Multiline } & =0 \\ \text { ScrollBars } & =0 \\ \text { TabIndex } & =58 \\ \text { Tabstop } & =-1\end{array}$




\begin{tabular}{|c|c|c|}
\hline & $\begin{array}{l}\text { Tag } \\
\text { Text } \\
\text { Top } \\
\text { Visible } \\
\text { Width }\end{array}$ & $\begin{array}{l}=" " \\
=" " \\
=\operatorname{Char}(14) \\
=-1 \\
=\operatorname{Char}(8)\end{array}$ \\
\hline \multirow{2}{*}{$\begin{array}{l}\text { END } \\
\text { BEGIN }\end{array}$} & & \\
\hline & $\begin{array}{l}\text { TextBox txtse } \\
\text { BackColor } \\
\text { BorderStyle } \\
\text { DragMode } \\
\text { Enabled } \\
\text { ForeColor } \\
\text { Height } \\
\text { Index } \\
\text { Left } \\
\text { MousePointer } \\
\text { Multiline } \\
\text { ScrollBars } \\
\text { TabIndex } \\
\text { TabStop } \\
\text { Tag } \\
\text { Text } \\
\text { Top } \\
\text { Visible } \\
\text { Width }\end{array}$ & $\begin{array}{l}\text { ector } \\
=Q B C o l o r(3) \\
=1 \\
=0 \\
=-1 \\
=Q B C o l o r(0) \\
=\text { Char }(3) \\
=37 \\
=\text { Char }(26) \\
=0 \\
=0 \\
=0 \\
=59 \\
=-1 \\
=" 1 \\
=" \text { " } \\
=\text { Char }(14) \\
=-1 \\
=\text { Char }(8)\end{array}$ \\
\hline \multicolumn{3}{|c|}{ WLCLLA } \\
\hline BEGIN & $\begin{array}{l}\text { TextBox txtse } \\
\text { BackColor } \\
\text { Borderstyle } \\
\text { DragMode } \\
\text { Enabled } \\
\text { ForeColor } \\
\text { Height } \\
\text { Index } \\
\text { Left } \\
\text { MousePointer } \\
\text { Multiline } \\
\text { ScrollBars } \\
\text { TabIndex } \\
\text { Tabstop } \\
\text { Tag } \\
\text { Text } \\
\text { Top } \\
\text { Visible } \\
\text { Width }\end{array}$ & $\begin{array}{l}\text { etor } \\
=\text { QBColor (3) } \\
=1 \\
=0 \\
=-1 \\
=Q B \operatorname{Color}(0) \\
=\text { Char }(3) \\
=38 \\
=\text { Char (33) } \\
=0 \\
=0 \\
=0 \\
=60 \\
=-1 \\
=" 1 \\
=" 1 \\
=\text { Char }(14) \\
=-1 \\
=\text { Char }(8)\end{array}$ \\
\hline \multirow{2}{*}{$\begin{array}{l}\text { END } \\
\text { BEGIN }\end{array}$} & & \\
\hline & $\begin{array}{l}\text { TextBox txtse } \\
\text { BackColor } \\
\text { Borderstyle } \\
\text { DragMode } \\
\text { Enabled } \\
\text { ForeColor } \\
\text { Height } \\
\text { Index } \\
\text { Left } \\
\text { MousePointer } \\
\text { Multiline } \\
\text { ScrollBars } \\
\text { TabIndex } \\
\text { Tabstop } \\
\text { Tag } \\
\text { Text } \\
\text { Top }\end{array}$ & $\begin{array}{l}\text { ector } \\
=\text { QBColor (3) } \\
=1 \\
=0 \\
=-1 \\
=\text { QBColor }(0) \\
=\text { Char }(3) \\
=39 \\
=\text { Char }(40) \\
=0 \\
=0 \\
=0 \\
=61 \\
=-1 \\
=" 1 \\
=" 1 \\
=\text { Char }(14)\end{array}$ \\
\hline
\end{tabular}




\begin{tabular}{|c|c|c|}
\hline & $\begin{array}{l}\text { Visible } \\
\text { width }\end{array}$ & $\begin{array}{l}=-1 \\
=\operatorname{Char}(8)\end{array}$ \\
\hline \multicolumn{3}{|l|}{ END } \\
\hline BEGIN & $\begin{array}{ll}\text { TextBox txtsec } & = \\
\text { BackColor } & = \\
\text { Borderstyle } & = \\
\text { DragMode } & = \\
\text { Enabled } & = \\
\text { ForeColor } & = \\
\text { Height } & = \\
\text { Index } & = \\
\text { Moft } & = \\
\text { MultiIine } & = \\
\text { ScrollBars } & = \\
\text { TabIndex } & = \\
\text { Tabstop } & = \\
\text { Text } & = \\
\text { Visible } & = \\
\text { Width } & =\end{array}$ & $\begin{array}{l}\text { etor } \\
=Q B \operatorname{Color}(3) \\
=1 \\
=0 \\
=-1 \\
=Q B \operatorname{Color}(0) \\
=\operatorname{Char}(3) \\
=40 \\
=\operatorname{Char}(47) \\
=0 \\
=0 \\
=0 \\
=62 \\
=-1 \\
=" " \\
=" \| \\
=\operatorname{Char}(14) \\
=-1 \\
=\operatorname{Char}(8)\end{array}$ \\
\hline \multicolumn{3}{|l|}{ END } \\
\hline BEGIN & $\begin{array}{l}\text { TextBox txtse } \\
\text { BackColor } \\
\text { Borderstyle } \\
\text { DragMode } \\
\text { Enabled } \\
\text { ForeColor } \\
\text { Height } \\
\text { Index } \\
\text { Left } \\
\text { MousePointer } \\
\text { Multiline } \\
\text { ScrollBars } \\
\text { TabIndex } \\
\text { Tabstop } \\
\text { Tag } \\
\text { Text } \\
\text { Top } \\
\text { Visible } \\
\text { Width }\end{array}$ & $\begin{array}{l}\text { ector } \\
=\text { QBColor (3) } \\
=1 \\
=0 \\
=-1 \\
=\text { QBColor }(0) \\
=\text { Char }(3) \\
=41 \\
=\text { Char }(54) \\
=0 \\
=0 \\
=0 \\
=63 \\
=-1 \\
=" n \\
=" n \\
=\text { Char (14) } \\
=-1 \\
=\text { Char (8) }\end{array}$ \\
\hline \multicolumn{3}{|l|}{ END } \\
\hline BEGIN & $\begin{array}{l}\text { TextBox txtse } \\
\text { BackColor } \\
\text { Borderstyle } \\
\text { DragMode } \\
\text { Enabled } \\
\text { ForeColor } \\
\text { Height } \\
\text { Index } \\
\text { Left } \\
\text { MousePointer } \\
\text { MultiLine } \\
\text { ScrollBars } \\
\text { TabIndex } \\
\text { Tabstop } \\
\text { Tag } \\
\text { Text } \\
\text { Top } \\
\text { Visible } \\
\text { Width }\end{array}$ & $\begin{array}{l}\text { ector } \\
=Q B \operatorname{Color}(3) \\
=1 \\
=0 \\
=-1 \\
=\text { QBColor }(0) \\
=\text { Char }(3) \\
=42 \\
=\text { Char }(12) \\
=0 \\
=0 \\
=0 \\
=64 \\
=-1 \\
=" 1 \\
=" n \\
=\text { Char }(16) \\
=-1 \\
=\text { Char }(8)\end{array}$ \\
\hline
\end{tabular}


BEGIN TextBox txtsector

$$
\begin{array}{ll}
\text { BackColor } & =\text { QBColor (3) } \\
\text { Borderstyle } & =1 \\
\text { DragMode } & =0 \\
\text { Enabled } & =-1 \\
\text { ForeColor } & =\text { QBColor (0) } \\
\text { Height } & =\text { Char (3) } \\
\text { Index } & =43 \\
\text { Left } & =\text { Char (19) } \\
\text { MousePointer } & =0 \\
\text { Multiline } & =0 \\
\text { ScrollBars } & =0 \\
\text { TabIndex } & =65 \\
\text { Tabstop } & =-1 \\
\text { Tag } & =" " \\
\text { Text } & =" \text { "Char (16) } \\
\text { Top } & =-1 \\
\text { Visible } & =\text { Char (8) } \\
\text { Width } &
\end{array}
$$

END

BEGIN TextBox txtsector

$$
\begin{array}{ll}
\text { BackColor } & =\text { QBColor (3) } \\
\text { BorderStyle } & =1 \\
\text { DragMode } & =0 \\
\text { Enabled } & =-1 \\
\text { EoreColor } & =\text { QBColor (0) } \\
\text { Height } & =\text { Char (3) } \\
\text { Index } & =44 \\
\text { Left } & =\text { Char (26) } \\
\text { MousePointer } & =0 \\
\text { Multiline } & =0 \\
\text { ScroliBars } & =0 \\
\text { TabIndex } & =66 \\
\text { Tabstop } & =-1 \\
\text { Tag } & =" " \\
\text { Text } & =" 1 \\
\text { Top } & =\text { Char (16) } \\
\text { Visible } & =-1 \\
\text { Width } & =\text { Char (8) }
\end{array}
$$

END

BEGIN TextBox txtsector

$$
\begin{array}{ll}
\text { BackColor } & =\text { QBColor (3) } \\
\text { Borderstyle } & =1 \\
\text { DragMode } & =0 \\
\text { Enabled } & =-1 \\
\text { EoreColor } & =\text { QBColor (0) } \\
\text { Height } & =\text { Char (3) } \\
\text { Index } & =45 \\
\text { Left } & =\text { Char (33) } \\
\text { MousePointer } & =0 \\
\text { Multiline } & =0 \\
\text { ScrollBars } & =0 \\
\text { TabIndex } & =67 \\
\text { Tabstop } & =-1 \\
\text { Tag } & =" n \\
\text { Text } & =" \text { " } \\
\text { Top } & =-1 \\
\text { Visible } & =\text { Char ( } 16) \\
\text { Width } &
\end{array}
$$

END

BEGIN TextBox txtsector

$$
\begin{aligned}
& \text { BackColor }=\text { QBColor }(3) \\
& \text { Borderstyle }=1
\end{aligned}
$$




\begin{tabular}{|c|c|c|}
\hline & DragMode & $=0$ \\
\hline & Enabled & $=-1$ \\
\hline & Forecolor & $=Q B C o l$ or $(0)$ \\
\hline & Height & $=\operatorname{Char}(3)$ \\
\hline & Index & $=46$ \\
\hline & Left & $=\operatorname{Char}(40)$ \\
\hline & MousePointer & $=0$ \\
\hline & Multiline & $=0$ \\
\hline & ScrollBars & $=0$ \\
\hline & TabIndex & $=68$ \\
\hline & Tabstop & $=-1$ \\
\hline & Tag & $=" n$ \\
\hline & Text & $=" 1 "$ \\
\hline & Top & $=\operatorname{Char}(16)$ \\
\hline & Visible & $=-1$ \\
\hline & width & $=\operatorname{Char}(8)$ \\
\hline END & & \\
\hline BEGIN & TextBox txtse & ector \\
\hline & BackColor & $=$ QBColor $(3)$ \\
\hline & Borderstyle & $=1$ \\
\hline & DragMode & $=\overline{0}$ \\
\hline & Enabled & $=-1$ \\
\hline & Forecolor & $=$ QBColor $(0)$ \\
\hline & Height & $=\operatorname{Char}(3)$ \\
\hline & Index & $=47$ \\
\hline & Left & $=\operatorname{Char}(47)$ \\
\hline & MousePointer & $=0$ \\
\hline & Multiline & $=0$ \\
\hline & ScrollBars & $=0$ \\
\hline & TabIndex & $=69$ \\
\hline & Tabstop & $=-1$ \\
\hline & Tag & $=n n$ \\
\hline & Text & $=" n$ \\
\hline & Top & $=\operatorname{Char}(16)$ \\
\hline & Visible & $=-1$ \\
\hline & Width & $=\operatorname{Char}(8)$ \\
\hline END & & \\
\hline BEGIN & TextBox txtse & ector \\
\hline & BackColor & $=$ QBColor (3) \\
\hline & Borderstyle & $=1$ \\
\hline & DragMode & $=0$ \\
\hline & Enabled & $=-1$ \\
\hline & Forecolor & $=$ QBColor $(0)$ \\
\hline & Height & $=\operatorname{Char}(3)$ \\
\hline & Index & $=48$ \\
\hline & Left & $=\operatorname{Char}(54)$ \\
\hline & MousePointer & $=0$ \\
\hline & MultiLine & $=0$ \\
\hline & ScrollBars & $=0$ \\
\hline & TabIndex & $=70$ \\
\hline & Tabstop & $=-1$ \\
\hline & Tag & $=n n$ \\
\hline & Text & $=" 1 "$ \\
\hline & Top & $=\operatorname{Char}(16)$ \\
\hline & Visible & $=-1$ \\
\hline & width & $=\operatorname{Char}(8)$ \\
\hline END & & \\
\hline BEGIN & Iabel Iblnum & radii \\
\hline & Alignment & $=1$ \\
\hline & Autosize & $=\overline{0}$ \\
\hline & BackColor & $=$ QBColor (3) \\
\hline & Borderstyle & $=0$ \\
\hline & Caption & $=" 50 "$ \\
\hline
\end{tabular}




$$
\begin{array}{ll}
\text { DragMode } & =0 \\
\text { Enabled } & =-1 \\
\text { ForeColor } & =\text { oBColor }(0) \\
\text { Height } & =\text { Char }(1) \\
\text { Left } & =\text { Char }(70) \\
\text { MousePointer } & =0 \\
\text { TabIndex } & =77 \\
\text { Tag } & =" " \\
\text { Top } & =\text { Char }(6) \\
\text { Visible } & =-1 \\
\text { Width } & =\text { Char }(3)
\end{array}
$$

END

OPTION EXPLICIT

'\$INCLUDE: 'secpop90.bi'

SUB cmaclear_click ()

DIM $i$ AS INTEGER, $j$ AS INTEGER

number econ regions $=0$

frmProb̄lem_data.lblNumber_of_regions. caption $=" n$

EOR $i=0$ TO number of segments - 1

FOR $j=2$ TO max number_of_radii NEXT $j$

NEXT i region_index $(j, i) \equiv 0^{-}$

$\mathrm{x}$ position $=0$

y_position $=0$

CALI paint_region $(0,0)$

END SUB

SUB cmaclose_Click ()

CAII cmasort Click

IF frmRegion.cmdSort.Tag = "F" THEN

EXIT SUB

ELSE

frmRegion. HIDE

END IF

END SUB

SUB cmdDefault_Click ()

CALL default_regions

$x$ position $=0$

Y_position $=0$

CALL paint_region $(0,0)$

END SUB

SUB cmdDown_Click ()

CAII paint_region $(0,1)$

END SUB 


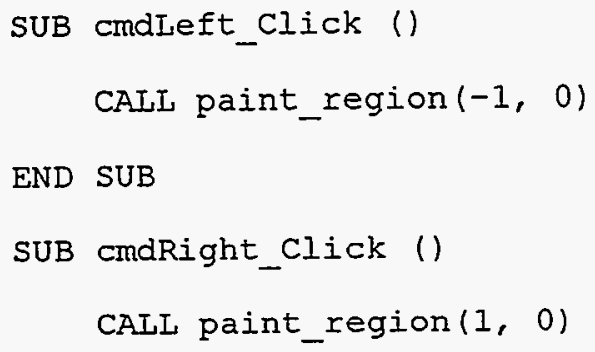


number econ regions = current region -1

frmprob̄lem_data.lblNumber_of_regions.caption = STRS(number_econ_regions)

CALL paint_region $(0,0)$

END SUB

SUB cmaUp_Click ()

CAIL paint_region $(0,-1)$

END SUB

SUB txtSector_LostFocus (index AS INTEGER)

DIM i AS INTEGER, j AS INTEGER, $k$ AS INTEGER

DIM Y AS INTEGER

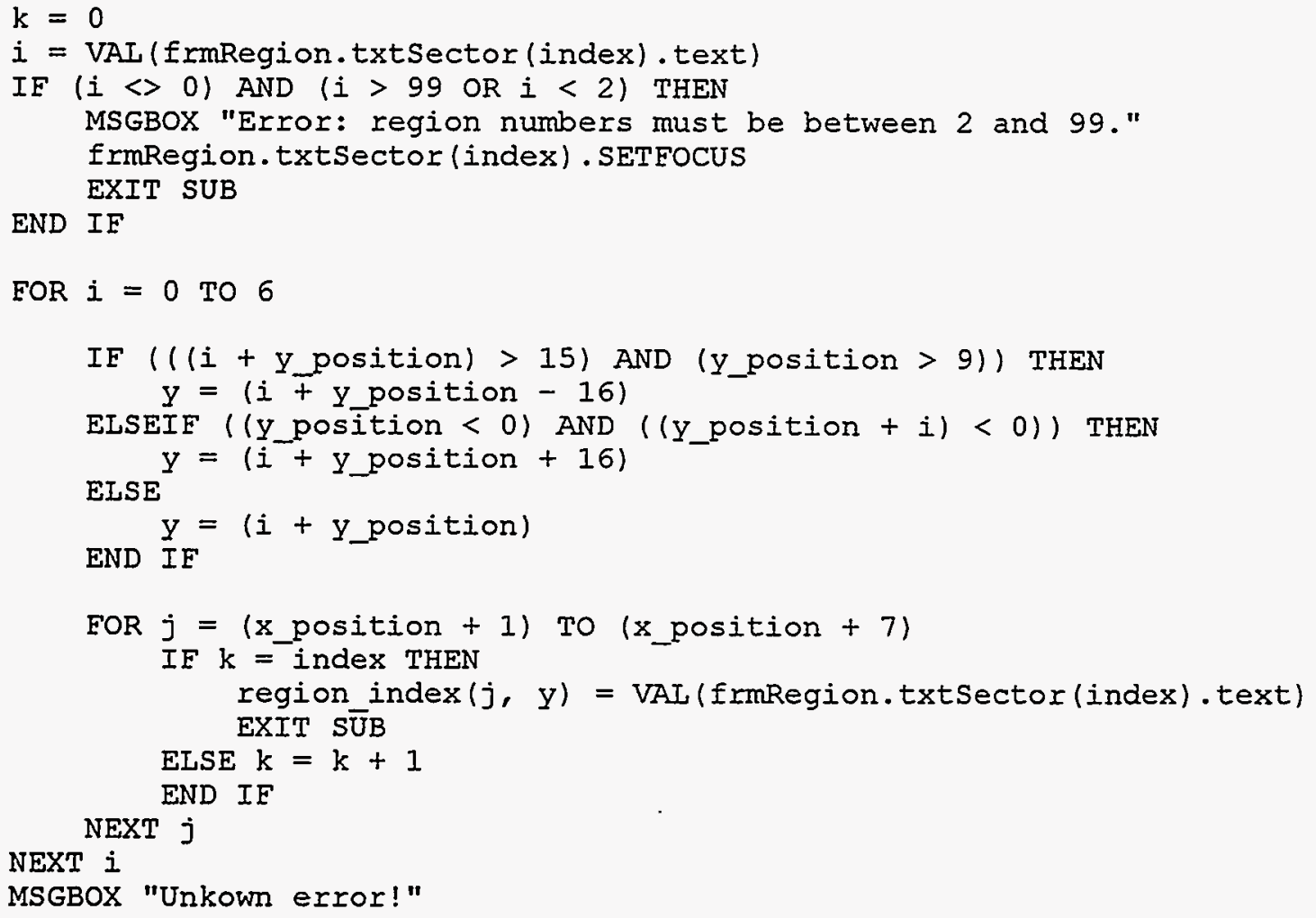

END SUB 


\section{OPTION EXPIICIT}

'\$INCLUDE: 'secpop 90. bi'

SUB print_graphic (text AS STRING, text_width AS INTEGER, centered AS INTEGER)

'This procedure allows the user to output text to the screen in a specified

'position when forms are not showing.

DIM next_line AS STRING, character AS STRING

DIM charācter_location AS INTEGER, character_found AS INTEGER

'Break the text into line sized pieces based upon length and upon

'special characters such as -, <cr> and ".

WHILE (LEN (text) > text_width)

next_line = LEFT\$(text, text_width)

charācter found = EALSE

character location = text width

WHILE ( (NOTT character fouñd) AND (character location > 0))

character $=\operatorname{MID}(\bar{n}$ ext_line, character_löcation, 1$)$

THEN

IF ((character $="$ ") OR (character $="-")$ OR (character $=\operatorname{CHRS(13))}$

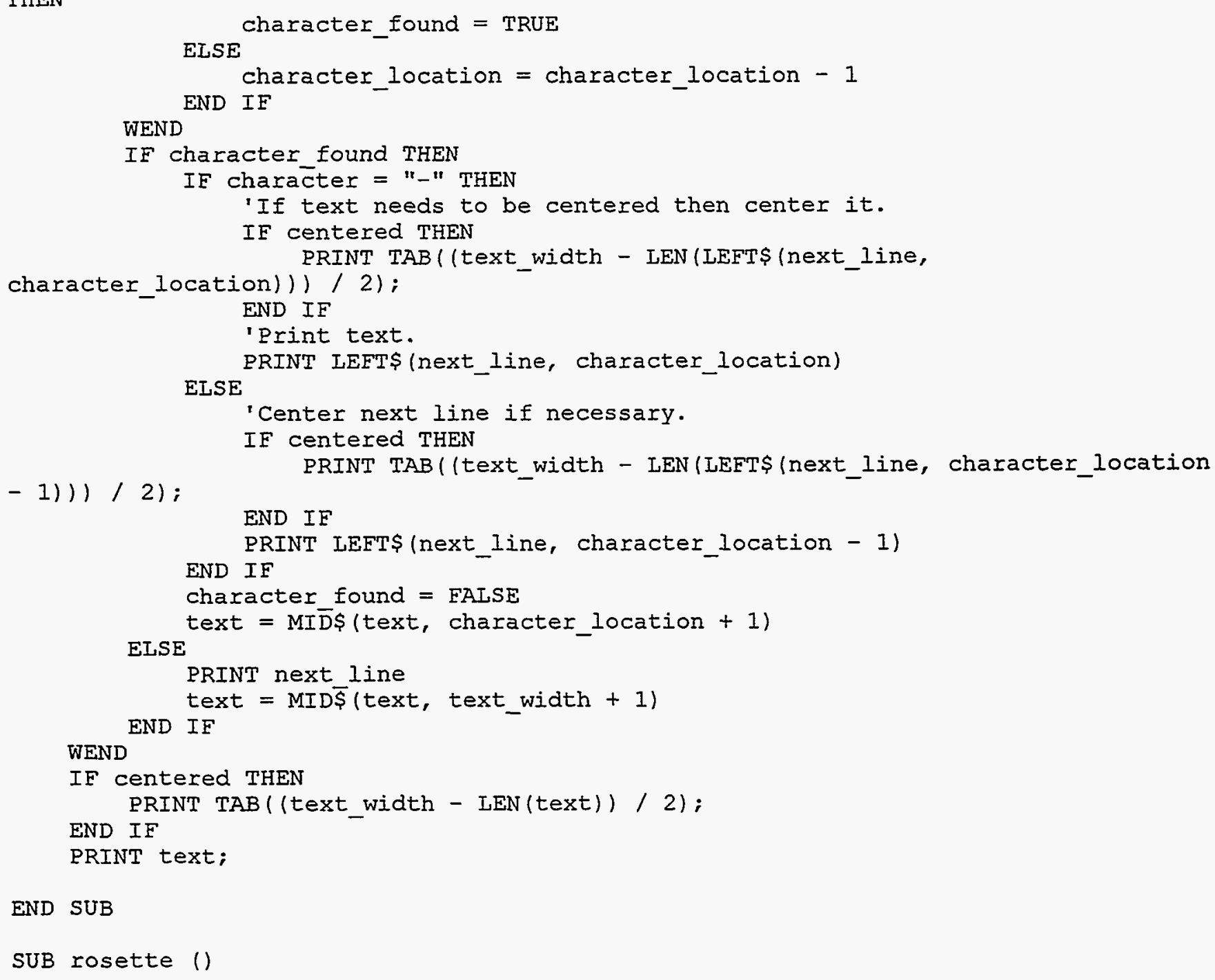


DIM paint value AS INTEGER, max pop AS LONG, pop scale AS LONG

DIM scale interval AS LONG, i AS INTEGER, $j$ AS INTEGER, titIe AS STRING

DIM max rädius AS SINGLE, rotation increment AS SINGLE, rotation AS SINGLE

DIM $y 2$ ĀS SINGLE, $y 1$ AS SINGLE, $x 2-A S$ SINGLE, $x 1$ AS SINGLE, r1 AS SINGLE

'Declare constants to determine if text is centered or not and pi.

CONST pi $=3.14159265358979 \#$

CONST paint_position $=.75$

CONST not centered = FAISE

CONST centered $=$ TRUE

UNLOAD frmDisclaimer

'Set max pop to the highest value in the population grid.

$\max \_$pop $=0$

FOR $i=1$ TO number of segments STEP 1

FOR $j=1$ TO numbe $\bar{r}$ of radii STEP 1

IF max pop < sector_population $(i, j)$ THEN END IF $\max$ pop $=$ sector_population $(i, j)$

NEXT i

NEXT $j$

'Determine which scale to used based upon max pop, and set max pop

'to that scale.

IF max_pop $<=15$ THEN

scale interval $=1$

max_pop $=15$

ELSEIE $\max$ pop $<=150$ THEN

scale interval $=10$

$\max \mathrm{p} \overline{\mathrm{op}}=150$

ELSEIE max_pop $<=1500$ THEN

scale_interval $=100$

ELSE

max_pop $=1500$

scale_interval $=7.5 *(10 \wedge(\operatorname{INT}(\operatorname{LOG}(\max$ pop $) / \operatorname{IOG}(10))-1))$

max_pop $=\left(\left(\max \_p o p \backslash\right.\right.$ scale_interval $\left.)+1\right) *$ scale_interval

scaIe interval $\equiv \max$ pop $\backslash \overline{15}$

END IF

'Set the outermost radius (max radius).

max_radius $=$ radial_distance (number_of_radii)

'Set screen to mode 12 - vga or mcga screen type.

SCREEN. HIDE

SCREEN 12

'set up and initialize color palate.

PALETTE I5, 0

$i=14$

FOR $j=21$ TO 63 STEP 21

PAIETTE $i, 256$ * $j+63$

$i=i-I$

NEXT $j$

FOR $j=42$ TO 0 STEP -21

PAIETTE i, 256 * $63+j$ 


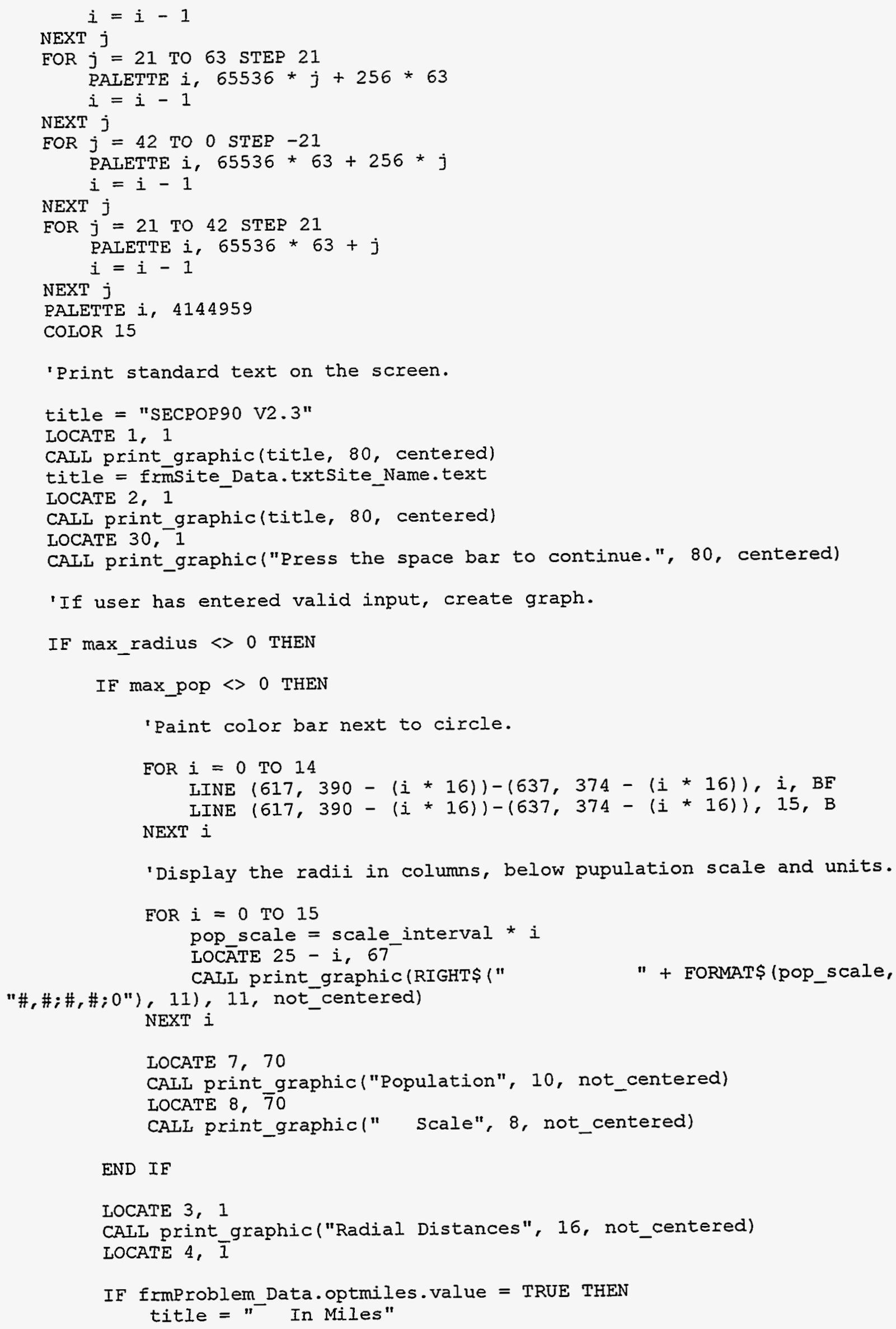


CALL print_graphic(title, 11, not_centered)

ELSE title = "In Kilometers"

END IF

CAII print_graphic(title, 13, not centered)

FOR $i=1$ TO number of radii STEP 2

LOCATE $4+(i+1) / 2,1$

CALL print_graphic(RIGHT\$(" "+FORMAT\$(radial_distance(i), ".0"),

6), 6, not centered)

IF $i<$ number of radii THEN LOCATE $4 \mp(\bar{i}+1) / 2,7$

CALI print_graphic(RIGHT\$(" " + FORMAT\$ (radial_distance $(i+1)$,

".0"), 6), 6, not centered)

END $I \bar{F}$

NEXT $i$

'Set up graphics view port, and coordinate system for it.

VIEW $(130,50)-(510,430)$

$\mathrm{y}^{2}=$ max radius

yl $=-\max$ radius

$\mathrm{x} 2=\max$ radius

$\mathrm{xl}=-\max$ radius

WINDOW $\left(\mathrm{x}_{\overline{1}}, \mathrm{y} 2\right)-(\mathrm{x} 2, \mathrm{y} 1)$

'Determine the number of degrees per segment.

rotation_increment $=2 * \mathrm{pi} /$ number_of_segments

'Divide the circle up into segments and label segments.

FOR $i=1$ TO number of segments STEP 1

rotation $=(i-1)^{*}$ rotation increment

$\mathrm{xI}=\operatorname{SIN}($ rotation) * max_radius

$y^{I}=\cos ($ rotation) $*$ max radius

$\mathrm{x} 2=\operatorname{PMAP}(\mathrm{x} 1,0)+(\operatorname{SIN}($ rotation $) * 20)$

$\mathrm{y}^{2}=\operatorname{PMAP}(\mathrm{y} 1,1)-(\cos ($ rotation $) * 15)$

LOCATE $\left(\left(y^{2}+50 !\right) / 16 !\right)+.5,((x 2+130 !) \backslash 8 !)+.5$

not_centered)

CAIL print_graphic(directions(i) + " ", len(directions (i)) + 1 ,

rotation = rotation + (rotation_increment / 2)

$x I=\operatorname{SIN}(r o t a t i o n) * \max$ radius

y1 $=\cos$ (rotation) * max_radius

NEXT i

$\operatorname{LINE}(0,0)-(x 1, y 1)$

'Draw circles to signify the different radii.

FOR $i=1$ TO number_of_radii STEP 1

NEXT i

CIRCLE $(0 !, 0 !)$, rädial_distance (i)

'Color all of the various circles.

FOR $i=1$ TO number of segments STEP 1

rotation $=((i=1) *$ rotation_increment $)$

FOR $j=1$ TO number_of radii STEP 1

- $r 1=(1-$ paint_position $) *$ radial distance $(j-1)$

$r 1=r 1+$ paint_position * radial_distance $(j)$

$x I=S I N($ rotation) * $r 1$

$\mathrm{y} I=\cos ($ rotation) $* \mathrm{r} 1$

IF max pop $=0$ THEN

paint_value $=0$ 
ELSE

paint_value $=\operatorname{FIX}($ sector_population $(i, j) / \max$ pop * 15)

IF paint_value $=15$ THEN

END IF

paint value $=14$

END IE

PAINT $(x 1, y 1)$, paint value, 15 NEXT $j$

NEXT i

END IF

'Wait until space bar is pressed.

WHILE INKEY\$ $<>$ " "

WEND

'Reset screen to default values, and show forms.

SCREEN 0

WIDTH 80

SCREEN . SHOW

END SUB 
FIIENAME: RPTABLE1.BAS

option explicit

'\$include: 'secpop90.bi'

'This function calculates and displays all of the fields for table one.

sub paint_table ()

$\operatorname{dim} i$ as integer, $j$ as integer, $k$ as integer

dim sum as long

'These two constants are the number of radii and sectors displayed.

const max_radii_displayed $=4$

const max_sectors_displayed $=6$

'Set the table caption to reflect the table type.

if (table_type $=1$ ) then

frmTable_1. Caption = "Population Table"

else

frmpable 1. Caption $=$

"Cumūlative (By Dírection) Population Table"

end if

'Determine the unit of measure.

! ! ! ! !

if (frmProblem_Data.optKilometers.value) then frmTable 1.1blDistance Heading.caption =

"Radi $\bar{i}$ (Kilometers)"

else

end if

ErmTable_1.IblDistance_Heading.caption = "Radii (Miles)"

'Verify that coordinates are within array.

if (x_position < 1 ) then

$x$ position $=1$

elsei $\bar{f}$ (number_of_radii <= max_radii_displayed) then $x$ position ${ }^{-}=\bar{I}$

elsei五 ( $(x$ position + max_radii_displayed - 1) > number of radii) then

end $i \bar{f}$

$x \_p o s i \overline{t i o n}=$ number_of_radii - max_radii_displayed + 1

if (y_position < 1 ) then

$y$ position $=1$

elseif (ly_position + max_sectors_displayed +1$)>$ number_of_segments) then 
end ${ }_{i \bar{f}}$ position $=$ number_of_segments - max_sectors_displayed +1

'Fill the radii label values.

for $i=0$ to max_radii_displayed -1 step 1

if ( $\left(x \_p o s i t i o n+i\right) \ll$ number_of_radii) then

frmTable_1.IblDistance arrāy $(\bar{i}) \cdot \operatorname{caption}=$

else

formats (radial_distance (x_position $+i \overline{)}$, "\#\#0.0000")

end if

frmTable_1.IblDistance_array $(i)$.caption $=" n$

next $i$

'Fill the direction label values.

for $i=0$ to max_sectors_displayed - 1 step 1

frmTable_1.Iblsector_array (i). caption $=$ directions (y_position $+i$ )

next $i$

'Fill the population sector label values with either the population

'data or the cumulative population data depending on which table

'type we're displaying. Also calculate and fill the sector sum

'labels.

for $i=0$ to max_sectors_displayed - I step 1

sum $=0$

frmTable_1.1blValue_array $(i)$. caption $=" n$

for $j=1$ to number_of_radii step 1

sum $=$ sum + sector_population $\left(y \_p o s i t i o n+i, j\right)$

if $((j>=x$ position $)$ and

$\left(j<=\left(\bar{x} \_\right.\right.$position + max_radii_displayed -1$\left.)\right)$ ) then

if (table type = 1) then

frmTable_l.IbIValue_array (i). caption = frmTāble_1.lblvālue array (i). captioñ + rights (fōmats (sectōr_population(

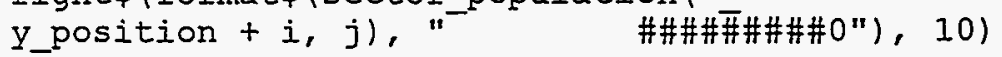

else

frmTable 1.1blValue array $(i) \cdot$ caption $=$ frmTable_1.Iblvalue_array(i) . caption + right (format\$(sum, "

end if

end if 
next j

frmTable_1.1blCsum_array $(i)$. caption $=$ format $($ sum, $" \# \# \# \# \# \# 0 ")$

next i

'Calculate and fill the radial sum labels.

for $i=0$ to max_radii_displayed - 1 step 1

if $\left(\left(x \_p o s i t i o n+i\right)<=\right.$ number_of_radii) then

sum $=0$

if (table type = 1) then

for $j^{-}=1$ to number of segments step 1 next $j$

sum $=$ sum + sector_population $(j, x$ position $+i)$

else

for $j=1$ to number of segments step 1

for $k=1$ to (xposition $+i$ ) step 1 next $k$ um $=$ sum + sector population $(j, k)$

next $j$

end if

frmable 1.1blRsum array (i). caption $=$ formats (sum, "\#\#\#\#\#\#\#0")

else

frmTable_1.lblRsum_array $(i)$.caption $=" "$

end if

next i

'Calculate and fill the total sum label.

sum $=0$

for $i=1$ to number_of_segments step 1

for $j=1$ to number_of_radii step 1

sum $=$ sum + sector_population $(i, j)$

next $j$

next $i$

frmTable_1.1blTotal.caption = format\$(sum, "\#\#\#\#\#\#\#0")

end sub 


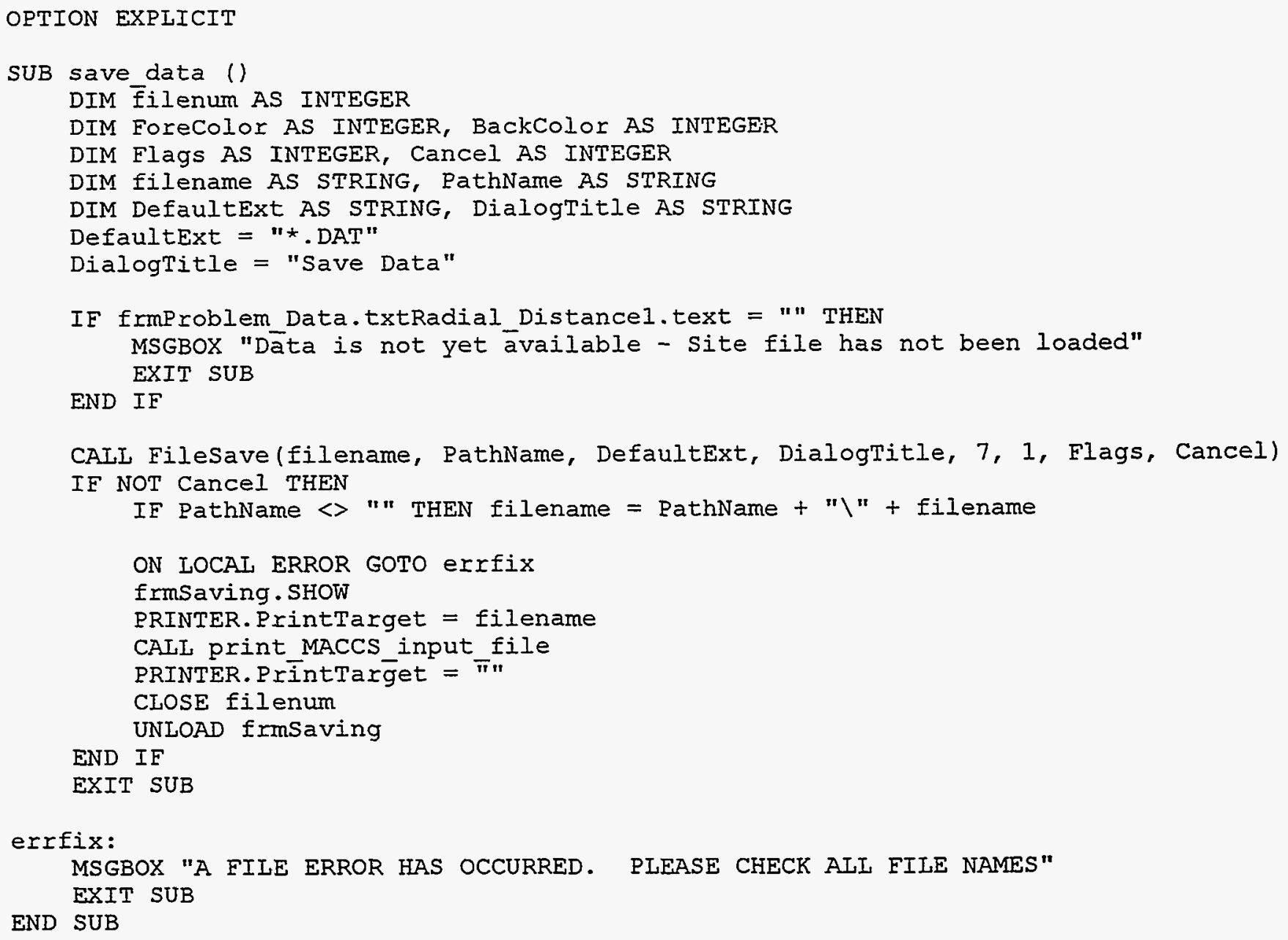

CAII FileSave (filename, PathName, DefaultExt, DialogTitle, 7, 1, Flags, Cancel) IF NOT Cancel THEN

IF PathName <> " "THEN filename = PathName + "\" + filename

ON IOCAI ERROR GOTO errfix

frmsaving. SHOW

PRINTER.PrintTarget $=$ filename

CAIL print MACCS input file

PRINTER. PrintTarḡet $=\bar{\pi} "$

CLOSE filenum

END IF UNLOAD frmsaving

EXIT SUB

errfix:

MSGBOX "A FIIE ERROR HAS OCCURRED. PIEASE CHECK ALI FILE NAMES" EXIT SUB

END SUB 


\section{OPTION EXPIICIT}

'\$INCLUDE: 'secpop90.bi'

'Define all of the dynamic arrays used in SECPOP9o.

REDIM SHARED radial distance (max number of radii) AS SINGLE REDIM SHARED raddis (max_number_of_zadii) A $\bar{S}$ SINGLE

REDIM SHARED regional_rādii (max̄ number of radii) AS SINGLE

REDIM SHARED population (max number of $\bar{r}$ adi $\bar{i}$ ) as long

REDIM SHARED population_threshold(max_number_of_radii) as long

REDIM SHARED region_index(max_number_of_radii, number of segments) AS INTĒGER

REDIM SHA $\bar{R} E D$ econ_data (max_econ_regions) AS economic_data

REDIM SHARED sector_population(number_of_segments, max number of radii) AS LONG

REDIM S̈HARED sector_area (number_of_segments, max number_of radii) AS LONG

REDIM STHRED Sector_frclnd (number_of_segments, max_number_of_radii) AS SINGLE

REDIM SHARED county_state (number_of_counties) AS STRING * 2

REDIM SHARED county_name (number_of_counties)

AS STRING * max county name Iength

REDIM SHARED countȳ_frclnd (number_of_counties) AS SINGLE

REDIM SHARED county_frmfrc (number of counties) AS SINGLE

REDIM SHARED county dpf (number of counties) AS SINGLE

REDIM SHARED county asfp (number of counties) AS SINGLE

REDIM SHARED county_vfrm(number_of_counties) AS SINGLE

REDIM SHARED county_vnfrm(number_of_counties) AS SINGLE

REDIM SHARED directions (number_of_segments) AS STRING

REDIM SHARED layer_number (max_layers) AS INTEGER

REDIM SHARED data $\bar{f}$ lag (max laȳers) AS INTEGER

REDIM SHARED display flag(max layers) AS INTEGER

REDIM SHARED active_layer(max_layers) AS INTEGER

REDIM SHARED layer ñame (max layers) AS STRING

REDIM SHARED pts_cōlor(max Iayers) AS INTEGER

REDIM SHARED pts-type (max_layers) AS INTEGER

REDIM SHARED pts_size (max_layers) AS INTEGER

REDIM SHARED pts mode (max layers) AS INTEGER

REDIM SHARED Iines_color(max_layers) AS INTEGER

REDIM SHARED Iines type (max Iayers) AS INTEGER

REDIM SHARED lines_size (max_layers) AS INTEGER

REDIM SHARED lines_mode (max layers) AS INTEGER

REDIM SHARED polyg_color (max layers) AS INTEGER

REDIM SHARED polyg_type (max_Iayers) AS INTEGER

REDIM SHARED polyg_size (max_layers) AS INTEGER

REDIM SHARED polyg mode (max layers) AS INTEGER

REDIM SHARED text_color(max layers) AS INTEGER

REDIM SHARED text_type (max_layers) AS INTEGER

REDIM SHARED text_size (max_layers) AS INTEGER

REDIM SHARED text_mode (max_layers) AS INTEGER

REDIM SHARED radial_area(max_number_of_radii) AS SINGLE

'Load and show the main SECPOP90 form. 
IOAD frmMain

frmMain. SHOW 


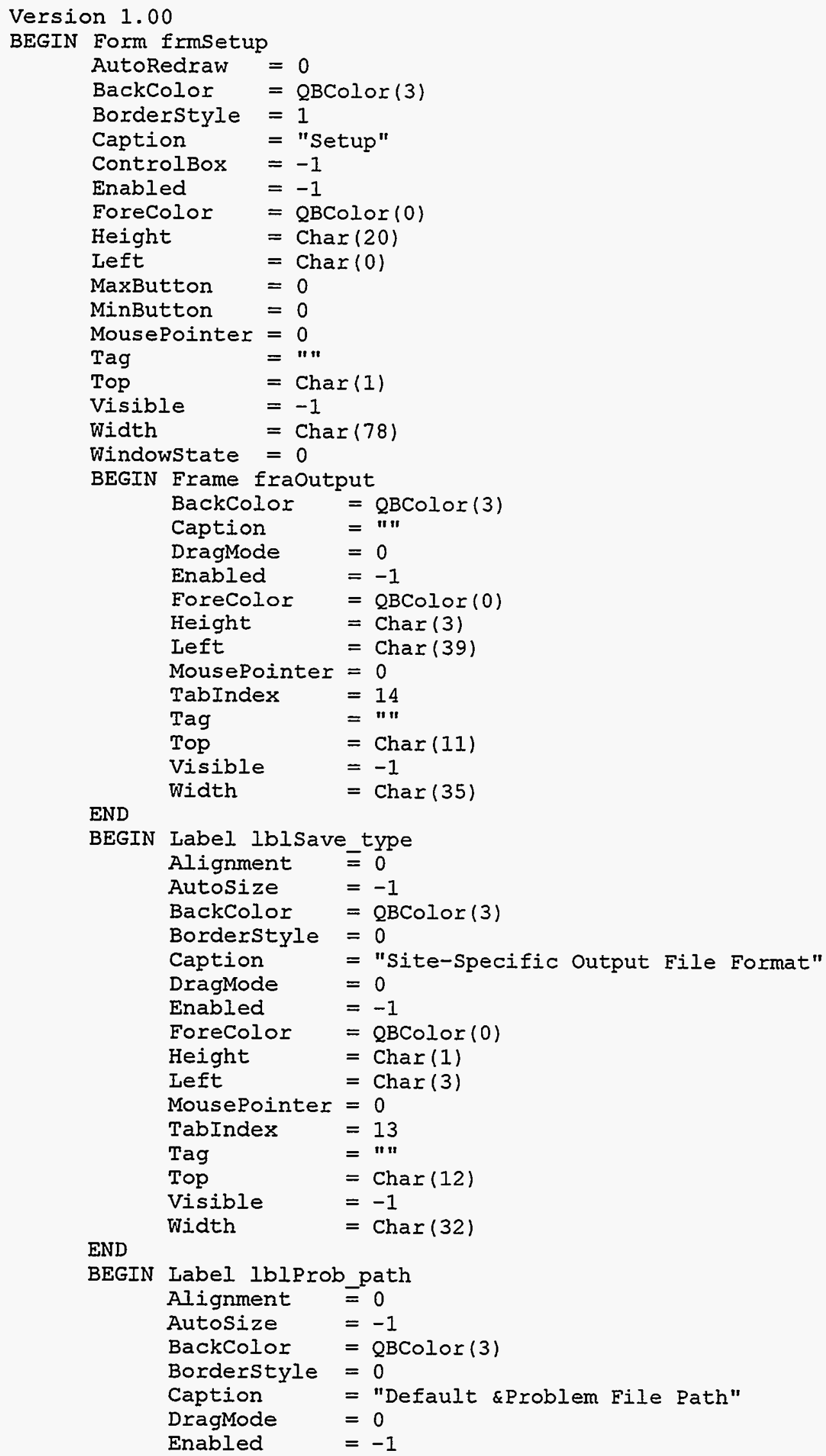




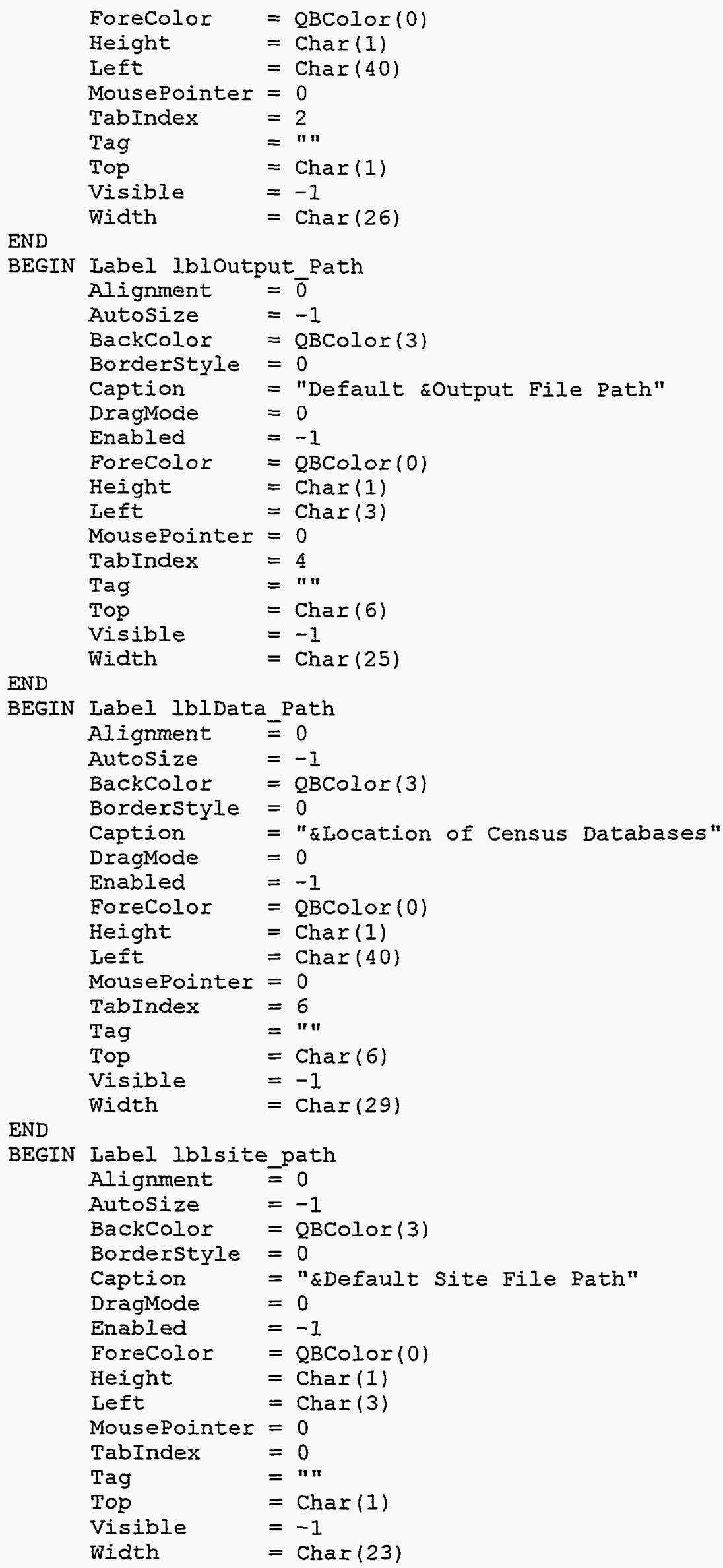


END

BEGIN TextBox txtsite Path

BackColor = $\bar{Q} B C o l o r(3)$

Borderstyle $=1$

DragMode $\quad=0$

Enabled $\quad=-1$

Forecolor = QBColor $(0)$

Height $=$ Char (3)

Left $=\operatorname{Char}(2)$

Mousepointer $=0$

Multiline $=0$

ScrollBars $=0$

TabIndex $\quad=1$

Tabstop $=-1$

Tag $=" n$

Text = "SITES"

Top $=\operatorname{Char}(2)$

Visible $\quad=-1$

Width $\quad=\operatorname{Char}(35)$

END

BEGIN TextBox txtProblem path

BackColor = QBCólor (3)

Borderstyle $=1$

DragMode $\quad=0$

Enabled $\quad=-1$

Forecolor $=$ QBColor $(0)$

Height $=\operatorname{Char}(3)$

Left $=$ Char (39)

MousePointer $=0$

Multiline $=0$

ScrollBars $=0$

TabIndex $\quad=3$

Tabstop $\quad=-1$

Tag = " "

Text $=$ "SITES"

Top $=\operatorname{Char}(2)$

Visible $\quad=-1$

Width $\quad=\operatorname{Char}(35)$

END

BEGIN TextBox txtoutput path

BackColor = QBColor (3)

Borderstyle $=1$

Dragmode $=0$

Enabled $\quad=-1$

ForeColor $=$ QBColor $(0)$

Height $=$ Char (3)

Left = Char (2)

MousePointer $=0$

Multiline $\doteq 0$

ScrollBars $=0$

TabIndex $=5$

Tabstop $\quad=-1$

Tag $="$ "

Text = "."

Top $=\operatorname{Char}(7)$

Visible $\quad=-1$

Width $=\operatorname{Char}(35)$

END

BEGIN TextBox txtData path

BackColor = QBColor (3)

Borderstyle $=1$

DragMode $=0$

Enabled $\quad=-1$ 


$$
\begin{array}{ll}
\text { ForeColor } & =\text { QBColor }(0) \\
\text { Height } & =\text { Char }(3) \\
\text { Left } & =\text { Char (39) } \\
\text { MousePointer } & =0 \\
\text { Multiline } & =0 \\
\text { ScrollBars } & =0 \\
\text { TabIndex } & =7 \\
\text { Tabstop } & =-1 \\
\text { Tag } & =" 1 \\
\text { Text } & =\text { "CENSUS" } \\
\text { Top } & =\text { Char }(7) \\
\text { Visible } & =-1 \\
\text { Width } & =\text { Char (35) }
\end{array}
$$

END

BEGIN OptionButton optCSV

$$
\begin{array}{ll}
\text { BackColor } & =\text { QBColor (3) } \\
\text { Caption } & =\text { "CS\&V" } \\
\text { DragMode } & =0 \\
\text { Enabled } & =-1 \\
\text { EoreColor } & =\text { QBColor (0) } \\
\text { Height } & =\text { Char (1) } \\
\text { Left } & =\text { Char (60) } \\
\text { MousePointer } & =0 \\
\text { TabIndex } & =9 \\
\text { Tabstop } & =0 \\
\text { Tag } & =" " \\
\text { Top } & =C h a r(12) \\
\text { Value } & =0 \\
\text { Visible } & =-1 \\
\text { Width } & =C h a r(7)
\end{array}
$$

END

BEGIN OptionButton optMACCS

$$
\begin{array}{ll}
\text { BackColor } & =\text { QBColor }(3) \\
\text { Caption } & =" \text { \&MCCS" } \\
\text { DragMode } & =0 \\
\text { Enabled } & =-1 \\
\text { EoreColor } & =\text { QBColor (0) } \\
\text { Height } & =\text { Char }(1) \\
\text { Left } & =\text { Char }(45) \\
\text { MousePointer } & =0 \\
\text { TabIndex } & =8 \\
\text { TabStop } & =-1 \\
\text { Tag } & =" 1 \\
\text { Top } & =\text { Char (12) } \\
\text { Value } & =-1 \\
\text { Visible } & =-1 \\
\text { Width } & =\text { Char (9) }
\end{array}
$$

END

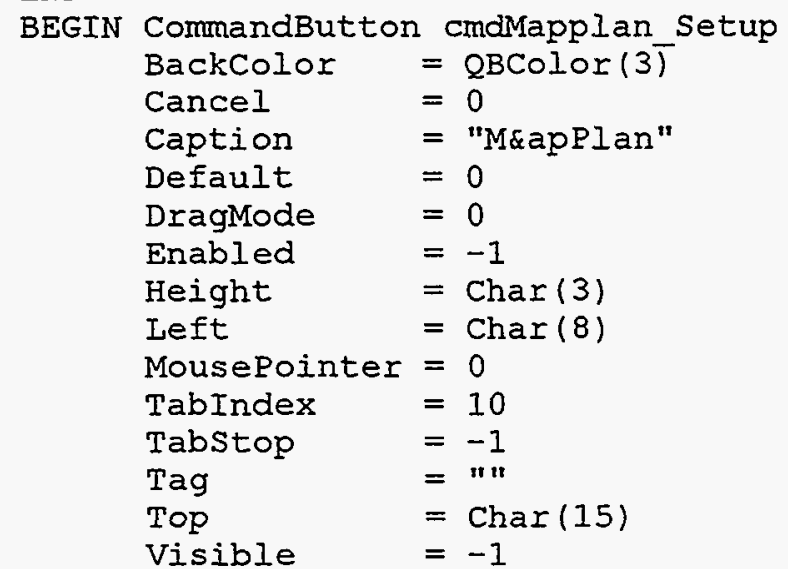


END

Width $=\operatorname{Char}(16)$

BEGIN CommandButton cmdSave Settings

BackColor $=$ QBColor $(3)$

Cancel $=0$

Caption $=$ "\&Save Changes"

Default $\quad=0$

Dragmode $\quad=0$

Enabled $\quad=-1$

Height $=\operatorname{Char}(3)$

Left $=$ Char (29)

MousePointer $=0$

TabIndex $=11$

Tabstop $\quad=-1$

Tag $=" 1$

Top $=\operatorname{Char}(15)$

Visible $\quad=-1$

END

Width $=\operatorname{Char}(16)$

BEGIN CommandButton cmdCancel

$\begin{array}{lll}\text { BackColor } & =\text { QBColor (3) } \\ \text { Cancel } & =-1 \\ \text { Caption } & =" \text { \&Close" } \\ \text { Default } & =-1 \\ \text { DragMode } & =0 \\ \text { Enabled } & =-1 \\ \text { Height } & =\text { Char (3) } \\ \text { Left } & =\text { Char (51) } \\ \text { MousePointer } & =0 \\ \text { TabIndex } & =12 \\ \text { TabStop } & =-1 \\ \text { Tag } & =" " \\ \text { Top } & =\text { Char (15) } \\ \text { Visible } & =-1 \\ \text { Width } & =\text { Char (16) }\end{array}$

END

END

OPTION EXPLICIT

'\$INCLUDE: 'secpop90.bi'

'This form allows the user to set certain paths which will be remembered

'by the program until they are changed. These paths include input and

'output directories, among other things. Setup also allows the user to

'specify which file format they would like to use with their output

'data files - MACCS input file or comma seperated variable formats.

SUB cmdCancel_Click ()

'If the user selects the cancel button from setup, the setup form is

'hidden, but the changes are retained until the program is exited.

frmsetup.HIDE

END SUB

SUB cmdMapplan_setup_Click ()

RUN "mp_setup.exe"

END SUB

SUB cmdSave_Settings_Click () 
'This routine saves setup data to a file so that the program has

'access to it every time the program is run.

DIM filenum AS INTEGER

'Locate first free file handle.

filenum $=$ FREEFILE

'Open file (called secpop90.cfg) and output all of the data to it. OPEN "SECPOP90.CFG" FOR OUTPUT AS \#filenum

PRINT \#filenum, frmsetup.txtsite path.text

PRINT \#filenum, frmsetup.txtProbIem path.text

RRINT \#filenum, frmsetup.txtoutput_path.text

PRINT \#filenum, frmsetup.txtData path.text

IF frmsetup.optMACCS.value = -1 THEN PRINT \#filenum, "MACCS" ELSE PRINT \#filenum, "CSV"

CLOSE \#filenum

END SUB

SUB txtData_path_KeyPress (KeyAscii AS INTEGER)

'Convert all input to upper case.

DIM C AS STRING

$c=\operatorname{CHRS(KeyAscii)}$

KeyAscii = ASC (UCASE\$ (c\$))

END SUB

SUB txtoutput_path_KeyPress (KeyAscii AS INTEGER)

' Convert all input to upper case.

DIM c AS STRING

$c=$ CHRS (KeyAscii)

KeyAsci $i=\operatorname{ASC}(\operatorname{UCASE}(c \$))$

END SUB

SUB txtProblem_path_KeyPress (KeyAscii AS INTEGER)

'Convert all input to upper case.

DIM C AS STRING

$c=\operatorname{CHR}($ KeyAscii)

KeyAscii = ASC (UCASE\$ (C\$))

END SUB

SUB txtSite_Path_KeyPress (KeyAscii AS INTEGER)

'Convert all input to upper case.

DIM C AS STRING 


$$
\begin{aligned}
& c=\text { CHR\$ (KeyAscii) } \\
& \text { KeyAsCi }=\text { ASC (UCASE\$ (c\$)) }
\end{aligned}
$$

END SUB 


\section{OPTION EXPLICIT}

'\$INCLUDE: 'secpop90.bi'

'This module allows the user to create, edit, and and view site files

SUB new_site ()

'This module initializes the new site form to its default values,

'and shows the form.

frmMain.mnuSave_SiteCom. Enabled = FALSE

frmMain.mnuSave As_SiteCom. Enabled = FALSE

frmsite Data.Caption = CURDIRS + "\" + "NEW_SITE.SIT"

frmProbIem Data.txtSite_File_Name.text = "NĒW_SITE.SIT"

frmsite Dā̌ta.txtSite Name.tex̄t = " "

frmsite-Data.txtLongitude_Degrees.text $=" n$

frmsite Data.txtLongitude Minutes.text $=" n$

frmsite-Data.txtIongitude- Seconds.text $=" n$

frmSite_Data.txtLatitude_Degrees.text = " "

frmsite-Data.txtLatitude Minutes.text $=" n$

frmsite - Data.txtLatitude Seconds.text $=" "$ "

frmsite-Data.txtSite Remārks. text $=" "$

frmsite_Data.SHOW MOD̄I

END SUB

SUB open_site ()

'This routine opens a previously created site file.

DIM site name AS STRING, site remarks AS STRING, dummy AS STRING

DIM longitude_degrees AS INTEḠER, longitude_minutes AS INTEGER,

longitude_seconds AS INTEGER

DIM lätitude_degrees AS INTEGER, latitude_minutes AS INTEGER, latitude_seconds

AS INTEGER

DIM ForeColor AS INTEGER, BackColor AS INTEGER

DIM Flags AS INTEGER, Cancel AS INTEGER

DIM filename AS STRING

STATIC Pathname AS STRING

DIM DefaultExt AS STRING, DialogTitle AS STRING

CONST site_file $=20$, file_buffer $=1024$

'Initialize file dialog box.

DefaultExt $=" \star$.SIT"

Pathname = frmsetup.txtsite_path.text

DialogTitle = "Open Site"

BackColor $=$ WHITE

ForeColor $=$ BLACK

'Enable local error checking.

ON IOCAI ERROR GOTO site_file_error

'Call file dialogue box.

CALL Fileopen(filename, Pathname, DefaultExt, DialogTitle, ForeColor, BackColor, Flags, Cancel) 
'If user did not select cancel from the file dialog box then proceed.

IF NOT Cancel THEN

'If file is not in current directory then add path to filename.

IF Pathname $<$ " "THEN filename = Pathname + "\" + filename

'Open site file and read in the site data, placing it into temp variables.

file_buffer

OPEN filename FOR INPUT ACCESS READ IOCK WRITE AS \#site file IEN =

IINE INPUT \#site_file, dummy

IINE INPUT \#site file, site name

LINE INPUT \#site_file, dummy

LINE INPUT \#site_file, dummy

INPUT \#site file, longitude degrees

INPUT \#site-file, longitude minutes

INPUT \#site-file, Iongitude seconds

LINE INPUT \#site_file, dummy

INPUT \#site file, latitude degrees

INPUT \#site_file, latitude minutes

INPUT \#site file, latitude seconds

IINE INPUT \#site file, dumy

LINE INPUT \#site_file, site_remarks

CLOSE site_file

'set window caption to filename.

frmsite_Data.Caption = filename

'Move file values from temporary variables to the correct form locations.

frmProblem_Data.txtSite_File_Name.text = filename

frmsite Dā̄a.txtsite Name.text = site name

frmsite_Data.txtLongītude_Degrees.tex $\bar{t}=$ STRS (longitude_degrees)

frmsite_Data.txtLongitude_Minutes.text = STR\$ (longitude_minutes)

frmsite_Data.txtLongitude_seconds.text = STRS (longitude_seconds)

frmsite Data.txtLatitude Degrees.text = STRS (latitude dégrees)

frmsite Data.txtlatitude Minutes.text = STRS (latitude minutes)

frmsite Data.txtIatitude_Seconds.text = STR\$ (latitude_seconds)

frmsite_Data.txtsite_Remārks.text = site_remarks

'If site form is not visible, show it.

IF (frmsite Data.Visible = FAISE) THEN

END IF frmsite_Data.SHOW MODAI

END IF

EXIT SUB

site_file_error:

'If an error occurs, inform the user and exit the sub routine.

MSGBOX "Error in opening or reading site file - check file name." EXIT SUB

END SUB

suB print_site () 


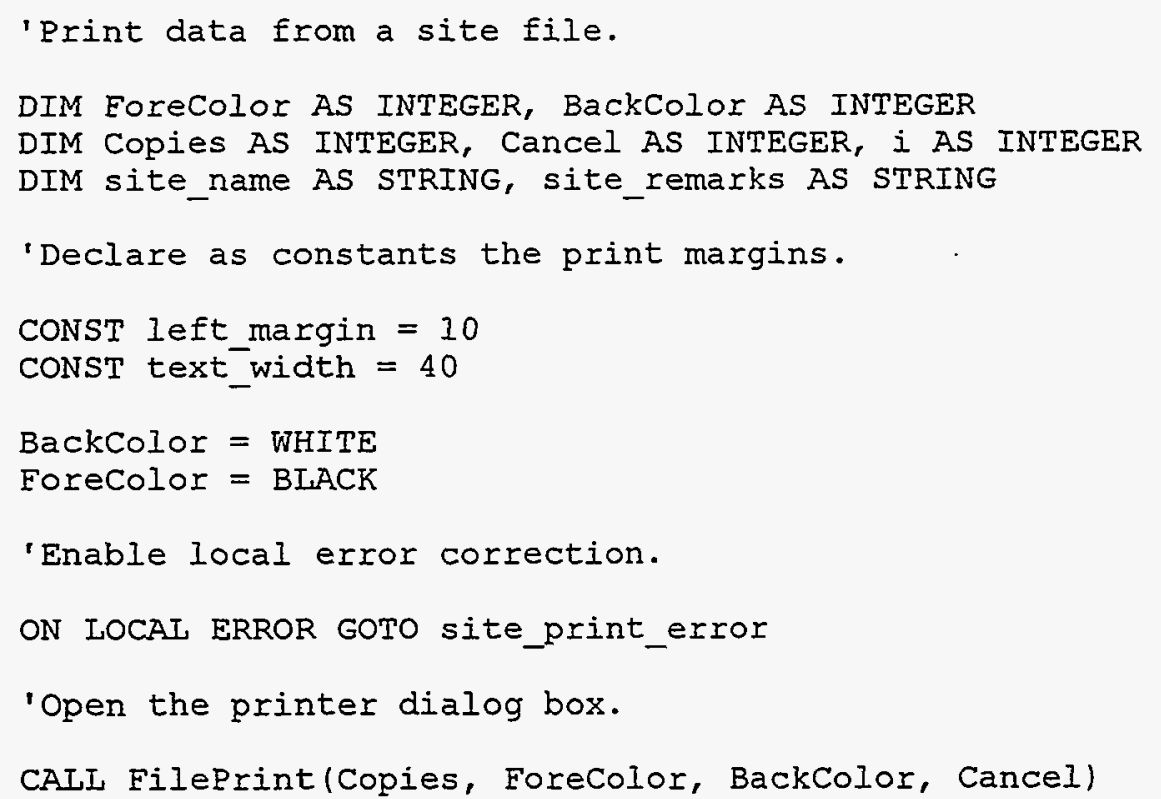

'If the user did not select cancel from the printer dialog then proceed.

IF NOT Cancel THEN

'Execute loop once for each copy the user has requested.

FOR $i=1$ TO Copies

PRINTER.PRINT TAB (left margin); "File Name:";

PRINTER.PRINT TAB (left_margin + IEN("Site Coordinates: "));

frmsite_Data.Caption

PRINTER. PRINT

PRINTER.PRINT TAB (left margin); "Site Name:";

site name = fmsite Data.txtsite Name.text

Coordinates: ") )

CAIL print text(site name, text width, left margin + LEN("Site

PRINTER. PRINT

PRINTER.PRINT TAB (left margin); "Site Coordinates:"; PRINTER. PRINT TAB (left_margin + LEN("Site Coordinates: ")); "Longitude

(Degrees, Minutes, Seconds)" PRINTER. PRINT USING " \#\#"; TAB(left margin + LEN("Site

Coordinates: ")); VAL(frmsite Data.txtLongitude Degrees.text); PRINTER.PRINT USING " \#";

VAI (Imsite_Data.txtLongitude Minutes.text); PRINTER.PRINT USIN̄G " \#\#";

VAI (Ermsite Data.txtIongitude Seconds.text) PRINTER.PRINT TAB (left_margin + LEN("Site Coordinates: ")); "Latitude

(Degrees, Minutes, Seconds)" PRINTER.PRINT USING " \#\#"; TAB (left margin + LEN("Site

Coordinates: ")); VAI(frmSite_Data.txtIatitude_Degrees.text); PRINTER.PRINT USING " \#\#";

VAI (frmsite Data.txtlatitude Minutes.text); PRINTER.PRINT USING " \#\#";

VAL (frmsite_Data.txtLatitude_seconds.text)

PRINTER. PRINT

PRINTER.PRINT TAB (left margin); "Site Remarks:";

site remarks = fmSite Data.txtsite Remarks.text

Coordinates: "))

CALI print_text(site_remarks, text_width, left_margin + IEN("Site

PRINTER. NEWPAGE

NEXT i 
PRINTER. ENDDOC

END IE

EXIT SUB

site_print_error:

'IF an error occurs, notify the user and exit the sub routine.

MSGBOX "Device error - Check printer or other print device." EXIT SUB

END SUB

SUB save_as_site ()

'Save a site file using a new name.

CONST site_file $=20$, file_buffer $=1024$ STRING

DIM site_name AS STRING, site_remarks AS STRING, dummy AS STRING, Caption AS

DIM longitude degrees AS INTEGER, longitude minutes AS INTEGER,

longitude seconds AS INTEGER

DIM lätitude_degrees AS INTEGER, latitude_minutes AS INTEGER, latitude_seconds AS INTEGER

DIM EoreColor AS INTEGER, BackColor AS INTEGER

DIM Elags AS INTEGER, Cancel AS INTEGER

DIM filename AS STRING, Pathname AS STRING

DIM DefaultExt AS STRING, DialogTitle AS STRING

DIM temp AS INTEGER

'Check validity of data entered by user, warn user if there is a problem.

temp = verify_input (2)

'set default file to name of site window.

Caption = frmsite_Data.Caption

'Remove path from filename.

CAIL parse_path_and_file(Caption, Pathname, filename)

'Initialize file dialog box.

Defaultext $=" *$.SIT"

Pathname $=$ frmsetup.txtsite path.text

DialogTitle = "Save As Site"

BackColor $=$ WHITE

ForeColor $=$ BLACK

'Call file dialogue box.

CALL FileSave(filename, Pathname, DefaultExt, DialogTitle, ForeColor, BackColor, Flags, Cancel)

'If user did not select cancel from the file dialog box, then proceed.

IF NOT Cancel THEN

'If file is not in the current directory, then add the path to 
'the filename.

IF Pathname $<>$ " THEN filename = Pathname + "\" + filename

frmsite Data. Caption = filename

frmProblem_Data,txtSite_File_Name.text = filename

'Call function to save the data.

CALL save_site

END IF

END SUB

SUB save_site ()

'Save data from a site form.

'Declare as constant the site file handle and the file buffer size.

CONST site_file $=20$, file_buffer $=1024$

DIM filename AS STRING, Pathname AS STRING

DIM DefaultExt AS STRING, DialogTitle AS STRING

'Set default filename to name of site window.

filename = frmsite_Data. Caption

'Enable local errror checking.

ON LOCAI ERROR GOTO site_save_error

'If the site has not yet been named, call save as.

IF filename $="$ "THEN

CALI save_as_site

EXIT SUB

END IF

'Open the site file and send the site info to it.

OPEN filename FOR OUTPUT ACCESS WRITE LOCK READ WRITE AS \#Site file LEN = file buffer

PRINT \#site file, "Site Name:"

PRINT \#site file, frmsite Data.txtsite Name.text

PRINT \#site-file, "Site coordinates:"

PRINT \#site-file, "Longitude (Degrees, Minutes, Seconds)"

PRINT \#site file, USING " \#\#";

VAL (frmsite Datā.txtlongitude Degrees.text); PRINT \#site file, USING "- \#\#";

VAL (frmsite Datā.txtLongitude Minutes.text);

PRINT \#Site file, USING "- \#\#"; VAL (frmsite Data.txtLongitude Seconds.text)

PRINT \#site file, "Latitude (Degrees, Minutes, sēconds)"

PRINT \#site file, USING "

VAI (frmsite Data.txtLatitude Degrees.text);

PRINT \#site file, USING " \#\#"; VAI (frmsite Data.txtlatitude Minutes.text);

PRINT \#site file, USING " \#\#"; VAL (frmSite_Data.txtLatitude_seconds.text)

PRINT \#site file, "Site Remarks:"

PRINT \#site file, frmsite Data.txtsite Remarks.text

CLOSE site $\bar{f}$ ile

'Reset window names to match site file name. 
frmsite Data. Caption = filename

frmProbİem_Data.txtsite_File_Name.text = filename

EXIT SUB

site_save_error:

'If an error occurs, inform the user and exit sub.

MSGBOX "Error opening or creating site file"

EXIT SUB

END SUB 


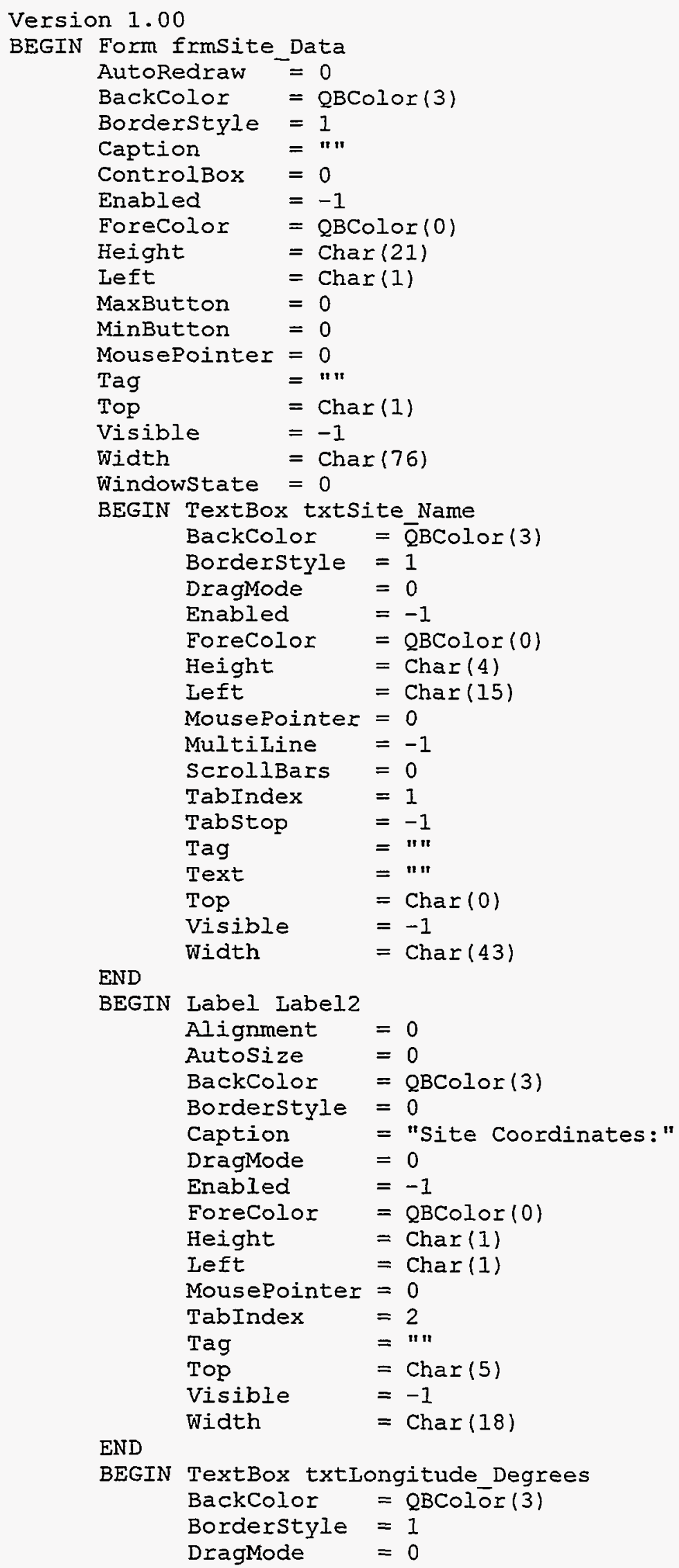




\begin{tabular}{|c|c|c|}
\hline & $\begin{array}{l}\text { Enabled } \\
\text { ForeColor } \\
\text { Height } \\
\text { Left } \\
\text { MousePointer } \\
\text { Multiline } \\
\text { ScrollBars } \\
\text { TabIndex } \\
\text { Tabstop } \\
\text { Tag } \\
\text { Text } \\
\text { Top } \\
\text { Visible } \\
\text { Width }\end{array}$ & $\begin{array}{l}=-1 \\
=\text { QBColor }(0) \\
=\text { Char }(3) \\
=\operatorname{Char}(48) \\
=0 \\
=0 \\
=0 \\
=14 \\
=-1 \\
=11 \\
=1 " \\
=\text { Char }(8) \\
=-1 \\
=\text { Char }(7)\end{array}$ \\
\hline \multirow{2}{*}{$\begin{array}{l}\text { END } \\
\text { BEGIN }\end{array}$} & & \\
\hline & $\begin{array}{l}\text { TextBox txtlo } \\
\text { BackColor } \\
\text { BorderStyle } \\
\text { DragMode } \\
\text { Enabled } \\
\text { ForeColor } \\
\text { Height } \\
\text { Left } \\
\text { MousePointer } \\
\text { Multiline } \\
\text { ScrollBars } \\
\text { TabIndex } \\
\text { TabStop } \\
\text { Tag } \\
\text { Text } \\
\text { Top } \\
\text { Visible } \\
\text { Width }\end{array}$ & $\begin{array}{l}\text { ongitude Minutes } \\
=\text { QBColor (3) } \\
=1 \\
=0 \\
=-1 \\
=\text { QBColor (0) } \\
=\text { Char (3) } \\
=\text { Char (58) } \\
=0 \\
=0 \\
=0 \\
=15 \\
=-1 \\
=" 1 \\
=" 1 \\
=\text { Char (8) } \\
=-1 \\
=\text { Char }(5)\end{array}$ \\
\hline \multirow{2}{*}{$\begin{array}{l}\text { END } \\
\text { BEGIN }\end{array}$} & & \\
\hline & $\begin{array}{l}\text { TextBox txtLo } \\
\text { BackColor } \\
\text { BorderStyle } \\
\text { DragMode } \\
\text { Enabled } \\
\text { ForeColor } \\
\text { Height } \\
\text { Left } \\
\text { MousePointer } \\
\text { Multiline } \\
\text { ScrollBars } \\
\text { TabIndex } \\
\text { Tabstop } \\
\text { Tag } \\
\text { Text } \\
\text { Top } \\
\text { Visible } \\
\text { Width }\end{array}$ & $\begin{array}{l}\text { ongitude Seconds } \\
=\text { QBColor (3) } \\
=1 \\
=0 \\
=-1 \\
=\text { QBColor }(0) \\
=\text { Char }(3) \\
=\text { Char }(67) \\
=0 \\
=0 \\
=0 \\
=16 \\
=-1 \\
=" 11 \\
=" 1 \\
=\text { Char }(8) \\
=-1 \\
=\text { Char }(5)\end{array}$ \\
\hline \multirow{2}{*}{$\begin{array}{l}\text { END } \\
\text { BEGIN }\end{array}$} & & \\
\hline & $\begin{array}{l}\text { TextBox txtIa } \\
\text { BackColor } \\
\text { Borderstyle } \\
\text { DragMode } \\
\text { Enabled } \\
\text { ForeColor } \\
\text { Height } \\
\text { Left } \\
\text { MousePointer } \\
\text { Multiline }\end{array}$ & $\begin{array}{l}\text { atitude Degrees } \\
=Q B C o \text { lor }(3) \\
=1 \\
=0 \\
=-1 \\
=Q B C o l o r(0) \\
=\text { Char }(3) \\
=\text { Char }(13) \\
=0 \\
=0\end{array}$ \\
\hline
\end{tabular}




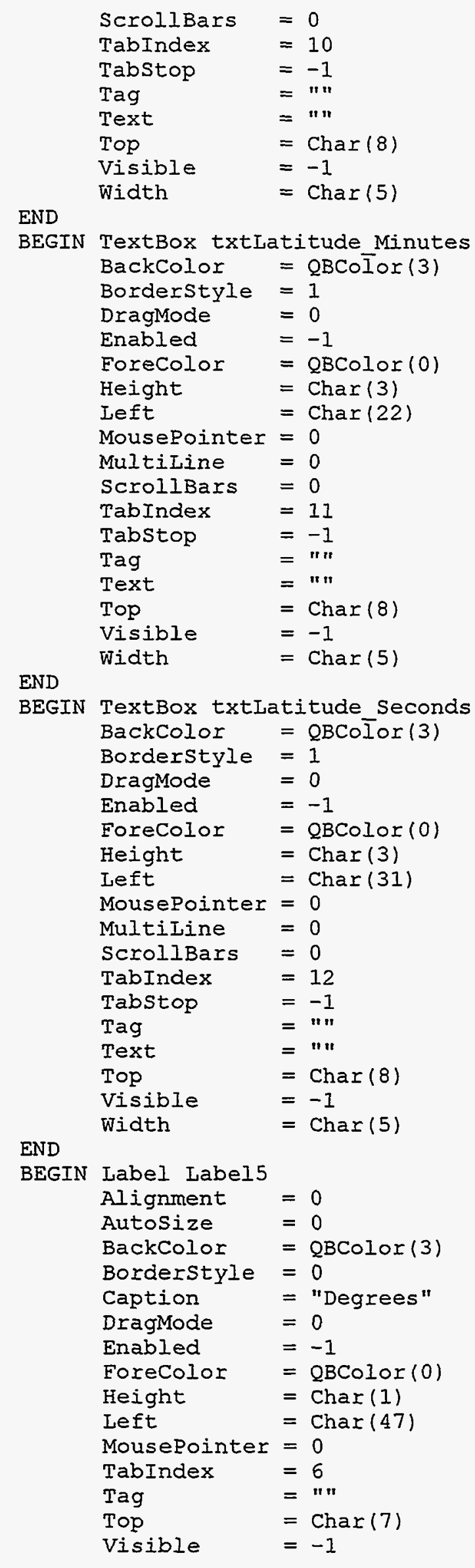




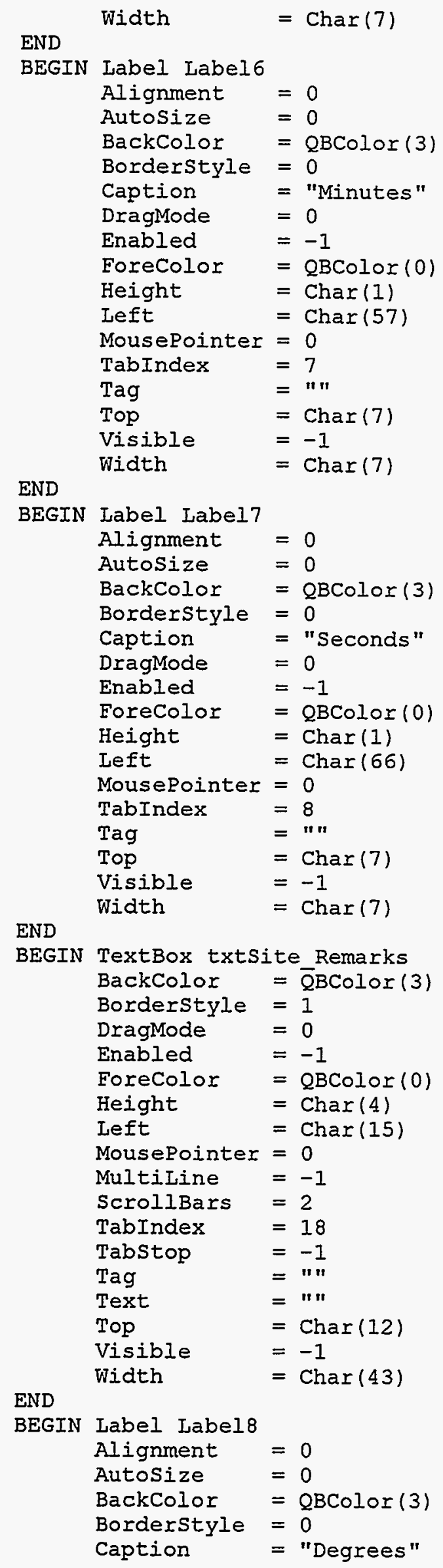




$$
\begin{array}{ll}
\text { DragMode } & =0 \\
\text { Enabled } & =-1 \\
\text { ForeColor } & =\text { QBColor }(0) \\
\text { Height } & =\text { Char }(1) \\
\text { Left } & =\text { Char }(12) \\
\text { MousePointer } & =0 \\
\text { TabIndex } & =3 \\
\text { Tag } & =" 1 \\
\text { Top } & =\text { Char }(7) \\
\text { Visible } & =-1 \\
\text { Width } & =\text { Char }(7)
\end{array}
$$

END

BEGIN Label Label9

$$
\begin{array}{ll}
\text { Alignment } & =0 \\
\text { Autosize } & =0 \\
\text { BackColor } & =\text { OBColor (3) } \\
\text { Borderstyle } & =0 \\
\text { Caption } & =\text { "Minutes" } \\
\text { DragMode } & =0 \\
\text { Enabled } & =-1 \\
\text { EoreColor } & =\text { QBColor (0) } \\
\text { Height } & =\text { Char (1) } \\
\text { Left } & =\text { Char (21) } \\
\text { MousePointer } & =0 \\
\text { TabIndex } & =4 \\
\text { Tag } & =" \text { " } \\
\text { Top } & =\text { Char (7) } \\
\text { Visible } & =-1 \\
\text { Width } & =\text { Char (7) }
\end{array}
$$

END

BEGIN Label Label10

$$
\begin{array}{ll}
\text { Alignment } & =0 \\
\text { Autosize } & =0 \\
\text { BackColor } & =\text { OBColor (3) } \\
\text { Borderstyle } & =0 \\
\text { Caption } & =\text { "Seconds" } \\
\text { DragMode } & =0 \\
\text { Enabled } & =-1 \\
\text { ForeColor } & =\text { QBColor (0) } \\
\text { Height } & =\text { Char }(1) \\
\text { Left } & =\text { Char (30) } \\
\text { MousePointer } & =0 \\
\text { TabIndex } & =5 \\
\text { Tag } & =" 1 \\
\text { Top } & =\text { Char }(7) \\
\text { Visible } & =-1 \\
\text { Width } & =\text { Char }(7)
\end{array}
$$

END

BEGIN CommandButton cmdopen

$$
\begin{array}{ll}
\text { BackColor } & =\text { QBColor (3) } \\
\text { Cancel } & =0 \\
\text { Caption } & =" \text { S\&ites" } \\
\text { Default } & =0 \\
\text { DragMode } & =0 \\
\text { Enabled } & =-1 \\
\text { Height } & =\text { Char (3) } \\
\text { Left } & =\text { Char (1) } \\
\text { Mousepointer } & =0 \\
\text { TabIndex } & =19 \\
\text { Tabstop } & =-1 \\
\text { Tag } & =" " \\
\text { Top } & =C h a r(16) \\
\text { Visible } & =-1
\end{array}
$$




\begin{tabular}{|c|c|c|}
\hline \multirow{3}{*}{$\begin{array}{l}\text { END } \\
\text { BEGIN }\end{array}$} & Width & $=\operatorname{Char}(11)$ \\
\hline & CommandButton & n cmasave \\
\hline & $\begin{array}{l}\text { BackColor } \\
\text { Cancel } \\
\text { Caption } \\
\text { Default } \\
\text { DragMode } \\
\text { Enabled } \\
\text { Height } \\
\text { Left } \\
\text { MousePointer } \\
\text { TabIndex } \\
\text { Tabstop } \\
\text { Tag } \\
\text { Top } \\
\text { Visible } \\
\text { Width }\end{array}$ & $\begin{array}{l}=\text { QBColor (3) } \\
=0 \\
=" \text { \&Save" } \\
=0 \\
=0 \\
=-1 \\
=\operatorname{Char}(3) \\
=\operatorname{Char}(31) \\
=0 \\
=21 \\
=-1 \\
=" 1 \\
=\text { Char }(16) \\
=-1 \\
=\text { Char }(11)\end{array}$ \\
\hline \multicolumn{3}{|l|}{ END } \\
\hline BEGIN & $\begin{array}{l}\text { CommandButton } \\
\text { BackColor } \\
\text { Cancel } \\
\text { Caption } \\
\text { Default } \\
\text { DragMode } \\
\text { Enabled } \\
\text { Height } \\
\text { Left } \\
\text { MousePointer } \\
\text { TabIndex } \\
\text { Tabstop } \\
\text { Tag } \\
\text { Top } \\
\text { Visible } \\
\text { Width }\end{array}$ & $\begin{array}{l}\text { cmdSave_As } \\
=\text { QBColor (3) } \\
=0 \\
=\text { "Save \&As" } \\
=0 \\
=0 \\
=-1 \\
=\operatorname{Char}(3) \\
=\text { Char (46) } \\
=0 \\
=22 \\
=-1 \\
=" " \\
=\text { Char (16) } \\
=-1 \\
=\operatorname{Char}(11)\end{array}$ \\
\hline \multicolumn{3}{|l|}{ END } \\
\hline BEGIN & $\begin{array}{l}\text { CommandButton } \\
\text { BackColor } \\
\text { Cancel } \\
\text { Caption } \\
\text { Default } \\
\text { DragMode } \\
\text { Enabled } \\
\text { Height } \\
\text { Left } \\
\text { MousePointer } \\
\text { TabIndex } \\
\text { Tabstop } \\
\text { Tag } \\
\text { Top } \\
\text { Visible } \\
\text { Width }\end{array}$ & $\begin{aligned} & \text { cmdPrint } \\
= & \text { QBColor (3) } \\
= & 0 \\
= & \text { "QPrint" } \\
= & 0 \\
= & 0 \\
= & -1 \\
= & C h a r(3) \\
= & C h a r(61) \\
= & 0 \\
= & 23 \\
= & -1 \\
= & " 1 \\
= & C h a r(16) \\
= & -1 \\
= & C h a r(11)\end{aligned}$ \\
\hline \multicolumn{3}{|l|}{ END } \\
\hline BEGIN & $\begin{array}{l}\text { CommandButton } \\
\text { BackColor } \\
\text { Cancel } \\
\text { Caption } \\
\text { Default } \\
\text { DragMode } \\
\text { Enabled } \\
\text { Height } \\
\text { Left } \\
\text { MousePointer }\end{array}$ & $\begin{array}{l}1 \text { cmdClose } \\
=\text { QBColor (3) } \\
=-1 \\
=\text { "\&Close" } \\
=-1 \\
=0 \\
=-1 \\
=C h a r(3) \\
=C h a r(16) \\
=0\end{array}$ \\
\hline
\end{tabular}




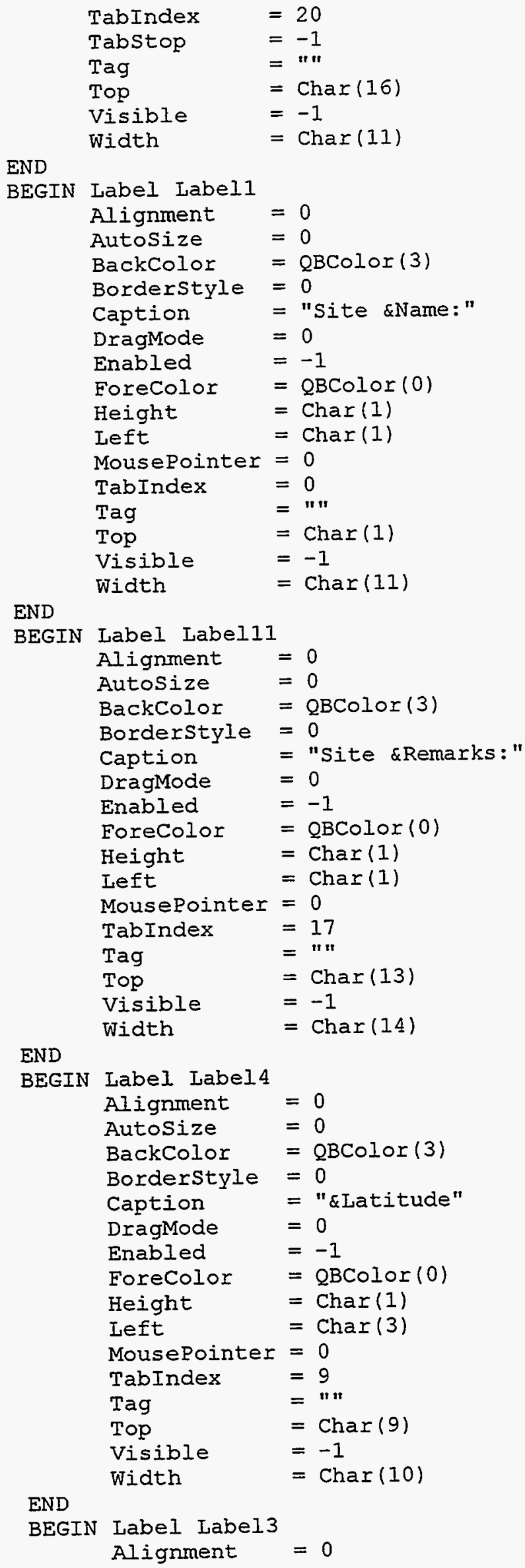




$\begin{array}{ll}\text { AutoSize } & =0 \\ \text { BackColor } & =\text { QBColor (3) } \\ \text { Borderstyle } & =0 \\ \text { Caption } & =\text { "Lon\&gitude" } \\ \text { DragMode } & =0 \\ \text { Enabled } & =-1 \\ \text { ForeColor } & =\text { OBColor }(0) \\ \text { Height } & =\text { Char }(1) \\ \text { Left } & =\text { Char (38) } \\ \text { MousePointer } & =0 \\ \text { TabIndex } & =13 \\ \text { Tag } & =" " \\ \text { Top } & =\text { Char (9) } \\ \text { Visible } & =-1 \\ \text { Width } & =\text { Char }(10)\end{array}$

\section{END}

END

\section{OPTION EXPLICIT}

'\$INCLUDE: 'secpop90.bi'

'This form allows the user to view and edit site files.

SUB cmdClose_Click ()

'This routine checks to see if the user has entered 'valid input and then unloads the site form. If the

'user data is incorrect, the program generates

'a warning.

DIM temp AS INTEGER

temp = verify_input (2)

frmsite_Data.HIDE

END SUB

SUB cmdOpen_Click ()

'This routine calls the function which opens a 'previously created site file.

CALL open_site

END SUB

SUB cmdPrint_Click ()

'This routine calls a procedure which prints

'the information in a site file

CAII print_site

END SUB

SUB cmasave_As_Click ()

'This routine calls a procedure which saves a file 'by a new name.

CALI save_as_site 
END SUB

SUB cmaSave_Click ()

'This routine calls a procedure which saves a

'site file.

CALI save_site

END SUB

SUB Form_Unload (Cancel AS INTEGER)

'This routine checks to see if the user has entered

'valid input and then unloads the site form. If the

'user data is incorrect, the program generates

'a warning.

DIM temp AS INTEGER

temp = verify_input (2)

frmsite_Data.HIDE

END SUB

SUB txtLatitude_Degrees_KeyPress (keyascii A.S INTEGER)

'Verify that the latitude degrees are a number,

'that the final number is two digits or shorter,

'and mark the site file as modified.

IF ( keyascii < 48) OR (keyascii > 57) OR

(IEN(frmsite_Data.txtLatitude_Degrees.text) $>=2$ )) AND (keyascii <> 8) AND keyascii <> 127 THEN

keyascii $=0$

ELSE

frmmain.mnuSave_SiteCom. Enabled = TRUE

frmmain.mnuSave_As_SiteCom. Enabled = TRUE

IF (INSTR(frmSite Data.Caption, " (Modified) ") =0) THEN frmSite_Data.C̄aption = frmSite_Data.Caption + " (Modified)"

END IF

IF (INSTR (frmProblem Data.txtSite File Name.text, " (Modified)") = 0) THEN END IF frmProblem_Data.ExtSite_File_Name.

END IF

END SUB

SUB txtLatitude_Minutes_KeyPress (keyascii AS INTEGER)

'For documentation, see latitude degrees.

IF ((keyascii < 48) OR (keyascii > 57) OR

(IEN (frmSite_Data.txtLatitude_Minutes.text) >= 2)) AND (keyascii <> 8) AND keyascii <> 127 THEN

keyascii $=0$ 
ELSE

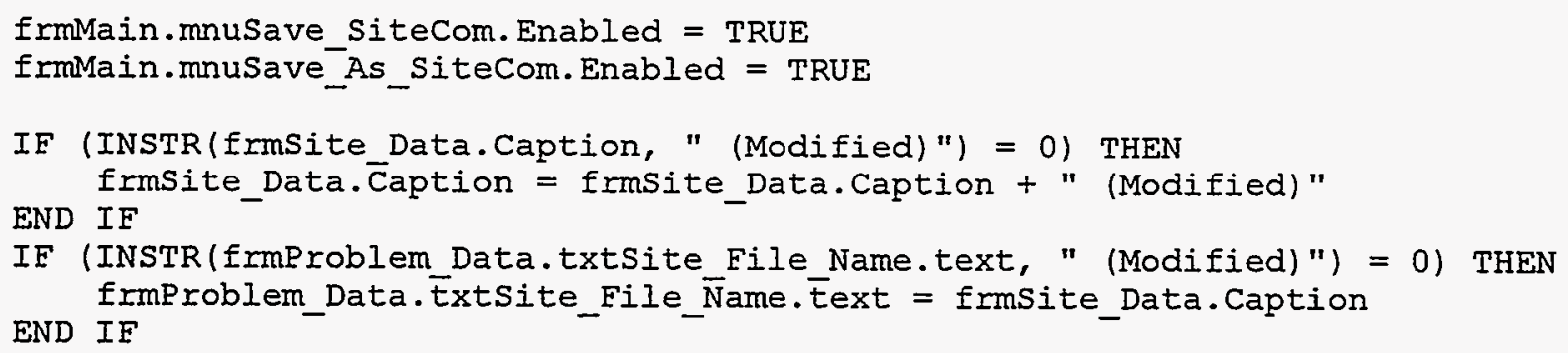

END IF

END SUB

SUB txtLatitude_Seconds_KeyPress (keyascii AS INTEGER)

'For documentation, see latitude degrees.

IF ((keyascii < 48) OR (keyascii > 57) OR

(LEN (frmSite_Data.txtLatitude_Seconds.text) >= 2)) AND (keyascii <> 8) AND keyascii <> 127 THEN

keyascii $=0$

ELSE

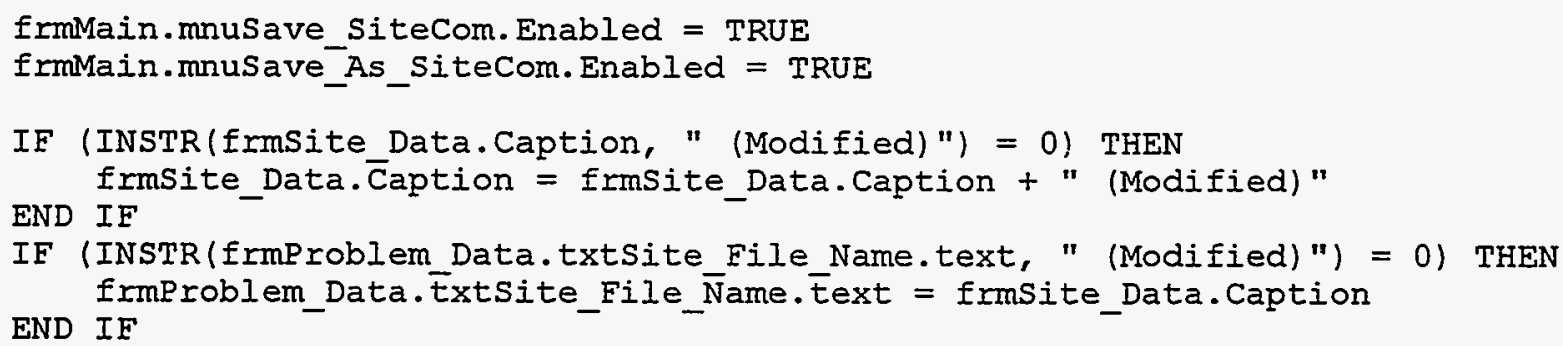

END IE

END SUB

SUB txtLongitude_Degrees_KeyPress (keyascii AS INTEGER)

'Verify that the longitude degrees are a number,

' that the final number is two digits or shorter,

'and mark the site file as modified.

IF ((keyascii < 48) OR (keyascii > 57) OR

(LEN (frmSite_Data.txtLongitude_Degrees.text) >=3)) AND (keyascii <> 8) AND keyascii <> 127 THEN

keyascii $=0$

ELSE

frmMain.mnusave SiteCom. Enabled = TRUE

frmmain.mnuSave As SiteCom. Enabled = TRUE

IF (INSTR(frmSite_Data.Caption, " (Modified)") =0) THEN 
END IF

frmsite_Data.Caption = Emsite_Data.Caption + " (Modified)"

IF (INSTR (frmproblem Data.txtSite File Name.text, " (Modified)") = 0) THEN END IF

END IE

END SUB

SUB txtIongitude_Minutes_KeyPress (keyascii AS INTEGER)

'For documentation, see longitude degrees.

IF ( (keyascii < 48) OR (keyascii > 57) OR

(LEN (frmSite_Data.txtLongitude_Minutes.text) > $=2$ )) AND (keyascii $<8$ ) AND keyascii <> 127 THEN

keyascii $=0$

ELSE

frmmain.mnusave Sitecom. Enabled = TRUE

frmmain.mnusave_As_SiteCom. Enabled = TRUE

IF (INSTR (frmsite Data.Caption, " (Modified)") = 0) THEN frmsite_Data.Caption = frmsite_Data.Caption + " (Modified)"

END IF

IF (INSTR (frmproblem_Data.txtsite_File Name.text, " (Modified)") = 0) THEN END IE frmProblem_Data.ExtSite_File_Name.Ẽext = Ermsite_Data.Caption

END IE

END SUB

SUB txtLongitude_Seconds_KeyPress (keyascii AS INTEGER)

'For documentation, see longitude degrees.

IF ( (keyascii < 48) OR (keyascii > 57) OR

(LEN (frmSite_Data.txtIongitude_Seconds.text) >=2)) AND (keyascii (> 8) AND keyascii <> 127 THEN

$$
\text { keyasci } i=0
$$

ELSE

frmMain.mnusave SiteCom. Enabled = TRUE

frmmain.musave_As_SiteCom.Enabled = TRUE

IF (INSTR(frmsite Data.Caption, " (Modified)") =0) THEN frmsite_Data.Caption = frmSite_Data.Caption + " (Modified)" END IF

IF (INSTR (ErmProblem Data.txtsite File Name.text, " (Modified)") = 0) THEN END IF frmProblem_Data.ExtSite_File_Name.Ẽext = frmsite_Data.Caption

END IF

END SUB

SUB txtSite_Name_KeyPress (keyascii AS INTEGER) 
'Disable the return key in the site field, and mark

' the site form as modified.

IF keyascii $=$ KEY_RETURN THEN

keyascii $=0$

MSGBOX "Sorry, no hard returns allowed." + CHR\$(13) + "Words will wrap automatically.", MB_OK, "Error"

ELSE

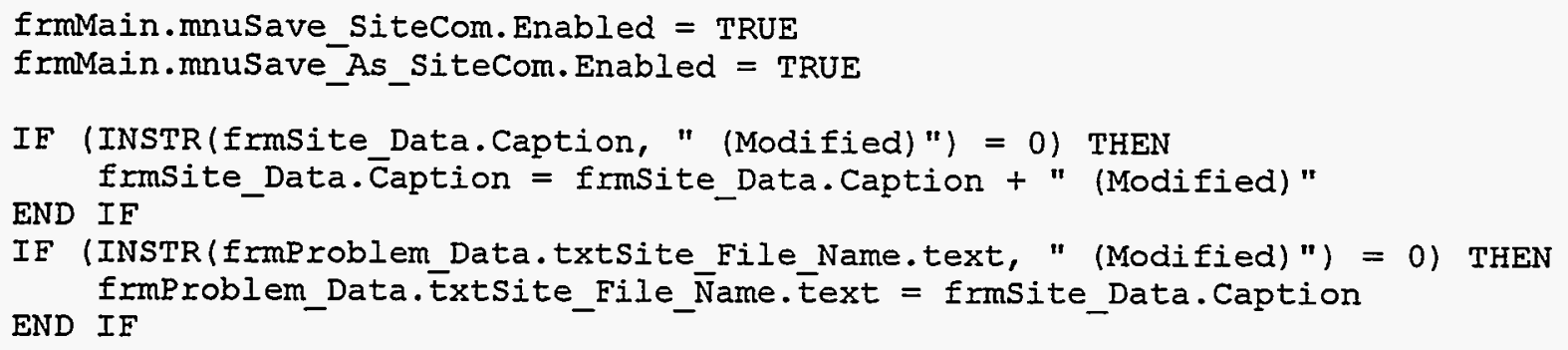

END IF

END SUB

SUB txtSite_Remarks_KeyPress (keyascii AS INTEGER)

'Disable the enter key in the remarks field, and

'Mark the site form as modified.

IF keyascii $=$ KEY_RETURN THEN

keyascii $=0$

MSGBOX "Sorry, no hard returns allowed." + CHRS(13) + "Words will wrap automatically.", MB_OK, "Error"

EISE

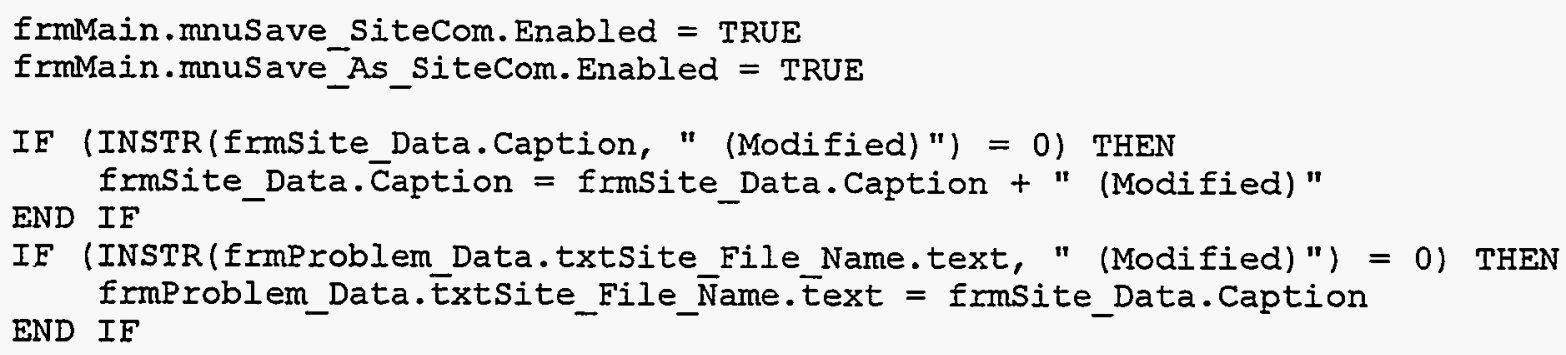

END IF

END SUB 


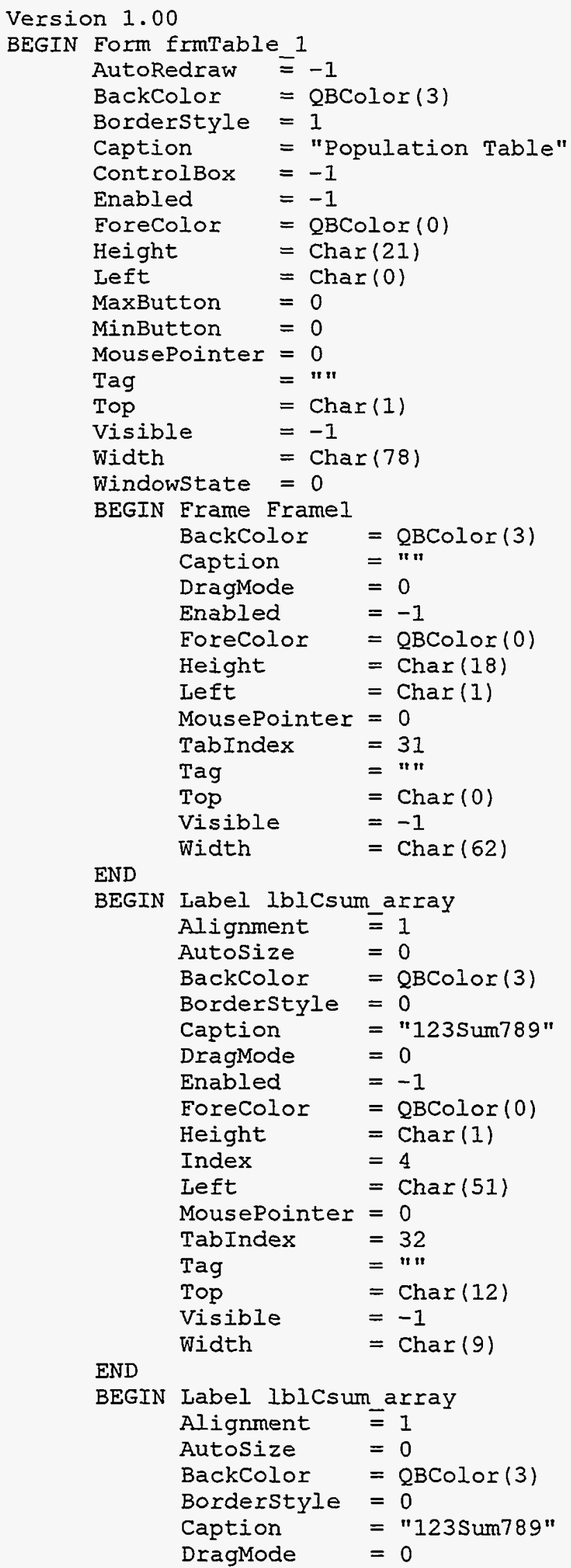




$$
\begin{array}{ll}
\text { Enabled } & =-1 \\
\text { ForeColor } & =\text { QBColor }(0) \\
\text { Height } & =\text { Char (1) } \\
\text { Index } & =5 \\
\text { Left } & =\text { Char (51) } \\
\text { MousePointer } & =0 \\
\text { TabIndex } & =33 \\
\text { Tag } & =" " \\
\text { Top } & =\text { Char (14) } \\
\text { Visible } & =-1 \\
\text { Width } & =\text { Char (9) }
\end{array}
$$

END

BEGIN Label lblCsum_array

$$
\begin{array}{ll}
\text { Alignment } & =1 \\
\text { AutoSize } & =0 \\
\text { BackColor } & =\text { oBColor (3) } \\
\text { Borderstyle } & =0 \\
\text { Caption } & =" 123 \operatorname{Sum} 789 " \\
\text { DragMode } & =0 \\
\text { Enabled } & =-1 \\
\text { EoreColor } & =\text { QBColor }(0) \\
\text { Height } & =\text { Char }(1) \\
\text { Index } & =3 \\
\text { Left } & =\text { Char }(51) \\
\text { MousePointer } & =0 \\
\text { TabIndex } & =24 \\
\text { Tag } & =" 1 \\
\text { Top } & =\text { Char (10) } \\
\text { Visible } & =-1 \\
\text { Width } & =\text { Char (9) }
\end{array}
$$

END

BEGIN Label 1blcsum_array

$$
\begin{array}{ll}
\text { Alignment } & =1 \\
\text { AutoSize } & =0 \\
\text { BackColor } & =\text { oBColor (3) } \\
\text { Borderstyle } & =0 \\
\text { Caption } & =" 123 \text { Sum789" } \\
\text { DragMode } & =0 \\
\text { Enabled } & =-1 \\
\text { EoreColor } & =\text { QBColor (0) } \\
\text { Height } & =\text { Char (1) } \\
\text { Index } & =2 \\
\text { Left } & =\text { Char (51) } \\
\text { MousePointer } & =0 \\
\text { TabIndex } & =25 \\
\text { Tag } & =" \text { " } \\
\text { Top } & =\text { Char (8) } \\
\text { Visible } & =-1 \\
\text { Width } & =\text { Char (9) }
\end{array}
$$

\section{END}

BEGIN Iabel lblcsum array

$$
\begin{array}{ll}
\text { Alignment } & \equiv 1 \\
\text { Autosize } & =0 \\
\text { BackColor } & =\text { QBColor }(3) \\
\text { Borderstyle } & =0 \\
\text { Caption } & =" 123 \text { Sum789" } \\
\text { DragMode } & =0 \\
\text { Enabled } & =-1 \\
\text { EoreColor } & =\text { QBColor }(0) \\
\text { Height } & =\text { Char }(1) \\
\text { Index } & =1 \\
\text { Left } & =\text { Char }(51) \\
\text { MousePointer } & =0
\end{array}
$$




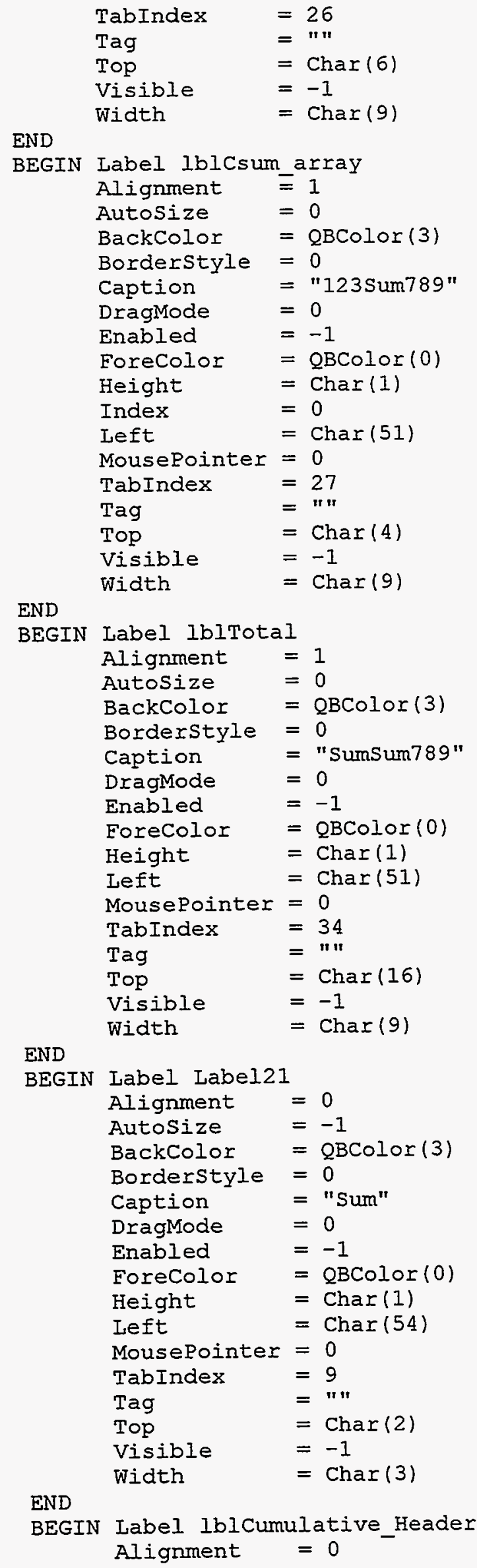




\begin{tabular}{|c|c|c|}
\hline & $\begin{array}{l}\text { AutoSize } \\
\text { BackColor } \\
\text { Borderstyle } \\
\text { Caption } \\
\text { DragMode } \\
\text { Enabled } \\
\text { ForeColor } \\
\text { Height } \\
\text { Left } \\
\text { MousePointer } \\
\text { TabIndex } \\
\text { Tag } \\
\text { Top } \\
\text { Visible } \\
\text { Width }\end{array}$ & $\begin{array}{l}=-1 \\
=Q B C o l o r(3) \\
=0 \\
=" \text { Sum" } \\
=0 \\
=-1 \\
=Q B \operatorname{Color}(0) \\
=\text { Char }(1) \\
=\text { Char }(3) \\
=0 \\
=14 \\
=" 1 \\
=\text { Char }(16) \\
=-1 \\
=\text { Char }(3)\end{array}$ \\
\hline \multicolumn{3}{|l|}{ END } \\
\hline BEGIN & $\begin{array}{l}\text { Label lblsect } \\
\text { Alignment } \\
\text { AutoSize } \\
\text { BackColor } \\
\text { Borderstyle } \\
\text { Caption } \\
\text { DragMode } \\
\text { Enabled } \\
\text { ForeColor } \\
\text { Height } \\
\text { Index } \\
\text { Left } \\
\text { MousePointer } \\
\text { TabIndex } \\
\text { Tag } \\
\text { Top } \\
\text { Visible } \\
\text { Width }\end{array}$ & $\begin{array}{l}\text { tor array } \\
=0 \\
=0 \\
=\text { QBColor (3) } \\
=0 \\
=" A " \\
=0 \\
=-1 \\
=\text { QBColor }(0) \\
=\text { Char }(1) \\
=0 \\
=\text { Char }(3) \\
=0 \\
=15 \\
=" 1 \\
=\text { Char }(4) \\
=-1 \\
=\text { Char }(3)\end{array}$ \\
\hline \multicolumn{3}{|c|}{ Width $\quad=$ Char $(3)$} \\
\hline BEGIN & $\begin{array}{l}\text { Label lblsect } \\
\text { Alignment } \\
\text { AutoSize } \\
\text { BackColor } \\
\text { BorderStyle } \\
\text { Caption } \\
\text { DragMode } \\
\text { Enabled } \\
\text { ForeColor } \\
\text { Height } \\
\text { Index } \\
\text { Left } \\
\text { MousePointer } \\
\text { TabIndex } \\
\text { Tag } \\
\text { Top } \\
\text { Visible } \\
\text { Width }\end{array}$ & $\begin{array}{l}\text { tor array } \\
=0 \\
=0 \\
=Q B C o l o r(3) \\
=0 \\
=" B^{\prime \prime} \\
=0 \\
=-1 \\
=Q B C o l o r(0) \\
=\text { Char }(1) \\
=1 \\
=\text { Char }(3) \\
=0 \\
=16 \\
=" 1 \\
=\text { Char }(6) \\
=-1 \\
=\text { Char }(3)\end{array}$ \\
\hline \multicolumn{3}{|c|}{ Mentat } \\
\hline BEGIN & $\begin{array}{l}\text { Label lblsect } \\
\text { Alignment } \\
\text { AutoSize } \\
\text { BackColor } \\
\text { BorderStyle } \\
\text { Caption } \\
\text { DragMode } \\
\text { Enabled } \\
\text { ForeColor }\end{array}$ & $\begin{array}{l}\text { tor array } \\
=0 \\
=0 \\
=0 B C \circ \operatorname{lor}(3) \\
=0 \\
=" C " \\
=0 \\
=-1 \\
=Q B C o l o r(0)\end{array}$ \\
\hline
\end{tabular}




\begin{tabular}{|c|c|c|}
\hline & Height & $=\operatorname{Char}(I)$ \\
\hline & Index & $=2$ \\
\hline & Left & $=\operatorname{Char}(3)$ \\
\hline & MousePointer & $=0$ \\
\hline & TabIndex & $=17$ \\
\hline & Tag & $=n n$ \\
\hline & TOP & $=\operatorname{Char}(8)$ \\
\hline & Visible & $=-1$ \\
\hline & width & $=\operatorname{Char}(3)$ \\
\hline END & & \\
\hline BEGIN & Label Iblsect & tor_array \\
\hline & Alignment & $=\overline{0}$ \\
\hline & Autosize & $=0$ \\
\hline & BackColor & $=$ QBColor $(3)$ \\
\hline & Borderstyle & $=0$ \\
\hline & Caption & $=" D "$ \\
\hline & DragMode & $=0$ \\
\hline & Enabled & $=-1$ \\
\hline & Forecolor & $=$ QBColor $(0)$ \\
\hline & Height & $=\operatorname{Char}(1)$ \\
\hline & Index & $=3$ \\
\hline & Left & $=\operatorname{Char}(3)$ \\
\hline & MousePointer & $=0$ \\
\hline & TabIndex & $=18$ \\
\hline & Tag & $=" n$ \\
\hline & Top & $=\operatorname{Char}(10)$ \\
\hline & Visible & $=-1$ \\
\hline & Width & $=\operatorname{Char}(3)$ \\
\hline END & & \\
\hline BEGIN & Label IbIsect & tor_array \\
\hline & Alignment & $=\overline{0}$ \\
\hline & Autosize & $=0$ \\
\hline & BackColor & $=$ QBColor (3) \\
\hline & Borderstyle & $=0$ \\
\hline & Caption & $=" E^{\prime \prime}$ \\
\hline & DragMode & $=0$ \\
\hline & Enabled & $=-1$ \\
\hline & ForeColor & $=$ QBColor $(0)$ \\
\hline & Height & $=\operatorname{Char}(1)$ \\
\hline & Index & $=4$ \\
\hline & Left & $=\operatorname{Char}(3)$ \\
\hline & MousePointer & $=0$ \\
\hline & TabIndex & $=19$ \\
\hline & $\operatorname{Tag}$ & $=n n$ \\
\hline & Top & $=\operatorname{Char}(12)$ \\
\hline & Visible & $=-1$ \\
\hline & Width & $=\operatorname{Char}(3)$ \\
\hline END & & \\
\hline BEGIN & Label Iblsect & tor_array \\
\hline & Alignment & $=\overline{0}$ \\
\hline & Autosize & $=0$ \\
\hline & BackColor & $=$ QBColor $(3)$ \\
\hline & Borderstyle & $=0$ \\
\hline & Caption & $=" E "$ \\
\hline & DragMode & $=0$ \\
\hline & Enabled & $=-1$ \\
\hline & EoreColor & $=$ QBColor $(0)$ \\
\hline & Height & $=\operatorname{Char}(1)$ \\
\hline & Index & $=5$ \\
\hline & Left & $=\operatorname{Char}(3)$ \\
\hline & MousePointer & $=0$ \\
\hline & TabIndex & $=28$ \\
\hline & $\operatorname{Tag}$ & $=" w$ \\
\hline
\end{tabular}




\begin{tabular}{|c|c|c|}
\hline & $\begin{array}{l}\text { Top } \\
\text { Visible } \\
\text { Width }\end{array}$ & $\begin{array}{l}=\operatorname{Char}(14) \\
=-1 \\
=\operatorname{Char}(3)\end{array}$ \\
\hline \multicolumn{3}{|l|}{ END } \\
\hline BEGIN & $\begin{array}{l}\text { Label lblRsun } \\
\text { Alignment } \\
\text { AutoSize } \\
\text { BackColor } \\
\text { Borderstyle } \\
\text { Caption } \\
\text { DragMode } \\
\text { Enabled } \\
\text { ForeColor } \\
\text { Height } \\
\text { Index } \\
\text { Left } \\
\text { MousePointer } \\
\text { TabIndex } \\
\text { Tag } \\
\text { Top } \\
\text { Visible } \\
\text { Width }\end{array}$ & $\begin{array}{l}\text { marray } \\
=1 \\
=0 \\
=\text { QBColor }(3) \\
=0 \\
=" \operatorname{Sum} 456789 " \\
=0 \\
=-1 \\
=Q B \operatorname{Color}(0) \\
=\text { Char }(1) \\
=0 \\
=\text { Char }(9) \\
=0 \\
=20 \\
=" " \\
=\text { Char (16) } \\
=-1 \\
=\text { Char }(9)\end{array}$ \\
\hline \multicolumn{3}{|l|}{ END } \\
\hline BEGIN & $\begin{array}{l}\text { Label lblRsum } \\
\text { Alignment } \\
\text { AutoSize } \\
\text { BackColor } \\
\text { Borderstyle } \\
\text { Caption } \\
\text { DragMode } \\
\text { Enabled } \\
\text { EoreColor } \\
\text { Height } \\
\text { Index } \\
\text { Left } \\
\text { MousePointer } \\
\text { TabIndex } \\
\text { Tag } \\
\text { Top } \\
\text { Visible } \\
\text { Width }\end{array}$ & $\begin{array}{l}\text { n_array } \\
\equiv 1 \\
=0 \\
=0 \text { QBColor (3) } \\
=0 \\
=\text { "Sum456789" } \\
=0 \\
=-1 \\
=0 B \operatorname{Color}(0) \\
=\text { Char }(1) \\
=1 \\
=\text { Char (19) } \\
=0 \\
=21 \\
=" " \\
=\text { Char (16) } \\
=-1 \\
=\text { Char (9) }\end{array}$ \\
\hline \multicolumn{3}{|l|}{ END } \\
\hline BEGIN & $\begin{array}{l}\text { Label lblRsum } \\
\text { Alignment } \\
\text { AutoSize } \\
\text { BackColor } \\
\text { Borderstyle } \\
\text { Caption } \\
\text { DragMode } \\
\text { Enabled } \\
\text { EoreColor } \\
\text { Height } \\
\text { Index } \\
\text { Left } \\
\text { MousePointer } \\
\text { TabIndex } \\
\text { Tag } \\
\text { Top } \\
\text { Visible } \\
\text { Width }\end{array}$ & $\begin{array}{l}n \text { array } \\
\equiv 1 \\
=0 \\
=\text { QBColor }(3) \\
=0 \\
=" \text { Sum } 456789 " \\
=0 \\
=-1 \\
=0 B \operatorname{Color}(0) \\
=\text { Char }(1) \\
=2 \\
=\text { Char }(29) \\
=0 \\
=22 \\
=" n \\
=\text { Char }(16) \\
=-1 \\
=\text { Char }(9)\end{array}$ \\
\hline \multicolumn{3}{|l|}{ END } \\
\hline DEGIN & $\begin{array}{l}\text { Label lblRsum } \\
\text { Alignment }\end{array}$ & $\begin{array}{l}n \text { array } \\
=1\end{array}$ \\
\hline
\end{tabular}




$$
\begin{array}{ll}
\text { Autosize } & =0 \\
\text { BackColor } & =\text { QBColor (3) } \\
\text { BorderStyle } & =0 \\
\text { Caption } & =" \text { Sum456789" } \\
\text { DragMode } & =0 \\
\text { Enabled } & =-1 \\
\text { EoreColor } & =\text { CBColor (0) } \\
\text { Height } & =\text { Char (1) } \\
\text { Index } & =3 \\
\text { Left } & =\text { Char (39) } \\
\text { MousePointer } & =0 \\
\text { TabIndex } & =23 \\
\text { Tag } & =" \text { "Char (16) } \\
\text { Top } & =-1 \\
\text { Visible } & =\text { Char (9) } \\
\text { Width } &
\end{array}
$$

END

BEGIN HScrollBar hsbRadii

$$
\begin{array}{ll}
\text { Attached } & =0 \\
\text { DragMode } & =0 \\
\text { Enabled } & =-1 \\
\text { Height } & =\text { Char (1) } \\
\text { LargeChange } & =1 \\
\text { Left } & =\text { Char (2) } \\
\text { Max } & =50 \\
\text { Min } & =1 \\
\text { MousePointer } & =0 \\
\text { SmallChange } & =1 \\
\text { TabIndex } & =29 \\
\text { Tabstop } & =-1 \\
\text { Tag } & =" 1 \\
\text { Top } & =\text { Char (17) } \\
\text { Value } & =1 \\
\text { Visible } & =-1 \\
\text { Width } & =\text { Char (60) }
\end{array}
$$

END

BEGIN VScrollBar vsbsectors

$$
\begin{array}{ll}
\text { Attached } & =0 \\
\text { DragMode } & =0 \\
\text { Enabled } & =-1 \\
\text { Height } & =\text { Char (16) } \\
\text { LargeChange } & =1 \\
\text { Left } & =\text { Char (62) } \\
\text { Max } & =16 \\
\text { Min } & =1 \\
\text { MousePointer } & =0 \\
\text { SmallChange } & =1 \\
\text { TabIndex } & =30 \\
\text { Tabstop } & =-1 \\
\text { Tag } & =" 1 \\
\text { Top } & =\text { Char (1) } \\
\text { Value } & =1 \\
\text { Visible } & =-1 \\
\text { Width } & =\text { Char (1) }
\end{array}
$$

\section{END}

BEGIN Iabel IbIDistance_array

$$
\begin{array}{ll}
\text { Alignment } & =1 \\
\text { Autosize } & =0 \\
\text { BackColor } & =\text { QBColor }(3) \\
\text { Borderstyle } & =0 \\
\text { Caption } & =" 1000.0000 " \\
\text { DragMode } & =0 \\
\text { Enabled } & =-1
\end{array}
$$




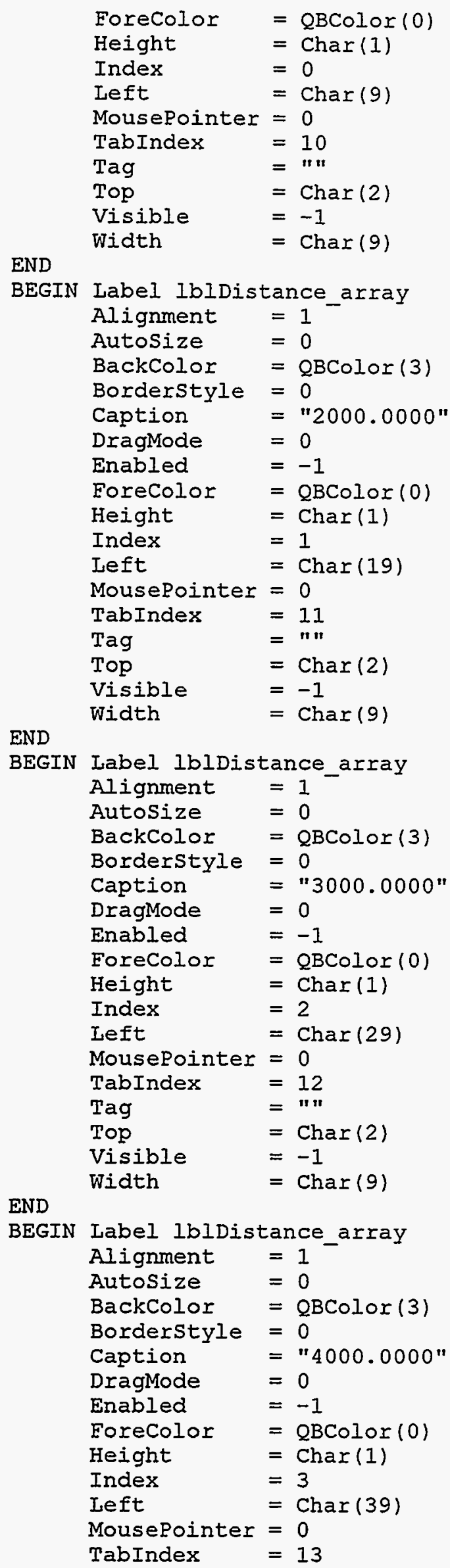




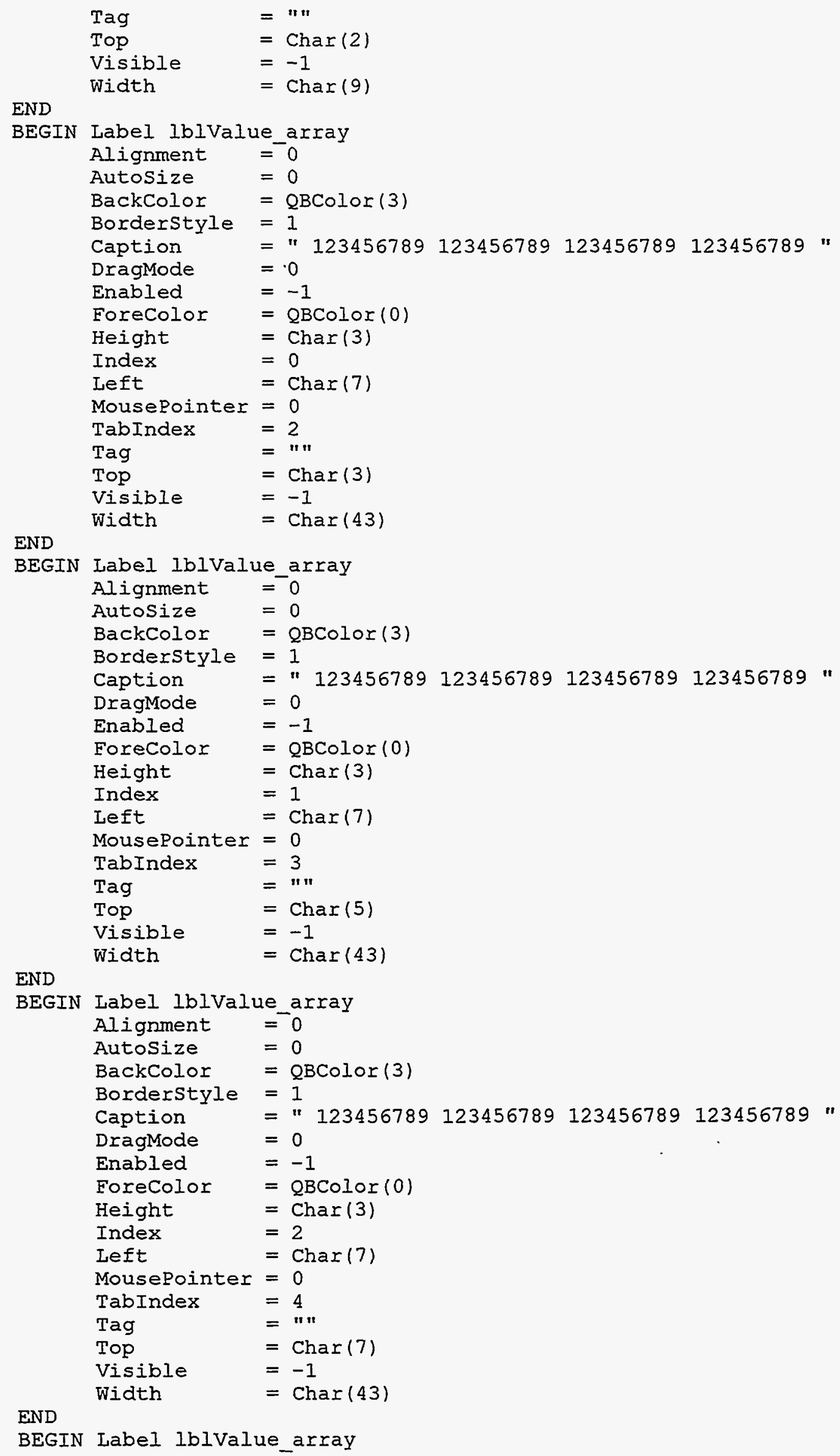




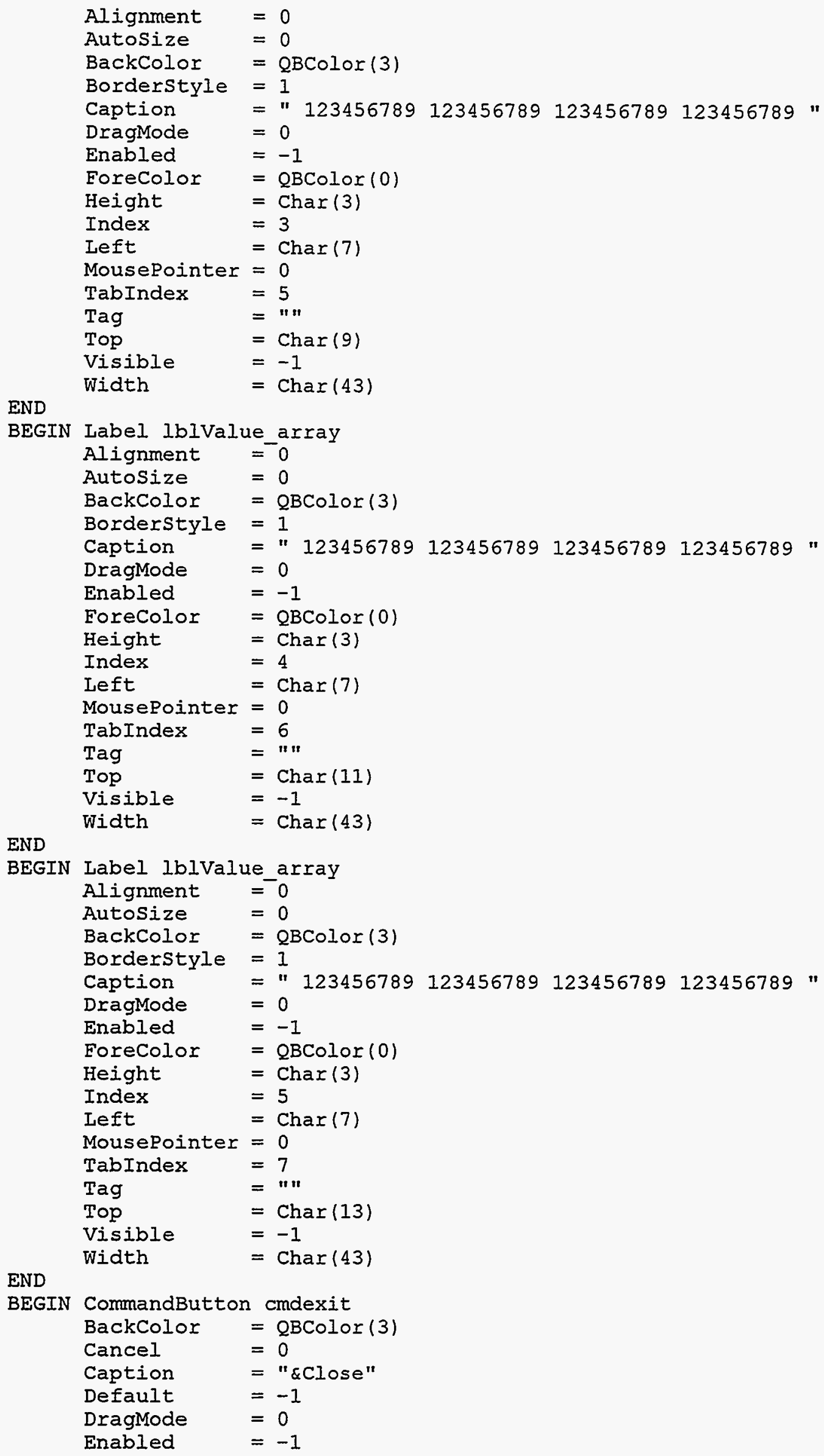




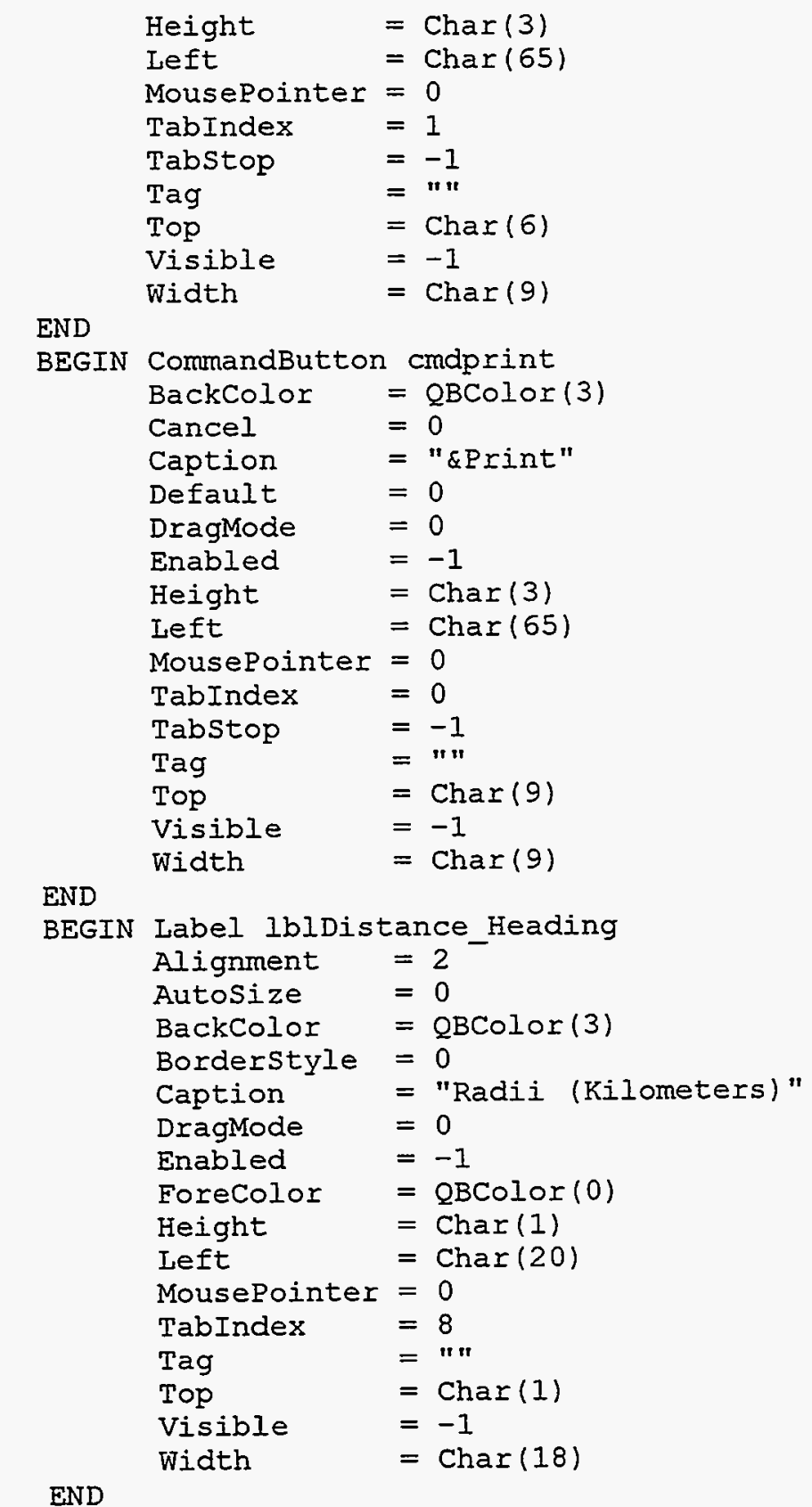

END

OPTION EXPLICIT

'\$INCLUDE: 'secpop90.bi'

'This is the actual table form for table 1 . See rptable_1 for more

info.

SUB cmaExit_Click ()

'Remove table 1 from memory.

UNLOAD frmTable_1

END SUB

sub cmdPrint_Click () 
'This routine prints the displayed population table.

dim Forecolor as integer, Backcolor as integer

dim Copies as integer, Cancel as integer

dim quote as string, comma as string

dim blank as string, degree as string

dim pages as integer, radius as integer

dim first_radius as integer, last_radius as integer

dim $i$ as integer, $j$ as integer, $k$ as integer

$\operatorname{dim} l$ as integer, $m$ as integer, $n$ as integer

dim sum as long

'Set quote and comma strings to null if MACCS input file format is

'selected or to '"' and ',' if comma separated variable (CSV) format

'is selected.

if (frmsetup.optmaccs.Value) then

quote $=" "$ "

comma $=" "$

blank = " "

elseif (frmsetup.optcsV.Value) then

quote $=" " n$

comma $=", "$

blank = ""

else

quote $=" n$

comma $=" "$

end if

blank = " "

'Set degree character.

degree $=\operatorname{chr} \$(248)$

'Set colors for Dialog box

BackColor $=$ WHITE

ForeColor $=$ BLACK

'Call the standard print dialog.

call FilePrint(Copies, ForeColor, BackColor, Cancel)

'If cancel is not selected by user in the printer dialogue box,

'then proceed.

if (not Cancel) then

'Enable local error checking.

on local error goto tablel_printer_error

'If the user is printing to a printer adjust the margins so that

'there are no extra line feeds after the lines that have 80

'columns otherwise prepend the output path to the printer

'target.

if ( printer.PrintTarget $=$ "IPT1:" ) or

(printer.PrintTarget $=$ "LPT2:") or

(printer.PrintTarget $=$ "LPT3:")) thēn

width printer.PrintTarget, 81 
else

printer.PrintTarget = frmsetup.txtoutput_path.text + "l" + printer.PrintTarget

end if

'Determine the number of "pages" that need to be printed per 'copy. A single page is considered to be 16 directions and a 'sum row long, by at most 6 radii or 5 radii and a sum column.

pages $=$ (number of radii +1$) \backslash 6$

if(( (number_of_radii +1$) \bmod 6)<0$ ) then

end if

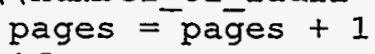

'Execute loop once for each copy requested by the user.

for $i=1$ to Copies step 1

'Print the first line.

printer.print quote;

printer.print "SECPOP90 V2.3 ";

if (table type $=1$ ) then

else

printer.print "Population Data";

printer.print "Cumulative Population Data";

end if

printer.print quote;

printer.print

'Print the second line.

printer.print quote;

printer.print "Date:";

printer.print quote; comma; blank;

printer.print quote;

printer.print format\$ (now, "mm/dd/yYYY");

printer.print quote; comma; blank; blank; blank;

printer.print quote;

printer.print "Time:";

printer.print quote; comma; blank;

printer.print quote:

printer.print formats (now, "hh:mm:ss");

printer.print quote;

printer.print

'Print the third line.

printer.print quote;

printer.print "Site Name:";

printer.print quote; comma; blank;

printer.print quote;

printer.print

ltrims (rtrims (ErmSite_data.txtsite_name.Text)) ;

printer.print quote;

printer.print

'Print the fourth line.

printer.print quote; 


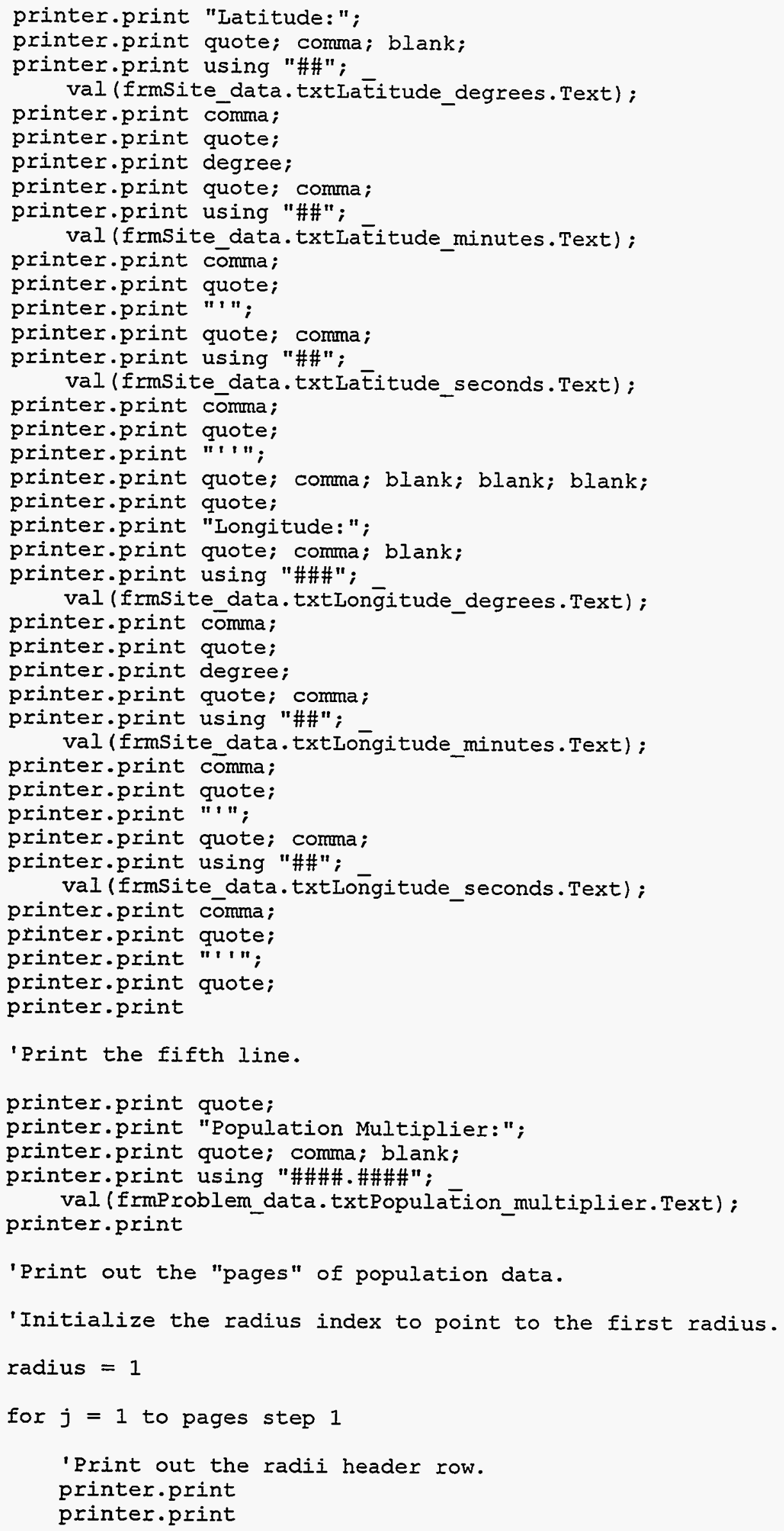


printer.print quote;

printer.print "Radii(";

if (frmproblem Data.optKilometers.value) then

else printer.print " $\mathrm{km}$ )";

end if

printer.print "mi)";

printer.print quote; comma;

'Print out the radii, setting the loop min and $\max$ to

'the radii to be printed for this page. Note: the final

'radii column is the sum column.

first radius $=$ radius

if ( (rädius +5$)<$ (number_of_radii +1$)$ ) then

else last_radius $=$ radius $+{ }^{-5}$

end if

last radius $=$ number of radii +1

for $k$ = first radius to last radius step 1

if $(k<>$ (number_of radi $\bar{i}+1)$ ) then printer.print using "\#\#\#\#\#.\#\#"; radial distance $(k)$;

if ( $\mathrm{k}<>$ last_radius) then

end if printer.print comma;

else

printer.print " "; blank;

printer.print quote;

printer.print "Sum";

end if

printer.print quote;

next $k$

printer.print

printer.print

'Print out the direction and the (cumulative)

'population values. The last column is the sum of the 'population values.

for $k=1$ to number_of_segments step 1

printer.print quote $\overline{\text {; }}$

printer.print using "\ $\backslash$ "; directions (k);

printer.print quote; comma;

for $I$ = first radius to last radius step 1

if $(1<>$ Tnumber of radi $\bar{i}+1)$ ) then

if (table type $\equiv 1$ ) then

printereprint using "\#\#\#\#\#\#\#\#"; -

else sector_population $(k, 1)$;

sum $=0$

for $m=1$ to 1 step 1

sum $=$ sum + sector_population $(k, m)$

next $m$

printer.print using "\#\#\#\#\#\#\#\#"; sum;

end if

if ( $I<>$ last radius) then printer.print comma;

else end if

sum $=0$ 


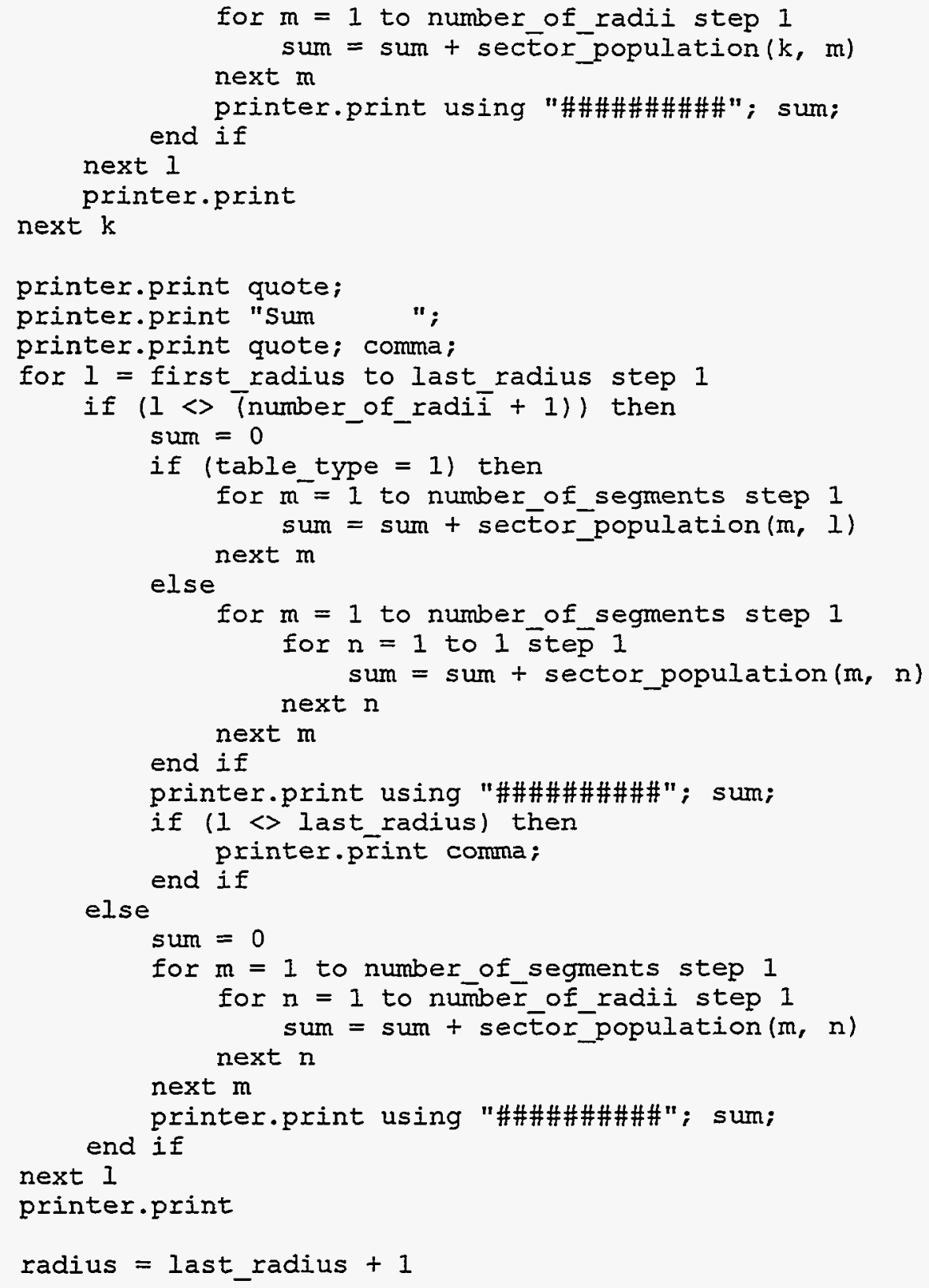


end sub

SUB Form_Load ()

'Perform first call to paint table to initialize the table.

UNLOAD frmDisclaimer

$x$ position $=1$

Y_position $=1$

CÁLI paint_table

END SUB

SUB hsbRadii_Change ()

$\mathrm{x}$ position $=$ hsbRadii.value

CÁLI paint_table

END SUB

SUB vsbSectors_Change ()

$y$ position = vsbSectors.value

CÁLI paint_table

END SUB 


\section{OPTION EXPLICIT}

'\$INCIUDE: 'secpop90.bi'

'Various general purpose utilities:

'1. Print stuff to a non-Form screen.

'2. Print stuff to the printer.

'3. Verify the user's input in one of several forms.

'4. Copy from one file to another.

SUB parse_path_and_file (caption AS STRING, path_name AS STRING, file_name AS STRING)

'This routine removes the path from a file name. This is necessary when 'passing a default filename to a file dialog box.

DIM temp AS STRING

DIM modified location AS INTEGER

DIM slash_foūnd AS INTEGER, slash_location AS INTEGER

'Remove the modified specifier from the file name if necessary.

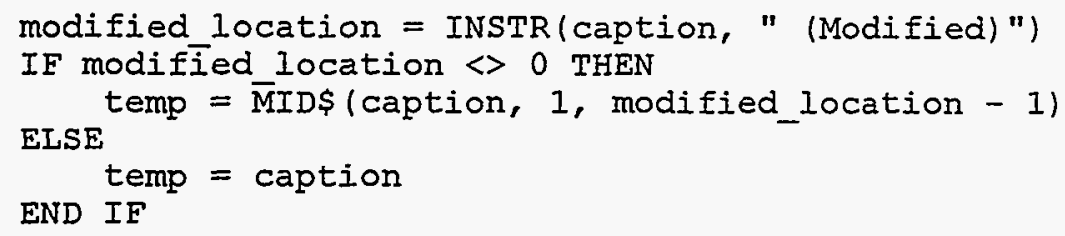

'Search backward from the end of the string to the first slash,

'and record the position of the first slash.

slash_found = FALSE

slash_location $=\operatorname{LEN}($ temp)

WHILE ((NOT slash_found) AND (slash_location >0))

IF MIDS(temp, slash location, $1 \bar{l}=$ " $\backslash$ " THEN

ELSE slash found $=T \bar{R} U E$

END IF

slash_location $=$ slash_location -1

WEND

'If a slash was found, break the name into two parts - path and file.

IF slash_found = TRUE THEN

path_name $=$ MIDS(temp, 1, slash_location - 1)

file_name $=$ MID\$(temp, slash_location + 1)

'Otherwise, return the unchanged filename.

ELSE

END IF

path_name $="$ "

file name $=$ temp

END SUB

SUB print_text (text AS STRING, text_width AS INTEGER, left_margin AS INTEGER)

'This routine prints formatted text strings in a non-form environment. 
DIM next line AS STRING, character AS STRING

DIM character_location AS INTEGER, character found AS INTEGER

'Execute loop while the lenght of text is greater

'than the user defined size.

WHIIE (IEN(text) > text_width)

'Put next string of length user has requested into next line.

next_line = LEET\$ (text, text_width)

charāter found = FALSE

character_location = text width

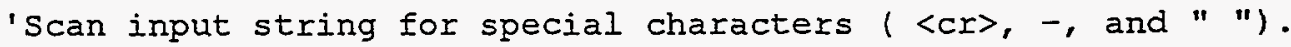

WHILE ((NOT character found) AND (character location > 0)) character = MIDȘ (ñext line, character löcation, 1)

THEN IE ((character = " ") OR (character = "-") OR (character $=\operatorname{CHR}(13))$ )

'Process special characters found in string.

IF character found THEN

PRINTER. $\bar{P}$ RINT TAB (left margin); LEFTŞ (next_line, character_location -

1):

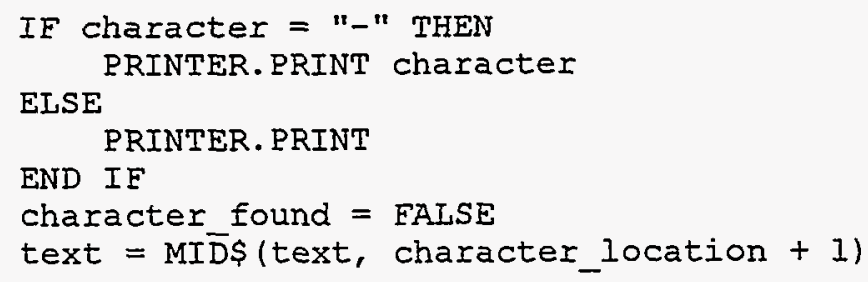




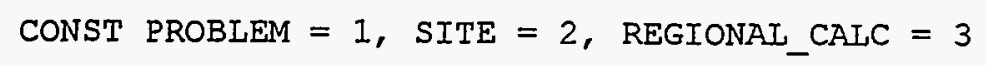

'Decide which form is calling, analyze the input on that form,

'display a message if necessary, and return a value to the calling

'function.

verify_input $=0$

SELECT CASE which_form

\section{CASE PROBLEM}

'Enable local error checking for the new problem form to deal 'with disk related errors check for the file then reset local

'error checking.

ON LOCAJ ERROR GOTO problem_bad_file_name

filenum = FREEFILE

OPEN frmProblem_Data.txtSite_Eile_Name.text FOR INPUT AS \#filenum CLOSE filenum

ON IOCAI ERROR GOTO 0

IF VAI(frmProblem_Data.txtPopulation_multiplier.text) $<=0$ THEN

MSGBOX "Error: Population multiplier must greater than 0 . "

verify_input $=-1$

THEN

ELSEIF VAI (frmProblem_Data.txtPopulation_multiplier.text) > 9999.9999

MSGBOX "Error: Population multiplier must be less than $10000 "$ verify_input $=-1$

ELSEIF (radial_distance (1) $<=0$ ) THEN

MSGBOX "Error: Radial distance 1 must be greater than 0." verify_input $=-2$

ELSEIF (radial distance $(2)<=0$ ) THEN

MSGBOX "Error: Radial distance 2 must be greater than $0 . "$ verify_input $=-3$

ELSEIF (VAI (frmProblem_Data.IblNumber_of_regions.caption) < 2 OR VAL (frmProblem_Data.IblNumber_of_régions.caption) $>\overline{9} 9$ ) THEN

MSGBOX "Invalid Economic region settings" verify_input $=-4$

ELSE

$$
\begin{aligned}
& \text { 'Everything's } 0 . K \\
& \text { verify_input }=1
\end{aligned}
$$

END IF

CASE SITE

IF (VAL (frmsite Data.txtLatitude Degrees.text) > 47) OR

(VAL (frmsite Data.txtlatitude Degrees.text) $<24$ ) OR

(VAL (frmsite Data.txtLongitude Degrees.text) >172) OR

(VAI (frmsite_Data.txtLongitude_Degrees.text) < 67) THEN 
MSGBOX "Warning: The Site that you have specified is outside of the continental United States. Data near the site may not be available." verify_input $=-1$

EISE

'Everything's O.K.

verify_input $=1$

END IF

case regional_calc

if ( (val (frmMake_a_circle.txtradii.text) < 1) or

(val (frmMake_a_circle.txtradii.text) >170)) then

msgbox

"Radii of Circles value must be greater than or" + $\operatorname{chr} \$(13)+\operatorname{chr} \$(10)+$

"equal to 1 mile and less than or equal to $170 "+$ $\operatorname{chr}(13)+\operatorname{chr} \$(10)+$

"miles.", MB_OK, "Error"

verify_input $=0$

elseif ((val (frmMake_a_circle.txtspacing.text) < 1) or -

(val (frmMake_a_cīrcile.txtspacing.text) > 340)) then

msgbox

"Loñgitudinal spacing of Circles value must" + $\operatorname{chr} \$(13)+\operatorname{chr} \$(10)+$

"be greater than or equal to 1 mile and less" + $\operatorname{chr} \$(13)+\operatorname{chr} \$(10)+$

"than or equal to 340 miles.", MB_OK, "Error"

verify input $=-1$

elseif ( (val (frmMake_a_circle.txtThreshold.text) < 1) or

(val (frmMake_a_círcle.txtThreshold.text) > 20000)) then

msgbox

"Population Density Threshold value must be" + $\operatorname{chr} \$(13)+\operatorname{chr} \$(10)+$

"greater than or equal to 1 person per square" + $\operatorname{chr} \$(13)+\operatorname{chr} \$(10)+$

"mile and less than or equal to 20000 persons" + $\operatorname{chr} \$(13)+\operatorname{chr} \$(10)+$

"per square mile.", MB_oK, "Error"

verify_input $=-2$

elseif (frmMake_a_circle.txtInput_map.text $=$ " ) then

msgbox

"Input Map Name missing. Please enter a valid" + $\operatorname{chr} \$(13)+\operatorname{chr} \$(10)+$

"filename.", MB OK, "Error"

verify_input $=-3$

else

'Enable local error checking for regional calc form 


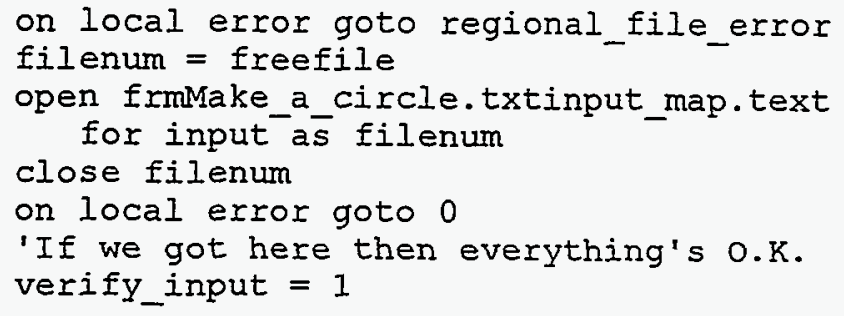

end if

END SELECT

EXIT FUNCTION

problem_bad_file_name:

MSGBOX "Error: Unable to open site file - check file name." verify_input $=0$

EXIT FUNNCTION

regional_file_error:

msgbox

"Inp̄ut Map Name invalid. Please enter a valid" + $\operatorname{chr} \$(13)+\operatorname{chr} \$(10)+$

"filename.", MB OK, "Ē̌rror"

verify_input $=-3$

exit function

END FUNCTION

sub copy_file(input_filename as string, output_filename as string)

$\operatorname{dim} i$ as long

dim my byte as string * 1

dim input_filenumber as integer, output filenumber as integer

on local error goto error_return

input filenumber $=$ freefile

open input_filename for binary access read as \#input_filenumber

output_filenumber $=$ freefile

'Truncate output file to 0 length if it already existed.

open output filename for output access write as \#output_filenumber close (outpuE_filenumber)

open output_filename for binary access write as \#output_filenumber

for $i=1$ to lof (input filenumber) step 1

get \#input_filenumber, , my_byte

next $i$

put \#output_filenumber, , my_byte

close (input filenumber)

close (output_filenumber)

normal_return: 
exit sub

error_return:

msgbox error\$ (err)

exit sub

end sub 
FILENAME: SECPOP90.BI

'\$INCIUDE: 'types.bi'

'\$INCLUDE: 'declare.bi'

'\$INCLUDE: 'const.bi'

'\$INCLUDE: 'common.bi'

'\$INCLUDE: 'forms.bi'

FILENAME：TYPES.BI

TYPE economic_data

region area AS IONG

region frmfrc AS SINGLE

region_dpE AS SINGLE

region_asfp AS SINGLE

region vfrm AS SINGLE

region_vnfrm AS SINGLE

END TYPE

FIIENAME: FORMS.BI

' \$FORM frmcalculate

' \$FORM frmCmnDlg

'\$FORM frmDensity

' \$FORM frmDisclaimer

' \$FORM frmMain

'\$FORM frmMake_a_Circle

' \$FORM frmoutpüt

' \$FORM frmProblem Data

'\$FORM frmRegion

' \$FORM frmsetup

' \$FORM frmsite data

'\$FORM frmTable_.1 


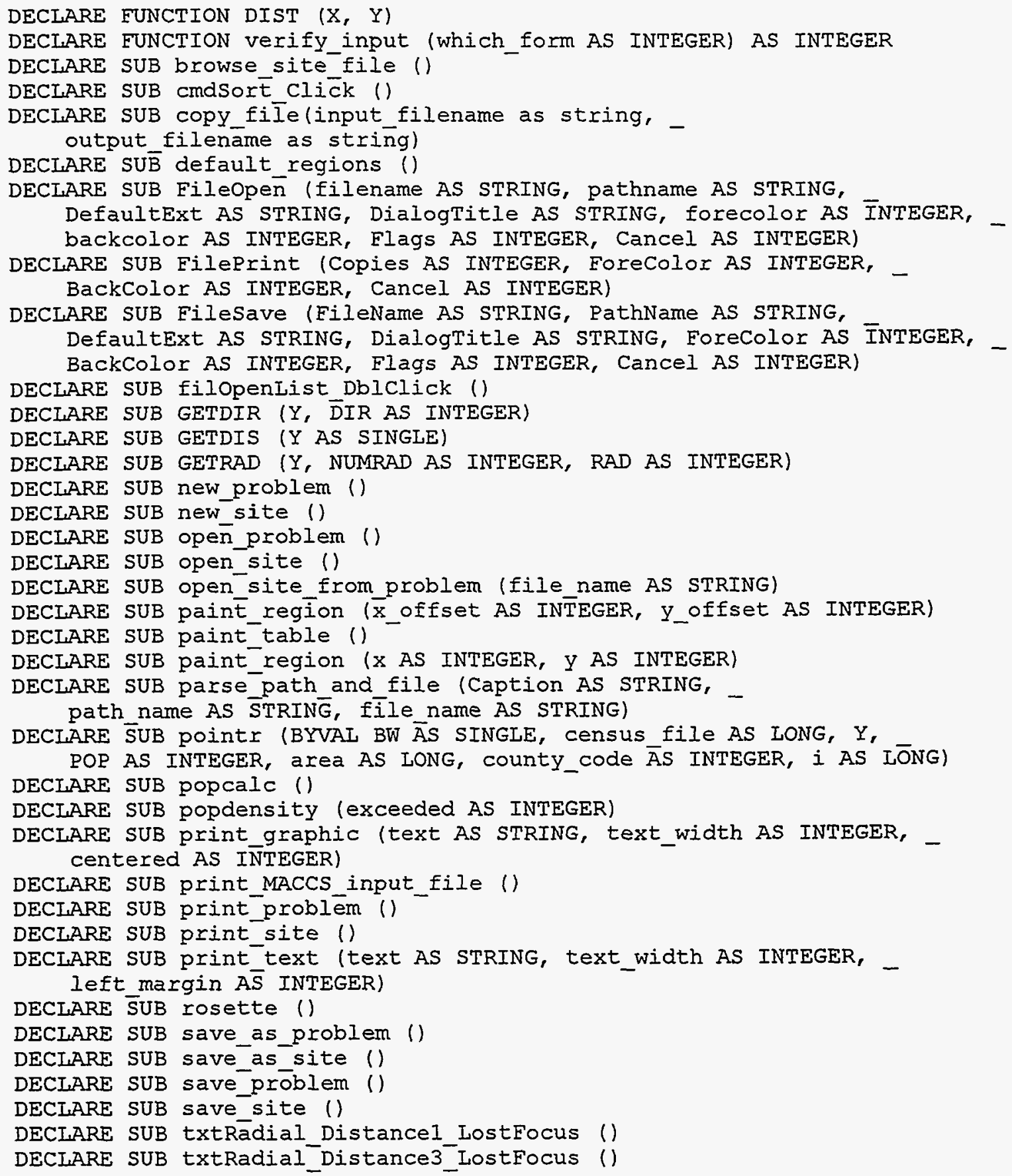


'Microsoft Visual Basic constants. Unused (by SECPOP90) constants are 'commented out to save memory.

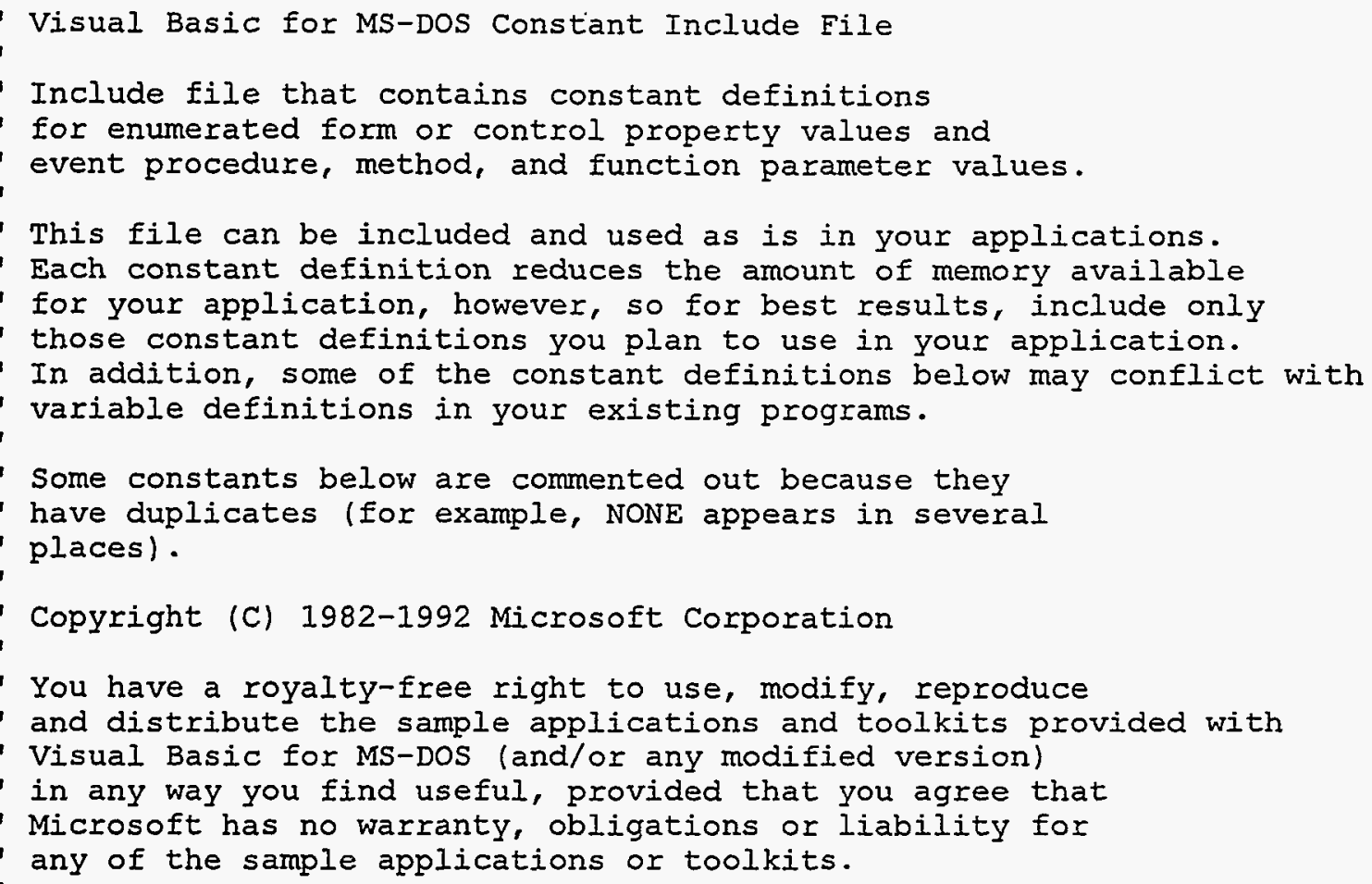

Event parameters

1 - - - -

' Button and Shift (KeyDown, KeyUp, MouseDown, MouseMove, MouseUp)

' CONST SHIET MASK $=1$

' CONST CTRL MAASK $=2$

' CONST AIT MASK $=4$

' CONST LEET BUTTON $=1$

'CONST RIGHT_BUTTON $=2$

- KeyCode (KeyDown, KeyUp)

' CONST KEY BACK $=8$

' CONST KEY TAB $=9$

' CONST KEY CLEAR $=12$

CONST KEY $\bar{R} E T U R N=13$

' CONST KEY SHIET $=16$

'CONST KEY_CONTROL $=17$

'CONST KEY MENU $=18$

' CONST KEY_PAUSE $=19$

- Enter key

- Alt key 


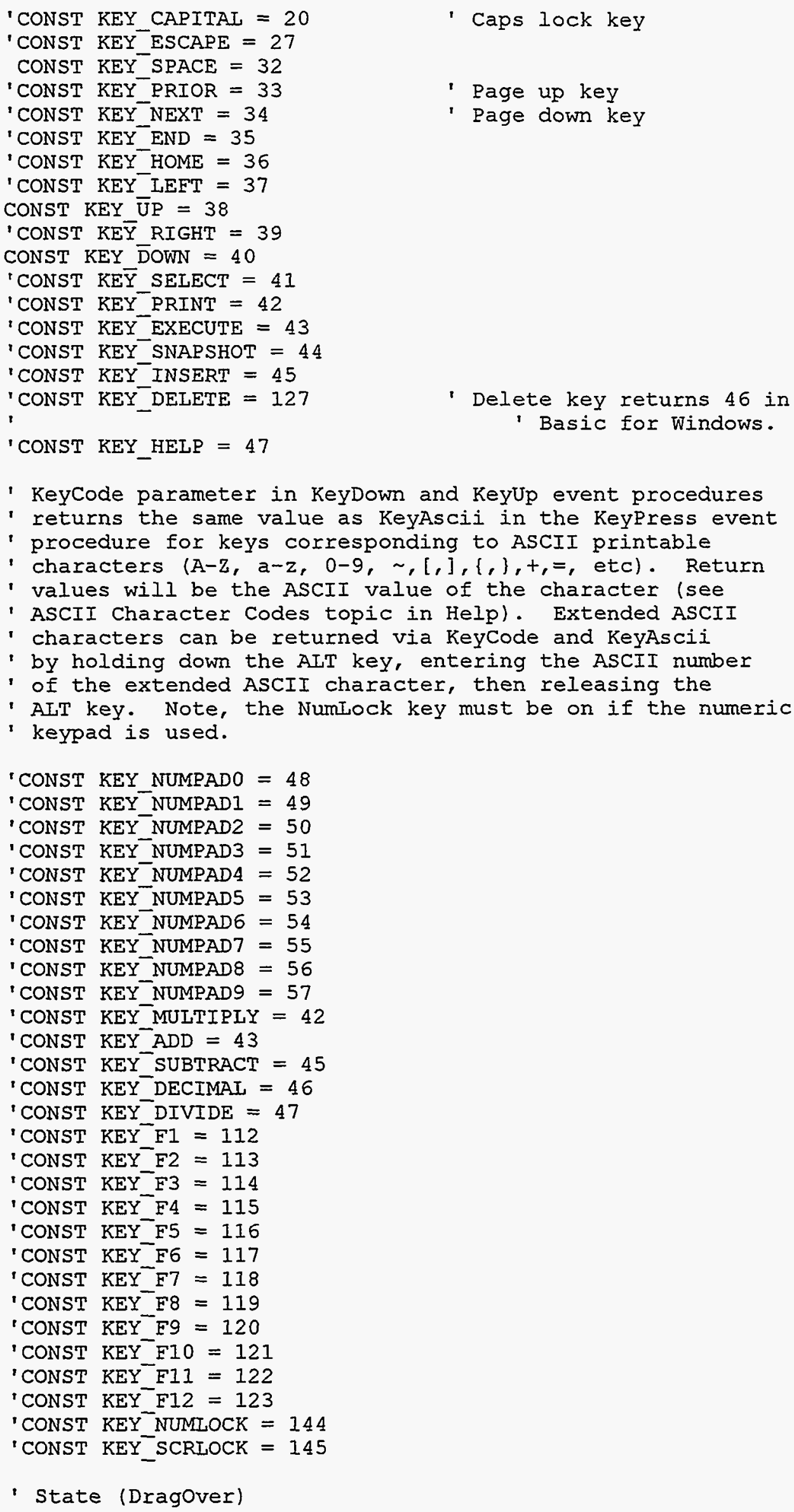

- State (Dragover) 


\begin{abstract}
' CONST ENTER $=0$
' CONST LEAVE $=1$

' CONST OVER $=2$
\end{abstract}

- Eunction parameters

- MSGBOX parameters

CONST MB OK $=0$

' CONST $M \bar{B}$ OKCANCEL $=1$

- OK button only

' CONST MB ABORTRETRYIGNORE $=2$

- $O K$ and Cancel buttons

- Abort, Retry, and Ignore buttons

CONST MB Y ESNOCANCEL $=3$

CONST MB YESNO $=4$

' CONST MB__RETRYCANCEL $=5$

- Yes, No, and Cancel buttons

- Yes and No buttons

- Retry and Cancel buttons

CONST MB DEFBUTTON $1=0$

CONST MB DEFBUTTON2 = 256

CONST $M \bar{B}$ DEFBUTTON3 $=512$

- First button is default

- Second button is default

- Third button is default

- MSGBOX return values

' CONST IDOK $=1$

CONST IDCANCEL $=2$

CONST IDABORT $=3$

' CONST IDRETRY $=4$

' CONST IDIGNORE $=5$

CONST IDYES $=6$

CONST IDNO $=7$

- OK button pressed

- Cancel button pressed

- Abort button pressed

- Retry button pressed

- Ignore button pressed

- Yes button pressed

- No button pressed

- Method parameters

' - - - --- - - - - - -

- DRAG (controls)

' CONST CANCEL DRAG $=0$

' CONST BEGIN $\bar{D} R A G=1$

CONST END_D $\overline{R A G}=2$

' SHOW (form)

CONST MODELESS $=0$

CONST MODAL $=1$

'

- Property values

, -

- Alignment (label)

' CONST LEET JUSTIFY $=0$

' CONST RIGHT JUSTIFY = I

' CONST CENTER $=2$

- 0 - Ieft Justify

- 1 - Right Justify

- 2 - Center

- BackColor, Eorecolor (form, controls)

CONST BLACK $=0$

CONST BLUE $=1$

' CONST GREEN $=2$

CONST CYAN $=3$

' CONST RED $=4$

CONST MAGENTA $=5$

CONST BROWN $=6$

CONST WHITE $=7$

' CONST GRAY $=8$ 


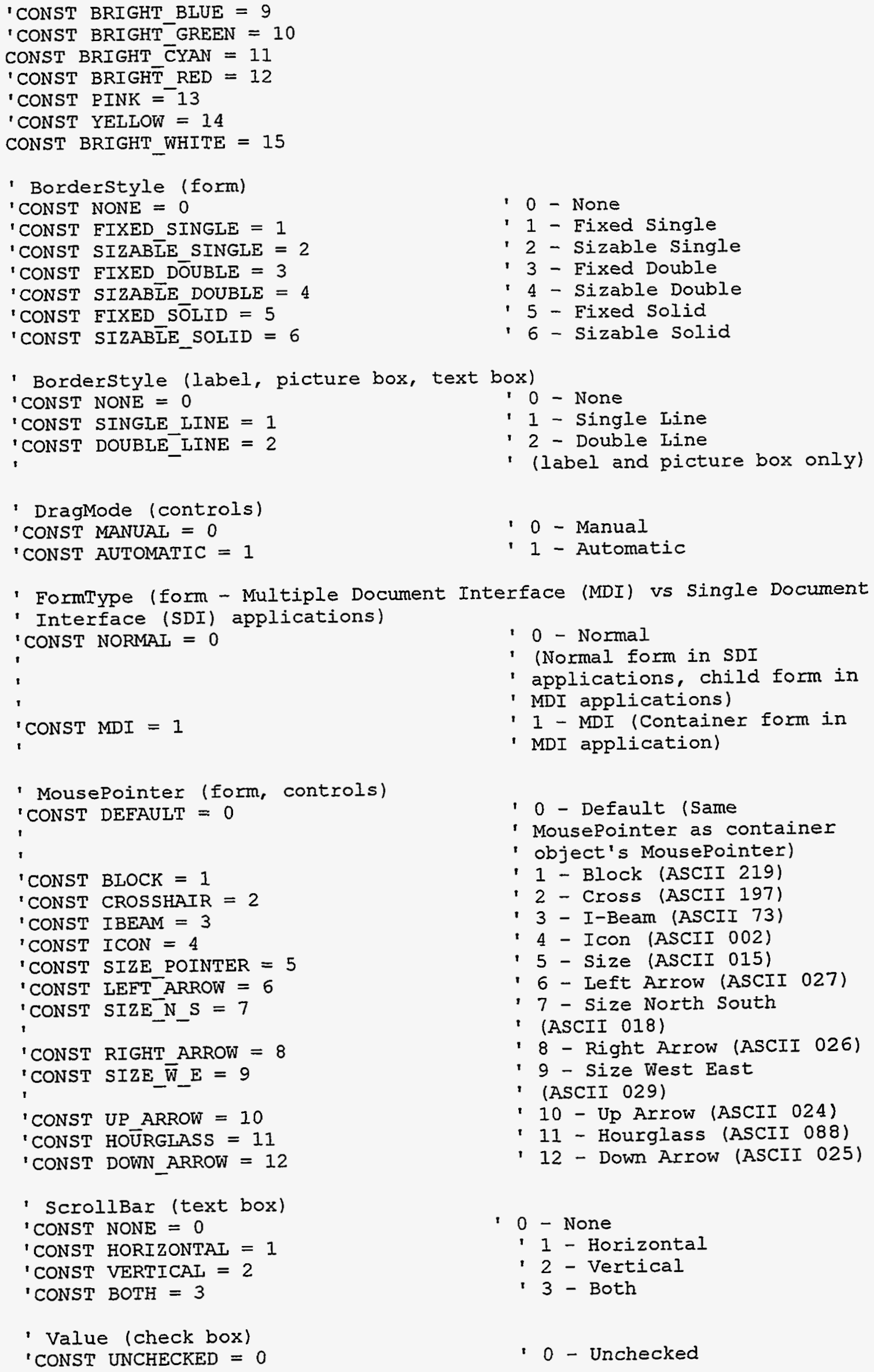


COMMON SHARED leave calculation AS INTEGER COMMON SHARED rec A $\bar{S}$ STRING * record length COMMON SHARED XION AS SINGLE, ylat AS SINGLE COMMON SHARED ipop AS INTEGER, area AS LONG, county_code AS INTEGER COMMON SHARED idir AS INTEGER, irad AS INTEGER, ibȳe AS LONG

COMMON SHARED number of radii AS INTEGER, numrad AS INTEGER COMMON SHARED Populatioñmultiplier AS SINGIE, scale AS SINGLE COMMON SHARED radial distance() AS SINGLE, raddis() AS SINGLE COMMON SHARED regionāl_radii() AS SINGLE COMMON SHARED population() AS long COMMON SHARED population threshold() AS long COMMON SHARED SIOn AS SIN̄GLE, slat AS SINGLE COMMON SHARED dismaX AS SINGLE, sitidS COMMON SHARED dpdlat AS SINGLE, dpdlon AS SINGLE, sdpdla AS SINGIE COMMON SHARED bNdrYW AS SINGLE, bndrYe AS SINGLE COMMON SHARED bndrYn AS SINGLE, bndrys AS SINGLE COMMON SHARED aminla AS SINGLE, dgtord AS SINGLE

COMMON SHARED region_index() AS INTEGER, number_econ_regions AS INTEGER COMMON SHARED econ_dāta() AS economic_data COMMON SHARED tablē type AS INTEGER COMMON SHARED $x$ _position AS INTEGER, y_position AS INTEGER

COMMON SHARED sector_population() AS LONG

COMMON SHARED sector_area() AS IONG

COMMON SHARED sector_frclnd() AS SINGLE

COMMON SHARED county_state() AS STRING * 2

COMMON SHARED county_name() AS STRING * max_county_name_length

COMMON SHARED county frclnd() AS SINGLE, county_frmfrc( $)$ AS SINGLE

COMMON SHARED county_dpf() AS SINGLE, county_asfp() AS SINGLE

COMMON SHARED county_vfrm() AS SINGLE, county_vnfrm() AS SINGLE

COMMON SHARED directions() AS STRING

COMMON SHARED layer_number() AS INTEGER

COMMON SHARED data flag() AS INTEGER

COMMON SHARED display flag() AS INTEGER

COMMON SHARED active_layer() AS INTEGER

COMMON SHARED layer_ñme() AS STRING

COMMON SHARED pts_cöIOr() AS INTEGER

COMMON SHARED pts_type() AS INTEGER

COMMON SHARED pts_size() AS INTEGER

COMMON SHARED pts mode() AS INTEGER

COMMON SHARED Iines color() AS INTEGER

COMMON SHARED lines_type() AS INTEGER

COMMON SHARED lines_size() AS INTEGER

COMMON SHARED lines_mode() AS INTEGER

COMMON SHARED pOIYg_color() AS INTEGER

COMMON SHARED polyg_type() AS INTEGER

COMMON SHARED polyg_size() AS INTEGER

COMMON SHARED polyg mode() AS INTEGER

COMMON SHARED text_color() AS INTEGER

COMMON SHARED text_type() AS INTEGER

COMMON SHARED text_size() AS INTEGER

COMMON SHARED text_mode() AS INTEGER

COMMON SHARED radial_area() AS SINGIE 
FIIENAME: SECPOP90.MAK

SECPOP 90. BAS

CALCCODE. BAS

CMNDLG. BAS

OUTPCODE.BAS

PROBCODE. BAS

ROSETTE. BAS

RPTABLE1. BAS

SITECODE. BAS

UTILITY.BAS

CALCFORM. ERM

CIRCLE. FRM

CMNDLGE. FRM

DENSFORM. FRM

DISCLAIM. FRM

MAIN . FRM

OUTPEORM. FRM

PROBFORM. FRM

REGION . ERM

SETUP . FRM

SITEFORM. ERM

TABLE1 . FRM 


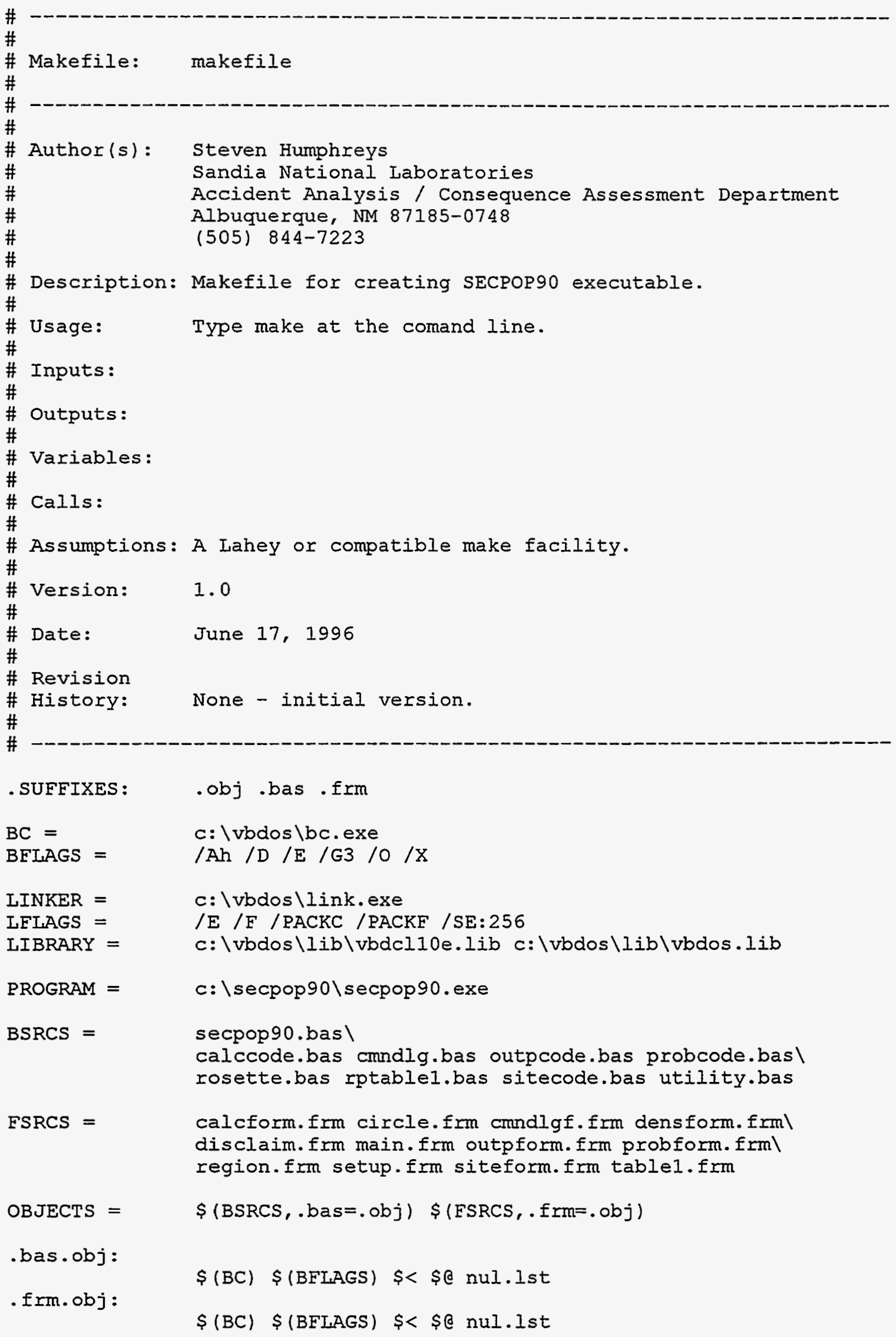




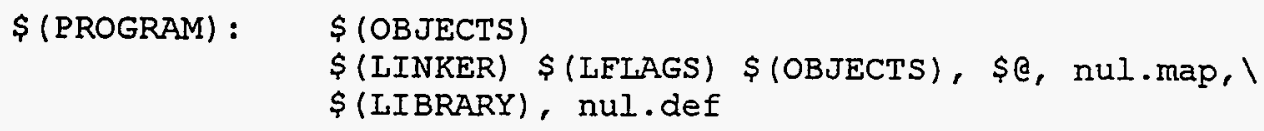


FIIENAME: MP_SETUP.BAS

CHDIR "mapplan"

SHELL "setup.exe"

CHDIR ".."

RUN "secpop90"

FIIENAME: MP_SETUP.MPR

MP_SETUP.BAS

FIIENAME: RUN_MPPR.BAS

CHDIR "mapplan"

SHELI "mppr.exe . map_out.spc"

CHDIR ".."

RUN "secpop90"

FIIFFAMME: RUN_MPPR.MAK

RUN_MPPR. BAS 


\begin{tabular}{|c|c|}
\hline $\begin{array}{l}\text { U.S. NUCLEAR REGULATORY COMMISSION } \\
\text { BIBLIOGRAPHIC DATA SHEET } \\
\text { (See instructions on the reverse) }\end{array}$ & $\begin{array}{l}\text { 1. REPORT NUMBER } \\
\text { (Assigned by NRC. Add Vol., Supo.. Rev.. } \\
\text { and Addendum Numbers, if any.) } \\
\text { NUREG/CR-6525 }\end{array}$ \\
\hline \multirow{2}{*}{\multicolumn{2}{|c|}{ 2. TITLE AND SUBTITLE }} \\
\hline & \\
\hline \multirow[t]{4}{*}{ Economic Estimation Program } & DATE REPORT PUBLISHED \\
\hline & MONTH \\
\hline & September $\quad 1997$ \\
\hline & $\begin{array}{l}\text { 4. FIN OR GRANT NUMBER } \\
\text { J6024 }\end{array}$ \\
\hline \multirow{3}{*}{$\begin{array}{l}\text { 5. AUTHOR(S) } \\
\text { Steven L. Humphreys } \\
\text { Judy A. Rollstin b. } \\
\text { John N. Ridgely C }\end{array}$} & ס. TYPE OF REPORT \\
\hline & Technical \\
\hline & $\begin{array}{l}\text { 7. PERIOD COVERED IInelusive Dates' } \\
\text { March } 1993 \text { to } \\
\text { August } 1997\end{array}$ \\
\hline
\end{tabular}

8. PERFORMING ORGANIZATION - NAME AND ADDRESS III NRC, provide Division, Office or Region, U.S. Nuelear Regulatory Commission, and mailhng aodress: if eontracter, providr a name and mailing address'

P.0. Box 5800 Mail Stop $0748 \quad 8500$ Menaul Blvd., NE

Albuquerque, NM 87185-0748

$C_{\text {Division of Systems Technology }}$

Office of Nuclear Regulatory Research

U.S. Nuclear Regulatory Commission

Mail Stop TIOK8

Washington, D.C. 20555-0001

9. SPONSORING ORGANIZATION - NAME AND ADDRESS II NRC. zype "Same as above": if contractor, provide NRC Oivision, Office or Region. U.S. Nuclear Regulatory Commission. and mailing address.)

Division of Systems Technology

Office of Nuclear Regulatory Research

U.S. Nuclear Regulatory Commission

Washington, D.C. 20555-0001

10. SUPPLEMENTARY NOTES

J. N. Ridgely, NRC Project Manager

11. AESTRACT (200 words or less)

In $1973 \mathrm{Mr}$. W. Athey of the Environmental Protection Agency wrote a computer program called SECPOP which calculated population estimates. Since that time, two things have changed which suggested the need for updating the original program--more recent population censuses and the widespread use of personal computers (PCs). The revised computer program uses the 1990 and 1992 Population Census information and runs on current PCs as "SECPOP90".

SECPOP90 consists of two parts: site and regional. The site analysis provides population and economic data estimates for any location within the continental United States. Siting analysis is relatively fast running. The regional portion assesses site availability for different siting policy decisions; i.e., the impact of available sites given specific population density criteria within the continental United States. Regional analysis is slow.

This report compares the SECPOP90 population estimates and the nuclear power reactor licensee-provided information. Although the source, and therefore, the accuracy of the licensee information is unknown, this comparison suggests SECPOP90 makes reasonable estimates.

12. KEY WORDS'DESCR!PTORS IList words or phrases that will assist researchers in locating the report. I

Reactor siting, population, economics, computer code, severe reactor accidents

\begin{tabular}{l} 
13. AVAILABILITY STATEMENT \\
Unlimited \\
\hline 34. SECURITY CLASSIFICATION \\
\hline (This PageI \\
Unclassified \\
(This ReDort \\
UnClas ified \\
\hline 15. NUMBER OF PAGES \\
\hline 16. PRICE \\
\hline
\end{tabular}

